\title{
FY2016 ILAW Glass Corrosion Testing with the Single-Pass Flow-Through Method
}

\section{April 2017}

JJ Neeway

RM Asmussen

BP Parruzot

EA Cordova
BD Williams

II Leavy

JR Stephenson

EM Mcelroy 


\title{
DISCLAIMER
}

This report was prepared as an account of work sponsored by an agency of the United States Government. Neither the United States Government nor any agency thereof, nor Battelle Memorial Institute, nor any of their employees, makes any warranty, express or implied, or assumes any legal liability or responsibility for the accuracy, completeness, or usefulness of any information, apparatus, product, or process disclosed, or represents that its use would not infringe privately owned rights. Reference herein to any specific commercial product, process, or service by trade name, trademark, manufacturer, or otherwise does not necessarily constitute or imply its endorsement, recommendation, or favoring by the United States Government or any agency thereof, or Battelle Memorial Institute. The views and opinions of authors expressed herein do not necessarily state or reflect those of the United States Government or any agency thereof.

\author{
PACIFIC NORTHWEST NATIONAL LABORATORY \\ operated by \\ BATTELLE \\ for the \\ UNITED STATES DEPARTMENT OF ENERGY \\ under Contract DE-AC05-76RL01830 \\ Printed in the United States of America \\ Available to DOE and DOE contractors from the \\ Office of Scientific and Technical Information, \\ P.O. Box 62, Oak Ridge, TN 37831-0062; \\ ph: (865) 576-8401 \\ fax: $(865) 576-5728$ \\ email: reports@adonis.osti.gov \\ Available to the public from the National Technical Information Service \\ 5301 Shawnee Rd., Alexandria, VA 22312 \\ ph: (800) 553-NTIS (6847) \\ email: orders@ntis.gov <http://www.ntis.gov/about/form.aspx> \\ Online ordering: http://www.ntis.gov
}




\title{
FY2016 ILAW Glass Corrosion Testing with the Single-Pass Flow- Through Method
}

\author{
JJ Neeway \\ RM Asmussen \\ BD Williams \\ BP Parruzot \\ II Leavy \\ EA Cordova \\ JR Stephenson \\ EM Mcelroy
}

April 2017

Prepared for

the U.S. Department of Energy

under Contract DE-AC05-76RL01830

Pacific Northwest National Laboratory

Richland, Washington 99352 


\section{Executive Summary}

The inventory of immobilized low-activity waste (ILAW) produced at the Hanford Tank Waste Treatment and Immobilization Plant (WTP) will be disposed of at the near-surface, on-site Integrated Disposal Facility (IDF). When groundwater comes into contact with the waste form, the glass will corrode and radionuclides will be released into the near-field environment. Because the release of the radionuclides is dependent on the dissolution rate of the glass, it is important that the performance assessment (PA) model accounts for the dissolution rate of the glass as a function of various conditions. To accomplish this, an IDF PA glass dissolution model based on Transition State Theory (TST) can be employed. The model is able to account for changes in temperature, exposed surface area, and $\mathrm{pH}$ of the contacting solution as well as the effect of silicon solution concentrations, specifically the activity of orthosilicic acid $\left(\mathrm{H}_{4} \mathrm{SiO}_{4}\right)$, whose concentration is directly linked to the glass dissolution rate. In addition, the IDF PA model accounts for the ion exchange process. The effect of temperature, $\mathrm{pH}, \mathrm{H}_{4} \mathrm{SiO}_{4}$ activity, and the rate of ion exchange can be parameterized and implemented directly into the PA rate model. The rate model parameters are derived from laboratory tests with the single-pass flow-through (SPFT) method. To date, full sets of rate model parameters have been determined for seven representative ILAW glass compositions, thus additional rate model parameters on a wider range of compositions will supplement the existing body of data for PA maintenance activities. In this report, we present SPFT results on two simulant immobilized low-activity waste (ILAW) glasses developed at the Vitreous State Laboratory at the Catholic University of America (VSL): IDF18-A161 and ORPLG9. The provided data can be used by ILAW glass scientists to further the understanding of ILAW glass behavior, by IDF PA modelers to use the rate model parameters in PA modeling efforts, and by Department of Energy (DOE) contractors and decision makers as they assess the IDF PA program.

Both the IDF18-A161 and ORPLG9 ILAW glass compositions were designed as part of a program to increase waste loading in ILAW glasses beyond the baseline compositions. The IDF18-A161 glass has a moderate alkali content (20.66 wt $\% \mathrm{Na}_{2} \mathrm{O}$ ) and a high target sulfate loading (1.1 wt\%). In contractual durability tests, the glass performed marginally in the Vapor Hydration Test (VHT) and passed the 7-day Product Consistency Test (PCT-A) ${ }^{\mathrm{a}}$. The IDF18-A161 glass was chosen for SPFT testing in order to increase the understanding of glasses that have a somewhat marginal response to VHT and to increase the amount of corrosion data available on glasses with intermediate, but higher than baseline glasses, sodium concentrations. The ORPLG9 glass has a high alkali content $\left(21.00 \mathrm{wt} \% \mathrm{Na}_{2} \mathrm{O}\right.$ and $\left.5.76 \mathrm{wt} \% \mathrm{~K}_{2} \mathrm{O}\right)$ and a low $\mathrm{SO}_{3}$ content $(0.20 \mathrm{wt} \%)$. ORPLG9 showed a high VHT alteration rate though it was still within acceptable contract limits. The ORPLG9 glass was selected for SPFT testing due to its high alkali concentration and because it allows for comparison of SPFT results with another high-alkali glass, ORPLB2, the only high sodium waste-loaded glass where a complete set of SPFT testing has been performed.

Several SPFT tests with IDF18-A161 and ORPLG9 were performed in a variety of chemically-controlled conditions and test temperatures. A full set of rate model parameters was obtained for IDF18-A161 and those values are provided in Table ES.1. The activation energy $\left(E_{a}\right)$, rate constant $\left(\mathrm{k}_{0}\right)$, and ion-exchange rate $\left(\mathrm{r}_{\mathrm{IEX}}\right)$ are all in a similar range as previously tested, lower waste-loaded ILAW glasses. However,

\footnotetext{
${ }^{\mathrm{a}}$ The contract durability requirements are for VHT $200^{\circ} \mathrm{C}$ alteration rate $<50 \mathrm{~g} \mathrm{~m}^{-2} \mathrm{~d}^{-1}$ and PCT normalized releases of $\mathrm{Na}, \mathrm{B}$, and $\mathrm{Si}<2 \mathrm{~g} \mathrm{~m}^{-2} \mathrm{~d}^{-1}$ according to DOE contract DE-AC27-01RV14136, US DOE, Office of River Protection, Richland, WA, as amended.
} 
performance of an uncertainty analysis on $\mathrm{r}_{\text {IEX }}$ shows that the uncertainty range of this parameter nearly bounds the entire range of previously-determined $\mathrm{r}_{\text {IEX }}$ values. Further studies on ion exchange in ILAW glasses should be able to refine this term. The $\mathrm{pH}$-dependent term $(\eta)$ is the lowest of any measured values and the significance of this remains unclear. In addition, the pseudo-equilibrium constant $\left(\mathrm{K}_{\mathrm{g}}\right)$ for this glass is relatively small indicating that the glass approaches a pseudo-saturation at much lower $\mathrm{H}_{4} \mathrm{SiO}_{4}$ activity than previously tested glasses.

The dissolution rate of the ORPLG9 was two to ten times lower than the dissolution rate of IDF18-A161 in similar test conditions. We were able to determine a set of dilute condition rate model parameters for ORPLG9, specifically $E_{a}, k_{0}$ and $\eta$. The values, which are listed in Table ES.1, for $E_{a}$ and $k_{0}$ were higher than any other glass composition determined thus far. The value of $\eta$ was within the range of previouslytested ILAW glass compositions. Again, the significance of this remains unclear and more testing of ILAW glasses would be necessary to be able to determine a correlation between the glass composition and the rate model parameters. On the other hand, the glass exhibited unexpected behavior as the $\mathrm{H}_{4} \mathrm{SiO}_{4}$ activity increased. Contrary to other ILAW glass compositions, the release rate of both boron and sodium was invariant with increasing solution $\mathrm{H}_{4} \mathrm{SiO}_{4}$ activity, though the release rate of aluminum decreased as the solution $\mathrm{H}_{4} \mathrm{SiO}_{4}$ activity increased. This behavior suggests that the glass contains both a B-rich and $\mathrm{Si}$ rich amorphous phase. The B-rich phase would not be affected by the $\mathrm{H}_{4} \mathrm{SiO}_{4}$ activity in solution while the Si-rich phase, where the aluminum presumably partitions, would be affected by the $\mathrm{H}_{4} \mathrm{SiO}_{4}$ activity. Upon further analysis of the pristine ORPLG9 glass solid, the presence of droplet in matrix phase separation was identified using Atomic Force Microscopy (AFM) imaging. At this point in time, we do not know the composition of the two immiscible phases. Because the boron and sodium release from the ORPLG9 glass are unresponsive to changes in $\mathrm{H}_{4} \mathrm{SiO}_{4}$ activity, we recommend using a $\log \mathrm{K}_{\mathrm{g}}$ value of 100 to reproduce the SPFT data. This will result in the glass corroding at a nominally forward rate as $\mathrm{H}_{4} \mathrm{SiO}_{4}$ activity increases, which is a conservative estimate of glass corrosion behavior. Further efforts could utilize Product Consistency Test (PCT-B) data to refine the value of $\mathrm{K}_{\mathrm{g}}$ to a more realistic value. Despite the presence of multiple amorphous phases, we were still able to determine a value of $\mathrm{r}_{\mathrm{IEX}}$ for ORPLG9, even if the significance of this value is convoluted because sodium and boron are being released from two separate phases. If the corrosion behavior of the two different glass compositions associated with the immiscibility curve is desired, each glass composition could be made separately and then tested separately to test the behavior of both glass compositions. This is not, however, within the scope of this report. Lastly, secondary phases should be further studied, through both experiment and geochemical modeling, in order be able to better predict the behavior of ORPLG9 and other ILAW glasses in the IDF repository environment. 
Table ES.1. Summary of Rate Model Parameters Obtained for Select ILAW Glasses at $15^{\circ} \mathrm{C}^{(\mathrm{a})}$

\begin{tabular}{ccccccc}
\hline & $k_{0}\left(\mathrm{~g} \mathrm{~m}^{-2} \mathrm{~d}^{-1}\right)$ & $\mathrm{K}_{\mathrm{g}}(\mathrm{T})$ & $\eta$ & $\mathrm{E}_{\mathrm{a}}\left(\mathrm{kJ} \mathrm{mol}^{-1}\right)$ & $\mathrm{r}_{\mathrm{IEX}}$ & Reference \\
\hline LD6-5412 & $9.7 \times 10^{6}$ & $1.14 \times 10^{-4}$ & $0.40 \pm 0.03$ & $74.8 \pm 1.0$ & $1.74 \times 10^{-11}$ & McGrail et al. 1997 \\
LAWABP1 & $3.4 \times 10^{6}$ & $4.90 \times 10^{-4}$ & $0.35 \pm 0.03$ & $68 \pm 3.0$ & $3.4 \times 10^{-11}$ & McGrail et al. 2001 \\
LAWA44 & $\begin{array}{c}1.3 \times 10^{4} \\
\left(\mathrm{R}^{2}=0.78\right)\end{array}$ & $\begin{array}{c}1.87 \times 10^{-3} \\
\left(\mathrm{R}^{2}=0.95\right)\end{array}$ & $0.49 \pm 0.08$ & $60 \pm 7$ & $5.3 \times 10^{-11}$ & Pierce et al. 2004 \\
LAWB45 & $\begin{array}{c}1.6 \times 10^{4} \\
\left(\mathrm{R}^{2}=0.90\right)\end{array}$ & $\begin{array}{c}1.79 \times 10^{-3} \\
\left(\mathrm{R}^{2}=0.78\right)\end{array}$ & $0.34 \pm 0.03$ & $53 \pm 3$ & $0.0 \times 10^{0}$ & Pierce et al. 2004 \\
LAWC22 & $\begin{array}{c}1.0 \times 10^{5} \\
\left(\mathrm{R}^{2}=0.98\right)\end{array}$ & $\begin{array}{c}1.80 \times 10^{-3} \\
\left(\mathrm{R}^{2}=0.94\right)\end{array}$ & $0.42 \pm 0.02$ & $64 \pm 2$ & $1.2 \times 10^{-10}$ & Pierce et al. 2004 \\
ORPLB2 & $4.5 \times 10^{4}$ & $1.70 \times 10^{-3}$ & $0.49 \pm 0.05$ & $58 \pm 3$ & $3.4 \times 10^{-10}$ & Papathanassiu et al. 2011 \\
IDF18-A161 & $\begin{array}{c}5.9 \times 10^{6} \\
\left(\mathrm{R}^{2}=0.89\right)\end{array}$ & $\begin{array}{c}2.3 \times 10^{-4} \\
\left(\mathrm{R}^{2}=0.98\right)\end{array}$ & $0.28 \pm 0.06$ & $61 \pm 6$ & $7.6 \times 10^{-11}$ & This report \\
ORPLG9 & $2.5 \times 10^{8}$ & $\log \mathrm{K}_{\mathrm{g}}=100^{(\mathrm{b})}$ & $0.36 \pm 0.07$ & $81 \pm 7$ & $5.3 \times 10^{-11(b)}$ & This report \\
\hline
\end{tabular}

(a) See Freedman et al. (2015) for additional details on rate model parameters presented in the table.

(b) See text for information concerning the $\mathrm{K}_{\mathrm{g}}$ and $\mathrm{r}_{\mathrm{IEX}}$ values of ORPLG9. 


\section{Acknowledgments}

This work was completed as part of the ILAW Glass Testing for Disposal at IDF project. Support for this project came through Washington River Protection Solutions, Inc. (WRPS). We thank Joe Ryan (PNNL) for his technical review of the document, Guzel Tartakovsky (PNNL) for review of the calculated values used in this report, Richard Daniel and Philip Schonewill (PNNL) for their assistance in SPFT data sorting, and Nathan Canfield (PNNL) for solids analysis. The authors also wish to acknowledge Dave Swanberg (WRPS), Steven Kelly (WRPS), Elvie Brown (WRPS), and Gary L. Smith (PNNL) for programmatic guidance, direction, and support. 


\section{Acronyms and Abbreviations}

\begin{tabular}{|c|c|}
\hline aq & aqueous \\
\hline AFM & Atomic Force Microscopy \\
\hline ASME & American Society of Mechanical Engineers \\
\hline ASTM & $\begin{array}{l}\text { ASTM International (consensus standards organization, West Conshohocken, PA } \\
\text { ( } \underline{\text { www.astm.org)) }}\end{array}$ \\
\hline BET & Brunauer-Emmett-Teller \\
\hline CCC & Canister Centerline Cooled \\
\hline DOE & U.S. Department of Energy \\
\hline EDS & Energy-Dispersive Spectroscopy \\
\hline EQL & estimated quantification limit \\
\hline FIO & for information only \\
\hline FY & $\begin{array}{l}\text { fiscal year (October } 1 \text { through September 30, e.g., FY } 2016 \text { ends on } \\
\text { September 30, 2016) }\end{array}$ \\
\hline $\mathrm{H}_{4} \mathrm{SiO}_{4}(\mathrm{aq})$ & aqueous orthosilicic acid \\
\hline HLW & high-level waste \\
\hline ICP-OES & Inductively Coupled Plasma-Optical Emission Spectroscopy \\
\hline IDF & Integrated Disposal Facility \\
\hline IEX & ion exchange \\
\hline ILAW & immobilized low-activity waste \\
\hline LAW & low-activity waste \\
\hline NBO & non-bridging oxygen \\
\hline ND & not determined \\
\hline NQA-1 & ASME nuclear quality assurance standard \\
\hline ORP & DOE Office of River Protection \\
\hline PA & performance assessment \\
\hline PCT & product consistency test \\
\hline $\mathrm{pH}(\mathrm{RT})$ & $\mathrm{pH}$ at room temperature \\
\hline PNNL & Pacific Northwest National Laboratory \\
\hline PTFE & polytetrafluoroethylene \\
\hline PUF & pressurized unsaturated flow (test) \\
\hline $\mathrm{q} / \mathrm{S}$ & solution-flow-rate-to-surface-area ratio \\
\hline QA & quality assurance \\
\hline $\mathrm{R} \& \mathrm{D}$ & research and development \\
\hline RCRA & Resource Conservation and Recovery Act \\
\hline SEM & scanning electron microscopy \\
\hline $\mathrm{SiO}_{2}(\mathrm{am})$ & amorphous silica \\
\hline
\end{tabular}


SPI

SPFT

$\mathrm{S} / \mathrm{V}$

TEM

TRIS

TST

VHT

VSL

WTP

WRPS

WWFTP

XRD
Structure Probe, Inc.

single-pass flow-through test

surface area to volume ratio

Transmission Electron Microscopy

tris(hydroxymethyl)aminomethane

transition state theory

vapor hydration test

Vitreous State Laboratory at The Catholic University of America

Hanford Tank Waste Treatment and Immobilization Plant

Washington River Protection Solutions

WRPS Waste Form Testing Program

$\mathrm{X}$-ray diffraction 


\section{Contents}

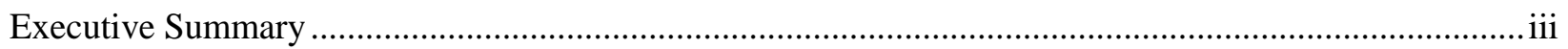

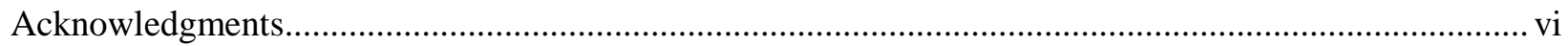

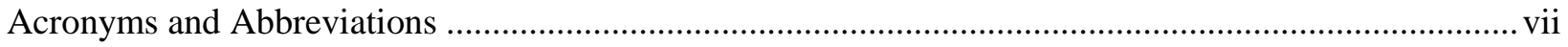

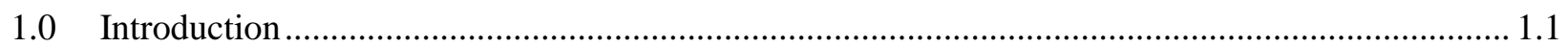

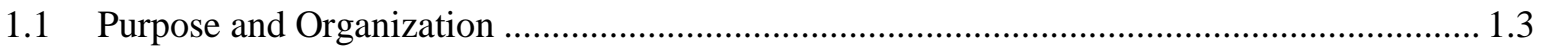

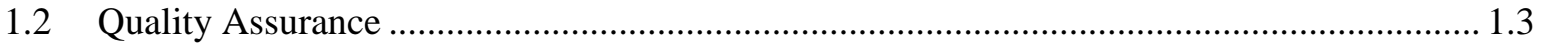

2.0 Glass Selection, Kinetic Rate Equation, and SPFT Test Method ................................................ 2.1

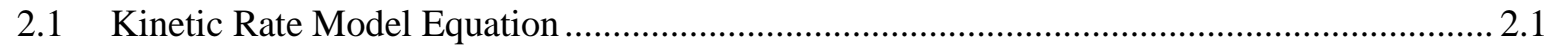

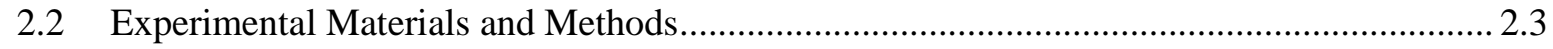

2.2.1 Glass Preparation and Characterization ............................................................. 2.3

2.2.2 Single-Pass Flow-Through Apparatus................................................................. 2.12

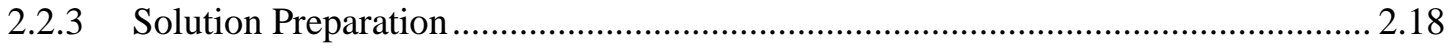

2.2.4 Dissolution Rate and Error Calculations ........................................................... 2.18

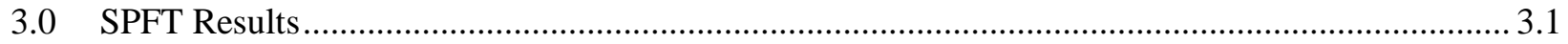

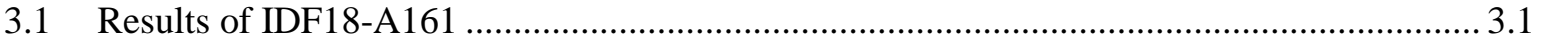

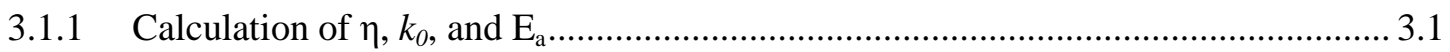

3.1.2 Effect of Silicon Concentration.............................................................................. 3.3

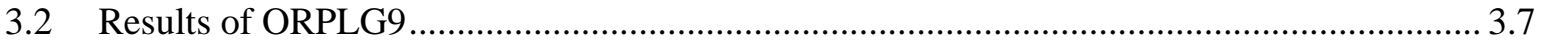

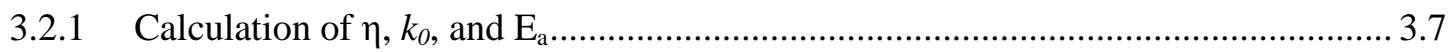

3.2.2 Effect of Silicon Concentration........................................................................... 3.9

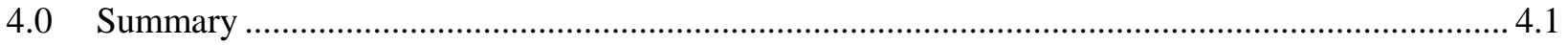

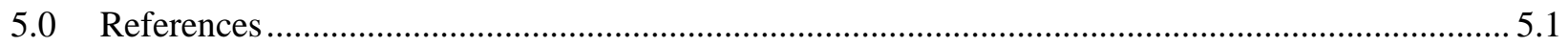

Appendix A - Steady-State Single-Pass Flow-Through Results .......................................................... A.1

Appendix B - Steady-State Single-Pass Flow-Through Results .............................................................1 


\section{Figures}

Figure 2.1. Optical photographs of ORPLG9 CCC (a) powder as received from VSL; (b) two pieces of glass powder polished to 600 grit on one face showing striations; and (c) observation of undissolved $\mathrm{Cr}_{2} \mathrm{O}_{3}$ particles in the glass.

Figure 2.2. XRD pattern of the as-received ORPLG9 CCC glass. The peaks in the diffraction pattern are due to the addition of a fluorite $\left(\mathrm{CaF}_{2}\right)$ standard........................................................ 2.6

Figure 2.3. Backscatter electron SEM (left) and EDS (right) of the as-received ORPLG9 CCC glass. Spectrum 1 is of a bright particle and Spectrum 3 is of the bulk glass.

Figure 2.4. Secondary electron SEM of $\mathrm{Cr}$ inclusions in the as-received ORPLG9 CCC glass

Figure 2.5. Photographs of (a) quenched IDF18-A161; (b) bottom view of CCC heat treatment of IDF18-A161; and (c) side view of CCC heat treatment of IDF18-A161.

Figure 2.6. XRD pattern of different preparations of IDF18-A161 glass. (a) quenched; (b) CCC heat treatment with crystals; and (c) CCC heat treatment where crystals have been physically separated from the glass.

Figure 2.7. Secondary electron SEM of the surface of a CCC heat treated sample of IDF18-A161 at two different magnifications. Images are from glass particles where large crystals have been physically separated.

Figure 2.8. Schematic of the single-pass flow-through (SPFT) apparatus for determining reaction rates in continuous flow solution

Figure 3.1. Normalized $\log _{10}$ boron release rates as a function of $\mathrm{pH}$ and temperature for IDF18A161. Measured test temperatures are provided in Appendix A. Errors are given as $2 \sigma$ from Eq. 2-10......

Figure 3.2. Normalized release rate with respect to $\mathrm{B}, \mathrm{Na}$, and $\mathrm{Al}$ as a function of $\mathrm{SiO}_{2}$ activity at 23, 40, 70, and $90^{\circ} \mathrm{C}$ for IDF18-A161. Measured test temperatures are provided in Appendix A. Data points with squares are from experiments used to calculate $\mathrm{K}_{\mathrm{g}}$ while data points with circles are experiments that are considered oversaturated with respect to $\mathrm{K}_{\mathrm{g}}$. The $\mathrm{SiO}_{2}$ activity is calculated from the measured $\mathrm{Si}$ in the effluent samples. Normalized dissolution rate errors (vertical error bars) are presented as $2 \sigma . a\left[\mathrm{H}_{4} \mathrm{SiO}_{4}\right]$ errors (horizontal error bars) are less than $10 \%$ and are not presented in the graph.

Figure 3.3. $\ln \mathrm{K}_{\mathrm{g}}$ vs. inverse temperature for IDF18-A161. The circles are from experimental data while the triangle is the $\ln \mathrm{K}_{\mathrm{g}}$ value calculated at $15^{\circ} \mathrm{C}$. The value of the triangle is the estimated value of $\mathrm{K}_{\mathrm{g}}$ at $15^{\circ} \mathrm{C}$.

Figure 3.4. The natural logarithm of the ion exchange rate as a function of inverse temperature for IDF18-A161

Figure 3.5. Normalized $\log _{10}$ boron release rates as a function of $\mathrm{pH}$ and temperature for ORPLG9. Measured test temperatures are provided in Appendix A. Errors are given as $2 \sigma$ from Eq. 2-10.

Figure 3.6. Normalized release rate with respect to $\mathrm{B}, \mathrm{Na}$, and $\mathrm{Al}$ as a function of $\mathrm{SiO}_{2}$ activity at 23, 40, 70, and $90^{\circ} \mathrm{C}$ for ORPLG9. Measured test temperatures are provided in Appendix A. Data points with squares are from experiments used to calculate $\mathrm{K}_{\mathrm{g}}$ while data points with circles are experiments that are considered oversaturated with respect to $\mathrm{K}_{\mathrm{g}}$. The $\mathrm{SiO}_{2}$ activity is calculated from the measured $\mathrm{Si}$ in the effluent samples. Normalized dissolution rate errors (vertical error bars) are presented as $2 \sigma . a\left[\mathrm{H}_{4} \mathrm{SiO}_{4}\right]$ errors (horizontal error bars) are less than $10 \%$ and are not presented in the graph. 
Figure 3.7. Normalized release rate with respect to boron as a function of $\mathrm{SiO}_{2}$ activity for ORPLG9. This is Figure 6.13 of Papathanassiu et al. (2011).

Figure 3.8. TEM (bright field) of an ORPLG9 glass particle rotated 90 degrees counter clockwise from the EDS maps

Figure 3.9. AFM images of chemically-mechanically polished samples of ORPLG9. The images on the top are topological maps of the sample at two different length scales. The image on the bottom is a phase map of the sample at a $2.0-\mu \mathrm{m}$ scale. The scales to the right of (a) and (b) are height in $\mathrm{nm}$. The scale to the right of (c) is the phase shift relative to the average phase in the horizontal direction.

Figure 3.10. AFM images of chemically-mechanically polished samples of IDF18-A161. The images on the top are topological maps and the images on the bottom are a phase maps at two different scales. The scales to the right of (a) and (b) are height in $\mathrm{nm}$. The scale to the right of (c) and (d) is the phase shift relative to the average phase in the horizontal direction.

Figure 3.11. The natural logarithm of $r_{\text {IEX }}$ as a function of inverse temperature for ORPLG9......

\section{Tables}

Table 2.1. Target composition (mass\%) of selected ILAW glasses......................................................... 2.4

Table 2.2. Centerline canister cooling (CCC) profile for ILAW glass. ..................................................... 2.5

Table 2.3. Target and measured composition of CCC samples of ORPLG9 and IDF18-A161. ............ 2.11

Table 2.4. List of SPFT conditions used for ORPLG9. Measured test temperatures are provided in Appendix A

Table 2.5. List of SPFT conditions used for IDF18-A161. Measured test temperatures are provided in Appendix A.

Table 2.6. Composition of solutions used in SPFT experiments where the effect of $\mathrm{pH}$ was investigated (Pierce et al. 2004).

Table 3.1. Forward dissolution rates for IDF18-A161. Boron release rates are used in Figure 3.1.

Table 3.2. Forward dissolution rates for ORPLG9. Boron release rates are used in Figure 3.5. Data are compared with data obtained in Papathanassiu et al. (2011) in similar conditions............. 3.8

Table 4.1. Summary of Rate Model Parameters Obtained for Select ILAW Glasses at $15^{\circ} \mathrm{C}^{(\mathrm{a})}$ 4.3 


\subsection{Introduction}

The Hanford Tank Waste Treatment and Immobilization Plant (WTP) will produce immobilized low activity waste (ILAW) glass as part of the clean-up mission at the U.S. Department of Energy (DOE) Hanford Site. This inventory of ILAW glass will be disposed of on-site at the Integrated Disposal Facility (IDF). The IDF was constructed in 2006 and is a shallow landfill design; however, it has not accepted any waste to date (Yabusaki et al. 2015). With its near-surface location, waste forms placed into the IDF will eventually come into contact with groundwater, which will initiate corrosion and release radionuclides into the near-field environment. The release rate of the radionuclides is dependent on the dissolution rate of the glass. The glass dissolution rate is known to be dependent on the glass composition, temperature, exposed surface area, and the $\mathrm{pH}$ of the contacting solution. For disposal at the IDF, only the temperature of $15{ }^{\circ} \mathrm{C}$ is assumed as a constant (McGrail et al. 2003). The contacting solution chemistry and $\mathrm{pH}$ are affected by infiltration rates, interactions with engineered barriers, gas-water equilibria, precipitation of secondary phases, alkali-ion exchange, and dissolution of the glass itself.

Per the radioactive waste management DOE Order 435.1, performance assessments (PA) must be performed. The PA describes the potential long-term impacts on human health and the environment resulting from disposal of wastes at the IDF. In order to asses these risks, predictions of the long-term release of radionuclides and other contaminants of concern from the glass are required.

The engineered system of the IDF contains multiple engineered components including a Resource Conservation and Recovery Act (RCRA) cap, back fill material, ILAW glass, and a leachate collection system (Mann et al. 2003). A reactive-chemical-transport-modelling framework must account for all facets of this system to describe influences of fluid flow and glass-water reactions on the chemistry of pore water contacting waste forms in the IDF. In this modelling approach, the fluid chemistry is coupled with kinetic rate equations developed to calculate the glass dissolution rate dependence on changes in liquid composition as a function of time and space. The kinetic rate equation assumes that (1) Transition State Theory (TST) arguments and assumptions describe dissolution and precipitation rates departing from equilibrium and (2) the displacement from thermodynamic equilibrium principally governs the driving force behind the transformation of unstable to stable silicate materials.

The input parameters to reactive transport codes are derived from laboratory testing. Specifically, singlepass flow-through (SPFT) (ASTM C1662-10) testing is utilized to supply ILAW kinetic rate model input parameters (McGrail et al. 1997, McGrail et al. 1998a, McGrail et al. 2000a, McGrail et al. 2001, Pierce et al. 2004, Papathanassiu et al. 2011). Three additional tests are utilized to obtain information on alteration phases that can form for a certain glass composition; (1) the Pressurized Unsaturated Flow (PUF) test (McGrail et al. 1996), (2) the Product Consistency Test (PCT) (ASTM C1285-14) and (3) the Vapor Hydration Test (VHT) (ASTM C1663-09). A combination of these approaches, amongst others, allows for a discretionary focus on specific components of the glass corrosion process. Further information on the techniques and their application in long-term glass corrosion can be found in McGrail et al. (1998b, 2000b), Neeway et al. (2014), and Ryan and Freedman (2016). This report will present rate model parameter measurements from SPFT testing on two ILAW glasses: IDF2-G9 (ORPLG9) and IDF18-A161.

The glasses selected for testing described in this report can be traced to the first ILAW glass PA in the mid 1990s (Rawlins et al. 1994, Mann et al. 1996). During this timeframe the low-activity waste (LAW) 
was split into three envelopes: A) highest $\mathrm{Na}^{+}: \mathrm{SO}_{4}{ }^{2-}$ ratio based on analysis of Tank AN-105 (Matlack et al. 2006a), B) lowest $\mathrm{Na}^{+}: \mathrm{SO}_{4}{ }^{2-}$ ratio based on analysis of Tank AZ-102 (Matlack et al. 2006a) and C) wastes with elevated levels of organic complexants and intermediate $\mathrm{Na}^{+}: \mathrm{SO}_{4}{ }^{2-}$ ratio based on analysis of Tank AN-102 (Matlack et al. 2006b). In 2003 the LAW envelopes were expanded to seven sub-envelopes (A1, A2, A3, B1, B2, C1, C2) to account for waste compositional differences.

The LAWA161 glass was developed at the Vitreous State Laboratory (VSL) at The Catholic University of America from the LAW Envelope A1 feed-containing glass and was part of a screening process of ILAW glasses at $20 \mathrm{wt} \% \mathrm{Na}_{2} \mathrm{O}$ and higher $\mathrm{Cl}\left(1.17 \mathrm{wt} \%\right.$ ) capable of increased $\mathrm{SO}_{3}$ loadings above the standard $0.19 \mathrm{wt} \%$ (Matlack et al. 2005). In crucible-scale screening tests, LAWA161 was identified, along with LAWA155 and LAWA160, of being capable of incorporating higher levels of $\mathrm{SO}_{3}$. For the crucible-scale tests, LAWA161 demonstrated results that were in between the performance of the three glasses for the PCT, VHT, and K-3 refractory corrosion test. Therefore, it was selected for further melter studies. The LAWA161 glass from the melter testing met all of the ILAW performance requirements (PCT-A B release of $0.672 \mathrm{~g} / \mathrm{m}^{2}$ and 7-day VHT alteration rate of $24.6 \mathrm{~g} / \mathrm{m}^{2} \mathrm{~d}^{-1}$ ) and had a $\mathrm{SO}_{3}$ concentration of $0.50 \mathrm{wt} \%$ (Matlack et al. 2005). In the subsequent melter studies, a $\mathrm{SO}_{3}$ loading of $1.1 \mathrm{wt} \%$ was achieved without secondary phase formation in the LAWA161 glass.

In a second phase of ILAW glass development, designed to expand the LAW glass compositional region toward higher waste loading, the LAWA161 formulation was used as the base formulation to generate IDF18-A161 (Muller and Pegg 2015). The $\mathrm{SO}_{3}$ content of IDF18-A161 was given as $1.1 \mathrm{wt} \%$. A centerline canister cooled (CCC) sample of IDF18-A161 was identified to have the highest VHT (24-day test, $68.0 \mathrm{~g} / \mathrm{m}^{2} / \mathrm{d}$ ) and 7-d PCT-B (value not given) ${ }^{\mathrm{a}}$ response in the series of Phase 2 glasses tested by Muller and Pegg (2015). In order to increase the understanding of glasses that have a somewhat marginal response to VHT, and to increase the amount of corrosion data available on glasses with intermediate sodium concentrations, IDF18-A161 was selected for SPFT testing (Muller and Pegg 2016).

The composition of ORPLG9 was designed in Phase 1 development of ILAW glasses because a noticable knowledge gap existed for increased waste loadings in the glass testing. A set of glasses designated 'ORP' (for the DOE Office of River Protection) were developed with higher waste loadings (Papathanassiu et al. 2011). From this Phase 1 ORP data set, bounding glasses were selected based on VHT and K-3 corrosion testing. From the set, ORPLB2 (IDF1-B2), was selected for a full suite of SPFT testing based on its performance in PCT, VHT, high sodium (25.0 wt\%) and melter testing performance. A second glass, ORPLF7 (IDF3-F7), was chosen for partial SPFT testing due to it having the highest $\mathrm{SO}_{3}$ and lowest $\mathrm{Na}_{2} \mathrm{O}$ concentration of the bounding Phase 1 glasses. Another glass, ORPLG9 (IDF2-G9) with a high $\mathrm{Na}_{2} \mathrm{O}(21.00 \mathrm{wt} \%)+\mathrm{K}_{2} \mathrm{O}(5.76 \mathrm{wt} \%)$ and low $\mathrm{SO}_{3}$ content $(0.20 \mathrm{wt} \%)$ was given partial SPFT testing as it had the highest K-3 corrosion rate and highest VHT alteration rate within acceptable limits of the high-alkali glasses tested as part of the ORP set. It was noted that it would be valuable to complete SPFT testing on ORPLG9 and ORPLF7 (Muller and Pegg 2016) ${ }^{\mathrm{b}}$. It was decided to select ORPLG9 due to its high sodium concentration. This would also allow comparison of SPFT results with ORPLB2, the only high waste-loaded glass where a complete set of SPFT testing has been performed.

\footnotetext{
${ }^{a}$ Of the glasses tested in Muller and Pegg (2015), the CCC sample of IDF18-A161 had the largest B release of the glasses tested in 7-day, $90^{\circ} \mathrm{C}, 20,000 \mathrm{~m}^{-1}$ PCT-B tests.

${ }^{\mathrm{b}}$ The partial set of SPFT tests includes tests in dilute conditions at $\mathrm{pH}(\mathrm{RT}) 9$ at 23,40 , and $90^{\circ} \mathrm{C}$ and at various inlet Si concentrations at $\mathrm{pH}(\mathrm{RT}) 9$ at $90^{\circ} \mathrm{C}$ (Papathanassiu et al. 2011).
} 


\subsection{Purpose and Organization}

The purpose of this report is to provide ILAW rate model parameters for PA maintenance for the glasses ORPLG9 and IDF18-A161. Where possible, the rate model parameters are tabulated with rate model parameters from past ILAW glasses. Section 2.0 descibes the fabrication and characterization of IDF18A161 and ORPLG9 and the SPFT test method that was used. Section 3.0 presents SPFT results collected on IDF18-A161 and ORPLG9. This effort attempts to use the SPFT data to provide input to the PA maintenance effort. The final portion of the report, Section 4.0, summarizes the key conclusions of this testing effort and, where possible, compares the ILAW rate model parameter values to a select number of previously-tested ILAW glasses. The data used to calculate the rate model parameters are provided in Appendix A. All raw data obtained from this effort are presented in Appendix B.

\subsection{Quality Assurance}

This work was conducted with funding from Washington River Protection Solutions (WRPS) under contract 36437-161, ILAW Glass Testing for Disposal at IDF. The work was conducted as part of Pacific Northwest National Laboratory (PNNL) Project 68391, ILAW Glass Testing for Disposal at IDF.

All research and development (R\&D) work at PNNL is performed in accordance with PNNL's laboratory-level Quality Management Program, which is based on a graded application of NQA-1-2000, Quality Assurance Requirements for Nuclear Facility Applications, to R\&D activities. In addition to the PNNL-wide quality assurance (QA) controls, the QA controls of the WRPS Waste Form Testing Program (WWFTP) QA program were also implemented for the work. The WWFTP QA program consists of the WWFTP Quality Assurance Plan (QA-WWFTP-001) and associated QA-NSLW-numbered procedures that provide detailed instructions for implementing NQA-1 requirements for R\&D work. The WWFTP QA program is based on the requirements of NQA-1-2008, Quality Assurance Requirements for Nuclear Facility Applications, and NQA-1a-2009, Addenda to ASME NQA-1-2008 Quality Assurance Requirements for Nuclear Facility Applications, graded on the approach presented in NQA-1-2008, Part IV, Subpart 4.2, "Guidance on Graded Application of Quality Assurance (QA) for Nuclear-Related Research and Development”. Performance of the FY2016 SPFT tests and preparation of this report were assigned the technology level "Applied Research" and were conducted in accordance with procedure QA-NSLW-1102, Scientific Investigation for Applied Research. All staff members contributing to the work have technical expertise in the subject matter and received QA training prior to performing qualityaffecting work. The "Applied Research" technology level provides adequate controls to ensure that the activities were performed correctly. Use of both the PNNL-wide and WWFTP QA controls ensured that all client QA expectations were addressed in performing the work. 


\subsection{Glass Selection, Kinetic Rate Equation, and SPFT Test Method}

\subsection{Kinetic Rate Model Equation}

The current understanding of the glass-water interaction has been summarized in two recent review articles (Gin et al. 2013, Vienna et al. 2013a). Based on these reviews, it has been concluded that when glass is contacted with initially-dilute water in a closed system, it corrodes at a relatively fast rate, known as the forward rate. Glass dissolution in dilute systems is described as Stage I behavior. As the reaction proceeds and species are released from the glass into solution, the glass dissolution rate slows and eventually reaches a rate that is three to four orders of magnitude slower than the forward rate. The slow residual rate of glass dissolution is described as Stage II behavior. As the reaction continues and the solution becomes saturated with respect to secondary phases, precipitation of certain phases may result in a marked acceleration in the reaction rate. This resumption in rate has been termed Stage III.

The entire glass-water interaction entails a complex set of coupled physicochemical mechanisms and a single equation or model that fully addresses the complexities and reflects observed behaviors has not yet been developed. However, a general rate equation based on arguments and assumptions of TST has been used to describe the dissolution of glass (and more ordered materials) in the presence of an aqueous solution and the general rate equation is described below. Transition State Theory is based on the concept that, in a set of elementary reactions, the overall reaction rate is governed by the slowest elementary reaction. Elementary reactions have simple stoichiometry and can be combined as an overall reaction. In many cases, the elementary reactions can only be inferred. Grambow (1985) argued that the rate-limiting reaction that best describes glass dissolution is the dissolution of $\mathrm{SiO}_{2}$ to form silicic acid:

$$
\mathrm{SiO}_{2}(s)+2 \mathrm{H}_{2} \mathrm{O} \leftrightarrow \mathrm{SiO}_{2} \cdot 2 \mathrm{H}_{2} \mathrm{O}^{\ddagger} \rightarrow \mathrm{H}_{4} \mathrm{SiO}_{4}(a q)
$$

where $\mathrm{SiO}_{2} \cdot 2 \mathrm{H}_{2} \mathrm{O}^{\ddagger}$ represents an activated complex. Note that a double-headed arrow links the reactants and the activated complex in Eq. 2-1, symbolizing a reversible reaction. The rate at which the activated complex (a transitional, short-lived aggregate of atoms that form during the collision process) changes from reactants to products limits the rate at which the overall reaction can proceed (Eyring 1935). As demonstrated in Eq. 2-1 with the single-direction arrow, the TST formulation assumes that the decay of the activated complex to form orthosilicic acid $\left[\mathrm{H}_{4} \mathrm{SiO}_{4(\mathrm{aq})}\right]$ is an irreversible reaction.

The general rate equation based on TST of chemical kinetics given as (Åagaard and Helgeson 1982):

$$
r_{\phi}=k_{\phi} \prod_{i} a_{i}^{-\hat{n}_{i, j}}\left(1-\exp \left(-\frac{A}{\sigma R T}\right)\right)
$$

where: $\quad r_{\varphi}=$ dissolution rate

$\mathrm{k}_{\varphi}=$ kinetic constant of hydrolysis of the mineral $\varphi$

$a_{i}=$ activity of the reactants $i$ in the limiting elementary step $j$

$\hat{n}_{i, j}=$ stoichiometric coefficient for reactant $i$ in the limiting reaction $j$

$\mathrm{A}=$ chemical affinity of the overall dissolution reaction 


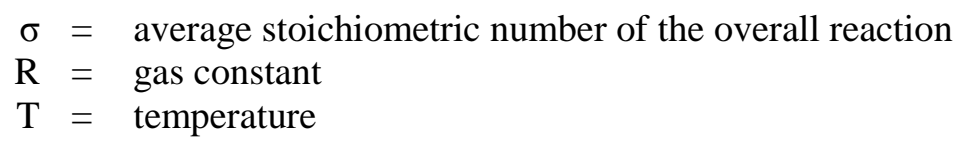

Eq. 22 relates the composition of water contacting the glass to the corrosion rate. Currently, $\mathrm{H}^{+}$is the only aqueous species that has been found to directly influence the rate via the product term of activities (McGrail et al. 1997). However, other species such as $\mathrm{Al}$ and $\mathrm{Na}$ have been proposed to influence the dissolution rate of waste glasses, basalt glasses, and amorphous silica (Abraitis et al. 2000a, Oelkers and Gislason 2001, Rimstidt et al. 2016). When applying the assumption made by Grambow (1985) where the dissolution of $\mathrm{SiO}_{2}$ is the rate-limiting reaction, Eq. 2-2 can be simplified to account for the flux of any element $i$ released from the glass into the aqueous phase by:

$$
r_{i}=k_{0} v_{i} a_{H^{+}}^{-\eta} \exp \left(\frac{-E_{a}}{R T}\right)\left[1-\left(\frac{Q}{K_{g}}\right)^{\sigma}\right]
$$

where: $\quad r_{i}=$ the dissolution rate $\left(\mathrm{g} \mathrm{m}^{-2} \mathrm{~d}^{-1}\right)$

$v_{\mathrm{i}}=$ the stoichiometric coefficient of element $i$ in the glass

$k_{0}=$ intrinsic rate constant $\left(\mathrm{g} \mathrm{m}^{-2} \mathrm{~d}^{-1}\right)$

$a_{\mathrm{H}^{+}}=$hydrogen ion activity

$\eta=\mathrm{pH}$ power law coefficient

$\mathrm{E}_{\mathrm{a}}=$ activation energy $\left(\mathrm{J} \mathrm{mol}^{-1}\right)^{\mathrm{a}}$

$\mathrm{R}=$ gas constant $\left(8.314 \mathrm{~J} \mathrm{~mol}^{-1} \cdot \mathrm{K}^{-1}\right)$

$\mathrm{T}=$ temperature, $\mathrm{K}$

$Q=$ ion-activity product

$K_{g}=$ pseudo-quilibrium constant for glass

$\sigma=$ Temkin coefficient

The ion activity product, $Q$, is a variable that must be computed by the numerical simulator as a function of time and space in the disposal system. Additionally, it has been argued that the Temkin coefficient, $\sigma$, is assumed to equal one because any value where $\sigma$ is not equal to one is inconsistent with TST (Lasaga 1995). The current assumption is this affinity-based rate model "best describes the majority of the experimental data that has been gathered over 35 years of studying glass/water reaction processes" (McGrail et al. 2000b). Therefore, it is necessary to determine the four remaining parameters, $k_{0}, \mathrm{E}_{\mathrm{a}}, \eta$, and $\mathrm{K}_{\mathrm{g}}$, for a range of glass formulations in order to implement Eq. 2-3 into a numerical simulator. A benefit of Eq. 2-3 is that it is solidly based on the TST of chemical kinetics, in which a series of reaction rates are governed by the slowest elementary reaction. Therefore, it is simply necessary to ascertain the rate-limiting step in dissolution rather than attempt to fully understand all of the possible reactions and kinetic pathways that can occur during the reaction of glass with aqueous solution. Because this ratelimiting step is an "elementary reaction," the stoichiometry of the reaction is typically simple and can be easily defined in a reactive transport model.

An additional term is necessary to describe the ILAW rate equation. The need for an additional term can be seen from Eq. 2-3, which suggests that the reaction rate will become zero when the ion-activity product, $Q$, is equal to the pseudo-equilibrium constant for glass, $\mathrm{K}_{\mathrm{g}}$. However, glass can never reach thermodynamic equilibrium with an aqueous solution and therefore the rate will not become zero

\footnotetext{
${ }^{a}$ Note, that $\mathrm{E}_{\mathrm{a}}$ in Eq. $2-2$ is included in the $\mathrm{k}_{\varphi}$ term.
} 
(Strachan and Neeway, 2014). One mechanism responsible for continued glass corrosion in silicasaturated solutions is ion exchange. This reaction results in the selective extraction of $\mathrm{Na}$, or other alkali, via the reaction:

$$
\text { Matrix-Na }+\mathrm{H}^{+} \rightarrow \text { Matrix- } \mathrm{H}+\mathrm{Na}^{+}
$$

where Matrix-Na represents the unreacted glass containing Na, and Matrix-H represents a hydrated glass where the Na has been replaced with an equimolar amount of hydrogen. This reaction occurs independently of matrix dissolution. The rate of this ion exchange reaction, referred to hereafter as $\mathrm{r}_{\mathrm{IEX}}$, has been determined from SPFT for numerous glasses (McGrail et al. 2000a, McGrail et al. 2001, Pierce et al. 2004, Papathanassiu et al. 2011). The $\mathrm{r}_{\mathrm{IEX}}$ value assumes a constant flux of Na from the glass into solution. However, this may be a simplification of the coupling of the actual diffusive process with a constantly dissolving dissolution front. Therefore, as identified in recent strategy documents, a more concerted effort should be made to understand the use of a diffusion-controlled alkali release term, which would increase the versatility and accuracy of the ion exchange model for implementation in future PA efforts (Ryan and Freedman, 2016). For ILAW glass, Na is used to represent alkali-ion exchange because $\mathrm{Na}$ is the single most abundant alkali contained in ILAW glass formulations. In saturated conditions in the glass-water reaction, $\mathrm{Na}$ is continuously introduced from the glass to solution, affecting the steady-state $\mathrm{pH}$ as Eq. 2-3 approaches or achieves saturation (i.e., $Q / \mathrm{K} \rightarrow 1$ ). In the absence of other reactions occurring in the near field at the IDF, this constant flux of Na results in a continuous change in solution $\mathrm{pH}$, thus affecting the long-term rate of glass corrosion.

\subsection{Experimental Materials and Methods}

\subsubsection{Glass Preparation and Characterization}

Both IDF18-A161 and ORPLG9 were prepared using reagent grade or higher purity chemicals. The target composition of each glass, along with other representative ILAW glasses that have undergone a full suite of SPFT testing, is given in Table 2.1. ORPLG9 was prepared and underwent CCC at VSL and was shipped to PNNL. Details of its preparation can be found elsewhere (Papathanassiu et al. 2011). IDF18A161 was prepared at PNNL and was batched to the composition provided in Table 2.1. The IDF18-A161 was prepared by batching and mixing the desired chemicals and melting in a furnace at $1150{ }^{\circ} \mathrm{C}$ for 60 min \pm 15 min in a $100 \%$ Pt crucible. Following the melt, the glass was air quenched on a stainless steel pour plate, ground in a tungsten disc mill, and the melting process repeated. No visible crystals were present in the quenched sample. 
Table 2.1. Target composition (mass\%) of selected ILAW glasses.

\begin{tabular}{|c|c|c|c|c|c|c|c|c|}
\hline Oxide & $\begin{array}{l}\text { LD6- } \\
5412^{(\text {a) }}\end{array}$ & LAWABP1 $1^{(\mathrm{b})}$ & LAWA44 ${ }^{(\mathrm{c})}$ & LAWB45 $5^{(\mathrm{d})}$ & LAWC22 $2^{(\mathrm{d})}$ & ORPLB2 $2^{(\mathrm{e})}$ & ORPLG9 & $\begin{array}{r}\text { IDF18 } \\
\text { A161 }\end{array}$ \\
\hline $\mathrm{Al}_{2} \mathrm{O}_{3}$ & 12 & 10 & 6.2 & 6.13 & 6.08 & 10 & 6.76 & 10.04 \\
\hline $\mathrm{B}_{2} \mathrm{O}_{3}$ & 5 & 9.25 & 8.9 & 12.34 & 10.06 & 7.3 & 8.51 & 13.51 \\
\hline $\mathrm{CaO}$ & 4 & NI & 1.99 & 6.63 & 5.12 & 1.1 & 2.7 & 7.9 \\
\hline $\mathrm{Cr}_{2} \mathrm{O}_{3}$ & 0.04 & 0.02 & 0.02 & 0.07 & 0.02 & 0.52 & 0.59 & 0.02 \\
\hline $\mathrm{Fe}_{2} \mathrm{O}_{3}$ & 0 & 2.5 & 6.98 & 5.26 & 5.43 & 1.1 & 0.2 & 0.99 \\
\hline $\mathrm{K}_{2} \mathrm{O}$ & 1.46 & 2.2 & 0.5 & 0.26 & 0.1 & 0.12 & 5.76 & 0.44 \\
\hline $\mathrm{La}_{2} \mathrm{O}_{3}$ & NI & 2 & NI & NI & NI & NI & NI & NI \\
\hline $\mathrm{Li}_{2} \mathrm{O}$ & NI & NI & NI & 4.62 & 2.51 & NI & NI & NI \\
\hline $\mathrm{MgO}$ & NI & 1 & 1.99 & 2.97 & 1.51 & 1.1 & 0.96 & 0.99 \\
\hline $\mathrm{Na}_{2} \mathrm{O}$ & 20 & 20 & 20 & 6.5 & 14.4 & 25 & 21 & 20.66 \\
\hline $\mathrm{SO}_{3}$ & 0.21 & 0.1 & 0.1 & 0.84 & 0.34 & 0.52 & 0.2 & 1.1 \\
\hline $\mathrm{SiO}_{2}$ & 55.91 & 41.89 & 44.55 & 47.86 & 46.67 & 39.98 & 40.82 & 36.05 \\
\hline $\mathrm{SnO}_{2}$ & NI & NI & NI & NI & NI & 1.08 & 2.84 & NI \\
\hline $\mathrm{TiO}_{2}$ & NI & 2.49 & 1.99 & 0 & 1.14 & NI & NI & NI \\
\hline $\mathrm{V}_{2} \mathrm{O}_{5}$ & NI & NI & NI & NI & NI & 2 & NI & 0.99 \\
\hline $\mathrm{ZnO}$ & NI & 2.6 & 2.96 & 3.15 & 3.07 & 3.65 & 3.4 & 2.95 \\
\hline $\mathrm{ZrO}_{2}$ & NI & 5.25 & 2.99 & 3.15 & 3.03 & 5.44 & 5.68 & 2.95 \\
\hline Others & 1.38 & 0.70 & 0.83 & 0.22 & 0.52 & 1.09 & 0.58 & 1.41 \\
\hline
\end{tabular}

(a) LD6-5412 - also known as HLP-46; see McGrail et al. (1998) and Vienna et al. (2001).

(b) LAWABP1 - also known as HLP-51; see McGrail et al. (2001).

(c) LAWA44 - also known as HLP-56; see Muller et al. (2001).

(d) See Muller et al. (2001) for LAWB45 and LAWC22.

(e) ORPLB2 - also known as IDF1-B2; see Papathanassiu et al. (2011).

$\mathrm{NI}=$ not included.

Others include minor amounts of $\mathrm{Ag}_{2} \mathrm{O}, \mathrm{BaO}, \mathrm{CdO}, \mathrm{Ce}_{2} \mathrm{O}_{3}, \mathrm{Cl}, \mathrm{Cr}_{2} \mathrm{O}_{3}, \mathrm{Cs}_{2} \mathrm{O}, \mathrm{F}, \mathrm{I}, \mathrm{MnO}, \mathrm{MoO}_{3}, \mathrm{Nd}_{2} \mathrm{O}_{3}, \mathrm{NiO}, \mathrm{P}_{2} \mathrm{O}_{5}$, $\mathrm{PbO}_{2}, \mathrm{Pr}_{2} \mathrm{O}_{3}, \mathrm{Re}_{2} \mathrm{O}_{7}, \mathrm{SeO}_{2}, \mathrm{SrO}, \mathrm{TeO}_{2}$, and $\mathrm{Y}_{2} \mathrm{O}_{3}$. Not all elements are present in every glass composition.

The quenched IDF18-A161 glass was next heat treated to simulate the CCC temperature profile. Before CCC heat treatment, the IDF18-A161 glass was crushed to allow pieces through a 3/8” sieve and $50 \mathrm{~g}$ was used for each CCC batch. The CCC heat treatment of the crushed glass involved placing the glass in a $100 \%$ Pt crucible in a furnace at the melt temperature for $30 \mathrm{~min}$ and the temperature was dropped according to the CCC cooling profile ${ }^{\mathrm{b}}$ shown in Table 2.2. At the end of the profile, the glass was cooled overnight in the furnace (after turning off) to room temperature.

\footnotetext{
${ }^{\mathrm{b}}$ Memorandum, Canister Centerline Cooling Data, Revision 1, CCN: 074181, RPP-WTP, October 29, 2003
} 
Table 2.2. Centerline canister cooling (CCC) profile for ILAW glass.

\begin{tabular}{rlrrr}
\hline Segment & Time (min) & Start Temp. $\left({ }^{\circ} \mathrm{C}\right)$ & Rate $\left({ }^{\circ} \mathrm{C} / \mathrm{min}\right)$ & End Temp. $\left({ }^{\circ} \mathrm{C}\right)$ \\
\hline 1 & $0-16$ & 1114 & -7.125 & 1000 \\
2 & $16-73$ & 1000 & -1.754 & 900 \\
3 & $73-195$ & 900 & -0.615 & 825 \\
4 & $195-355$ & 825 & -0.312 & 775 \\
5 & $355-640$ & 775 & -0.175 & 725 \\
6 & $640-1600$ & 725 & -0.13 & 600 \\
7 & $1600-3710$ & 600 & -0.095 & 400 \\
\hline
\end{tabular}

A summary of the solids analyses that were performed on the glasses prior to SPFT testing is provided below. As will be explained in the Section 3.0, based on the SPFT results, further solids analysis were performed in order to explain the dissolution behavior of the glasses. Because those solids analyses are imperative to understand the dissolution behavior of these glasses, a description of the methods and results of those analyses will also be provided in Section 3.0.

The CCC heat-treated samples of ORPLG9 and IDF18-A161 both contained crystalline inclusions. Images of the as-received ORPLG9 CCC glass and an ORPLG9 CCC particle that has been polished to 600 grit are provided in Figure 2.1. Figure 2.1b shows striations in the glass. At the higher magnification of Figure 2.1c, these striations appear to be composed of inclusions, most likely a $\mathrm{Cr}_{2} \mathrm{O}_{3}$ phase, as discussed below. An X-ray Diffraction (XRD) pattern of a sample of the as-received ORPLG9 glass is shown in Figure 2.2. The pattern was obtained with a $\mathrm{Cu}$ K-alpha wavelength source ( $40 \mathrm{~mA}, 40 \mathrm{kV})$ from $5^{\circ}$ to $70^{\circ}(2 \theta)$ with a step size of $0.03^{\circ}$ and a dwell time of 4.0 seconds. Besides the peaks that are present from the addition of a fluorite $\left(\mathrm{CaF}_{2}\right)$ standard, the main feature in the diffraction pattern is the amorphous hump near $26^{\circ}$ to $32^{\circ} 2 \theta$, which is indicative of an amorphous material. For the analysis conditions, the amount of crystalline material in the glass sample is under the assumed detection limit of $1.0 \mathrm{wt} \%$. Figure 2.3 shows a Scanning Electron Microscopy (SEM) image and Energy-Dispersive Spectroscopy (EDS) spectra of the as-received ORPLG9 sample. The backscatter electron SEM image demonstrates that the surface is largely homogeneous. However, there are areas of higher atomic number as evidenced by small areas with brighter shading. By referencing the EDS obtained on the brighter areas, it is observed that the areas are Cr-rich. Based on the morphology of the particles observed at higher magnification with secondary electrons (see Figure 2.4), the Cr-rich phases are most likely eskolaite $\left(\mathrm{Cr}_{2} \mathrm{O}_{3}\right)$ (Vienna et al. 2013b). 


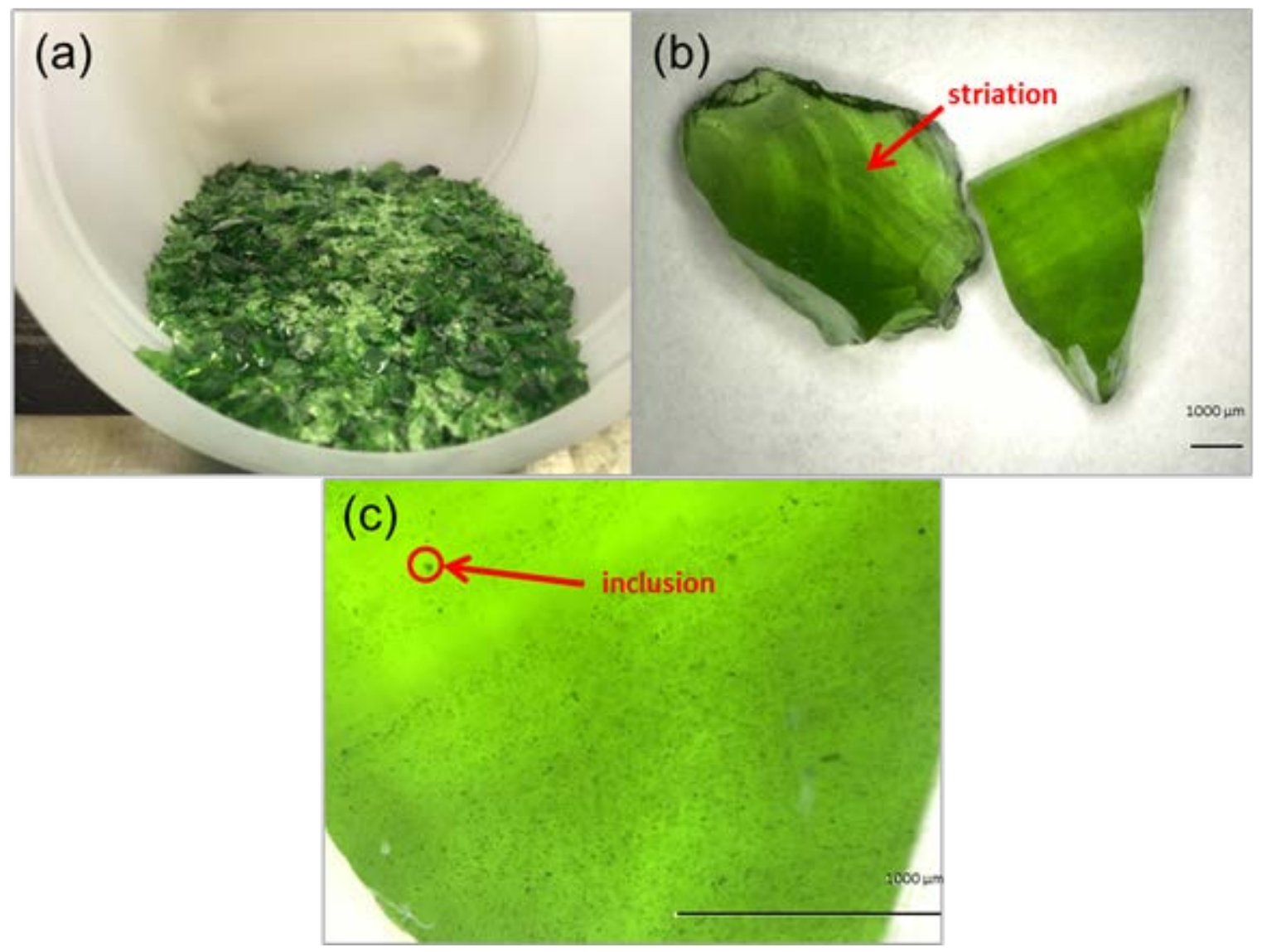

Figure 2.1. Optical photographs of ORPLG9 CCC (a) powder as received from VSL; (b) two pieces of glass powder polished to 600 grit on one face showing striations; and (c) observation of undissolved $\mathrm{Cr}_{2} \mathrm{O}_{3}$ particles in the glass.

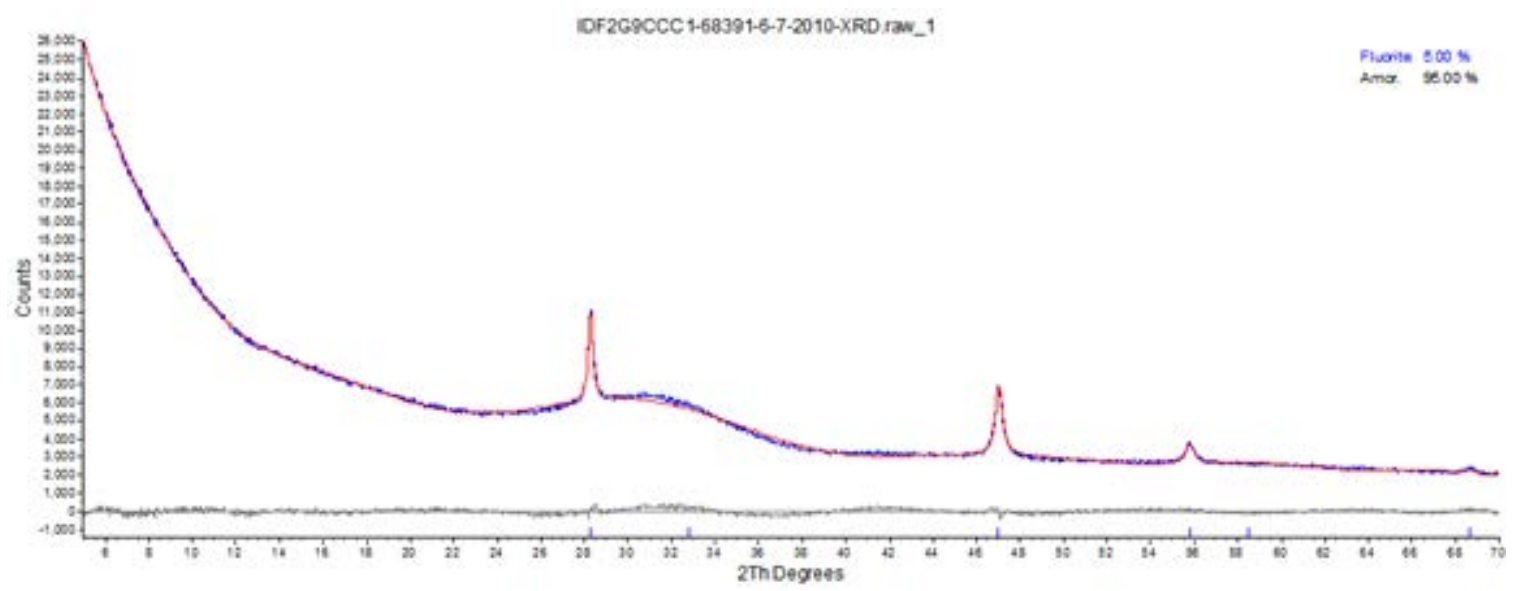

Figure 2.2. XRD pattern of the as-received ORPLG9 CCC glass. The peaks in the diffraction pattern are due to the addition of a fluorite $\left(\mathrm{CaF}_{2}\right)$ standard. 


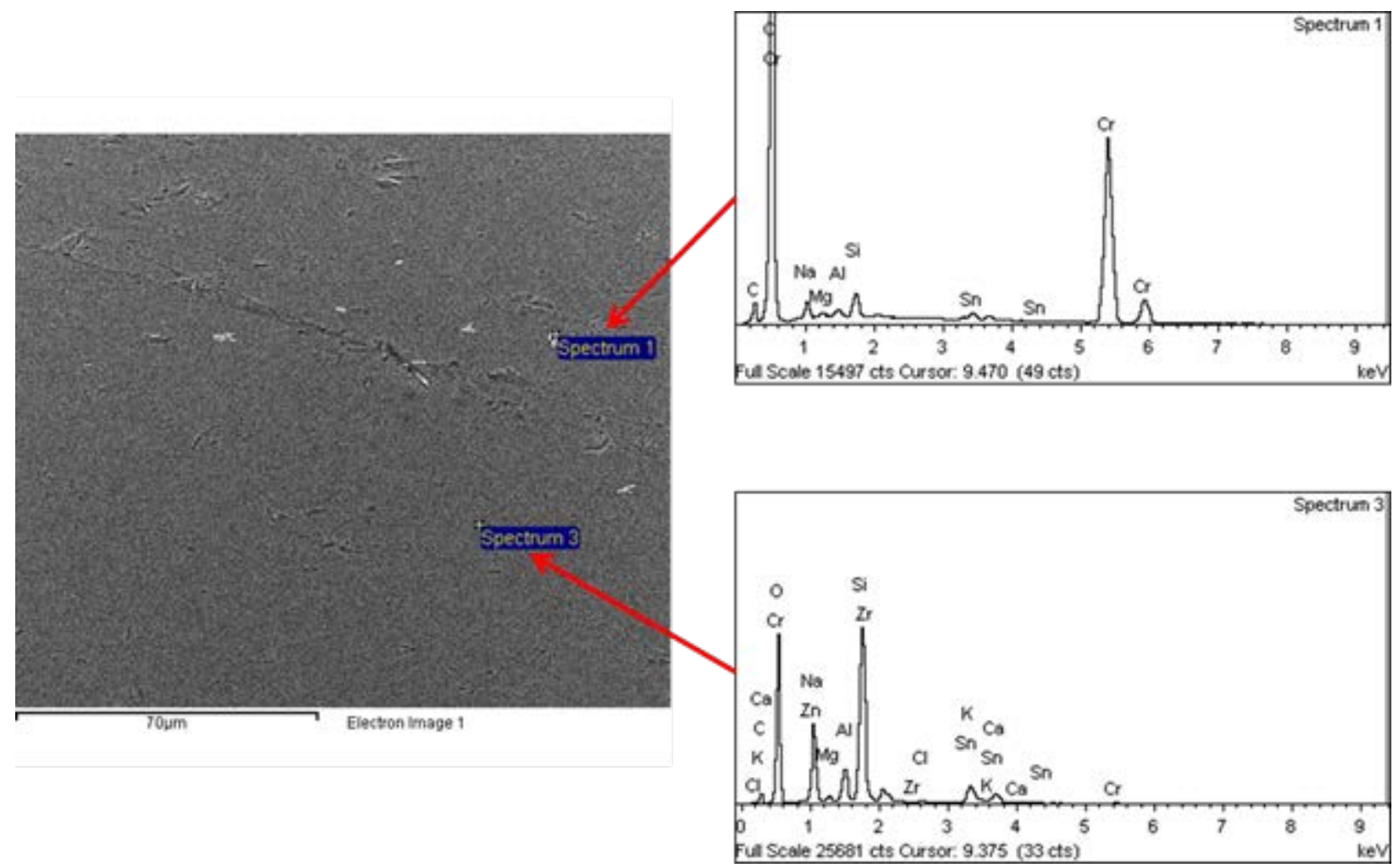

Figure 2.3. Backscatter electron SEM (left) and EDS (right) of the as-received ORPLG9 CCC glass. Spectrum 1 is of a bright particle and Spectrum 3 is of the bulk glass.

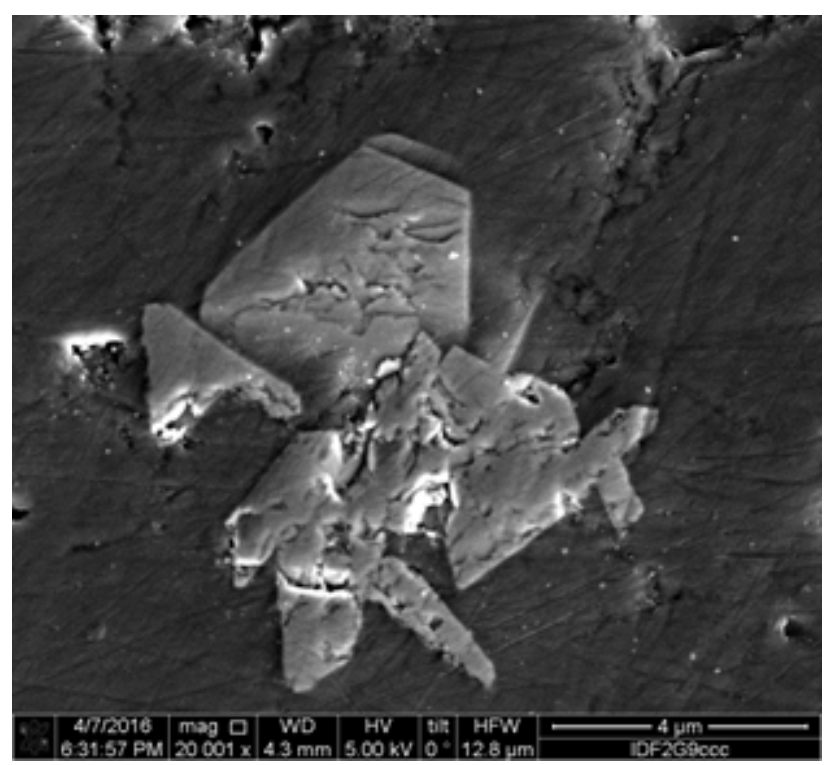

Figure 2.4. Secondary electron SEM of Cr inclusions in the as-received ORPLG9 CCC glass.

Photographs of IDF18-A161 (quenched and after CCC) are provided in Figure 2.5. The quenched glass appears to be homogenous while the CCC heat-treated sample contains a large amount of crystals. Though the crystals are primarily at the crucible/glass interface, they also appear in the bulk of the sample. Even so, the large majority of the volume of the sample appears to be glass. XRD patterns are presented in Figure 2.6 for (a) IDF18-A161 as quenched, (b) a portion of the IDF18-A161 CCC sample 
that contains crystals, and (c) a portion of the IDF18-A161 CCC sample where the crystals were physically separated from the glass by hand sorting. In both the quenched sample and the CCC sample where crystals were manually removed, the XRD patterns indicate that these samples are x-ray amorphous; conservatively, the crystalline detection limit is 2 wt\%.
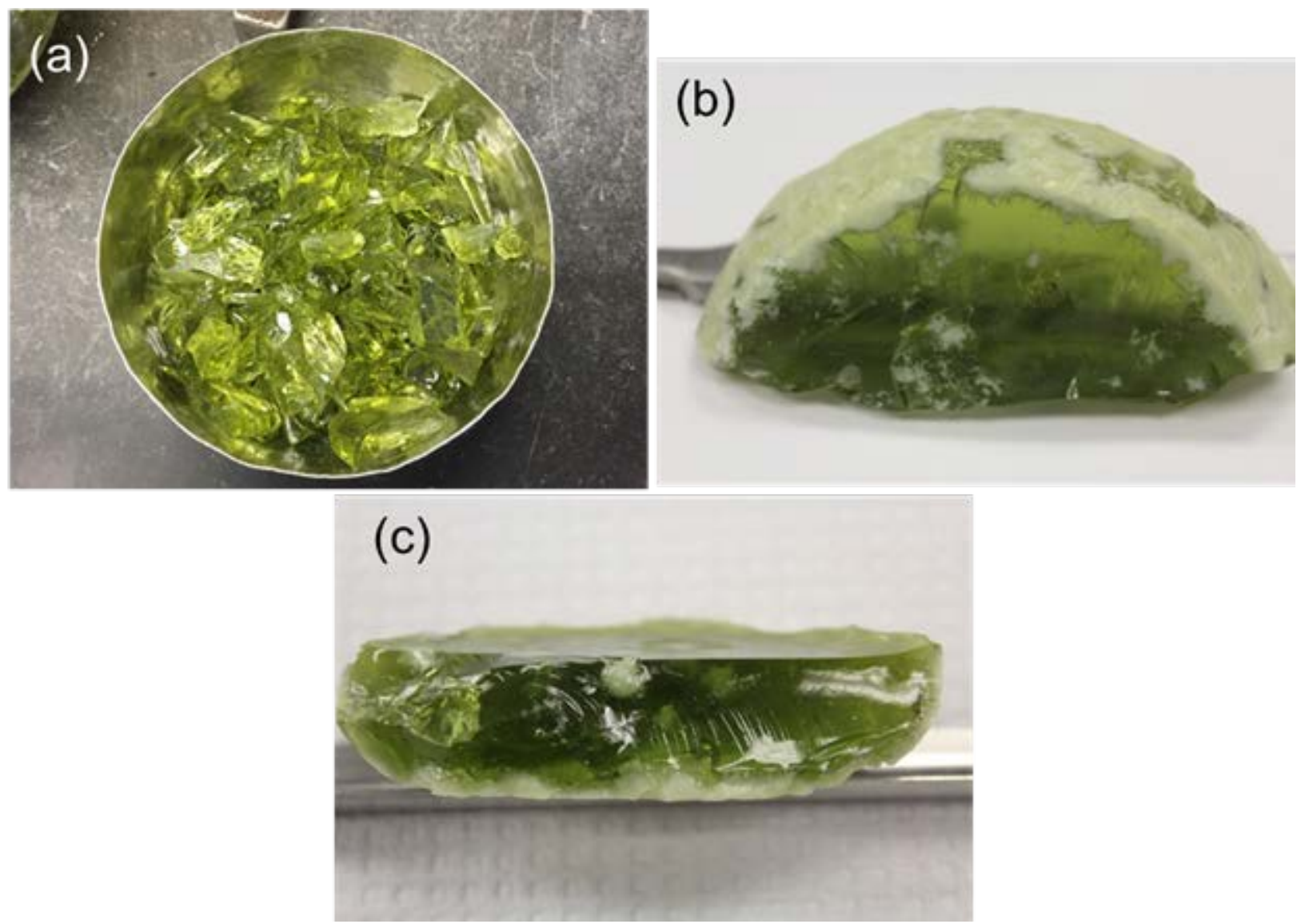

Figure 2.5. Photographs of (a) quenched IDF18-A161; (b) bottom view of CCC heat treatment of IDF18A161; and (c) side view of CCC heat treatment of IDF18-A161. 

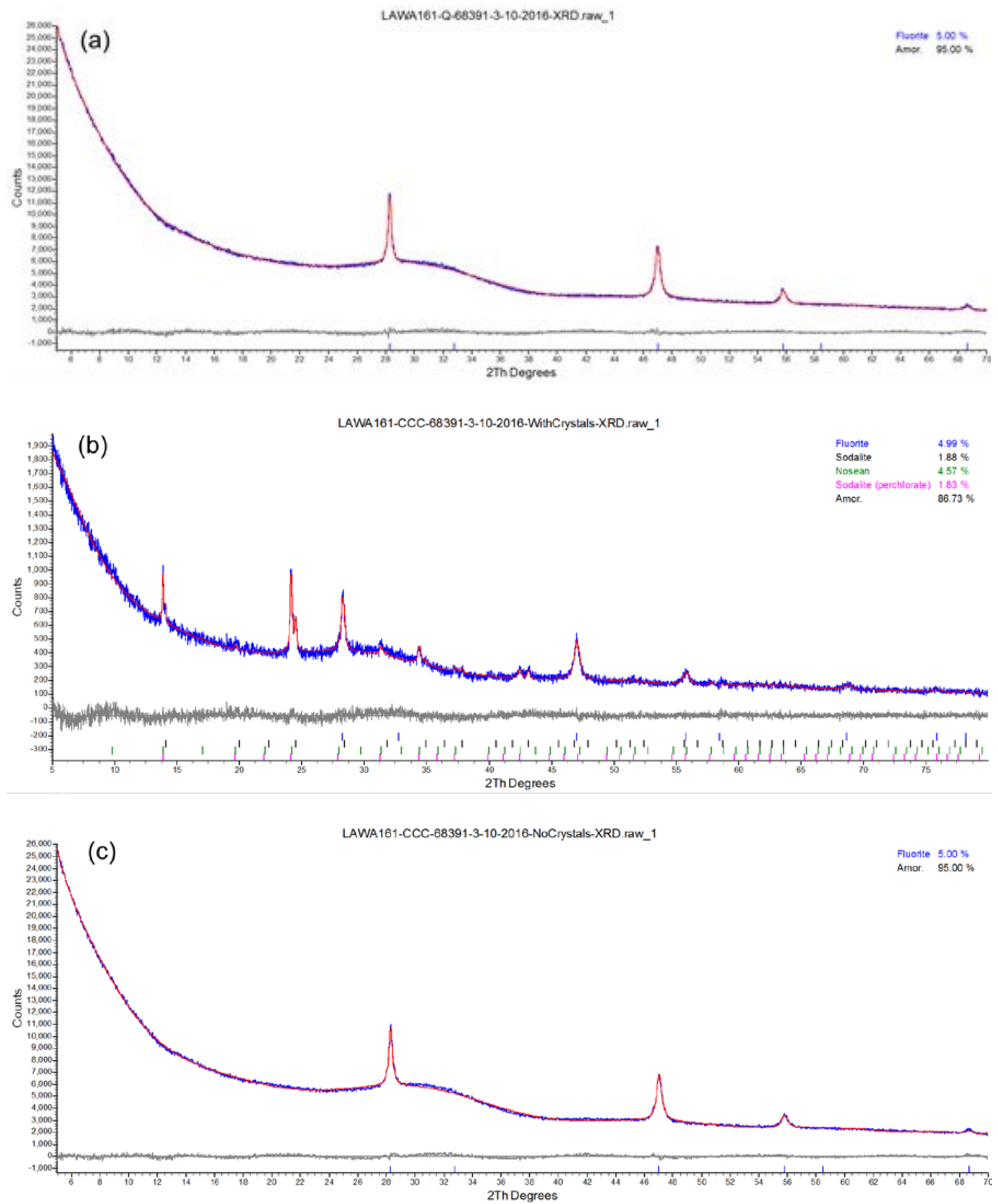

Figure 2.6. XRD pattern of different preparations of IDF18-A161 glass. (a) quenched; (b) CCC heat treatment with crystals; and (c) CCC heat treatment where crystals have been physically separated from the glass.

The crystalline portion contains sodalite $\left(\mathrm{Na}_{8} \mathrm{Al}_{6} \mathrm{Si}_{6} \mathrm{O}_{24} \mathrm{Cl}_{2}\right)$ and nosean $\left(\mathrm{Na}_{8} \mathrm{Al}_{6} \mathrm{Si}_{6} \mathrm{O}_{24} \mathrm{SO}_{4}\right)$. Thus, the two major anions, $\mathrm{Cl}^{-}$and $\mathrm{SO}_{4}{ }^{2-}$, appear to have partitioned to these crystalline phases. The relative percentages of each phase in Figure 2.6b are for a random portion of the CCC sample and may not necessarily be the representative proportion of each crystalline phase for the entire sample. The 
partitioning of $\mathrm{SO}_{4}{ }^{2-}$ to the crystalline phase is supported by the chemical analysis of a crystal-free sample of IDF18-A161 (Table 2.3). The measured $\mathrm{SO}_{4}{ }^{2-}$ value is $19 \%$ below the target value. Thus, the loss of $\mathrm{SO}_{4}{ }^{2-}$ compared to the target value is due to a certain fraction partitioning to the nosean phase, though volatilization may also cause significant loss of $\mathrm{SO}_{4}{ }^{2-}$. The concentration of $\mathrm{Cl}^{-}$in the glasses was not determined.

Secondary electron SEM images of a polished IDF18-A161 (Figure 2.7) glass particle are provided in Figure 2.7. The surface is heterogeneous, with the presence of a bulk phase and a second phase evenly distributed across the surface. EDS of the glass surface did not show any obvious differences in composition between the two phases.

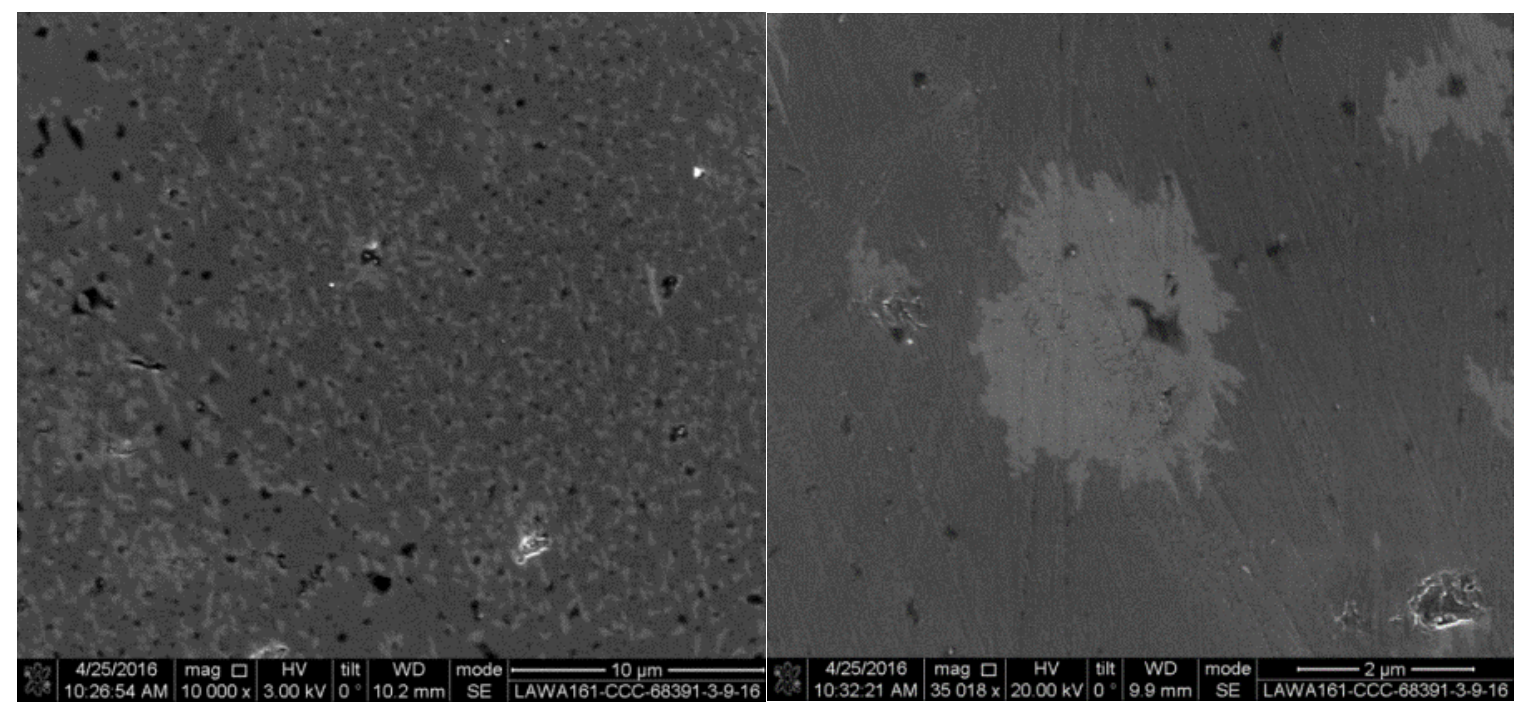

Figure 2.7. Secondary electron SEM of the surface of a CCC heat treated sample of IDF18-A161 at two different magnifications. Images are from glass particles where large crystals have been physically separated.

A chemical analysis was performed on both glasses using a combination of a flux fusion and acid digestion. The results of this analysis confirm that there was no large change in composition for the major elements between the as-batched composition and the glass after treatment (Table 2.3). For glasses used in SPFT testing, the crystalline inclusions for the CCC sample of ORPLG9 were left in the glass while any crystals present in the CCC sample of IDF18-A161 were manually removed.

Removal of the crystalline portion of the IDF18-A161 can be justified for several reasons. First, because the crystals present in the CCC sample were largely located at the crucible-glass interface, the presence of the crystals is most likely an artifact of this interface, which acts as a nucleation site for crystal growth. Second, in an actual ILAW glass container, the portion of the glass melt that is subject to the exact CCC heat treatment profile will be largely confined to the center of the canister. Thus possible crystal formation that results from the CCC heat treatment will be largely confined to the center of the canister and will only represent a small volume fraction of ILAW in the canister. Lastly, because the purpose of SPFT testing is to understand the dissolution behavior of ILAW glass, the removal of these crystals, which are an artifact of the CCC heat treatment setup, is justified as their presence in the test may confound the experimental results. 
The CCC glasses were prepared for SPFT testing by crushing with a tungsten carbide disc mill. After sieving the glass, the $149 \mu \mathrm{m}$ to $74 \mu \mathrm{m}$ (- 100 to +200 mesh) fraction was collected. This size fraction was washed according to Section 19.6 of ASTM C1285-14 and stored in a desiccator until use in SPFT testing. Due to the relatively fast dissolution rate of IDF18-A161 in dilute conditions, monolithic samples were used in some cases to decrease the relative amount of surface area lost in the test duration. Tests where this was done included those with a large solution-flow-rate-to-surface-area ratio $(q / S)$ (i.e. high flow rate, low surface area) or high temperature/high $\mathrm{pH}$. A single monolithic was used in the place of the crushed glass. Monolithic samples can be used in SPFT tests per Section 6.4 of ASTM C1662-10. The monoliths were polished to a colloidal silica finish $(0.05 \mu \mathrm{m})$, and had dimensions of $\sim 2 \times 2 \times 0.2 \mathrm{~cm}$. It is assumed that polishing did not largely affect the composition of the surface in comparison to the bulk glass. The monolith was placed on a mesh cage in the reactor to expose all faces.

Table 2.3. Target and measured composition of CCC samples of ORPLG9 and IDF18-A161.

\begin{tabular}{|c|c|c|c|c|c|c|}
\hline Oxide & $\begin{array}{c}\text { ORPLG9 } \\
\text { Target }\end{array}$ & $\begin{array}{c}\text { measured } \\
\text { wt } \%\end{array}$ & $\begin{array}{c}\text { relative } \\
\% \\
\text { difference } \\
\end{array}$ & $\begin{array}{l}\text { IDF18- } \\
\text { A161 } \\
\text { Target }\end{array}$ & $\begin{array}{c}\text { measured } \\
\text { wt } \%\end{array}$ & $\begin{array}{c}\text { relative } \\
\% \\
\text { difference } \\
\end{array}$ \\
\hline $\mathrm{Al}_{2} \mathrm{O}_{3}$ & 6.76 & 6.84 & $1 \%$ & 10.04 & 9.75 & $3 \%$ \\
\hline $\mathbf{B}_{2} \mathbf{O}_{3}$ & 8.51 & 8.39 & $1 \%$ & 13.51 & 13.42 & $1 \%$ \\
\hline $\mathrm{CaO}$ & 2.70 & 2.71 & $0 \%$ & 7.90 & 7.83 & $1 \%$ \\
\hline $\mathrm{Cr}_{2} \mathrm{O}_{3}$ & 0.59 & 0.62 & $4 \%$ & 0.02 & 0.02 & $11 \%$ \\
\hline $\mathrm{Cs}_{2} \mathrm{O}$ spike & --- & --- & --- & 0.15 & --- & --- \\
\hline $\mathrm{Fe}_{2} \mathbf{O}_{3}$ & 0.20 & 0.20 & $2 \%$ & 0.99 & 1.29 & $30 \%$ \\
\hline $\mathbf{K}_{2} \mathbf{O}$ & 5.76 & 5.66 & $2 \%$ & 0.44 & 0.81 & $84 \%$ \\
\hline MgO & 0.96 & 0.88 & $8 \%$ & 0.99 & 0.94 & $5 \%$ \\
\hline $\mathrm{Na}_{2} \mathrm{O}$ & 21.00 & 20.47 & $3 \%$ & 20.66 & 20.17 & $2 \%$ \\
\hline $\mathrm{NiO}$ & 0.01 & --- & --- & - & --- & --- \\
\hline PbO & 0.01 & --- & --- & - & --- & --- \\
\hline $\mathrm{SiO}_{2}$ & 40.82 & 42.05 & $3 \%$ & 36.05 & 36.20 & $0 \%$ \\
\hline $\mathrm{SnO}_{2}$ & 2.84 & 2.74 & $3 \%$ & - & --- & --- \\
\hline $\mathbf{V}_{2} \mathbf{O}_{5}$ & --- & --- & --- & 0.99 & 0.99 & $0 \%$ \\
\hline $\mathrm{ZnO}$ & 3.40 & 3.45 & $1 \%$ & 2.95 & 2.85 & $3 \%$ \\
\hline $\mathrm{ZrO}_{2}$ & 5.68 & 5.35 & $6 \%$ & 2.95 & 2.63 & $11 \%$ \\
\hline Cl & 0.23 & NM & --- & 1.17 & NM & --- \\
\hline $\mathbf{F}$ & 0.09 & NM & --- & - & NM & --- \\
\hline $\mathbf{P}_{2} \mathbf{O}_{5}$ & 0.14 & NM & --- & - & NM & --- \\
\hline $\mathrm{SO}_{3}$ & 0.20 & --- & --- & 1.10 & 0.89 & $19 \%$ \\
\hline $\begin{array}{c}\mathbf{R e}_{2} \mathbf{O}_{7} \\
\text { spike }\end{array}$ & 0.10 & 0.04 & $57 \%$ & 0.10 & --- & --- \\
\hline Sum & 100 & 99.40 & & 100 & 97.79 & \\
\hline
\end{tabular}


The specific surface area, $S\left(\mathrm{~m}^{2} \mathrm{~g}^{-1}\right)$, of the glass powder samples is estimated based on the following geometric formula (McGrail et al. 1997):

$$
S=\frac{3}{\rho r}
$$

where: $\quad \rho=$ the glass density $\left(\mathrm{g} \mathrm{m}^{-3}\right)$

$r=$ the average particle radius $(\mathrm{m})$.

With the average particle radius for the -100 to +200 mesh fraction of $5.63 \times 10^{-5} \mathrm{~m}$, the resulting specific surface area for ORPLG9 $\left(\rho=2.7106 \mathrm{~g} \mathrm{~cm}^{-3}\right)$ and IDF18-A161 $\left(\rho=2.6403 \mathrm{~g} \mathrm{~cm}^{-3}\right)$ is $1.97 \times 10^{-2}$ and $2.02 \times 10^{-2} \mathrm{~m}^{2} \mathrm{~g}^{-1}$, respectively. The use of the geometric surface area formula was recently shown to be more similar to the solution-derived dissolution rate than when the Brunauer-Emmett-Teller (BET) surface area was used in calculations (Icenhower and Steefel 2013).

\subsubsection{Single-Pass Flow-Through Apparatus}

The SPFT system is designed to enable a continuous flow of fresh solution into a vessel containing the material of interest. The method is governed by ASTM C1662-10. Collection of the effluent from the vessel allows for monitoring of release of elements from the material as a function of time. The work performed in this report utilizes a "well-mixed” reactor design commonly used in studies of glasses.

The SPFT apparatus is shown in Figure 2.8. A syringe pump (Norgren Kloehn, Las Vegas, NV, USA, Model 55022) is used to draw buffered solution from a reservoir and transfer it at a steady flow rate to the reactor vessels and effluent collection. All connections are made with polytetrafluoroethylene (PTFE) tubing. The $60-\mathrm{mL}$ reactor vessels used in this study were Teflon reactors (Savillex, Minnetonka, MN) with a 40.8-mm inner diameter and 63.6-mm height. The crushed glass (mass typically between 0.02 and $4.0 \mathrm{~g}$ ) is placed into the reactor and settles to form a thin layer at the bottom of the reactor. In accordance with ASTM C1662-10, these conditions are designed to yield a well-mixed solution due to the relatively high flow rates and convection at elevated test temperatures. 


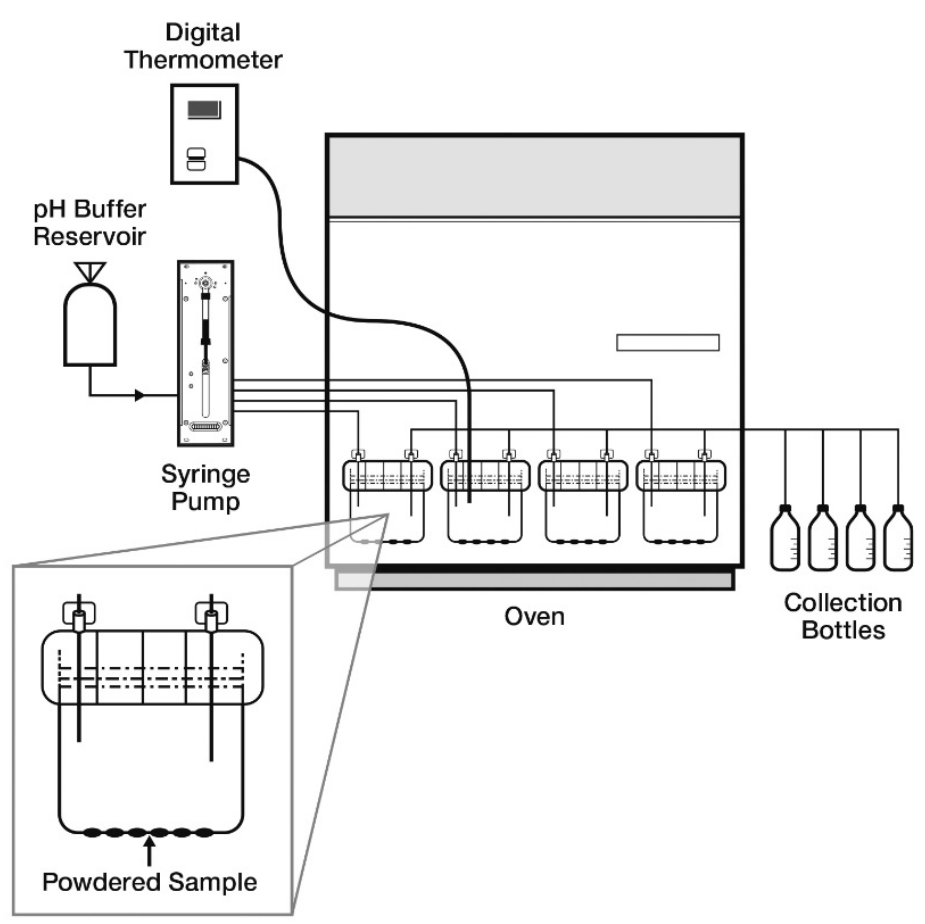

Figure 2.8. Schematic of the single-pass flow-through (SPFT) apparatus for determining reaction rates in continuous flow solution

A testing matrix for the SPFT was designed for both glasses covering a range of temperature, $\mathrm{pH}$, and inlet Si concentration. The full matrix is provided in Table 2.4 and Table 2.5. The raw data from each test are presented in Appendix B. As seen from Table 2.4 and Table 2.5, the ORPLG9 test underwent more testing than IDF18-A161. In particular, a q/S sweep was performed with ORPLG9 across all $\mathrm{pH} /$ temperature combinations whereas a q/S sweep was performed with IDF18-A161 only at pH(RT) 9 at each temperature. By performing a large set of q/S sweeps, we were able to demonstrate that the rates calculated at each $\mathrm{pH} /$ temperature combination were indeed at, or very near, the forward rate in those conditions. The forward rate from $\mathrm{q} / \mathrm{S}$ sweeps at various $\mathrm{pH} /$ temperature combinations was assigned to the reactor with the highest value of $\mathrm{q} / \mathrm{S}$ where effluent concentrations of $\mathrm{Al}, \mathrm{B}, \mathrm{Na}$, and Si were above the estimated quantification limit (EQL) and the release of these four was congruent within error. The q/S value that is used to determine the forward rate will change with test temperature and $\mathrm{pH}$ because the degree of system saturation will change as these experimental parameters are varied. Thus, it is not possible to assign one $\mathrm{q} / \mathrm{S}$ value across all test conditions as the $\mathrm{q} / \mathrm{S}$ value that can be used to determine the forward rate. In addition to q/S sweeps, certain tests were performed at $\mathrm{pH}(\mathrm{RT}) 9$ where 1-100 $\mathrm{ppm} \mathrm{Si}$ was added to the solution. The steady-state release concentrations, along with the values used to calculate the steady-state release rates, are provided in Appendix A.

During testing, the effluent samples were collected at regular intervals, where the frequency of sampling intervals depended on the testing conditions. Three blank effluent samples were collected from each reactor prior to the addition of the glass to determine background concentrations of the analytes. A blank reactor was not run with each experiment. This is because, for solutions without Si added to the inlet solution, the blank effluent concentrations for $\mathrm{Al}, \mathrm{B}, \mathrm{Na}$, and $\mathrm{Si}$ were well below values measured for the outlet solutions collected after the glass was added to the reactor vessels. Inlet reservoirs for experiments with $\mathrm{Si}$ added to the inlet were placed in ovens at the test temperature to avoid Si precipitation. The 
influent reservoir $\mathrm{pH}$ was checked twice per week to ensure that it was within $\pm 0.1 \mathrm{pH}$ units of the target $\mathrm{pH}$. Flow rates were determined gravimetrically and the collected effluent was analyzed with inductively coupled plasma optical emission spectroscopy (ICP-OES) for $\mathrm{Na}, \mathrm{Si}, \mathrm{Al}$, and $\mathrm{B}$. Based on the dilution of the ICP-OES sample an EQL was determined by the lowest calibration standard that can be reproducibly determined during an analytical run within $10 \%$ of the certified value multiplied by the dilution factor. For cases where the effluent concentration of the analyte of interest was below the EQL, the dissolution rate based on that element was calculated using the value of the EQL. The use of the EQL gives a maximum dissolution rate in those conditions. The calculated dissolution rates from EQL values are provided in Appendix A. In a few instances, the boron release rate calculated from the EQL was used in rate parameter calculations. This was the case when the boron release was above the instrument detection limit (25.2 ppb) and the release rate could be compared with the measurable release rate of the other analytes of interest, $\mathrm{Na}, \mathrm{Si}$, and $\mathrm{Al}$. The use of the EQL compared to the use of the value above the instrument detection limit but below the EQL (see Appendix B) does not change the calculated rate law parameters within uncertainty.

Effluent samples that varied from the average flow rate of the test by more than $10 \%$ were not used in calculations. A steady-state dissolution rate was defined as three samples collected near the end of the test, which did not deviate by more than $15 \%$ of their mean. A surface area correction at each sampling interval was performed according to Appendix A of ASTM C1662-10. If more than 30\% of the sample surface area was lost during the experiment, the samples collected after the loss of $30 \%$ were not used to calculate the steady-state release. We note that Section 6.3 of ASTM C1662-10 states, "the particle size of crushed samples must be large enough that the decrease in surface area during the test is less than 15 mass percent.” From this statement, it is unclear if mass percent or percent surface area was intended. However, for certain experiments provided in Appendix B, it can be seen that the assignment of a 30\%-maximum surface area loss is reasonable because release rates remain relatively constant until this threshold is reached. Also, steady-state release rates for tests performed at nearly-equivalent $q / S$ with powders and coupons show that this assumption is merited. 
Table 2.4. List of SPFT conditions used for ORPLG9. Measured test temperatures are provided in Appendix A.

\begin{tabular}{|c|c|c|c|c|c|c|c|}
\hline $\begin{array}{l}\text { Temp } \\
{ }^{\circ} \mathrm{C}\end{array}$ & $\begin{array}{c}\mathrm{pH} \\
@ 23^{\circ} \mathrm{C}\end{array}$ & $\begin{array}{c}\text { Nominal Inlet Si } \\
\text { ppm }\end{array}$ & $\begin{array}{c}\text { Initial } \mathrm{q} / \mathrm{S} \\
\mathrm{m} / \mathrm{s}\end{array}$ & $\begin{array}{l}\text { Temp } \\
{ }^{\circ} \mathrm{C}\end{array}$ & $\begin{array}{c}\mathrm{pH} \\
@ 23^{\circ} \mathrm{C}\end{array}$ & $\begin{array}{c}\text { Nominal Inlet Si } \\
\text { ppm }\end{array}$ & $\begin{array}{c}\text { Initial } \mathrm{q} / \mathrm{S} \\
\mathrm{m} / \mathrm{s}\end{array}$ \\
\hline \multirow[t]{8}{*}{23} & 9 & 0 & 6.69E-09 & 40 & 9 & 0 & $1.15 \mathrm{E}-09$ \\
\hline & & 0 & $3.24 \mathrm{E}-08$ & & & 0 & 7.45E-09 \\
\hline & & 0 & 1.57E-07 & & & 0 & 2.93E-08 \\
\hline & & 0 & 7.46E-07 & & & 0 & 1.49E-07 \\
\hline & & 5 & 2.91E-09 & & & 0 & 7.85E-07 \\
\hline & & 20 & 2.61E-09 & & & 10 & $5.38 \mathrm{E}-09$ \\
\hline & & 35 & 2.82E-09 & & & 30 & 3.08E-09 \\
\hline & & & & & & 50 & 5.89E-09 \\
\hline \multirow[t]{5}{*}{23} & 10 & 0 & $1.08 \mathrm{E}-08$ & 40 & 10 & 0 & 1.17E-09 \\
\hline & & 0 & $2.82 \mathrm{E}-08$ & & & 0 & 6.96E-09 \\
\hline & & 0 & 1.47E-07 & & & 0 & 3.04E-08 \\
\hline & & 0 & $7.70 \mathrm{E}-07$ & & & 0 & $1.47 \mathrm{E}-07$ \\
\hline & & & & & & 0 & 7.35E-07 \\
\hline \multirow[t]{5}{*}{23} & 11 & 0 & $3.60 \mathrm{E}-09$ & 40 & 11 & 0 & $1.64 \mathrm{E}-09$ \\
\hline & & 0 & $1.51 \mathrm{E}-08$ & & & 0 & 3.58E-09 \\
\hline & & 0 & $9.19 \mathrm{E}-08$ & & & 0 & $1.84 \mathrm{E}-08$ \\
\hline & & 0 & $4.25 \mathrm{E}-07$ & & & 0 & 7.95E-08 \\
\hline & & & & & & 0 & 4.89E-07 \\
\hline \multirow[t]{5}{*}{23} & 12 & 0 & 4.42E-09 & 40 & 12 & 0 & 1.61E-09 \\
\hline & & 0 & $1.40 \mathrm{E}-08$ & & & 0 & 3.80E-09 \\
\hline & & 0 & 5.91E-08 & & & 0 & 1.53E-08 \\
\hline & & 0 & 4.53E-07 & & & 0 & $9.03 \mathrm{E}-08$ \\
\hline & & & & & & 0 & 4.56E-07 \\
\hline
\end{tabular}


Table 2.4. (cont.) List of SPFT conditions used for ORPLG9. Measured test temperatures are provided in Appendix A.

\begin{tabular}{|c|c|c|c|c|c|c|c|}
\hline $\begin{array}{l}\text { Temp } \\
{ }^{\circ} \mathrm{C}\end{array}$ & $\begin{array}{c}\mathrm{pH} \\
@ 23^{\circ} \mathrm{C}\end{array}$ & $\begin{array}{c}\text { Nominal Inlet Si } \\
\text { ppm }\end{array}$ & $\begin{array}{c}\text { Initial } \mathrm{q} / \mathrm{S} \\
\mathrm{m} / \mathrm{s}\end{array}$ & $\begin{array}{l}\text { Temp } \\
{ }^{\circ} \mathrm{C}\end{array}$ & $\begin{array}{c}\mathrm{pH} \\
@ 23^{\circ} \mathrm{C}\end{array}$ & $\begin{array}{c}\text { Nominal Inlet Si } \\
\text { ppm }\end{array}$ & $\begin{array}{c}\text { Initial } \mathrm{q} / \mathrm{S} \\
\mathrm{m} / \mathrm{s}\end{array}$ \\
\hline \multirow[t]{9}{*}{70} & 9 & 0 & $1.12 \mathrm{E}-08$ & 90 & 9 & 0 & $1.18 \mathrm{E}-08$ \\
\hline & & 0 & 7.83E-08 & & & 0 & 7.38E-08 \\
\hline & & 0 & 4.35E-07 & & & 0 & 4.42E-07 \\
\hline & & 0 & $1.90 \mathrm{E}-06$ & & & 0 & 1.82E-06 \\
\hline & & 30 & 4.55E-09 & & & 40 & 2.79E-09 \\
\hline & & 60 & $6.22 \mathrm{E}-09$ & & & 70 & 8.11E-09 \\
\hline & & 90 & 7.04E-09 & & & 100 & 8.20E-09 \\
\hline & & & & & & 100 & $1.68 \mathrm{E}-08$ \\
\hline & & & & & & 100 & 2.77E-08 \\
\hline \multirow[t]{4}{*}{70} & 10 & 0 & 1.03E-08 & 90 & 10 & 0 & 1.09E-08 \\
\hline & & 0 & 7.90E-08 & & & 0 & $7.26 \mathrm{E}-08$ \\
\hline & & 0 & 4.62E-07 & & & 0 & 4.36E-07 \\
\hline & & 0 & $1.78 \mathrm{E}-06$ & & & 0 & $1.79 \mathrm{E}-06$ \\
\hline \multirow[t]{4}{*}{70} & 11 & 0 & 2.12E-09 & 90 & 11 & 0 & $1.24 \mathrm{E}-08$ \\
\hline & & 0 & 3.73E-08 & & & 0 & 3.59E-08 \\
\hline & & 0 & $1.53 \mathrm{E}-07$ & & & 0 & $1.47 \mathrm{E}-07$ \\
\hline & & 0 & 8.89E-07 & & & 0 & 8.70E-07 \\
\hline \multirow[t]{4}{*}{70} & 12 & 0 & $6.00 \mathrm{E}-09$ & 90 & 12 & 0 & $4.89 \mathrm{E}-09$ \\
\hline & & 0 & 3.49E-08 & & & 0 & 2.67E-08 \\
\hline & & 0 & $1.57 \mathrm{E}-07$ & & & 0 & $1.40 \mathrm{E}-07$ \\
\hline & & 0 & 8.97E-07 & & & 0 & 8.59E-07 \\
\hline
\end{tabular}


Table 2.5. List of SPFT conditions used for IDF18-A161. Measured test temperatures are provided in Appendix A.

\begin{tabular}{|c|c|c|c|c|c|c|c|}
\hline $\begin{array}{l}\text { Temp } \\
{ }^{\circ} \mathrm{C}\end{array}$ & $\begin{array}{c}\mathrm{pH} \\
@ 23^{\circ} \mathrm{C}\end{array}$ & $\begin{array}{c}\text { Nominal Inlet Si } \\
\text { ppm }\end{array}$ & $\begin{array}{c}\text { Initial q/S } \\
\mathrm{m} / \mathrm{s}\end{array}$ & $\begin{array}{l}\text { Temp } \\
{ }^{\circ} \mathrm{C}\end{array}$ & $\begin{array}{c}\mathrm{pH} \\
@ 23^{\circ} \mathrm{C}\end{array}$ & $\begin{array}{c}\text { Nominal Inlet Si } \\
\text { ppm }\end{array}$ & $\begin{array}{c}\text { Initial } \mathrm{q} / \mathrm{S} \\
\mathrm{m} / \mathrm{s}\end{array}$ \\
\hline \multirow[t]{11}{*}{23} & 9 & 0 & 9.95E-09 & 40 & 9 & 0 & 1.17E-09 \\
\hline & & 0 & 3.01E-08 & & & 0 & 7.34E-09 \\
\hline & & 0 & 1.37E-07 & & & 0 & 2.90E-08 \\
\hline & & 0 & $7.84 \mathrm{E}-07$ & & & 0 & $1.40 \mathrm{E}-07$ \\
\hline & & 0 & 5.62E-07 & & & 0 & 6.70E-07 \\
\hline & & 1 & 3.38E-09 & & & 0 & 7.47E-07 \\
\hline & & 3 & 3.09E-09 & & & 3 & 1.19E-08 \\
\hline & & 5 & 2.91E-09 & & & 5 & $1.18 \mathrm{E}-08$ \\
\hline & & 20 & $3.21 \mathrm{E}-09$ & & & 10 & 5.83E-09 \\
\hline & & 30 & 3.04E-09 & & & 30 & 4.48E-09 \\
\hline & & & & & & 50 & 5.86E-09 \\
\hline \multirow[t]{2}{*}{23} & 10 & 0 & 7.98E-07 & 40 & 10 & 0 & 7.28E-07 \\
\hline & & 0 & $6.42 \mathrm{E}-07$ & & & 0 & 9.20E-07 \\
\hline \multirow[t]{2}{*}{23} & 11 & 0 & 1.62E-07 & 40 & 11 & 0 & 4.29E-07 \\
\hline & & 0 & 4.40E-07 & & & 0 & 3.09E-07 \\
\hline \multirow[t]{2}{*}{23} & 12 & 0 & 4.30E-07 & 40 & 12 & 0 & $4.20 \mathrm{E}-07$ \\
\hline & & 0 & 4.42E-07 & & & 0 & 3.54E-07 \\
\hline \multirow[t]{10}{*}{70} & 9 & 0 & $6.28 \mathrm{E}-09$ & 90 & 9 & 0 & $1.25 \mathrm{E}-08$ \\
\hline & & 0 & 8.29E-08 & & & 0 & 7.03E-08 \\
\hline & & 0 & 4.29E-07 & & & 0 & 4.29E-07 \\
\hline & & 0 & 1.64E-06 & & & 0 & 1.70E-06 \\
\hline & & 0 & 1.85E-06 & & & 0 & 9.72E-07 \\
\hline & & 5 & 6.35E-09 & & & 10 & $1.84 \mathrm{E}-08$ \\
\hline & & 10 & $1.19 \mathrm{E}-08$ & & & 20 & $1.89 \mathrm{E}-08$ \\
\hline & & 30 & 5.11E-09 & & & 40 & 7.26E-09 \\
\hline & & 60 & 4.41E-09 & & & 70 & 7.43E-09 \\
\hline & & 90 & 5.50E-09 & & & 100 & 8.53E-09 \\
\hline \multirow[t]{2}{*}{70} & 10 & 0 & $1.73 \mathrm{E}-06$ & 90 & 10 & 0 & 1.69E-06 \\
\hline & & 0 & 2.40E-06 & & & 0 & $9.61 \mathrm{E}-07$ \\
\hline \multirow[t]{2}{*}{70} & 11 & 0 & $8.39 \mathrm{E}-07$ & 90 & 11 & 0 & 8.81E-07 \\
\hline & & 0 & 7.67E-07 & & & 0 & 4.58E-08 \\
\hline \multirow[t]{2}{*}{70} & 12 & 0 & $6.52 \mathrm{E}-07$ & 90 & 12 & 0 & 8.88E-07 \\
\hline & & 0 & 7.19E-07 & & & 0 & 4.09E-07 \\
\hline
\end{tabular}




\subsubsection{Solution Preparation}

The solutions used to control the $\mathrm{pH}$ were $0.05 \mathrm{M}$ tris(hydroxymethyl)aminomethane (TRIS) (pH 9 and 10) or $\mathrm{LiOH} / \mathrm{LiCl}(\mathrm{pH} 11$ and 12) made with deionized water $(18.2 \mathrm{M} \Omega \cdot \mathrm{cm})$ and adjusted to the desired $\mathrm{pH}$ with either $15.8 \mathrm{M} \mathrm{HNO}_{3}$ or $1 \mathrm{M} \mathrm{LiOH}$. Because the in situ $\mathrm{pH}$ of the solution can vary by as much as $1.5 \mathrm{pH}$ units over the temperature range from $23^{\circ} \mathrm{C}$ to $90^{\circ} \mathrm{C}$, the modeled composition of the influent at the various temperatures and $\mathrm{pH}$ values is presented in Table 2.6. The table also includes the computed in situ $\mathrm{pH}$ of the solution at the various temperatures. The values are taken from Pierce et al. (2004) and were computed from the chemical composition of the buffered solution with the EQ3NR thermodynamic software package (Wolery and Daveler 1992).

Table 2.6. Composition of solutions used in SPFT experiments where the effect of pH was investigated (Pierce et al. 2004).

\begin{tabular}{cccccc}
\hline & & \multicolumn{4}{c}{$\mathrm{pH} @$} \\
Solution & Composition & $23{ }^{\circ} \mathrm{C}$ & $40{ }^{\circ} \mathrm{C}$ & $70{ }^{\circ} \mathrm{C}$ & $90{ }^{\circ} \mathrm{C}$ \\
\hline 1 & $0.05 \mathrm{M}$ TRIS + 0.004 $\mathrm{M} \mathrm{HNO}_{3}$ & 8.99 & 8.67 & 8.08 & 7.72 \\
2 & $0.05 \mathrm{M}$ TRIS $+0.034 \mathrm{M} \mathrm{LiOH}$ & 9.99 & 9.55 & 8.88 & 8.52 \\
3 & $0.0010 \mathrm{M} \mathrm{LiOH}+0.001 \mathrm{M} \mathrm{LiCl}$ & 11.00 & 10.89 & 10.43 & 10.06 \\
4 & $0.0107 \mathrm{M} \mathrm{LiOH}+0.01 \mathrm{M} \mathrm{LiCl}$ & 12.02 & 11.74 & 11.08 & 10.70 \\
\hline
\end{tabular}

For select experiments performed at pH (RT) 9, the influent solution Si concentration was varied from dilute to saturated with respect to amorphous silica $\left[\mathrm{SiO}_{2}(\mathrm{am})\right]$ solubility at various temperatures. To produce the desired concentration of $\mathrm{Si}$, analytical grade silicic acid powder (Fisher, $\mathrm{SiO}_{2} \cdot \mathrm{H}_{2} \mathrm{O}$ ) was dissolved in a solution of $\mathrm{pH}$ (RT) 9 and $0.05 \mathrm{M}$ TRIS and the mixture was heated at $90{ }^{\circ} \mathrm{C}$ for three days to achieve saturation ( $140 \mathrm{ppm}$ ). This solution was then diluted to the target Si concentration for the desired test. Some of the calculations for the pseudo-equilibrium constant for glass, $\mathrm{K}_{\mathrm{g}}$, used $\mathrm{Si}$ concentration values that were imposed by the dissolving glass. The measured Si values used in these calculations can be determined from the blank analyses in Appendix B.

\subsubsection{Dissolution Rate and Error Calculations}

In flow-through experiments with high solution residence times, the normalized dissolution rate can be calculated from two consecutive samplings, $i$ and $i+1$, from the following expression (Lasaga 1998):

$$
r_{i+1}=\frac{\left(\frac{C_{i+1}-C_{i}}{\left(t_{i+1}-t_{i}\right)}\right)+\left(\frac{q}{V} \cdot C_{i+1}\right)}{f_{i} \cdot S / V}
$$

where $\quad r_{i+1}=$ the normalized leaching rate $\left(\mathrm{g} \mathrm{m}^{-2} \cdot \mathrm{d}^{-1}\right)$ at time $t$ in days,

$q=$ the flow rate $\left(\mathrm{m}^{3} \mathrm{~d}^{-1}\right)$,

$V=$ the volume of solution $\left(\mathrm{m}^{3}\right)$,

$S=$ the glass surface area available for leaching $\left(\mathrm{m}^{2}\right)$

$C_{i}=$ the concentration of the element $i$ in the solution $\left(\mathrm{g} \mathrm{m}^{-3}\right)$

$f_{i}=$ the mass fraction of element $i$ in the pristine glass (unitless) 


$$
\left(t_{i+1}-t_{i}\right)=\text { the time difference between two samples }(\mathrm{d})
$$

When the system has reached a steady state (i.e., $C_{i+1}=C_{i}$ ), the first term in the numerator of Eq. 2-6 becomes zero. The steady-state dissolution rate, which also accounts for background concentrations of the analyst of interest, is then presented as:

$$
r_{i}=\frac{\left(C_{i, s}-\bar{C}_{i, b}\right) \cdot q}{f_{i} \cdot S}
$$

where $C_{i, s}$ is the steady-state concentration of element $i\left(\mathrm{~g} \mathrm{~m}^{-3}\right)$ and $\bar{C}_{i, b}$ is the average background concentration of element $i$ in the blank solutions collected at the beginning of the experiment $\left(\mathrm{g} \mathrm{m}^{-3}\right)$. The target concentration listed in Table 2.1 was used to calculate $f_{i}$. In the cases where the background concentration of an element was above the EQL, the average concentration of element $i$ in the three blank samples was used as the value of $\bar{C}_{i, b}$. For the case where all three blank samples were below the EQL, a background of zero was assumed. This gives the most conservative calculation of the glass dissolution rate.

Determination of the experimental uncertainty of the dissolution rate takes into account uncertainties of each parameter in Eq. 2-7. For uncorrelated random errors, the standard deviation of a function of the form $f\left(x_{1}, x_{2}, x_{n}\right)$ is estimated using the standard, first-order error propagation method:

$$
\sigma_{f}=\sqrt{\sum_{i=1}^{n}\left(\frac{\partial f}{\partial x_{i}}\right)^{2} \sigma_{i}^{2}}
$$

where

$$
\begin{aligned}
\sigma_{f} & =\text { standard deviation of the function } f \\
x_{i} & =\text { parameter } i \\
\sigma_{i} & =\text { standard deviation of parameter } i
\end{aligned}
$$

Substitution of Eq.2-8 in Eq.2-7 results in:

$$
\sigma_{r_{i}}=\sqrt{\left(\left(\frac{q}{f_{i}^{2} S}\right)^{2}\left(\sigma_{C_{i, s}}^{2}+\sigma_{\bar{C}_{i, b}}^{2}\right)+\left(\frac{C_{i, s}-\bar{C}_{i, b}}{f_{i} S}\right)^{2} \sigma_{q}^{2}+\left(\frac{\left(C_{i, s}-\bar{C}_{i, b}\right) q}{f_{i}^{2} S}\right)^{2} \sigma_{f_{i}}^{2}+\left(\frac{\left(C_{i, s}-\bar{C}_{i, b}\right) q}{f_{i} S^{2}}\right)^{2} \sigma_{S}^{2}\right)}
$$

If the relative error, $\hat{\sigma}_{r_{i}}=\sigma_{r_{i}} / r_{i}$, is used in Eq.2-9, the following equation is obtained:

$$
\hat{\sigma}_{r_{i}}=\sqrt{\left(\frac{\left(\hat{\sigma}_{C_{i}, s} C_{i, s}\right)^{2}+\left(\hat{\sigma}_{\bar{C}_{i}, b} \bar{C}_{i, b}\right)^{2}}{\left(C_{i, s}-\bar{C}_{i, b}\right)^{2}}+\hat{\sigma}_{q}^{2}+\hat{\sigma}_{f_{i}}^{2}+\hat{\sigma}_{S}^{2}\right)}
$$

Relative errors of $10 \%, 10 \%, 3 \%$, and $15 \%$ are assigned to $C_{i}, \bar{C}_{i, b}, f_{i}$, and $S$, respectively. Although the absolute error in $f_{i}$ is likely higher than $3 \%$, this error is systematic and therefore does not contribute 
significantly to sample-to-sample uncertainty, which is the principal error of interest here. The $10 \%$ for $C_{i}$ and $\bar{C}_{i, b}$, are from analytical uncertainty from ICP. In cases where all three blanks were below the EQL, we have made the $\bar{C}_{i, b}$ term equal to the EQL. 


\subsection{SPFT Results}

\subsection{Results of IDF18-A161}

\subsubsection{Calculation of $\eta, k_{0}$, and $E_{a}$}

As described in the previous section, the forward dissolution rate of IDF18-A161 was determined at solution $\mathrm{pH}(\mathrm{RT})$ values varied by one $\mathrm{pH}$ unit between 9 and 12 at temperatures of 23, 40, 70, and $90^{\circ} \mathrm{C}$. The in situ solution $\mathrm{pH}$ was corrected by geochemical modeling previously performed by Pierce et al. (2004) (see Table 2.6). The choice of this $\mathrm{pH}$ range is informed from previous glass dissolution experiments that have been conducted as a function of varying $\mathrm{pH}$. These studies typically show minima in the dissolution rate at near-neutral $\mathrm{pH}$ and increases in the dissolution rate as the $\mathrm{pH}$ becomes either more acidic or more alkaline (Inagaki et al. 2006, Inagaki et al. 2013). This "V-shaped” behavior is typically described with the use of Eq. 2-3 by changes in the sign of the $\eta$ value from a positive to a negative value. However, to avoid conditions where the sign on the $\eta$ term needs to be changed, and because the pore water solution at the IDF is expected to be neutral to slightly basic (Brown et al 2006), our focus in this study is on alkaline conditions.

With the set of forward rate values, it is possible to determine the three forward-rate parameters, $k_{0}, \eta$, and $E_{a}$. Because the forward rate is determined from dilute solutions [i.e. the chemical affinity term $1-$ $(\mathrm{Q} / \mathrm{K})=1$ ], Eq. 2-3 simplifies to:

$$
r=k_{0} 10^{\eta[p H]} \exp \left(\frac{-E_{a}}{R T}\right)
$$

Note that the activity of $\mathrm{H}^{+}$from Eq. 2-3 is now expressed as a pH instead of hydrogen ion activity. Upon further rearrangement of Eq. 3-1 to a linear form the following expression is obtained:

$$
\log (r)=\log k_{0}+\eta[p H]-\log [e]\left(\frac{E_{a}}{R}\right)\left(\frac{1}{T}\right)
$$

When performing a linear regression with boron release rates on Eq. 3-2, the coefficients $k_{0}$, $\eta$, and $E_{a}$, which must be solved by rearranging the various terms in Eq. 3-2, are determined. A global fit of the boron data leads to more robust uncertainty calculations because it allows for a calculation of coefficients without ignoring possible correlation among data and coefficients that may occur if the various parameters were to be fit separately. From the regression the parameter values for IDF18-A161 are $\eta$ of $0.28 \pm 0.06, \log \left(k_{0}\right)=6.8 \pm 0.9\left[\mathrm{~g} \mathrm{~m}^{-2} \mathrm{~d}^{-1}\right]$ and $\mathrm{E}_{\mathrm{a}}=61 \pm 6 \mathrm{~kJ} \mathrm{~mol}^{-1}$ with a correlation coefficient $\left(\mathrm{R}^{2}\right)$ of 0.89 . The $k_{0}$ value with the common units for glass dissolution of $\mathrm{g} \mathrm{m}^{-2} \mathrm{~d}^{-1}$ is then given as $k_{0}=5.9 \times 10^{6} \mathrm{~g} \mathrm{~m}^{-2} \mathrm{~d}^{-1} \pm 13 \%$. The global fit results, along with the forward rate boron release rates across all examined temperature and $\mathrm{pH}$ values, is presented in Figure 3.1. The forward boron release rates used to perform the fit are provided in Table 3.1 and Appendix A. 
Table 3.1. Forward dissolution rates for IDF18-A161. Boron release rates are used in Figure 3.1.

\begin{tabular}{|c|c|c|c|c|c|c|c|c|c|c|c|c|c|}
\hline \multirow{2}{*}{$\begin{array}{c}\text { Mean } \\
\text { Temperature } \\
{ }^{\circ} \mathrm{C} \\
22.7\end{array}$} & \multirow{2}{*}{$\begin{array}{c}\text { Nominal } \\
\mathrm{pH}(\mathrm{RT}) \\
9\end{array}$} & \multicolumn{3}{|c|}{$\begin{array}{c}\text { B release rate } \\
\mathrm{g} \mathrm{m}^{-2} \mathrm{~d}^{-1}\end{array}$} & \multicolumn{3}{|c|}{$\begin{array}{l}\text { Al release rate } \\
\mathrm{g} \mathrm{m}^{-2} \mathrm{~d}^{-1}\end{array}$} & \multicolumn{3}{|c|}{$\begin{array}{l}\text { Na release rate } \\
\mathrm{g} \mathrm{m}^{-2} \mathrm{~d}^{-1}\end{array}$} & \multicolumn{3}{|c|}{$\begin{array}{c}\text { Si release rate } \\
\mathrm{g} \mathrm{m}^{-2} \mathrm{~d}^{-1} \\
\end{array}$} \\
\hline & & 7.61E-03 & \pm & 2.01E-03 & 7.64E-03 & \pm & 2.33E-03 & $9.18 \mathrm{E}-03$ & \pm & $2.94 \mathrm{E}-03$ & 7.07E-03 & \pm & $2.10 \mathrm{E}-03$ \\
\hline 22.7 & 10 & $1.37 E-01^{*}$ & \pm & --- & 9.99E-02 & \pm & $2.41 \mathrm{E}-02$ & 1.32E-01 & \pm & 3.38E-02 & $1.14 \mathrm{E}-01$ & \pm & 2.76E-02 \\
\hline 22.7 & 11 & $1.14 \mathrm{E}-01$ & \pm & 2.71E-02 & 9.33E-02 & \pm & 2.39E-02 & 1.66E-01 & \pm & 4.78E-02 & 9.19E-02 & \pm & 2.32E-02 \\
\hline 22.7 & 12 & 3.64E-01 & \pm & 7.96E-02 & 3.88E-01 & \pm & 9.31E-02 & 4.98E-01 & \pm & $1.04 \mathrm{E}-01$ & 4.29E-01 & \pm & $1.04 \mathrm{E}-01$ \\
\hline 40.9 & 9 & 5.74E-02 & \pm & $1.58 \mathrm{E}-02$ & 4.69E-02 & \pm & $1.41 \mathrm{E}-02$ & 5.34E-02 & \pm & 1.65E-02 & 3.60E-02 & \pm & $1.02 \mathrm{E}-02$ \\
\hline 40.9 & 10 & $1.63 \mathrm{E}-01$ & \pm & 3.98E-02 & 2.79E-01 & \pm & 8.18E-02 & 2.53E-01 & \pm & 7.40E-02 & 2.26E-01 & \pm & 6.38E-02 \\
\hline 40.2 & 11 & 3.32E-01 & \pm & $9.81 \mathrm{E}-02$ & 3.10E-01 & \pm & $9.79 \mathrm{E}-02$ & 3.13E-01 & \pm & 9.94E-02 & 3.60E-01 & \pm & 1.15E-01 \\
\hline 39.9 & 12 & $5.11 \mathrm{E}-01$ & \pm & $1.37 \mathrm{E}-01$ & $6.79 \mathrm{E}-01$ & \pm & $1.94 \mathrm{E}-01$ & $7.40 \mathrm{E}-01$ & \pm & $1.87 \mathrm{E}-01$ & 7.04E-01 & \pm & $1.88 \mathrm{E}-01$ \\
\hline 71.0 & 9 & $8.50 \mathrm{E}-01$ & \pm & $1.68 \mathrm{E}-01$ & $6.25 \mathrm{E}-01$ & \pm & $1.32 \mathrm{E}-01$ & 6.34E-01 & \pm & $1.36 \mathrm{E}-01$ & 7.54E-01 & \pm & 1.63E-01 \\
\hline 71.0 & 10 & $1.56 \mathrm{E}+00$ & \pm & 4.97E-01 & $1.68 \mathrm{E}+00$ & \pm & 5.95E-01 & $1.47 \mathrm{E}+00$ & \pm & $5.20 \mathrm{E}-01$ & $1.79 \mathrm{E}+00$ & \pm & 6.37E-01 \\
\hline 70.1 & 11 & $2.94 \mathrm{E}+00$ & \pm & 8.22E-01 & $2.62 \mathrm{E}+00$ & \pm & 7.45E-01 & $2.76 \mathrm{E}+00$ & \pm & 7.86E-01 & $2.72 \mathrm{E}+00$ & \pm & 7.75E-01 \\
\hline 70.1 & 12 & $3.93 \mathrm{E}+00$ & \pm & $1.28 \mathrm{E}+00$ & $4.06 \mathrm{E}+00$ & \pm & $1.34 \mathrm{E}+00$ & $3.67 \mathrm{E}+00$ & \pm & $1.18 \mathrm{E}+00$ & $3.75 \mathrm{E}+00$ & \pm & $1.20 \mathrm{E}+00$ \\
\hline 90.6 & 9 & $1.61 \mathrm{E}+00$ & \pm & 2.97E-01 & $1.52 \mathrm{E}+00$ & \pm & 3.03E-01 & $1.58 \mathrm{E}+00$ & \pm & 3.19E-01 & $1.79 \mathrm{E}+00$ & \pm & 3.62E-01 \\
\hline 90.6 & 10 & $2.73 E+00$ & \pm & $6.28 \mathrm{E}-01$ & $2.59 \mathrm{E}+00$ & \pm & 6.23E-01 & $3.41 \mathrm{E}+00$ & \pm & 8.31E-01 & $3.10 \mathrm{E}+00$ & \pm & 7.49E-01 \\
\hline 90.5 & 11 & $4.92 \mathrm{E}+00$ & \pm & $1.48 \mathrm{E}+00$ & $3.78 \mathrm{E}+00$ & \pm & $1.15 \mathrm{E}+00$ & $5.15 \mathrm{E}+00$ & \pm & $1.57 \mathrm{E}+00$ & $4.29 \mathrm{E}+00$ & \pm & $1.30 \mathrm{E}+00$ \\
\hline 90.5 & 12 & $3.15 E+00$ & \pm & $1.26 \mathrm{E}+00$ & $2.40 \mathrm{E}+00$ & \pm & 9.65E-01 & $3.36 \mathrm{E}+00$ & \pm & $1.35 \mathrm{E}+00$ & $2.54 \mathrm{E}+00$ & \pm & $1.02 \mathrm{E}+00$ \\
\hline
\end{tabular}

* This value is calculated from the Boron EQL. The measured value, which is below the EQL, is provided in Appendix B. 


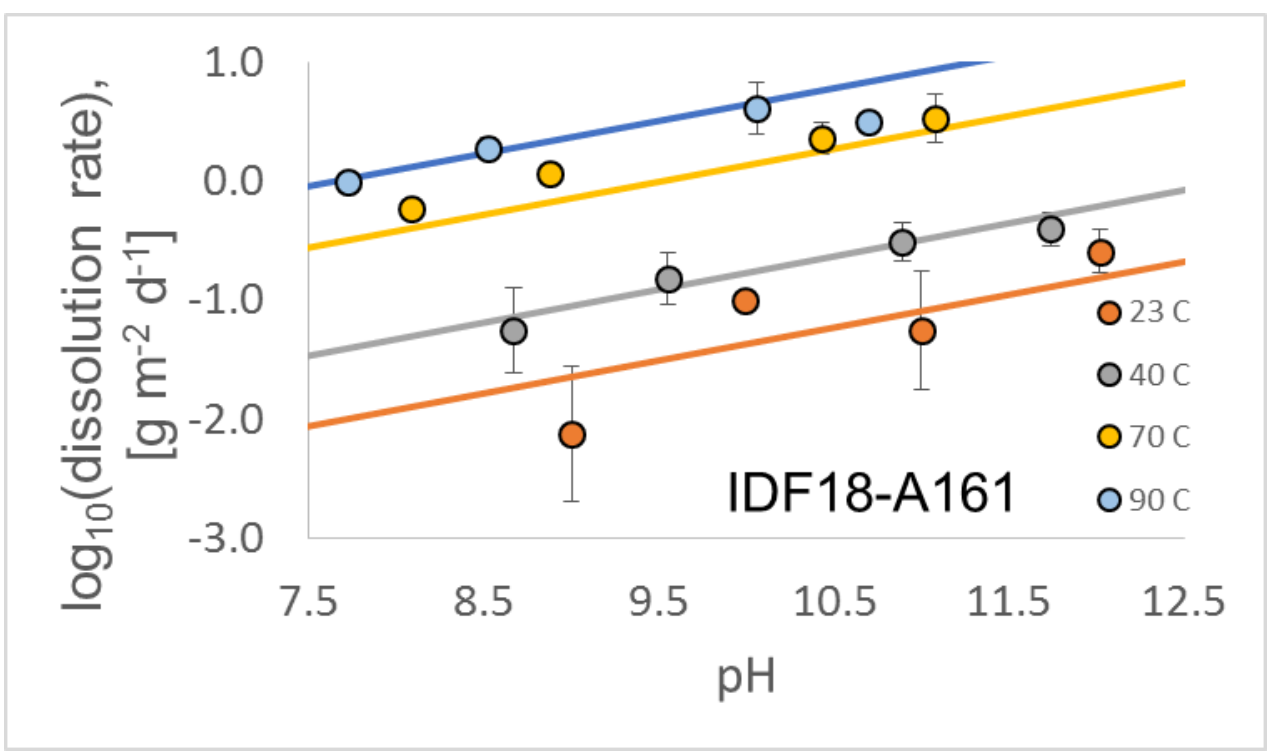

Figure 3.1. Normalized $\log _{10}$ boron release rates as a function of $\mathrm{pH}$ and temperature for IDF18-A161. Measured test temperatures are provided in Appendix A. Errors are given as $2 \sigma$ from Eq. $2-10$.

The value of $\eta=0.28 \pm 0.06$ is slightly lower than the values obtained for other ILAW glasses (see Table 4.1) and other high-level waste glass formulations (Vernaz et al. 1988, Abraitis et al. 2000b). The value of $k_{0}\left(5.9 \times 10^{6} \mathrm{~g} \mathrm{~m}^{-2} \mathrm{~d}^{-1} \pm 13 \%\right)$ is on the same order of magnitude as LD6-5412 and LAWABP1 and one to three orders of magnitude greater than LAWA44, LAWB45, LAWC22, and ORPBL2. In a sensitivity analysis by Bacon and Pierce (2010), it was shown that the glass dissolution and resulting Tc flux into the near-field environment is insensitive to the $k_{0}$ parameter at field conditions of $15{ }^{\circ} \mathrm{C}$ and a water flux of $0.9 \mathrm{~mm} / \mathrm{yr}$. The value of $\mathrm{E}_{\mathrm{a}}\left(61 \pm 6 \mathrm{~kJ} \mathrm{~mol}^{-1}\right)$ is similar to the other ILAW glasses (see Table 4.1), slightly lower than several $\mathrm{SiO}_{2}$ polymorphs [quartz: 66-83 $\mathrm{kJ} \mathrm{mol}^{-1}$ (Dove 1994, Rimstidt et al. 2016), $\mathrm{SiO}_{2}(\mathrm{am}$ ): $74.5 \mathrm{~kJ} \mathrm{~mol}^{-1}$ (Icenhower and Dove 2000), cristobalite: $69 \mathrm{~kJ} \mathrm{~mol}^{-1}$ (Renders et al. 1995)] and simulated high-level waste (HLW) glasses [SON68: $76 \mathrm{~kJ} \mathrm{~mol}^{-1}$ (Frugier et al. 2008), PNL 76-68: $74 \mathrm{~kJ} \mathrm{~mol}^{-1}$ (Strachan 1983), ISG: 62-77 $\mathrm{kJ} \mathrm{mol}^{-1}$ (Inagaki et al. 2013)].

\subsubsection{Effect of Silicon Concentration}

The normalized release rates for $\mathrm{B}, \mathrm{Na}$, and $\mathrm{Al}$ as a function of $a\left[\mathrm{H}_{4} \mathrm{SiO}_{4}\right]$ for experiments performed at $\mathrm{pH}(\mathrm{RT}) 9$ are presented in Figure 3.2. The data used to make the plots in Figure 3.2 are provided in Appendix A. The plots contains normalized release rates for B, Na, and Al that were determined from q/S sweeps as well as tests where $\mathrm{Si}$ was added to the influent solution. Values of $a\left[\mathrm{H}_{4} \mathrm{SiO}_{4}\right]$ were calculated from the measured $\mathrm{Si}$ in the effluent solution and not Si added to the influent solution. The value of $a\left[\mathrm{H}_{4} \mathrm{SiO}_{4}\right]$ was calculated from the silicic acid activity at the temperature and $\mathrm{pH}$ of these experiments being $\sim 89 \%$ of the total $\mathrm{Si}$ species, the other major species being $\mathrm{H}_{3} \mathrm{SiO}_{4}{ }^{-}(\sim 11 \%)$ and $\mathrm{H}_{2} \mathrm{SiO}_{4}{ }^{2-}(<<1 \%)$ (McGrail et al. 1997). Overall, the general trend in Figure 3.2 for room temperature (nominally $23^{\circ} \mathrm{C}$ ), 40,70 , and $90{ }^{\circ} \mathrm{C}$ is a decreasing dissolution rate with increasing $a\left[\mathrm{H}_{4} \mathrm{SiO}_{4}\right]$ until the dissolution rate remains relatively constant as $a\left[\mathrm{H}_{4} \mathrm{SiO}_{4}\right]$ increases. 

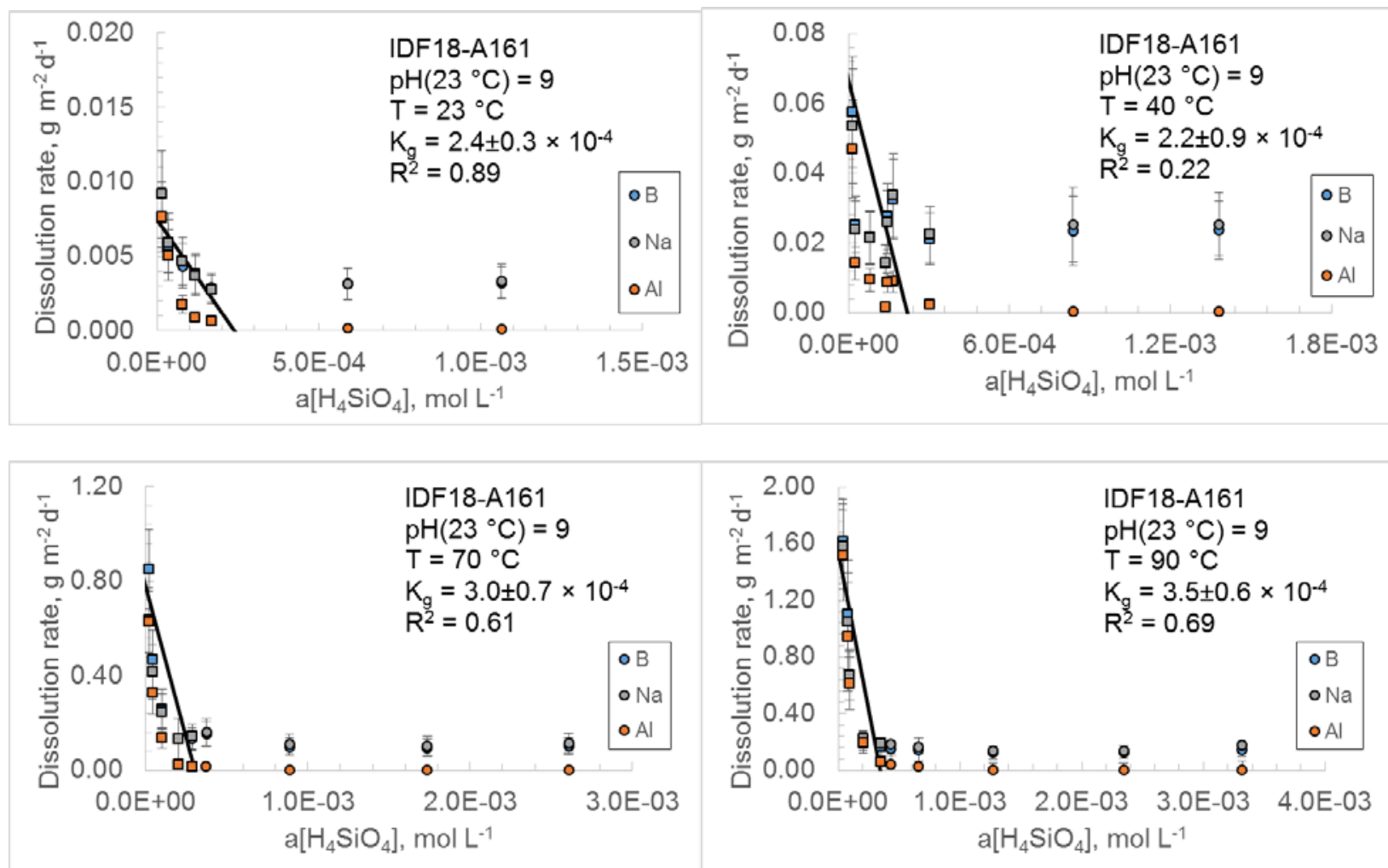

Figure 3.2. Normalized release rate with respect to $\mathrm{B}, \mathrm{Na}$, and $\mathrm{Al}$ as a function of $\mathrm{SiO}_{2}$ activity at 23, 40, 70, and $90^{\circ} \mathrm{C}$ for IDF18-A161. Measured test temperatures are provided in Appendix A. Data points with squares are from experiments used to calculate $\mathrm{K}_{\mathrm{g}}$ while data points with circles are experiments that are considered oversaturated with respect to $\mathrm{K}_{\mathrm{g}}$. The $\mathrm{SiO}_{2}$ activity is calculated from the measured $\mathrm{Si}$ in the effluent samples. Normalized dissolution rate errors (vertical error bars) are presented as $2 \sigma . a\left[\mathrm{H}_{4} \mathrm{SiO}_{4}\right]$ errors (horizontal error bars) are less than $10 \%$ and are not presented in the graph.

The data presented in Figure 3.2 are used to calculate both $\mathrm{K}_{\mathrm{g}}$ and $\mathrm{r}_{\mathrm{IEX}}$ at the assumed IDF temperature of $15^{\circ} \mathrm{C}$. In order to obtain $\mathrm{K}_{\mathrm{g}}$ at $15^{\circ} \mathrm{C}$, we first calculate $\mathrm{K}_{\mathrm{g}}$ at each individual temperature and then extrapolate to the desired temperature. The data used to calculate $\mathrm{K}_{\mathrm{g}}$ at each individual temperature are identified with square markers in Figure 3.2. The square markers are elemental release data where a decrease in the dissolution rate with increasing $a\left[\mathrm{H}_{4} \mathrm{SiO}_{4}\right]$ was observed. For the circle markers in Figure 3.2 , these values showed no response in the dissolution rate with increasing $a\left[\mathrm{H}_{4} \mathrm{SiO}_{4}\right]$ and thus were not used in the regression to determine $\mathrm{K}_{\mathrm{g}}$ at the individual temperatures. The fits at the individual temperatures are relatively poor with $\mathrm{R}^{2}$ values less than 0.90 . The poorest fit comes from the experiments at $40{ }^{\circ} \mathrm{C}$ where the $\mathrm{R}^{2}$ value is 0.22 and the argument of correlation between dissolution rates and $a\left[\mathrm{H}_{4} \mathrm{SiO}_{4}\right]$ is tenuous. For this reason, the $40{ }^{\circ} \mathrm{C}$ data were removed from the regression of $\ln \mathrm{K}_{\mathrm{g}}$ versus the inverse temperature that is presented in Figure 3.3 and this fit results in an r-squared of 0.98 . The regression of $\mathrm{K}_{\mathrm{g}}$ to $15^{\circ} \mathrm{C}$ results in a value of $2.3 \times 10^{-4}$, which may be used as $\mathrm{K}_{\mathrm{g}}$ for IDF18-A161 at the IDF temperature. 


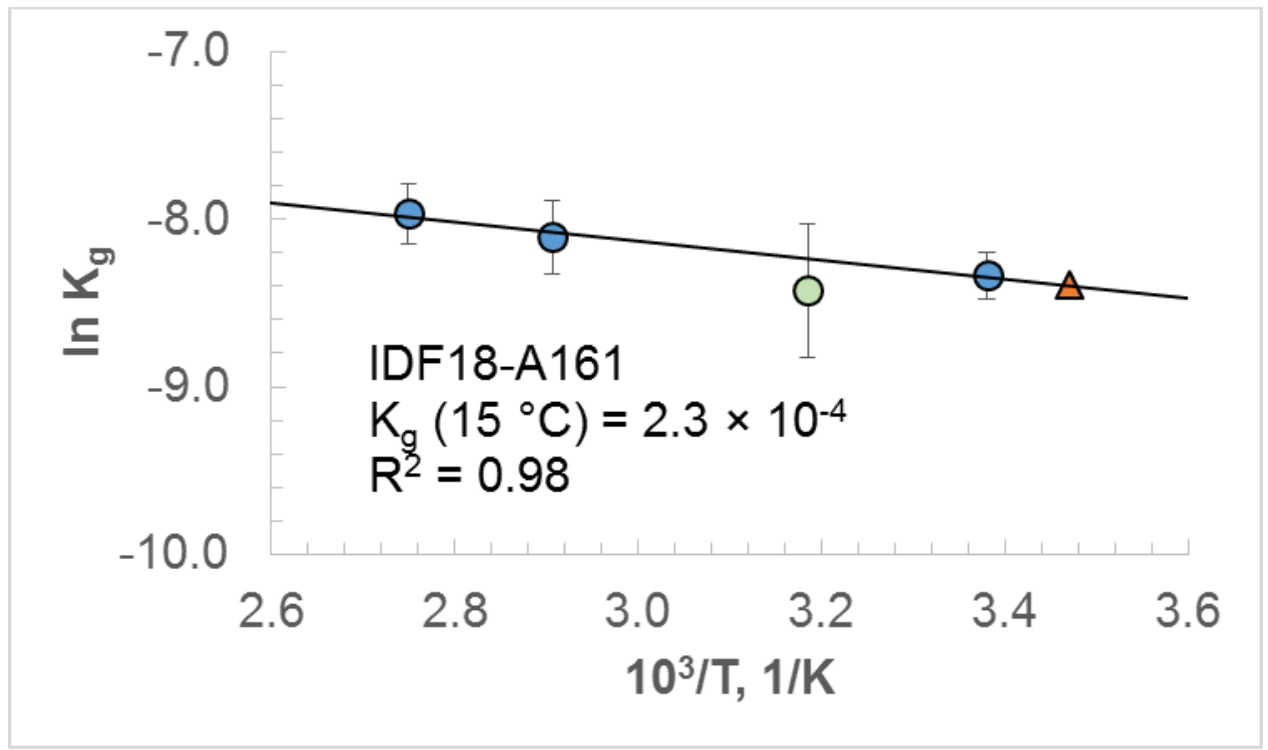

Figure 3.3. In $\mathrm{K}_{\mathrm{g}}$ vs. inverse temperature for IDF18-A161. The circles are from experimental data while the triangle is the $\ln \mathrm{K}_{\mathrm{g}}$ value calculated at $15^{\circ} \mathrm{C}$. The value of the triangle is the estimated value of $\mathrm{K}_{\mathrm{g}}$ at $15^{\circ} \mathrm{C}$.

This $\mathrm{K}_{\mathrm{g}}$ value is roughly $7 \mathrm{x}$ lower than values calculated for the ILAW glasses, LAWA44, LAWB45, LAWC22, and ORPLB2 and roughly half the value calculated and reported for LAWABP1. The $\mathrm{K}_{\mathrm{g}}$ value is $2 x$ greater than the value assigned for LD6-5412. It is noted that the $K_{g}$ value for LD6-5412 was not determined experimentally. Rather it was assigned as being equal to the $\mathrm{K}$ value for chalcedony because previous assignments for $\mathrm{K}$ values of silica polymorphs had yielded good agreement for most glasses (McGrail et al. 1997). Thus, the IDF18-A161 glass has a relatively low value of $\mathrm{K}_{\mathrm{g}}$, slightly greater than the value for chalcedony.

Because of the relatively low value of $\mathrm{K}_{\mathrm{g}}$, "saturation” would be achieved at lower orthosilicic acid concentrations compared to other ILAW glasses. Despite this, IDF18-A161 appears to have reached Stage III resumption between 56 and 119 days for a PCT-B performed at 20,000 m¹ (Muller and Pegg 2015). This result reaffirms the importance of understanding the role of secondary phases, which are allowed to form in PCT-B tests and not in SPFT tests, on the long-term performance of ILAW glass. It is unclear at this time why this particular glass composition would have a lower value of $\mathrm{K}_{\mathrm{g}}$ compared to previous ILAW glasses. Further experiments should attempt to isolate the effects of various glass components on the relative durability of the glass in various tests. The value of $\mathrm{K}_{\mathrm{g}}$ has previously been determined as the most sensitive parameter as it relates to the long-term weathering of glass (Bacon and Pierce 2010).

The last parameter investigated for IDF18-A161 is the ion-exchange rate. The ion exchange rate ( $\left.\mathrm{r}_{\text {IEX }}\right)$ is calculated with the following equation:

$$
r_{I E X}=\frac{\left(r_{N a}-r_{B}\right)}{86400 \cdot M W_{N a}}
$$

where $\mathrm{r}_{\mathrm{Na}}$ is the glass dissolution rate indexed by $\mathrm{Na}\left(\mathrm{g} \mathrm{m}^{-2} \mathrm{~d}^{-1}\right), \mathrm{r}_{\mathrm{B}}$ is the glass dissolution rate indexed by $\mathrm{B}\left(\mathrm{g} \mathrm{m}^{-2} \mathrm{~d}^{-1}\right), \mathrm{MW}_{\mathrm{Na}}$ is the molecular mass of $\mathrm{Na}\left(22.9898 \mathrm{~g} \mathrm{~mol}^{-1}\right)$, and 86400 is a factor to convert seconds to days. This calculation was performed for various tests at different temperatures where the 
normalized sodium release was in excess of the normalized boron release. The results of the natural logarithm of the ion exchange rate plotted as a function of inverse temperature are presented in Figure 3.4. The figure presents results from tests that were performed in unsaturated $\left(a\left[\mathrm{H}_{4} \mathrm{SiO}_{4}\right]<\mathrm{K}_{\mathrm{g}}\right)$ and saturated $\left(a\left[\mathrm{H}_{4} \mathrm{SiO}_{4}\right]>\mathrm{K}_{\mathrm{g}}\right)$ conditions. Though the bulk of the data in unsaturated conditions correlate well with the data from saturated conditions, the unsaturated values show more scatter. Indeed, McGrail et al. (2001) states, "under conditions of dilute solution compositions, matrix dissolution dominates so normalized boron and sodium release rates are equal. As silicon is added to solution, matrix dissolution rates decrease.” Therefore, the use of solely experimental data from saturated experiments is merited. It should be noted that previous tests on ILAW glasses have used data collected in unsaturated conditions (Pierce et al. 2004, Papathanassiu et al. 2011), however, visual inspection of those fits does not show any outlying data points that may have occurred from using ion exchange rates calculated from tests conducted in saturated conditions.

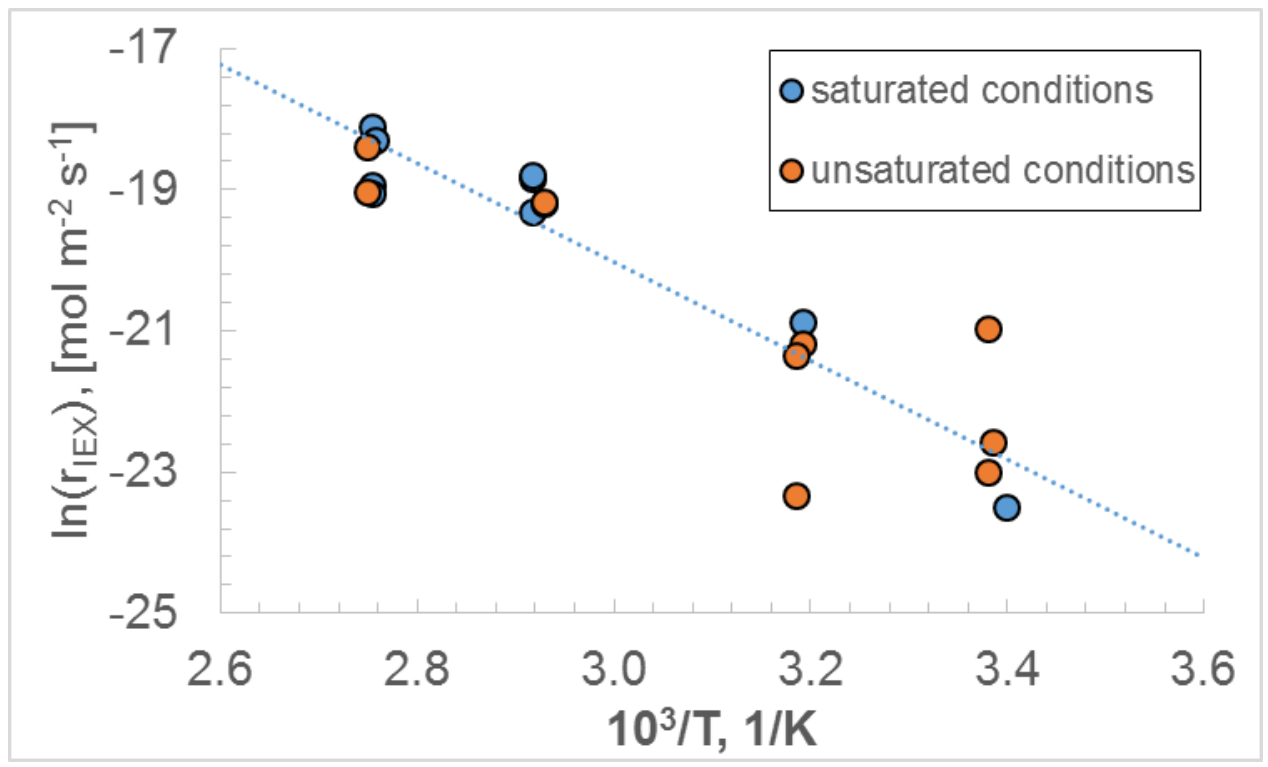

Figure 3.4. The natural logarithm of the ion exchange rate as a function of inverse temperature for IDF18-A161.

A linear regression was performed on the results from the tests performed in saturated conditions and the ion exchange rate at $15^{\circ} \mathrm{C}$ is calculated to be $7.6 \times 10^{-11} \mathrm{~mol} \mathrm{~m}^{-2} \mathrm{~s}^{-1}$. Through $1 \sigma$ error propagation of the calculated $\mathrm{r}_{\text {IEX }}$ value, the lower bound of this value is $1.8 \times 10^{-11}$ and the upper bound of this value is $3.2 \times$ $10^{-10}$, indicating a high degree of uncertainty for this parameter. The determined value of $\mathrm{r}_{\mathrm{IEX}}$ is nearest to the value of LAWA44 but when one considers the upper and lower bound, the value of $\mathrm{r}_{\text {IEX }}$ for IDF18A161 overlaps with the majority of the previously tested ILAW glasses. Indeed, a more in depth look into the physical meaning and calculation of the ion exchange rate for future ILAW glasses should be performed to ensure that this parameter is presented in the most useful fashion for future PA modeling efforts (Ryan and Freedman, 2016). 


\subsection{Results of ORPLG9}

\subsubsection{Calculation of $\eta, k_{0}$, and $\mathrm{E}_{\mathrm{a}}$}

The results for experiments performed at the four temperatures and at various $\mathrm{pH}$ values for ORPLG9 are presented in Figure 3.5. The forward dissolution rate data used to make the plots in Figure 3.5 are provided in Table 3.2 and Appendix A. In comparison to IDF18-A161 the forward boron release rates for ORPLG9 are anywhere from 2 to 10 times smaller in the same experimental conditions. The boron release rate values presented here for $\mathrm{pH}(\mathrm{RT}) 9$ at 23, 40, and $90^{\circ} \mathrm{C}$ for ORPLG9 can be compared with those obtained by VSL in that set of partial SPFT testing (Table 3.2) ${ }^{\mathrm{a}}$. From Table 6.4 in Papathanassiu et al. (2011), forward dissolution rates are given as $1.2 \pm 0.0 \times 10^{-3}, 1.28 \pm 0.11 \times 10^{-2}$, and $2.112 \pm 0.055 \times 10^{-1} \mathrm{~g} \mathrm{~m}^{-2} \mathrm{~d}^{-1}$ at 23,40 , and $90^{\circ} \mathrm{C}$, respectively. The values at these temperatures can be compared to the $\mathrm{pH}(\mathrm{RT}) 9$ values obtained in this report of $1.66 \pm 0.0 \times 10^{-3}, 7.44 \pm 2.07 \times 10^{-3}$, and $3.18 \pm 0.78 \times 10^{-1} \mathrm{~g} \mathrm{~m}^{-2} \mathrm{~d}^{-1}$ at 23,40 , and $90^{\circ} \mathrm{C}$, respectively. The calculated values from the two research groups are in good agreement, generally within a factor of two of one another.

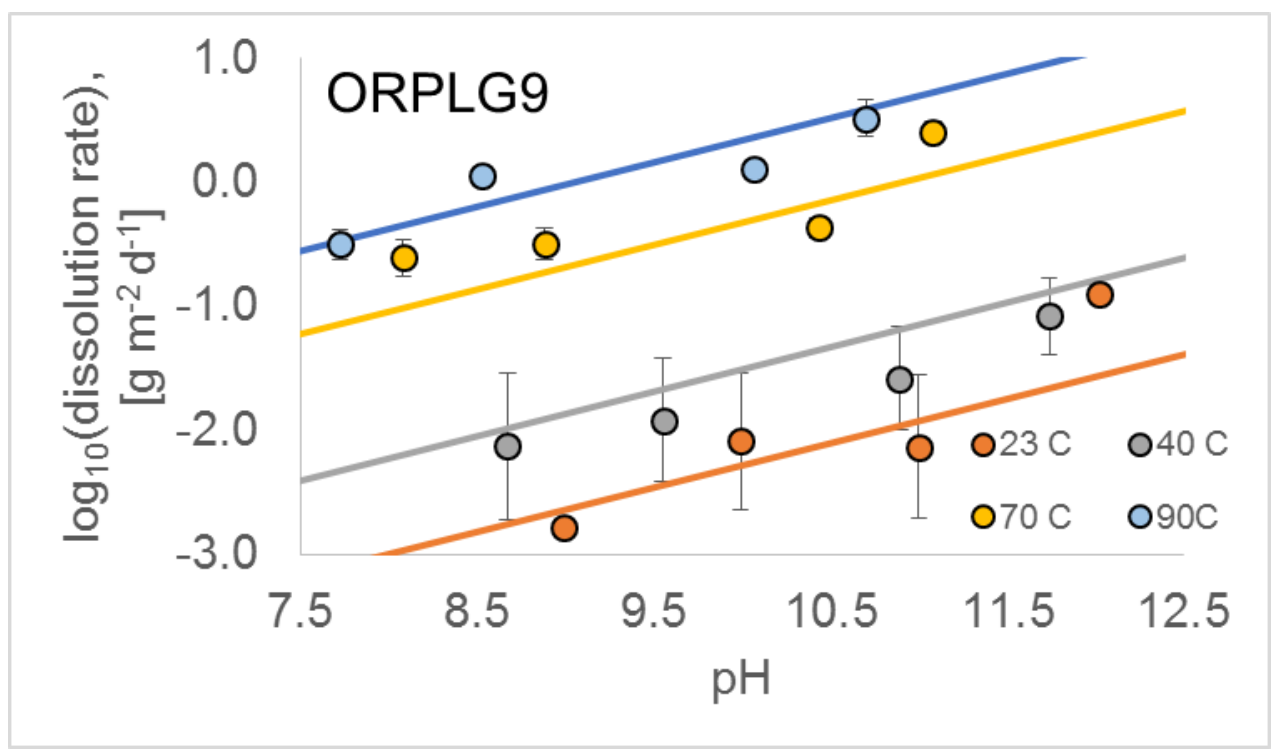

Figure 3.5. Normalized $\log _{10}$ boron release rates as a function of $\mathrm{pH}$ and temperature for ORPLG9. Measured test temperatures are provided in Appendix A. Errors are given as $2 \sigma$ from Eq. $2-10$.

\footnotetext{
${ }^{a}$ Papathanassiu et al. (2011) conducted SPFT experiments on the IDF2-G9 glass composition. From Tables 3.3 and 3.5 in that report there appear to be negligible differences between ORPLG9 and IDF2-G9.
} 
Table 3.2. Forward dissolution rates for ORPLG9. Boron release rates are used in Figure 3.5. Data are compared with data obtained in Papathanassiu et al. (2011) in similar conditions.

\begin{tabular}{|c|c|c|c|c|c|c|c|c|c|c|c|c|c|c|c|}
\hline \multirow[t]{2}{*}{$\begin{array}{c}\text { Mean } \\
\text { Temperature } \\
\\
{ }^{\circ} \mathrm{C} \\
22.7\end{array}$} & \multirow[t]{2}{*}{$\begin{array}{c}\text { Nominal } \\
\mathrm{pH}(\mathrm{RT})\end{array}$} & \multicolumn{3}{|c|}{$\begin{array}{l}\text { Data from this report } \\
\text { B release rate } \\
\qquad \mathrm{g} \mathrm{m}^{-2} \mathrm{~d}^{-1}\end{array}$} & \multicolumn{2}{|c|}{$\begin{array}{l}\text { Data from Papathanassiu et al. } \\
\qquad \begin{array}{c}\text { (2011) } \\
\text { B release rate } \\
\text { g m}^{-2} \mathrm{~d}^{-1} \\
\end{array}\end{array}$} & \multicolumn{6}{|c|}{$\begin{array}{l}\text { Data from this report } \\
\text { Na release rate } \\
\mathrm{g} \mathrm{m}^{-2} \mathrm{~d}^{-1} \\
\end{array}$} & \multicolumn{3}{|c|}{$\begin{array}{c}\text { Si release rate } \\
\mathrm{g} \mathrm{m}^{-2} \mathrm{~d}^{-1} \\
\end{array}$} \\
\hline & & $1.66 E-03^{*}$ & \pm & --- & $1.2 \mathrm{E}-03 \pm$ & 0 & $1.14 \mathrm{E}-03$ & \pm & $3.00 \mathrm{E}-04$ & $1.42 \mathrm{E}-03$ & \pm & 4.24E-04 & $6.70 \mathrm{E}-04$ & \pm & $1.76 \mathrm{E}-04$ \\
\hline 22.7 & 10 & 8.14E-03 & $I$ & 2.17E-03 & --- & & 5.74E-03 & \pm & $1.53 \mathrm{E}-03$ & 6.70E-03 & \pm & 2.05E-03 & 6.59E-03 & \pm & $2.01 \mathrm{E}-03$ \\
\hline 22.7 & 11 & 7.39E-03 & \pm & $1.99 \mathrm{E}-03$ & --- & & $6.42 \mathrm{E}-03$ & \pm & $1.94 \mathrm{E}-03$ & 8.67E-03 & \pm & $2.90 \mathrm{E}-03$ & 7.29E-03 & \pm & $2.40 \mathrm{E}-03$ \\
\hline 22.7 & 12 & $1.27 E-01^{*}$ & 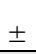 & --- & --- & & $9.36 \mathrm{E}-02$ & \pm & $2.21 \mathrm{E}-02$ & $1.68 \mathrm{E}-01$ & \pm & $3.92 \mathrm{E}-02$ & 8.33E-02 & \pm & $2.06 \mathrm{E}-02$ \\
\hline 40.9 & 9 & 7.44E-03 & $I$ & 2.07E-03 & $1.28 \mathrm{E}-02 \pm$ & 1.1E-03 & 4.68E-03 & \pm & $1.22 \mathrm{E}-03$ & 7.60E-03 & 1 & 2.31E-03 & 2.90E-03 & \pm & 7.57E-04 \\
\hline 40.9 & 10 & $1.22 \mathrm{E}-02$ & \pm & $3.14 \mathrm{E}-03$ & --- & & $1.54 \mathrm{E}-02$ & \pm & $4.78 \mathrm{E}-03$ & $1.31 \mathrm{E}-02$ & \pm & 4.22E-03 & 1.33E-02 & \pm & 4.31E-03 \\
\hline 40.2 & 11 & 2.62E-02 & \pm & $6.91 \mathrm{E}-03$ & --- & & 4.63E-02 & \pm & $1.61 \mathrm{E}-02$ & 4.19E-02 & \pm & 1.53E-02 & 4.43E-02 & \pm & $1.63 \mathrm{E}-02$ \\
\hline 39.9 & 12 & 8.35E-02 & \pm & 2.43E-02 & --- & & $1.26 \mathrm{E}-01$ & \pm & 4.17E-02 & $1.36 \mathrm{E}-01$ & \pm & 4.15E-02 & $1.19 \mathrm{E}-01$ & \pm & $3.98 \mathrm{E}-02$ \\
\hline 71.0 & 9 & 2.49E-01 & \pm & 6.15E-02 & --- & & 5.87E-02 & \pm & 1.49E-02 & 2.35E-01 & \pm & 7.00E-02 & 7.18E-02 & \pm & $1.76 \mathrm{E}-02$ \\
\hline 71.0 & 10 & 3.18E-01 & \pm & $8.21 \mathrm{E}-02$ & --- & & 3.41E-01 & \pm & $1.00 \mathrm{E}-01$ & 3.07E-01 & \pm & 9.37E-02 & 3.65E-01 & \pm & $1.13 \mathrm{E}-01$ \\
\hline 70.1 & 11 & 4.31E-01 & \pm & $1.01 \mathrm{E}-01$ & --- & & 4.28E-01 & \pm & 1.15E-01 & 3.42E-01 & \pm & $9.56 \mathrm{E}-02$ & 4.68E-01 & \pm & $1.36 \mathrm{E}-01$ \\
\hline 70.1 & 12 & $2.55 \mathrm{E}+00$ & \pm & $5.40 \mathrm{E}-01$ & --- & & $2.55 \mathrm{E}+00$ & \pm & 5.72E-01 & $2.43 E+00$ & \pm & 5.12E-01 & $2.56 \mathrm{E}+00$ & \pm & 5.74E-01 \\
\hline 90.6 & 9 & 3.18E-01 & \pm & 7.75E-02 & $2.112 \mathrm{E}-01 \pm$ & 5.5E-03 & $1.92 \mathrm{E}-01$ & \pm & 4.90E-02 & 4.36E-01 & \pm & $1.29 \mathrm{E}-01$ & 1.95E-01 & \pm & 5.37E-02 \\
\hline 90.6 & 10 & $1.15 \mathrm{E}+00$ & \pm & 2.31E-01 & --- & & $1.14 \mathrm{E}+00$ & \pm & 2.63E-01 & $1.42 \mathrm{E}+00$ & \pm & 3.55E-01 & $1.30 \mathrm{E}+00$ & \pm & 3.23E-01 \\
\hline 90.5 & 11 & $1.29 \mathrm{E}+00$ & \pm & 3.24E-01 & -- & & $1.30 \mathrm{E}+00$ & \pm & 3.52E-01 & $1.41 \mathrm{E}+00$ & \pm & 3.93E-01 & $1.43 \mathrm{E}+00$ & \pm & 3.99E-01 \\
\hline 90.5 & 12 & $3.31 \mathrm{E}+00$ & \pm & $9.27 \mathrm{E}-01$ & --- & & $3.38 \mathrm{E}+00$ & \pm & $9.75 \mathrm{E}-01$ & $4.02 \mathrm{E}+00$ & \pm & $1.07 \mathrm{E}+00$ & $3.34 \mathrm{E}+00$ & \pm & $9.72 \mathrm{E}-01$ \\
\hline
\end{tabular}

* These values are calculated from the Boron EQL. The measured value, which is below the EQL, is provided in Appendix B. 
When all boron data are regressed across all temperatures and $\mathrm{pH}$, we obtain a $\eta$ value of $0.36 \pm 0.07$ for ORPLG9. If the regression is performed for the other elements, $\eta$ values of $0.45 \pm 0.05$ (Al), $0.39 \pm 0.07$ $(\mathrm{Na})$, and $0.47 \pm 0.06(\mathrm{Si})$ are obtained. Thus, for ORPLG9, there may be a trend wherein network modifying and soluble intermediate elements (i.e. Na and B) have a slightly different pH dependence compared to the less-soluble intermediate elements and network-forming elements (i.e. $\mathrm{Al}$ and $\mathrm{Si}$ ). Reasons for this may be related to the inhomogeneity of this glass, which will be discussed below. However, given the uncertainties in the $\eta$ values, this trend may need further investigation to examine if there is indeed a pH-dependent release of elements based on their structural role in the glass. Regardless of these differences, to remain consistent with past ILAW glass parameter determination efforts, we use the value from boron and set a value of $0.36 \pm 0.07$ for ORPLG9 for the anticipated repository temperature of $15{ }^{\circ} \mathrm{C}$. The value of $0.36 \pm 0.07$ is similar to $\eta$ values obtained for other ILAW glasses (see Table 4.1).

The remaining dilute-condition variables, $k_{0}$ and $\mathrm{E}_{\mathrm{a}}$, were also determined from the boron data linear regression. The values are $\log \left(k_{0}\right)=8.4 \pm 1.0\left[\mathrm{~g} \mathrm{~m}^{-2} \mathrm{~d}^{-1}\right]$ and $\mathrm{E}_{\mathrm{a}}=81 \pm 7 \mathrm{~kJ} \mathrm{~mol}^{-1}$ with a correlation coefficient $\left(\mathrm{R}^{2}\right)$ of 0.91 . The $k_{0}$ value with the common units for glass dissolution of $\mathrm{g} \mathrm{m}^{-2} \mathrm{~d}^{-1}$ is then given as $k_{0}=2.5 \times 10^{8} \mathrm{~g} \mathrm{~m}^{-2} \mathrm{~d}^{-1} \pm 13 \%$. The fit, along with the boron release rates across all experimental temperature and $\mathrm{pH}$ values, is presented in Figure 3.5. The values of $k_{0}$ and $\mathrm{E}_{\mathrm{a}}$ are higher than any of the previously tested ILAW glasses.

\subsubsection{Effect of Silicon Concentration}

The normalized release rates for $\mathrm{B}, \mathrm{Na}$, and $\mathrm{Al}$ as a function of $a\left[\mathrm{H}_{4} \mathrm{SiO}_{4}\right]$ at $\mathrm{pH}(\mathrm{RT}) 9$ for ORPLG9 are presented in Figure 3.6. The data used to make the plots in Figure 3.6 are provided in Appendix A. Unlike IDF18-A161, there does not appear to be any linear decrease in B or Na release rates for the range of $a\left[\mathrm{H}_{4} \mathrm{SiO}_{4}\right]$ tested here. The reason for this lack of response may be the result of glass-in-glass phase separation in this glass, which will be explained in further detail below. Though an effort was made to determine $\mathrm{K}_{\mathrm{g}}$ values at the various temperatures from $\mathrm{B}$ release rates (thick black lines in Figure 3.6), the correlation between the $\mathrm{B}$ release rate and $a\left[\mathrm{H}_{4} \mathrm{SiO}_{4}\right]$ was poor, as can be seen from the low r-squared values presented in Figure 3.6. As a result, calculating a $\mathrm{K}_{\mathrm{g}}$ value for ORPLG9 was not possible. For this reason, we recommend using a large value $\left(\log \mathrm{K}_{\mathrm{g}}=100\right)$ in the thermodynamic portion of the rate model equation when modeling this glass. With a large value of $\mathrm{K}_{\mathrm{g}}$, the thermodynamic portion is essentially eliminated [i.e. 1-(Q/K) $\rightarrow 1$ ] and the dissolution rate of this glass will simply become a function of $\mathrm{pH}$ at a fixed temperature. Thus the forward rate model parameters provided above can still be used for this glass along with the $\mathrm{r}_{\mathrm{IEX}}$ term provided below and will result in a constant release rate at fixed $\mathrm{pH}$ and temperature. Stage II behavior could then only be modeled with a knowledge of the equilibrium value of the rate-controlling phase or a solid knowledge of the secondary phase reaction network.

A similar absence of an effect of the glass dissolution rate with increases in $a\left[\mathrm{H}_{4} \mathrm{SiO}_{4}\right]$ was observed by Papathanassiu et al. (2011). They performed experiments in the presence of various concentrations of $a\left[\mathrm{H}_{4} \mathrm{SiO}_{4}\right]$ at $90^{\circ} \mathrm{C}$ and $\mathrm{pH}(\mathrm{RT}) 9$ in a set of partial SPFT testing (Figure 3.7). The results in Figure 3.7 can be directly compared to the values in the bottom right plot in Figure 3.6. The results from the two groups show a similar trend and the boron release rates from both groups are within $2 \times$ of one another. Papathanassiu et al. (2011) also stated that it was not possible to estimate a $\mathrm{K}_{\mathrm{g}}$ value from the data 
obtained for ORPLG9. Similarly to what was measured in this report, the Na release rate values measured by Papathanassiu et al. (2011) were similar to B release rates.
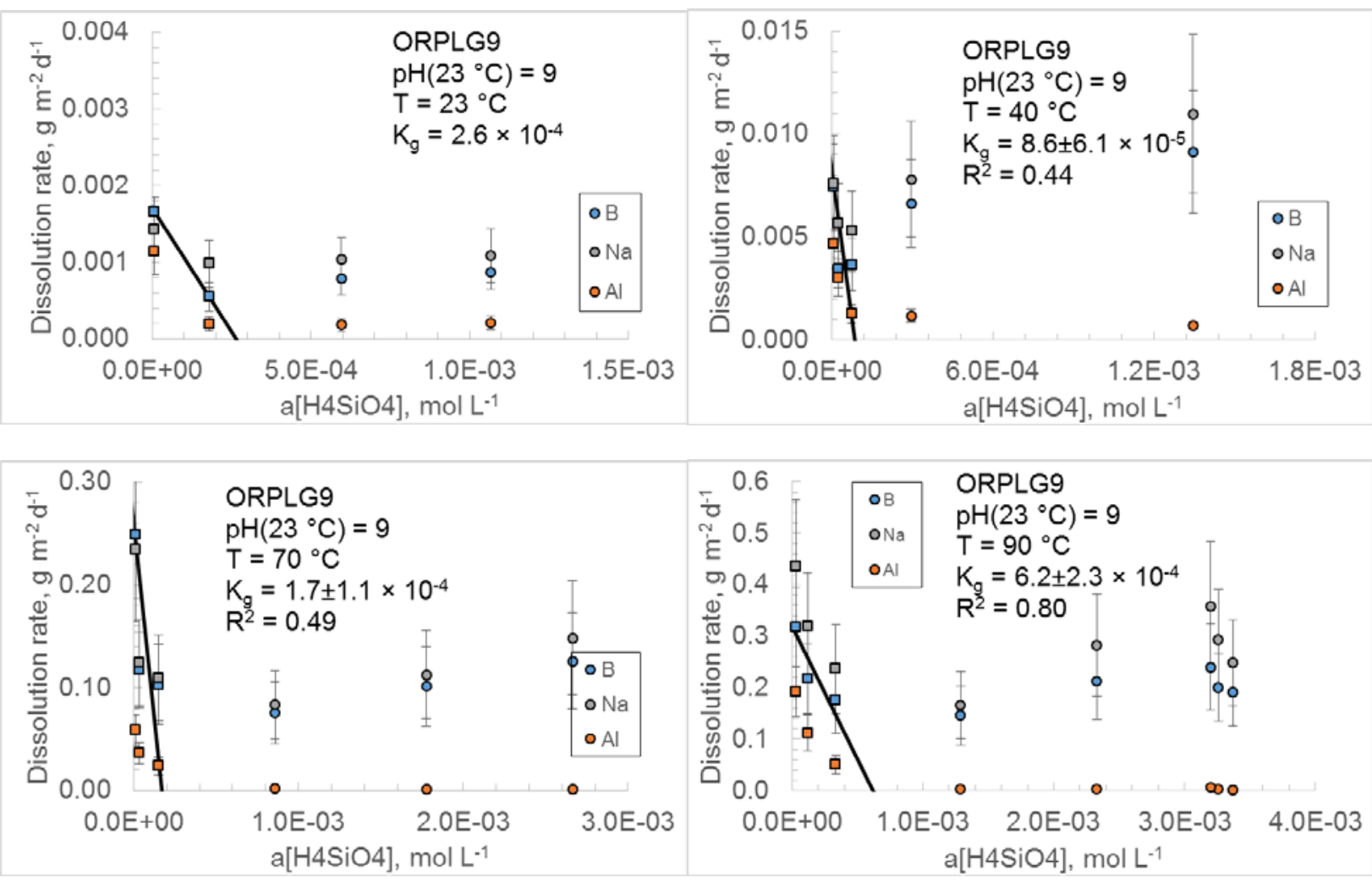

Figure 3.6. Normalized release rate with respect to $\mathrm{B}, \mathrm{Na}$, and $\mathrm{Al}$ as a function of $\mathrm{SiO}_{2}$ activity at 23, 40, 70 , and $90{ }^{\circ} \mathrm{C}$ for ORPLG9. Measured test temperatures are provided in Appendix A. Data points with squares are from experiments used to calculate $\mathrm{K}_{\mathrm{g}}$ while data points with circles are experiments that are considered oversaturated with respect to $\mathrm{K}_{\mathrm{g}}$. The $\mathrm{SiO}_{2}$ activity is calculated from the measured $\mathrm{Si}$ in the effluent samples. Normalized dissolution rate errors (vertical error bars) are presented as $2 \sigma . a\left[\mathrm{H}_{4} \mathrm{SiO}_{4}\right]$ errors (horizontal error bars) are less than $10 \%$ and are not presented in the graph. 


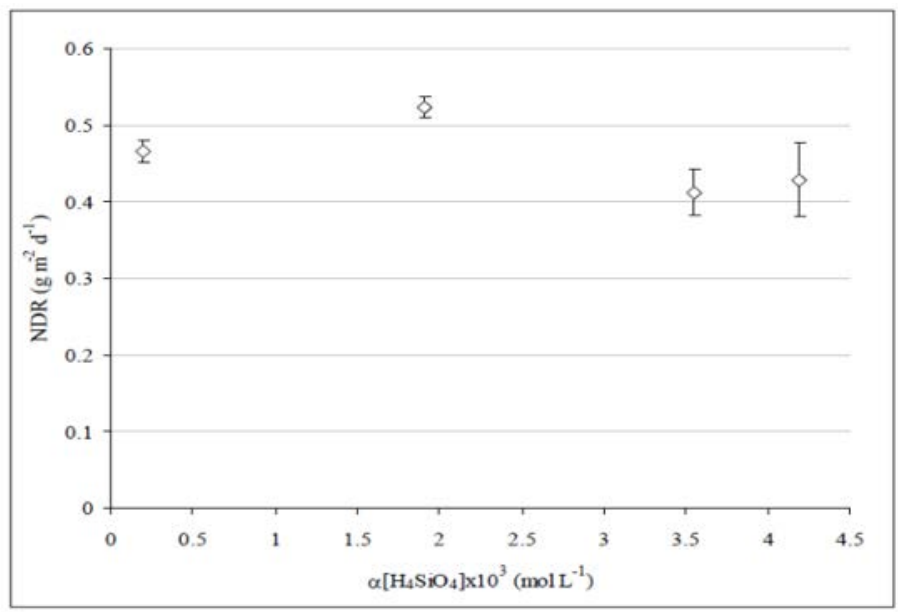

Figure 3.7. Normalized release rate with respect to boron as a function of $\mathrm{SiO}_{2}$ activity for ORPLG9. This is Figure 6.13 of Papathanassiu et al. (2011).

In addition to $\mathrm{B}$ and Na release rates, Figure 3.6 also presents the $\mathrm{Al}$ release rates for ORPLG9. For Al, there appears to be a correlation between increased $a\left[\mathrm{H}_{4} \mathrm{SiO}_{4}\right]$ and a decrease in the release rate of this element. This correlation between $\mathrm{Al}$ and increased $a\left[\mathrm{H}_{4} \mathrm{SiO}_{4}\right]$ can also be seen from the data provided in Appendix E of Papathanassiu et al. (2011).

A possible reason for a response in the $\mathrm{Al}$ release rate and not for the $\mathrm{B}$ or Na release rates is that ORPLG9 may contain more than one non-crystalline phase, principally boron-rich and silica-rich portions. Previous glasses with no response to $a\left[\mathrm{H}_{4} \mathrm{SiO}_{4}\right]$ were also believed to contain liquid-liquid phase separation (McGrail et al. 2001, Icenhower et al. 2003). We note that these amorphous phases would be in addition to the eskolaite inclusions presented in Section 2.0. Microscopic phase separated glasses, where the phases may be either crystalline or amorphous, can show agglomerations of Na accommodated in the glass structure at non-bridging oxygen (NBO) sites (Fleet and Muthupari, 1999). This creates boron-rich regions where the dissolution rate would not be affected by the activity of $\mathrm{H}_{4} \mathrm{SiO}_{4}$. Evidence of boron-rich regions and silica-rich regions is also presented in Figure 3.6, where the $\mathrm{Al}$ data have been plotted along with the $\mathrm{B}$ and $\mathrm{Na}$ data. Unlike $\mathrm{B}$ and $\mathrm{Na}$, the $\mathrm{Al}$ release rate is responsive to increases in $a\left[\mathrm{H}_{4} \mathrm{SiO}_{4}\right]$. This may be due to $\mathrm{Al}$ partitioning to the Si-rich phase and the solubility of this phase being responsive to the solution activity of $\mathrm{H}_{4} \mathrm{SiO}_{4}$.

From the solids analysis conducted before the start of the SPFT testing (Section 2.0), we were unable to identify any inhomogeneity in the glass beyond the crystalline eskolaite phase, a crystalline phase that should not affect the overall durability of the glass. However, identification of amorphous phase separation is not a straightforward task as the scale of phase separation is difficult to predict, especially with the complex glasses being used here. For the SEM/EDS analyses performed in Section 2.0, the information attainable by that instrument may have been the wrong scale to identify phase separation for the glass compositions studied here. However, due to the unexpected dissolution behavior of ORPLG9, which is indicative of phase separation, further efforts were conducted to be able to unambiguously identify amorphous phase separation in this glass.

To accomplish this, two additional techniques that were employed to be able to identify phase separation in these glasses were Transmission Electron Microscopy (TEM) and Atomic Force Microscopy (AFM). Both techniques are capable of imaging on the nm scale. The TEM samples were prepared by crushing 
the glass to a very fine powder in a tungsten ball mall and adding the crushed powder by the drop method onto lacy carbon grids. A pin-head amount of powder of each sample was placed between two glass slides, which were rubbed vigorously to further separate the powders into the smallest possible size. A lacy carbon grid was then placed onto the sheared powders in order to adhere the particles to the lacy carbon. Samples were analyzed in both TEM and Scanning TEM modes in a JEOL ARM200F aberration corrected (probe) TEM. Only ORPLG9 was analyzed with TEM.

For the AFM imaging, glass particles with a thickness of greater than $500 \mu \mathrm{m}$ in one dimension were polished with 0.25 um diamond compound [Structure Probe Inc. (SPI)] followed by chemical-mechanical polishing with $50 \mathrm{~nm}$ colloidal silica (Buehler MasterMet). The chemical-mechanical polishing is, in effect, a leach of the glass surface as it would dissolve the more soluble phase and leave behind a more durable material. After polishing, samples were cleaned with First Contact, a gel that dries onto the surface and is then peeled off to remove carbon contamination. AFM images were taken on a Veeco Icon AFM in tapping mode using Bruker RTESPA-300 probes ( $8 \mathrm{~nm}$ nominal tip radius, $40 \mathrm{~N} / \mathrm{m}$ nominal spring constant). Sample preparation and imaging with AFM was performed with both ORPLG9 and IDF18-A161.

An example of a bright field TEM image is provided in Figure 3.8 along with the EDS map from the same particle. The bright field image allows for identification of areas of differing density in the sample, principally based on differences in beam interactions with light and heavy atoms. The bright field image may also be influenced by the thickness of the sample. On the other hand, the EDS maps allow for identification of the elemental distribution in the sample. The technique remains challenging in terms of identifying phase separation in the samples because the presumed separated phases would not differ greatly in density and because light elements, including boron, are not easily identifiable by EDS. Thus, the aim was to identify areas of enrichment in either $\mathrm{Na}, \mathrm{Al}$, or $\mathrm{Si}$. Of the regions analyzed with TEM, there appears to be no differences in density or elemental distribution. Though there does not appear to be any chemical segregation of the glass in these samples, it is difficult to sort for particles which exhibit this microstructure in TEM due to: a) the small sample volume in relation to the sample size and random sampling due to the crushing technique; b) the notion that the presumably phase-separated regions are amorphous and presumably only a few percentage different in Na content; and c) the length scale of the phase separation required to be observed in these samples may preclude TEM from being the optimum analysis technique. With the only measure of phase separation being chemical mapping, combined with the idea that these glass samples are highly beam sensitive, counting statistics require regions of interest be collected for longer periods of time. This greatly decreases the chances of observing any phase separation. Regardless, of the regions analyzed, there was no indication of chemical separation, Na or otherwise. 

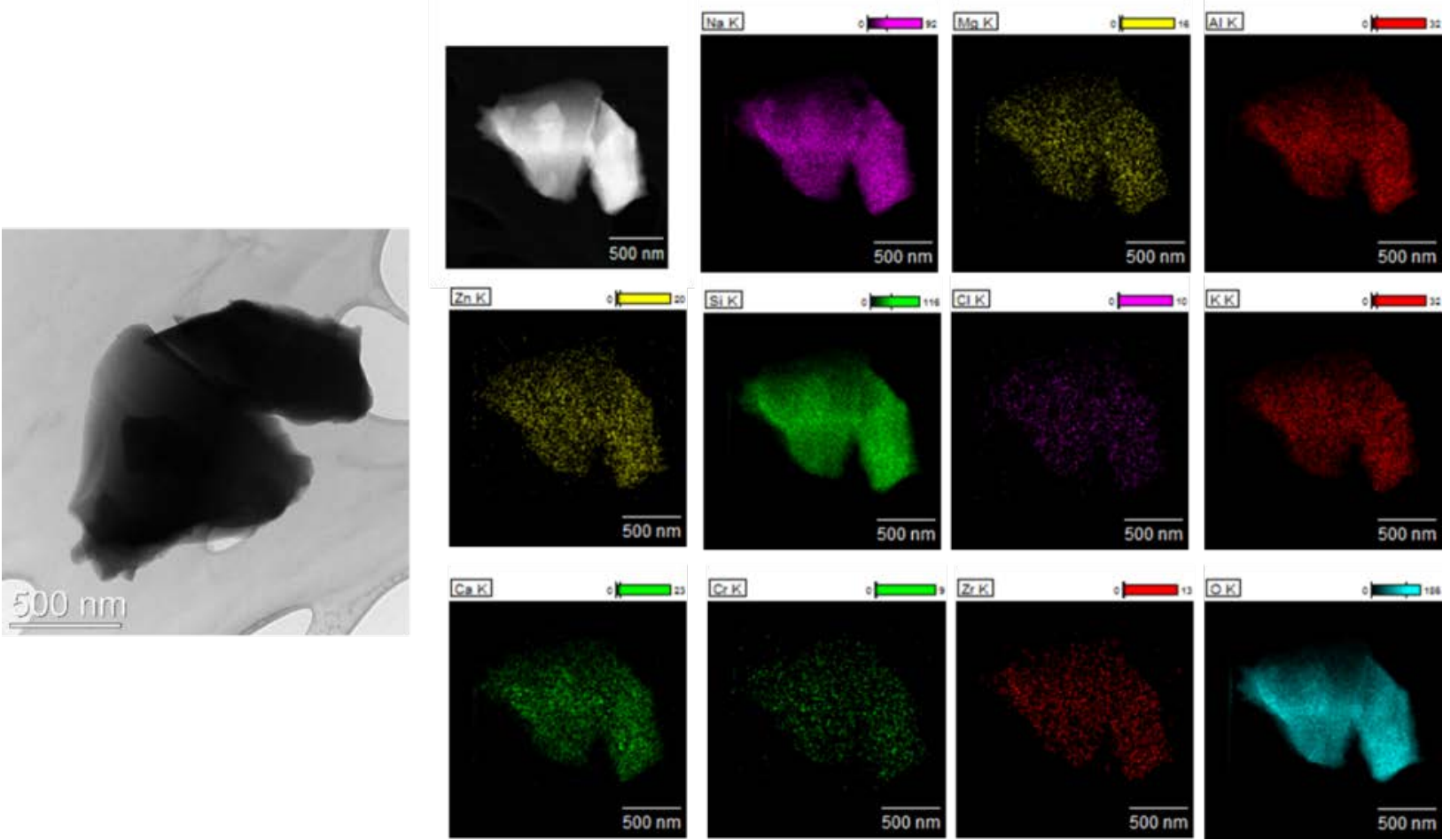

Figure 3.8. TEM (bright field) of an ORPLG9 glass particle rotated 90 degrees counter clockwise from the EDS maps.

The AFM images acquired for polished ORPLG9 and IDF18-A161 are provided in Figure 3.9 and Figure 3.10, respectively. Each figure includes a topological map and a phase map ${ }^{a}$ taken at two different length scales. The topological map provides a 3D map of the sample surface whereas the phase map provides information on surface structure and elasticity. The two methods can be used to elucidate both height and physical differences in the sample. The $2.0 \times 2.0$ - $\mu \mathrm{m}$ topological map images for ORPLG9 (Figure 3.9a) show height differences on the order of $10 \mathrm{~nm}$. The light regions of the figure are areas where that were less affected by the chemical mechanical polish. These height differences appear to correspond with, in terms of scale, differences in the corresponding phase map (Figure 3.9c). These data suggest that the ORPLG9 sample exhibits droplet in matrix phase separation and that the size of the droplets are on the order of $100 \mathrm{~nm}$. The image at the $80 \times 80-\mu \mathrm{m}$ scale (Figure $3.9 \mathrm{~b}$ ) is dominated by two large protrusions, which are presumably the chromium oxide crystalline phase that was previously identified in ORPLG9. Due to the dimensions of the protrusions, a phase map on the same scale is not presented.

\footnotetext{
${ }^{\mathrm{a}}$ The AFM phase maps are acquired with the use of tapping mode where the cantilever is excited to a resonance oscillation with a piezoelectric driver. As the cantilever moves across the sample, it interacts with the sample surface. If there is a difference in the cantilever resonance with respect to the piezo driver due to interactions, then the will be a phase lag. The method is qualitative but can be used to map differences beyond topological differences on a sample surface
} 

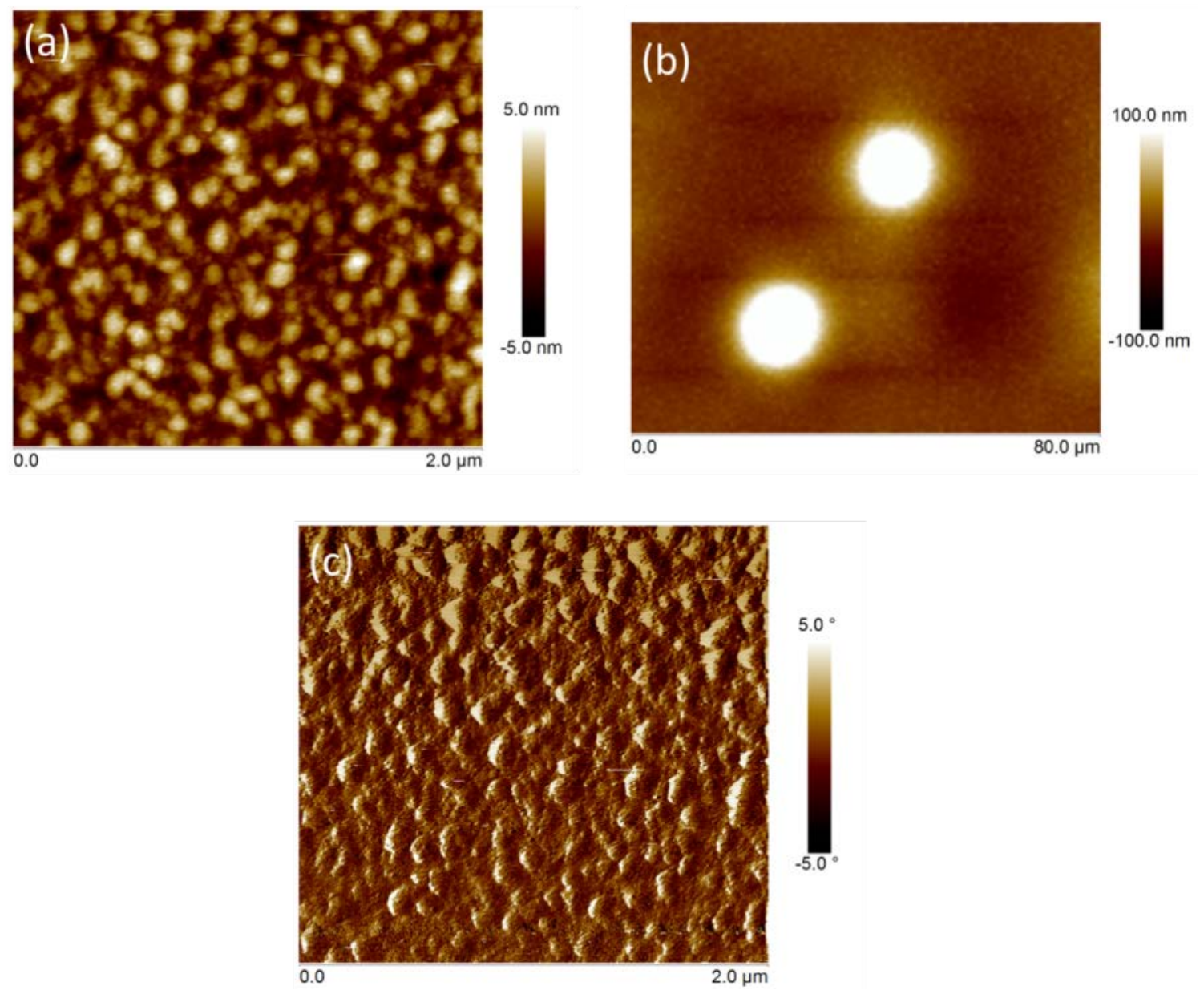

Figure 3.9. AFM images of chemically-mechanically polished samples of ORPLG9. The images on the top are topological maps of the sample at two different length scales. The image on the bottom is a phase map of the sample at a $2.0-\mu \mathrm{m}$ scale. The scales to the right of (a) and (b) are height in $\mathrm{nm}$. The scale to the right of (c) is the phase shift relative to the average phase in the horizontal direction.

In order to make a complete comparison with the features presented from the ORPLG9 sample, similar images from IDF18-A161 are presented in Figure 3.10. In contrast with ORPLG9, the images from IDF18-A161 are flatter and do not demonstrate any evidence of structure with consistent size and morphology. The $2.0 \times 2.0$ - $\mu$ m scale images (Figure 3.10a and Figure 3.10c) show the presence of some height differences, but those are on the order of $5 \mathrm{~nm}$ and the features are not evenly distributed about the surface as they are with the ORPLG9 images. The lack of features, beyond residual scratch marks from polishing, in this material is confirmed in the corresponding phase map. The $80 \times 80-\mu \mathrm{m}$ scale images (Figure 3.10b and Figure 3.10d), which are used in order to determine if the scale of phase separation is at a larger scale, show areas that differ by roughly $20 \mathrm{~nm}$ in height, however, the associated phase map does not correspond with the areas of differing height. Thus, the height differences may be an artifact of polishing. We did not observe any areas in the AFM images of IDF18-A161 that are similar to the features observed in the SEM images of this glass (Figure 2.7). The absence of evidence of phase separation in this glass is in agreement with the SPFT results, which show expected boron release rates that are linear with increasing $a\left[\mathrm{H}_{4} \mathrm{SiO}_{4}\right]$ until $\mathrm{K}_{\mathrm{g}}$ is achieved, at which point the boron release is invariant with $a\left[\mathrm{H}_{4} \mathrm{SiO}_{4}\right]$. 

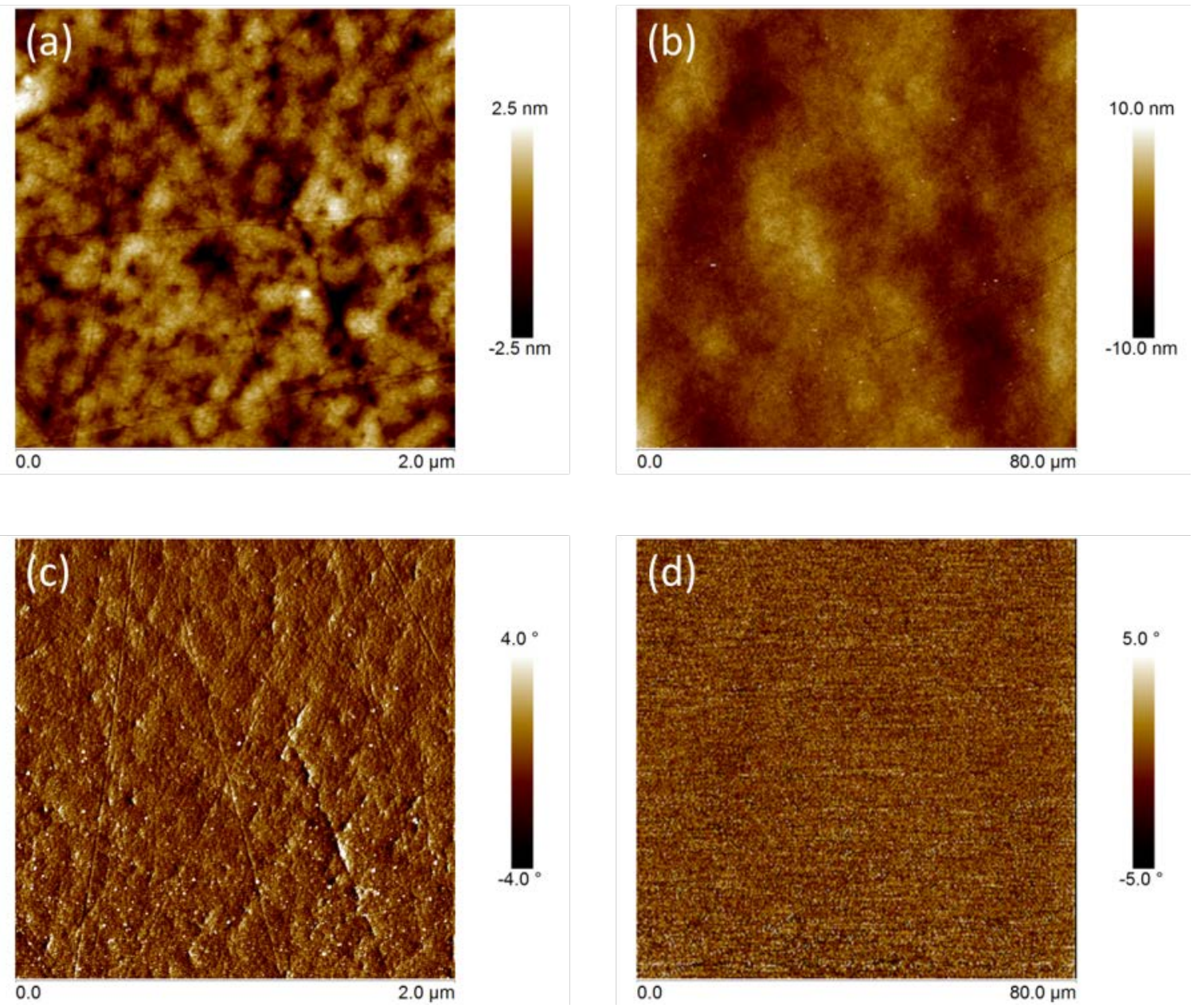

Figure 3.10. AFM images of chemically-mechanically polished samples of IDF18-A161. The images on the top are topological maps and the images on the bottom are a phase maps at two different scales. The scales to the right of (a) and (b) are height in $\mathrm{nm}$. The scale to the right of (c) and (d) is the phase shift relative to the average phase in the horizontal direction.

As noted from the SPFT data, even though ORPLG9 exhibits phase separation, the dissolution rate of this glass, in terms of B and Na release, is lower than IDF18-A161 in dilute conditions and similar to IDF18A161 in saturated conditions. To understand this, it is necessary to describe the consequences of phase separation in terms of the overall durability of the glass. As described above, when a borosilicate glass undergoes phase separation, it usually forms a Si-rich phase and a B-rich phase, with the latter presumably being less durable. Tomozawa et al. (1979) describe three microstructural types, which include phase separation from both spinodal decomposition and droplet in matrix (nucleation and growth):

Type A: Both phases have interconnected, continuous structures (spinodal decomposition).

Type B: The more durable phase is dispersed as discrete particles, or droplets, in a continuous matrix of the less durable phase.

Type C. The less durable phase is dispersed in a continuous matrix of the more durable phase, i.e., the converse of Type B. 
If a glass exhibits Type A or B microstructure the overall durability of the glass would be less than if the glass exhibits Type $\mathrm{C}$ microstructure. Thus, for some compositions, phase separation can markedly improve durability because dissolution of the more durable (matrix) phase is rate-controlling (Taylor et al. 1987). The ORPLG9 glass may exhibit Type C microstructure due to its relatively high durability in PCTB tests and the relatively low elemental releases observed from SPFT testing. Reasons for inhomogeneity in the glass structure are likely related to the high-Na content of ORPLG9. However, the exact chemical composition of each amorphous phase remains unknown. This means it is not possible to find a $\mathrm{K}_{\mathrm{g}}$ value for the TST rate equation for ORPLG9. However, when modeling ORPLG9 we recommend using a log $\mathrm{K}_{\mathrm{g}}$ value of 100 to reproduce the SPFT data. This assignment of $\mathrm{K}_{\mathrm{g}}$ is merely used so that the model can reproduce the glass corroding at a nominally forward rate as the $\mathrm{H}_{4} \mathrm{SiO}_{4}$ activity increases, which is a conservative estimate of glass corrosion behavior. Using the forward reaction rate was also recommended to model the behavior of the HLP-31 glass, though that value was merely given as a constant and no $\mathrm{pH}-$ dependence was determined (McGrail et al. 2001). Further efforts could utilize PCT-B data to refine the value of $\mathrm{K}_{\mathrm{g}}$ to a more realistic value. This is because the PCT-B data from ORPLG9 show that the dissolution rate slows with reaction time. Therefore, a rate-controlling species is released into solution, which is causing the glass dissolution rate to slow. Because the ILAW rate equation currently only accounts for $\mathrm{H}_{4} \mathrm{SiO}_{4}$ activity as the rate-controlling species, the model could attempt to vary the $\mathrm{K}_{\mathrm{g}}$ value until a value is found that fits the releases of boron from the glass. Additionally, though unexpected behavior was observed for ORPLG9, the B and Na release rates in the high-Si portion of Figure 3.6 are nearly equivalent for IDF18-A161 and ORPLG9. Thus, even if a $\mathrm{K}_{\mathrm{g}}$ value cannot be determined through SPFT testing for ORPLG9, this does not mean that the glass will behave poorly in a repository environment. One possible approach to determine the corrosion behavior of the two different glass compositions associated with the immiscibility curve, is to fabricate each glass composition separately and then test each glass to show both glass compositions are acceptable. However, that testing is not within the scope of this report.

Despite the inability to determine a value of $\mathrm{K}_{\mathrm{g}}$, a value of $\mathrm{r}_{\mathrm{IEX}}$ for ORPLG9 can be calculated by using Eq. 3-3. However, assigning a value of $\mathrm{r}_{\mathrm{IEX}}$ for a glass that possibly has more than one phase may be convoluted because $\mathrm{B}$ and $\mathrm{Na}$ would be released from two separate phases. The results of the natural logarithm of $r_{\text {IEX }}$ as a function of inverse temperature are presented in Figure 3.11. A linear regression was performed on the results from the tests performed in saturated conditions and $\mathrm{r}_{\mathrm{IEX}}$ at $15^{\circ} \mathrm{C}$ can be calculated to be $5.3 \times 10^{-11} \mathrm{~mol} \mathrm{~m}^{-2} \mathrm{~s}^{-1}$. Through $1 \sigma$ error propagation of the calculated $\mathrm{r}_{\mathrm{IEX}}$ value, the lower bound of this value is $1.7 \times 10^{-11} \mathrm{~mol} \mathrm{~m}^{-2} \mathrm{~s}^{-1}$ and the upper bound of this value is $1.7 \times 10^{-10} \mathrm{~mol} \mathrm{~m}^{-2}$ $\mathrm{s}^{-1}$. Because ORPLG9 is a high-Na glass, we would expect $\mathrm{r}_{\text {IEX }}$ to be more similar to the other high-Na glass, ORPLB2. However, as mentioned previously, ion exchange may be convoluted with this glass because B and Na would be dissolving from the B-rich phase and Na would only undergo ion exchange from the Si-rich phase. This would result in a relatively high release of B compared to Na, which would result in a lower value of $\mathrm{r}_{\mathrm{IEX}}$. 


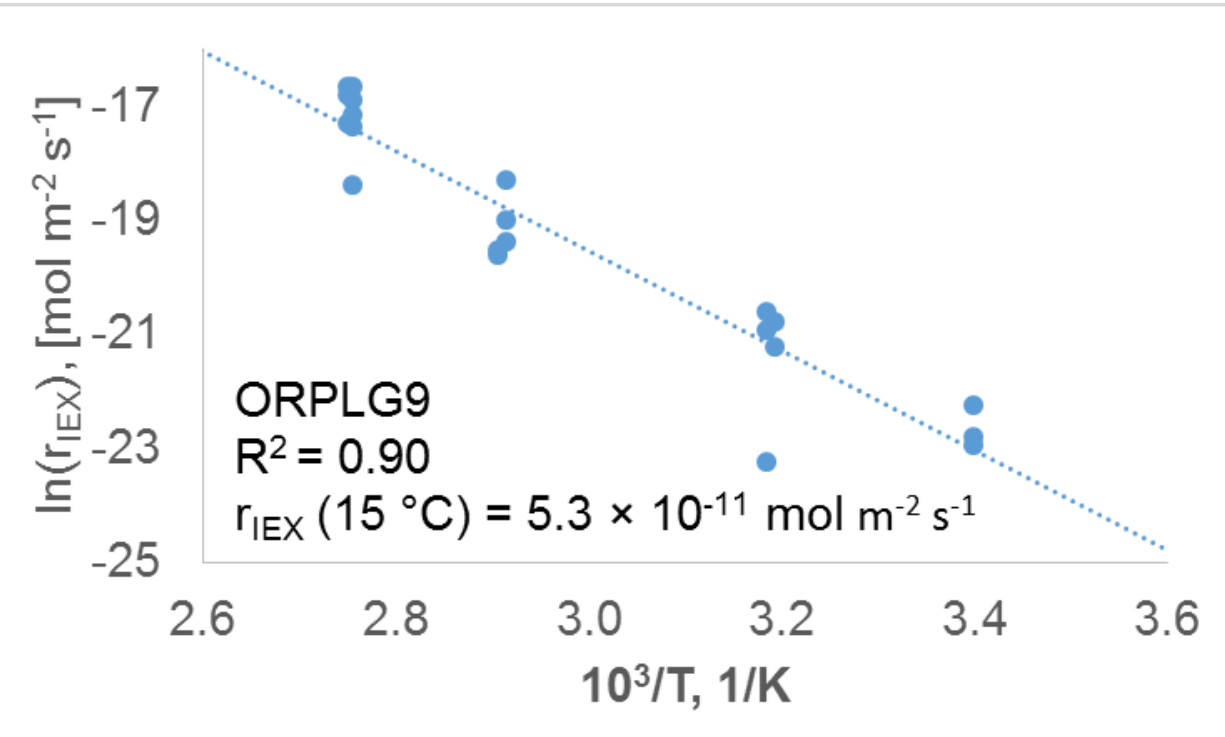

Figure 3.11. The natural logarithm of $\mathrm{r}_{\mathrm{IEX}}$ as a function of inverse temperature for ORPLG9. 


\subsection{Summary}

For the purpose of PA maintenance activities, ILAW rate model parameters that can be used in the IDF PA model for glass dissolution were determined. The rate model parameters are used to model the effect of temperature, $\mathrm{pH}, \mathrm{H}_{4} \mathrm{SiO}_{4}$ activity, and the rate of ion-exchange on the glass dissolution rate. Two advanced, high-waste loaded glasses, IDF18-A161 and ORPLG9, were studied using the SPFT method, which is capable of deriving the ILAW rate model parameters for ILAW glasses. The IDF18-A161 glass is of interest because of its moderate alkali content $\left(20.66 \mathrm{wt} \% \mathrm{Na}_{2} \mathrm{O}\right)$, a high target sulfate loading (1.1 wt\%), and marginal response to VHT. This glass showed a large crystalline fraction at the crucible interface after CCC heat treatment, but the measured composition of the glass portion was still very near the target composition. The ORPLG9 glass is of interest because it has a high alkali content (21.00 wt $\%$ $\mathrm{Na}_{2} \mathrm{O}$ and $5.76 \mathrm{wt} \% \mathrm{~K}_{2} \mathrm{O}$ ), low $\mathrm{SO}_{3}$ content $(0.20 \mathrm{wt} \%$ ), and a high VHT alteration rate though still within acceptable contract limits. In addition, partial SPFT testing had previously been performed on ORPLG9 and this allowed comparison of SPFT results from two different labs.

The SPFT tests with IDF18-A161 and ORPLG9 were performed in a variety of chemically-controlled conditions and test temperatures. A full set of ILAW rate model parameters was obtained for IDF18-A161 and those values are provided in Table 4.1. The IDF18-A161 is most interesting due to its relatively low $\mathrm{K}_{\mathrm{g}}$ value, which would cause the glass to achieve a residual rate observed in static tests at much lower $\mathrm{H}_{4} \mathrm{SiO}_{4}$ concentrations. However, this glass appears to have reached Stage III resumption between 56 and 119 days for a PCT-B performed at 20,000 $\mathrm{m}^{-1}$ (Muller and Pegg 2015).

Dilute-condition rate model parameters were determined for ORPLG9. However, ORPLG9 exhibited unexpected behavior as the release rate of both $\mathrm{B}$ and $\mathrm{Na}$ did not appear to be affected by increases in $\mathrm{H}_{4} \mathrm{SiO}_{4}$ activity. This behavior was presumed to be the result of amorphous phase separation. This was confirmed by the identification of droplet in matrix phase separation upon further analysis of the starting ORPLG9 glass solid by AFM; however, no definitive determination of the chemical composition of these phases was achieved. In light of these findings, when modeling ORPLG9 we recommend using a $\log \mathrm{K}_{\mathrm{g}}$ value of 100 to reproduce the SPFT data. This will result in the glass corroding at a nominally forward rate as $\mathrm{H}_{4} \mathrm{SiO}_{4}$ activity increases, which is a conservative estimate of glass corrosion behavior. This will make the glass dissolution rate simply a function of $\mathrm{pH}$ at a fixed temperature. Further efforts could utilize PCT-B data to refine the value of $\mathrm{K}_{\mathrm{g}}$ to a more realistic value and allow the identification of secondary phases that would also influence the glass corrosion rate. The dilute condition ILAW rate model parameters can still be used for the ORPLG9 glass along with the $\mathrm{r}_{\mathrm{IEX}}$ term. We emphasize again that ORPLG9 is a relatively durable glass; from a set of tests of glasses with high waste loadings, it has one of the lowest measured B releases from PCT-B tests $\left(90^{\circ} \mathrm{C}, \sim 1,800\right.$ days, $\left.\mathrm{S} / \mathrm{V} 2,000 \mathrm{~m}^{-1}\right)$ and has not reached Stage III after 272 days in a PCT-B test at a higher S/V ratio $\left(90^{\circ} \mathrm{C}, \mathrm{S} / \mathrm{V} 20,000 \mathrm{~m}^{-1}\right)$ (Muller and Pegg 2015). Thus, the use of the parameters from this report will be a conservative estimate of the boron release rate. In its present form, the ILAW rate model is only designed to describe the dissolution behavior of a homogenous glass and a modified model would be needed in order to describe the behavior of a glasses containing multiple amorphous phases.

To conclude, we have determined ILAW rate model parameters for the advanced ILAW glasses, IDF18A161 and ORPLG9. These parameters can be used in a TST rate model though it should be emphasized that the $\mathrm{K}_{\mathrm{g}}$ value for ORPLG9 was recommended to reproduce SPFT data and the $\mathrm{r}_{\mathrm{IEX}}$ value for this glass is convoluted from the presence of multiple amorphous phases with different dissolution characteristics. 
In addition, accurate long-term modeling will rely on a comprehensive set of secondary phases for any glass composition that is to be modeled, which should be able to account for the solution concentration of all species in solution. Additionally, we have remarked on the large uncertainties associated with the $\mathrm{r}_{\text {IEX }}$ term used in the rate model. A more focused examination of the ion exchange process should help refine this parameter into a term that either has less uncertainty or is more technically sound. 
Table 4.1. Summary of Rate Model Parameters Obtained for Select ILAW Glasses at $15^{\circ} \mathrm{C}^{(\mathrm{a})}$

\begin{tabular}{|c|c|c|c|c|c|c|}
\hline & $k_{0}\left(\mathrm{~g} \mathrm{~m}^{-2} \mathrm{~d}^{-1}\right)$ & $\mathrm{K}_{\mathrm{g}}(\mathrm{T})$ & $\eta$ & $\mathrm{E}_{\mathrm{a}}\left(\mathrm{kJ} \mathrm{mol}^{-1}\right)$ & $\mathrm{r}_{\text {IEX }}$ & Reference \\
\hline LD6-5412 & $9.7 \times 10^{6}$ & $1.14 \times 10^{-4}$ & $0.40 \pm 0.03$ & $74.8 \pm 1.0$ & $1.74 \times 10^{-11}$ & McGrail et al. 1997 \\
\hline LAWABP1 & $3.4 \times 10^{6}$ & $4.90 \times 10^{-4}$ & $0.35 \pm 0.03$ & $68 \pm 3.0$ & $3.4 \times 10^{-11}$ & McGrail et al. 2001 \\
\hline LAWA44 & $\begin{array}{c}1.3 \times 10^{4} \\
\left(\mathrm{R}^{2}=0.78\right)\end{array}$ & $\begin{array}{l}1.87 \times 10^{-3} \\
\left(R^{2}=0.95\right)\end{array}$ & $0.49 \pm 0.08$ & $60 \pm 7$ & $5.3 \times 10^{-11}$ & Pierce et al. 2004 \\
\hline LAWB45 & $\begin{array}{c}1.6 \times 10^{4} \\
\left(R^{2}=0.90\right)\end{array}$ & $\begin{array}{l}1.79 \times 10^{-3} \\
\left(R^{2}=0.78\right)\end{array}$ & $0.34 \pm 0.03$ & $53 \pm 3$ & $0.0 \times 10^{0}$ & Pierce et al. 2004 \\
\hline LAWC22 & $\begin{array}{c}1.0 \times 10^{5} \\
\left(R^{2}=0.98\right)\end{array}$ & $\begin{array}{l}1.80 \times 10^{-3} \\
\left(R^{2}=0.94\right)\end{array}$ & $0.42 \pm 0.02$ & $64 \pm 2$ & $1.2 \times 10^{-10}$ & Pierce et al. 2004 \\
\hline ORPLB2 & $4.5 \times 10^{4}$ & $1.70 \times 10^{-3}$ & $0.49 \pm 0.05$ & $58 \pm 3$ & $3.4 \times 10^{-10}$ & Papathanassiu et al. 2011 \\
\hline IDF18-A161 & $\begin{array}{c}5.9 \times 10^{6} \\
\left(\mathrm{R}^{2}=0.90\right)\end{array}$ & $\begin{array}{c}2.3 \times 10^{-4} \\
\left(R^{2}=0.98\right)\end{array}$ & $0.28 \pm 0.06$ & $61 \pm 6$ & $7.6 \times 10^{-11}$ & This report \\
\hline ORPLG9 & $\begin{array}{c}2.5 \times 10^{8} \\
\left(\mathrm{R}^{2}=0.91\right)\end{array}$ & $\log K_{g}=100^{(b)}$ & $0.36 \pm 0.07$ & $81 \pm 7$ & $5.3 \times 10^{-11(b)}$ & This report \\
\hline
\end{tabular}

(a) See Freedman et al. (2015) for additional details on rate model parameters presented in the table.

(b) See text for information concerning the $\mathrm{K}_{\mathrm{g}}$ and $\mathrm{r}_{\mathrm{IEX}}$ values of ORPLG9. 


\subsection{References}

Åagaard P and HC Helgeson. 1982. Thermodynamic and Kinetic Constraints on Reaction Rates among Minerals and Aqueous Solutions I. Theoretical Considerations. American Journal of Science 282 237285.

Abraitis PK, BP McGrail, DP Trivedi, FR Livens and DJ Vaughan. 2000a. Single-Pass Flow-Through Experiments on a Simulated Waste Glass in Alkaline Media At $40^{\circ} \mathrm{C}$. II. Experiments Conducted with Buffer Solutions Containing Controlled Quantities of Si and Al. Journal of Nuclear Materials 280(2) 206-215.

Abraitis PK, FR Livens, JE Monteith, JS Small, DP Trivedi, DJ Vaughan and RA Wogelius. 2000b. The Kinetics and Mechanisms of Simulated British Magnox Waste Glass Dissolution as a Function of $\mathrm{pH}$, Silicic Acid Activity, and Time in Low Temperature Aqueous Systems. Applied Geochemistry 15(9) 1399-1416.

ASTM. C1285-14 Standard Test Method for Determining the Chemical Durability of Nuclear Waste Glasses: The Product Consistency Test (PCT). ASTM International, West Conshohocken, PA.

ASTM. C1662-10 Standard Practice for Measurement of the Glass Dissolution Rate Using the SinglePass Flow-Through Test Method. ASTM International, West Conshohocken, PA.

ASTM. C1663-09 Standard Test Method for Measuring Waste Glass or Glass Ceramic Durability by Vapor Hydration Test. ASTM International, West Conshohocken, PA.

Bacon DH and EM Pierce. 2010. Sensitivity Analysis of Kinetic Rate-Law Parameters Used to Simulate Long-Term Weathering of ILAW Glass. PNNL-19472, Pacific Northwest National Laboratory, Richland, WA.

Brown CF, RJ Serne, BN Bjornstad, DG Horton, DC Lanigan, RE Clayton, MM Valenta, TS Vickerman, IV Kutnyakov, KN Geiszler. 2006. Characterization of Vadose Zone Sediments Below the C Tank Farm: Borehole C4297 and RCRA Borehole 299-E27-22, PNNL-15503, Rev. 1, Pacific Northwest National Laboratory, Richland, WA.

Dove PM. 1994. The Dissolution Kinetics of Quartz in Sodium Chloride Solutions at $25^{\circ} \mathrm{C}$ to $300^{\circ} \mathrm{C}$. American Journal of Science 294 665-712.

Eyring H. 1935. The Activated Complex in Chemical Reactions. Journal of Chemical Physics 3 107-115.

Fleet ME, S Muthupari. Coordination of boron in alkali borosilicate glasses using XANES. Journal of Non-Crystalline Solids 255 233-241.

Freedman VL, JV Ryan and DH Bacon. 2015. Immobilized Low-Activity Waste Glass Release Data Package for the Integrated Disposal Facility Performance Assessment. PNNL-24615, Pacific Northwest National Laboratory, Richland, WA. 
Frugier P, S Gin, Y Minet, T Chave, B Bonin, N Godon, JE Lartigue, P Jollivet, A Ayral, L De Windt and G Santarini. 2008. SON68 Nuclear Glass Dissolution Kinetics: Current State of Knowledge and Basis of the New GRAAL Model. Journal of Nuclear Materials 380(1-3) 8-21.

Gin S, A Abdelouas, LJ Criscenti, WL Ebert, K Ferrand, T Geisler, MT Harrison, Y Inagaki, S Mitsui, KT Mueller, JC Marra, CG Pantano, EM Pierce, JV Ryan, JM Schofield, CI Steefel and JD Vienna. 2013. An International Initiative on Long-Term Behavior of High-Level Nuclear Waste Glass. Materials Today 16(6) 243-248.

Grambow B. 1985. A General Rate Equation for Nuclear Waste Glass Corrosion. Scientific Basis for Nuclear Waste Management VIII, Pittsburgh, Pennsylvania, Materials Research Society.

Icenhower J and PM Dove. 2000. The Dissolution Kinetics of Amorphous Silica Into Sodium Chloride Solutions: Effects of Temperature and Ionic Strength. Geochimica et Cosmochimica Acta 64(24) 41934203.

Icenhower JP, A Luttge, BP McGrail, MS Beig, RS Arvidson, EA Rodriguez, JL Steele and SR Baum 2003. Results of Vertical Scanning Interferometry (VSI) of Dissolved Borosilicate Glass: Evidence for Variable Surface Features and Global Surface Retreat. Scientific Basis for Nuclear Waste Management XXVI, Boston, Massachussetts, Materials Research Society.

Icenhower JP and CI Steefel. 2013. Experimentally Determined Dissolution Kinetics of SON68 Glass at $90{ }^{\circ} \mathrm{C}$ Over a Silica Saturation Interval: Evidence Against a Linear Rate Law. Journal of Nuclear Materials 439 137-147.

Inagaki Y, A Shinkai, K Idemistu, T Arima, H Yoshikawa and M Yui. 2006. Aqueous Alteration of Japanese Simulated Waste Glass P0798: Effects of Alteration-Phase Formation on Alteration Rate and Cesium Retention. Journal of Nuclear Materials 354(1-3)171-84.

Inagaki Y, T Kikunaga, K Idemistu and T Arima. 2013. Initial Dissolution Rate of the International Simple Glass as a Function of $\mathrm{pH}$ and Temperature Measured Using Microchannel Flow-Through Test Method. International Journal of Applied Glass Science 4 317-327.

Lasaga AC. 1995. Fundamental Approaches in Describing Mineral Dissolution and Precipitation Rates. Chemical Weathering Rates of Silicate Minerals. AF White and SL Brantley. Washington, DC, Mineralogical Society of America. 31 23-86.

Lasaga AC 1998. Kinetic Theory in the Earth Sciences. Princeton, NJ, Princeton University Press.

Mann FM, CR Eiholzer, AH Lu, PD Rittmann, NW Kline, Y Chen and BP McGrail. 1996. Hanford LowLevel Tank Waste Interim Performance Assessment. WHC-EP-0884, Rev. 0, Richland, WA.

Mann FM, KC Burgard, WR Root, RP Puigh, SH Finfrock, R Khaleel, DH Bacon, EJ Freeman, BP McGrail, SK Wurstner and PE LaMont. 2001. Hanford Immobilization Low-Activity Tank Waste Performance Assessment: 2001 Version. DOE/ORP-2000-24, US Department of Energy, Office of River Protection, Richland, WA. 
Mann, FM, RJ Puigh, SH Finfrock, R Khaleel, MI Wood. 2003. Integrated Disposal Facility Risk Assessment. RPP-15834, Rev. 0, CH2M Hill Hanford Group, Inc., Richland, WA.

Matlack KS, M Chaudhuri, H Gan, IS Muller, W Gong and IL Pegg. 2005. Glass Formulation Testing to Increase Sulfate Incorporation. VSL-04R4960-1, ORP-51808, Vitreous State Laboratory, The Catholic University of America, Washington, D.C.

Matlack KS, W Gong, IS Muller, I Joseph and IL Pegg. 2006a. LAW Envelope A and B Glass Formulations Testing to Increase Waste Loading. VSL-06R6900-1, Vitreous State Laboratory, The Catholic University of America, Washington, D.C.

Matlack KS, W Gong, IS Muller, I Joseph and IL Pegg. 2006b. LAW Envelope C Glass Formulation Testing to Increase Waste Loading. VSL-05R5900-1, Rev. 0, Vitreous State Laboratory, The Catholic University of America, Washington, D.C.

McGrail BP, CW Lindenmeier, PF Martin and GW Gee. 1996. The Pressurized Unsaturated Flow (PUF) Test for Accelerated Ageing of Waste Forms. Transactions of the American Ceramics Society 72 317329.

McGrail BP, WL Ebert, AJ Bakel and DK Peeler. 1997. Measurement of kinetic rate law parameters on a Na-Ca-Al borosilicate glass for low-activity waste. Journal of Nuclear Materials 249(2-3) 175-189.

McGrail BP, PF Martin, CW Lindenmeier and HT Schaef. 1998a. Corrosion Testing of Low-Activity Waste Glasses: Fiscal Year 1998 Summary Report. PNNL-12014, Pacific Northwest National Laboratory, Richland, WA.

McGrail BP, WL Ebert, DH Bacon and DM Strachan. 1998b. A Strategy to Conduct an Analysis of the Long-Term Performance of Low-Activity Waste Glass in a Shallow Subsurface Disposal System at Hanford. PNNL-11834, Pacific Northwest National Laboratory, Richland, WA.

McGrail BP, JP Icenhower, PF Martin, DR Rector, HT Schaef, EA Rodriguez and JL Steele. 2000a. LowActivity Waste Glass Studies: FY2000 Summary Report. PNNL-13381, Pacific Northwest National Laboratory, Richland, WA.

McGrail BP, DH Bacon, WL Ebert and KP Saripalli. 2000b. A Strategy to Conduct an Analysis of the Long-Term Performance of Low-Activity Waste Glass in a Shallow Subsurface Disposal System at Hanford. PNNL-11834 Rev. 1, Pacific Northwest National Laboratory, Richland, WA.

McGrail BP, JP Icenhower, PF Martin, HT Schaef, MJ O'Hara, EA Rodriguez and JL Steele. 2001. Waste Form Release Data Package for the 2001 Immobilized Low-Activity Waste Performance Assessment. PNNL-13043 Rev. 2, Pacific Northwest National Laboratory, Richland, WA.

McGrail BP, D Bacon, R Serne, E Pierce. 2003. A Strategy to Assess Performance of Selected LowActivity Waste Forms in an Integrated Disposal Facility. PNNL-14362, Pacific Northwest National Laboratory, Richland, WA. 
Muller IS, AC Buechele and IL Pegg. 2001. Glass Formulation and Testing with RPP-WTP-LAW Simulants. VSL-01R3560-2, Vitreous State Laboratory, The Catholic University of America, Washington, D.C.

Muller IS and IL Pegg. 2015. FY 2015 ILAW Glass Testing for Disposal at IDF. VSL-15R3790-1, Rev. 0, Vitreous State Laboratory, The Catholic University of America, Washington, D.C.

Muller IS and IL Pegg. 2016. Selection of LAW Glasses for FY 2016 SPFT Testing. VSL16R3860-1, Rev. A, Vitreous State Laboratory, The Catholic University of America, Washington, D.C.

Neeway JJ, EM Pierce, VL Freedman, JV Ryan and NP Qafoku. 2014. A Strategy to Conduct an Analysis of the Long-Term Performance of Low-Activity Waste Glass in a Shallow Subsurface Disposal System at Hanford. PNNL-23503, Pacific Northwest National Laboratory, Richland, WA.

Oelkers EH and SR Gislason. 2001. The Mechanism, Rates and Consequences of Basaltic Glass Dissolution: I. An Experimental Study of the Dissolution Rates of Basaltic Glass as a Function of Aqueous Al, Si and Oxalic Acid Concentration At 25 Degrees C And Ph=3 And 11. Geochimica et Cosmochimica Acta 65(21) 3671-3681.

Papathanassiu A, IS Muller, M Brandys, K Gilbo, A Barkatt, I Joseph and IL Pegg. 2011. ILAW Glass Testing for Disposal at IDF: Phase 1 Testing. VSL-11R2270-1, Vitreous State Laboratory, The Catholic University of America, Washington, D.C.

Pierce EM, BP McGrail, EA Rodriguez, HT Schaef, KP Saripalli, RJ Serne, PF Martin, SR Baum, KN Geizler, LR Reed and WJ Shaw. 2004. Waste Form Release Data Package for the 2005 Integrated Disposal Facility Performance Assessment. PNNL-14805, Pacific Northwest National Laboratory, Richland, WA.

Rawlins JA, RA Karnesky, R Khaleel, FM Mann, BP McGrail, WJ McMahon, MG Piepho, PD Rittmann and F Schmittroth. 1994. Impacts of Disposal System Design Options on Low-Level Glass Waste Disposal System Performance. WHC-EP-0810, Rev. 0, Westinghouse Hanford Company, Richland, WA.

Renders PJN, CH Gammons and HL Barnes. 1995. Precipitation and Dissolution Rate Constants for Cristobalite from $150^{\circ} \mathrm{C}$ to $300^{\circ} \mathrm{C}$. Geochimica et Cosmochimica Acta 59(1) 77-85.

Rimstidt JD, Y Zhang and C Zhu. 2016. Rate Equations for Sodium Catalyzed Amorphous Silica Dissolution. Geochimica et Cosmochimica Acta 195 120-125.

Ryan JV, VL Freedman. 2016. A Strategy and Technical Approach for Maintenance of the Long-Term Performance Assessment of Immobilized Low-Activity Waste Glass. PNNL-23503, Rev. 1, Pacific Northwest National Laboratory, Richland, WA.

Strachan DM. 1983. Results from Long-Term Use of the MCC-1 Static Leach Test Method. Nuclear and Chemical Waste Management 4 177-188.

Strachan DM, JJ Neeway. 2014. Effects of Alteration Product Precipitation on Glass Dissolution. Applied Geochemistry 45 144-157. 
Taylor P, SD Ashmore and DG Owen. 1987. Chemical durability of some sodium borosilicate glasses improved by phase separation. Journal of the American Ceramics Society 70 333-338.

Tomozawa M, GM Singer, Y Oka and JT Varden. 1979. Phase separation in nuclear waste glasses. Ceramics in Nuclear Waste Management. Cincinnati, OH,

Vernaz EY, JL Dussossoy and S Fillet. 1988. Temperature Dependence of R7T7 Nuclear Waste Glass Alteration Mechanism. Materials Research Society Symposium Proceedings 112 555-563.

Vienna JD, A Jiricka, PR Hrma, DE Smith, TH Lorier, RL Schulz and IA Reamer. 2001. Hanford Immobilized LAW Product Acceptance Testing: Tanks Focus Area Results. PNNL-13744, Pacific Northwest National Laboratory, Richland, WA.

Vienna JD, JV Ryan, S Gin and Y Inagaki. 2013a. Current Understanding and Remaining Challenges in Modeling Long-Term Degradation of Borosilicate Nuclear Waste Glasses. International Journal of Applied Glass Science 4(4) 283-294.

Vienna JD, DC Skorski, DS Kim, J Matyas. 2013b. Glass Property Models and Constraints for Estimating the Glass to be Produced at Hanford by Implementing Current Advanced Glass Formulation Efforts. PNNL-22631, Rev. 1, Pacific Northwest National Laboratory, Richland, WA.

Wolery TJ and SA Daveler. 1992. EQ6, A Computer Program for Reaction-Path Modeling of Aqueous Geochemical Systems: Theoretical Manual, User's Guide, and Documentation., Lawrence Livermore National Laboratory, Livermore, CA.

Yabusaki S, RJ Serne, M Rockhold, G Wang and JH Westsik Jr. 2015. Technical Approach for Determining Key Parameters Needed for Modeling the Performance of Cast Stone for the Integrated Disposal Facility Performance Assessment. PNNL-24022, Pacific Northwest National Laboratory, Richland, WA. 


\section{Appendix A}

\section{Steady-State Single-Pass Flow-Through Results}

When the average measured value is below the EQL, the EQL value is provided in brackets. 


\begin{tabular}{|c|c|c|c|c|c|c|c|c|c|c|c|c|c|}
\hline Reactor & $\begin{array}{l}\text { Glass Mass } \\
(\mathrm{g})^{(\mathrm{a})}\end{array}$ & $\begin{array}{l}\text { Steady- } \\
\text { State q/S, } \\
\mathrm{m} \mathrm{s}^{-1}\end{array}$ & $\begin{array}{l}\text { [B] Steady } \\
\text { State, } \\
\mu \mathrm{g} \mathrm{L}^{-1}\end{array}$ & $\begin{array}{l}\text { [Al] Steady } \\
\text { State, } \\
\mu^{-1} \mathrm{~L}^{-1}\end{array}$ & $\begin{array}{l}{[\mathrm{Na}]} \\
\text { Steady } \\
\text { State, } \\
\mu \mathrm{g} \mathrm{L}^{-1} \\
\end{array}$ & $\begin{array}{l}\text { [Si] Steady } \\
\text { State, } \\
\mu \mathrm{g} \mathrm{L}^{-1}\end{array}$ & $\begin{array}{l}\text { B steady-state rate, } \\
\mathrm{g} \mathrm{m}^{-2} \mathrm{~d}^{-1}\end{array}$ & $\begin{array}{l}2 \sigma \mathrm{B} \text { rate } \\
\text { error }^{(b)}\end{array}$ & $\begin{array}{l}\text { Avg. Flow } \\
\text { Rate, } \mathrm{m}^{3} \mathrm{~d}^{-1} \\
\text { (c) }\end{array}$ & $\begin{array}{l}\text { Deviation } \\
\text { of Flow } \\
\text { Rate }^{(\mathrm{d})}\end{array}$ & $\begin{array}{l}\text { Avg. Test } \\
\text { Temp. }\left({ }^{\circ} \mathrm{C}\right) \\
\text { (e) }\end{array}$ & $\begin{array}{l}\text { Temp. } \\
\text { Dev. }\end{array}$ & $\begin{array}{l}\text { Steady- } \\
\text { state } \\
\text { samples }{ }^{(f)}\end{array}$ \\
\hline \multicolumn{14}{|c|}{ LG9 $40^{\circ} \mathrm{C}$ pH 9} \\
\hline Reac. 1 & 4.0016 & 1.15E-09 & 970 & 461 & 8297 & 2380 & 3.64E-03 & $1.28 \mathrm{E}-03$ & 7.80E-06 & $\pm 3.33 \%$ & 40.87 & \pm 0.08 & \\
\hline Reac. 2 & 1.5057 & 7.47E-09 & 140 & 166 & 1367 & 767 & 3.42E-03 & 9.13E-04 & $1.91 \mathrm{E}-05$ & $\pm 1.48 \%$ & 40.87 & \pm 0.08 & \\
\hline Reac. 3 & 0.7505 & $2.94 \mathrm{E}-08$ & 77 & 66 & 465 & 217 & $7.44 \mathrm{E}-03$ & 2.07E-03 & $3.74 \mathrm{E}-05$ & $\pm 3.61 \%$ & 40.87 & \pm 0.08 & \\
\hline Reac. 4 & 0.3001 & $1.52 \mathrm{E}-07$ & [75.6] & [49.4] & 172 & [164] & $3.75 E-02$ & --- & 7.62E-05 & $\pm 0.94 \%$ & 40.87 & \pm 0.08 & \\
\hline Reac. 5 & 0.0581 & 8.72E-07 & {$[75.6]$} & [49.4] & [134] & [164] & $2.16 E-01$ & --- & 7.75E-05 & $\pm 0.74 \%$ & 40.87 & \pm 0.08 & \\
\hline \multicolumn{14}{|c|}{ LG9 $40^{\circ} \mathrm{C}$ pH 10} \\
\hline Reac. 1 & 3.9995 & 1.17E-09 & 681 & 879 & 7043 & 4673 & 2.61E-03 & 8.90E-04 & 7.95E-06 & $\pm 2.78 \%$ & 40.87 & \pm 0.11 & \\
\hline Reac. 2 & 1.4984 & 6.98E-09 & 276 & 440 & 1957 & 2197 & $6.29 \mathrm{E}-03$ & $1.92 \mathrm{E}-03$ & $1.77 \mathrm{E}-05$ & $\pm 2.13 \%$ & 40.87 & \pm 0.11 & \\
\hline Reac. 3 & 0.7559 & 3.06E-08 & 122 & 228 & 902 & 1060 & $1.22 \mathrm{E}-02$ & 3.17E-03 & 3.90E-05 & $\pm 1.32 \%$ & 40.87 & \pm 0.11 & \\
\hline Reac. 4 & 0.3033 & $1.51 \mathrm{E}-07$ & [75.6] & 101 & 360 & 404 & $3.72 E-02$ & --- & 7.59E-05 & $\pm 2.09 \%$ & 40.87 & \pm 0.11 & \\
\hline Reac. 5 & 0.0615 & $8.00 \mathrm{E}-07$ & [75.6] & {$[49.4]$} & [134] & [164] & $1.98 E-01$ & --- & 7.69E-05 & $\pm 0.84 \%$ & 40.87 & \pm 0.11 & \\
\hline \multicolumn{14}{|c|}{ LG9 $40^{\circ} \mathrm{C}$ pH 11} \\
\hline Reac. 1 & 2.0000 & 1.65E-09 & 1440 & 1727 & 10950 & 9263 & 7.77E-03 & 4.84E-03 & 5.59E-06 & $\pm 27.23 \%$ & 40.20 & \pm 2.60 & \\
\hline Reac. 2 & 1.5020 & 3.59E-09 & 676 & 928 & 4387 & 4787 & 7.93E-03 & 3.49E-03 & $9.15 \mathrm{E}-06$ & $\pm 15.96 \%$ & 40.20 & \pm 2.60 & \\
\hline Reac. 3 & 0.7493 & $1.85 \mathrm{E}-08$ & 305 & 489 & 2087 & 2550 & $1.85 \mathrm{E}-02$ & 5.85E-03 & 2.35E-05 & $\pm 5.17 \%$ & 40.20 & \pm 2.60 & $12-14$ \\
\hline Reac. 4 & 0.2511 & 7.99E-08 & 100 & 240 & 944 & 1223 & 2.62E-02 & $6.91 \mathrm{E}-03$ & 3.39E-05 & $\pm 9.32 \%$ & 40.20 & \pm 2.60 & $7,9,14$ \\
\hline Reac. 5 & 0.0501 & 5.07E-07 & [75.6] & 91 & 363 & 461 & $1.25 E-01$ & --- & 4.17E-05 & $\pm 6.48 \%$ & 40.20 & \pm 2.60 & $9,13,14$ \\
\hline \multicolumn{14}{|c|}{ LG9 $40^{\circ} \mathrm{C}$ pH 12} \\
\hline Reac. 1 & 3.9999 & 1.62E-09 & 3167 & 4333 & 19900 & 20933 & $1.67 \mathrm{E}-02$ & $6.15 \mathrm{E}-03$ & 1.09E-05 & $\pm 4.48 \%$ & 39.93 & \pm 2.96 & \\
\hline Reac. 2 & 3.0199 & 3.83E-09 & 1733 & 2603 & 11867 & 12567 & 2.17E-02 & 7.68E-03 & $1.95 \mathrm{E}-05$ & $\pm 2.18 \%$ & 39.93 & \pm 2.96 & \\
\hline Reac. 3 & 1.5077 & $1.56 \mathrm{E}-08$ & 732 & 1227 & 5360 & 5990 & 3.73E-02 & $1.25 \mathrm{E}-02$ & 3.92E-05 & $\pm 1.75 \%$ & 39.93 & \pm 2.96 & \\
\hline Reac. 4 & 0.5008 & $9.40 \mathrm{E}-08$ & 272 & 556 & 2613 & 2787 & 8.35E-02 & 2.43E-02 & 7.69E-05 & $\pm 0.98 \%$ & 39.93 & \pm 2.96 & \\
\hline Reac. 5 & 0.1023 & 4.93E-07 & [75.6] & 243 & 1367 & 1213 & $1.22 E-01$ & --- & 7.94E-05 & $\pm 0.99 \%$ & 39.93 & \pm 2.96 & \\
\hline \multicolumn{14}{|c|}{ LG9 $70^{\circ} \mathrm{C}$ pH 9} \\
\hline Reac. 1 & 0.3991 & $1.16 \mathrm{E}-08$ & 2720 & 848 & 16967 & 4777 & $1.03 \mathrm{E}-01$ & $3.88 \mathrm{E}-02$ & 7.63E-06 & $\pm 7.65 \%$ & 70.97 & \pm 0.39 & \\
\hline Reac. 2 & 0.1519 & $8.21 \mathrm{E}-08$ & 437 & 182 & 2723 & 1100 & $1.17 \mathrm{E}-01$ & 3.66E-02 & $2.02 \mathrm{E}-05$ & $\pm 2.60 \%$ & 70.97 & \pm 0.39 & \\
\hline Reac. 3 & 0.0514 & $4.81 \mathrm{E}-07$ & 158 & 51 & 882 & 330 & 2.49E-01 & 6.15E-02 & $3.80 \mathrm{E}-05$ & $\pm 0.71 \%$ & 70.97 & \pm 0.39 & \\
\hline Reac. 4 & 0.0241 & $2.26 \mathrm{E}-06$ & [75.6] & [49.4] & 174 & [164] & $5.59 E-01$ & --- & 7.77E-05 & $\pm 0.55 \%$ & 70.97 & \pm 0.39 & \\
\hline \multicolumn{14}{|c|}{ LG9 $70{ }^{\circ} \mathrm{C}$ pH 10} \\
\hline Reac. 1 & 0.4005 & $1.04 \mathrm{E}-08$ & 976 & 1280 & 7490 & 7643 & 3.32E-02 & 1.17E-02 & 7.01E-06 & $\pm 4.61 \%$ & 70.97 & \pm 0.38 & \\
\hline
\end{tabular}




\begin{tabular}{|c|c|c|c|c|c|c|c|c|c|c|c|c|c|}
\hline Reactor & $\begin{array}{l}\text { Glass Mass } \\
(\mathrm{g})^{(\mathrm{a})}\end{array}$ & $\begin{array}{l}\text { Steady- } \\
\text { State q/S, } \\
\mathrm{m} \mathrm{s}^{-1}\end{array}$ & $\begin{array}{l}{[\mathrm{B}] \text { Steady }} \\
\text { State, } \\
\mu \mathrm{g} \mathrm{L}^{-1}\end{array}$ & $\begin{array}{l}\text { [Al] Steady } \\
\text { State, } \\
\mu \mathrm{g} \mathrm{L}^{-1}\end{array}$ & $\begin{array}{l}{[\mathrm{Na}]} \\
\text { Steady } \\
\text { State, } \\
\mu \mathrm{g} \mathrm{L}^{-1}\end{array}$ & $\begin{array}{l}\text { [Si] Steady } \\
\text { State, } \\
\mu \mathrm{g} \mathrm{L}^{-1}\end{array}$ & $\begin{array}{l}\text { B steady-state rate, } \\
\mathrm{g} \mathrm{m}^{-2} \mathrm{~d}^{-1}\end{array}$ & $\begin{array}{l}2 \sigma \mathrm{B} \text { rate } \\
\text { error }^{(b)}\end{array}$ & $\begin{array}{l}\text { Avg. Flow } \\
\text { Rate, } \mathrm{m}^{3} \mathrm{~d}^{-1} \\
\text { (c) }\end{array}$ & $\begin{array}{l}\text { Deviation } \\
\text { of Flow } \\
\text { Rate }\end{array}$ & $\begin{array}{l}\text { Avg. Test } \\
\text { Temp. }\left({ }^{\circ} \mathrm{C}\right) \\
\text { (e) }\end{array}$ & $\begin{array}{l}\text { Temp. } \\
\text { Dev. }\end{array}$ & $\begin{array}{l}\text { Steady- } \\
\text { state } \\
\text { samples }{ }^{(\mathrm{f})}\end{array}$ \\
\hline Reac. 2 & 0.1500 & 8.19E-08 & 433 & 610 & 2603 & 3550 & $1.16 \mathrm{E}-01$ & 3.65E-02 & 2.02E-05 & $\pm 2.29 \%$ & 70.97 & \pm 0.38 & \\
\hline Reac. 3 & 0.0499 & $5.08 \mathrm{E}-07$ & 192 & 278 & 1090 & 1587 & 3.18E-01 & $8.21 \mathrm{E}-02$ & 3.91E-05 & $\pm 0.62 \%$ & 70.97 & \pm 0.38 & \\
\hline Reac. 4 & 0.0257 & $2.08 \mathrm{E}-06$ & [75.6] & 112 & 372 & 625 & 5.15E-01 & --- & 7.79E-05 & $\pm 0.28 \%$ & 70.97 & \pm 0.38 & \\
\hline \multicolumn{14}{|c|}{ LG9 $70^{\circ} \mathrm{C}$ pH 11} \\
\hline Reac. 1 & 0.7511 & 2.13E-09 & 4743 & 4487 & 31333 & 24600 & 3.31E-02 & 8.18E-02 & 2.71E-06 & $\pm 124.98 \%$ & 70.09 & \pm 1.51 & \\
\hline Reac. 2 & 0.3748 & 3.87E-08 & 1130 & 1397 & 6220 & 7260 & $1.43 \mathrm{E}-01$ & $5.08 \mathrm{E}-02$ & 2.37E-05 & $\pm 6.45 \%$ & 70.09 & \pm 1.51 & $9,11,13$ \\
\hline Reac. 3 & 0.1499 & $1.66 \mathrm{E}-07$ & 511 & 665 & 2783 & 3720 & $2.78 \mathrm{E}-01$ & 8.47E-02 & 3.91E-05 & $\pm 1.33 \%$ & 70.09 & \pm 1.51 & \\
\hline Reac. 4 & 0.0513 & $1.01 \mathrm{E}-06$ & 131 & 176 & 612 & 1028 & 4.31E-01 & $1.01 \mathrm{E}-01$ & 7.76E-05 & $\pm 2.14 \%$ & 70.09 & \pm 1.51 & \\
\hline \multicolumn{14}{|c|}{ LG9 $70^{\circ} \mathrm{C}$ pH 12} \\
\hline Reac. 1 & 0.7509 & 6.27E-09 & 7840 & 9257 & 43433 & 44333 & $1.61 \mathrm{E}-01$ & $1.61 \mathrm{E}-01$ & 7.66E-06 & $\pm 49.73 \%$ & 70.09 & \pm 1.51 & \\
\hline Reac. 2 & 0.3687 & 3.84E-08 & 3223 & 4270 & 19267 & 20300 & 4.05E-01 & 1.49E-01 & 2.19E-05 & $\pm 9.70 \%$ & 70.09 & \pm 1.51 & $9,11,12$ \\
\hline Reac. 3 & 0.1495 & $1.86 \mathrm{E}-07$ & 911 & 1197 & 4917 & 6027 & 5.52E-01 & $1.62 \mathrm{E}-01$ & 4.00E-05 & $\pm 1.78 \%$ & 70.09 & \pm 1.51 & \\
\hline Reac. 4 & 0.0493 & $1.50 \mathrm{E}-06$ & 521 & 707 & 2923 & 3783 & $2.55 E+00$ & $5.40 \mathrm{E}-01$ & 7.52E-05 & $\pm 7.27 \%$ & 70.09 & \pm 1.51 & $7,9,10$ \\
\hline \multicolumn{14}{|c|}{ LG9 90 ${ }^{\circ} \mathrm{C}$ pH 9} \\
\hline Reac. 1 & 0.3984 & $1.24 \mathrm{E}-08$ & 4340 & 1690 & 34500 & 10567 & $1.75 \mathrm{E}-01$ & 6.38E-02 & 7.90E-06 & $\pm 6.87 \%$ & 90.63 & \pm 1.71 & \\
\hline Reac. 2 & 0.1514 & 8.13E-08 & 820 & 569 & 7070 & 3707 & $2.18 \mathrm{E}-01$ & $6.80 \mathrm{E}-02$ & $1.90 \mathrm{E}-05$ & $\pm 1.39 \%$ & 90.63 & \pm 1.71 & \\
\hline Reac. 3 & 0.0998 & $5.14 \mathrm{E}-07$ & 189 & 155 & 1530 & 838 & 3.18E-01 & 7.75E-02 & 7.50E-05 & $\pm 0.62 \%$ & 90.63 & \pm 1.71 & \\
\hline Reac. 4 & 0.0490 & $2.28 \mathrm{E}-06$ & [75.6] & 59 & 426 & [164] & 5.63E-01 & --- & $1.52 \mathrm{E}-04$ & $\pm 4.15 \%$ & 90.63 & \pm 1.71 & \\
\hline \multicolumn{14}{|c|}{ LG9 $90{ }^{\circ} \mathrm{C}$ pH 10} \\
\hline Reac. 1 & 0.3999 & $1.10 \mathrm{E}-08$ & 1920 & 2233 & 22000 & 13500 & 6.93E-02 & 2.52E-02 & 7.38E-06 & $\pm 5.16 \%$ & 90.63 & \pm 1.71 & \\
\hline Reac. 2 & 0.1490 & 7.70E-08 & 953 & 1203 & 6703 & 7343 & 2.40E-01 & 7.97E-02 & $1.84 \mathrm{E}-05$ & $\pm 3.70 \%$ & 90.63 & \pm 1.71 & \\
\hline Reac. 3 & 0.1002 & 5.15E-07 & 393 & 504 & 2657 & 3090 & $6.61 \mathrm{E}-01$ & $1.80 \mathrm{E}-01$ & 7.43E-05 & $\pm 1.77 \%$ & 90.63 & \pm 1.71 & \\
\hline Reac. 4 & 0.0489 & 2.40E-06 & 147 & 197 & 1067 & 1197 & $1.15 \mathrm{E}+00$ & 2.31E-01 & 1.49E-04 & $\pm 1.09 \%$ & 90.63 & \pm 1.71 & \\
\hline \multicolumn{14}{|c|}{ LG9 $90^{\circ} \mathrm{C}$ pH 11} \\
\hline Reac. 1 & 0.7481 & 1.27E-08 & 1443 & 1156 & 5847 & 4840 & 5.99E-02 & 2.15E-02 & 1.58E-05 & $\pm 5.37 \%$ & 90.52 & \pm 1.63 & \\
\hline Reac. 2 & 0.3760 & $3.78 \mathrm{E}-08$ & 2837 & 2937 & 17233 & 17433 & $3.50 \mathrm{E}-01$ & $1.21 \mathrm{E}-01$ & $2.28 \mathrm{E}-05$ & $\pm 3.33 \%$ & 90.52 & \pm 1.63 & \\
\hline Reac. 3 & 0.2981 & $1.63 \mathrm{E}-07$ & 1173 & 1377 & 7657 & 7913 & $6.24 \mathrm{E}-01$ & 1.97E-01 & 7.44E-05 & $\pm 1.22 \%$ & 90.52 & \pm 1.63 & \\
\hline Reac. 4 & 0.1009 & $1.10 \mathrm{E}-06$ & 360 & 491 & 2320 & 2883 & $1.29 \mathrm{E}+00$ & 3.24E-01 & 1.49E-04 & $\pm 0.38 \%$ & 90.52 & \pm 1.63 & \\
\hline \multicolumn{14}{|c|}{ LG9 90 ${ }^{\circ} \mathrm{C}$ pH 12} \\
\hline Reac. 1 & 0.7500 & 5.19E-09 & 19050 & 14700 & 75095 & 95450 & 3.23E-01 & 1.97E-01 & 6.23E-06 & $\pm 26.89 \%$ & 90.52 & \pm 1.63 & \\
\hline
\end{tabular}




\begin{tabular}{|c|c|c|c|c|c|c|c|c|c|c|c|c|c|}
\hline Reactor & $\begin{array}{l}\text { Glass Mass } \\
(\mathrm{g})^{(\mathrm{a})}\end{array}$ & $\begin{array}{l}\text { Steady- } \\
\text { State q/S, } \\
\mathrm{m} \mathrm{s}^{-1}\end{array}$ & $\begin{array}{l}\text { [B] Steady } \\
\text { State, } \\
\text { ug L }^{-1}\end{array}$ & $\begin{array}{l}\text { [Al] Steady } \\
\text { State, } \\
\mu \mathrm{g} \mathrm{L}^{-1}\end{array}$ & $\begin{array}{l}{[\mathrm{Na}]} \\
\text { Steady } \\
\text { State, } \\
\mu \mathrm{g} \mathrm{L}^{-1}\end{array}$ & $\begin{array}{l}\text { [Si] Steady } \\
\text { State, } \\
\text { ug L L }^{-1}\end{array}$ & $\begin{array}{l}\text { B steady-state rate, } \\
\mathrm{g} \mathrm{m}^{-2} \mathrm{~d}^{-1}\end{array}$ & $\begin{array}{l}2 \sigma \mathrm{B} \text { rate } \\
\text { error }^{(b)}\end{array}$ & $\begin{array}{l}\text { Avg. Flow } \\
\text { Rate, } \mathrm{m}^{3} \mathrm{~d}^{-1} \\
\text { (c) }\end{array}$ & $\begin{array}{l}\text { Deviation } \\
\text { of Flow } \\
\text { Rate }\end{array}$ & $\begin{array}{l}\text { Avg. Test } \\
\text { Temp. }\left({ }^{\circ} \mathrm{C}\right) \\
\text { (e) }\end{array}$ & $\begin{array}{l}\text { Temp. } \\
\text { Dev. }\end{array}$ & $\begin{array}{l}\text { Steady- } \\
\text { state } \\
\text { samples }{ }^{(\mathrm{f})}\end{array}$ \\
\hline Reac. 2 & 0.3759 & 3.00E-08 & 9373 & 9517 & 57900 & 52133 & 9.19E-01 & 3.52E-01 & $1.70 \mathrm{E}-05$ & $\pm 9.06 \%$ & 90.52 & \pm 1.63 & $5,7,8$ \\
\hline Reac. 3 & 0.3008 & $1.87 \mathrm{E}-07$ & 2537 & 3277 & 16800 & 16800 & $1.55 \mathrm{E}+00$ & 4.19E-01 & 7.16E-05 & $\pm 2.14 \%$ & 90.52 & \pm 1.63 & \\
\hline Reac. 4 & 0.0993 & $1.07 \mathrm{E}-06$ & 950 & 1313 & 6793 & 6913 & $3.31 \mathrm{E}+00$ & $9.27 \mathrm{E}-01$ & 1.45E-04 & $\pm 1.88 \%$ & 90.52 & \pm 1.63 & $3,4,5$ \\
\hline \multicolumn{14}{|c|}{ LG9 $23^{\circ} \mathrm{C}$ pH 9} \\
\hline Reac. 1 & 0.7494 & 6.70E-09 & {$[75.6]$} & 71 & 383 & 221 & $1.66 E-03$ & --- & 8.52E-06 & $\pm 5.26 \%$ & 22.70 & \pm 0.63 & $20,22,24$ \\
\hline Reac. 2 & 0.3757 & 3.26E-08 & {$[75.6]$} & [49.4] & 147 & [164] & 8.06E-03 & --- & 2.07E-05 & $\pm 2.22 \%$ & 22.70 & \pm 0.63 & \\
\hline Reac. 3 & 0.1503 & $1.64 \mathrm{E}-07$ & {$[75.6]$} & {$[49.4]$} & [134] & [164] & $4.04 E-02$ & --- & 4.01E-05 & $\pm 2.07 \%$ & 22.70 & \pm 0.63 & \\
\hline Reac. 4 & 0.0298 & $9.36 \mathrm{E}-07$ & {$[75.6]$} & [49.4] & [134] & [164] & $2.31 E-01$ & --- & $3.78 \mathrm{E}-05$ & $\pm 4.70 \%$ & 22.70 & \pm 0.63 & \\
\hline \multicolumn{14}{|c|}{ LG9 $23^{\circ} \mathrm{C}$ pH 10} \\
\hline Reac. 1 & 0.7494 & 1.09E-08 & 108 & 168 & 668 & 890 & 3.83E-03 & $9.97 \mathrm{E}-04$ & $1.38 \mathrm{E}-05$ & $\pm 1.86 \%$ & 22.70 & \pm 0.63 & \\
\hline Reac. 2 & 0.3744 & $2.84 \mathrm{E}-08$ & 88 & 84 & 425 & 512 & 8.14E-03 & 2.17E-03 & $1.80 \mathrm{E}-05$ & $\pm 6.30 \%$ & 22.70 & \pm 0.63 & \\
\hline Reac. 3 & 0.1501 & $1.53 \mathrm{E}-07$ & {$[75.6]$} & {$[49.4]$} & 154 & 186 & 3.79E-02 & --- & $3.76 \mathrm{E}-05$ & $\pm 2.33 \%$ & 22.70 & \pm 0.63 & \\
\hline Reac. 4 & 0.0298 & $9.78 \mathrm{E}-07$ & [75.6] & [49.4] & [134] & [164] & $2.42 E-01$ & --- & 3.90E-05 & $\pm 2.16 \%$ & 22.70 & \pm 0.63 & \\
\hline \multicolumn{14}{|c|}{ LG9 $23^{\circ} \mathrm{C}$ pH 11} \\
\hline Reac. 1 & 1.5007 & 3.61E-09 & 318 & 400 & 2047 & 2577 & 3.75E-03 & $1.43 \mathrm{E}-03$ & 9.18E-06 & $\pm 14.73 \%$ & 22.70 & \pm 0.63 & \\
\hline Reac. 2 & 0.7497 & $1.52 \mathrm{E}-08$ & 148 & 175 & 1026 & 1057 & 7.39E-03 & $1.99 \mathrm{E}-03$ & 1.93E-05 & $\pm 2.65 \%$ & 22.70 & \pm 0.63 & \\
\hline Reac. 3 & 0.2506 & $9.42 \mathrm{E}-08$ & {$[75.6]$} & 55 & 459 & 225 & 2.33E-02 & --- & 3.91E-05 & $\pm 4.91 \%$ & 22.70 & \pm 0.63 & \\
\hline Reac. 4 & 0.0500 & 4.80E-07 & {$[75.6]$} & [49.4] & 348 & [164] & 1.19E-01 & --- & 3.61E-05 & $\pm 2.55 \%$ & 22.70 & \pm 0.63 & \\
\hline \multicolumn{14}{|c|}{ LG9 $23^{\circ} \mathrm{C}$ pH 12} \\
\hline Reac. 1 & 1.4985 & 4.47E-09 & 643 & 929 & 4280 & 4983 & $9.41 \mathrm{E}-03$ & 4.92E-03 & $1.13 \mathrm{E}-05$ & $\pm 23.16 \%$ & 22.70 & \pm 0.63 & \\
\hline Reac. 2 & 0.7492 & $1.42 \mathrm{E}-08$ & 456 & 650 & 2893 & 3777 & $2.12 \mathrm{E}-02$ & $8.42 \mathrm{E}-03$ & 1.78E-05 & $\pm 14.28 \%$ & 22.70 & \pm 0.63 & \\
\hline Reac. 3 & 0.2497 & $6.08 \mathrm{E}-08$ & 149 & 211 & 1190 & 1220 & $2.96 \mathrm{E}-02$ & $1.65 \mathrm{E}-02$ & $2.51 \mathrm{E}-05$ & $\pm 51.43 \%$ & 22.70 & \pm 0.63 & \\
\hline Reac. 4 & 0.0499 & 5.15E-07 & {$[75.6]$} & 75 & 587 & 357 & $1.27 E-01$ & --- & $3.84 \mathrm{E}-05$ & $\pm 8.77 \%$ & 22.70 & \pm 0.63 & \\
\hline \multicolumn{14}{|c|}{ LG9 $23^{\circ} \mathrm{C}$ Si pH 9} \\
\hline Reac. 1 & 4.0053 & 2.92E-09 & 58 & 28 & 606 & 5643 & 5.49E-04 & $1.89 \mathrm{E}-04$ & $1.98 \mathrm{E}-05$ & $\pm 1.53 \%$ & 21.15 & \pm 0.72 & \\
\hline Reac. 2 & 3.9944 & 2.61E-09 & 92 & 29 & 715 & 18800 & 7.82E-04 & 2.07E-04 & $1.77 \mathrm{E}-05$ & $\pm 2.87 \%$ & 21.15 & \pm 0.72 & \\
\hline Reac. 3 & 3.9944 & 2.83E-09 & 95 & 31 & 695 & 33667 & 8.76E-04 & $2.30 \mathrm{E}-04$ & $1.92 \mathrm{E}-05$ & $\pm 2.79 \%$ & 21.15 & \pm 0.72 & \\
\hline \multicolumn{14}{|c|}{ LG9 $40^{\circ} \mathrm{C}$ Si pH 9} \\
\hline Reac. 1 & 1.9959 & 5.43E-09 & 373 & 89 & 2593 & 9343 & $6.61 \mathrm{E}-03$ & $2.14 \mathrm{E}-03$ & 1.83E-05 & $\pm 4.16 \%$ & 40.12 & \pm 1.46 & \\
\hline Reac. 2 & 1.9790 & 3.30E-09 & 869 & 58 & 5897 & 26367 & 9.38E-03 & 8.06E-03 & $1.11 \mathrm{E}-05$ & $\pm 43.40 \%$ & 40.12 & \pm 1.46 & \\
\hline Reac. 3 & 1.9921 & 5.93E-09 & 470 & {$[49.4]$} & 3337 & 42467 & $9.11 \mathrm{E}-03$ & $2.98 \mathrm{E}-03$ & $1.99 \mathrm{E}-05$ & $\pm 2.44 \%$ & 40.12 & \pm 1.46 & \\
\hline
\end{tabular}




\begin{tabular}{|c|c|c|c|c|c|c|c|c|c|c|c|c|c|}
\hline Reactor & $\begin{array}{l}\text { Glass Mass } \\
(\mathrm{g})^{(\mathrm{a})}\end{array}$ & $\begin{array}{l}\text { Steady- } \\
\text { State q/S, } \\
\mathrm{m} \mathrm{s}^{-1}\end{array}$ & $\begin{array}{l}{[\mathrm{B}] \text { Steady }} \\
\text { State, } \\
\mu \mathrm{g} \mathrm{L}^{-1}\end{array}$ & $\begin{array}{l}\text { [Al] Steady } \\
\text { State, } \\
\mu \mathrm{g} \mathrm{L}^{-1}\end{array}$ & $\begin{array}{l}{[\mathrm{Na}]} \\
\text { Steady } \\
\text { State, } \\
\mu \mathrm{g} \mathrm{L}^{-1}\end{array}$ & $\begin{array}{l}\text { [Si] Steady } \\
\text { State, } \\
\mu \mathrm{g} \mathrm{L}^{-1}\end{array}$ & $\begin{array}{l}\text { B steady-state rate, } \\
\mathrm{g} \mathrm{m}^{-2} \mathrm{~d}^{-1}\end{array}$ & $\begin{array}{l}2 \sigma \mathrm{B} \text { rate } \\
\text { error }^{(b)}\end{array}$ & $\begin{array}{l}\text { Avg. Flow } \\
\text { Rate, } \mathrm{m}^{3} \mathrm{~d}^{-1} \\
\text { (c) }\end{array}$ & $\begin{array}{l}\text { Deviation } \\
\text { of Flow } \\
\text { Rate }\end{array}$ & $\begin{array}{l}\text { Avg. Test } \\
\text { Temp. }\left({ }^{\circ} \mathrm{C}\right) \\
\text { (e) }\end{array}$ & $\begin{array}{l}\text { Temp. } \\
\text { Dev. }\end{array}$ & $\begin{array}{l}\text { Steady- } \\
\text { state } \\
\text { samples }{ }^{\left({ }^{f}\right.}\end{array}$ \\
\hline \multicolumn{14}{|c|}{ LG9 $70^{\circ} \mathrm{C}$ Si pH9 } \\
\hline Reac. 1 & 1.9900 & 4.71E-09 & 4910 & 216 & 31867 & 27100 & 7.55E-02 & $3.00 \mathrm{E}-02$ & $1.54 \mathrm{E}-05$ & $\pm 9.88 \%$ & 69.99 & \pm 2.01 & $11,12,14$ \\
\hline Reac. 2 & 2.0059 & $6.45 \mathrm{E}-09$ & 4815 & 63 & 31500 & 56033 & $1.01 \mathrm{E}-01$ & $3.86 \mathrm{E}-02$ & 2.12E-05 & $\pm 7.97 \%$ & 69.99 & \pm 2.01 & $8,10,13$ \\
\hline Reac. 3 & 1.4754 & 7.45E-09 & 5177 & 70 & 35967 & 84033 & $1.26 \mathrm{E}-01$ & 4.68E-02 & $1.77 \mathrm{E}-05$ & $\pm 7.73 \%$ & 69.99 & \pm 2.01 & $11,12,14$ \\
\hline \multicolumn{14}{|c|}{ LG9 $90^{\circ} \mathrm{C}$ Si pH 9} \\
\hline Reac. 1 & 1.3113 & 2.85E-09 & 15633 & 448 & 105133 & 40667 & $1.46 \mathrm{E}-01$ & 5.77E-02 & $6.24 \mathrm{E}-06$ & $\pm 8.80 \%$ & 89.93 & \pm 2.17 & $4,5,6$ \\
\hline Reac. 2 & 1.3009 & 8.74E-09 & 7440 & 106 & 58367 & 73533 & 2.12E-01 & 7.39E-02 & $1.80 \mathrm{E}-05$ & $\pm 4.71 \%$ & 89.93 & \pm 2.17 & \\
\hline Reac. 3 & 1.2875 & 8.85E-09 & 6600 & 83 & 50633 & 106333 & $1.91 \mathrm{E}-01$ & $6.42 \mathrm{E}-02$ & $1.80 \mathrm{E}-05$ & $\pm 1.16 \%$ & 89.93 & \pm 2.17 & \\
\hline Reac. 4 & 1.3096 & $1.84 \mathrm{E}-08$ & 3327 & 83 & 28733 & 102833 & $2.00 \mathrm{E}-01$ & 6.57E-02 & 3.73E-05 & $\pm 1.35 \%$ & 89.93 & \pm 2.17 & \\
\hline Reac. 5 & 1.2861 & 3.05E-08 & 2410 & 81 & 21167 & 100967 & $2.41 \mathrm{E}-01$ & 8.43E-02 & $6.05 \mathrm{E}-05$ & $\pm 7.61 \%$ & 89.93 & \pm 2.17 & \\
\hline \multicolumn{14}{|c|}{$\mathrm{A} 16123^{\circ} \mathrm{C}$ pH 9} \\
\hline Reac. 1 & 0.7493 & $1.00 \mathrm{E}-08$ & 276 & 311 & 1044 & 1113 & 5.70E-03 & $1.76 \mathrm{E}-03$ & $1.30 \mathrm{E}-05$ & $\pm 4.39 \%$ & 22.70 & \pm 0.65 & \\
\hline Reac. 2 & 0.3751 & $3.04 \mathrm{E}-08$ & 122 & 155 & 536 & 454 & 7.61E-03 & $2.01 \mathrm{E}-03$ & 1.97E-05 & $\pm 6.52 \%$ & 22.70 & \pm 0.65 & \\
\hline Reac. 3 & 0.1499 & $1.40 \mathrm{E}-07$ & {$[75.6]$} & [49.4] & 172 & [164] & $2.18 E-02$ & --- & 3.58E-05 & $\pm 2.09 \%$ & 22.70 & \pm 0.65 & \\
\hline Reac. 4 & 0.0298 & $9.09 \mathrm{E}-07$ & [75.6] & [49.4] & [134] & [164] & $1.41 E-01$ & --- & 4.08E-05 & $\pm 1.79 \%$ & 22.70 & \pm 0.65 & \\
\hline Reac. 5 & 0.0203 & 6.23E-07 & [75.6] & [49.4] & [134] & [164] & $9.71 E-02$ & --- & 3.58E-05 & $\pm 3.21 \%$ & 22.70 & \pm 0.65 & $\mathrm{CP}^{(\mathrm{g})}$ \\
\hline \multicolumn{14}{|c|}{ A161 $23^{\circ} \mathrm{C}$ pH 10} \\
\hline Reac. 1 & 0.0300 & 8.79E-07 & {$[75.6]$} & 70 & 266 & 252 & $1.37 E-01$ & --- & 4.18E-05 & $\pm 8.33 \%$ & 22.70 & \pm 0.65 & $14,16,18$ \\
\hline Reac. 2 & 0.0218 & $7.21 \mathrm{E}-07$ & [75.6] & 61 & 257 & 175 & $1.12 E-01$ & --- & 4.05E-05 & $\pm 3.43 \%$ & 22.70 & \pm 0.65 & $\begin{array}{l}20,22,24 \\
\mathrm{CP}^{(\mathrm{g})}\end{array}$ \\
\hline \multicolumn{14}{|c|}{ A161 $23^{\circ} \mathrm{C}$ pH 11} \\
\hline Reac. 1 & 0.0501 & $1.71 \mathrm{E}-07$ & 167 & 194 & 867 & 582 & 5.89E-02 & 2.21E-02 & $1.42 \mathrm{E}-05$ & $\pm 25.83 \%$ & 22.70 & \pm 0.65 & \\
\hline Reac. 2 & 0.0399 & 4.86E-07 & 114 & 118 & 605 & 368 & $1.14 \mathrm{E}-01$ & $2.71 \mathrm{E}-02$ & 4.14E-05 & $\pm 3.26 \%$ & 22.70 & \pm 0.65 & $\mathrm{CP}^{(\mathrm{g})}$ \\
\hline \multicolumn{14}{|c|}{$\mathrm{A} 16123^{\circ} \mathrm{C}$ pH 12} \\
\hline Reac. 1 & 0.0501 & $5.74 \mathrm{E}-07$ & 266 & 356 & 1297 & 1533 & 3.14E-01 & 7.58E-02 & 3.76E-05 & $\pm 7.69 \%$ & 22.70 & \pm 0.65 & $20,22,26$ \\
\hline Reac. 2 & 0.0406 & $6.23 \mathrm{E}-07$ & 283 & 382 & 1417 & 1343 & 3.64E-01 & 7.96E-02 & 4.21E-05 & $\pm 3.26 \%$ & 22.70 & \pm 0.65 & $\begin{array}{l}20,22,23 \\
\mathrm{CP}^{(\mathrm{g})}\end{array}$ \\
\hline \multicolumn{14}{|c|}{$\mathrm{A} 16140^{\circ} \mathrm{C}$ pH 9} \\
\hline Reac. 1 & 3.6672 & $1.18 \mathrm{E}-09$ & 5807 & 795 & 21433 & 4357 & $1.41 \mathrm{E}-02$ & 5.22E-03 & 7.51E-06 & $\pm 4.36 \%$ & 40.86 & \pm 0.21 & \\
\hline Reac. 2 & 1.4929 & 7.43E-09 & 1413 & 785 & 5093 & 2533 & 2.16E-02 & 7.53E-03 & $1.91 \mathrm{E}-05$ & $\pm 1.10 \%$ & 40.86 & \pm 0.21 & \\
\hline Reac. 3 & 0.7577 & 2.94E-08 & 416 & 297 & 1437 & 822 & $2.52 \mathrm{E}-02$ & 8.04E-03 & 3.83E-05 & $\pm 2.05 \%$ & 40.86 & \pm 0.21 & \\
\hline Reac. 4 & 0.3122 & $1.45 \mathrm{E}-07$ & 193 & 199 & 655 & 485 & 5.74E-02 & $1.58 \mathrm{E}-02$ & 7.65E-05 & $\pm 0.18 \%$ & 40.86 & \pm 0.21 & \\
\hline Reac. 5 & 0.0652 & 7.08E-07 & 83 & [49.4] & 195 & [164] & $1.21 \mathrm{E}-01$ & 3.11E-02 & 7.62E-05 & $\pm 0.09 \%$ & 40.86 & \pm 0.21 & \\
\hline
\end{tabular}




\begin{tabular}{|c|c|c|c|c|c|c|c|c|c|c|c|c|c|}
\hline Reactor & $\begin{array}{l}\text { Glass Mass } \\
(\mathrm{g})^{(\mathrm{a})}\end{array}$ & $\begin{array}{l}\text { Steady- } \\
\text { State q/S, } \\
\mathrm{m} \mathrm{s}^{-1}\end{array}$ & $\begin{array}{l}\text { [B] Steady } \\
\text { State, } \\
\mu \mathrm{g} \mathrm{L}^{-1}\end{array}$ & $\begin{array}{l}{[\mathrm{Al}] \text { Steady }} \\
\text { State, } \\
\mu \mathrm{g} \mathrm{L}^{-1}\end{array}$ & $\begin{array}{l}{[\mathrm{Na}]} \\
\text { Steady } \\
\text { State, } \\
\mu \mathrm{g} \mathrm{L}^{-1}\end{array}$ & $\begin{array}{l}\text { [Si] Steady } \\
\text { State, } \\
\mu \mathrm{g} \mathrm{L}^{-1}\end{array}$ & $\begin{array}{l}\text { B steady-state rate, } \\
\mathrm{g} \mathrm{m}^{-2} \mathrm{~d}^{-1}\end{array}$ & $\begin{array}{l}2 \sigma \mathrm{B} \text { rate } \\
\text { error }^{(b)}\end{array}$ & $\begin{array}{l}\text { Avg. Flow } \\
\text { Rate, } \mathrm{m}^{3} \mathrm{~d}^{-1} \\
\text { (c) }\end{array}$ & $\begin{array}{l}\text { Deviation } \\
\text { of Flow } \\
\text { Rate }^{(d)}\end{array}$ & $\begin{array}{l}\text { Avg. Test } \\
\text { Temp. }\left({ }^{\circ} \mathrm{C}\right) \\
\text { (e) }\end{array}$ & $\begin{array}{l}\text { Temp. } \\
\text { Dev. }\end{array}$ & $\begin{array}{l}\text { Steady- } \\
\text { state } \\
\text { samples }{ }^{(f)}\end{array}$ \\
\hline Reac. 6 & 0 & 7.47E-07 & [75.6] & {$[49.4]$} & [134] & [164] & $1.16 E-01$ & --- & $1.87 \mathrm{E}-05$ & $\pm 0.91 \%$ & 40.86 & \pm 0.21 & \\
\hline \multicolumn{14}{|c|}{$\mathrm{A} 16140^{\circ} \mathrm{C}$ pH 10} \\
\hline Reac. 1 & 0.0594 & 7.79E-07 & 102 & 220 & 577 & 567 & 1.63E-01 & 3.98E-02 & 7.54E-05 & $\pm 1.38 \%$ & 40.86 & \pm 0.18 & \\
\hline Reac. 2 & 0 & $9.20 \mathrm{E}-07$ & [75.6] & 159 & 371 & 385 & $1.43 E-01$ & --- & 2.29E-05 & $\pm 3.13 \%$ & 40.86 & \pm 0.18 & \\
\hline \multicolumn{14}{|c|}{$\mathrm{A161} 40^{\circ} \mathrm{C}$ pH 11} \\
\hline Reac. 1 & 0.0501 & 4.62E-07 & 348 & 412 & 1200 & 1520 & 3.32E-01 & $9.81 \mathrm{E}-02$ & $3.75 \mathrm{E}-05$ & $\pm 2.93 \%$ & 40.20 & \pm 2.60 & $9,10,11$ \\
\hline Reac. 2 & 0 & $3.09 \mathrm{E}-07$ & 345 & 404 & 1183 & 1657 & 2.19E-01 & 1.15E-01 & 7.70E-06 & $\pm 26.98 \%$ & 40.20 & \pm 2.60 & \\
\hline \multicolumn{14}{|c|}{$\mathrm{A} 16140^{\circ} \mathrm{C}$ pH 12} \\
\hline Reac. 1 & 0.1042 & 5.43E-07 & 457 & 769 & 2420 & 2530 & $5.11 \mathrm{E}-01$ & 1.37E-01 & 7.63E-05 & $\pm 6.99 \%$ & 39.93 & \pm 2.96 & $13,15,17$ \\
\hline Reac. 2 & 0 & 3.54E-07 & 711 & 1100 & 3337 & 3640 & $5.18 \mathrm{E}-01$ & $1.87 \mathrm{E}-01$ & 9.49E-06 & $\pm 6.91 \%$ & 39.93 & \pm 2.96 & $9,13,15$ \\
\hline \multicolumn{14}{|c|}{$\mathrm{A161} 70^{\circ} \mathrm{C} \mathrm{pH} 9$} \\
\hline Reac. 1 & 0.3992 & 6.53E-09 & 9873 & 1947 & 35767 & 6467 & 1.33E-01 & $8.51 \mathrm{E}-02$ & 4.38E-06 & $\pm 28.11 \%$ & 70.99 & \pm 0.19 & (h) \\
\hline Reac. 2 & 0.1545 & $9.35 \mathrm{E}-08$ & 1340 & 885 & 4613 & 3233 & $2.58 \mathrm{E}-01$ & 8.32E-02 & $2.24 \mathrm{E}-05$ & $\pm 5.02 \%$ & 70.99 & \pm 0.19 & \\
\hline Reac. 3 & 0.0509 & $5.26 \mathrm{E}-07$ & 432 & 384 & 1400 & 1380 & 4.68E-01 & $1.24 \mathrm{E}-01$ & 3.81E-05 & $\pm 1.62 \%$ & 70.99 & \pm 0.19 & \\
\hline Reac. 4 & 0.0272 & 2.33E-06 & 177 & 165 & 484 & 632 & $8.50 \mathrm{E}-01$ & $1.68 \mathrm{E}-01$ & 7.79E-05 & $\pm 0.71 \%$ & 70.99 & \pm 0.19 & \\
\hline Reac. 5 & 0 & $1.85 \mathrm{E}-06$ & [75.6] & 59 & 197 & 255 & $2.88 E-01$ & --- & 3.87E-05 & $\pm 0.46 \%$ & 70.99 & \pm 0.19 & \\
\hline \multicolumn{14}{|c|}{$\mathrm{A161} 70^{\circ} \mathrm{C}$ pH 10} \\
\hline Reac. 1 & 0.0249 & 2.93E-06 & 326 & 439 & 1157 & 1540 & $1.97 \mathrm{E}+00$ & 3.66E-01 & 7.52E-05 & $\pm 2.46 \%$ & 70.99 & \pm 0.19 & $\begin{array}{l}\text { No steady } \\
\text { state }^{(i)}\end{array}$ \\
\hline Reac. 2 & 0 & 2.83E-06 & 268 & 365 & 925 & 1235 & $1.56 \mathrm{E}+00$ & 4.97E-01 & 3.70E-05 & $\pm 7.21 \%$ & 70.99 & \pm 0.19 & $10,11^{(\mathrm{i})}$ \\
\hline \multicolumn{14}{|c|}{$\mathrm{A161} 70^{\circ} \mathrm{C}$ pH 11} \\
\hline Reac. 1 & 0.0499 & 1.07E-06 & 1330 & 1500 & 4557 & 4950 & $2.94 \mathrm{E}+00$ & 8.22E-01 & 7.31E-05 & $\pm 3.54 \%$ & 70.09 & \pm 1.51 & $4,5,6$ \\
\hline Reac. 2 & 0 & 7.67E-07 & 1153 & 1163 & 3610 & 4113 & $1.82 \mathrm{E}+00$ & 6.89E-01 & 1.75E-05 & $\pm 7.74 \%$ & 70.09 & \pm 1.51 & \\
\hline \multicolumn{14}{|c|}{$\mathrm{A161} 70^{\circ} \mathrm{C}$ pH 12} \\
\hline Reac. 1 & 0.0500 & 7.49E-07 & 2547 & 3337 & 8683 & 9767 & $3.93 \mathrm{E}+00$ & $1.28 \mathrm{E}+00$ & 5.69E-05 & $\pm 5.72 \%$ & 70.09 & \pm 1.51 & $2,3,4$ \\
\hline Reac. 2 & 0 & 7.19E-07 & 1487 & 1803 & 5000 & 5440 & $2.20 \mathrm{E}+00$ & $1.28 \mathrm{E}+00$ & $1.62 \mathrm{E}-05$ & $\pm 24.47 \%$ & 70.09 & \pm 1.51 & \\
\hline \multicolumn{14}{|c|}{$\mathrm{A} 16190^{\circ} \mathrm{C}$ pH 9} \\
\hline Reac. 1 & 0.4001 & $1.32 \mathrm{E}-08$ & 6103 & 2713 & 25067 & 11167 & $1.66 \mathrm{E}-01$ & $6.11 \mathrm{E}-02$ & 8.70E-06 & $\pm 7.34 \%$ & 90.63 & \pm 1.71 & $6,7,8$ \\
\hline Reac. 2 & 0.1514 & 7.81E-08 & 1300 & 1533 & 4993 & 6447 & 2.09E-01 & 6.62E-02 & $1.86 \mathrm{E}-05$ & $\pm 1.35 \%$ & 90.63 & \pm 1.71 & \\
\hline Reac. 3 & 0.1007 & $5.41 \mathrm{E}-07$ & 604 & 699 & 2187 & 2970 & 6.73E-01 & 1.79E-01 & 7.53E-05 & $\pm 0.55 \%$ & 90.63 & \pm 1.71 & \\
\hline Reac. 4 & 0.0512 & $2.80 \mathrm{E}-06$ & 279 & 333 & 1000 & 1247 & $1.61 \mathrm{E}+00$ & 2.97E-01 & $1.52 \mathrm{E}-04$ & $\pm 0.17 \%$ & 90.63 & \pm 1.71 & \\
\hline Reac. 5 & 0 & $9.72 \mathrm{E}-07$ & 550 & 599 & 1917 & 2490 & $1.10 \mathrm{E}+00$ & 3.83E-01 & 3.61E-05 & $\pm 6.06 \%$ & 90.63 & \pm 1.71 & \\
\hline
\end{tabular}




\begin{tabular}{|c|c|c|c|c|c|c|c|c|c|c|c|c|c|}
\hline Reactor & $\begin{array}{l}\text { Glass Mass } \\
(\mathrm{g})^{(\mathrm{a})}\end{array}$ & $\begin{array}{l}\text { Steady- } \\
\text { State q/S, } \\
\mathrm{m} \mathrm{s}^{-1}\end{array}$ & $\begin{array}{l}\text { [B] Steady } \\
\text { State, } \\
\mu \mathrm{g} \mathrm{L}^{-1}\end{array}$ & $\begin{array}{l}\text { [Al] Steady } \\
\text { State, } \\
\mu \mathrm{g} \mathrm{L}^{-1}\end{array}$ & $\begin{array}{l}{[\mathrm{Na}]} \\
\text { Steady } \\
\text { State, } \\
\mu \mathrm{g} \mathrm{L}^{-1}\end{array}$ & $\begin{array}{l}\text { [Si] Steady } \\
\text { State, } \\
\mu \mathrm{g} \mathrm{L}^{-1}\end{array}$ & $\begin{array}{l}\text { B steady-state rate, } \\
\mathrm{g} \mathrm{m}^{-2} \mathrm{~d}^{-1}\end{array}$ & $\begin{array}{l}2 \sigma \mathrm{B} \text { rate } \\
\text { error }^{(b)}\end{array}$ & $\begin{array}{l}\text { Avg. Flow } \\
\text { Rate, } \mathrm{m}^{3} \mathrm{~d}^{-1} \\
\text { (c) }\end{array}$ & $\begin{array}{l}\text { Deviation } \\
\text { of Flow } \\
\text { Rate }^{(\mathrm{d})}\end{array}$ & $\begin{array}{l}\text { Avg. Test } \\
\text { Temp. }\left({ }^{\circ} \mathrm{C}\right) \\
\text { (e) }\end{array}$ & $\begin{array}{l}\text { Temp. } \\
\text { Dev. }\end{array}$ & $\begin{array}{l}\text { Steady- } \\
\text { state } \\
\text { samples }{ }^{(f)}\end{array}$ \\
\hline \multicolumn{14}{|c|}{$\mathrm{A} 16190^{\circ} \mathrm{C}$ pH 10} \\
\hline Reac. 1 & 0.0507 & $2.46 \mathrm{E}-06$ & 540 & 649 & 2460 & 2457 & $2.73 \mathrm{E}+00$ & $6.28 \mathrm{E}-01$ & $1.50 \mathrm{E}-04$ & $\pm 2.01 \%$ & 90.63 & \pm 1.71 & $3,4,5$ \\
\hline Reac. 2 & 0 & 9.61E-07 & 984 & 1187 & 4237 & 4280 & $1.95 \mathrm{E}+00$ & 7.38E-01 & 3.64E-05 & $\pm 8.34 \%$ & 90.63 & \pm 1.71 & $6,7,9$ \\
\hline \multicolumn{14}{|c|}{$\mathrm{A} 16190^{\circ} \mathrm{C}$ pH 11} \\
\hline Reac. 1 & 0.0989 & $1.05 \mathrm{E}-06$ & 2277 & 2220 & 8717 & 7983 & $4.92 \mathrm{E}+00$ & $1.48 \mathrm{E}+00$ & $1.52 \mathrm{E}-04$ & $\pm 1.17 \%$ & 90.52 & \pm 1.63 & $2,3,4$ \\
\hline Reac. 2 & 0 & $4.58 \mathrm{E}-08$ & 8493 & 6317 & 59533 & 40533 & $8.01 \mathrm{E}-01$ & $9.01 \mathrm{E}-01$ & 1.69E-06 & $\pm 53.72 \%$ & 90.52 & \pm 1.63 & \\
\hline \multicolumn{14}{|c|}{$\mathrm{A} 16190^{\circ} \mathrm{C}$ pH 12} \\
\hline Reac. 1 & 0.1008 & 5.47E-06 & 438 & 617 & 2043 & 2607 & $4.93 \mathrm{E}+00$ & $2.60 \mathrm{E}-01$ & $1.56 \mathrm{E}-04$ & $\pm 0.59 \%$ & 90.52 & \pm 1.63 & $\begin{array}{l}\text { No steady } \\
\text { state }^{(i)}\end{array}$ \\
\hline Reac. 2 & 0 & 4.09E-07 & 3747 & 3607 & 14600 & 12133 & $3.15 \mathrm{E}+00$ & $1.26 \mathrm{E}+00$ & $1.40 \mathrm{E}-05$ & $\pm 8.95 \%$ & 90.52 & \pm 1.63 & $5,6,7$ \\
\hline \multicolumn{14}{|c|}{ A161 $23^{\circ} \mathrm{C}$ Si pH 9} \\
\hline Reac. 1 & 4.0047 & 2.92E-09 & 481 & 139 & 1680 & 5313 & 2.89E-03 & $9.49 \mathrm{E}-04$ & $2.04 \mathrm{E}-05$ & $\pm 1.74 \%$ & 21.15 & \pm 0.72 & \\
\hline Reac. 2 & 4.0004 & 3.24E-09 & 476 & 30 & 1723 & 18600 & 3.17E-03 & $1.04 \mathrm{E}-03$ & 2.25E-05 & $\pm 2.09 \%$ & 21.15 & \pm 0.72 & \\
\hline Reac. 3 & 3.9968 & $3.04 \mathrm{E}-09$ & 515 & 29 & 1953 & 33600 & 3.23E-03 & $1.07 \mathrm{E}-03$ & $2.12 \mathrm{E}-05$ & $\pm 2.89 \%$ & 21.15 & \pm 0.72 & \\
\hline Reac. 4 & 2.0078 & 3.39E-09 & 624 & 321 & 2443 & 2493 & 4.36E-03 & 9.49E-04 & 1.19E-05 & $\pm 1.73 \%$ & 21.81 & \pm 0.51 & \\
\hline Reac. 5 & 2.0001 & 3.10E-09 & 599 & 184 & 2117 & 3760 & 3.82E-03 & $1.04 \mathrm{E}-03$ & $1.08 \mathrm{E}-05$ & $\pm 6.14 \%$ & 21.81 & \pm 0.51 & \\
\hline \multicolumn{14}{|c|}{$\mathrm{A161} 40^{\circ} \mathrm{C}$ Si pH 9} \\
\hline Reac. 1 & 2.0025 & 5.91E-09 & 1737 & 240 & 6720 & 9553 & $2.11 \mathrm{E}-02$ & 7.40E-03 & $2.04 \mathrm{E}-05$ & $\pm 1.28 \%$ & 40.12 & \pm 1.46 & \\
\hline Reac. 2 & 2.0473 & 4.56E-09 & 2493 & 52 & 9823 & 26400 & 2.34E-02 & $9.85 \mathrm{E}-03$ & $1.61 \mathrm{E}-05$ & $\pm 11.87 \%$ & 40.12 & \pm 1.46 & \\
\hline Reac. 3 & 1.9732 & 5.95E-09 & 1930 & [49.4] & 7563 & 43567 & $2.37 \mathrm{E}-02$ & 8.32E-03 & 2.02E-05 & $\pm 1.70 \%$ & 40.12 & \pm 1.46 & \\
\hline Reac. 4 & 1.0009 & $1.19 \mathrm{E}-08$ & 1333 & 461 & 5030 & 5283 & $3.26 \mathrm{E}-02$ & $1.14 \mathrm{E}-02$ & 2.05E-05 & $\pm 1.30 \%$ & 40.82 & \pm 0.76 & \\
\hline Reac. 5 & 1.0004 & $1.21 \mathrm{E}-08$ & 1110 & 439 & 3810 & 4587 & $2.76 \mathrm{E}-02$ & $9.50 \mathrm{E}-03$ & $2.08 \mathrm{E}-05$ & $\pm 0.49 \%$ & 40.82 & \pm 0.76 & \\
\hline \multicolumn{14}{|c|}{$\mathrm{A} 1617^{\circ} \mathrm{C}$ Si pH 9} \\
\hline Reac. 1 & 1.9973 & 5.42E-09 & 8713 & 187 & 36100 & 28000 & $9.73 \mathrm{E}-02$ & $3.66 \mathrm{E}-02$ & $1.78 \mathrm{E}-05$ & $\pm 8.38 \%$ & 69.67 & \pm 0.50 & \\
\hline Reac. 2 & 2.0047 & 4.61E-09 & 10030 & 55 & 39800 & 54800 & 9.52E-02 & 3.76E-02 & $1.54 \mathrm{E}-05$ & $\pm 9.87 \%$ & 69.67 & \pm 0.50 & $9,11,12$ \\
\hline Reac. 3 & 2.0006 & 5.76E-09 & 8487 & {$[49.4]$} & 35267 & 82267 & $1.01 \mathrm{E}-01$ & 3.64E-02 & $1.92 \mathrm{E}-05$ & $\pm 5.30 \%$ & 69.67 & \pm 0.50 & \\
\hline Reac. 4 & 0.9996 & $6.56 \mathrm{E}-09$ & 9773 & 1247 & 38167 & 9093 & $1.32 \mathrm{E}-01$ & 4.77E-02 & $1.11 \mathrm{E}-05$ & $\pm 4.25 \%$ & 68.40 & \pm 0.57 & \\
\hline Reac. 5 & 1.0002 & $1.24 \mathrm{E}-08$ & 5937 & 766 & 23033 & 11833 & $1.52 \mathrm{E}-01$ & 5.30E-02 & 2.08E-05 & $\pm 1.76 \%$ & 68.40 & \pm 0.57 & \\
\hline \multicolumn{14}{|c|}{ A161 $90^{\circ} \mathrm{C}$ Si pH 9} \\
\hline Reac. 1 & 1.2549 & $9.17 \mathrm{E}-09$ & 7887 & 69 & 34100 & 104667 & 1.49E-01 & 5.15E-02 & $1.87 \mathrm{E}-05$ & $\pm 3.96 \%$ & 89.93 & \pm 2.17 & \\
\hline Reac. 2 & 1.2604 & 7.67E-09 & 7903 & 452 & 31600 & 40100 & $1.25 \mathrm{E}-01$ & $4.41 \mathrm{E}-02$ & $1.60 \mathrm{E}-05$ & $\pm 4.32 \%$ & 89.93 & \pm 2.17 & \\
\hline Reac. 3 & 1.2545 & 7.89E-09 & 7867 & 179 & 31100 & 73933 & $1.28 \mathrm{E}-01$ & 4.66E-02 & $1.63 \mathrm{E}-05$ & $\pm 6.80 \%$ & 89.93 & \pm 2.17 & \\
\hline
\end{tabular}




\begin{tabular}{|c|c|c|c|c|c|c|c|c|c|c|c|c|c|}
\hline Reactor & $\begin{array}{l}\text { Glass Mass } \\
(\mathrm{g})^{(\mathrm{a})}\end{array}$ & $\begin{array}{l}\text { Steady- } \\
\text { State q/S, } \\
\mathrm{m} \mathrm{s}^{-1}\end{array}$ & $\begin{array}{l}\text { [B] Steady } \\
\text { State, } \\
\mu \mathrm{g} \mathrm{L}^{-1}\end{array}$ & $\begin{array}{l}\text { [Al] Steady } \\
\text { State, } \\
\mu \mathrm{g} \mathrm{L}^{-1}\end{array}$ & $\begin{array}{l}{[\mathrm{Na}]} \\
\text { Steady } \\
\text { State, } \\
\mu \mathrm{g} \mathrm{L}^{-1}\end{array}$ & $\begin{array}{l}\text { [Si] Steady } \\
\text { State, } \\
\mu \mathrm{g} \mathrm{L}^{-1}\end{array}$ & $\begin{array}{l}\text { B steady-state rate, } \\
\mathrm{g} \mathrm{m}^{-2} \mathrm{~d}^{-1}\end{array}$ & $\begin{array}{l}2 \sigma \mathrm{B} \text { rate } \\
\text { error }^{(b)}\end{array}$ & $\begin{array}{l}\text { Avg. Flow } \\
\text { Rate, } \mathrm{m}^{3} \mathrm{~d}^{-1} \\
\text { (c) }\end{array}$ & $\begin{array}{l}\text { Deviation } \\
\text { of Flow } \\
\text { Rate }^{(d)}\end{array}$ & $\begin{array}{l}\text { Avg. Test } \\
\text { Temp. }\left({ }^{\circ} \mathrm{C}\right) \\
\text { (e) }\end{array}$ & $\begin{array}{l}\text { Temp. } \\
\text { Dev. }\end{array}$ & $\begin{array}{l}\text { Steady- } \\
\text { state } \\
\text { samples }{ }^{(\mathrm{f})}\end{array}$ \\
\hline Reac. 4 & 0.6248 & $1.98 \mathrm{E}-08$ & 3787 & 1290 & 16400 & 13700 & $1.55 \mathrm{E}-01$ & 4.86E-02 & 2.00E-05 & $\pm 1.70 \%$ & 89.60 & \pm 0.77 & \\
\hline Reac. 5 & 0.6253 & 2.02E-08 & 3493 & 851 & 14733 & 20867 & $1.45 \mathrm{E}-01$ & 4.93E-02 & 2.06E-05 & $\pm 1.93 \%$ & 89.60 & \pm 0.77 & \\
\hline
\end{tabular}

Notes:

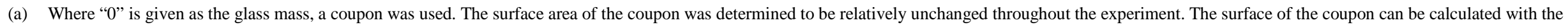
given $\mathrm{q} / \mathrm{S}$ of the experiment.

(b) When "---" is provided as the $2 \sigma \mathrm{B}$ rate error, the corresponding B steady-state rate value is a maximum value calculated from the EQL. When a rate is a maximum value it is also italicized.

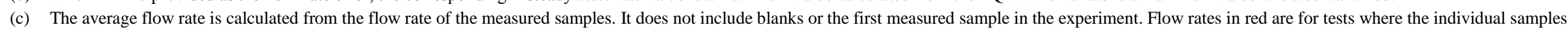
in the test varied by $>10 \%$. For tests where the flow rate relative standard deviation was $>10 \%$, the steady-state values that are presented are from the last three samplings from that reactor.

(d) The deviation of the flow rate is determined as the standard deviation of the average flow rate and does not include blanks or the first measured sample in the experiment.

(e) Average test temperature determined from hourly thermocouple readings.

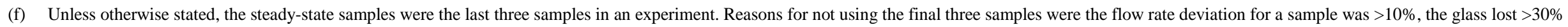

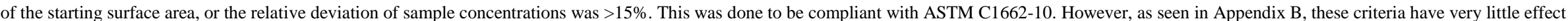
on release rate calculated values of consecutive samples.

(g) "CP" means the reactor contained both powder and coupons to ensure enough surface area was present to have a measurable analyte concentration in the effluent.

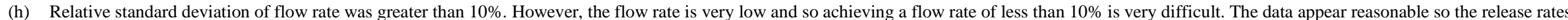
calculated here were used in the $\mathrm{K}_{\mathrm{g}}$ regression. The last three data points were used for steady-state values.

(i) A steady-state ( $<15 \%$ relative standard deviation between samples) was never achieved. The steady-state values given are from the last three samplings from that reactor.

(j) The last three reactors were at steady-state release concentrations. However, the flow rate of sample 9 was $11 \%$ from the average flow rate for that reactor so it was not used in the calculation. 


\section{Appendix B}

\section{Steady-State Single-Pass Flow-Through Results}

In the following tables, a value with a "** is a value below the EQL but above the instrument detection limit. The value is FIO. The EQL value is provided in brackets. Due to the relatively high Si concentration in tests with Si added to the inlet, no Si release rates are provided from these tests. 


\begin{tabular}{|c|c|c|c|c|c|c|c|c|c|c|c|c|c|c|}
\hline Sample ID & $\begin{array}{l}\text { Influent } \\
{[\mathrm{Si}]} \\
(\mathrm{ppm})\end{array}$ & $\begin{array}{l}\text { Flow } \\
\text { Rate, } q \\
\left(\mathrm{~m}^{3} \mathrm{~d}^{-1}\right)\end{array}$ & $\begin{array}{l}\text { Time } \\
\text { (d) }\end{array}$ & $\begin{array}{l}\text { Glass } \\
\text { Mass } \\
\text { (g) }\end{array}$ & $\begin{array}{l}\text { Surface } \\
\text { Area, S } \\
\left(\mathrm{m}^{2}\right)\end{array}$ & $\begin{array}{l}{[\mathrm{Al}]} \\
\left(\mu \mathrm{g} \mathrm{L}^{-1}\right)\end{array}$ & $\begin{array}{l}\text { Al Norm. } \\
\text { Diss. Rate } \\
\left(\mathrm{g} \mathrm{m}^{-2} \mathrm{~d}^{-1}\right)\end{array}$ & $\begin{array}{l}{[\mathrm{B}]} \\
\left(\mu \mathrm{g} \mathrm{L}^{-1}\right)\end{array}$ & $\begin{array}{l}\text { B Norm. } \\
\text { Diss. Rate } \\
\left(\mathrm{g} \mathrm{m}^{-2} \mathrm{~d}^{-1}\right)\end{array}$ & $\begin{array}{l}{[\mathrm{Si}]} \\
\left(\mu \mathrm{g} \mathrm{L}^{-1}\right)\end{array}$ & $\begin{array}{l}\text { Si Norm. } \\
\text { Diss. Rate } \\
\left(\mathrm{g} \mathrm{m}^{-2} \mathrm{~d}^{-1}\right)\end{array}$ & $\begin{array}{l}{[\mathrm{Na}]} \\
\left(\mu \mathrm{g} \mathrm{L}{ }^{-}\right. \\
\left.{ }^{1}\right)\end{array}$ & $\begin{array}{l}\text { Na Norm. } \\
\text { Diss. Rate, } \\
\mathrm{r} \\
\left(\mathrm{g} \mathrm{m}^{-2} \mathrm{~d}^{-1}\right)\end{array}$ & $\begin{array}{l}\mathrm{r}_{\mathrm{IEX}} \\
\left(\mathrm{mol} \mathrm{Na} \mathrm{No}^{-1}\right)\end{array}$ \\
\hline \multicolumn{15}{|c|}{ LG9 $23^{\circ} \mathrm{C}$ pH 9 Reactor \#1 } \\
\hline LG9.23.9.20.Blank1 & & $1.02 \mathrm{E}-05$ & --- & --- & --- & {$[49.4]$} & --- & [75.6] & --- & [164] & -.- & [134] & --- & -.- \\
\hline LG9.23.9.20.Blank2 & & $9.88 \mathrm{E}-06$ & --- & --- & --- & {$[49.4]$} & --- & [75.6] & --- & [164] & --- & [134] & --- & -.- \\
\hline LG9.23.9.20.Blank3 & & $7.51 \mathrm{E}-06$ & 0 & --- & --- & {$[49.4]$} & --- & [75.6] & --- & [164] & -.- & [134] & --- & --. \\
\hline LG9.23.9.20.2 & & 7.68E-06 & 6 & 0.749 & 1.47E-02 & $\begin{array}{l}{[49.4]} \\
46.6^{*}\end{array}$ & 7.19E-04 & $\begin{array}{l}{[75.6]} \\
47.6^{*}\end{array}$ & $1.49 \mathrm{E}-03$ & [164] & 4.48E-04 & 1,580 & $5.28 \mathrm{E}-03$ & $1.91 \mathrm{E}-09$ \\
\hline LG9.23.9.20.4 & & 7.66E-06 & 13 & 0.749 & $1.47 E-02$ & 66 & $9.65 \mathrm{E}-04$ & $\begin{array}{l}{[75.6]} \\
70.8^{*}\end{array}$ & 1.49E-03 & [164] & 4.47E-04 & 1,400 & 4.67E-03 & $1.60 E-09$ \\
\hline LG9.23.9.20.6 & & 7.69E-06 & 20 & 0.749 & 1.47E-02 & 71 & $1.04 \mathrm{E}-03$ & $\begin{array}{l}{[75.6]} \\
56.3^{*}\end{array}$ & 1.49E-03 & [164] & 4.49E-04 & 1,070 & $3.59 \mathrm{E}-03$ & $1.05 \mathrm{E}-09$ \\
\hline LG9.23.9.20.8 & & $8.48 \mathrm{E}-06$ & 27 & 0.749 & $1.47 \mathrm{E}-02$ & 75 & $1.20 \mathrm{E}-03$ & $\begin{array}{l}{[75.6]} \\
42.0^{*}\end{array}$ & $1.65 \mathrm{E}-03$ & [164] & $4.94 \mathrm{E}-04$ & 887 & $3.27 \mathrm{E}-03$ & $8.20 \mathrm{E}-10$ \\
\hline LG9.23.9.20.10 & & $8.53 \mathrm{E}-06$ & 34 & 0.749 & $1.47 \mathrm{E}-02$ & 70 & $1.12 \mathrm{E}-03$ & $\begin{array}{l}{[75.6]} \\
28.8^{*}\end{array}$ & $1.66 \mathrm{E}-03$ & [164] & $4.97 E-04$ & 706 & $2.62 \mathrm{E}-03$ & $4.87 \mathrm{E}-10$ \\
\hline LG9.23.9.20.12 & & $8.35 \mathrm{E}-06$ & 41 & 0.748 & $1.47 E-02$ & 74 & $1.18 \mathrm{E}-03$ & $\begin{array}{l}{[75.6]} \\
58.9^{*}\end{array}$ & $1.62 \mathrm{E}-03$ & 265 & 7.87E-04 & 630 & $2.29 \mathrm{E}-03$ & $3.38 \mathrm{E}-10$ \\
\hline LG9.23.9.20.14 & & $8.39 \mathrm{E}-06$ & 48 & 0.748 & $1.47 \mathrm{E}-02$ & 70 & $1.12 \mathrm{E}-03$ & $\begin{array}{l}{[75.6]} \\
49.2^{*}\end{array}$ & $1.63 \mathrm{E}-03$ & 225 & $6.71 \mathrm{E}-04$ & 574 & $2.10 \mathrm{E}-03$ & $2.36 \mathrm{E}-10$ \\
\hline LG9.23.9.20.16 & & $9.01 \mathrm{E}-06$ & 55 & 0.748 & $1.47 \mathrm{E}-02$ & 70 & $1.20 \mathrm{E}-03$ & $\begin{array}{l}{[75.6]} \\
48.5^{\star}\end{array}$ & $1.75 \mathrm{E}-03$ & 255 & $8.18 E-04$ & 519 & $2.04 \mathrm{E}-03$ & 1.45E-10 \\
\hline LG9.23.9.20.18 & & $8.50 \mathrm{E}-06$ & 62 & 0.748 & $1.47 E-02$ & 70 & $1.13 \mathrm{E}-03$ & $\begin{array}{l}{[75.6]} \\
42.1^{*}\end{array}$ & $1.65 \mathrm{E}-03$ & 219 & $6.62 E-04$ & 422 & $1.56 \mathrm{E}-03$ & $<0$ \\
\hline LG9.23.9.20.20 & & $8.75 E-06$ & 69 & 0.748 & $1.47 E-02$ & 72 & $1.20 \mathrm{E}-03$ & $\begin{array}{l}{[75.6]} \\
35.6^{*}\end{array}$ & $1.70 \mathrm{E}-03$ & 228 & 7.10E-04 & 406 & $1.55 \mathrm{E}-03$ & $<0$ \\
\hline LG9.23.9.20.22 & & $9.13 \mathrm{E}-06$ & 76 & 0.748 & $1.47 \mathrm{E}-02$ & 67 & $1.17 \mathrm{E}-03$ & $\begin{array}{l}{[75.6]} \\
35.6^{*}\end{array}$ & $1.77 \mathrm{E}-03$ & 205 & $6.66 \mathrm{E}-04$ & 365 & $1.45 \mathrm{E}-03$ & $<0$ \\
\hline LG9.23.9.20.23 & & $8.98 \mathrm{E}-06$ & 79 & 0.747 & $1.47 \mathrm{E}-02$ & 87 & $1.48 \mathrm{E}-03$ & $\begin{array}{l}{[75.6]} \\
62.8^{*}\end{array}$ & $1.74 \mathrm{E}-03$ & 373 & $1.19 \mathrm{E}-03$ & 409 & $1.60 \mathrm{E}-03$ & $<0$ \\
\hline LG9.23.9.20.24 & & $8.77 \mathrm{E}-06$ & 83 & 0.747 & $1.47 \mathrm{E}-02$ & 72 & $1.21 \mathrm{E}-03$ & $\begin{array}{l}{[75.6]} \\
42.6^{*}\end{array}$ & $1.70 \mathrm{E}-03$ & 230 & $7.18 \mathrm{E}-04$ & 379 & $1.45 \mathrm{E}-03$ & $<0$ \\
\hline \multicolumn{15}{|c|}{ LG9 $23^{\circ} \mathrm{C}$ pH 9 Reactor $\# 2$} \\
\hline LG9.23.9.40.Blank1 & & $1.92 \mathrm{E}-05$ & --- & --- & --- & [49.4] & --- & [75.6] & --- & [164] & -- & [134] & --- & -- \\
\hline LG9.23.9.40.Blank2 & & $1.95 \mathrm{E}-05$ & --- & --- & --- & {$[49.4]$} & --- & {$[75.6]$} & --- & [164] & --- & [134] & --- & --- \\
\hline LG9.23.9.40.Blank3 & & $2.14 \mathrm{E}-05$ & 0 & --- & --- & [49.4] & --- & [75.6] & --- & [164] & --- & [134] & --- & -- \\
\hline LG9.23.9.40.2 & & $1.45 \mathrm{E}-05$ & 6 & 0.375 & $7.39 E-03$ & $\begin{array}{l}{[49.4]} \\
23.1^{*}\end{array}$ & $2.70 \mathrm{E}-03$ & {$[75.6]$} & $5.60 \mathrm{E}-03$ & [164] & $1.68 \mathrm{E}-03$ & 597 & $7.50 \mathrm{E}-03$ & $9.58 \mathrm{E}-10$ \\
\hline LG9.23.9.40.4 & & $1.93 \mathrm{E}-05$ & 13 & 0.375 & $7.38 \mathrm{E}-03$ & $\begin{array}{l}{[49.4]} \\
27.9^{*}\end{array}$ & $3.60 \mathrm{E}-03$ & [75.6] & $7.46 \mathrm{E}-03$ & [164] & $2.24 \mathrm{E}-03$ & 344 & $5.76 \mathrm{E}-03$ & $<0$ \\
\hline LG9.23.9.40.6 & & 2.09E-05 & 20 & 0.375 & $7.38 \mathrm{E}-03$ & $\begin{array}{l}{[49.4]} \\
22.6^{*}\end{array}$ & $3.90 \mathrm{E}-03$ & {$[75.6]$} & $8.08 \mathrm{E}-03$ & [164] & $2.43 E-03$ & 216 & $3.92 \mathrm{E}-03$ & $<0$ \\
\hline LG9.23.9.40.8 & & 2.07E-05 & 27 & 0.374 & 7.37E-03 & $\begin{array}{l}{[49.4]} \\
20.9^{*}\end{array}$ & $3.88 \mathrm{E}-03$ & [75.6] & 8.03E-03 & [164] & $2.41 \mathrm{E}-03$ & 169 & 3.05E-03 & $<0$ \\
\hline LG9.23.9.40.10 & & 2.09E-05 & 34 & 0.374 & 7.37E-03 & $\begin{array}{l}{[49.4]} \\
20.6^{*}\end{array}$ & $3.91 \mathrm{E}-03$ & [75.6] & $8.11 \mathrm{E}-03$ & [164] & 2.44E-03 & 155 & $2.82 \mathrm{E}-03$ & $<0$ \\
\hline LG9.23.9.40.12 & & $2.09 \mathrm{E}-05$ & 41 & 0.373 & $7.36 \mathrm{E}-03$ & $\begin{array}{l}{[49.4]} \\
17.7^{*}\end{array}$ & $3.91 \mathrm{E}-03$ & [75.6] & $8.11 \mathrm{E}-03$ & [164] & $2.44 \mathrm{E}-03$ & 171 & $3.11 \mathrm{E}-03$ & $<0$ \\
\hline LG9.23.9.40.14 & & $2.11 \mathrm{E}-05$ & 48 & 0.373 & $7.35 \mathrm{E}-03$ & [49.4] & $3.96 \mathrm{E}-03$ & [75.6] & $8.20 \mathrm{E}-03$ & [164] & $2.46 \mathrm{E}-03$ & 164 & $3.02 \mathrm{E}-03$ & $<0$ \\
\hline
\end{tabular}




\begin{tabular}{|c|c|c|c|c|c|c|c|c|c|c|c|c|c|c|}
\hline Sample ID & $\begin{array}{l}\text { Influent } \\
\text { [Si] } \\
\text { (ppm) }\end{array}$ & $\begin{array}{l}\text { Flow } \\
\text { Rate, q } \\
\left(\mathrm{m}^{3} \mathrm{~d}^{-1}\right)\end{array}$ & $\begin{array}{l}\text { Time } \\
\text { (d) }\end{array}$ & $\begin{array}{l}\text { Glass } \\
\text { Mass } \\
\text { (g) }\end{array}$ & $\begin{array}{l}\text { Surface } \\
\text { Area, } \mathrm{S} \\
\left(\mathrm{m}^{2}\right)\end{array}$ & $\begin{array}{l}{[\mathrm{Al}]} \\
\left(\mu \mathrm{g} \mathrm{L}^{-1}\right)\end{array}$ & $\begin{array}{l}\text { Al Norm. } \\
\text { Diss. Rate } \\
\left(\mathrm{g} \mathrm{m}^{-2} \mathrm{~d}^{-1}\right)\end{array}$ & $\begin{array}{l}{[\mathrm{B}]} \\
\left(\mu \mathrm{L} \mathrm{L}^{-1}\right)\end{array}$ & $\begin{array}{l}\text { B Norm. } \\
\text { Diss. Rate } \\
\left(\mathrm{g} \mathrm{m}^{-2} \mathrm{~d}^{-1}\right)\end{array}$ & $\begin{array}{l}{[\mathrm{Si}]} \\
\left(\mu \mathrm{L}^{-1}\right)\end{array}$ & $\begin{array}{l}\text { Si Norm. } \\
\text { Diss. Rate } \\
\left(\mathrm{g} \mathrm{m}^{-2} \mathrm{~d}^{-1}\right)\end{array}$ & $\begin{array}{l}{[\mathrm{Na}]} \\
(\mu \mathrm{gg} \mathrm{L} \\
\left.{ }^{1}\right)\end{array}$ & $\begin{array}{l}\text { Na Norm. } \\
\text { Diss. Rate, } \\
\mathrm{r} \\
\left(\mathrm{g} \mathrm{m}^{-2} \mathrm{~d}^{-1}\right)\end{array}$ & $\begin{array}{l}\mathrm{r}_{\mathrm{IEX}} \\
\left(\mathrm{mol} \mathrm{Na} \mathrm{a}^{-2} \mathrm{~s}^{-1}\right)\end{array}$ \\
\hline & & & & & & $19.2^{\star}$ & & & & & & & & \\
\hline LG9.23.9.40.16 & & 2.05E-05 & 55 & 0.372 & 7.35E-03 & $\begin{array}{l}{[49.4]} \\
18.2^{\star}\end{array}$ & $3.85 E-03$ & {$[75.6]$} & 7.99E-03 & [164] & $2.40 \mathrm{E}-03$ & 148 & $2.65 \mathrm{E}-03$ & $<0$ \\
\hline LG9.23.9.40.18 & & 2.10E-05 & 62 & 0.372 & 7.34E-03 & $\begin{array}{l}{[49.4]} \\
19.3^{\star}\end{array}$ & $3.94 \mathrm{E}-03$ & {$[75.6]$} & 8.17E-03 & [164] & $2.45 \mathrm{E}-03$ & $\begin{array}{l}{[134]} \\
118^{\star}\end{array}$ & $2.46 \mathrm{E}-03$ & $<0$ \\
\hline LG9.23.9.40.20 & & 2.06E-05 & 69 & 0.372 & 7.34E-03 & $\begin{array}{l}{[49.4]} \\
17.8^{\star}\end{array}$ & $3.88 \mathrm{E}-03$ & [75.6] & 8.05E-03 & [164] & $2.42 \mathrm{E}-03$ & 142 & $2.56 \mathrm{E}-03$ & $<0$ \\
\hline LG9.23.9.40.22 & & 2.09E-05 & 76 & 0.371 & 7.33E-03 & $\begin{array}{l}{[49.4]} \\
17.8^{\star}\end{array}$ & 3.94E-03 & {$[75.6]$} & 8.17E-03 & [164] & $2.46 \mathrm{E}-03$ & $\begin{array}{l}{[134]} \\
130^{*}\end{array}$ & $2.46 \mathrm{E}-03$ & $<0$ \\
\hline LG9.23.9.40.23 & & $2.07 E-05$ & 79 & 0.371 & 7.33E-03 & $\begin{array}{l}{[49.4]} \\
17.4^{*}\end{array}$ & $3.90 \mathrm{E}-03$ & [75.6] & $8.09 E-03$ & [164] & 2.43E-03 & 147 & $2.67 \mathrm{E}-03$ & $<0$ \\
\hline LG9.23.9.40.24 & & $2.05 E-05$ & 83 & 0.371 & $7.33 \mathrm{E}-03$ & $\begin{array}{l}{[49.4]} \\
17.7^{\star}\end{array}$ & $3.87 \mathrm{E}-03$ & {$[75.6]$} & $8.01 E-03$ & [164] & $2.41 \mathrm{E}-03$ & $\begin{array}{l}{[134]} \\
133^{\star}\end{array}$ & $2.41 \mathrm{E}-03$ & $<0$ \\
\hline \multicolumn{15}{|c|}{ LG9 $23^{\circ} \mathrm{C} \mathrm{pH} 9$ Reactor \#3 } \\
\hline LG9.23.9.80A.Blank1 & & $3.99 \mathrm{E}-05$ & -.- & --- & -.- & [49.4] & --- & {$[75.6]$} & --- & [164] & --- & [134] & --- & --- \\
\hline LG9.23.9.80A.Blank2 & & 3.96E-05 & --- & --- & --- & [49.4] & --- & {$[75.6]$} & --- & [164] & --- & [134] & --- & --- \\
\hline LG9.23.9.80A.Blank3 & & $3.92 E-05$ & 0 & --- & -- & [49.4] & --- & {$[75.6]$} & --- & [164] & -- & [134] & -- & -- \\
\hline LG9.23.9.80A.2 & & $2.99 \mathrm{E}-05$ & 6 & 0.150 & $2.95 \mathrm{E}-03$ & [49.4] & $1.40 \mathrm{E}-02$ & [75.6] & $2.90 \mathrm{E}-02$ & [164] & $8.71 \mathrm{E}-03$ & 135 & $8.78 \mathrm{E}-03$ & $<0$ \\
\hline LG9.23.9.80A.4 & & 3.74E-05 & 13 & 0.149 & $2.94 \mathrm{E}-03$ & [49.4] & $1.76 \mathrm{E}-02$ & {$[75.6]$} & 3.64E-02 & [164] & 1.09E-02 & [134] & $1.09 \mathrm{E}-02$ & $<0$ \\
\hline LG9.23.9.80A.6 & & $4.01 \mathrm{E}-05$ & 20 & 0.148 & 2.93E-03 & [49.4] & $1.89 \mathrm{E}-02$ & {$[75.6]$} & 3.92E-02 & [164] & 1.18E-02 & [134] & $1.18 \mathrm{E}-02$ & $<0$ \\
\hline LG9.23.9.80A.8 & & $4.02 \mathrm{E}-05$ & 27 & 0.147 & $2.92 \mathrm{E}-03$ & [49.4] & $1.90 \mathrm{E}-02$ & {$[75.6]$} & $3.94 \mathrm{E}-02$ & [164] & $1.18 \mathrm{E}-02$ & {$[134]$} & $1.18 \mathrm{E}-02$ & $<0$ \\
\hline LG9.23.9.80A.10 & & 4.05E-05 & 34 & 0.146 & $2.91 \mathrm{E}-03$ & [49.4] & $1.92 E-02$ & {$[75.6]$} & $3.98 \mathrm{E}-02$ & [164] & $1.20 \mathrm{E}-02$ & $\begin{array}{l}{[134]} \\
50.1^{*}\end{array}$ & $1.20 \mathrm{E}-02$ & $<0$ \\
\hline LG9.23.9.80A.12 & & $4.02 E-05$ & 41 & 0.146 & $2.90 \mathrm{E}-03$ & [49.4] & $1.92 \mathrm{E}-02$ & [75.6] & $3.97 \mathrm{E}-02$ & [164] & 1.19E-02 & $\begin{array}{l}{[134]} \\
84.8^{\star}\end{array}$ & $1.19 \mathrm{E}-02$ & $<0$ \\
\hline LG9.23.9.80A.14 & & 4.04E-05 & 48 & 0.145 & $2.88 \mathrm{E}-03$ & [49.4] & $1.94 \mathrm{E}-02$ & [75.6] & $4.01 E-02$ & [164] & $1.20 \mathrm{E}-02$ & $\begin{array}{l}{[134]} \\
93.8^{*}\end{array}$ & $1.21 \mathrm{E}-02$ & $<0$ \\
\hline LG9.23.9.80A.16 & & 4.06E-05 & 55 & 0.144 & 2.87E-03 & [49.4] & $1.95 \mathrm{E}-02$ & {$[75.6]$} & 4.05E-02 & [164] & $1.22 \mathrm{E}-02$ & $\begin{array}{l}{[134]} \\
79.6^{*}\end{array}$ & $1.22 \mathrm{E}-02$ & $<0$ \\
\hline LG9.23.9.80A.18 & & 4.04E-05 & 62 & 0.143 & $2.86 \mathrm{E}-03$ & [49.4] & $1.95 \mathrm{E}-02$ & {$[75.6]$} & 4.03E-02 & [164] & $1.21 \mathrm{E}-02$ & $\begin{array}{l}{[134]} \\
74.0^{*}\end{array}$ & $1.21 \mathrm{E}-02$ & $<0$ \\
\hline LG9.23.9.80A.20 & & $4.02 E-05$ & 69 & 0.142 & $2.85 \mathrm{E}-03$ & [49.4] & $1.94 \mathrm{E}-02$ & [75.6] & $4.03 E-02$ & [164] & $1.21 \mathrm{E}-02$ & $\begin{array}{l}{[134]} \\
48.3^{*}\end{array}$ & $1.21 \mathrm{E}-02$ & $<0$ \\
\hline LG9.23.9.80A.22 & & 4.03E-05 & 76 & 0.142 & $2.84 \mathrm{E}-03$ & [49.4] & $1.96 \mathrm{E}-02$ & {$[75.6]$} & $4.06 \mathrm{E}-02$ & [164] & 1.22E-02 & $\begin{array}{l}{[134]} \\
63.7^{\star}\end{array}$ & $1.22 \mathrm{E}-02$ & $<0$ \\
\hline LG9.23.9.80A.23 & & 4.05E-05 & 79 & 0.141 & 2.84E-03 & [49.4] & $1.97 \mathrm{E}-02$ & {$[75.6]$} & 4.08E-02 & [164] & $1.23 \mathrm{E}-02$ & $\begin{array}{l}{[134]} \\
85.2^{\star}\end{array}$ & $1.23 \mathrm{E}-02$ & $<0$ \\
\hline LG9.23.9.80A.24 & & 4.05E-05 & 83 & 0.141 & 2.83E-03 & [49.4] & $1.97 E-02$ & [75.6] & 4.09E-02 & [164] & $1.23 \mathrm{E}-02$ & $\begin{array}{l}{[134]} \\
81.0^{\star}\end{array}$ & $1.23 \mathrm{E}-02$ & $<0$ \\
\hline \multicolumn{15}{|c|}{ LG9 $23^{\circ} \mathrm{C}$ pH 9 Reactor \#4 } \\
\hline LG9.23.9.80B.Blank1 & & 3.50E-05 & --- & --- & --- & [49.4] & --- & {$[75.6]$} & --- & [164] & --- & {$[134]$} & --- & --- \\
\hline LG9.23.9.80B.Blank2 & & $2.75 \mathrm{E}-05$ & --- & --- & --- & [49.4] & --- & {$[75.6]$} & --- & [164] & --- & {$[134]$} & --- & -- \\
\hline LG9.23.9.80B.Blank3 & & $3.52 E-05$ & 0 & --- & --- & [49.4] & --- & {$[75.6]$} & --- & [164] & -- & {$[134]$} & --- & --- \\
\hline LG9.23.9.80B.2 & & $2.78 \mathrm{E}-05$ & 6 & 0.029 & $5.78 \mathrm{E}-04$ & [49.4] & $6.63 E-02$ & {$[75.6]$} & 1.37E-01 & [164] & $4.13 \mathrm{E}-02$ & [134] & 4.13E-02 & $<0$ \\
\hline LG9.23.9.80B.4 & & 3.22E-05 & 13 & 0.029 & 5.69E-04 & [49.4] & $7.81 E-02$ & {$[75.6]$} & 1.62E-01 & [164] & $4.86 \mathrm{E}-02$ & {$[134]$} & 4.86E-02 & $<0$ \\
\hline LG9.23.9.80B.6 & & $3.89 \mathrm{E}-05$ & 20 & 0.028 & $5.58 \mathrm{E}-04$ & [49.4] & $9.62 \mathrm{E}-02$ & {$[75.6]$} & $1.99 \mathrm{E}-01$ & [164] & $5.99 \mathrm{E}-02$ & {$[134]$} & $5.99 \mathrm{E}-02$ & $<0$ \\
\hline
\end{tabular}




\begin{tabular}{|c|c|c|c|c|c|c|c|c|c|c|c|c|c|c|}
\hline Sample ID & $\begin{array}{l}\text { Influent } \\
{[\mathrm{Si}]} \\
(\mathrm{ppm})\end{array}$ & $\begin{array}{l}\text { Flow } \\
\text { Rate, } \mathrm{q} \\
\left(\mathrm{m}^{3} \mathrm{~d}^{-1}\right)\end{array}$ & $\begin{array}{l}\text { Time } \\
\text { (d) }\end{array}$ & $\begin{array}{l}\text { Glass } \\
\text { Mass } \\
\text { (g) }\end{array}$ & $\begin{array}{l}\text { Surface } \\
\text { Area, S } \\
\left(\mathrm{m}^{2}\right)\end{array}$ & $\begin{array}{l}{[\mathrm{Al}]} \\
\left(\mu \mathrm{g} \mathrm{L}^{-1}\right)\end{array}$ & $\begin{array}{l}\text { Al Norm. } \\
\text { Diss. Rate } \\
\left(\mathrm{g} \mathrm{m}^{-2} \mathrm{~d}^{-1}\right)\end{array}$ & $\begin{array}{l}{[\mathrm{B}]} \\
\left(\mu \mathrm{g} \mathrm{L}^{-1}\right)\end{array}$ & $\begin{array}{l}\text { B Norm. } \\
\text { Diss. Rate } \\
\left(\mathrm{g} \mathrm{m}^{-2} \mathrm{~d}^{-1}\right)\end{array}$ & $\begin{array}{l}{[\mathrm{Si}]} \\
\left(\mu \mathrm{g} \mathrm{L}^{-1}\right)\end{array}$ & $\begin{array}{l}\text { Si Norm. } \\
\text { Diss. Rate } \\
\left(\mathrm{g} \mathrm{m}^{-2} \mathrm{~d}^{-1}\right)\end{array}$ & $\begin{array}{l}{[\mathrm{Na}]} \\
(\mu \mathrm{g} \mathrm{L} \\
\left.{ }^{1}\right)\end{array}$ & $\begin{array}{l}\text { Na Norm. } \\
\text { Diss. Rate, } \\
\mathrm{r} \\
\left(\mathrm{g} \mathrm{m}^{-2} \mathrm{~d}^{-1}\right)\end{array}$ & $\begin{array}{l}\mathrm{r}_{\mathrm{IEX}} \\
\left(\mathrm{mol} \mathrm{Na} \mathrm{No}^{-1}\right)\end{array}$ \\
\hline LG9.23.9.80B.8 & & $3.80 \mathrm{E}-05$ & 27 & 0.027 & $5.48 \mathrm{E}-04$ & [49.4] & $9.57 \mathrm{E}-02$ & [75.6] & $1.98 \mathrm{E}-01$ & [164] & $5.96 \mathrm{E}-02$ & [134] & $5.96 \mathrm{E}-02$ & $<0$ \\
\hline LG9.23.9.80B.10 & & $3.82 \mathrm{E}-05$ & 34 & 0.026 & $5.38 \mathrm{E}-04$ & [49.4] & $9.82 \mathrm{E}-02$ & [75.6] & 2.03E-01 & [164] & $6.11 \mathrm{E}-02$ & [134] & $6.12 \mathrm{E}-02$ & $<0$ \\
\hline LG9.23.9.80B.12 & & 3.94E-05 & 41 & 0.025 & $5.27 \mathrm{E}-04$ & [49.4] & $1.03 \mathrm{E}-01$ & [75.6] & $2.14 \mathrm{E}-01$ & [164] & $6.43 \mathrm{E}-02$ & [134] & $6.43 E-02$ & $<0$ \\
\hline LG9.23.9.80B.14 & & 3.79E-05 & 48 & 0.025 & $5.16 \mathrm{E}-04$ & [49.4] & $1.01 \mathrm{E}-01$ & {$[75.6]$} & 2.10E-01 & [164] & $6.31 \mathrm{E}-02$ & $\begin{array}{l}{[134]} \\
59.5^{*}\end{array}$ & $6.32 \mathrm{E}-02$ & $<0$ \\
\hline LG9.23.9.80B.16 & & 3.83E-05 & 55 & 0.024 & $5.06 \mathrm{E}-04$ & [49.4] & $1.05 \mathrm{E}-01$ & [75.6] & 2.17E-01 & [164] & $6.51 E-02$ & $\begin{array}{l}{[134]} \\
62.7^{*}\end{array}$ & $6.51 \mathrm{E}-02$ & $<0$ \\
\hline LG9.23.9.80B.18 & & 3.86E-05 & 62 & 0.023 & $4.95 E-04$ & [49.4] & $1.08 \mathrm{E}-01$ & {$[75.6]$} & 2.23E-01 & {$[164]$} & $6.71 \mathrm{E}-02$ & $\begin{array}{l}{[134]} \\
52.7^{*}\end{array}$ & $6.72 \mathrm{E}-02$ & $<0$ \\
\hline LG9.23.9.80B.20 & & $3.87 E-05$ & 69 & 0.022 & 4.83E-04 & [49.4] & $1.11 \mathrm{E}-01$ & [75.6] & $2.29 \mathrm{E}-01$ & [164] & $6.89 \mathrm{E}-02$ & [134] & $6.89 \mathrm{E}-02$ & $<0$ \\
\hline LG9.23.9.80B.22 & & 3.72E-05 & 76 & 0.022 & $4.73 \mathrm{E}-04$ & [49.4] & $1.09 \mathrm{E}-01$ & [75.6] & $2.25 \mathrm{E}-01$ & [164] & $6.77 \mathrm{E}-02$ & $\begin{array}{l}{[134]} \\
53.2^{*}\end{array}$ & $6.77 \mathrm{E}-02$ & $<0$ \\
\hline LG9.23.9.80B.23 & & 3.82E-05 & 79 & 0.021 & $4.68 \mathrm{E}-04$ & [49.4] & $1.13 \mathrm{E}-01$ & [75.6] & 2.33E-01 & [164] & $7.01 E-02$ & $\begin{array}{l}{[134]} \\
47.9^{\star}\end{array}$ & 7.02E-02 & $<0$ \\
\hline LG9.23.9.80B.24 & & $3.79 \mathrm{E}-05$ & 83 & 0.021 & 4.61E-04 & [49.4] & $1.13 \mathrm{E}-01$ & [75.6] & $2.35 \mathrm{E}-01$ & [164] & $7.06 \mathrm{E}-02$ & $\begin{array}{l}{[134]} \\
45.1^{*}\end{array}$ & 7.07E-02 & $<0$ \\
\hline \multicolumn{15}{|c|}{ LG9 $23^{\circ} \mathrm{C} \mathrm{pH} 10$ Reactor $\# 1$} \\
\hline LG9.23.10.20.Blank1 & & $1.48 \mathrm{E}-05$ & -.- & --- & --- & 82 & --- & {$[75.6]$} & --- & [164] & --- & {$[134]$} & --- & --- \\
\hline LG9.23.10.20.Blank2 & & $1.48 \mathrm{E}-05$ & --- & --- & --- & 125 & --- & [75.6] & --- & [164] & -.- & {$[134]$} & --- & --. \\
\hline LG9.23.10.20.Blank3 & & $1.31 \mathrm{E}-05$ & 0 & --- & --- & 109 & --- & {$[75.6]$} & --- & [164] & --- & [134] & --- & --- \\
\hline LG9.23.10.20.2 & & $1.37 \mathrm{E}-05$ & 6 & 0.749 & $1.47 E-02$ & 248 & $6.42 \mathrm{E}-03$ & [75.6] & 2.65E-03 & $\begin{array}{l}{[164]} \\
101^{*}\end{array}$ & 7.97E-04 & 930 & $5.53 \mathrm{E}-03$ & $1.45 \mathrm{E}-09$ \\
\hline LG9.23.10.20.4 & & $1.36 \mathrm{E}-05$ & 13 & 0.749 & $1.47 \mathrm{E}-02$ & 206 & $5.33 \mathrm{E}-03$ & {$[75.6]$} & $2.65 \mathrm{E}-03$ & 438 & $2.12 \mathrm{E}-03$ & 725 & $4.31 \mathrm{E}-03$ & $8.36 \mathrm{E}-10$ \\
\hline LG9.23.10.20.6 & & $1.37 \mathrm{E}-05$ & 20 & 0.749 & $1.47 \mathrm{E}-02$ & 196 & $5.11 \mathrm{E}-03$ & [75.6] & $2.67 \mathrm{E}-03$ & 720 & $3.52 \mathrm{E}-03$ & 654 & $3.91 \mathrm{E}-03$ & $6.28 \mathrm{E}-10$ \\
\hline LG9.23.10.20.8 & & $1.39 \mathrm{E}-05$ & 27 & 0.748 & $1.47 \mathrm{E}-02$ & 221 & $5.84 \mathrm{E}-03$ & {$[75.6]$} & $2.70 \mathrm{E}-03$ & 712 & $3.53 \mathrm{E}-03$ & 697 & $4.23 \mathrm{E}-03$ & $7.68 \mathrm{E}-10$ \\
\hline LG9.23.10.20.10 & & $1.41 \mathrm{E}-05$ & 34 & 0.748 & $1.47 \mathrm{E}-02$ & 203 & $5.44 \mathrm{E}-03$ & [75.6] & $2.74 \mathrm{E}-03$ & 669 & $3.36 \mathrm{E}-03$ & 693 & $4.26 \mathrm{E}-03$ & $7.66 \mathrm{E}-10$ \\
\hline LG9.23.10.20.12 & & $1.41 \mathrm{E}-05$ & 41 & 0.748 & $1.47 E-02$ & 165 & 4.42E-03 & 101 & $3.66 \mathrm{E}-03$ & 902 & 4.53E-03 & 697 & $4.29 \mathrm{E}-03$ & $3.15 E-10$ \\
\hline LG9.23.10.20.14 & & $1.41 \mathrm{E}-05$ & 48 & 0.747 & $1.47 \mathrm{E}-02$ & 176 & $4.73 \mathrm{E}-03$ & 128 & $4.65 \mathrm{E}-03$ & 922 & 4.64E-03 & 689 & $4.25 \mathrm{E}-03$ & $<0$ \\
\hline LG9.23.10.20.16 & & $1.36 \mathrm{E}-05$ & 55 & 0.747 & $1.47 \mathrm{E}-02$ & 150 & $3.89 \mathrm{E}-03$ & 117 & $4.11 \mathrm{E}-03$ & 869 & $4.22 \mathrm{E}-03$ & 645 & $3.84 \mathrm{E}-03$ & $<0$ \\
\hline LG9.23.10.20.18 & & $1.38 \mathrm{E}-05$ & 62 & 0.746 & $1.47 \mathrm{E}-02$ & 174 & $4.55 \mathrm{E}-03$ & 136 & $4.82 \mathrm{E}-03$ & 778 & $3.82 \mathrm{E}-03$ & 617 & $3.71 \mathrm{E}-03$ & $<0$ \\
\hline LG9.23.10.20.20 & & $1.32 E-05$ & 69 & 0.746 & $1.47 E-02$ & 161 & 4.03E-03 & 103 & 3.49E-03 & 812 & $3.82 E-03$ & 660 & 3.80E-03 & 1.53E-10 \\
\hline LG9.23.10.20.22 & & $1.38 \mathrm{E}-05$ & 76 & 0.745 & $1.47 \mathrm{E}-02$ & 184 & $4.83 \mathrm{E}-03$ & 116 & $4.12 \mathrm{E}-03$ & 1,030 & $5.07 \mathrm{E}-03$ & 704 & $4.25 \mathrm{E}-03$ & $6.14 \mathrm{E}-11$ \\
\hline LG9.23.10.20.23 & & $1.38 \mathrm{E}-05$ & 79 & 0.745 & $1.47 \mathrm{E}-02$ & 172 & $4.53 \mathrm{E}-03$ & 114 & $4.07 E-03$ & 836 & $4.13 \mathrm{E}-03$ & 679 & $4.11 \mathrm{E}-03$ & $2.14 \mathrm{E}-11$ \\
\hline LG9.23.10.20.24 & & $1.37 \mathrm{E}-05$ & 83 & 0.745 & $1.47 \mathrm{E}-02$ & 147 & 3.83E-03 & 94 & $3.30 \mathrm{E}-03$ & 805 & $3.93 \mathrm{E}-03$ & 621 & $3.71 \mathrm{E}-03$ & $2.07 \mathrm{E}-10$ \\
\hline \multicolumn{15}{|c|}{ LG9 $23^{\circ} \mathrm{C} \mathrm{pH} 10$ Reactor \#2 } \\
\hline LG9.23.10.40.Blank1 & & 2.03E-05 & --- & --. & --- & [49.4] & -.- & [75.6] & --- & [164] & -.- & [134] & --- & -.- \\
\hline LG9.23.10.40.Blank2 & & $2.11 \mathrm{E}-05$ & --- & --- & --- & [49.4] & --- & {$[75.6]$} & --- & [164] & --- & {$[134]$} & --- & --- \\
\hline LG9.23.10.40.Blank3 & & $2.02 \mathrm{E}-05$ & 0 & --- & --- & [49.4] & -.- & [75.6] & --- & [164] & -.- & [134] & --- & -.- \\
\hline LG9.23.10.40.2 & & $2.08 \mathrm{E}-05$ & 6 & 0.374 & $7.36 \mathrm{E}-03$ & 54 & $4.26 \mathrm{E}-03$ & {$[75.6]$} & $8.08 \mathrm{E}-03$ & [164] & $2.43 E-03$ & 406 & 7.36E-03 & $<0$ \\
\hline LG9.23.10.40.4 & & $1.97 \mathrm{E}-05$ & 13 & 0.374 & $7.36 \mathrm{E}-03$ & 104 & $7.78 \mathrm{E}-03$ & [75.6] & $7.66 \mathrm{E}-03$ & 312 & $4.38 \mathrm{E}-03$ & 369 & $6.34 \mathrm{E}-03$ & $<0$ \\
\hline LG9.23.10.40.6 & & $1.73 \mathrm{E}-05$ & 20 & 0.373 & $7.35 \mathrm{E}-03$ & 121 & $7.95 \mathrm{E}-03$ & [75.6] & $6.72 \mathrm{E}-03$ & 417 & $5.14 \mathrm{E}-03$ & 421 & $6.35 \mathrm{E}-03$ & $<0$ \\
\hline LG9.23.10.40.8 & & $1.69 \mathrm{E}-05$ & 27 & 0.373 & $7.35 \mathrm{E}-03$ & 117 & $7.50 \mathrm{E}-03$ & [75.6] & $6.56 \mathrm{E}-03$ & 414 & $4.98 \mathrm{E}-03$ & 425 & $6.26 \mathrm{E}-03$ & $<0$ \\
\hline LG9.23.10.40.10 & & $1.66 \mathrm{E}-05$ & 34 & 0.373 & 7.34E-03 & 118 & 7.47E-03 & [75.6] & $6.48 \mathrm{E}-03$ & 413 & 4.90E-03 & 456 & $6.63 \mathrm{E}-03$ & 7.59E-11 \\
\hline LG9.23.10.40.12 & & $1.73 \mathrm{E}-05$ & 41 & 0.372 & $7.34 \mathrm{E}-03$ & 98 & $6.49 \mathrm{E}-03$ & 80 & 7.13E-03 & 554 & $6.85 \mathrm{E}-03$ & 463 & $7.01 \mathrm{E}-03$ & $<0$ \\
\hline
\end{tabular}




\begin{tabular}{|c|c|c|c|c|c|c|c|c|c|c|c|c|c|c|}
\hline Sample ID & $\begin{array}{l}\text { Influent } \\
\text { [Si] } \\
\text { (ppm) }\end{array}$ & $\begin{array}{l}\text { Flow } \\
\text { Rate, q } \\
\left(\mathrm{m}^{3} \mathrm{~d}^{-1}\right)\end{array}$ & $\begin{array}{l}\text { Time } \\
\text { (d) }\end{array}$ & $\begin{array}{l}\text { Glass } \\
\text { Mass } \\
\text { (g) }\end{array}$ & $\begin{array}{l}\text { Surface } \\
\text { Area, } \mathrm{S} \\
\left(\mathrm{m}^{2}\right)\end{array}$ & $\begin{array}{l}{[\mathrm{Al}]} \\
\left(\mu \mathrm{g} \mathrm{L}^{-1}\right)\end{array}$ & $\begin{array}{l}\text { Al Norm. } \\
\text { Diss. Rate } \\
\left(\mathrm{g} \mathrm{m}^{-2} \mathrm{~d}^{-1}\right)\end{array}$ & $\begin{array}{l}{[\mathrm{B}]} \\
\left(\mu \mathrm{L} \mathrm{L}^{-1}\right)\end{array}$ & $\begin{array}{l}\text { B Norm. } \\
\text { Diss. Rate } \\
\left(\mathrm{g} \mathrm{m}^{-2} \mathrm{~d}^{-1}\right)\end{array}$ & $\begin{array}{l}{[\mathrm{Si}]} \\
\left(\mu \mathrm{L}^{-1}\right)\end{array}$ & $\begin{array}{l}\text { Si Norm. } \\
\text { Diss. Rate } \\
\left(\mathrm{g} \mathrm{m}^{-2} \mathrm{~d}^{-1}\right)\end{array}$ & $\begin{array}{l}{[\mathrm{Na}]} \\
(\mu \mathrm{g} \mathrm{L} \\
\left.{ }^{1}\right)\end{array}$ & $\begin{array}{l}\text { Na Norm. } \\
\text { Diss. Rate, } \\
\mathrm{r} \\
\left(\mathrm{g} \mathrm{m}^{-2} \mathrm{~d}^{-1}\right)\end{array}$ & $\begin{array}{l}\mathrm{r}_{\mathrm{IEX}} \\
\left(\mathrm{mol} \mathrm{Na} \mathrm{a}^{-2} \mathrm{~s}^{-1}\right)\end{array}$ \\
\hline LG9.23.10.40.14 & & $1.69 \mathrm{E}-05$ & 48 & 0.372 & $7.33 \mathrm{E}-03$ & 113 & $7.28 \mathrm{E}-03$ & 77 & $6.69 \mathrm{E}-03$ & 567 & $6.85 \mathrm{E}-03$ & 460 & $6.80 \mathrm{E}-03$ & $5.88 \mathrm{E}-11$ \\
\hline LG9.23.10.40.16 & & $1.96 \mathrm{E}-05$ & 55 & 0.371 & 7.33E-03 & 91 & $6.77 \mathrm{E}-03$ & 80 & $8.14 \mathrm{E}-03$ & 527 & $7.39 \mathrm{E}-03$ & 409 & 7.03E-03 & $<0$ \\
\hline LG9.23.10.40.18 & & $1.89 \mathrm{E}-05$ & 62 & 0.371 & $7.32 \mathrm{E}-03$ & 67 & 4.83E-03 & $\begin{array}{l}{[75.6]} \\
66^{\star}\end{array}$ & 7.37E-03 & 430 & $5.81 \mathrm{E}-03$ & 360 & $5.96 \mathrm{E}-03$ & $<0$ \\
\hline LG9.23.10.40.20 & & $1.96 \mathrm{E}-05$ & 69 & 0.371 & $7.32 \mathrm{E}-03$ & 84 & $6.30 \mathrm{E}-03$ & $\begin{array}{l}{[75.6]} \\
68^{*}\end{array}$ & $7.66 \mathrm{E}-03$ & 466 & $6.54 \mathrm{E}-03$ & 381 & $6.55 \mathrm{E}-03$ & $<0$ \\
\hline LG9.23.10.40.22 & & 1.69E-05 & 76 & 0.370 & $7.31 \mathrm{E}-03$ & 79 & $5.12 \mathrm{E}-03$ & $\begin{array}{l}{[75.6]} \\
75^{\star}\end{array}$ & $6.62 \mathrm{E}-03$ & 453 & $5.50 \mathrm{E}-03$ & 375 & $5.57 \mathrm{E}-03$ & $<0$ \\
\hline LG9.23.10.40.23 & & $1.77 \mathrm{E}-05$ & 79 & 0.370 & $7.31 \mathrm{E}-03$ & 94 & $6.38 \mathrm{E}-03$ & 88 & $8.04 \mathrm{E}-03$ & 574 & $7.29 \mathrm{E}-03$ & 486 & $7.56 \mathrm{E}-03$ & $<0$ \\
\hline LG9.23.10.40.24 & & $1.81 E-05$ & 83 & 0.370 & $7.31 \mathrm{E}-03$ & 78 & $5.37 \mathrm{E}-03$ & $\begin{array}{l}{[75.6]} \\
75^{\star}\end{array}$ & $7.09 E-03$ & 509 & $6.62 \mathrm{E}-03$ & 414 & $6.59 \mathrm{E}-03$ & $<0$ \\
\hline \multicolumn{15}{|c|}{ LG9 $23^{\circ} \mathrm{C} \mathrm{pH} 10$ Reactor \#3 } \\
\hline LG9.23.10.80A.Blank1 & & $3.29 \mathrm{E}-05$ & --- & --- & -.- & [49.4] & --- & {$[75.6]$} & --- & [164] & --- & [134] & --- & -- \\
\hline LG9.23.10.80A.Blank2 & & $3.74 \mathrm{E}-05$ & --- & --- & --- & [49.4] & --- & [75.6] & --- & [164] & --- & [134] & --- & --- \\
\hline LG9.23.10.80A.Blank3 & & $3.75 \mathrm{E}-05$ & 0 & --- & --- & [49.4] & --- & {$[75.6]$} & --- & [164] & -- & [134] & --- & -- \\
\hline LG9.23.10.80A.2 & & 3.68E-05 & 6 & 0.149 & $2.94 \mathrm{E}-03$ & $\begin{array}{l}{[49.4]} \\
24^{*}\end{array}$ & $1.73 \mathrm{E}-02$ & [75.6] & $3.57 \mathrm{E}-02$ & [164] & 1.07E-02 & [134] & 1.07E-02 & $<0$ \\
\hline LG9.23.10.80A.4 & & $3.78 \mathrm{E}-05$ & 13 & 0.149 & 2.93E-03 & $\begin{array}{l}{[49.4]} \\
32^{*}\end{array}$ & $1.78 \mathrm{E}-02$ & {$[75.6]$} & 3.68E-02 & [164] & $1.11 \mathrm{E}-02$ & [134] & $1.11 \mathrm{E}-02$ & $<0$ \\
\hline LG9.23.10.80A.6 & & $3.86 \mathrm{E}-05$ & 20 & 0.148 & $2.92 \mathrm{E}-03$ & $\begin{array}{l}{[49.4]} \\
31^{*}\end{array}$ & $1.82 \mathrm{E}-02$ & [75.6] & $3.78 \mathrm{E}-02$ & [164] & $1.14 \mathrm{E}-02$ & {$[134]$} & 1.14E-02 & $<0$ \\
\hline LG9.23.10.80A.8 & & $3.92 E-05$ & 27 & 0.147 & $2.91 \mathrm{E}-03$ & $\begin{array}{l}{[49.4]} \\
34^{*}\end{array}$ & $1.86 \mathrm{E}-02$ & [75.6] & $3.85 \mathrm{E}-02$ & [164] & $1.16 \mathrm{E}-02$ & [134] & $1.16 \mathrm{E}-02$ & $<0$ \\
\hline LG9.23.10.80A.10 & & 3.66E-05 & 34 & 0.146 & $2.90 \mathrm{E}-03$ & $\begin{array}{l}{[49.4]} \\
32^{\star}\end{array}$ & $1.74 \mathrm{E}-02$ & [75.6] & $3.60 \mathrm{E}-02$ & [164] & $1.08 \mathrm{E}-02$ & [134] & $1.08 \mathrm{E}-02$ & $<0$ \\
\hline LG9.23.10.80A.12 & & $3.78 \mathrm{E}-05$ & 41 & 0.145 & $2.89 \mathrm{E}-03$ & $\begin{array}{l}{[49.4]} \\
19^{*}\end{array}$ & $1.80 \mathrm{E}-02$ & $\begin{array}{l}{[75.6]} \\
26^{\star}\end{array}$ & $3.74 \mathrm{E}-02$ & $\begin{array}{l}{[164]} \\
148^{*}\end{array}$ & $1.12 \mathrm{E}-02$ & 157 & $1.32 \mathrm{E}-02$ & $<0$ \\
\hline LG9.23.10.80A.14 & & 3.82E-05 & 48 & 0.145 & $2.88 \mathrm{E}-03$ & $\begin{array}{l}{[49.4]} \\
21^{*}\end{array}$ & $1.83 E-02$ & $\begin{array}{l}{[75.6]} \\
26^{*}\end{array}$ & $3.79 E-02$ & $\begin{array}{l}{[164]} \\
158^{*}\end{array}$ & $1.14 \mathrm{E}-02$ & 153 & 1.30E-02 & $<0$ \\
\hline LG9.23.10.80A.16 & & $3.62 E-05$ & 55 & 0.144 & 2.87E-03 & $\begin{array}{l}{[49.4]} \\
17^{\star}\end{array}$ & $1.74 \mathrm{E}-02$ & $\begin{array}{l}{[75.6]} \\
25^{\star}\end{array}$ & $3.60 \mathrm{E}-02$ & $\begin{array}{l}{[164]} \\
142^{*}\end{array}$ & $1.08 \mathrm{E}-02$ & 154 & 1.25E-02 & $<0$ \\
\hline LG9.23.10.80A.18 & & 3.64E-05 & 62 & 0.143 & $2.86 \mathrm{E}-03$ & $\begin{array}{l}{[49.4]} \\
19^{\star}\end{array}$ & $1.76 \mathrm{E}-02$ & {$[75.6]$} & $3.64 \mathrm{E}-02$ & $\begin{array}{l}{[164]} \\
137^{*}\end{array}$ & $1.09 \mathrm{E}-02$ & [134] & $1.09 \mathrm{E}-02$ & $<0$ \\
\hline LG9.23.10.80A.20 & & $3.73 E-05$ & 69 & 0.143 & $2.85 \mathrm{E}-03$ & $\begin{array}{l}{[49.4]} \\
29^{\star}\end{array}$ & $1.80 \mathrm{E}-02$ & $\begin{array}{l}{[75.6]} \\
29^{\star}\end{array}$ & $3.74 \mathrm{E}-02$ & $\begin{array}{l}{[164]} \\
103^{*}\end{array}$ & $1.12 \mathrm{E}-02$ & 135 & $1.13 \mathrm{E}-02$ & $<0$ \\
\hline LG9.23.10.80A.22 & & 3.83E-05 & 76 & 0.142 & $2.84 \mathrm{E}-03$ & [49.4] & $1.86 \mathrm{E}-02$ & {$[75.6]$} & $3.85 E-02$ & $\begin{array}{l}{[164]} \\
126^{*}\end{array}$ & 1.16E-02 & 142 & 1.23E-02 & $<0$ \\
\hline LG9.23.10.80A.23 & & $3.81 E-05$ & 79 & 0.141 & $2.84 \mathrm{E}-03$ & $\begin{array}{l}{[49.4]} \\
25^{\star}\end{array}$ & $1.85 \mathrm{E}-02$ & [75.6] & $3.84 \mathrm{E}-02$ & 186 & $1.31 \mathrm{E}-02$ & 167 & $1.44 \mathrm{E}-02$ & $<0$ \\
\hline \multicolumn{15}{|c|}{ LG9 $23^{\circ} \mathrm{C} \mathrm{pH} 10$ Reactor $\# 4$} \\
\hline LG9.23.10.80B.Blank1 & & $3.58 \mathrm{E}-05$ & --- & --- & --- & [49.4] & --- & {$[75.6]$} & --- & [164] & --- & [134] & --- & -- \\
\hline LG9.23.10.80B.Blank2 & & $3.72 E-05$ & --- & --- & --- & [49.4] & --- & {$[75.6]$} & --- & [164] & --- & [134] & --- & --- \\
\hline LG9.23.10.80B.Blank3 & & $3.75 \mathrm{E}-05$ & 0 & --. & --- & [49.4] & --- & [75.6] & --- & [164] & --- & [134] & --- & --- \\
\hline LG9.23.10.80B.2 & & $3.75 \mathrm{E}-05$ & 6 & 0.029 & $5.75 \mathrm{E}-04$ & [49.4] & $9.00 \mathrm{E}-02$ & {$[75.6]$} & $1.86 \mathrm{E}-01$ & [164] & $5.60 \mathrm{E}-02$ & [134] & $5.61 \mathrm{E}-02$ & $<0$ \\
\hline LG9.23.10.80B.4 & & $3.79 \mathrm{E}-05$ & 13 & 0.028 & $5.65 \mathrm{E}-04$ & [49.4] & $9.26 \mathrm{E}-02$ & {$[75.6]$} & $1.92 \mathrm{E}-01$ & [164] & $5.77 \mathrm{E}-02$ & [134] & $5.77 \mathrm{E}-02$ & $<0$ \\
\hline
\end{tabular}




\begin{tabular}{|c|c|c|c|c|c|c|c|c|c|c|c|c|c|c|}
\hline Sample ID & $\begin{array}{l}\text { Influent } \\
\text { [Si] } \\
\text { (ppm) }\end{array}$ & $\begin{array}{l}\text { Flow } \\
\text { Rate, } \mathrm{q} \\
\left(\mathrm{m}^{3} \mathrm{~d}^{-1}\right)\end{array}$ & $\begin{array}{l}\text { Time } \\
\text { (d) }\end{array}$ & $\begin{array}{l}\text { Glass } \\
\text { Mass } \\
\text { (g) }\end{array}$ & $\begin{array}{l}\text { Surface } \\
\text { Area, S } \\
\left(\mathrm{m}^{2}\right)\end{array}$ & $\begin{array}{l}{[\mathrm{Al}]} \\
\left(\mu \mathrm{L} \mathrm{L}^{-1}\right)\end{array}$ & $\begin{array}{l}\text { Al Norm. } \\
\text { Diss. Rate } \\
\left(\mathrm{g} \mathrm{m}^{-2} \mathrm{~d}^{-1}\right)\end{array}$ & $\begin{array}{l}{[B]} \\
\left(\mu g L^{-1}\right)\end{array}$ & $\begin{array}{l}\text { B Norm. } \\
\text { Diss. Rate } \\
\left(\mathrm{g} \mathrm{m}^{-2} \mathrm{~d}^{-1}\right)\end{array}$ & $\begin{array}{l}{[\mathrm{Si}]} \\
\left(\mu \mathrm{L} \mathrm{L}^{-1}\right)\end{array}$ & $\begin{array}{l}\text { Si Norm. } \\
\text { Diss. Rate } \\
\left(\mathrm{g} \mathrm{m}^{-2} \mathrm{~d}^{-1}\right)\end{array}$ & $\begin{array}{l}{[\mathrm{Na}]} \\
(\mu \mathrm{gg} \mathrm{L} \\
\left.{ }^{2}\right)\end{array}$ & $\begin{array}{l}\text { Na Norm. } \\
\text { Diss. Rate, } \\
\mathrm{r} \\
\left(\mathrm{g} \mathrm{m}^{-2} \mathrm{~d}^{-1}\right)\end{array}$ & $\begin{array}{l}\mathrm{r}_{\mathrm{IEX}} \\
\left(\mathrm{mol} \mathrm{Na} \mathrm{s}^{-1}\right)\end{array}$ \\
\hline LG9.23.10.80B.6 & & $3.77 \mathrm{E}-05$ & 20 & 0.027 & $5.55 \mathrm{E}-04$ & {$[49.4]$} & $9.37 \mathrm{E}-02$ & {$[75.6]$} & $1.94 \mathrm{E}-01$ & [164] & $5.83 \mathrm{E}-02$ & [134] & $5.84 \mathrm{E}-02$ & $<0$ \\
\hline LG9.23.10.80B.8 & & $3.88 \mathrm{E}-05$ & 27 & 0.027 & $5.44 \mathrm{E}-04$ & {$[49.4]$} & $9.84 \mathrm{E}-02$ & {$[75.6]$} & $2.04 \mathrm{E}-01$ & [164] & $6.12 \mathrm{E}-02$ & [134] & $6.13 \mathrm{E}-02$ & $<0$ \\
\hline LG9.23.10.80B.10 & & $3.81 \mathrm{E}-05$ & 34 & 0.026 & $5.34 \mathrm{E}-04$ & [49.4] & $9.85 \mathrm{E}-02$ & {$[75.6]$} & $2.04 \mathrm{E}-01$ & [164] & $6.13 \mathrm{E}-02$ & [134] & $6.14 \mathrm{E}-02$ & $<0$ \\
\hline LG9.23.10.80B.12 & & 3.85E-05 & 41 & 0.025 & $5.23 E-04$ & {$[49.4]$} & $1.02 \mathrm{E}-01$ & {$[75.6]$} & $2.11 \mathrm{E}-01$ & [164] & $6.33 E-02$ & $\begin{array}{l}{[134]} \\
67^{\star}\end{array}$ & $6.34 \mathrm{E}-02$ & $<0$ \\
\hline LG9.23.10.80B.14 & & 3.89E-05 & 48 & 0.024 & $5.12 \mathrm{E}-04$ & {$[49.4]$} & $1.05 \mathrm{E}-01$ & [75.6] & 2.17E-01 & [164] & $6.53 E-02$ & $\begin{array}{l}{[134]} \\
77^{\star}\end{array}$ & $6.53 E-02$ & $<0$ \\
\hline LG9.23.10.80B.16 & & 3.99E-05 & 55 & 0.024 & $5.01 \mathrm{E}-04$ & [49.4] & $1.10 \mathrm{E}-01$ & {$[75.6]$} & $2.28 \mathrm{E}-01$ & [164] & $6.85 \mathrm{E}-02$ & $\begin{array}{l}{[134]} \\
54^{\star}\end{array}$ & $6.85 \mathrm{E}-02$ & $<0$ \\
\hline LG9.23.10.80B.18 & & $3.90 \mathrm{E}-05$ & 62 & 0.023 & $4.90 \mathrm{E}-04$ & [49.4] & $1.10 \mathrm{E}-01$ & [75.6] & $2.28 \mathrm{E}-01$ & [164] & $6.85 E-02$ & $\begin{array}{l}{[134]} \\
75^{\star}\end{array}$ & $6.85 \mathrm{E}-02$ & $<0$ \\
\hline LG9.23.10.80B.20 & & $4.01 \mathrm{E}-05$ & 69 & 0.022 & $4.78 \mathrm{E}-04$ & {$[49.4]$} & $1.16 \mathrm{E}-01$ & {$[75.6]$} & 2.40E-01 & [164] & $7.20 \mathrm{E}-02$ & [134] & $7.21 \mathrm{E}-02$ & $<0$ \\
\hline LG9.23.10.80B.22 & & $3.90 \mathrm{E}-05$ & 76 & 0.021 & 4.67E-04 & [49.4] & $1.15 \mathrm{E}-01$ & {$[75.6]$} & $2.39 \mathrm{E}-01$ & [164] & $7.17 \mathrm{E}-02$ & [134] & 7.17E-02 & $<0$ \\
\hline LG9.23.10.80B.23 & & 4.03E-05 & 79 & 0.021 & 4.63E-04 & {$[49.4]$} & $1.20 \mathrm{E}-01$ & {$[75.6]$} & 2.49E-01 & [164] & 7.49E-02 & $\begin{array}{l}{[134]} \\
65^{\star}\end{array}$ & 7.49E-02 & $<0$ \\
\hline LG9.23.10.80B.24 & & 3.99E-05 & 83 & 0.020 & 4.55E-04 & {$[49.4]$} & $1.21 \mathrm{E}-01$ & {$[75.6]$} & $2.51 \mathrm{E}-01$ & {$[164]$} & $7.53 E-02$ & $\begin{array}{l}{[134]} \\
70^{\star}\end{array}$ & $7.54 \mathrm{E}-02$ & $<0$ \\
\hline \multicolumn{15}{|c|}{ LG9 $23^{\circ} \mathrm{C} \mathrm{pH} 11$ Reactor \#1 } \\
\hline LG9.23.11.20.Blank1 & & $9.82 \mathrm{E}-06$ & --- & --- & --- & {$[49.4]$} & --- & [75.6] & --- & 176 & -.- & [134] & --- & --. \\
\hline LG9.23.11.20.Blank2 & & $1.01 \mathrm{E}-05$ & --- & --- & --- & {$[49.4]$} & --- & {$[75.6]$} & --- & [164] & --- & [134] & --- & --- \\
\hline LG9.23.11.20.Blank3 & & $1.01 \mathrm{E}-05$ & 0 & --- & --- & {$[49.4]$} & --- & [75.6] & --- & [164] & --- & [134] & --- & --- \\
\hline LG9.23.11.20.2 & & $9.27 \mathrm{E}-06$ & 2 & 1.501 & $2.95 \mathrm{E}-02$ & 275 & $2.41 \mathrm{E}-03$ & [75.6] & $8.98 E-04$ & 1,200 & $1.98 \mathrm{E}-03$ & 1,660 & $3.35 \mathrm{E}-03$ & $1.23 \mathrm{E}-09$ \\
\hline LG9.23.11.20.4 & & 7.98E-06 & 9 & 1.500 & 2.95E-02 & 615 & $4.65 \mathrm{E}-03$ & $\begin{array}{l}{[75.6]} \\
41^{\star}\end{array}$ & 7.73E-04 & 2,940 & 4.16E-03 & 2,740 & $4.75 \mathrm{E}-03$ & 2.00E-09 \\
\hline LG9.23.11.20.6 & & $7.65 \mathrm{E}-06$ & 16 & 1.500 & $2.95 \mathrm{E}-02$ & 642 & $4.65 \mathrm{E}-03$ & 108 & $1.06 \mathrm{E}-03$ & 3,080 & $4.18 \mathrm{E}-03$ & 3,020 & $5.02 \mathrm{E}-03$ & $2.00 \mathrm{E}-09$ \\
\hline LG9.23.11.20.8 & & $6.66 \mathrm{E}-06$ & 23 & 1.500 & $2.95 \mathrm{E}-02$ & 621 & $3.92 \mathrm{E}-03$ & 90 & $7.67 \mathrm{E}-04$ & 3,060 & $3.62 \mathrm{E}-03$ & 2,730 & $3.96 \mathrm{E}-03$ & $1.61 \mathrm{E}-09$ \\
\hline LG9.23.11.20.10 & & $9.43 E-06$ & 30 & 1.500 & $2.95 \mathrm{E}-02$ & 564 & $5.04 \mathrm{E}-03$ & $\begin{array}{l}{[75.6]} \\
61^{*}\end{array}$ & $9.14 \mathrm{E}-04$ & 2,720 & $4.55 \mathrm{E}-03$ & 2,390 & $4.90 \mathrm{E}-03$ & $2.01 \mathrm{E}-09$ \\
\hline LG9.23.11.20.12 & & $8.52 E-06$ & 37 & 1.499 & 2.95E-02 & 475 & 3.83E-03 & 391 & 4.27E-03 & 3,040 & 4.60E-03 & 2,340 & $4.34 \mathrm{E}-03$ & $3.29 \mathrm{E}-11$ \\
\hline LG9.23.11.20.14 & & $1.07 E-05$ & 44 & 1.498 & $2.95 \mathrm{E}-02$ & 397 & $4.03 \mathrm{E}-03$ & 329 & $4.52 \mathrm{E}-03$ & 2,510 & $4.77 \mathrm{E}-03$ & 2,180 & $5.08 \mathrm{E}-03$ & $2.82 \mathrm{E}-10$ \\
\hline LG9.23.11.20.16 & & 1.13E-05 & 51 & 1.497 & 2.95E-02 & 422 & $4.51 \mathrm{E}-03$ & 345 & $5.00 \mathrm{E}-03$ & 2,730 & $5.48 \mathrm{E}-03$ & 2,090 & $5.13 \mathrm{E}-03$ & $6.97 \mathrm{E}-11$ \\
\hline LG9.23.11.20.18 & & $9.45 \mathrm{E}-06$ & 58 & 1.496 & $2.95 \mathrm{E}-02$ & 373 & $3.34 \mathrm{E}-03$ & 312 & $3.79 \mathrm{E}-03$ & 2,440 & $4.10 \mathrm{E}-03$ & 1,900 & $3.91 \mathrm{E}-03$ & $6.31 \mathrm{E}-11$ \\
\hline LG9.23.11.20.20 & & 8.92E-06 & 65 & 1.495 & $2.95 E-02$ & 390 & $3.30 \mathrm{E}-03$ & 311 & 3.56E-03 & 2,470 & $3.92 \mathrm{E}-03$ & 1,960 & $3.81 \mathrm{E}-03$ & $1.24 \mathrm{E}-10$ \\
\hline LG9.23.11.20.22 & & $1.12 \mathrm{E}-05$ & 72 & 1.494 & $2.94 \mathrm{E}-02$ & 382 & $4.08 \mathrm{E}-03$ & 304 & $4.39 \mathrm{E}-03$ & 2,410 & $4.82 \mathrm{E}-03$ & 1,760 & $4.32 \mathrm{E}-03$ & $<0$ \\
\hline LG9.23.11.20.24 & & $9.44 \mathrm{E}-06$ & 79 & 1.494 & $2.94 \mathrm{E}-02$ & 410 & 3.67E-03 & 331 & $4.02 \mathrm{E}-03$ & 2,670 & $4.49 \mathrm{E}-03$ & 2,060 & $4.24 \mathrm{E}-03$ & 1.13E-10 \\
\hline LG9.23.11.20.25 & & $8.86 \mathrm{E}-06$ & 83 & 1.493 & $2.94 \mathrm{E}-02$ & 409 & $3.44 \mathrm{E}-03$ & 318 & $3.62 \mathrm{E}-03$ & 2,650 & $4.18 \mathrm{E}-03$ & 2,320 & $4.48 \mathrm{E}-03$ & $4.33 \mathrm{E}-10$ \\
\hline \multicolumn{15}{|c|}{ LG9 $23^{\circ} \mathrm{C} \mathrm{pH} 11$ Reactor $\# 2$} \\
\hline LG9.23.11.40.Blank1 & & $1.83 \mathrm{E}-05$ & --- & --- & --- & [49.4] & --- & {$[75.6]$} & --- & [164] & --- & [134] & --- & --- \\
\hline LG9.23.11.40.Blank2 & & 2.02E-05 & --- & --- & --- & {$[49.4]$} & --- & [75.6] & --- & [164] & --- & [134] & --- & --- \\
\hline LG9.23.11.40.Blank3 & & 2.05E-05 & 0 & --- & --- & {$[49.4]$} & --- & {$[75.6]$} & --- & [164] & --- & [134] & --- & --- \\
\hline LG9.23.11.40.2 & & $2.11 \mathrm{E}-05$ & 1 & 0.750 & $1.47 \mathrm{E}-02$ & 92 & $3.70 \mathrm{E}-03$ & [75.6] & $4.10 \mathrm{E}-03$ & 330 & $2.48 \mathrm{E}-03$ & 727 & $6.69 \mathrm{E}-03$ & $1.30 \mathrm{E}-09$ \\
\hline LG9.23.11.40.4 & & $1.97 \mathrm{E}-05$ & 6 & 0.749 & $1.47 E-02$ & 250 & $9.33 \mathrm{E}-03$ & {$[75.6]$} & 3.82E-03 & 1,100 & $7.70 \mathrm{E}-03$ & 1,020 & $8.74 \mathrm{E}-03$ & 2.48E-09 \\
\hline LG9.23.11.40.6 & & $1.96 \mathrm{E}-05$ & 13 & 0.749 & 1.47E-02 & 259 & $9.62 \mathrm{E}-03$ & [75.6] & 3.80E-03 & 1,150 & $8.01 \mathrm{E}-03$ & 1,090 & $9.29 \mathrm{E}-03$ & 2.77E-09 \\
\hline LG9.23.11.40.8 & & $1.92 \mathrm{E}-05$ & 20 & 0.748 & $1.47 \mathrm{E}-02$ & 238 & $8.68 \mathrm{E}-03$ & {$[75.6]$} & 3.73E-03 & 1,070 & 7.32E-03 & 1,040 & $8.71 \mathrm{E}-03$ & 2.51E-09 \\
\hline LG9.23.11.40.10 & & $1.99 \mathrm{E}-05$ & 27 & 0.748 & $1.47 \mathrm{E}-02$ & 236 & $8.90 \mathrm{E}-03$ & [75.6] & $3.86 \mathrm{E}-03$ & 1,080 & $7.64 \mathrm{E}-03$ & 981 & $8.50 \mathrm{E}-03$ & $2.34 \mathrm{E}-09$ \\
\hline
\end{tabular}




\begin{tabular}{|c|c|c|c|c|c|c|c|c|c|c|c|c|c|c|}
\hline Sample ID & $\begin{array}{l}\text { Influent } \\
\text { [Si] } \\
\text { (ppm) }\end{array}$ & $\begin{array}{l}\text { Flow } \\
\text { Rate, } q \\
\left(\mathrm{~m}^{3} \mathrm{~d}^{-1}\right)\end{array}$ & $\begin{array}{l}\text { Time } \\
\text { (d) }\end{array}$ & $\begin{array}{l}\text { Glass } \\
\text { Mass } \\
\text { (g) }\end{array}$ & $\begin{array}{l}\text { Surface } \\
\text { Area, S } \\
\left(\mathrm{m}^{2}\right)\end{array}$ & $\begin{array}{l}{[\mathrm{Al}]} \\
\left(\mu \mathrm{g} \mathrm{L}^{-1}\right)\end{array}$ & $\begin{array}{l}\text { Al Norm. } \\
\text { Diss. Rate } \\
\left(\mathrm{g} \mathrm{m}^{-2} \mathrm{~d}^{-1}\right)\end{array}$ & $\begin{array}{l}{[\mathrm{B}]} \\
\left(\mu \mathrm{g} \mathrm{L}^{-1}\right)\end{array}$ & $\begin{array}{l}\text { B Norm. } \\
\text { Diss. Rate } \\
\left(\mathrm{g} \mathrm{m}^{-2} \mathrm{~d}^{-1}\right)\end{array}$ & $\begin{array}{l}{[\mathrm{Si}]} \\
\left(\mu \mathrm{L} \mathrm{L}^{-1}\right)\end{array}$ & $\begin{array}{l}\text { Si Norm. } \\
\text { Diss. Rate } \\
\left(\mathrm{g} \mathrm{m}^{-2} \mathrm{~d}^{-1}\right)\end{array}$ & $\begin{array}{l}{[\mathrm{Na}]} \\
(\mu \mathrm{g} \mathrm{L} \\
\left.{ }^{1}\right)\end{array}$ & $\begin{array}{l}\text { Na Norm. } \\
\text { Diss. Rate, } \\
\mathrm{r} \\
\left(\mathrm{g} \mathrm{m}^{-2} \mathrm{~d}^{-1}\right)\end{array}$ & $\begin{array}{l}\mathrm{r}_{\mathrm{IEX}} \\
\left(\mathrm{mol} \mathrm{Na} \mathrm{s}^{-1}\right)\end{array}$ \\
\hline LG9.23.11.40.12 & & $1.95 \mathrm{E}-05$ & 34 & 0.747 & $1.47 \mathrm{E}-02$ & 204 & $7.56 \mathrm{E}-03$ & 171 & $8.58 \mathrm{E}-03$ & 1,260 & $8.75 \mathrm{E}-03$ & 1,140 & $9.70 \mathrm{E}-03$ & $5.66 \mathrm{E}-10$ \\
\hline LG9.23.11.40.14 & & $1.92 \mathrm{E}-05$ & 41 & 0.746 & $1.47 \mathrm{E}-02$ & 201 & $7.34 \mathrm{E}-03$ & 167 & $8.26 \mathrm{E}-03$ & 1,280 & $8.77 \mathrm{E}-03$ & 1,120 & $9.39 \mathrm{E}-03$ & $5.73 \mathrm{E}-10$ \\
\hline LG9.23.11.40.16 & & $1.91 \mathrm{E}-05$ & 48 & 0.745 & $1.47 \mathrm{E}-02$ & 198 & $7.20 \mathrm{E}-03$ & 163 & $8.02 \mathrm{E}-03$ & 1,220 & $8.32 \mathrm{E}-03$ & 1,140 & $9.52 \mathrm{E}-03$ & $7.53 \mathrm{E}-10$ \\
\hline LG9.23.11.40.18 & & $1.91 \mathrm{E}-05$ & 55 & 0.745 & 1.47E-02 & 185 & $6.73 \mathrm{E}-03$ & 159 & 7.83E-03 & 1,110 & 7.57E-03 & 999 & $8.34 \mathrm{E}-03$ & 2.60E-10 \\
\hline LG9.23.11.40.20 & & $1.97 \mathrm{E}-05$ & 62 & 0.744 & $1.47 \mathrm{E}-02$ & 164 & $6.16 \mathrm{E}-03$ & 142 & $7.22 \mathrm{E}-03$ & 992 & $6.99 \mathrm{E}-03$ & 960 & $8.28 \mathrm{E}-03$ & $5.34 \mathrm{E}-10$ \\
\hline LG9.23.11.40.22 & & $1.96 \mathrm{E}-05$ & 69 & 0.743 & $1.47 \mathrm{E}-02$ & 167 & $6.23 \mathrm{E}-03$ & 138 & $6.97 \mathrm{E}-03$ & 1,010 & 7.07E-03 & 925 & 7.93E-03 & $4.82 \mathrm{E}-10$ \\
\hline LG9.23.11.40.24 & & $1.88 \mathrm{E}-05$ & 76 & 0.742 & 1.47E-02 & 171 & $6.13 \mathrm{E}-03$ & 142 & $6.89 \mathrm{E}-03$ & 1,030 & $6.92 \mathrm{E}-03$ & 1,070 & $8.80 \mathrm{E}-03$ & $9.65 \mathrm{E}-10$ \\
\hline LG9.23.11.40.25 & & $1.94 \mathrm{E}-05$ & 79 & 0.742 & $1.47 \mathrm{E}-02$ & 183 & $6.77 \mathrm{E}-03$ & 156 & $7.82 \mathrm{E}-03$ & 1,110 & $7.70 \mathrm{E}-03$ & 1,060 & $9.01 \mathrm{E}-03$ & $6.01 \mathrm{E}-10$ \\
\hline LG9.23.11.40.26 & & $1.78 \mathrm{E}-05$ & 83 & 0.742 & $1.46 \mathrm{E}-02$ & 170 & $5.78 \mathrm{E}-03$ & 147 & $6.77 \mathrm{E}-03$ & 1,030 & $6.57 \mathrm{E}-03$ & 949 & 7.41E-03 & $3.25 \mathrm{E}-10$ \\
\hline \multicolumn{15}{|c|}{ LG9 $23^{\circ} \mathrm{C} \mathrm{pH} 11$ Reactor $\# 3$} \\
\hline LG9.23.11.80A.Blank1 & & 4.04E-05 & --- & --- & --- & [49.4] & --- & {$[75.6]$} & --- & [164] & --- & {$[134]$} & --- & --- \\
\hline LG9.23.11.80A.Blank2 & & $4.10 \mathrm{E}-05$ & --- & --- & --- & [49.4] & --- & [75.6] & --- & [164] & --- & [134] & --- & --- \\
\hline LG9.23.11.80A.Blank3 & & 4.04E-05 & 0 & --- & --- & [49.4] & --- & [75.6] & --- & [164] & --- & [134] & --- & --- \\
\hline LG9.23.11.80A.2 & & 4.19E-05 & 1 & 0.250 & 4.93E-03 & $\begin{array}{l}{[49.4]} \\
35^{*}\end{array}$ & $1.17 \mathrm{E}-02$ & [75.6] & 2.43E-02 & [164] & $7.31 \mathrm{E}-03$ & 260 & $1.42 \mathrm{E}-02$ & $<0$ \\
\hline LG9.23.11.80A.4 & & $3.79 \mathrm{E}-05$ & 6 & 0.250 & $4.92 \mathrm{E}-03$ & 82 & $1.77 \mathrm{E}-02$ & [75.6] & $2.20 \mathrm{E}-02$ & 329 & $1.33 \mathrm{E}-02$ & 369 & $1.82 \mathrm{E}-02$ & $<0$ \\
\hline LG9.23.11.80A.6 & & 3.73E-05 & 13 & 0.249 & $4.91 \mathrm{E}-03$ & 77 & $1.64 \mathrm{E}-02$ & [75.6] & $2.17 \mathrm{E}-02$ & 330 & $1.31 \mathrm{E}-02$ & 351 & $1.71 \mathrm{E}-02$ & $<0$ \\
\hline LG9.23.11.80A.8 & & $3.50 \mathrm{E}-05$ & 20 & 0.248 & $4.90 \mathrm{E}-03$ & 76 & $1.51 \mathrm{E}-02$ & [75.6] & $2.04 \mathrm{E}-02$ & 280 & $1.05 \mathrm{E}-02$ & 362 & $1.66 \mathrm{E}-02$ & $<0$ \\
\hline LG9.23.11.80A.10 & & 4.15E-05 & 27 & 0.247 & 4.89E-03 & 70 & $1.66 \mathrm{E}-02$ & $\begin{array}{l}{[75.6]} \\
66^{*}\end{array}$ & 2.43E-02 & 265 & $1.18 \mathrm{E}-02$ & 338 & $1.84 \mathrm{E}-02$ & $<0$ \\
\hline LG9.23.11.80A.12 & & 4.09E-05 & 34 & 0.247 & $4.88 \mathrm{E}-03$ & 53 & $1.23 \mathrm{E}-02$ & $\begin{array}{l}{[75.6]} \\
58^{\star}\end{array}$ & $2.40 \mathrm{E}-02$ & 314 & $1.38 \mathrm{E}-02$ & 500 & 2.69E-02 & $1.47 \mathrm{E}-09$ \\
\hline LG9.23.11.80A.14 & & 4.16E-05 & 41 & 0.246 & $4.87 \mathrm{E}-03$ & 59 & $1.40 \mathrm{E}-02$ & $\begin{array}{l}{[75.6]} \\
49^{\star}\end{array}$ & 2.45E-02 & 280 & $1.26 \mathrm{E}-02$ & 496 & $2.72 \mathrm{E}-02$ & $1.39 \mathrm{E}-09$ \\
\hline LG9.23.11.80A.16 & & 4.04E-05 & 48 & 0.245 & $4.86 \mathrm{E}-03$ & 58 & $1.36 \mathrm{E}-02$ & {$[75.6]$} & $2.38 \mathrm{E}-02$ & 246 & $1.07 E-02$ & 460 & $2.45 E-02$ & $3.86 \mathrm{E}-10$ \\
\hline LG9.23.11.80A.18 & & 3.94E-05 & 55 & 0.244 & $4.85 \mathrm{E}-03$ & $\begin{array}{l}{[49.4]} \\
39^{*}\end{array}$ & $1.12 \mathrm{E}-02$ & $\begin{array}{l}{[75.6]} \\
49^{\star}\end{array}$ & 2.33E-02 & $\begin{array}{l}{[164]} \\
79^{*}\end{array}$ & $6.99 \mathrm{E}-03$ & 457 & $2.39 \mathrm{E}-02$ & $2.99 \mathrm{E}-10$ \\
\hline LG9.23.11.80A.20 & & 3.96E-05 & 62 & 0.243 & $4.84 \mathrm{E}-03$ & $\begin{array}{l}{[49.4]} \\
44^{*}\end{array}$ & $1.13 \mathrm{E}-02$ & {$[75.6]$} & 2.34E-02 & [164] & 7.04E-03 & 443 & $2.33 E-02$ & $<0$ \\
\hline LG9.23.11.80A.22 & & 3.82E-05 & 69 & 0.243 & $4.83 \mathrm{E}-03$ & 55 & $1.22 \mathrm{E}-02$ & $\begin{array}{l}{[75.6]} \\
49^{\star}\end{array}$ & $2.26 \mathrm{E}-02$ & 227 & $9.40 \mathrm{E}-03$ & 445 & $2.26 \mathrm{E}-02$ & $<0$ \\
\hline LG9.23.11.80A.24 & & 4.04E-05 & 76 & 0.242 & $4.82 \mathrm{E}-03$ & 53 & $1.24 \mathrm{E}-02$ & $\begin{array}{l}{[75.6]} \\
54^{\star}\end{array}$ & $2.40 \mathrm{E}-02$ & 202 & $8.87 \mathrm{E}-03$ & 460 & 2.47E-02 & $3.89 \mathrm{E}-10$ \\
\hline LG9.23.11.80A.25 & & 3.99E-05 & 79 & 0.242 & $4.81 \mathrm{E}-03$ & 57 & $1.33 \mathrm{E}-02$ & $\begin{array}{l}{[75.6]} \\
52^{\star}\end{array}$ & $2.37 \mathrm{E}-02$ & 230 & $1.00 \mathrm{E}-02$ & 475 & $2.53 \mathrm{E}-02$ & $7.88 \mathrm{E}-10$ \\
\hline LG9.23.11.80A.26 & & 3.68E-05 & 83 & 0.241 & $4.81 \mathrm{E}-03$ & 54 & $1.15 \mathrm{E}-02$ & $\begin{array}{l}{[75.6]} \\
55^{*}\end{array}$ & 2.19E-02 & 242 & $9.70 \mathrm{E}-03$ & 442 & $2.17 \mathrm{E}-02$ & $<0$ \\
\hline \multicolumn{15}{|c|}{ LG9 $23^{\circ} \mathrm{C} \mathrm{pH} 11$ Reactor \#4 } \\
\hline LG9.23.11.80B.Blank1 & & $3.75 \mathrm{E}-05$ & --- & --- & --- & [49.4] & --- & {$[75.6]$} & --- & [164] & --- & [134] & --- & --- \\
\hline LG9.23.11.80B.Blank2 & & $3.82 \mathrm{E}-05$ & --- & --- & --- & [49.4] & --- & [75.6] & --- & [164] & --- & {$[134]$} & --- & --- \\
\hline LG9.23.11.80B.Blank3 & & $3.65 \mathrm{E}-05$ & 0 & --- & --- & [49.4] & --- & [75.6] & --- & [164] & --- & [134] & --- & --- \\
\hline LG9.23.11.80B.2 & & 4.66E-05 & 1 & 0.050 & $9.82 E-04$ & $\begin{array}{l}{[49.4]} \\
17^{\star}\end{array}$ & $6.55 \mathrm{E}-02$ & {$[75.6]$} & $1.36 \mathrm{E}-01$ & [164] & $4.08 E-02$ & $\begin{array}{l}{[134]} \\
121^{*}\end{array}$ & $4.08 \mathrm{E}-02$ & $<0$ \\
\hline LG9.23.11.80B.4 & & $3.64 \mathrm{E}-05$ & 6 & 0.049 & $9.72 \mathrm{E}-04$ & $\begin{array}{l}{[49.4]} \\
32^{*}\end{array}$ & $5.17 \mathrm{E}-02$ & [75.6] & 1.07E-01 & [164] & $3.22 \mathrm{E}-02$ & 158 & $3.80 \mathrm{E}-02$ & $<0$ \\
\hline
\end{tabular}




\begin{tabular}{|c|c|c|c|c|c|c|c|c|c|c|c|c|c|c|}
\hline Sample ID & $\begin{array}{l}\text { Influent } \\
\text { [Si] } \\
\text { (ppm) }\end{array}$ & $\begin{array}{l}\text { Flow } \\
\text { Rate, } \mathrm{q} \\
\left(\mathrm{m}^{3} \mathrm{~d}^{-1}\right)\end{array}$ & $\begin{array}{l}\text { Time } \\
\text { (d) }\end{array}$ & $\begin{array}{l}\text { Glass } \\
\text { Mass } \\
\text { (g) }\end{array}$ & $\begin{array}{l}\text { Surface } \\
\text { Area, S } \\
\left(\mathrm{m}^{2}\right)\end{array}$ & $\begin{array}{l}{[\mathrm{Al}]} \\
\left(\mu \mathrm{L} \mathrm{L}^{-1}\right)\end{array}$ & $\begin{array}{l}\text { Al Norm. } \\
\text { Diss. Rate } \\
\left(\mathrm{g} \mathrm{m}^{-2} \mathrm{~d}^{-1}\right)\end{array}$ & $\begin{array}{l}{[\mathrm{B}]} \\
\left(\mu \mathrm{g} \mathrm{L}^{-1}\right)\end{array}$ & $\begin{array}{l}\text { B Norm. } \\
\text { Diss. Rate } \\
\left(\mathrm{g} \mathrm{m}^{-2} \mathrm{~d}^{-1}\right)\end{array}$ & $\begin{array}{l}{[\mathrm{Si}]} \\
\left(\mu \mathrm{g} \mathrm{L}^{-1}\right)\end{array}$ & $\begin{array}{l}\text { Si Norm. } \\
\text { Diss. Rate } \\
\left(\mathrm{g} \mathrm{m}^{-2} \mathrm{~d}^{-1}\right)\end{array}$ & $\begin{array}{l}{[\mathrm{Na}]} \\
\left(\mu \mathrm{g} \mathrm{L}{ }^{-}\right. \\
\left.{ }^{1}\right)\end{array}$ & $\begin{array}{l}\text { Na Norm. } \\
\text { Diss. Rate, } \\
\mathrm{r} \\
\left(\mathrm{g} \mathrm{m}^{-2} \mathrm{~d}^{-1}\right)\end{array}$ & $\begin{array}{l}\mathrm{r}_{\mathrm{IEX}} \\
\left(\mathrm{mol} \mathrm{Na} \mathrm{No}^{-1}\right)\end{array}$ \\
\hline LG9.23.11.80B.6 & & 3.53E-05 & 13 & 0.048 & $9.63 \mathrm{E}-04$ & $\begin{array}{l}{[49.4]} \\
32^{\star}\end{array}$ & $5.05 \mathrm{E}-02$ & {$[75.6]$} & $1.05 \mathrm{E}-01$ & $\begin{array}{l}{[164]} \\
92^{\star}\end{array}$ & $3.15 E-02$ & 184 & $4.32 \mathrm{E}-02$ & $<0$ \\
\hline LG9.23.11.80B.8 & & 3.52E-05 & 20 & 0.048 & $9.54 \mathrm{E}-04$ & $\begin{array}{l}{[49.4]} \\
31^{\star}\end{array}$ & $5.09 \mathrm{E}-02$ & {$[75.6]$} & $1.05 E-01$ & $\begin{array}{l}{[164]} \\
78^{\star}\end{array}$ & $3.17 E-02$ & 193 & $4.57 \mathrm{E}-02$ & $<0$ \\
\hline LG9.23.11.80B.10 & & 3.62E-05 & 27 & 0.047 & $9.44 E-04$ & $\begin{array}{l}{[49.4]} \\
32^{\star}\end{array}$ & $5.30 \mathrm{E}-02$ & {$[75.6]$} & $1.10 \mathrm{E}-01$ & $\begin{array}{l}{[164]} \\
93^{\star}\end{array}$ & $3.30 \mathrm{E}-02$ & 208 & $5.12 \mathrm{E}-02$ & $<0$ \\
\hline LG9.23.11.80B.12 & & $3.69 \mathrm{E}-05$ & 34 & 0.046 & $9.34 \mathrm{E}-04$ & $\begin{array}{l}{[49.4]} \\
29^{\star}\end{array}$ & $5.45 E-02$ & $\begin{array}{l}{[75.6]} \\
31^{\star}\end{array}$ & 1.13E-01 & $\begin{array}{l}{[164]} \\
71^{\star}\end{array}$ & $3.39 \mathrm{E}-02$ & 379 & $9.60 \mathrm{E}-02$ & $<0$ \\
\hline LG9.23.11.80B.14 & & 3.69E-05 & 41 & 0.046 & $9.24 \mathrm{E}-04$ & $\begin{array}{l}{[49.4]} \\
28^{\star}\end{array}$ & $5.51 \mathrm{E}-02$ & $\begin{array}{l}{[75.6]} \\
33^{\star}\end{array}$ & 1.14E-01 & $\begin{array}{l}{[164]} \\
85^{\star}\end{array}$ & $3.43 \mathrm{E}-02$ & 357 & $9.15 E-02$ & $<0$ \\
\hline LG9.23.11.80B.16 & & $3.51 \mathrm{E}-05$ & 48 & 0.045 & $9.15 \mathrm{E}-04$ & $\begin{array}{l}{[49.4]} \\
17^{\star}\end{array}$ & $5.30 \mathrm{E}-02$ & $\begin{array}{l}{[75.6]} \\
41^{\star}\end{array}$ & 1.10E-01 & $\begin{array}{l}{[164]} \\
143^{\star}\end{array}$ & $3.30 \mathrm{E}-02$ & 318 & 7.83E-02 & $<0$ \\
\hline LG9.23.11.80B.18 & & 3.64E-05 & 55 & 0.044 & $9.05 E-04$ & $\begin{array}{l}{[49.4]} \\
25^{\star}\end{array}$ & $5.55 \mathrm{E}-02$ & $\begin{array}{l}{[75.6]} \\
31^{\star}\end{array}$ & $1.15 \mathrm{E}-01$ & [164] & 3.46E-02 & 332 & $8.57 \mathrm{E}-02$ & $<0$ \\
\hline LG9.23.11.80B.20 & & 3.57E-05 & 62 & 0.043 & $8.95 E-04$ & $\begin{array}{l}{[49.4]} \\
25^{\star}\end{array}$ & $5.51 \mathrm{E}-02$ & $\begin{array}{l}{[75.6]} \\
30^{*}\end{array}$ & 1.14E-01 & [164] & $3.43 E-02$ & 328 & 8.40E-02 & $<0$ \\
\hline LG9.23.11.80B.22 & & 3.74E-05 & 69 & 0.043 & $8.85 E-04$ & $\begin{array}{l}{[49.4]} \\
23^{\star}\end{array}$ & $5.84 \mathrm{E}-02$ & $\begin{array}{l}{[75.6]} \\
35^{\star}\end{array}$ & $1.21 \mathrm{E}-01$ & [164] & $3.64 \mathrm{E}-02$ & 338 & $9.18 \mathrm{E}-02$ & $<0$ \\
\hline LG9.23.11.80B.24 & & 3.74E-05 & 76 & 0.042 & $8.74 \mathrm{E}-04$ & $\begin{array}{l}{[49.4]} \\
24^{\star}\end{array}$ & $5.91 \mathrm{E}-02$ & $\begin{array}{l}{[75.6]} \\
36^{\star}\end{array}$ & $1.23 E-01$ & {$[164]$} & $3.68 E-02$ & 341 & $9.37 \mathrm{E}-02$ & $<0$ \\
\hline LG9.23.11.80B.25 & & 3.59E-05 & 79 & 0.042 & $8.71 E-04$ & $\begin{array}{l}{[49.4]} \\
17^{\star}\end{array}$ & $5.70 \mathrm{E}-02$ & $\begin{array}{l}{[75.6]} \\
30^{\star}\end{array}$ & $1.18 \mathrm{E}-01$ & $\begin{array}{l}{[164]} \\
69^{\star}\end{array}$ & $3.55 E-02$ & 354 & $9.38 \mathrm{E}-02$ & $<0$ \\
\hline LG9.23.11.80B.26 & & 3.43E-05 & 83 & 0.041 & $8.65 \mathrm{E}-04$ & $\begin{array}{l}{[49.4]} \\
41^{\star}\end{array}$ & $5.48 \mathrm{E}-02$ & $\begin{array}{l}{[75.6]} \\
36^{*}\end{array}$ & 1.13E-01 & $\begin{array}{l}{[164]} \\
94^{\star}\end{array}$ & $3.41 \mathrm{E}-02$ & 349 & $8.88 \mathrm{E}-02$ & $<0$ \\
\hline \multicolumn{15}{|c|}{ LG9 $23{ }^{\circ} \mathrm{C} \mathrm{pH} 12$ Reactor \#1 } \\
\hline LG9.23.12.20.Blank1 & & $7.90 \mathrm{E}-06$ & --- & --- & --- & [49.4] & --. & [75.6] & --- & [164] & -.- & 503 & --- & -.- \\
\hline LG9.23.12.20.Blank2 & & $1.39 \mathrm{E}-05$ & --- & --- & --- & {$[49.4]$} & --- & {$[75.6]$} & --- & [164] & --- & 518 & --- & --- \\
\hline LG9.23.12.20.Blank3 & & $1.30 \mathrm{E}-05$ & 0 & --- & -.- & [49.4] & --. & [75.6] & --- & 215 & --- & 523 & --- & -.- \\
\hline LG9.23.12.20.2 & & $3.71 \mathrm{E}-06$ & 2 & 1.498 & $2.95 \mathrm{E}-02$ & 608 & $2.14 \mathrm{E}-03$ & 247 & $1.17 \mathrm{E}-03$ & 2,630 & $1.73 \mathrm{E}-03$ & 2,840 & $2.29 \mathrm{E}-03$ & $5.62 \mathrm{E}-10$ \\
\hline LG9.23.12.20.4 & & 8.07E-06 & 9 & 1.496 & $2.95 \mathrm{E}-02$ & 1,610 & $1.23 \mathrm{E}-02$ & 964 & $1.00 \mathrm{E}-02$ & 8,000 & $1.15 \mathrm{E}-02$ & 6,960 & $1.22 \mathrm{E}-02$ & $1.13 \mathrm{E}-09$ \\
\hline LG9.23.12.20.6 & & $1.04 \mathrm{E}-05$ & 16 & 1.493 & $2.94 \mathrm{E}-02$ & 1,330 & $1.32 \mathrm{E}-02$ & 847 & $1.14 \mathrm{E}-02$ & 6,840 & $1.27 \mathrm{E}-02$ & 6,130 & $1.39 \mathrm{E}-02$ & $1.30 \mathrm{E}-09$ \\
\hline LG9.23.12.20.8 & & $9.46 \mathrm{E}-06$ & 23 & 1.492 & $2.94 \mathrm{E}-02$ & 1,240 & $1.12 \mathrm{E}-02$ & 817 & $9.95 \mathrm{E}-03$ & 6,290 & $1.06 \mathrm{E}-02$ & 5,800 & $1.20 \mathrm{E}-02$ & $1.02 \mathrm{E}-09$ \\
\hline LG9.23.12.20.10 & & $1.02 \mathrm{E}-05$ & 30 & 1.489 & $2.94 \mathrm{E}-02$ & 1,170 & $1.13 \mathrm{E}-02$ & 804 & $1.05 \mathrm{E}-02$ & 6,050 & $1.10 \mathrm{E}-02$ & 5,570 & $1.24 \mathrm{E}-02$ & $9.30 \mathrm{E}-10$ \\
\hline LG9.23.12.20.12 & & $1.03 E-05$ & 37 & 1.487 & $2.93 \mathrm{E}-02$ & 1,250 & $1.23 \mathrm{E}-02$ & 877 & $1.16 \mathrm{E}-02$ & 6,720 & $1.24 \mathrm{E}-02$ & 5,780 & $1.30 \mathrm{E}-02$ & $6.92 \mathrm{E}-10$ \\
\hline LG9.23.12.20.14 & & $9.05 E-06$ & 44 & 1.485 & 2.93E-02 & 1,290 & $1.11 \mathrm{E}-02$ & 912 & $1.07 E-02$ & 6,940 & $1.12 \mathrm{E}-02$ & 5,420 & $1.07 E-02$ & 4.41E-11 \\
\hline LG9.23.12.20.16 & & $1.06 \mathrm{E}-05$ & 51 & 1.481 & $2.93 \mathrm{E}-02$ & 1,600 & $1.63 \mathrm{E}-02$ & 1,100 & $1.51 \mathrm{E}-02$ & 8,980 & $1.71 \mathrm{E}-02$ & 5,640 & $1.32 \mathrm{E}-02$ & $<0$ \\
\hline LG9.23.12.20.18 & & $9.88 \mathrm{E}-06$ & 58 & 1.480 & $2.92 E-02$ & 1,250 & $1.18 \mathrm{E}-02$ & 856 & $1.09 \mathrm{E}-02$ & 6,700 & $1.19 \mathrm{E}-02$ & 5,520 & $1.20 \mathrm{E}-02$ & $5.18 \mathrm{E}-10$ \\
\hline LG9.23.12.20.20 & & $1.09 \mathrm{E}-05$ & 65 & 1.477 & $2.92 \mathrm{E}-02$ & 1,180 & $1.23 \mathrm{E}-02$ & 810 & $1.15 \mathrm{E}-02$ & 6,140 & $1.20 \mathrm{E}-02$ & 5,080 & $1.22 \mathrm{E}-02$ & $3.69 \mathrm{E}-10$ \\
\hline LG9.23.12.20.22 & & $1.75 \mathrm{E}-05$ & 72 & 1.474 & $2.92 \mathrm{E}-02$ & 863 & $1.45 \mathrm{E}-02$ & 581 & $1.32 \mathrm{E}-02$ & 4,570 & $1.44 \mathrm{E}-02$ & 4,020 & $1.55 \mathrm{E}-02$ & $1.15 \mathrm{E}-09$ \\
\hline LG9.23.12.20.24 & & $1.41 \mathrm{E}-05$ & 79 & 1.472 & $2.91 \mathrm{E}-02$ & 998 & $1.35 \mathrm{E}-02$ & 693 & $1.27 \mathrm{E}-02$ & 5,370 & $1.36 \mathrm{E}-02$ & 4,590 & $1.42 \mathrm{E}-02$ & $7.88 \mathrm{E}-10$ \\
\hline LG9.23.12.20.25 & & $1.46 \mathrm{E}-05$ & 83 & 1.470 & $2.91 \mathrm{E}-02$ & 925 & $1.30 \mathrm{E}-02$ & 656 & $1.25 \mathrm{E}-02$ & 5,010 & $1.32 \mathrm{E}-02$ & 4,230 & $1.36 \mathrm{E}-02$ & $5.90 \mathrm{E}-10$ \\
\hline \multicolumn{15}{|c|}{ LG9 $23^{\circ} \mathrm{C} \mathrm{pH} 12$ Reactor \#2 } \\
\hline LG9.23.12.40.2 & & $5.49 \mathrm{E}-06$ & 1 & 0.749 & $1.47 \mathrm{E}-02$ & 286 & $2.98 \mathrm{E}-03$ & [75.6] & $1.07 E-03$ & 1,040 & 2.03E-03 & 1,570 & 3.75E-03 & $1.35 \mathrm{E}-09$ \\
\hline LG9.23.12.40.6 & & 2.00E-05 & 13 & 0.748 & $1.47 \mathrm{E}-02$ & 305 & $1.16 \mathrm{E}-02$ & [75.6] & 3.89E-03 & 1,240 & 8.83E-03 & 1,450 & $1.26 \mathrm{E}-02$ & 4.41E-09 \\
\hline LG9.23.12.40.8 & & $2.07 E-05$ & 20 & 0.748 & $1.47 \mathrm{E}-02$ & 276 & $1.09 \mathrm{E}-02$ & [75.6] & $4.02 \mathrm{E}-03$ & 987 & $7.28 \mathrm{E}-03$ & 1,280 & $1.16 \mathrm{E}-02$ & $3.79 \mathrm{E}-09$ \\
\hline
\end{tabular}




\begin{tabular}{|c|c|c|c|c|c|c|c|c|c|c|c|c|c|c|}
\hline Sample ID & $\begin{array}{l}\text { Influent } \\
\text { [Si] } \\
\text { (ppm) }\end{array}$ & $\begin{array}{l}\text { Flow } \\
\text { Rate, } q \\
\left(\mathrm{~m}^{3} \mathrm{~d}^{-1}\right)\end{array}$ & $\begin{array}{l}\text { Time } \\
\text { (d) }\end{array}$ & $\begin{array}{l}\text { Glass } \\
\text { Mass } \\
\text { (g) }\end{array}$ & $\begin{array}{l}\text { Surface } \\
\text { Area, S } \\
\left(\mathrm{m}^{2}\right)\end{array}$ & $\begin{array}{l}{[\mathrm{Al}]} \\
\left(\mu \mathrm{g} \mathrm{L}^{-1}\right)\end{array}$ & $\begin{array}{l}\text { Al Norm. } \\
\text { Diss. Rate } \\
\left(\mathrm{g} \mathrm{m}^{-2} \mathrm{~d}^{-1}\right)\end{array}$ & $\begin{array}{l}{[\mathrm{B}]} \\
\left(\mu \mathrm{g} \mathrm{L}^{-1}\right)\end{array}$ & $\begin{array}{l}\text { B Norm. } \\
\text { Diss. Rate } \\
\left(\mathrm{g} \mathrm{m}^{-2} \mathrm{~d}^{-1}\right)\end{array}$ & $\begin{array}{l}{[\mathrm{Si}]} \\
\left(\mu \mathrm{g} \mathrm{L}^{-1}\right)\end{array}$ & $\begin{array}{l}\text { Si Norm. } \\
\text { Diss. Rate } \\
\left(\mathrm{g} \mathrm{m}^{-2} \mathrm{~d}^{-1}\right)\end{array}$ & $\begin{array}{l}{[\mathrm{Na}]} \\
\left(\mu \mathrm{g} \mathrm{L}{ }^{-}\right. \\
\left.{ }^{1}\right)\end{array}$ & $\begin{array}{l}\text { Na Norm. } \\
\text { Diss. Rate, } \\
\mathrm{r} \\
\left(\mathrm{g} \mathrm{m}^{-2} \mathrm{~d}^{-1}\right)\end{array}$ & $\begin{array}{l}\mathrm{r}_{\mathrm{IEX}} \\
\left(\mathrm{mol} \mathrm{Na} \mathrm{No}^{-1}\right)\end{array}$ \\
\hline LG9.23.12.40.10 & & $1.95 \mathrm{E}-05$ & 27 & 0.747 & $1.47 \mathrm{E}-02$ & 319 & $1.18 \mathrm{E}-02$ & 77 & $3.83 \mathrm{E}-03$ & 1,300 & $9.01 \mathrm{E}-03$ & 1,470 & $1.25 \mathrm{E}-02$ & $4.35 \mathrm{E}-09$ \\
\hline LG9.23.12.40.12 & & $1.68 \mathrm{E}-05$ & 34 & 0.745 & $1.47 \mathrm{E}-02$ & 744 & $2.38 \mathrm{E}-02$ & 521 & $2.26 \mathrm{E}-02$ & 4,310 & $2.59 \mathrm{E}-02$ & 3,610 & $2.65 \mathrm{E}-02$ & 1.99E-09 \\
\hline LG9.23.12.40.14 & & $1.35 \mathrm{E}-05$ & 41 & 0.743 & $1.47 \mathrm{E}-02$ & 786 & 2.02E-02 & 550 & $1.91 \mathrm{E}-02$ & 4,550 & $2.19 \mathrm{E}-02$ & 3,810 & $2.25 \mathrm{E}-02$ & 1.69E-09 \\
\hline LG9.23.12.40.16 & & $1.73 \mathrm{E}-05$ & 48 & 0.740 & $1.46 \mathrm{E}-02$ & 741 & $2.45 \mathrm{E}-02$ & 517 & $2.31 \mathrm{E}-02$ & 4,160 & $2.58 \mathrm{E}-02$ & 3,920 & $2.97 \mathrm{E}-02$ & 3.33E-09 \\
\hline LG9.23.12.40.18 & & $1.47 \mathrm{E}-05$ & 55 & 0.738 & $1.46 \mathrm{E}-02$ & 741 & $2.08 \mathrm{E}-02$ & 509 & $1.94 \mathrm{E}-02$ & 4,250 & $2.24 \mathrm{E}-02$ & 3,510 & $2.26 \mathrm{E}-02$ & $1.65 \mathrm{E}-09$ \\
\hline LG9.23.12.40.20 & & $1.34 \mathrm{E}-05$ & 62 & 0.737 & $1.46 \mathrm{E}-02$ & 746 & $1.91 \mathrm{E}-02$ & 507 & $1.76 \mathrm{E}-02$ & 4,180 & $2.01 \mathrm{E}-02$ & 3,530 & $2.08 \mathrm{E}-02$ & 1.60E-09 \\
\hline LG9.23.12.40.22 & & $1.96 \mathrm{E}-05$ & 69 & 0.734 & $1.45 \mathrm{E}-02$ & 682 & 2.57E-02 & 446 & $2.28 \mathrm{E}-02$ & 3,720 & $2.63 \mathrm{E}-02$ & 3,200 & $2.77 \mathrm{E}-02$ & 2.49E-09 \\
\hline LG9.23.12.40.24 & & $1.99 \mathrm{E}-05$ & 76 & 0.732 & $1.45 \mathrm{E}-02$ & 645 & 2.47E-02 & 443 & $2.30 \mathrm{E}-02$ & 3,700 & $2.66 \mathrm{E}-02$ & 2,890 & $2.54 \mathrm{E}-02$ & $1.23 \mathrm{E}-09$ \\
\hline LG9.23.12.40.25 & & 1.93E-05 & 79 & 0.731 & $1.45 \mathrm{E}-02$ & 681 & $2.53 \mathrm{E}-02$ & 481 & 2.42E-02 & 3,960 & $2.76 \mathrm{E}-02$ & 2,870 & $2.45 \mathrm{E}-02$ & 1.49E-10 \\
\hline LG9.23.12.40.26 & & $1.92 \mathrm{E}-05$ & 83 & 0.730 & $1.45 \mathrm{E}-02$ & 625 & $2.32 \mathrm{E}-02$ & 444 & 2.23E-02 & 3,670 & $2.56 \mathrm{E}-02$ & 2,920 & $2.49 \mathrm{E}-02$ & $1.30 \mathrm{E}-09$ \\
\hline \multicolumn{15}{|c|}{ LG9 $23^{\circ} \mathrm{C} \mathrm{pH} 12$ Reactor $\# 3$} \\
\hline LG9.23.12.80A.Blank1 & - & $2.40 \mathrm{E}-05$ & --- & --- & --- & [49.4] & --- & [75.6] & --- & [164] & --- & 501 & --- & --- \\
\hline LG9.23.12.80A.Blank2 & - & $2.60 \mathrm{E}-05$ & -.- & --. & --. & [49.4] & -.- & [75.6] & --- & 206 & --- & 486 & --- & --- \\
\hline LG9.23.12.80A.Blank3 & - & $2.23 \mathrm{E}-05$ & 0 & --- & --- & [49.4] & --- & [75.6] & --- & 264 & --- & 508 & --- & -- \\
\hline LG9.23.12.80A.2 & & $1.00 \mathrm{E}-05$ & 1 & 0.250 & $4.91 \mathrm{E}-03$ & 84 & $4.81 \mathrm{E}-03$ & [75.6] & $5.84 \mathrm{E}-03$ & [164] & $1.76 \mathrm{E}-03$ & 360 & $4.72 \mathrm{E}-03$ & $<0$ \\
\hline LG9.23.12.80A.4 & & 2.97E-05 & 6 & 0.249 & $4.90 \mathrm{E}-03$ & 131 & $2.22 \mathrm{E}-02$ & [75.6] & $1.73 \mathrm{E}-02$ & 372 & $1.18 \mathrm{E}-02$ & 661 & $2.57 \mathrm{E}-02$ & $4.22 \mathrm{E}-09$ \\
\hline LG9.23.12.80A.6 & & 3.46E-05 & 13 & 0.248 & $4.90 \mathrm{E}-03$ & 195 & $3.86 \mathrm{E}-02$ & $\begin{array}{l}{[75.6]} \\
61^{\star}\end{array}$ & 2.02E-02 & 837 & $3.10 E-02$ & 727 & $3.30 \mathrm{E}-02$ & $6.43 E-09$ \\
\hline LG9.23.12.80A.8 & & $3.51 \mathrm{E}-05$ & 20 & 0.248 & $4.89 \mathrm{E}-03$ & 92 & $1.84 \mathrm{E}-02$ & [75.6] & $2.06 \mathrm{E}-02$ & 203 & $7.65 \mathrm{E}-03$ & 505 & $2.33 \mathrm{E}-02$ & $1.38 \mathrm{E}-09$ \\
\hline LG9.23.12.80A.10 & & $3.14 \mathrm{E}-05$ & 27 & 0.246 & $4.87 \mathrm{E}-03$ & 236 & $4.26 \mathrm{E}-02$ & 131 & $3.20 \mathrm{E}-02$ & 1,060 & $3.59 \mathrm{E}-02$ & 1,230 & $5.10 \mathrm{E}-02$ & $9.55 \mathrm{E}-09$ \\
\hline LG9.23.12.80A.12 & & $2.24 \mathrm{E}-05$ & 34 & 0.245 & $4.85 \mathrm{E}-03$ & 299 & $3.85 \mathrm{E}-02$ & 212 & $3.70 \mathrm{E}-02$ & 1,740 & $4.20 \mathrm{E}-02$ & 1,790 & $5.30 \mathrm{E}-02$ & $8.05 \mathrm{E}-09$ \\
\hline LG9.23.12.80A.14 & & $1.09 \mathrm{E}-05$ & 41 & 0.245 & $4.85 \mathrm{E}-03$ & 346 & $2.17 \mathrm{E}-02$ & 238 & $2.02 \mathrm{E}-02$ & 2,010 & $2.37 \mathrm{E}-02$ & 1,940 & $2.80 \mathrm{E}-02$ & $3.90 \mathrm{E}-09$ \\
\hline LG9.23.12.80A.16 & & $1.76 \mathrm{E}-05$ & 48 & 0.243 & $4.82 E-03$ & 424 & $4.34 \mathrm{E}-02$ & 311 & $4.30 \mathrm{E}-02$ & 2,540 & $4.87 \mathrm{E}-02$ & 2,330 & $5.47 \mathrm{E}-02$ & $5.87 E-09$ \\
\hline LG9.23.12.80A.18 & & $1.36 \mathrm{E}-06$ & 55 & 0.243 & $4.83 \mathrm{E}-03$ & 603 & $4.76 \mathrm{E}-03$ & 417 & $4.45 \mathrm{E}-03$ & 3,500 & $5.18 \mathrm{E}-03$ & 3,070 & $5.56 \mathrm{E}-03$ & $5.58 \mathrm{E}-10$ \\
\hline LG9.23.12.80A.20 & & $7.51 \mathrm{E}-08$ & 62 & 0.243 & 4.83E-03 & 756 & 3.29E-04 & 560 & $3.30 \mathrm{E}-04$ & 820 & $6.68 \mathrm{E}-05$ & 4,080 & 4.07E-04 & $3.92 \mathrm{E}-11$ \\
\hline LG9.23.12.80A.22 & & $3.38 \mathrm{E}-05$ & 69 & 0.241 & $4.80 \mathrm{E}-03$ & 288 & $5.66 \mathrm{E}-02$ & 178 & $4.74 \mathrm{E}-02$ & 1,530 & $5.64 \mathrm{E}-02$ & 1,480 & $6.68 \mathrm{E}-02$ & $9.79 \mathrm{E}-09$ \\
\hline LG9.23.12.80A.24 & & 3.50E-05 & 76 & 0.240 & $4.78 \mathrm{E}-03$ & 210 & $4.30 \mathrm{E}-02$ & 151 & $4.18 \mathrm{E}-02$ & 1,250 & $4.79 E-02$ & 1,280 & $6.01 \mathrm{E}-02$ & $9.22 \mathrm{E}-09$ \\
\hline LG9.23.12.80A.25 & & $3.72 \mathrm{E}-05$ & 79 & 0.239 & 4.77E-03 & 225 & $4.90 \mathrm{E}-02$ & 157 & $4.62 \mathrm{E}-02$ & 1,280 & $5.22 \mathrm{E}-02$ & 1,240 & $6.20 \mathrm{E}-02$ & $7.91 \mathrm{E}-09$ \\
\hline LG9.23.12.80A.26 & & 3.67E-05 & 83 & 0.238 & $4.76 \mathrm{E}-03$ & 197 & $4.24 \mathrm{E}-02$ & 139 & $4.05 E-02$ & 1,130 & $4.56 \mathrm{E}-02$ & 1,050 & $5.19 \mathrm{E}-02$ & $5.74 \mathrm{E}-09$ \\
\hline \multicolumn{15}{|c|}{ LG9 $23^{\circ} \mathrm{C} \mathrm{pH} 12$ Reactor \#4 } \\
\hline LG9.23.12.80B.Blank1 & & $1.22 \mathrm{E}-05$ & --- & --- & --- & [49.4] & --- & [75.6] & --- & [164] & --- & 446 & --- & --- \\
\hline LG9.23.12.80B.Blank2 & & $1.76 \mathrm{E}-05$ &.-- & --- & -.- & [49.4] & -.- & [75.6] & --- & 183 & -.- & 489 & --. & -.- \\
\hline LG9.23.12.80B.Blank3 & & $2.04 \mathrm{E}-05$ & 0 & --- & --- & [49.4] & --- & [75.6] & --- & 212 & --- & 501 & --- & --- \\
\hline LG9.23.12.80B.2 & & $4.41 \mathrm{E}-06$ & 1 & 0.050 & $9.82 \mathrm{E}-04$ & $\begin{array}{l}{[49.4]} \\
29^{\star}\end{array}$ & $6.20 \mathrm{E}-03$ & [75.6] & $1.29 \mathrm{E}-02$ & [164] & $3.86 \mathrm{E}-03$ & 358 & $1.03 \mathrm{E}-02$ & $<0$ \\
\hline LG9.23.12.80B.4 & & 4.37E-05 & 6 & 0.049 & $9.70 \mathrm{E}-04$ & 84 & $1.05 \mathrm{E}-01$ & [75.6] & $1.29 \mathrm{E}-01$ & 236 & $5.57 \mathrm{E}-02$ & 673 & $1.95 \mathrm{E}-01$ & $3.31 \mathrm{E}-08$ \\
\hline LG9.23.12.80B.6 & & $4.25 \mathrm{E}-05$ & 13 & 0.048 & $9.59 \mathrm{E}-04$ & 65 & $7.98 \mathrm{E}-02$ & [75.6] & $1.27 \mathrm{E}-01$ & $\begin{array}{l}{[164]} \\
84^{*}\end{array}$ & $3.80 \mathrm{E}-02$ & 347 & $9.86 \mathrm{E}-02$ & $<0$ \\
\hline LG9.23.12.80B.8 & & $4.35 \mathrm{E}-05$ & 20 & 0.047 & $9.48 \mathrm{E}-04$ & 64 & 8.17E-02 & [75.6] & $1.31 \mathrm{E}-01$ & $\begin{array}{l}{[164]} \\
143^{*}\end{array}$ & $3.94 \mathrm{E}-02$ & 644 & $1.90 \mathrm{E}-01$ & $2.94 \mathrm{E}-08$ \\
\hline LG9.23.12.80B.10 & & $3.98 \mathrm{E}-05$ & 27 & 0.047 & $9.37 \mathrm{E}-04$ & 66 & $7.82 \mathrm{E}-02$ & [75.6] & $1.22 \mathrm{E}-01$ & 190 & 4.23E-02 & 790 & $2.15 \mathrm{E}-01$ & $4.73 \mathrm{E}-08$ \\
\hline LG9.23.12.80B.12 & & 3.76E-05 & 34 & 0.046 & $9.27 \mathrm{E}-04$ & 73 & $8.28 \mathrm{E}-02$ & $\begin{array}{l}{[75.6]} \\
56^{*}\end{array}$ & 1.16E-01 & 469 & $9.98 \mathrm{E}-02$ & 807 & $2.10 \mathrm{E}-01$ & $4.74 \mathrm{E}-08$ \\
\hline LG9.23.12.80B.14 & & $3.38 \mathrm{E}-05$ & 41 & 0.045 & $9.18 \mathrm{E}-04$ & 86 & $8.87 \mathrm{E}-02$ & {$[75.6]$} & $1.05 \mathrm{E}-01$ & 470 & $9.07 E-02$ & 811 & $1.92 \mathrm{E}-01$ & $4.35 \mathrm{E}-08$ \\
\hline
\end{tabular}




\begin{tabular}{|c|c|c|c|c|c|c|c|c|c|c|c|c|c|c|}
\hline Sample ID & $\begin{array}{l}\text { Influent } \\
{[\mathrm{Si}]} \\
(\mathrm{ppm})\end{array}$ & $\begin{array}{l}\text { Flow } \\
\text { Rate, } \mathrm{q} \\
\left(\mathrm{m}^{3} \mathrm{~d}^{-1}\right)\end{array}$ & $\begin{array}{l}\text { Time } \\
\text { (d) }\end{array}$ & $\begin{array}{l}\text { Glass } \\
\text { Mass } \\
\text { (g) }\end{array}$ & $\begin{array}{l}\text { Surface } \\
\text { Area, S } \\
\left(\mathrm{m}^{2}\right)\end{array}$ & $\begin{array}{l}{[\mathrm{Al}]} \\
\left(\mu \mathrm{g} \mathrm{L}^{-1}\right)\end{array}$ & $\begin{array}{l}\text { Al Norm. } \\
\text { Diss. Rate } \\
\left(\mathrm{g} \mathrm{m}^{-2} \mathrm{~d}^{-1}\right)\end{array}$ & $\begin{array}{l}{[B]} \\
\left(\mu g L^{-1}\right)\end{array}$ & $\begin{array}{l}\text { B Norm. } \\
\text { Diss. Rate } \\
\left(\mathrm{g} \mathrm{m}^{-2} \mathrm{~d}^{-1}\right)\end{array}$ & $\begin{array}{l}{[\mathrm{Si}]} \\
\left(\mu \mathrm{g} \mathrm{L}^{-1}\right)\end{array}$ & $\begin{array}{l}\text { Si Norm. } \\
\text { Diss. Rate } \\
\left(\mathrm{g} \mathrm{m}^{-2} \mathrm{~d}^{-1}\right)\end{array}$ & $\begin{array}{l}{[\mathrm{Na}]} \\
(\mu \mathrm{gg} \mathrm{L} \\
\left.{ }^{1}\right)\end{array}$ & $\begin{array}{l}\text { Na Norm. } \\
\text { Diss. Rate, } \\
\mathrm{r} \\
\left(\mathrm{g} \mathrm{m}^{-2} \mathrm{~d}^{-1}\right)\end{array}$ & $\begin{array}{l}\mathrm{r}_{\mathrm{IEX}} \\
\left(\mathrm{mol} \mathrm{Na} \mathrm{No}^{-1}\right)\end{array}$ \\
\hline & & & & & & & & $56^{\star}$ & & & & & & \\
\hline LG9.23.12.80B.16 & & 3.72E-05 & 48 & 0.044 & $9.08 \mathrm{E}-04$ & 76 & $8.65 \mathrm{E}-02$ & $\begin{array}{l}{[75.6]} \\
50^{*}\end{array}$ & 1.17E-01 & 393 & $8.44 E-02$ & 712 & $1.87 \mathrm{E}-01$ & $3.53 \mathrm{E}-08$ \\
\hline LG9.23.12.80B.18 & & $3.31 \mathrm{E}-05$ & 55 & 0.044 & $8.99 E-04$ & 81 & $8.34 \mathrm{E}-02$ & $\begin{array}{l}{[75.6]} \\
60^{\star}\end{array}$ & $1.05 E-01$ & 394 & 7.61E-02 & 673 & $1.59 \mathrm{E}-01$ & $2.71 \mathrm{E}-08$ \\
\hline LG9.23.12.80B.20 & & 3.37E-05 & 62 & 0.043 & $8.90 E-04$ & 90 & $9.51 \mathrm{E}-02$ & $\begin{array}{l}{[75.6]} \\
53^{*}\end{array}$ & $1.08 \mathrm{E}-01$ & 396 & 7.86E-02 & 625 & $1.52 \mathrm{E}-01$ & 2.19E-08 \\
\hline LG9.23.12.80B.22 & & 3.95E-05 & 69 & 0.042 & $8.78 \mathrm{E}-04$ & 79 & $9.96 \mathrm{E}-02$ & $\begin{array}{l}{[75.6]} \\
44^{\star}\end{array}$ & $1.29 \mathrm{E}-01$ & 336 & $7.91 E-02$ & 613 & $1.77 \mathrm{E}-01$ & 2.43E-08 \\
\hline LG9.23.12.80B.24 & & 3.79E-05 & 76 & 0.041 & $8.68 \mathrm{E}-04$ & 77 & $9.41 \mathrm{E}-02$ & $\begin{array}{l}{[75.6]} \\
43^{*}\end{array}$ & $1.25 \mathrm{E}-01$ & 328 & $7.51 \mathrm{E}-02$ & 631 & $1.77 \mathrm{E}-01$ & 2.62E-08 \\
\hline LG9.23.12.80B.25 & & $3.92 \mathrm{E}-05$ & 79 & 0.041 & $8.64 \mathrm{E}-04$ & 77 & $9.74 \mathrm{E}-02$ & $\begin{array}{l}{[75.6]} \\
46^{\star}\end{array}$ & $1.30 \mathrm{E}-01$ & 391 & $9.29 \mathrm{E}-02$ & 600 & $1.75 \mathrm{E}-01$ & $2.26 \mathrm{E}-08$ \\
\hline LG9.23.12.80B.26 & & 3.78E-05 & 83 & 0.041 & $8.57 E-04$ & 72 & $8.86 \mathrm{E}-02$ & $\begin{array}{l}{[75.6]} \\
52^{\star}\end{array}$ & $1.26 \mathrm{E}-01$ & 353 & $8.16 \mathrm{E}-02$ & 530 & $1.50 \mathrm{E}-01$ & $1.20 \mathrm{E}-08$ \\
\hline \multicolumn{15}{|c|}{ LG9 $40^{\circ} \mathrm{C} \mathrm{pH} 9$ Reactor $\# 1$} \\
\hline LG9.40.9.10.Blank1 & & $8.76 \mathrm{E}-06$ & --- & --- & --- & [49.4] & --- & [75.6] & --- & [164] & --- & [134] & --- & --- \\
\hline LG9.40.9.10.Blank2 & & $1.09 \mathrm{E}-05$ & --- & --- & --- & [49.4] & --- & {$[75.6]$} & --- & [164] & --- & [134] & --- & -- \\
\hline LG9.40.9.10.Blank3 & & $1.09 E-05$ & --- & --- & --- & [49.4] & --- & [75.6] & --- & [164] & --- & [134] & --- & --- \\
\hline LG9.40.9.10.2 & & $1.18 \mathrm{E}-05$ & 3.8 & 4.000 & $7.87 \mathrm{E}-02$ & 261 & $1.10 \mathrm{E}-03$ & 901 & $5.12 \mathrm{E}-03$ & 2,000 & $1.57 \mathrm{E}-03$ & 14,000 & $1.35 \mathrm{E}-02$ & $4.22 \mathrm{E}-09$ \\
\hline LG9.40.9.10.4 & & 7.64E-06 & 8.7 & 3.998 & 7.87E-02 & 388 & $1.05 \mathrm{E}-03$ & 1,120 & $4.12 \mathrm{E}-03$ & 2,720 & $1.38 \mathrm{E}-03$ & 15,100 & $9.42 \mathrm{E}-03$ & 2.67E-09 \\
\hline LG9.40.9.10.6 & & 7.73E-06 & 13.7 & 3.996 & $7.87 \mathrm{E}-02$ & 430 & $1.18 \mathrm{E}-03$ & 1,170 & $4.35 \mathrm{E}-03$ & 2,800 & $1.44 \mathrm{E}-03$ & 13,900 & $8.77 \mathrm{E}-03$ & $2.22 \mathrm{E}-09$ \\
\hline LG9.40.9.10.8 & & 7.96E-06 & 17.9 & 3.995 & $7.86 \mathrm{E}-02$ & 469 & $1.33 \mathrm{E}-03$ & 1,190 & 4.55E-03 & 2,670 & $1.42 \mathrm{E}-03$ & 12,300 & $7.99 \mathrm{E}-03$ & 1.73E-09 \\
\hline LG9.40.9.10.10 & & $7.91 \mathrm{E}-06$ & 23.6 & 3.993 & $7.86 \mathrm{E}-02$ & 479 & $1.35 \mathrm{E}-03$ & 1,120 & $4.26 \mathrm{E}-03$ & 4,640 & $2.45 \mathrm{E}-03$ & 10,300 & $6.65 \mathrm{E}-03$ & $1.20 \mathrm{E}-09$ \\
\hline LG9.40.9.10.12 & & $8.01 \mathrm{E}-06$ & 27.8 & 3.992 & $7.86 \mathrm{E}-02$ & 468 & $1.33 \mathrm{E}-03$ & 1,050 & 4.05E-03 & 2,470 & $1.32 \mathrm{E}-03$ & 9,650 & $6.32 \mathrm{E}-03$ & $1.14 \mathrm{E}-09$ \\
\hline LG9.40.9.10.14 & & 7.22E-06 & 31.9 & 3.991 & 7.86E-02 & 471 & $1.21 \mathrm{E}-03$ & 1,000 & 3.47E-03 & 2,420 & $1.16 \mathrm{E}-03$ & 8,690 & $5.12 \mathrm{E}-03$ & $8.30 \mathrm{E}-10$ \\
\hline LG9.40.9.10.15 & & $8.10 \mathrm{E}-06$ & 34.6 & 3.990 & $7.86 \mathrm{E}-02$ & 459 & $1.32 \mathrm{E}-03$ & 958 & $3.74 \mathrm{E}-03$ & 2,360 & $1.27 \mathrm{E}-03$ & 8,220 & $5.44 \mathrm{E}-03$ & $8.57 \mathrm{E}-10$ \\
\hline LG9.40.9.10.16 & & $7.83 \mathrm{E}-06$ & 36.6 & 3.989 & $7.86 \mathrm{E}-02$ & 453 & $1.26 \mathrm{E}-03$ & 952 & $3.59 \mathrm{E}-03$ & 2,360 & $1.23 \mathrm{E}-03$ & 7,980 & $5.10 \mathrm{E}-03$ & $7.63 \mathrm{E}-10$ \\
\hline \multicolumn{15}{|c|}{ LG9 $40^{\circ} \mathrm{C} \mathrm{pH} 9$ Reactor $\# 2$} \\
\hline LG9.40.9.20.Blank1 & & $2.11 \mathrm{E}-05$ & --- & --- & --- & [49.4] & --- & {$[75.6]$} & --- & [164] & --- & [134] & --- & --- \\
\hline LG9.40.9.20.Blank2 & & $1.90 \mathrm{E}-05$ & --- & --- & --- & [49.4] & --- & {$[75.6]$} & --- & [164] & --- & [134] & --- & --- \\
\hline LG9.40.9.20.Blank3 & & $1.65 \mathrm{E}-05$ & --- & --- & --- & [49.4] & --- & {$[75.6]$} & --- & [164] & --- & [134] & --- & --- \\
\hline LG9.40.9.20.2 & & $2.33 \mathrm{E}-05$ & 3.8 & 1.505 & $2.96 \mathrm{E}-02$ & 114 & $2.50 \mathrm{E}-03$ & 266 & $7.91 \mathrm{E}-03$ & 675 & $2.78 \mathrm{E}-03$ & 4,170 & $2.10 \mathrm{E}-02$ & $6.61 \mathrm{E}-09$ \\
\hline LG9.40.9.20.4 & & $1.88 \mathrm{E}-05$ & 8.7 & 1.504 & $2.96 \mathrm{E}-02$ & 161 & $2.86 \mathrm{E}-03$ & 265 & $6.36 \mathrm{E}-03$ & 878 & $2.92 E-03$ & 3,250 & $1.32 \mathrm{E}-02$ & $3.46 E-09$ \\
\hline LG9.40.9.20.6 & & $1.88 \mathrm{E}-05$ & 13.7 & 1.503 & $2.96 \mathrm{E}-02$ & 165 & $2.92 \mathrm{E}-03$ & 213 & $5.11 \mathrm{E}-03$ & 839 & $2.79 \mathrm{E}-03$ & 2,530 & $1.03 E-02$ & 2.61E-09 \\
\hline LG9.40.9.20.8 & & $1.93 E-05$ & 17.9 & 1.502 & $2.96 \mathrm{E}-02$ & 170 & $3.10 \mathrm{E}-03$ & 186 & $4.59 \mathrm{E}-03$ & 730 & $2.49 E-03$ & 1,960 & $8.20 \mathrm{E}-03$ & $1.82 E-09$ \\
\hline LG9.40.9.20.10 & & $1.87 \mathrm{E}-05$ & 23.6 & 1.502 & $2.96 \mathrm{E}-02$ & 169 & $2.99 \mathrm{E}-03$ & 168 & $4.02 \mathrm{E}-03$ & 783 & $2.59 \mathrm{E}-03$ & 1,750 & $7.10 \mathrm{E}-03$ & $1.55 \mathrm{E}-09$ \\
\hline LG9.40.9.20.12 & & 1.92E-05 & 27.8 & 1.501 & $2.96 \mathrm{E}-02$ & 160 & $2.90 \mathrm{E}-03$ & 139 & $3.41 \mathrm{E}-03$ & 728 & $2.47 E-03$ & 1,520 & $6.32 \mathrm{E}-03$ & 1.47E-09 \\
\hline LG9.40.9.20.14 & & $1.91 \mathrm{E}-05$ & 31.9 & 1.501 & $2.96 \mathrm{E}-02$ & 166 & $3.00 \mathrm{E}-03$ & 140 & $3.42 \mathrm{E}-03$ & 792 & $2.68 \mathrm{E}-03$ & 1,430 & $5.93 \mathrm{E}-03$ & $1.26 \mathrm{E}-09$ \\
\hline LG9.40.9.20.15 & & $1.92 E-05$ & 34.6 & 1.501 & $2.96 \mathrm{E}-02$ & 167 & $3.04 \mathrm{E}-03$ & 147 & 3.62E-03 & 749 & $2.56 \mathrm{E}-03$ & 1,360 & $5.68 \mathrm{E}-03$ & 1.04E-09 \\
\hline LG9.40.9.20.16 & & $1.95 \mathrm{E}-05$ & 36.6 & 1.500 & $2.96 \mathrm{E}-02$ & 166 & $3.07 \mathrm{E}-03$ & 133 & 3.33E-03 & 759 & $2.63 \mathrm{E}-03$ & 1,310 & $5.56 \mathrm{E}-03$ & $1.12 \mathrm{E}-09$ \\
\hline \multicolumn{15}{|c|}{ LG9 $40^{\circ} \mathrm{C} \mathrm{pH} 9$ Reactor $\# 3$} \\
\hline LG9.40.9.40.Blank1 & & 4.42E-05 & --- & --- & --- & [49.4] & --- & [75.6] & --- & [164] & -.- & [134] & --- & -.- \\
\hline LG9.40.9.40.Blank2 & & 3.60E-05 & --- & --- & --- & [49.4] & --- & [75.6] & --- & [164] & --- & [134] & --- & --- \\
\hline LG9.40.9.40.Blank3 & & 3.99E-05 & --. & --- & --- & [49.4] & --- & [75.6] & --- & [164] & --- & [134] & --- & --- \\
\hline
\end{tabular}




\begin{tabular}{|c|c|c|c|c|c|c|c|c|c|c|c|c|c|c|}
\hline Sample ID & $\begin{array}{l}\text { Influent } \\
\text { [Si] } \\
\text { (ppm) }\end{array}$ & $\begin{array}{l}\text { Flow } \\
\text { Rate, } q \\
\left(\mathrm{~m}^{3} \mathrm{~d}^{-1}\right)\end{array}$ & $\begin{array}{l}\text { Time } \\
\text { (d) }\end{array}$ & $\begin{array}{l}\text { Glass } \\
\text { Mass } \\
\text { (g) }\end{array}$ & $\begin{array}{l}\text { Surface } \\
\text { Area, S } \\
\left(\mathrm{m}^{2}\right)\end{array}$ & $\begin{array}{l}{[\mathrm{Al}]} \\
\left(\mu \mathrm{L} \mathrm{L}^{-1}\right)\end{array}$ & $\begin{array}{l}\text { Al Norm. } \\
\text { Diss. Rate } \\
\left(\mathrm{g} \mathrm{m}^{-2} \mathrm{~d}^{-1}\right)\end{array}$ & $\begin{array}{l}{[\mathrm{B}]} \\
\left(\mu \mathrm{g} \mathrm{L}^{-1}\right)\end{array}$ & $\begin{array}{l}\text { B Norm. } \\
\text { Diss. Rate } \\
\left(\mathrm{g} \mathrm{m}^{-2} \mathrm{~d}^{-1}\right)\end{array}$ & $\begin{array}{l}{[\mathrm{Si}]} \\
\left(\mu \mathrm{g} \mathrm{L}^{-1}\right)\end{array}$ & $\begin{array}{l}\text { Si Norm. } \\
\text { Diss. Rate } \\
\left(\mathrm{g} \mathrm{m}^{-2} \mathrm{~d}^{-1}\right)\end{array}$ & $\begin{array}{l}{[\mathrm{Na}]} \\
(\mu \mathrm{g} \mathrm{L} \\
\left.{ }^{1}\right)\end{array}$ & $\begin{array}{l}\text { Na Norm. } \\
\text { Diss. Rate, } \\
\mathrm{r} \\
\left(\mathrm{g} \mathrm{m}^{-2} \mathrm{~d}^{-1}\right)\end{array}$ & $\begin{array}{l}\mathrm{r}_{\mathrm{IEX}} \\
\left(\mathrm{mol} \mathrm{Na} \mathrm{No}^{-1}\right)\end{array}$ \\
\hline LG9.40.9.40.2 & & $4.69 \mathrm{E}-05$ & 3.8 & 0.750 & $1.48 \mathrm{E}-02$ & {$[49.4]$} & $4.39 \mathrm{E}-03$ & {$[75.6]$} & $9.09 \mathrm{E}-03$ & [164] & $2.73 \mathrm{E}-03$ & 550 & $1.12 \mathrm{E}-02$ & $1.07 E-09$ \\
\hline LG9.40.9.40.4 & & $3.79 \mathrm{E}-05$ & 8.7 & 0.749 & $1.47 \mathrm{E}-02$ & 69 & $4.95 \mathrm{E}-03$ & 98 & $9.52 \mathrm{E}-03$ & 286 & $3.86 \mathrm{E}-03$ & 910 & $1.50 \mathrm{E}-02$ & $2.77 \mathrm{E}-09$ \\
\hline LG9.40.9.40.6 & & $3.41 \mathrm{E}-05$ & 13.7 & 0.749 & $1.47 \mathrm{E}-02$ & 68 & $4.40 \mathrm{E}-03$ & 91 & $7.98 \mathrm{E}-03$ & 273 & $3.31 \mathrm{E}-03$ & 770 & $1.14 \mathrm{E}-02$ & $1.75 \mathrm{E}-09$ \\
\hline LG9.40.9.40.8 & & $3.87 \mathrm{E}-05$ & 17.9 & 0.748 & $1.47 \mathrm{E}-02$ & 73 & $5.34 \mathrm{E}-03$ & 85 & $8.47 \mathrm{E}-03$ & 218 & $3.00 \mathrm{E}-03$ & 622 & $1.05 \mathrm{E}-02$ & $1.02 \mathrm{E}-09$ \\
\hline LG9.40.9.40.10 & & $3.79 \mathrm{E}-05$ & 23.6 & 0.747 & $1.47 \mathrm{E}-02$ & 69 & $5.00 \mathrm{E}-03$ & 86 & $8.37 \mathrm{E}-03$ & 231 & $3.12 \mathrm{E}-03$ & 578 & $9.56 \mathrm{E}-03$ & $6.02 \mathrm{E}-10$ \\
\hline LG9.40.9.40.12 & & $3.77 \mathrm{E}-05$ & 27.8 & 0.747 & $1.47 \mathrm{E}-02$ & 66 & $4.73 \mathrm{E}-03$ & 78 & $7.52 \mathrm{E}-03$ & 219 & $2.94 \mathrm{E}-03$ & 504 & $8.29 \mathrm{E}-03$ & $3.86 \mathrm{E}-10$ \\
\hline LG9.40.9.40.14 & & $3.75 \mathrm{E}-05$ & 31.9 & 0.746 & $1.47 \mathrm{E}-02$ & 64 & $4.55 \mathrm{E}-03$ & $\begin{array}{l}{[75.6]} \\
67^{*}\end{array}$ & 7.30E-03 & 228 & 3.05E-03 & 482 & 7.89E-03 & $3.00 E-10$ \\
\hline LG9.40.9.40.15 & & $3.68 \mathrm{E}-05$ & 34.6 & 0.746 & $1.47 E-02$ & 68 & $4.78 \mathrm{E}-03$ & $\begin{array}{l}{[75.6]} \\
74^{\star}\end{array}$ & 7.16E-03 & 197 & $2.59 \mathrm{E}-03$ & 465 & 7.48E-03 & $1.57 \mathrm{E}-10$ \\
\hline LG9.40.9.40.16 & & $3.86 \mathrm{E}-05$ & 36.6 & 0.746 & $1.47 \mathrm{E}-02$ & 65 & $4.79 \mathrm{E}-03$ & 77 & $7.67 \mathrm{E}-03$ & 227 & $3.12 \mathrm{E}-03$ & 449 & $7.56 \mathrm{E}-03$ & $<0$ \\
\hline \multicolumn{15}{|c|}{ LG9 $40^{\circ} \mathrm{C} \mathrm{pH} 9$ Reactor $\# 4$} \\
\hline LG9.40.9.80A.Blank1 & & $8.48 \mathrm{E}-05$ & --- & --- & --- & [49.4] & --- & [75.6] & --- & [164] & --- & [134] & --- & --- \\
\hline LG9.40.9.80A.Blank2 & & 7.97E-05 & --- & --- & --- & [49.4] & -.- & [75.6] & --- & [164] & --- & [134] & --- & -.- \\
\hline LG9.40.9.80A.Blank3 & & $7.28 \mathrm{E}-05$ & --- & --- & --- & {$[49.4]$} & --- & [75.6] & --- & [164] & --- & [134] & --- & --- \\
\hline LG9.40.9.80A.2 & & $5.17 \mathrm{E}-05$ & 3.8 & 0.299 & $5.90 \mathrm{E}-03$ & $\begin{array}{l}{[49.4]} \\
28^{\star}\end{array}$ & $1.21 \mathrm{E}-02$ & $\begin{array}{l}{[75.6]} \\
59^{\star}\end{array}$ & $2.51 \mathrm{E}-02$ & $\begin{array}{l}{[164]} \\
66^{\star}\end{array}$ & $7.53 \mathrm{E}-03$ & 544 & $3.06 \mathrm{E}-02$ & $2.79 \mathrm{E}-09$ \\
\hline LG9.40.9.80A.4 & & 7.59E-05 & 8.7 & 0.298 & $5.88 \mathrm{E}-03$ & {$[49.4]$} & $1.78 \mathrm{E}-02$ & $\begin{array}{l}{[75.6]} \\
40^{*}\end{array}$ & 3.69E-02 & [164] & $1.11 \mathrm{E}-02$ & 227 & $1.88 \mathrm{E}-02$ & $<0$ \\
\hline LG9.40.9.80A.6 & & $7.66 \mathrm{E}-05$ & 13.7 & 0.297 & $5.86 \mathrm{E}-03$ & [49.4] & $1.80 \mathrm{E}-02$ & $\begin{array}{l}{[75.6]} \\
39^{\star}\end{array}$ & $3.74 \mathrm{E}-02$ & [164] & $1.12 \mathrm{E}-02$ & 237 & $1.99 \mathrm{E}-02$ & $<0$ \\
\hline LG9.40.9.80A.8 & & $7.53 \mathrm{E}-05$ & 17.9 & 0.296 & $5.85 \mathrm{E}-03$ & [49.4] & $1.77 \mathrm{E}-02$ & $\begin{array}{l}{[75.6]} \\
59^{\star}\end{array}$ & $3.68 \mathrm{E}-02$ & [164] & $1.10 \mathrm{E}-02$ & 206 & $1.70 \mathrm{E}-02$ & $<0$ \\
\hline LG9.40.9.80A.10 & & 7.64E-05 & 23.6 & 0.295 & $5.84 \mathrm{E}-03$ & {$[49.4]$} & $1.81 \mathrm{E}-02$ & $\begin{array}{l}{[75.6]} \\
55^{*}\end{array}$ & $3.74 \mathrm{E}-02$ & [164] & $1.12 \mathrm{E}-02$ & 197 & $1.66 \mathrm{E}-02$ & $<0$ \\
\hline LG9.40.9.80A.12 & & $7.68 \mathrm{E}-05$ & 27.8 & 0.294 & $5.82 E-03$ & {$[49.4]$} & $1.82 \mathrm{E}-02$ & $\begin{array}{l}{[75.6]} \\
42^{\star}\end{array}$ & $3.77 \mathrm{E}-02$ & [164] & $1.13 E-02$ & 144 & $1.22 \mathrm{E}-02$ & $<0$ \\
\hline LG9.40.9.80A.14 & & 7.66E-05 & 31.9 & 0.293 & $5.81 \mathrm{E}-03$ & [49.4] & $1.82 \mathrm{E}-02$ & $\begin{array}{l}{[75.6]} \\
44^{\star}\end{array}$ & 3.77E-02 & [164] & 1.13E-02 & 184 & $1.56 \mathrm{E}-02$ & $<0$ \\
\hline LG9.40.9.80A.15 & & $7.70 \mathrm{E}-05$ & 34.6 & 0.293 & $5.80 \mathrm{E}-03$ & [49.4] & $1.83 \mathrm{E}-02$ & $\begin{array}{l}{[75.6]} \\
38^{\star}\end{array}$ & $3.79 \mathrm{E}-02$ & [164] & $1.14 \mathrm{E}-02$ & 138 & $1.17 \mathrm{E}-02$ & $<0$ \\
\hline LG9.40.9.80A.16 & & 7.48E-05 & 36.6 & 0.292 & $5.80 \mathrm{E}-03$ & [49.4] & $1.78 \mathrm{E}-02$ & $\begin{array}{l}{[75.6]} \\
35^{*}\end{array}$ & 3.69E-02 & [164] & $1.11 \mathrm{E}-02$ & 194 & $1.61 \mathrm{E}-02$ & $<0$ \\
\hline \multicolumn{15}{|c|}{ LG9 $40^{\circ} \mathrm{C} \mathrm{pH} 9$ Reactor \#5 } \\
\hline LG9.40.9.80B.Blank1 & & $9.88 \mathrm{E}-05$ & --- & --- & --- & [49.4] & --- & [75.6] & --- & [164] & --- & [134] & --- & --- \\
\hline LG9.40.9.80B.Blank2 & & $7.90 \mathrm{E}-05$ & --- & --- & --- & [49.4] & --. & [75.6] & --- & [164] & --- & {$[134]$} & --- & -.- \\
\hline LG9.40.9.80B.Blank3 & & $8.37 \mathrm{E}-05$ & --- & --- & --- & [49.4] & --- & [75.6] & --- & [164] & --- & [134] & --- & --- \\
\hline LG9.40.9.80B.2 & & $1.35 \mathrm{E}-04$ & 3.8 & 0.056 & $1.12 \mathrm{E}-03$ & [49.4] & $1.67 \mathrm{E}-01$ & [75.6] & $3.46 \mathrm{E}-01$ & [164] & $1.04 \mathrm{E}-01$ & $\begin{array}{l}{[134} \\
\left.66^{\star}\right]\end{array}$ & $1.04 \mathrm{E}-01$ & $<0$ \\
\hline LG9.40.9.80B.4 & & $7.79 \mathrm{E}-05$ & 8.7 & 0.055 & $1.11 \mathrm{E}-03$ & [49.4] & $9.72 \mathrm{E}-02$ & $\begin{array}{l}{[75.6]} \\
44^{\star}\end{array}$ & $2.01 \mathrm{E}-01$ & [164] & $6.05 E-02$ & $\begin{array}{l}{[134]} \\
85^{\star}\end{array}$ & $6.05 \mathrm{E}-02$ & $<0$ \\
\hline LG9.40.9.80B.6 & & $7.71 \mathrm{E}-05$ & 13.7 & 0.054 & $1.09 \mathrm{E}-03$ & {$[49.4]$} & $9.77 \mathrm{E}-02$ & {$[75.6]$} & $2.02 \mathrm{E}-01$ & [164] & $6.08 \mathrm{E}-02$ & {$[134]$} & $6.08 \mathrm{E}-02$ & $<0$ \\
\hline LG9.40.9.80B.8 & & $7.86 \mathrm{E}-05$ & 17.9 & 0.053 & $1.08 \mathrm{E}-03$ & [49.4] & $1.01 \mathrm{E}-01$ & [75.6] & $2.08 \mathrm{E}-01$ & [164] & $6.26 \mathrm{E}-02$ & $\begin{array}{l}{[134]} \\
106^{*}\end{array}$ & $6.27 \mathrm{E}-02$ & $<0$ \\
\hline LG9.40.9.80B.10 & & 7.73E-05 & 23.6 & 0.052 & $1.06 \mathrm{E}-03$ & [49.4] & $1.01 \mathrm{E}-01$ & {$[75.6]$} & 2.09E-01 & [164] & $6.27 \mathrm{E}-02$ & $\begin{array}{l}{[134]} \\
62^{\star}\end{array}$ & $6.28 \mathrm{E}-02$ & $<0$ \\
\hline
\end{tabular}

B.10 


\begin{tabular}{|c|c|c|c|c|c|c|c|c|c|c|c|c|c|c|}
\hline Sample ID & $\begin{array}{l}\text { Influent } \\
\text { [Si] } \\
\text { (ppm) }\end{array}$ & $\begin{array}{l}\text { Flow } \\
\text { Rate, } \mathrm{q} \\
\left(\mathrm{m}^{3} \mathrm{~d}^{-1}\right)\end{array}$ & $\begin{array}{l}\text { Time } \\
\text { (d) }\end{array}$ & $\begin{array}{l}\text { Glass } \\
\text { Mass } \\
\text { (g) }\end{array}$ & $\begin{array}{l}\text { Surface } \\
\text { Area, } \mathrm{S} \\
\left(\mathrm{m}^{2}\right)\end{array}$ & $\begin{array}{l}{[\mathrm{Al}]} \\
\left(\mu \mathrm{L} \mathrm{L}^{-1}\right)\end{array}$ & $\begin{array}{l}\text { Al Norm. } \\
\text { Diss. Rate } \\
\left(\mathrm{g} \mathrm{m}^{-2} \mathrm{~d}^{-1}\right)\end{array}$ & $\begin{array}{l}{[\mathrm{B}]} \\
\left(\mu \mathrm{g} \mathrm{L}^{-1}\right)\end{array}$ & $\begin{array}{l}\text { B Norm. } \\
\text { Diss. Rate } \\
\left(\mathrm{g} \mathrm{m}^{-2} \mathrm{~d}^{-1}\right)\end{array}$ & $\begin{array}{l}{[\mathrm{Si}]} \\
\left(\mu \mathrm{L} \mathrm{L}^{-1}\right)\end{array}$ & $\begin{array}{l}\text { Si Norm. } \\
\text { Diss. Rate } \\
\left(\mathrm{g} \mathrm{m}^{-2} \mathrm{~d}^{-1}\right)\end{array}$ & $\begin{array}{l}{[\mathrm{Na}]} \\
\left(\mu \mathrm{gg} \mathrm{L}^{-}\right. \\
\left.{ }^{1}\right)\end{array}$ & $\begin{array}{l}\text { Na Norm. } \\
\text { Diss. Rate, } \\
\mathrm{r} \\
\left(\mathrm{g} \mathrm{m}^{-2} \mathrm{~d}^{-1}\right)\end{array}$ & $\begin{array}{l}\mathrm{r}_{\mathrm{IEX}} \\
\left(\mathrm{mol} \mathrm{Na} \mathrm{No}^{-1}\right)\end{array}$ \\
\hline LG9.40.9.80B.12 & & $7.69 \mathrm{E}-05$ & 27.8 & 0.051 & $1.05 \mathrm{E}-03$ & [49.4] & $1.01 \mathrm{E}-01$ & [75.6] & $2.10 \mathrm{E}-01$ & [164] & $6.31 \mathrm{E}-02$ & $\begin{array}{l}{[134]} \\
84^{*}\end{array}$ & $6.32 \mathrm{E}-02$ & $<0$ \\
\hline LG9.40.9.80B.14 & & 7.70E-05 & 31.9 & 0.050 & $1.04 \mathrm{E}-03$ & [49.4] & 1.03E-01 & {$[75.6]$} & $2.13 E-01$ & [164] & $6.39 \mathrm{E}-02$ & $\begin{array}{l}{[134]} \\
56^{*}\end{array}$ & $6.39 \mathrm{E}-02$ & $<0$ \\
\hline LG9.40.9.80B.15 & & $7.72 \mathrm{E}-05$ & 34.6 & 0.049 & 1.03E-03 & [49.4] & 1.04E-01 & $\begin{array}{l}{[75.6]} \\
36^{\star}\end{array}$ & $2.15 \mathrm{E}-01$ & [164] & $6.46 \mathrm{E}-02$ & $\begin{array}{l}{[134]} \\
69^{\star}\end{array}$ & 6.46E-02 & $<0$ \\
\hline LG9.40.9.80B.16 & & $7.82 \mathrm{E}-05$ & 36.6 & 0.049 & $1.02 \mathrm{E}-03$ & [49.4] & $1.06 \mathrm{E}-01$ & {$[75.6]$} & $2.19 \mathrm{E}-01$ & [164] & $6.58 \mathrm{E}-02$ & $\begin{array}{l}{[134]} \\
69^{\star}\end{array}$ & $6.58 \mathrm{E}-02$ & $<0$ \\
\hline \multicolumn{15}{|c|}{ LG9 $40^{\circ} \mathrm{C} \mathrm{pH} 10$ Reactor $\# 1$} \\
\hline LG9.40.10.10.Blank1 & & $8.59 \mathrm{E}-06$ & --- & -- & --- & {$[49.4]$} & -- & [75.6] & --- & [164] & --- & [134] & --- & --- \\
\hline LG9.40.10.10.Blank2 & & $8.51 \mathrm{E}-06$ & --- & --- & --- & [49.4] & --- & [75.6] & --- & [164] & --- & [134] & --- & --- \\
\hline LG9.40.10.10.Blank3 & & $7.72 \mathrm{E}-06$ & -.- & --- & --- & [49.4] & --- & [75.6] & --- & [164] & --- & [134] & --- & -.- \\
\hline LG9.40.10.10.2 & & $8.10 \mathrm{E}-06$ & 4 & 3.999 & 7.87E-02 & 180 & $5.18 \mathrm{E}-04$ & 263 & $1.02 \mathrm{E}-03$ & 1,120 & $6.04 \mathrm{E}-04$ & 6,150 & $4.06 \mathrm{E}-03$ & $1.53 \mathrm{E}-09$ \\
\hline LG9.40.10.10.4 & & $8.36 \mathrm{E}-06$ & 9 & 3.998 & 7.87E-02 & 734 & $2.18 \mathrm{E}-03$ & 757 & $3.04 \mathrm{E}-03$ & 4,840 & $2.69 \mathrm{E}-03$ & 11,300 & $7.70 \mathrm{E}-03$ & $2.35 \mathrm{E}-09$ \\
\hline LG9.40.10.10.6 & & 8.05E-06 & 14 & 3.996 & 7.87E-02 & 850 & $2.43 \mathrm{E}-03$ & 796 & $3.08 \mathrm{E}-03$ & 5,320 & $2.85 \mathrm{E}-03$ & 11,300 & 7.43E-03 & 2.19E-09 \\
\hline LG9.40.10.10.8 & & $8.00 \mathrm{E}-06$ & 18 & 3.996 & 7.86E-02 & 893 & $2.54 \mathrm{E}-03$ & 761 & 2.93E-03 & 5,020 & $2.68 \mathrm{E}-03$ & 9,280 & $6.06 \mathrm{E}-03$ & $1.58 \mathrm{E}-09$ \\
\hline LG9.40.10.10.10 & & $8.02 \mathrm{E}-06$ & 24 & 3.994 & $7.86 \mathrm{E}-02$ & 885 & $2.52 \mathrm{E}-03$ & 724 & $2.80 \mathrm{E}-03$ & 4,790 & $2.56 \mathrm{E}-03$ & 8,250 & $5.40 \mathrm{E}-03$ & $1.31 \mathrm{E}-09$ \\
\hline LG9.40.10.10.12 & & 7.83E-06 & 28 & 3.993 & 7.86E-02 & 869 & $2.42 \mathrm{E}-03$ & 706 & $2.66 \mathrm{E}-03$ & 4,750 & $2.48 \mathrm{E}-03$ & 7,580 & $4.85 \mathrm{E}-03$ & $1.10 \mathrm{E}-09$ \\
\hline LG9.40.10.10.14 & & $7.60 \mathrm{E}-06$ & 32 & 3.993 & $7.86 \mathrm{E}-02$ & 869 & $2.35 \mathrm{E}-03$ & 682 & $2.49 \mathrm{E}-03$ & 4,590 & $2.33 \mathrm{E}-03$ & 7,030 & $4.36 \mathrm{E}-03$ & $9.40 \mathrm{E}-10$ \\
\hline LG9.40.10.10.16 & & 7.80E-06 & 37 & 3.992 & 7.86E-02 & 898 & $2.49 \mathrm{E}-03$ & 654 & $2.46 \mathrm{E}-03$ & 4,680 & $2.43 \mathrm{E}-03$ & 6,520 & 4.15E-03 & $8.55 \mathrm{E}-10$ \\
\hline \multicolumn{15}{|c|}{ LG9 $40^{\circ} \mathrm{C} \mathrm{pH} 10$ Reactor $\# 2$} \\
\hline LG9.40.10.20.Blank1 & & $1.85 \mathrm{E}-05$ & --. & -- & --- & [49.4] & --- & [75.6] & --- & [164] & --- & [134] & -- & --- \\
\hline LG9.40.10.20.Blank2 & & $1.93 \mathrm{E}-05$ & --- & --- & --- & [49.4] & --- & [75.6] & --- & [164] & --- & [134] & --- & --- \\
\hline LG9.40.10.20.Blank3 & & $1.91 \mathrm{E}-05$ & -- & -- & -- & {$[49.4]$} & -- & [75.6] & --- & [164] & --- & [134] & -- & --- \\
\hline LG9.40.10.20.2 & & $1.86 \mathrm{E}-05$ & 4 & 1.498 & $2.95 \mathrm{E}-02$ & 84 & $1.47 \mathrm{E}-03$ & 92 & 2.19E-03 & 378 & $1.25 \mathrm{E}-03$ & 1,700 & $6.87 \mathrm{E}-03$ & $2.36 \mathrm{E}-09$ \\
\hline LG9.40.10.20.4 & & $1.83 \mathrm{E}-05$ & 9 & 1.497 & $2.95 \mathrm{E}-02$ & 403 & $7.00 \mathrm{E}-03$ & 321 & $7.54 \mathrm{E}-03$ & 2,300 & $7.49 \mathrm{E}-03$ & 3,220 & $1.28 \mathrm{E}-02$ & $2.67 \mathrm{E}-09$ \\
\hline LG9.40.10.20.6 & & $1.81 \mathrm{E}-05$ & 14 & 1.495 & $2.94 \mathrm{E}-02$ & 451 & 7.77E-03 & 357 & $8.32 \mathrm{E}-03$ & 2,540 & $8.20 \mathrm{E}-03$ & 2,830 & $1.12 \mathrm{E}-02$ & $1.44 \mathrm{E}-09$ \\
\hline LG9.40.10.20.8 & & $1.72 \mathrm{E}-05$ & 18 & 1.495 & $2.94 \mathrm{E}-02$ & 461 & $7.53 \mathrm{E}-03$ & 311 & $6.88 \mathrm{E}-03$ & 2,350 & $7.20 \mathrm{E}-03$ & 2,440 & $9.16 \mathrm{E}-03$ & $1.15 \mathrm{E}-09$ \\
\hline LG9.40.10.20.10 & & $1.75 \mathrm{E}-05$ & 24 & 1.494 & $2.94 \mathrm{E}-02$ & 455 & 7.57E-03 & 299 & $6.74 \mathrm{E}-03$ & 2,310 & $7.21 \mathrm{E}-03$ & 2,210 & 8.45E-03 & $8.61 \mathrm{E}-10$ \\
\hline LG9.40.10.20.12 & & $1.79 \mathrm{E}-05$ & 28 & 1.493 & $2.94 \mathrm{E}-02$ & 432 & $7.34 \mathrm{E}-03$ & 262 & $6.03 \mathrm{E}-03$ & 2,170 & $6.91 \mathrm{E}-03$ & 2,010 & $7.84 \mathrm{E}-03$ & $9.15 \mathrm{E}-10$ \\
\hline LG9.40.10.20.14 & & $1.73 \mathrm{E}-05$ & 32 & 1.492 & $2.94 \mathrm{E}-02$ & 435 & $7.16 \mathrm{E}-03$ & 280 & $6.24 \mathrm{E}-03$ & 2,180 & $6.73 \mathrm{E}-03$ & 1,930 & 7.30E-03 & $5.32 \mathrm{E}-10$ \\
\hline LG9.40.10.20.16 & & $1.78 \mathrm{E}-05$ & 37 & 1.491 & 2.94E-02 & 453 & 7.66E-03 & 285 & $6.52 \mathrm{E}-03$ & 2,240 & 7.10E-03 & 1,930 & $7.50 \mathrm{E}-03$ & 4.89E-10 \\
\hline \multicolumn{15}{|c|}{ LG9 $40^{\circ} \mathrm{C} \mathrm{pH} 10$ Reactor $\# 3$} \\
\hline LG9.40.10.40.Blank1 & & $3.92 \mathrm{E}-05$ & -- & -- & -- & [49.4] & -- & {$[75.6]$} & -- & [164] & --- & [134] & --- & --- \\
\hline LG9.40.10.40.Blank2 & & $3.91 \mathrm{E}-05$ & --. & --- & --- & [49.4] & --. & [75.6] & --- & [164] & --- & [134] & --- & --. \\
\hline LG9.40.10.40.Blank3 & & 3.89E-05 & -- & --- & --- & [49.4] & --- & {$[75.6]$} & --- & [164] & --- & [134] & --- & --- \\
\hline LG9.40.10.40.2 & & $3.90 \mathrm{E}-05$ & 4 & 0.755 & 1.49E-02 & [49.4] & $3.62 \mathrm{E}-03$ & [75.6] & $7.51 \mathrm{E}-03$ & [164] & $2.26 \mathrm{E}-03$ & 567 & $9.55 \mathrm{E}-03$ & $1.03 \mathrm{E}-09$ \\
\hline LG9.40.10.40.4 & & 3.87E-05 & 9 & 0.754 & $1.48 \mathrm{E}-02$ & 246 & $1.79 \mathrm{E}-02$ & 181 & $1.78 \mathrm{E}-02$ & 1,300 & $1.77 \mathrm{E}-02$ & 1,240 & 2.07E-02 & $1.46 \mathrm{E}-09$ \\
\hline LG9.40.10.40.6 & & $3.92 \mathrm{E}-05$ & 14 & 0.752 & $1.48 \mathrm{E}-02$ & 239 & $1.76 \mathrm{E}-02$ & 189 & $1.89 \mathrm{E}-02$ & 1,280 & $1.77 \mathrm{E}-02$ & 1,120 & $1.90 \mathrm{E}-02$ & $5.06 \mathrm{E}-11$ \\
\hline LG9.40.10.40.8 & & 3.99E-05 & 18 & 0.752 & 1.48E-02 & 230 & 1.73E-02 & 147 & $1.50 \mathrm{E}-02$ & 1,070 & $1.51 \mathrm{E}-02$ & 1,000 & 1.73E-02 & $1.16 \mathrm{E}-09$ \\
\hline LG9.40.10.40.10 & & $3.94 \mathrm{E}-05$ & 24 & 0.750 & $1.48 \mathrm{E}-02$ & 219 & 1.63E-02 & 124 & $1.25 \mathrm{E}-02$ & 1,040 & $1.45 \mathrm{E}-02$ & 880 & $1.50 \mathrm{E}-02$ & $1.28 \mathrm{E}-09$ \\
\hline LG9.40.10.40.12 & & 3.87E-05 & 28 & 0.750 & $1.48 \mathrm{E}-02$ & 218 & $1.59 \mathrm{E}-02$ & 109 & $1.08 \mathrm{E}-02$ & 1,010 & $1.38 \mathrm{E}-02$ & 882 & $1.48 \mathrm{E}-02$ & $2.02 \mathrm{E}-09$ \\
\hline LG9.40.10.40.16 & & $3.84 \mathrm{E}-05$ & 37 & 0.749 & $1.48 \mathrm{E}-02$ & 247 & $1.79 \mathrm{E}-02$ & 132 & $1.30 \mathrm{E}-02$ & 1,130 & $1.54 \mathrm{E}-02$ & 943 & 1.57E-02 & $1.39 \mathrm{E}-09$ \\
\hline \multicolumn{15}{|c|}{ LG9 $40^{\circ} \mathrm{C} \mathrm{pH} 10$ Reactor $\# 4$} \\
\hline
\end{tabular}




\begin{tabular}{|c|c|c|c|c|c|c|c|c|c|c|c|c|c|c|}
\hline Sample ID & $\begin{array}{l}\text { Influent } \\
\text { [Si] } \\
\text { (ppm) }\end{array}$ & $\begin{array}{l}\text { Flow } \\
\text { Rate, } \mathrm{q} \\
\left(\mathrm{m}^{3} \mathrm{~d}^{-1}\right)\end{array}$ & $\begin{array}{l}\text { Time } \\
\text { (d) }\end{array}$ & $\begin{array}{l}\text { Glass } \\
\text { Mass } \\
\text { (g) }\end{array}$ & $\begin{array}{l}\text { Surface } \\
\text { Area, S } \\
\left(\mathrm{m}^{2}\right)\end{array}$ & $\begin{array}{l}{[\mathrm{Al}]} \\
\left(\mu \mathrm{g} \mathrm{L}^{-1}\right)\end{array}$ & $\begin{array}{l}\text { Al Norm. } \\
\text { Diss. Rate } \\
\left(\mathrm{g} \mathrm{m}^{-2} \mathrm{~d}^{-1}\right)\end{array}$ & $\begin{array}{l}{[\mathrm{B}]} \\
\left(\mu \mathrm{g} \mathrm{L}^{-1}\right)\end{array}$ & $\begin{array}{l}\text { B Norm. } \\
\text { Diss. Rate } \\
\left(\mathrm{g} \mathrm{m}^{-2} \mathrm{~d}^{-1}\right)\end{array}$ & $\begin{array}{l}{[\mathrm{Si}]} \\
\left(\mu \mathrm{g} \mathrm{L}^{-1}\right)\end{array}$ & $\begin{array}{l}\text { Si Norm. } \\
\text { Diss. Rate } \\
\left(\mathrm{g} \mathrm{m}^{-2} \mathrm{~d}^{-1}\right)\end{array}$ & $\begin{array}{l}{[\mathrm{Na}]} \\
(\mu \mathrm{g} \mathrm{L} \\
\left.{ }^{1}\right)\end{array}$ & $\begin{array}{l}\text { Na Norm. } \\
\text { Diss. Rate, } \\
\mathrm{r} \\
\left(\mathrm{g} \mathrm{m}^{-2} \mathrm{~d}^{-1}\right)\end{array}$ & $\begin{array}{l}\mathrm{r}_{\mathrm{IEX}} \\
\left(\mathrm{mol} \mathrm{Na} \mathrm{No}^{-1}\right)\end{array}$ \\
\hline LG9.40.10.80A.Blank1 & & $7.36 \mathrm{E}-05$ & --- & --- & --- & [49.4] & --- & {$[75.6]$} & --- & [164] & --- & [134] & --- & --- \\
\hline LG9.40.10.80A.Blank2 & & $7.53 \mathrm{E}-05$ & --- & --- & --- & [49.4] & --- & {$[75.6]$} & --- & [164] & --- & [134] & --- & --- \\
\hline LG9.40.10.80A.Blank3 & & $7.30 \mathrm{E}-05$ & --- & --- & --- & [49.4] & --- & {$[75.6]$} & --- & [164] & --- & [134] & --- & --- \\
\hline LG9.40.10.80A.2 & & $7.67 \mathrm{E}-05$ & 4 & 0.302 & $5.95 \mathrm{E}-03$ & [49.4] & $1.78 \mathrm{E}-02$ & [75.6] & $3.69 \mathrm{E}-02$ & [164] & $1.11 \mathrm{E}-02$ & [134] & $1.11 \mathrm{E}-02$ & $<0$ \\
\hline LG9.40.10.80A.4 & & $7.24 \mathrm{E}-05$ & 9 & 0.299 & $5.91 \mathrm{E}-03$ & 254 & $8.69 \mathrm{E}-02$ & 203 & $9.40 \mathrm{E}-02$ & 811 & $5.20 \mathrm{E}-02$ & 803 & $6.31 \mathrm{E}-02$ & $<0$ \\
\hline LG9.40.10.80A.6 & & $7.70 \mathrm{E}-05$ & 14 & 0.296 & $5.88 \mathrm{E}-03$ & 232 & $8.50 \mathrm{E}-02$ & 186 & $9.22 \mathrm{E}-02$ & 778 & $5.34 \mathrm{E}-02$ & 709 & $5.96 \mathrm{E}-02$ & $<0$ \\
\hline LG9.40.10.80A.8 & & $7.76 \mathrm{E}-05$ & 18 & 0.296 & $5.87 \mathrm{E}-03$ & 95 & 3.52E-02 & [75.6] & $3.78 \mathrm{E}-02$ & 363 & $2.51 \mathrm{E}-02$ & 342 & $2.90 \mathrm{E}-02$ & $<0$ \\
\hline LG9.40.10.80A.10 & & $7.63 \mathrm{E}-05$ & 24 & 0.295 & $5.85 \mathrm{E}-03$ & 97 & 3.54E-02 & [75.6] & $3.73 \mathrm{E}-02$ & 382 & $2.61 \mathrm{E}-02$ & 336 & $2.81 \mathrm{E}-02$ & $<0$ \\
\hline LG9.40.10.80A.12 & & $7.65 \mathrm{E}-05$ & 28 & 0.294 & $5.84 \mathrm{E}-03$ & 93 & $3.39 \mathrm{E}-02$ & [75.6] & $3.75 \mathrm{E}-02$ & 346 & $2.37 \mathrm{E}-02$ & 335 & $2.82 \mathrm{E}-02$ & $<0$ \\
\hline LG9.40.10.80A.14 & & $7.52 \mathrm{E}-05$ & 32 & 0.293 & $5.83 \mathrm{E}-03$ & 102 & $3.68 \mathrm{E}-02$ & [75.6] & $3.69 \mathrm{E}-02$ & 429 & $2.90 \mathrm{E}-02$ & 349 & $2.89 \mathrm{E}-02$ & $<0$ \\
\hline LG9.40.10.80A.16 & & $7.64 \mathrm{E}-05$ & 37 & 0.292 & $5.82 \mathrm{E}-03$ & 107 & 3.93E-02 & [75.6] & $3.76 \mathrm{E}-02$ & 436 & $3.00 \mathrm{E}-02$ & 396 & $3.34 \mathrm{E}-02$ & $<0$ \\
\hline \multicolumn{15}{|c|}{ LG9 $40^{\circ} \mathrm{C} \mathrm{pH} 10$ Reactor $\# 5$} \\
\hline LG9.40.10.80B.Blank1 & & $7.88 \mathrm{E}-05$ & --. & --- & --- & [49.4] & --- & [75.6] & --- & [164] & --- & [134] & --- & --- \\
\hline LG9.40.10.80B.Blank2 & & $7.98 \mathrm{E}-05$ & --- & --- & --- & [49.4] & --- & {$[75.6]$} & --- & [164] & --- & [134] & --- & --- \\
\hline LG9.40.10.80B.Blank3 & & $7.90 \mathrm{E}-05$ & --- & --- & --- & [49.4] & --- & [75.6] & --- & [164] & --- & [134] & --- & --- \\
\hline LG9.40.10.80B.2 & & $7.76 \mathrm{E}-05$ & 4 & 0.060 & $1.20 \mathrm{E}-03$ & [49.4] & $8.96 \mathrm{E}-02$ & {$[75.6]$} & $1.86 \mathrm{E}-01$ & [164] & $5.58 \mathrm{E}-02$ & [134] & $5.58 \mathrm{E}-02$ & $<0$ \\
\hline LG9.40.10.80B.4 & & 7.77E-05 & 9 & 0.059 & $1.18 \mathrm{E}-03$ & $\begin{array}{l}{[49.4]} \\
28^{*}\end{array}$ & $9.08 \mathrm{E}-02$ & {$[75.6]$} & $1.88 \mathrm{E}-01$ & $\begin{array}{l}{[164]} \\
72^{\star}\end{array}$ & $5.65 E-02$ & $\begin{array}{l}{[134]} \\
121^{*}\end{array}$ & $5.66 \mathrm{E}-02$ & $<0$ \\
\hline LG9.40.10.80B.6 & & 7.59E-05 & 14 & 0.058 & $1.17 E-03$ & $\begin{array}{l}{[49.4]} \\
31^{*}\end{array}$ & 8.99E-02 & {$[75.6]$} & $1.86 \mathrm{E}-01$ & $\begin{array}{l}{[164]} \\
69^{\star}\end{array}$ & $5.60 E-02$ & $\begin{array}{l}{[134]} \\
117^{*}\end{array}$ & $5.60 \mathrm{E}-02$ & $<0$ \\
\hline LG9.40.10.80B.8 & & $7.76 \mathrm{E}-05$ & 18 & 0.057 & $1.15 \mathrm{E}-03$ & $\begin{array}{l}{[49.4]} \\
30^{*}\end{array}$ & $9.28 \mathrm{E}-02$ & [75.6] & $1.92 \mathrm{E}-01$ & [164] & $5.78 \mathrm{E}-02$ & $\begin{array}{l}{[134]} \\
105^{*}\end{array}$ & $5.78 \mathrm{E}-02$ & $<0$ \\
\hline LG9.40.10.80B.10 & & 7.64E-05 & 24 & 0.056 & $1.14 \mathrm{E}-03$ & $\begin{array}{l}{[49.4]} \\
32^{*}\end{array}$ & $9.28 \mathrm{E}-02$ & {$[75.6]$} & $1.92 \mathrm{E}-01$ & $\begin{array}{l}{[164]} \\
51^{\star}\end{array}$ & $5.78 \mathrm{E}-02$ & $\begin{array}{l}{[134]} \\
111^{*}\end{array}$ & $5.78 \mathrm{E}-02$ & $<0$ \\
\hline LG9.40.10.80B.12 & & 7.65E-05 & 28 & 0.055 & $1.12 \mathrm{E}-03$ & [49.4] & $9.40 \mathrm{E}-02$ & {$[75.6]$} & $1.95 \mathrm{E}-01$ & [164] & $5.85 E-02$ & $\begin{array}{l}{[134]} \\
47^{\star}\end{array}$ & $5.85 \mathrm{E}-02$ & $<0$ \\
\hline LG9.40.10.80B.14 & & 7.74E-05 & 32 & 0.054 & $1.11 \mathrm{E}-03$ & $\begin{array}{l}{[49.4]} \\
38^{*}\end{array}$ & $9.61 \mathrm{E}-02$ & {$[75.6]$} & 1.99E-01 & [164] & $5.98 E-02$ & $\begin{array}{l}{[134]} \\
68^{*}\end{array}$ & $5.99 \mathrm{E}-02$ & $<0$ \\
\hline LG9.40.10.80B.16 & & $7.66 \mathrm{E}-05$ & 37 & 0.053 & $1.10 \mathrm{E}-03$ & $\begin{array}{l}{[49.4]} \\
39^{*}\end{array}$ & $9.62 \mathrm{E}-02$ & [75.6] & $1.99 \mathrm{E}-01$ & [164] & $5.99 \mathrm{E}-02$ & $\begin{array}{l}{[134]} \\
103^{*}\end{array}$ & $5.99 \mathrm{E}-02$ & $<0$ \\
\hline \multicolumn{15}{|c|}{ LG9 $40^{\circ} \mathrm{C} \mathrm{pH} 11$ Reactor \#1 } \\
\hline LG9.40.11.10.Blank1 & & $4.60 \mathrm{E}-06$ & --- & --- & --- & 56 & --- & [75.6] & --- & [164] & -.- & [134] & --- & -.- \\
\hline LG9.40.11.10.Blank2 & & $6.70 \mathrm{E}-06$ & --- & --- & --- & 56 & --- & [75.6] & --- & [164] & --- & [134] & --- & --- \\
\hline LG9.40.11.10.Blank3 & & 4.92E-06 & --- & --- & --- & [49.4] & --- & [75.6] & --- & [164] & -.- & [134] & --- & -.- \\
\hline LG9.40.11.10.1 & & $5.00 \mathrm{E}-08$ & 1 & 2.000 & $3.94 \mathrm{E}-02$ & [165] & $5.86 \mathrm{E}-06$ & [252] & $1.21 \mathrm{E}-05$ & [548] & $3.65 \mathrm{E}-06$ & [447] & $3.64 \mathrm{E}-06$ & $<0$ \\
\hline LG9.40.11.10.5 & & $4.60 \mathrm{E}-06$ & 10 & 1.997 & $3.93 E-02$ & 2,020 & $6.61 \mathrm{E}-03$ & 1,530 & $6.78 \mathrm{E}-03$ & 10,300 & $6.32 \mathrm{E}-03$ & 13,400 & $1.01 \mathrm{E}-02$ & $1.66 \mathrm{E}-09$ \\
\hline LG9.40.11.10.7 & & 4.14E-06 & 15 & 1.996 & 3.93E-02 & 2,000 & $5.89 \mathrm{E}-03$ & 1,570 & $6.26 \mathrm{E}-03$ & 10,200 & $5.63 \mathrm{E}-03$ & 12,600 & $8.52 \mathrm{E}-03$ & 1.14E-09 \\
\hline LG9.40.11.10.11 & & $8.08 \mathrm{E}-06$ & 24 & 1.991 & $3.92 \mathrm{E}-02$ & 1,870 & $1.08 \mathrm{E}-02$ & 1,620 & $1.26 \mathrm{E}-02$ & 10,200 & $1.10 \mathrm{E}-02$ & 12,900 & $1.70 \mathrm{E}-02$ & $2.23 \mathrm{E}-09$ \\
\hline LG9.40.11.10.12 & & $5.55 \mathrm{E}-06$ & 27 & 1.991 & $3.92 \mathrm{E}-02$ & 1,670 & $6.60 \mathrm{E}-03$ & 1,310 & $7.01 \mathrm{E}-03$ & 8,960 & $6.64 \mathrm{E}-03$ & 10,500 & $9.53 \mathrm{E}-03$ & $1.27 \mathrm{E}-09$ \\
\hline LG9.40.11.10.13 & & $0.00 \mathrm{E}+00$ & 29 & 1.991 & $3.92 E-02$ & 1,640 & $0.00 E+00$ & 1,390 & $0.00 \mathrm{E}+00$ & 8,630 & $0.00 \mathrm{E}+00$ & 9,450 & $0.00 \mathrm{E}+00$ & $<0$ \\
\hline \multicolumn{15}{|c|}{ LG9 $40^{\circ} \mathrm{C} \mathrm{pH} 11$ Reactor $\# 2$} \\
\hline LG9.40.11.20.Blank1 & & $1.44 \mathrm{E}-05$ & --- & --- & --- & [49.4] & --- & {$[75.6]$} & --- & [164] & --- & [134] & --- & --- \\
\hline LG9.40.11.20.Blank2 & & $1.72 \mathrm{E}-05$ & --- & --- & --- & [49.4] & --- & [75.6] & --- & [164] & -.- & [134] & --- & -.- \\
\hline LG9.40.11.20.Blank3 & & $1.72 \mathrm{E}-05$ & --- & --- & --- & [49.4] & --- & [75.6] & --- & [164] & --- & [134] & --- & --- \\
\hline
\end{tabular}

B.12 


\begin{tabular}{|c|c|c|c|c|c|c|c|c|c|c|c|c|c|c|}
\hline Sample ID & $\begin{array}{l}\text { Influent } \\
\text { [Si] } \\
\text { (ppm) }\end{array}$ & $\begin{array}{l}\text { Flow } \\
\text { Rate, q } \\
\left(\mathrm{m}^{3} \mathrm{~d}^{-1}\right)\end{array}$ & $\begin{array}{l}\text { Time } \\
\text { (d) }\end{array}$ & $\begin{array}{l}\text { Glass } \\
\text { Mass } \\
\text { (g) }\end{array}$ & $\begin{array}{l}\text { Surface } \\
\text { Area, } \mathrm{S} \\
\left(\mathrm{m}^{2}\right)\end{array}$ & $\begin{array}{l}{[\mathrm{Al}]} \\
\left(\mu \mathrm{g} \mathrm{L}^{-1}\right)\end{array}$ & $\begin{array}{l}\text { Al Norm. } \\
\text { Diss. Rate } \\
\left(\mathrm{g} \mathrm{m}^{-2} \mathrm{~d}^{-1}\right)\end{array}$ & $\begin{array}{l}{[\mathrm{B}]} \\
\left(\mu \mathrm{g} \mathrm{L}^{-1}\right)\end{array}$ & $\begin{array}{l}\text { B Norm. } \\
\text { Diss. Rate } \\
\left(\mathrm{g} \mathrm{m}^{-2} \mathrm{~d}^{-1}\right)\end{array}$ & $\begin{array}{l}{[\mathrm{Si}]} \\
\left(\mu g \mathrm{~L}^{-1}\right)\end{array}$ & $\begin{array}{l}\text { Si Norm. } \\
\text { Diss. Rate } \\
\left(\mathrm{g} \mathrm{m}^{-2} \mathrm{~d}^{-1}\right)\end{array}$ & $\begin{array}{l}{[\mathrm{Na}]} \\
(\mu \mathrm{g} \mathrm{L} \\
\left.{ }^{1}\right)\end{array}$ & $\begin{array}{l}\text { Na Norm. } \\
\text { Diss. Rate, } \\
\mathrm{r} \\
\left(\mathrm{g} \mathrm{m}^{-2} \mathrm{~d}^{-1}\right)\end{array}$ & $\begin{array}{l}\mathrm{r}_{\mathrm{IEX}} \\
\left(\mathrm{mol} \mathrm{Na} \mathrm{s}^{-1}\right)\end{array}$ \\
\hline LG9.40.11.20.1 & & $6.53 \mathrm{E}-07$ & 1 & 1.502 & $2.96 \mathrm{E}-02$ & 91 & $5.59 \mathrm{E}-05$ & {$[75.6]$} & $6.32 \mathrm{E}-05$ & 416 & $4.81 \mathrm{E}-05$ & 1,200 & $1.70 \mathrm{E}-04$ & $5.38 \mathrm{E}-11$ \\
\hline LG9.40.11.20.3 & & 7.36E-06 & 6 & 1.501 & 2.95E-02 & 863 & $6.01 \mathrm{E}-03$ & 588 & $5.54 \mathrm{E}-03$ & 4,470 & $5.84 \mathrm{E}-03$ & 4,920 & 7.87E-03 & 1.17E-09 \\
\hline LG9.40.11.20.5 & & $7.75 \mathrm{E}-06$ & 10 & 1.500 & $2.95 \mathrm{E}-02$ & 1,020 & $7.48 \mathrm{E}-03$ & 734 & $7.28 \mathrm{E}-03$ & 5,190 & $7.13 \mathrm{E}-03$ & 5,670 & $9.55 \mathrm{E}-03$ & 1.14E-09 \\
\hline LG9.40.11.20.7 & & $8.53 \mathrm{E}-06$ & 15 & 1.499 & 2.95E-02 & 1,040 & $8.40 \mathrm{E}-03$ & 745 & $8.15 \mathrm{E}-03$ & 5,310 & $8.04 E-03$ & 5,310 & $9.85 \mathrm{E}-03$ & $8.58 \mathrm{E}-10$ \\
\hline LG9.40.11.20.9 & & $1.08 \mathrm{E}-05$ & 20 & 1.498 & $2.95 \mathrm{E}-02$ & 770 & 7.91E-03 & 429 & $5.96 \mathrm{E}-03$ & 3,480 & $6.70 \mathrm{E}-03$ & 2,610 & $6.16 \mathrm{E}-03$ & $9.65 \mathrm{E}-11$ \\
\hline LG9.40.11.20.10 & & $1.16 \mathrm{E}-05$ & 22 & 1.497 & 2.95E-02 & 961 & $1.06 \mathrm{E}-02$ & 688 & $1.02 \mathrm{E}-02$ & 4,770 & $9.83 \mathrm{E}-03$ & 4,420 & $1.12 \mathrm{E}-02$ & 4.63E-10 \\
\hline LG9.40.11.20.11 & & $8.50 \mathrm{E}-06$ & 24 & 1.497 & 2.95E-02 & 929 & 7.49E-03 & 711 & $7.76 \mathrm{E}-03$ & 4,780 & $7.22 \mathrm{E}-03$ & 4,390 & $8.13 \mathrm{E}-03$ & $1.85 \mathrm{E}-10$ \\
\hline LG9.40.11.20.12 & & $9.46 \mathrm{E}-06$ & 27 & 1.496 & 2.95E-02 & 935 & $8.39 \mathrm{E}-03$ & 667 & $8.10 \mathrm{E}-03$ & 4,830 & $8.12 E-03$ & 4,500 & $9.27 \mathrm{E}-03$ & $5.89 \mathrm{E}-10$ \\
\hline LG9.40.11.20.13 & & $0.00 E+00$ & 29 & 1.496 & 2.95E-02 & 920 & $0.00 E+00$ & 649 & $0.00 E+00$ & 4,750 & $0.00 \mathrm{E}+00$ & 4,270 & $0.00 E+00$ & $<0$ \\
\hline \multicolumn{15}{|c|}{ LG9 $40^{\circ} \mathrm{C} \mathrm{pH} 11$ Reactor $\# 3$} \\
\hline LG9.40.11.40.Blank1 & & $3.61 \mathrm{E}-05$ & --- & --- & --- & [49.4] & --- & [75.6] & --- & [164] & --- & [134] & --- & --- \\
\hline LG9.40.11.40.Blank2 & & 4.18E-05 & --- & --- & --- & 51 & --- & [75.6] & --- & [164] & --- & [134] & --- & --- \\
\hline LG9.40.11.40.Blank3 & & $4.23 \mathrm{E}-05$ & --- & --- & --- & [49.4] & --- & [75.6] & --- & [164] & --- & [134] & --- & --- \\
\hline LG9.40.11.40.1 & & $1.12 \mathrm{E}-06$ & 1 & 0.749 & $1.47 \mathrm{E}-02$ & [49.4] & $1.05 \mathrm{E}-04$ & [75.6] & $2.18 \mathrm{E}-04$ & 251 & $1.00 \mathrm{E}-04$ & 624 & 3.05E-04 & 4.39E-11 \\
\hline LG9.40.11.40.3 & & $2.31 \mathrm{E}-05$ & 2 & 0.749 & $1.47 \mathrm{E}-02$ & 354 & $1.55 \mathrm{E}-02$ & 148 & $8.79 \mathrm{E}-03$ & 1,700 & $1.40 \mathrm{E}-02$ & 1,670 & $1.68 \mathrm{E}-02$ & 4.04E-09 \\
\hline LG9.40.11.40.5 & & $2.41 \mathrm{E}-05$ & 6 & 0.748 & $1.47 \mathrm{E}-02$ & 570 & $2.61 \mathrm{E}-02$ & 333 & $2.06 \mathrm{E}-02$ & 2,880 & $2.47 \mathrm{E}-02$ & 2,500 & 2.63E-02 & $2.84 \mathrm{E}-09$ \\
\hline LG9.40.11.40.7 & & $2.31 \mathrm{E}-05$ & 10 & 0.747 & $1.47 \mathrm{E}-02$ & 572 & $2.51 \mathrm{E}-02$ & 322 & $1.91 \mathrm{E}-02$ & 2,830 & $2.33 \mathrm{E}-02$ & 2,490 & $2.51 \mathrm{E}-02$ & 3.01E-09 \\
\hline LG9.40.11.40.9 & & $2.18 \mathrm{E}-05$ & 15 & 0.745 & $1.47 \mathrm{E}-02$ & 554 & $2.29 \mathrm{E}-02$ & 312 & $1.75 \mathrm{E}-02$ & 2,720 & $2.11 \mathrm{E}-02$ & 2,250 & $2.14 \mathrm{E}-02$ & $1.97 \mathrm{E}-09$ \\
\hline LG9.40.11.40.11 & & $2.52 \mathrm{E}-05$ & 20 & 0.744 & $1.47 \mathrm{E}-02$ & 516 & $2.48 \mathrm{E}-02$ & 293 & $1.91 \mathrm{E}-02$ & 2,600 & $2.34 \mathrm{E}-02$ & 2,060 & $2.27 \mathrm{E}-02$ & $1.85 \mathrm{E}-09$ \\
\hline LG9.40.11.40.12 & & $2.48 \mathrm{E}-05$ & 22 & 0.743 & $1.47 \mathrm{E}-02$ & 496 & $2.34 \mathrm{E}-02$ & 258 & $1.65 \mathrm{E}-02$ & 2,510 & $2.22 \mathrm{E}-02$ & 2,040 & $2.21 \mathrm{E}-02$ & $2.84 \mathrm{E}-09$ \\
\hline LG9.40.11.40.13 & & $2.17 \mathrm{E}-05$ & 24 & 0.743 & $1.47 \mathrm{E}-02$ & 486 & $2.01 \mathrm{E}-02$ & 321 & $1.80 \mathrm{E}-02$ & 2,570 & $1.99 \mathrm{E}-02$ & 2,090 & $1.98 \mathrm{E}-02$ & $9.46 \mathrm{E}-10$ \\
\hline LG9.40.11.40.14 & & $2.39 \mathrm{E}-05$ & 27 & 0.742 & $1.46 \mathrm{E}-02$ & 485 & $2.22 \mathrm{E}-02$ & 336 & $2.08 \mathrm{E}-02$ & 2,570 & $2.20 \mathrm{E}-02$ & 2,130 & 2.24E-02 & 7.90E-10 \\
\hline LG9.40.11.40.15 & & $0.00 E+00$ & 29 & 1 & 0 & 483 & $0.00 E+00$ & 322 & $0.00 E+00$ & 2,480 & $0.00 \mathrm{E}+00$ & 1,990 & $0.00 E+00$ & $<0$ \\
\hline \multicolumn{15}{|c|}{ LG9 $40{ }^{\circ} \mathrm{C} \mathrm{pH} 11$ Reactor $\# 4$} \\
\hline LG9.40.11.80A.Blank1 & & $6.57 \mathrm{E}-05$ & --- & --- & 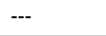 & [49.4] & --- & [75.6] & --- & [164] & --- & [134] & --- & --- \\
\hline LG9.40.11.80A.Blank2 & & $7.53 \mathrm{E}-05$ & --- & --- & --- & [49.4] & --- & [75.6] & --- & [164] & --- & [134] & --- & --- \\
\hline LG9.40.11.80A.Blank3 & & $7.19 \mathrm{E}-05$ & --- & --- & --- & [49.4] & --- & {$[75.6]$} & --- & [164] & --- & [134] & --- & --- \\
\hline LG9.40.11.80A.1 & & $1.55 \mathrm{E}-06$ & 1 & 0.251 & $4.94 \mathrm{E}-03$ & [49.4] & $4.34 \mathrm{E}-04$ & {$[75.6]$} & $9.00 \mathrm{E}-04$ & 208 & 3.43E-04 & 250 & $5.05 E-04$ & $<0$ \\
\hline LG9.40.11.80A.3 & & 3.42E-05 & 2 & 0.251 & $4.94 \mathrm{E}-03$ & 166 & $3.21 \mathrm{E}-02$ & {$[75.6]$} & $1.98 \mathrm{E}-02$ & 867 & $3.14 \mathrm{E}-02$ & 694 & $3.08 \mathrm{E}-02$ & $5.55 \mathrm{E}-09$ \\
\hline LG9.40.11.80A.5 & & 3.26E-05 & 6 & 0.250 & 4.93E-03 & 174 & $3.22 \mathrm{E}-02$ & [75.6] & $1.89 \mathrm{E}-02$ & 886 & $3.07 \mathrm{E}-02$ & 354 & $1.50 \mathrm{E}-02$ & $<0$ \\
\hline LG9.40.11.80A.7 & & 3.62E-05 & 10 & 0.250 & $4.93 \mathrm{E}-03$ & 247 & $5.07 \mathrm{E}-02$ & 98 & $2.72 \mathrm{E}-02$ & 1,270 & $4.89 \mathrm{E}-02$ & 949 & $4.48 \mathrm{E}-02$ & $8.83 E-09$ \\
\hline LG9.40.11.80A.9 & & $3.61 \mathrm{E}-05$ & 15 & 0.249 & $4.92 \mathrm{E}-03$ & 248 & $5.09 \mathrm{E}-02$ & 76 & $2.12 \mathrm{E}-02$ & 1,190 & $4.58 \mathrm{E}-02$ & 966 & $4.55 \mathrm{E}-02$ & $1.22 \mathrm{E}-08$ \\
\hline LG9.40.11.80A.11 & & $2.88 \mathrm{E}-05$ & 20 & 0.249 & $4.91 \mathrm{E}-03$ & 237 & $3.88 \mathrm{E}-02$ & $\begin{array}{l}{[75.6]} \\
62^{\star}\end{array}$ & $1.68 \mathrm{E}-02$ & 1,230 & $3.78 \mathrm{E}-02$ & 762 & $2.87 \mathrm{E}-02$ & $5.99 \mathrm{E}-09$ \\
\hline LG9.40.11.80A.12 & & $2.95 \mathrm{E}-05$ & 22 & 0.249 & $4.91 \mathrm{E}-03$ & 229 & $3.84 \mathrm{E}-02$ & $\begin{array}{l}{[75.6]} \\
50^{*}\end{array}$ & $1.72 \mathrm{E}-02$ & 1,140 & $3.59 \mathrm{E}-02$ & 764 & $2.95 \mathrm{E}-02$ & $6.18 \mathrm{E}-09$ \\
\hline LG9.40.11.80A.13 & & $3.82 \mathrm{E}-05$ & 24 & 0.248 & $4.90 \mathrm{E}-03$ & 232 & $5.05 E-02$ & 147 & 4.33E-02 & 1,250 & $5.10 \mathrm{E}-02$ & 1,020 & $5.10 \mathrm{E}-02$ & $3.86 \mathrm{E}-09$ \\
\hline LG9.40.11.80A.14 & & 3.60E-05 & 27 & 0.248 & $4.90 \mathrm{E}-03$ & 224 & $4.60 \mathrm{E}-02$ & 126 & 3.50E-02 & 1,210 & $4.66 \mathrm{E}-02$ & 917 & $4.32 \mathrm{E}-02$ & 4.14E-09 \\
\hline LG9.40.11.80A.15 & & $0.00 E+00$ & 29 & 0 & 0 & 239 & $0.00 E+00$ & 156 & $0.00 E+00$ & 1,350 & $0.00 E+00$ & 990 & $0.00 E+00$ & $<0$ \\
\hline \multicolumn{15}{|c|}{ LG9 $40^{\circ} \mathrm{C} \mathrm{pH} 11$ Reactor $\# 5$} \\
\hline LG9.40.11.80B.Blank1 & & $6.44 \mathrm{E}-05$ & --- & --- & --- & [49.4] & --- & {$[75.6]$} & --- & [164] & --- & [134] & --- & --- \\
\hline LG9.40.11.80B.Blank2 & & $7.55 \mathrm{E}-05$ & --- & --- & --- & [49.4] & --- & [75.6] & --- & [164] & --- & [134] & --- & --- \\
\hline LG9.40.11.80B.Blank3 & & $7.81 \mathrm{E}-05$ & --- & --- & --- & [49.4] & --- & [75.6] & --- & [164] & --- & [134] & --- & $-\cdots$ \\
\hline
\end{tabular}

B.13 


\begin{tabular}{|c|c|c|c|c|c|c|c|c|c|c|c|c|c|c|}
\hline Sample ID & $\begin{array}{l}\text { Influent } \\
{[\mathrm{Si}]} \\
(\mathrm{ppm})\end{array}$ & $\begin{array}{l}\text { Flow } \\
\text { Rate, } q \\
\left(\mathrm{~m}^{3} \mathrm{~d}^{-1}\right)\end{array}$ & $\begin{array}{l}\text { Time } \\
\text { (d) }\end{array}$ & $\begin{array}{l}\text { Glass } \\
\text { Mass } \\
\text { (g) }\end{array}$ & $\begin{array}{l}\text { Surface } \\
\text { Area, S } \\
\left(\mathrm{m}^{2}\right)\end{array}$ & $\begin{array}{l}{[\mathrm{Al}]} \\
\left(\mu \mathrm{L} \mathrm{L}^{-1}\right)\end{array}$ & $\begin{array}{l}\text { Al Norm. } \\
\text { Diss. Rate } \\
\left(\mathrm{g} \mathrm{m}^{-2} \mathrm{~d}^{-1}\right)\end{array}$ & $\begin{array}{l}{[B]} \\
\left(\mu \mathrm{L} \mathrm{L}^{-1}\right)\end{array}$ & $\begin{array}{l}\text { B Norm. } \\
\text { Diss. Rate } \\
\left(\mathrm{g} \mathrm{m}^{-2} \mathrm{~d}^{-1}\right)\end{array}$ & $\begin{array}{l}{[\mathrm{Si}]} \\
\left(\mu \mathrm{L} \mathrm{L}^{-1}\right)\end{array}$ & $\begin{array}{l}\text { Si Norm. } \\
\text { Diss. Rate } \\
\left(\mathrm{g} \mathrm{m}^{-2} \mathrm{~d}^{-1}\right)\end{array}$ & $\begin{array}{l}{[\mathrm{Na}]} \\
(\mu \mathrm{g} \mathrm{L} \\
\left.{ }^{1}\right)\end{array}$ & $\begin{array}{l}\text { Na Norm. } \\
\text { Diss. Rate, } \\
\mathrm{r} \\
\left(\mathrm{g} \mathrm{m}^{-2} \mathrm{~d}^{-1}\right)\end{array}$ & $\begin{array}{l}\mathrm{r}_{\mathrm{IEX}} \\
\left(\mathrm{mol} \mathrm{Na} \mathrm{s}^{-1}\right)\end{array}$ \\
\hline LG9.40.11.80B.1 & & $1.72 \mathrm{E}-06$ & 1 & 0.050 & $9.85 \mathrm{E}-04$ & [49.4] & $2.41 \mathrm{E}-03$ & {$[75.6]$} & $5.00 \mathrm{E}-03$ & [164] & $1.50 \mathrm{E}-03$ & [134] & $1.50 \mathrm{E}-03$ & $<0$ \\
\hline LG9.40.11.80B.3 & & 3.96E-05 & 2 & 0.050 & $9.84 \mathrm{E}-04$ & 63 & $7.11 \mathrm{E}-02$ & {$[75.6]$} & $1.15 \mathrm{E}-01$ & 291 & $6.13 \mathrm{E}-02$ & 249 & $6.43 \mathrm{E}-02$ & $<0$ \\
\hline LG9.40.11.80B.5 & & 4.16E-05 & 6 & 0.049 & $9.76 \mathrm{E}-04$ & 97 & $1.16 \mathrm{E}-01$ & {$[75.6]$} & $1.22 \mathrm{E}-01$ & 497 & $1.11 \mathrm{E}-01$ & 424 & $1.16 \mathrm{E}-01$ & $<0$ \\
\hline LG9.40.11.80B.7 & & $3.98 \mathrm{E}-05$ & 10 & 0.049 & $9.71 \mathrm{E}-04$ & 103 & $1.18 \mathrm{E}-01$ & {$[75.6]$} & $1.17 \mathrm{E}-01$ & 514 & 1.10E-01 & 403 & $1.06 \mathrm{E}-01$ & $<0$ \\
\hline LG9.40.11.80B.9 & & 3.93E-05 & 15 & 0.048 & 9.63E-04 & 100 & 1.14E-01 & {$[75.6]$} & 1.17E-01 & 487 & 1.04E-01 & 372 & $9.74 E-02$ & $<0$ \\
\hline LG9.40.11.80B.11 & & $4.64 \mathrm{E}-05$ & 20 & 0.048 & $9.53 \mathrm{E}-04$ & 95 & $1.29 \mathrm{E}-01$ & {$[75.6]$} & $1.39 \mathrm{E}-01$ & 485 & $1.24 \mathrm{E}-01$ & 372 & $1.16 \mathrm{E}-01$ & $<0$ \\
\hline LG9.40.11.80B.12 & & 4.59E-05 & 22 & 0.047 & $9.50 \mathrm{E}-04$ & 90 & $1.22 \mathrm{E}-01$ & [75.6] & $1.38 \mathrm{E}-01$ & 461 & 1.17E-01 & 348 & $1.08 \mathrm{E}-01$ & $<0$ \\
\hline LG9.40.11.80B.13 & & 3.95E-05 & 24 & 0.047 & $9.47 \mathrm{E}-04$ & 88 & $1.02 \mathrm{E}-01$ & {$[75.6]$} & $1.19 \mathrm{E}-01$ & 439 & $9.59 \mathrm{E}-02$ & 360 & $9.64 \mathrm{E}-02$ & $<0$ \\
\hline LG9.40.11.80B.14 & & 4.13E-05 & 27 & 0.047 & $9.42 \mathrm{E}-04$ & 86 & $1.05 \mathrm{E}-01$ & {$[75.6]$} & $1.26 \mathrm{E}-01$ & 456 & 1.05E-01 & 356 & 1.00E-01 & $<0$ \\
\hline \multicolumn{15}{|c|}{ LG9 $40^{\circ} \mathrm{C} \mathrm{pH} 12$ Reactor $\# 1$} \\
\hline LG9.40.12.10.Blank1 & & $1.10 \mathrm{E}-05$ & --- & --- & --- & [49.4] & --- & {$[75.6]$} & --- & 325 & --- & 726 & --- & --- \\
\hline LG9.40.12.10.Blank2 & & $1.09 \mathrm{E}-05$ & --- & --- & --- & {$[49.4]$} & --- & {$[75.6]$} & --- & 299 & --- & 702 & --- & --. \\
\hline LG9.40.12.10.Blank3 & & $1.01 E-05$ & --- & --- & --- & [49.4] & --- & {$[75.6]$} & --- & 354 & --- & 666 & --- & --- \\
\hline LG9.40.12.10.1 & & $7.49 \mathrm{E}-07$ & 1.0 & 4.000 & $7.87 \mathrm{E}-02$ & 427 & $1.14 \mathrm{E}-04$ & 241 & $8.68 \mathrm{E}-05$ & 2,310 & 1.15E-04 & 6,190 & $3.78 \mathrm{E}-04$ & $1.47 \mathrm{E}-10$ \\
\hline LG9.40.12.10.2 & & $1.07 E-05$ & 1.9 & 3.999 & 7.87E-02 & 1,560 & $5.91 \mathrm{E}-03$ & 947 & $4.86 \mathrm{E}-03$ & 8,300 & 5.90E-03 & 9,750 & $8.49 E-03$ & 1.83E-09 \\
\hline LG9.40.12.10.3 & & $7.56 \mathrm{E}-06$ & 3.9 & 3.998 & $7.87 \mathrm{E}-02$ & 3,040 & $8.16 \mathrm{E}-03$ & 2,110 & $7.67 \mathrm{E}-03$ & 16,500 & $8.31 \mathrm{E}-03$ & 15,700 & $9.68 \mathrm{E}-03$ & $1.01 E-09$ \\
\hline LG9.40.12.10.4 & & 7.70E-06 & 6.8 & 3.995 & $7.86 E-02$ & 4,130 & 1.13E-02 & 3,030 & $1.12 \mathrm{E}-02$ & 22,500 & 1.15E-02 & 21,300 & $1.34 \mathrm{E}-02$ & 1.09E-09 \\
\hline LG9.40.12.10.5 & & $1.05 E-05$ & 8.8 & 3.992 & $7.86 E-02$ & 4,430 & 1.65E-02 & 3,310 & $1.67 E-02$ & 23,900 & 1.67E-02 & 22,800 & $1.95 \mathrm{E}-02$ & $1.42 \mathrm{E}-09$ \\
\hline LG9.40.12.10.7 & & $1.13 E-05$ & 13.8 & 3.984 & $7.85 E-02$ & 4,260 & $1.71 \mathrm{E}-02$ & 3,250 & $1.77 \mathrm{E}-02$ & 23,000 & 1.73E-02 & 22,600 & 2.09E-02 & 1.60E-09 \\
\hline LG9.40.12.10.9 & & $1.15 \mathrm{E}-05$ & 18.0 & 3.979 & $7.84 \mathrm{E}-02$ & 4,190 & $1.72 \mathrm{E}-02$ & 2,970 & $1.65 \mathrm{E}-02$ & 20,100 & $1.54 \mathrm{E}-02$ & 19,400 & $1.82 \mathrm{E}-02$ & $8.96 \mathrm{E}-10$ \\
\hline LG9.40.12.10.11 & & $1.12 \mathrm{E}-05$ & 23.7 & 3.971 & $7.83 \mathrm{E}-02$ & 4,410 & $1.76 \mathrm{E}-02$ & 3,130 & $1.69 \mathrm{E}-02$ & 20,900 & 1.56E-02 & 20,300 & $1.86 \mathrm{E}-02$ & $8.52 \mathrm{E}-10$ \\
\hline LG9.40.12.10.13 & & 1.12E-05 & 27.9 & 3.965 & 7.82E-02 & 4,440 & $1.77 E-02$ & 3,230 & $1.74 \mathrm{E}-02$ & 21,400 & 1.60E-02 & 20,600 & $1.89 E-02$ & 7.20E-10 \\
\hline LG9.40.12.10.15 & & $1.09 \mathrm{E}-05$ & 32.0 & 3.960 & $7.82 \mathrm{E}-02$ & 4,370 & $1.70 \mathrm{E}-02$ & 3,270 & $1.72 \mathrm{E}-02$ & 21,300 & 1.55E-02 & 19,800 & $1.76 \mathrm{E}-02$ & $2.36 \mathrm{E}-10$ \\
\hline LG9.40.12.10.17 & & $9.97 \mathrm{E}-06$ & 36.7 & 3.956 & $7.81 \mathrm{E}-02$ & 4,190 & $1.49 \mathrm{E}-02$ & 3,000 & $1.45 \mathrm{E}-02$ & 20,100 & $1.34 \mathrm{E}-02$ & 19,300 & $1.58 \mathrm{E}-02$ & $6.66 \mathrm{E}-10$ \\
\hline \multicolumn{15}{|c|}{ LG9 $40^{\circ} \mathrm{C} \mathrm{pH} 12$ Reactor $\# 2$} \\
\hline LG9.40.12.20.Blank1 & & 2.00E-05 & --- & --- & --- & {$[49.4]$} & --- & {$[75.6]$} & --- & 320 & --- & 624 & -- & --- \\
\hline LG9.40.12.20.Blank2 & & $2.04 \mathrm{E}-05$ & --- & --- & --- & [49.4] & --- & {$[75.6]$} & --- & 266 & --- & 605 & --- & --. \\
\hline LG9.40.12.20.Blank3 & & $1.93 E-05$ & --- & --- & --- & {$[49.4]$} & --- & {$[75.6]$} & --- & 232 & -- & 647 & -- & --- \\
\hline LG9.40.12.20.1 & & $1.93 \mathrm{E}-06$ & 1.0 & 3.020 & $5.94 \mathrm{E}-02$ & 259 & $2.36 \mathrm{E}-04$ & 198 & $2.44 \mathrm{E}-04$ & 1,400 & 2.39E-04 & 4,510 & $9.42 \mathrm{E}-04$ & $3.52 \mathrm{E}-10$ \\
\hline LG9.40.12.20.2 & & $2.01 \mathrm{E}-05$ & 1.9 & 3.019 & $5.94 \mathrm{E}-02$ & 1,240 & 1.17E-02 & 771 & $9.85 \mathrm{E}-03$ & 6,520 & 1.15E-02 & 6,830 & $1.48 \mathrm{E}-02$ & 2.49E-09 \\
\hline LG9.40.12.20.3 & & $1.99 \mathrm{E}-05$ & 3.9 & 3.016 & $5.94 \mathrm{E}-02$ & 2,150 & $2.01 E-02$ & 1,500 & $1.90 \mathrm{E}-02$ & 11,700 & 2.05E-02 & 10,600 & $2.28 \mathrm{E}-02$ & $1.90 \mathrm{E}-09$ \\
\hline LG9.40.12.20.4 & & 1.87E-05 & 6.8 & 3.011 & $5.93 \mathrm{E}-02$ & 2,610 & $2.30 \mathrm{E}-02$ & 1,890 & $2.26 \mathrm{E}-02$ & 14,300 & $2.36 \mathrm{E}-02$ & 12,900 & $2.61 \mathrm{E}-02$ & 1.79E-09 \\
\hline LG9.40.12.20.5 & & $1.97 \mathrm{E}-05$ & 8.8 & 3.009 & $5.93 \mathrm{E}-02$ & 2,720 & $2.53 \mathrm{E}-02$ & 1,980 & $2.49 \mathrm{E}-02$ & 14,600 & $2.55 \mathrm{E}-02$ & 13,800 & $2.95 \mathrm{E}-02$ & 2.29E-09 \\
\hline LG9.40.12.20.7 & & $1.95 \mathrm{E}-05$ & 13.8 & 3.001 & $5.92 E-02$ & 2,610 & $2.41 E-02$ & 1,950 & 2.44E-02 & 14,200 & $2.46 \mathrm{E}-02$ & 12,800 & $2.71 \mathrm{E}-02$ & $1.39 \mathrm{E}-09$ \\
\hline LG9.40.12.20.9 & & $2.00 \mathrm{E}-05$ & 18.0 & 2.996 & $5.91 \mathrm{E}-02$ & 2,530 & $2.39 \mathrm{E}-02$ & 1,690 & $2.16 \mathrm{E}-02$ & 12,100 & 2.15E-02 & 10,900 & $2.37 \mathrm{E}-02$ & $1.03 \mathrm{E}-09$ \\
\hline LG9.40.12.20.11 & & $1.92 \mathrm{E}-05$ & 23.7 & 2.988 & $5.90 E-02$ & 2,700 & $2.46 \mathrm{E}-02$ & 1,850 & $2.28 \mathrm{E}-02$ & 13,200 & $2.26 \mathrm{E}-02$ & 12,200 & $2.55 E-02$ & $1.37 E-09$ \\
\hline LG9.40.12.20.13 & & $1.91 \mathrm{E}-05$ & 27.9 & 2.983 & $5.89 E-02$ & 2,710 & $2.46 E-02$ & 1,810 & $2.22 \mathrm{E}-02$ & 13,200 & $2.24 \mathrm{E}-02$ & 12,400 & $2.58 \mathrm{E}-02$ & $1.81 \mathrm{E}-09$ \\
\hline LG9.40.12.20.15 & & $1.91 \mathrm{E}-05$ & 32.0 & 2.978 & $5.89 E-02$ & 2,540 & $2.30 \mathrm{E}-02$ & 1,690 & 2.07E-02 & 12,100 & 2.05E-02 & 11,800 & $2.45 E-02$ & $1.92 \mathrm{E}-09$ \\
\hline LG9.40.12.20.17 & & $1.96 \mathrm{E}-05$ & 36.7 & 2.972 & $5.88 E-02$ & 2,560 & $2.39 \mathrm{E}-02$ & 1,700 & 2.15E-02 & 12,400 & 2.17E-02 & 11,400 & $2.44 E-02$ & 1.49E-09 \\
\hline \multicolumn{15}{|c|}{ LG9 $40^{\circ} \mathrm{C}$ pH 12 Reactor $\# 3$} \\
\hline LG9.40.12.40.Blank1 & & 3.89E-05 & --- & --- & --- & {$[49.4]$} & --- & [75.6] & --- & 405 & --- & 647 & --- & --- \\
\hline LG9.40.12.40.Blank2 & & 3.98E-05 & --- & --- & --- & {$[49.4]$} & --- & {$[75.6]$} & --- & 270 & --- & 529 & -- & --- \\
\hline LG9.40.12.40.Blank3 & & $3.76 \mathrm{E}-05$ & --- & --- & --- & [49.4] & --- & {$[75.6]$} & --- & 245 & --- & 535 & --- & --- \\
\hline
\end{tabular}




\begin{tabular}{|c|c|c|c|c|c|c|c|c|c|c|c|c|c|c|}
\hline Sample ID & $\begin{array}{l}\text { Influent } \\
\text { [Si] } \\
\text { (ppm) }\end{array}$ & $\begin{array}{l}\text { Flow } \\
\text { Rate, } q \\
\left(\mathrm{~m}^{3} \mathrm{~d}^{-1}\right)\end{array}$ & $\begin{array}{l}\text { Time } \\
\text { (d) }\end{array}$ & $\begin{array}{l}\text { Glass } \\
\text { Mass } \\
\text { (g) }\end{array}$ & $\begin{array}{l}\text { Surface } \\
\text { Area, S } \\
\left(\mathrm{m}^{2}\right)\end{array}$ & $\begin{array}{l}{[\mathrm{Al}]} \\
\left(\mu \mathrm{g} \mathrm{L}^{-1}\right)\end{array}$ & $\begin{array}{l}\text { Al Norm. } \\
\text { Diss. Rate } \\
\left(\mathrm{g} \mathrm{m}^{-2} \mathrm{~d}^{-1}\right)\end{array}$ & $\begin{array}{l}{[\mathrm{B}]} \\
\left(\mu \mathrm{L} \mathrm{L}^{-1}\right)\end{array}$ & $\begin{array}{l}\text { B Norm. } \\
\text { Diss. Rate } \\
\left(\mathrm{g} \mathrm{m}^{-2} \mathrm{~d}^{-1}\right)\end{array}$ & $\begin{array}{l}{[\mathrm{Si}]} \\
\left(\mu \mathrm{L} \mathrm{L}^{-1}\right)\end{array}$ & $\begin{array}{l}\text { Si Norm. } \\
\text { Diss. Rate } \\
\left(\mathrm{g} \mathrm{m}^{-2} \mathrm{~d}^{-1}\right)\end{array}$ & $\begin{array}{l}{[\mathrm{Na}]} \\
(\mu \mathrm{g} \mathrm{L}- \\
\left.{ }^{1}\right)\end{array}$ & $\begin{array}{l}\text { Na Norm. } \\
\text { Diss. Rate, } \\
\mathrm{r} \\
\left(\mathrm{g} \mathrm{m}^{-2} \mathrm{~d}^{-1}\right)\end{array}$ & $\begin{array}{l}\mathrm{r}_{\mathrm{IEX}} \\
\left(\mathrm{mol} \mathrm{Na} \mathrm{s}^{-2} \mathrm{~s}^{-1}\right)\end{array}$ \\
\hline LG9.40.12.40.1 & & $1.48 \mathrm{E}-06$ & 0.6 & 1.508 & 2.97E-02 & 226 & $3.16 \mathrm{E}-04$ & 136 & 2.57E-04 & 1,290 & $3.38 \mathrm{E}-04$ & 2,640 & $8.47 \mathrm{E}-04$ & $2.97 \mathrm{E}-10$ \\
\hline LG9.40.12.40.2 & & 3.92E-05 & 1.0 & 1.508 & $2.97 \mathrm{E}-02$ & 332 & $1.23 \mathrm{E}-02$ & 195 & $9.75 \mathrm{E}-03$ & 1,820 & $1.26 \mathrm{E}-02$ & 2,540 & $2.15 \mathrm{E}-02$ & $5.94 \mathrm{E}-09$ \\
\hline LG9.40.12.40.3 & & 3.96E-05 & 1.7 & 1.507 & $2.97 \mathrm{E}-02$ & 752 & $2.80 \mathrm{E}-02$ & 488 & $2.46 \mathrm{E}-02$ & 4,110 & $2.87 \mathrm{E}-02$ & 3,790 & $3.25 \mathrm{E}-02$ & $3.94 \mathrm{E}-09$ \\
\hline LG9.40.12.40.4 & & 3.90E-05 & 3.9 & 1.503 & $2.96 \mathrm{E}-02$ & 1,190 & $4.38 \mathrm{E}-02$ & 835 & $4.16 \mathrm{E}-02$ & 6,530 & $4.51 \mathrm{E}-02$ & 5,650 & $4.77 \mathrm{E}-02$ & $3.10 \mathrm{E}-09$ \\
\hline LG9.40.12.40.5 & & 3.97E-05 & 6.8 & 1.499 & $2.95 \mathrm{E}-02$ & 1,260 & 4.73E-02 & 910 & 4.63E-02 & 6,960 & $4.90 \mathrm{E}-02$ & 6,200 & $5.35 \mathrm{E}-02$ & 3.63E-09 \\
\hline LG9.40.12.40.7 & & 3.87E-05 & 11.1 & 1.493 & 2.95E-02 & 1,240 & $4.55 \mathrm{E}-02$ & 897 & 4.46E-02 & 6,810 & $4.69 \mathrm{E}-02$ & 6,040 & $5.09 \mathrm{E}-02$ & $3.19 \mathrm{E}-09$ \\
\hline LG9.40.12.40.9 & & $3.82 \mathrm{E}-05$ & 15.7 & 1.489 & $2.94 \mathrm{E}-02$ & 1,190 & $4.32 \mathrm{E}-02$ & 734 & $3.60 \mathrm{E}-02$ & 5,820 & $3.96 \mathrm{E}-02$ & 5,240 & $4.36 \mathrm{E}-02$ & $3.83 \mathrm{E}-09$ \\
\hline LG9.40.12.40.11 & & 3.91E-05 & 20.7 & 1.482 & 2.93E-02 & 1,300 & $4.84 \mathrm{E}-02$ & 793 & $4.00 \mathrm{E}-02$ & 6,330 & $4.42 E-02$ & 5,770 & 4.93E-02 & $4.72 \mathrm{E}-09$ \\
\hline LG9.40.12.40.13 & & 4.04E-05 & 25.0 & 1.478 & $2.93 \mathrm{E}-02$ & 1,290 & $4.98 \mathrm{E}-02$ & 818 & 4.27E-02 & 6,370 & 4.61E-02 & 5,940 & $5.27 \mathrm{E}-02$ & 4.99E-09 \\
\hline LG9.40.12.40.15 & & 3.95E-05 & 29.7 & 1.472 & $2.92 \mathrm{E}-02$ & 1,250 & $4.73 \mathrm{E}-02$ & 766 & 3.92E-02 & 6,180 & $4.38 \mathrm{E}-02$ & 5,430 & $4.71 \mathrm{E}-02$ & 4.00E-09 \\
\hline LG9.40.12.40.17 & & $4.01 \mathrm{E}-05$ & 34.8 & 1.467 & $2.91 \mathrm{E}-02$ & 1,170 & $4.50 \mathrm{E}-02$ & 693 & $3.61 \mathrm{E}-02$ & 5,690 & 4.10E-02 & 5,020 & 4.43E-02 & 4.16E-09 \\
\hline LG9.40.12.40.18 & & 3.82E-05 & 36.7 & 1.465 & $2.91 \mathrm{E}-02$ & 1,260 & 4.62E-02 & 738 & $3.66 \mathrm{E}-02$ & 6,100 & 4.19E-02 & 5,630 & $4.74 \mathrm{E}-02$ & $5.42 E-09$ \\
\hline \multicolumn{15}{|c|}{ LG9 $40{ }^{\circ} \mathrm{C} \mathrm{pH} 12$ Reactor \#4 } \\
\hline LG9.40.12.80A.Blank1 & & 7.92E-05 & --- & --- & --- & [49.4] & --- & [75.6] & --- & 213 & --- & 616 & --- & --- \\
\hline LG9.40.12.80A.Blank2 & & 8.00E-05 & --- & --- & --- & [49.4] & --- & [75.6] & --- & [164] & --- & 509 & --- & --- \\
\hline LG9.40.12.80A.Blank3 & & $7.60 \mathrm{E}-05$ & --- & --- & --- & [49.4] & --- & {$[75.6]$} & --- & [164] & --- & 523 & --- & --- \\
\hline LG9.40.12.80A.1 & & $4.30 \mathrm{E}-06$ & 0.6 & 0.501 & $9.85 \mathrm{E}-03$ & 105 & $1.28 \mathrm{E}-03$ & 102 & $1.68 \mathrm{E}-03$ & 669 & $1.53 \mathrm{E}-03$ & 1,040 & $2.91 \mathrm{E}-03$ & $6.19 \mathrm{E}-10$ \\
\hline LG9.40.12.80A.2 & & 7.89E-05 & 1.0 & 0.501 & $9.85 \mathrm{E}-03$ & 152 & 3.40E-02 & 130 & 3.94E-02 & 901 & 3.78E-02 & 1,090 & $5.60 \mathrm{E}-02$ & $8.38 \mathrm{E}-09$ \\
\hline LG9.40.12.80A.3 & & 7.64E-05 & 1.7 & 0.500 & $9.84 \mathrm{E}-03$ & 340 & 7.37E-02 & 249 & $7.31 \mathrm{E}-02$ & 1,880 & 7.65E-02 & 1,890 & $9.41 \mathrm{E}-02$ & $1.06 \mathrm{E}-08$ \\
\hline LG9.40.12.80A.4 & & 7.64E-05 & 3.9 & 0.497 & $9.80 \mathrm{E}-03$ & 497 & $1.08 \mathrm{E}-01$ & 365 & $1.08 \mathrm{E}-01$ & 2,770 & 1.13E-01 & 2,750 & $1.38 \mathrm{E}-01$ & $1.51 \mathrm{E}-08$ \\
\hline LG9.40.12.80A.5 & & $7.68 \mathrm{E}-05$ & 6.8 & 0.493 & $9.75 \mathrm{E}-03$ & 528 & $1.16 \mathrm{E}-01$ & 392 & $1.17 E-01$ & 3,030 & $1.25 \mathrm{E}-01$ & 2,810 & $1.42 \mathrm{E}-01$ & $1.27 \mathrm{E}-08$ \\
\hline LG9.40.12.80A.7 & & $7.74 \mathrm{E}-05$ & 11.1 & 0.488 & $9.69 \mathrm{E}-03$ & 523 & $1.17 \mathrm{E}-01$ & 408 & $1.23 \mathrm{E}-01$ & 2,960 & $1.24 \mathrm{E}-01$ & 2,840 & $1.46 \mathrm{E}-01$ & $1.12 \mathrm{E}-08$ \\
\hline LG9.40.12.80A.9 & & $7.64 \mathrm{E}-05$ & 15.7 & 0.486 & $9.66 \mathrm{E}-03$ & 509 & $1.13 \mathrm{E}-01$ & 221 & $6.61 \mathrm{E}-02$ & 2,520 & $1.04 \mathrm{E}-01$ & 2,510 & $1.27 \mathrm{E}-01$ & $3.09 E-08$ \\
\hline LG9.40.12.80A.11 & & 7.69E-05 & 20.7 & 0.481 & $9.59 \mathrm{E}-03$ & 577 & $1.29 \mathrm{E}-01$ & 292 & $8.86 E-02$ & 2,880 & $1.21 \mathrm{E}-01$ & 2,640 & $1.36 \mathrm{E}-01$ & $2.38 \mathrm{E}-08$ \\
\hline LG9.40.12.80A.13 & & 7.69E-05 & 25.0 & 0.478 & $9.56 \mathrm{E}-03$ & 566 & $1.27 \mathrm{E}-01$ & 276 & $8.40 \mathrm{E}-02$ & 2,800 & $1.18 \mathrm{E}-01$ & 2,780 & $1.44 \mathrm{E}-01$ & $3.00 E-08$ \\
\hline LG9.40.12.80A.15 & & $7.65 \mathrm{E}-05$ & 29.7 & 0.474 & $9.50 \mathrm{E}-03$ & 561 & $1.26 \mathrm{E}-01$ & 280 & $8.53 \mathrm{E}-02$ & 2,800 & $1.18 \mathrm{E}-01$ & 2,670 & $1.38 \mathrm{E}-01$ & $2.65 \mathrm{E}-08$ \\
\hline LG9.40.12.80A.17 & & 7.70E-05 & 34.8 & 0.471 & $9.45 \mathrm{E}-03$ & 524 & $1.19 \mathrm{E}-01$ & 237 & $7.31 \mathrm{E}-02$ & 2,620 & $1.12 \mathrm{E}-01$ & 2,450 & $1.28 \mathrm{E}-01$ & $2.77 \mathrm{E}-08$ \\
\hline LG9.40.12.80A.18 & & $7.58 \mathrm{E}-05$ & 36.7 & 0.469 & $9.43 \mathrm{E}-03$ & 584 & $1.31 \mathrm{E}-01$ & 298 & $9.06 \mathrm{E}-02$ & 2,940 & $1.24 \mathrm{E}-01$ & 2,720 & $1.40 \mathrm{E}-01$ & $2.50 \mathrm{E}-08$ \\
\hline \multicolumn{15}{|c|}{ LG9 $40{ }^{\circ} \mathrm{C} \mathrm{pH} 12$ Reactor $\# 5$} \\
\hline LG9.40.12.80B.Blank1 & & $7.81 \mathrm{E}-05$ & --- & --- & --- & [49.4] & --- & [75.6] & --- & 200 & --- & 531 & --- & --. \\
\hline LG9.40.12.80B.Blank2 & & 8.17E-05 & --- & --- & --- & [49.4] & --- & {$[75.6]$} & --- & [164] & --- & 502 & --- & --- \\
\hline LG9.40.12.80B.Blank3 & & $7.59 \mathrm{E}-05$ & --- & --- & --- & [49.4] & --- & [75.6] & --- & [164] & --- & 537 & --- & --- \\
\hline LG9.40.12.80B.1 & & $4.62 \mathrm{E}-06$ & 0.6 & 0.102 & $2.01 E-03$ & [49.4] & $3.17 \mathrm{E}-03$ & $\begin{array}{l}{[75.6]} \\
60^{*}\end{array}$ & $6.57 E-03$ & 346 & 4.17E-03 & 712 & $1.05 E-02$ & $1.98 \mathrm{E}-09$ \\
\hline LG9.40.12.80B.2 & & $8.00 \mathrm{E}-05$ & 1.0 & 0.102 & $2.01 \mathrm{E}-03$ & 60 & $6.69 \mathrm{E}-02$ & $\begin{array}{l}{[75.6]} \\
61^{*}\end{array}$ & $1.14 \mathrm{E}-01$ & 415 & $8.65 E-02$ & 732 & $1.87 E-01$ & $3.68 \mathrm{E}-08$ \\
\hline LG9.40.12.80B.3 & & $7.76 \mathrm{E}-05$ & 1.7 & 0.102 & $2.01 E-03$ & 127 & $1.37 \mathrm{E}-01$ & 111 & 1.62E-01 & 758 & $1.54 \mathrm{E}-01$ & 1,080 & $2.68 \mathrm{E}-01$ & $5.32 \mathrm{E}-08$ \\
\hline LG9.40.12.80B.4 & & 7.89E-05 & 3.9 & 0.100 & $1.99 \mathrm{E}-03$ & 222 & 2.47E-01 & 169 & 2.54E-01 & 1,250 & 2.60E-01 & 1,480 & 3.77E-01 & $6.21 \mathrm{E}-08$ \\
\hline LG9.40.12.80B.5 & & 7.93E-05 & 6.8 & 0.098 & $1.96 \mathrm{E}-03$ & 237 & $2.68 \mathrm{E}-01$ & 210 & 3.22E-01 & 1,420 & $3.01 \mathrm{E}-01$ & 1,540 & 4.00E-01 & $3.95 \mathrm{E}-08$ \\
\hline LG9.40.12.80B.7 & & $7.94 \mathrm{E}-05$ & 11.1 & 0.096 & $1.93 E-03$ & 241 & $2.78 \mathrm{E}-01$ & 193 & 3.01E-01 & 1,400 & $3.02 \mathrm{E}-01$ & 1,530 & $4.05 E-01$ & $5.22 \mathrm{E}-08$ \\
\hline LG9.40.12.80B.9 & & 7.88E-05 & 15.7 & 0.095 & $1.92 \mathrm{E}-03$ & 241 & $2.76 \mathrm{E}-01$ & $\begin{array}{l}{[75.6]} \\
58^{\star}\end{array}$ & $1.17 E-01$ & 1,180 & $2.54 \mathrm{E}-01$ & 1,270 & 3.34E-01 & 1.09E-07 \\
\hline LG9.40.12.80B.11 & & $7.91 \mathrm{E}-05$ & 20.7 & 0.094 & $1.90 \mathrm{E}-03$ & 259 & $3.01 \mathrm{E}-01$ & $\begin{array}{l}{[75.6]} \\
70^{*}\end{array}$ & 1.19E-01 & 1,300 & 2.83E-01 & 1,460 & 3.89E-01 & $1.36 \mathrm{E}-07$ \\
\hline
\end{tabular}




\begin{tabular}{|c|c|c|c|c|c|c|c|c|c|c|c|c|c|c|}
\hline Sample ID & $\begin{array}{l}\text { Influent } \\
\text { [Si] } \\
\text { (ppm) }\end{array}$ & $\begin{array}{l}\text { Flow } \\
\text { Rate, } \mathrm{q} \\
\left(\mathrm{m}^{3} \mathrm{~d}^{-1}\right)\end{array}$ & $\begin{array}{l}\text { Time } \\
\text { (d) }\end{array}$ & $\begin{array}{l}\text { Glass } \\
\text { Mass } \\
\text { (g) }\end{array}$ & $\begin{array}{l}\text { Surface } \\
\text { Area, S } \\
\left(\mathrm{m}^{2}\right)\end{array}$ & $\begin{array}{l}{[\mathrm{Al}]} \\
\left(\mu \mathrm{g} \mathrm{L}^{-1}\right)\end{array}$ & $\begin{array}{l}\text { Al Norm. } \\
\text { Diss. Rate } \\
\left(\mathrm{g} \mathrm{m}^{-2} \mathrm{~d}^{-1}\right)\end{array}$ & $\begin{array}{l}{[B]} \\
\left(\mu g L^{-1}\right)\end{array}$ & $\begin{array}{l}\text { B Norm. } \\
\text { Diss. Rate } \\
\left(\mathrm{g} \mathrm{m}^{-2} \mathrm{~d}^{-1}\right)\end{array}$ & $\begin{array}{l}{[\mathrm{Si}]} \\
\left(\mu \mathrm{g} \mathrm{L}^{-1}\right)\end{array}$ & $\begin{array}{l}\text { Si Norm. } \\
\text { Diss. Rate } \\
\left(\mathrm{g} \mathrm{m}^{-2} \mathrm{~d}^{-1}\right)\end{array}$ & $\begin{array}{l}{[\mathrm{Na}]} \\
(\mu \mathrm{gg} \mathrm{L} \\
\left.{ }^{1}\right)\end{array}$ & $\begin{array}{l}\text { Na Norm. } \\
\text { Diss. Rate, } \\
\mathrm{r} \\
\left(\mathrm{g} \mathrm{m}^{-2} \mathrm{~d}^{-1}\right)\end{array}$ & $\begin{array}{l}\mathrm{r}_{\mathrm{IEX}} \\
\left(\mathrm{mol} \mathrm{Na} \mathrm{No}^{-1}\right)\end{array}$ \\
\hline LG9.40.12.80B.13 & & $8.08 \mathrm{E}-05$ & 25.0 & 0.093 & $1.89 \mathrm{E}-03$ & 263 & $3.14 \mathrm{E}-01$ & 77 & $1.25 \mathrm{E}-01$ & 1,310 & $2.93 \mathrm{E}-01$ & 1,450 & $3.98 \mathrm{E}-01$ & $1.37 \mathrm{E}-07$ \\
\hline LG9.40.12.80B.15 & & 8.01E-05 & 29.7 & 0.092 & $1.88 \mathrm{E}-03$ & 253 & 3.02E-01 & $\begin{array}{l}{[75.6]} \\
55^{\star}\end{array}$ & $1.22 \mathrm{E}-01$ & 1,250 & $2.80 \mathrm{E}-01$ & 1,440 & $3.94 \mathrm{E}-01$ & $1.37 \mathrm{E}-07$ \\
\hline LG9.40.12.80B.17 & & $7.98 \mathrm{E}-05$ & 34.8 & 0.091 & $1.86 \mathrm{E}-03$ & 222 & 2.66E-01 & $\begin{array}{l}{[75.6]} \\
37^{\star}\end{array}$ & $1.23 \mathrm{E}-01$ & 1,130 & $2.54 \mathrm{E}-01$ & 1,240 & $3.41 \mathrm{E}-01$ & 1.10E-07 \\
\hline LG9.40.12.80B.18 & & 7.93E-05 & 36.7 & 0.090 & $1.85 \mathrm{E}-03$ & 253 & 3.02E-01 & $\begin{array}{l}{[75.6]} \\
57^{*}\end{array}$ & $1.22 \mathrm{E}-01$ & 1,260 & $2.82 \mathrm{E}-01$ & 1,420 & $3.90 \mathrm{E}-01$ & $1.35 \mathrm{E}-07$ \\
\hline \multicolumn{15}{|c|}{ LG9 $70^{\circ} \mathrm{C}$ pH 9 Reactor $\# 1$} \\
\hline LG9.70.9.20.Blank1 & & $2.01 \mathrm{E}-05$ & --- & --- & --- & [49.4] & --- & [75.6] & --- & [164] & --- & [134] & --- & --- \\
\hline LG9.70.9.20.Blank2 & & $1.19 \mathrm{E}-05$ & --- & --- & --- & 127 & --- & {$[75.6]$} & --- & [164] & --- & [134] & --- & --- \\
\hline LG9.70.9.20.Blank3 & & $1.79 \mathrm{E}-05$ & --- & --- & --- & [49.4] & --- & [75.6] & --- & [164] & --- & [134] & --- & --- \\
\hline LG9.70.9.20.1 & & $8.34 \mathrm{E}-06$ & 2 & 0.397 & $7.83 \mathrm{E}-03$ & 391 & $1.16 \mathrm{E}-02$ & 1,700 & $6.85 \mathrm{E}-02$ & 2,380 & $1.33 \mathrm{E}-02$ & 12,400 & $8.47 \mathrm{E}-02$ & $8.19 \mathrm{E}-09$ \\
\hline LG9.70.9.20.2 & & $8.60 \mathrm{E}-06$ & 4 & 0.395 & $7.80 \mathrm{E}-03$ & 691 & $2.13 \mathrm{E}-02$ & 2,630 & $1.10 \mathrm{E}-01$ & 3,950 & $2.28 \mathrm{E}-02$ & 18,000 & $1.27 \mathrm{E}-01$ & $8.90 \mathrm{E}-09$ \\
\hline LG9.70.9.20.3 & & $7.90 \mathrm{E}-06$ & 7 & 0.393 & $7.77 \mathrm{E}-03$ & 768 & $2.18 \mathrm{E}-02$ & 2,800 & $1.08 \mathrm{E}-01$ & 4,340 & $2.31 \mathrm{E}-02$ & 19,700 & $1.29 \mathrm{E}-01$ & $1.05 \mathrm{E}-08$ \\
\hline LG9.70.9.20.5 & & $7.60 \mathrm{E}-06$ & 11 & 0.389 & $7.72 \mathrm{E}-03$ & 870 & $2.40 \mathrm{E}-02$ & 3,040 & $1.13 \mathrm{E}-01$ & 4,990 & $2.58 \mathrm{E}-02$ & 20,000 & $1.26 \mathrm{E}-01$ & $6.62 \mathrm{E}-09$ \\
\hline LG9.70.9.20.7 & & $6.48 \mathrm{E}-06$ & 16 & 0.385 & $7.67 \mathrm{E}-03$ & 927 & $2.19 \mathrm{E}-02$ & 3,130 & $1.00 \mathrm{E}-01$ & 5,320 & $2.36 \mathrm{E}-02$ & 19,900 & $1.08 \mathrm{E}-01$ & $3.96 \mathrm{E}-09$ \\
\hline LG9.70.9.20.9 & & $7.72 \mathrm{E}-06$ & 21 & 0.381 & $7.61 \mathrm{E}-03$ & 888 & $2.52 \mathrm{E}-02$ & 2,920 & $1.12 \mathrm{E}-01$ & 5,000 & $2.66 \mathrm{E}-02$ & 18,100 & $1.18 \mathrm{E}-01$ & $2.91 \mathrm{E}-09$ \\
\hline LG9.70.9.20.10 & & $7.68 \mathrm{E}-06$ & 23 & 0.380 & $7.60 \mathrm{E}-03$ & 856 & $2.42 \mathrm{E}-02$ & 2,700 & $1.03 \mathrm{E}-01$ & 4,780 & $2.53 \mathrm{E}-02$ & 16,400 & $1.06 \mathrm{E}-01$ & $1.58 \mathrm{E}-09$ \\
\hline LG9.70.9.20.11 & & 7.42E-06 & 24 & 0.379 & $7.59 \mathrm{E}-03$ & 799 & $2.18 \mathrm{E}-02$ & 2,540 & $9.40 \mathrm{E}-02$ & 4,550 & $2.33 \mathrm{E}-02$ & 16,400 & $1.03 E-01$ & 4.51E-09 \\
\hline \multicolumn{15}{|c|}{ LG9 $70^{\circ} \mathrm{C} \mathrm{pH} 9$ Reactor $\# 2$} \\
\hline LG9.70.9.40.Blank1 & & 3.72E-05 & --- & --- & --- & [49.4] & --- & [75.6] & --- & [164] & --- & [134] & --- & --- \\
\hline LG9.70.9.40.Blank2 & & $1.85 \mathrm{E}-05$ & -.- & --- & --- & [49.4] & --- & [75.6] & --- & [164] & --- & [134] & --- & --- \\
\hline LG9.70.9.40.Blank3 & & 3.63E-05 & --- & --- & --- & [49.4] & --- & [75.6] & --- & [164] & --- & [134] & --- & --- \\
\hline LG9.70.9.40.1 & & $2.00 \mathrm{E}-05$ & 2 & 0.151 & $2.97 \mathrm{E}-03$ & 117 & $2.20 \mathrm{E}-02$ & 493 & $1.26 \mathrm{E}-01$ & 664 & $2.34 \mathrm{E}-02$ & 3,570 & $1.54 \mathrm{E}-01$ & $1.44 \mathrm{E}-08$ \\
\hline LG9.70.9.40.2 & & $1.98 \mathrm{E}-05$ & 4 & 0.149 & 2.95E-03 & 215 & 4.02E-02 & 810 & 2.05E-01 & 1,240 & 4.35E-02 & 5,290 & 2.27E-01 & $1.12 \mathrm{E}-08$ \\
\hline LG9.70.9.40.3 & & $1.96 \mathrm{E}-05$ & 7 & 0.147 & $2.93 \mathrm{E}-03$ & 242 & $4.51 \mathrm{E}-02$ & 787 & $1.99 \mathrm{E}-01$ & 1,400 & $4.90 \mathrm{E}-02$ & 5,080 & $2.18 \mathrm{E}-01$ & $9.51 \mathrm{E}-09$ \\
\hline LG9.70.9.40.5 & & 2.01E-05 & 11 & 0.146 & $2.90 \mathrm{E}-03$ & 230 & $4.46 \mathrm{E}-02$ & 637 & $1.67 \mathrm{E}-01$ & 1,340 & 4.87E-02 & 3,900 & $1.74 \mathrm{E}-01$ & $3.25 \mathrm{E}-09$ \\
\hline LG9.70.9.40.7 & & $2.12 \mathrm{E}-05$ & 16 & 0.144 & $2.88 \mathrm{E}-03$ & 205 & $4.22 \mathrm{E}-02$ & 522 & $1.46 \mathrm{E}-01$ & 1,220 & $4.71 \mathrm{E}-02$ & 3,240 & $1.53 \mathrm{E}-01$ & $3.88 \mathrm{E}-09$ \\
\hline LG9.70.9.40.9 & & $2.05 \mathrm{E}-05$ & 21 & 0.142 & $2.86 \mathrm{E}-03$ & 181 & 3.63E-02 & 441 & $1.20 \mathrm{E}-01$ & 1,090 & $4.10 \mathrm{E}-02$ & 2,900 & $1.33 \mathrm{E}-01$ & $6.96 \mathrm{E}-09$ \\
\hline LG9.70.9.40.10 & & $1.99 \mathrm{E}-05$ & 23 & 0.141 & $2.85 \mathrm{E}-03$ & 174 & $3.39 \mathrm{E}-02$ & 423 & $1.12 \mathrm{E}-01$ & 1,070 & $3.91 \mathrm{E}-02$ & 2,710 & $1.21 \mathrm{E}-01$ & $4.88 \mathrm{E}-09$ \\
\hline LG9.70.9.40.11 & & $2.05 E-05$ & 24 & 0.141 & $2.85 \mathrm{E}-03$ & 192 & $3.87 \mathrm{E}-02$ & 448 & $1.22 \mathrm{E}-01$ & 1,140 & $4.31 \mathrm{E}-02$ & 2,560 & $1.19 \mathrm{E}-01$ & $<0$ \\
\hline \multicolumn{15}{|c|}{ LG9 $70^{\circ} \mathrm{C} \mathrm{pH} 9$ Reactor $\# 3$} \\
\hline LG9.70.9.80A.Blank1 & & 7.44E-05 & --- & --- & -.- & [49.4] & --- & [75.6] & --- & [164] & --. & [134] & --. & -.- \\
\hline LG9.70.9.80A.Blank2 & & 3.52E-05 & --- & --- & --- & [49.4] & --- & {$[75.6]$} & --- & [164] & --- & [134] & --- & --- \\
\hline LG9.70.9.80A.Blank3 & & $7.25 \mathrm{E}-05$ & --- & --- & --- & [49.4] & --- & [75.6] & --- & [164] & --- & [134] & --- & -.- \\
\hline LG9.70.9.80A.1 & & $3.30 \mathrm{E}-05$ & 2 & 0.050 & $9.98 \mathrm{E}-04$ & 51 & $4.69 \mathrm{E}-02$ & 269 & $3.36 \mathrm{E}-01$ & 321 & $5.56 \mathrm{E}-02$ & 1,860 & $3.94 \mathrm{E}-01$ & $2.93 \mathrm{E}-08$ \\
\hline LG9.70.9.80A.2 & & $3.81 \mathrm{E}-05$ & 4 & 0.049 & $9.83 \mathrm{E}-04$ & 66 & $7.10 \mathrm{E}-02$ & 304 & $4.46 \mathrm{E}-01$ & 412 & $8.36 \mathrm{E}-02$ & 1,970 & $4.90 \mathrm{E}-01$ & $2.23 \mathrm{E}-08$ \\
\hline LG9.70.9.80A.3 & & $3.82 \mathrm{E}-05$ & 7 & 0.048 & $9.68 \mathrm{E}-04$ & 83 & $9.20 \mathrm{E}-02$ & 279 & $4.17 \mathrm{E}-01$ & 438 & $9.06 \mathrm{E}-02$ & 1,600 & $4.05 \mathrm{E}-01$ & $<0$ \\
\hline LG9.70.9.80A.5 & & 3.79E-05 & 11 & 0.047 & $9.51 \mathrm{E}-04$ & 61 & $6.84 \mathrm{E}-02$ & 220 & $3.32 \mathrm{E}-01$ & 394 & $8.24 \mathrm{E}-02$ & 1,250 & $3.20 \mathrm{E}-01$ & $<0$ \\
\hline LG9.70.9.80A.7 & & 3.81E-05 & 16 & 0.046 & $9.35 \mathrm{E}-04$ & 54 & $6.17 \mathrm{E}-02$ & 181 & $2.79 \mathrm{E}-01$ & 352 & $7.51 \mathrm{E}-02$ & 1,050 & $2.74 \mathrm{E}-01$ & $<0$ \\
\hline LG9.70.9.80A.9 & & $3.84 \mathrm{E}-05$ & 21 & 0.044 & $9.18 \mathrm{E}-04$ & 51 & $6.00 \mathrm{E}-02$ & 169 & $2.67 \mathrm{E}-01$ & 335 & 7.33E-02 & 916 & $2.46 \mathrm{E}-01$ & $<0$ \\
\hline LG9.70.9.80A.10 & & $3.78 \mathrm{E}-05$ & 23 & 0.044 & $9.13 E-04$ & 51 & $5.84 \mathrm{E}-02$ & 162 & $2.54 \mathrm{E}-01$ & 332 & 7.20E-02 & 883 & $2.35 \mathrm{E}-01$ & $<0$ \\
\hline LG9.70.9.80A.11 & & 3.75E-05 & 24 & 0.044 & $9.12 \mathrm{E}-04$ & 50 & $5.73 \mathrm{E}-02$ & 144 & $2.24 \mathrm{E}-01$ & 323 & $6.96 \mathrm{E}-02$ & 846 & $2.23 \mathrm{E}-01$ & $<0$ \\
\hline \multicolumn{15}{|c|}{ LG9 $70^{\circ} \mathrm{C} \mathrm{pH} 9$ Reactor $\# 4$} \\
\hline
\end{tabular}

B. 16 


\begin{tabular}{|c|c|c|c|c|c|c|c|c|c|c|c|c|c|c|}
\hline Sample ID & $\begin{array}{l}\text { Influent } \\
{[\mathrm{Si}]} \\
(\mathrm{ppm})\end{array}$ & $\begin{array}{l}\text { Flow } \\
\text { Rate, q } \\
\left(\mathrm{m}^{3} \mathrm{~d}^{-1}\right)\end{array}$ & $\begin{array}{l}\text { Time } \\
\text { (d) }\end{array}$ & $\begin{array}{l}\text { Glass } \\
\text { Mass } \\
\text { (g) }\end{array}$ & $\begin{array}{l}\text { Surface } \\
\text { Area, S } \\
\left(\mathrm{m}^{2}\right)\end{array}$ & $\begin{array}{l}{[\mathrm{Al}]} \\
\left(\mu \mathrm{L} \mathrm{L}^{-1}\right)\end{array}$ & $\begin{array}{l}\text { Al Norm. } \\
\text { Diss. Rate } \\
\left(\mathrm{g} \mathrm{m}^{-2} \mathrm{~d}^{-1}\right)\end{array}$ & $\begin{array}{l}{[\mathrm{B}]} \\
\left(\mu \mathrm{g} \mathrm{L}^{-1}\right)\end{array}$ & $\begin{array}{l}\text { B Norm. } \\
\text { Diss. Rate } \\
\left(\mathrm{g} \mathrm{m}^{-2} \mathrm{~d}^{-1}\right)\end{array}$ & $\begin{array}{l}{[\mathrm{Si}]} \\
\left(\mu \mathrm{g} \mathrm{L}^{-1}\right)\end{array}$ & $\begin{array}{l}\text { Si Norm. } \\
\text { Diss. Rate } \\
\left(\mathrm{g} \mathrm{m}^{-2} \mathrm{~d}^{-1}\right)\end{array}$ & $\begin{array}{l}{[\mathrm{Na}]} \\
(\mu \mathrm{g} \mathrm{L} \\
\left.{ }^{1}\right)\end{array}$ & $\begin{array}{l}\text { Na Norm. } \\
\text { Diss. Rate, } \\
\mathrm{r} \\
\left(\mathrm{g} \mathrm{m}^{-2} \mathrm{~d}^{-1}\right)\end{array}$ & $\begin{array}{l}\mathrm{r}_{\mathrm{IEX}} \\
\left(\mathrm{mol} \mathrm{Na}^{-2} \mathrm{~s}^{-1}\right)\end{array}$ \\
\hline LG9.70.9.80B.Blank1 & & $7.51 \mathrm{E}-05$ & --- & --- & --- & [49.4] & --- & {$[75.6]$} & --- & [164] & --- & [134] & --- & --- \\
\hline LG9.70.9.80B.Blank2 & & $3.61 \mathrm{E}-05$ & --- & --- & --- & [49.4] & --- & {$[75.6]$} & --- & [164] & -- & [134] & --- & --- \\
\hline LG9.70.9.80B.Blank3 & & $7.26 \mathrm{E}-05$ & --- & --- & --- & [49.4] & -.- & {$[75.6]$} & --- & [164] & -- & [134] & --- & -.- \\
\hline LG9.70.9.80B.1 & & $7.51 \mathrm{E}-05$ & 2 & 0.023 & $4.62 \mathrm{E}-04$ & [49.4] & $2.25 \mathrm{E}-01$ & 108 & $6.65 \mathrm{E}-01$ & 165 & 1.41E-01 & 615 & $6.42 \mathrm{E}-01$ & $<0$ \\
\hline LG9.70.9.80B.2 & & 7.76E-05 & 4 & 0.023 & 4.54E-04 & [49.4] & $2.36 \mathrm{E}-01$ & 93 & $6.00 \mathrm{E}-01$ & $\begin{array}{l}{[164]} \\
160^{*}\end{array}$ & 1.47E-01 & 575 & $6.31 \mathrm{E}-01$ & $1.58 \mathrm{E}-08$ \\
\hline LG9.70.9.80B.3 & & 7.77E-05 & 7 & 0.022 & $4.45 E-04$ & {$[49.4]$} & $2.41 E-01$ & 81 & $5.32 \mathrm{E}-01$ & 170 & 1.56E-01 & 398 & $4.46 \mathrm{E}-01$ & $<0$ \\
\hline LG9.70.9.80B.5 & & 7.75E-05 & 11 & 0.021 & $4.32 E-04$ & {$[49.4]$} & 2.47E-01 & $\begin{array}{l}{[75.6]} \\
59^{\star}\end{array}$ & $5.13 E-01$ & $\begin{array}{l}{[164]} \\
153^{*}\end{array}$ & 1.54E-01 & 272 & $3.13 E-01$ & $<0$ \\
\hline LG9.70.9.80B.7 & & 7.79E-05 & 16 & 0.020 & $4.18 \mathrm{E}-04$ & {$[49.4]$} & $2.57 E-01$ & $\begin{array}{l}{[75.6]} \\
55^{\star}\end{array}$ & $5.33 \mathrm{E}-01$ & $\begin{array}{l}{[164]} \\
152^{\star}\end{array}$ & 1.60E-01 & 208 & $2.49 E-01$ & $<0$ \\
\hline LG9.70.9.80B.9 & & $7.80 \mathrm{E}-05$ & 21 & 0.019 & $4.02 \mathrm{E}-04$ & [49.4] & $2.68 \mathrm{E}-01$ & $\begin{array}{l}{[75.6]} \\
48^{\star}\end{array}$ & $5.55 \mathrm{E}-01$ & $\begin{array}{l}{[164]} \\
148^{*}\end{array}$ & 1.67E-01 & 191 & $2.38 \mathrm{E}-01$ & $<0$ \\
\hline LG9.70.9.80B.10 & & $7.83 \mathrm{E}-05$ & 23 & 0.018 & $3.96 \mathrm{E}-04$ & [49.4] & $2.73 \mathrm{E}-01$ & $\begin{array}{l}{[75.6]} \\
41^{\star}\end{array}$ & $5.65 \mathrm{E}-01$ & $\begin{array}{l}{[164]} \\
134^{\star}\end{array}$ & $1.70 \mathrm{E}-01$ & 170 & $2.15 \mathrm{E}-01$ & $<0$ \\
\hline LG9.70.9.80B.11 & & $7.68 \mathrm{E}-05$ & 24 & 0.018 & $3.94 \mathrm{E}-04$ & [49.4] & 2.69E-01 & $\begin{array}{l}{[75.6]} \\
38^{\star}\end{array}$ & $5.57 \mathrm{E}-01$ & $\begin{array}{l}{[164]} \\
141^{\star}\end{array}$ & 1.67E-01 & 161 & $2.01 E-01$ & $<0$ \\
\hline \multicolumn{15}{|c|}{ LG9 $70^{\circ} \mathrm{C} \mathrm{pH} 10$ Reactor $\# 1$} \\
\hline LG9.70.10.20.Blank1 & & $1.77 \mathrm{E}-05$ & --- & --- & --- & [49.4] & --- & {$[75.6]$} & --- & [164] & --- & [134] & --- & --- \\
\hline LG9.70.10.20.Blank2 & & $1.86 \mathrm{E}-05$ & --- & --- & --- & [49.4] & --- & {$[75.6]$} & --- & [164] & --- & [134] & --- & -.- \\
\hline LG9.70.10.20.Blank3 & & $1.81 \mathrm{E}-05$ & --- & --- & --- & [49.4] & --- & {$[75.6]$} & --- & [164] & --- & [134] & --- & --- \\
\hline LG9.70.10.20.1 & & $7.59 \mathrm{E}-06$ & 2 & 0.400 & $7.87 \mathrm{E}-03$ & 648 & $1.75 \mathrm{E}-02$ & 477 & $1.74 \mathrm{E}-02$ & 3,760 & 1.90E-02 & 5,500 & $3.40 \mathrm{E}-02$ & 8.37E-09 \\
\hline LG9.70.10.20.2 & & $7.45 \mathrm{E}-06$ & 4 & 0.399 & 7.87E-03 & 1,200 & $3.18 \mathrm{E}-02$ & 915 & $3.28 \mathrm{E}-02$ & 7,120 & 3.53E-02 & 9,010 & $5.48 \mathrm{E}-02$ & $1.11 \mathrm{E}-08$ \\
\hline LG9.70.10.20.3 & & $6.63 \mathrm{E}-06$ & 7 & 0.399 & $7.86 \mathrm{E}-03$ & 1,270 & $3.00 \mathrm{E}-02$ & 987 & $3.15 \mathrm{E}-02$ & 7,630 & 3.37E-02 & 9,310 & $5.04 \mathrm{E}-02$ & $9.52 \mathrm{E}-09$ \\
\hline LG9.70.10.20.5 & & $6.56 \mathrm{E}-06$ & 11 & 0.398 & $7.84 \mathrm{E}-03$ & 1,330 & $3.11 \mathrm{E}-02$ & 1,030 & $3.26 \mathrm{E}-02$ & 7,960 & 3.49E-02 & 9,110 & $4.89 \mathrm{E}-02$ & $8.22 \mathrm{E}-09$ \\
\hline LG9.70.10.20.7 & & $6.83 \mathrm{E}-06$ & 16 & 0.396 & $7.83 \mathrm{E}-03$ & 1,290 & $3.15 \mathrm{E}-02$ & 994 & $3.28 \mathrm{E}-02$ & 7,770 & 3.56E-02 & 8,150 & $4.57 \mathrm{E}-02$ & $6.46 \mathrm{E}-09$ \\
\hline LG9.70.10.20.9 & & $7.10 \mathrm{E}-06$ & 21 & 0.395 & $7.81 \mathrm{E}-03$ & 1,320 & $3.36 \mathrm{E}-02$ & 1,010 & 3.48E-02 & 7,910 & 3.77E-02 & 8,020 & $4.68 \mathrm{E}-02$ & $6.08 \mathrm{E}-09$ \\
\hline LG9.70.10.20.10 & & $7.09 \mathrm{E}-06$ & 23 & 0.395 & $7.80 \mathrm{E}-03$ & 1,280 & $3.25 \mathrm{E}-02$ & 968 & 3.33E-02 & 7,570 & $3.61 \mathrm{E}-02$ & 7,320 & $4.27 \mathrm{E}-02$ & $4.74 \mathrm{E}-09$ \\
\hline LG9.70.10.20.11 & & $7.39 \mathrm{E}-06$ & 24 & 0.394 & $7.80 \mathrm{E}-03$ & 1,240 & $3.28 \mathrm{E}-02$ & 951 & $3.41 \mathrm{E}-02$ & 7,450 & 3.70E-02 & 7,130 & $4.34 \mathrm{E}-02$ & 4.67E-09 \\
\hline \multicolumn{15}{|c|}{ LG9 $70^{\circ} \mathrm{C}$ pH 10 Reactor \#2 } \\
\hline LG9.70.10.40.Blank1 & & 3.82E-05 & --- & --- & --- & {$[49.4]$} & --- & {$[75.6]$} & --- & [164] & --- & [134] & --- & --- \\
\hline LG9.70.10.40.Blank2 & & 3.97E-05 & --- & --- & --- & {$[49.4]$} & --- & {$[75.6]$} & --- & [164] & --- & [134] & --- & --- \\
\hline LG9.70.10.40.Blank3 & & 3.90E-05 & --- & --- & --- & [49.4] & --- & {$[75.6]$} & --- & [164] & --- & [134] & --- & --- \\
\hline LG9.70.10.40.1 & & $1.95 \mathrm{E}-05$ & 2 & 0.149 & $2.94 \mathrm{E}-03$ & 390 & $7.24 \mathrm{E}-02$ & 272 & $6.83 \mathrm{E}-02$ & 2,240 & 7.79E-02 & 2,350 & $1.00 \mathrm{E}-01$ & $1.60 \mathrm{E}-08$ \\
\hline LG9.70.10.40.2 & & 2.02E-05 & 4 & 0.148 & $2.93 \mathrm{E}-03$ & 627 & $1.21 \mathrm{E}-01$ & 457 & $1.19 \mathrm{E}-01$ & 3,650 & 1.32E-01 & 3,310 & $1.47 \mathrm{E}-01$ & $1.37 \mathrm{E}-08$ \\
\hline LG9.70.10.40.3 & & $2.01 \mathrm{E}-05$ & 7 & 0.147 & $2.92 \mathrm{E}-03$ & 647 & $1.25 \mathrm{E}-01$ & 493 & $1.29 \mathrm{E}-01$ & 3,750 & $1.36 \mathrm{E}-01$ & 3,090 & $1.37 \mathrm{E}-01$ & 4.10E-09 \\
\hline LG9.70.10.40.5 & & $2.05 \mathrm{E}-05$ & 11 & 0.146 & $2.90 \mathrm{E}-03$ & 616 & 1.22E-01 & 445 & $1.19 \mathrm{E}-01$ & 3,600 & 1.34E-01 & 2,700 & $1.23 \mathrm{E}-01$ & $1.76 \mathrm{E}-09$ \\
\hline LG9.70.10.40.7 & & $2.10 \mathrm{E}-05$ & 16 & 0.144 & $2.88 \mathrm{E}-03$ & 573 & $1.17 \mathrm{E}-01$ & 408 & $1.13 \mathrm{E}-01$ & 3,320 & $1.27 \mathrm{E}-01$ & 2,460 & $1.15 \mathrm{E}-01$ & $1.30 \mathrm{E}-09$ \\
\hline LG9.70.10.40.9 & & $1.96 \mathrm{E}-05$ & 21 & 0.143 & $2.86 \mathrm{E}-03$ & 603 & $1.15 \mathrm{E}-01$ & 421 & $1.09 \mathrm{E}-01$ & 3,480 & 1.25E-01 & 2,580 & $1.13 \mathrm{E}-01$ & $2.18 \mathrm{E}-09$ \\
\hline LG9.70.10.40.10 & & $1.98 \mathrm{E}-05$ & 23 & 0.142 & $2.85 \mathrm{E}-03$ & 608 & $1.18 \mathrm{E}-01$ & 439 & $1.15 \mathrm{E}-01$ & 3,570 & $1.30 \mathrm{E}-01$ & 2,590 & $1.16 \mathrm{E}-01$ & $5.16 \mathrm{E}-11$ \\
\hline LG9.70.10.40.11 & & $1.98 \mathrm{E}-05$ & 24 & 0.142 & $2.85 \mathrm{E}-03$ & 618 & $1.20 \mathrm{E}-01$ & 439 & $1.16 \mathrm{E}-01$ & 3,600 & 1.32E-01 & 2,640 & $1.18 \mathrm{E}-01$ & $1.18 \mathrm{E}-09$ \\
\hline \multicolumn{15}{|c|}{ LG9 $70^{\circ} \mathrm{C} \mathrm{pH} 10$ Reactor $\# 3$} \\
\hline LG9.70.10.80A.Blank1 & & $7.56 \mathrm{E}-05$ & --- & --- & --- & {$[49.4]$} & --- & {$[75.6]$} & --- & {$[164]$} & --- & [134] & --- & --- \\
\hline
\end{tabular}




\begin{tabular}{|c|c|c|c|c|c|c|c|c|c|c|c|c|c|c|}
\hline Sample ID & $\begin{array}{l}\text { Influent } \\
\text { [Si] } \\
\text { (ppm) }\end{array}$ & $\begin{array}{l}\text { Flow } \\
\text { Rate, } \mathrm{q} \\
\left(\mathrm{m}^{3} \mathrm{~d}^{-1}\right)\end{array}$ & $\begin{array}{l}\text { Time } \\
\text { (d) }\end{array}$ & $\begin{array}{l}\text { Glass } \\
\text { Mass } \\
\text { (g) }\end{array}$ & $\begin{array}{l}\text { Surface } \\
\text { Area, S } \\
\left(\mathrm{m}^{2}\right)\end{array}$ & $\begin{array}{l}{[\mathrm{Al}]} \\
\left(\mu \mathrm{g} \mathrm{L}^{-1}\right)\end{array}$ & $\begin{array}{l}\text { Al Norm. } \\
\text { Diss. Rate } \\
\left(\mathrm{g} \mathrm{m}^{-2} \mathrm{~d}^{-1}\right)\end{array}$ & $\begin{array}{l}{[\mathrm{B}]} \\
\left(\mu \mathrm{g} \mathrm{L}^{-1}\right)\end{array}$ & $\begin{array}{l}\text { B Norm. } \\
\text { Diss. Rate } \\
\left(\mathrm{g} \mathrm{m}^{-2} \mathrm{~d}^{-1}\right)\end{array}$ & $\begin{array}{l}{[\mathrm{Si}]} \\
\left(\mu \mathrm{g} \mathrm{L}^{-1}\right)\end{array}$ & $\begin{array}{l}\text { Si Norm. } \\
\text { Diss. Rate } \\
\left(\mathrm{g} \mathrm{m}^{-2} \mathrm{~d}^{-1}\right)\end{array}$ & $\begin{array}{l}{[\mathrm{Na}]} \\
(\mu \mathrm{g} \mathrm{L} \\
\left.{ }^{1}\right)\end{array}$ & $\begin{array}{l}\text { Na Norm. } \\
\text { Diss. Rate, } \\
\mathrm{r} \\
\left(\mathrm{g} \mathrm{m}^{-2} \mathrm{~d}^{-1}\right)\end{array}$ & $\begin{array}{l}\mathrm{r}_{\mathrm{IEX}} \\
\left(\mathrm{mol} \mathrm{Na} \mathrm{No}^{-1}\right)\end{array}$ \\
\hline LG9.70.10.80A.Blank2 & & $7.66 \mathrm{E}-05$ & --- & --- & --- & [49.4] & --- & {$[75.6]$} & --- & [164] & --- & [134] & --- & --- \\
\hline LG9.70.10.80A.Blank3 & & 7.67E-05 & --- & --- & --- & [49.4] & --- & [75.6] & --- & [164] & --- & [134] & --- & --- \\
\hline LG9.70.10.80A.1 & & $3.69 \mathrm{E}-05$ & 2 & 0.049 & $9.75 \mathrm{E}-04$ & 190 & $2.01 \mathrm{E}-01$ & 124 & $1.77 \mathrm{E}-01$ & 1,070 & $2.12 \mathrm{E}-01$ & 918 & $2.23 \mathrm{E}-01$ & $2.29 \mathrm{E}-08$ \\
\hline LG9.70.10.80A.2 & & 3.90E-05 & 4 & 0.049 & $9.64 \mathrm{E}-04$ & 299 & $3.38 \mathrm{E}-01$ & 199 & $3.04 \mathrm{E}-01$ & 1,690 & $3.58 \mathrm{E}-01$ & 1,180 & $3.06 \mathrm{E}-01$ & $9.12 \mathrm{E}-10$ \\
\hline LG9.70.10.80A.3 & & 3.92E-05 & 7 & 0.048 & $9.52 \mathrm{E}-04$ & 297 & $3.42 \mathrm{E}-01$ & 205 & $3.19 \mathrm{E}-01$ & 1,750 & $3.78 \mathrm{E}-01$ & 1,210 & $3.20 \mathrm{E}-01$ & $2.15 \mathrm{E}-10$ \\
\hline LG9.70.10.80A.5 & & $3.90 \mathrm{E}-05$ & 11 & 0.046 & $9.36 \mathrm{E}-04$ & 292 & 3.40E-01 & 198 & $3.12 \mathrm{E}-01$ & 1,680 & $3.67 \mathrm{E}-01$ & 1,230 & $3.29 \mathrm{E}-01$ & $8.46 \mathrm{E}-09$ \\
\hline LG9.70.10.80A.7 & & 3.96E-05 & 16 & 0.045 & $9.18 \mathrm{E}-04$ & 293 & 3.53E-01 & 191 & $3.12 \mathrm{E}-01$ & 1,700 & $3.84 \mathrm{E}-01$ & 1,140 & $3.16 \mathrm{E}-01$ & $1.97 \mathrm{E}-09$ \\
\hline LG9.70.10.80A.9 & & $3.89 \mathrm{E}-05$ & 21 & 0.044 & 8.97E-04 & 289 & $3.51 \mathrm{E}-01$ & 200 & $3.28 \mathrm{E}-01$ & 1,650 & $3.75 \mathrm{E}-01$ & 1,160 & $3.23 \mathrm{E}-01$ & $<0$ \\
\hline LG9.70.10.80A.10 & & 3.94E-05 & 23 & 0.043 & $8.90 \mathrm{E}-04$ & 280 & 3.47E-01 & 196 & $3.28 \mathrm{E}-01$ & 1,580 & $3.67 \mathrm{E}-01$ & 1,100 & 3.13E-01 & $<0$ \\
\hline LG9.70.10.80A.11 & & 3.90E-05 & 24 & 0.043 & $8.88 \mathrm{E}-04$ & 264 & 3.24E-01 & 179 & $2.97 \mathrm{E}-01$ & 1,530 & $3.52 \mathrm{E}-01$ & 1,010 & $2.85 \mathrm{E}-01$ & $<0$ \\
\hline \multicolumn{15}{|c|}{ LG9 $70^{\circ} \mathrm{C} \mathrm{pH} 10$ Reactor $\# 4$} \\
\hline LG9.70.10.80B.Blank1 & & $7.55 \mathrm{E}-05$ & --- & --- & --- & [49.4] & --- & [75.6] & --- & [164] & --- & [134] & --- & -- \\
\hline LG9.70.10.80B.Blank2 & & 7.63E-05 & --- & --- & --- & [49.4] & --- & [75.6] & --- & [164] & --- & [134] & --- & --- \\
\hline LG9.70.10.80B.Blank3 & & $7.65 \mathrm{E}-05$ & --- & --- & --- & [49.4] & --- & [75.6] & --- & [164] & --- & [134] & --- & -- \\
\hline LG9.70.10.80B.1 & & 7.33E-05 & 2 & 0.025 & $4.97 \mathrm{E}-04$ & 103 & $4.25 \mathrm{E}-01$ & $\begin{array}{l}{[75.6]} \\
66^{*}\end{array}$ & 4.22E-01 & 581 & $4.49 E-01$ & 417 & $3.95 \mathrm{E}-01$ & $<0$ \\
\hline LG9.70.10.80B.2 & & 7.80E-05 & 4 & 0.025 & $4.90 \mathrm{E}-04$ & 129 & $5.74 \mathrm{E}-01$ & $\begin{array}{l}{[75.6]} \\
68^{\star}\end{array}$ & 4.55E-01 & 732 & $6.11 \mathrm{E}-01$ & 414 & 4.23E-01 & $<0$ \\
\hline LG9.70.10.80B.3 & & 7.77E-05 & 7 & 0.024 & $4.81 \mathrm{E}-04$ & 128 & $5.78 \mathrm{E}-01$ & $\begin{array}{l}{[75.6]} \\
74^{\star}\end{array}$ & $4.62 \mathrm{E}-01$ & 689 & $5.83 \mathrm{E}-01$ & 454 & $4.70 \mathrm{E}-01$ & 4.37E-09 \\
\hline LG9.70.10.80B.5 & & 7.77E-05 & 11 & 0.023 & 4.69E-04 & 125 & $5.79 \mathrm{E}-01$ & $\begin{array}{l}{[75.6]} \\
72^{\star}\end{array}$ & 4.74E-01 & 698 & $6.06 \mathrm{E}-01$ & 447 & $4.75 \mathrm{E}-01$ & $7.35 E-10$ \\
\hline LG9.70.10.80B.7 & & $7.81 \mathrm{E}-05$ & 16 & 0.022 & 4.53E-04 & 121 & $5.84 \mathrm{E}-01$ & 81 & $5.29 \mathrm{E}-01$ & 662 & $5.99 \mathrm{E}-01$ & 384 & $4.25 \mathrm{E}-01$ & $<0$ \\
\hline LG9.70.10.80B.9 & & $7.82 \mathrm{E}-05$ & 21 & 0.021 & 4.37E-04 & 112 & $5.60 \mathrm{E}-01$ & $\begin{array}{l}{[75.6]} \\
72^{\star}\end{array}$ & $5.12 \mathrm{E}-01$ & 589 & $5.53 \mathrm{E}-01$ & 372 & 4.27E-01 & $<0$ \\
\hline LG9.70.10.80B.10 & & 7.79E-05 & 23 & 0.020 & $4.32 E-04$ & 112 & $5.64 \mathrm{E}-01$ & $\begin{array}{l}{[75.6]} \\
62^{\star}\end{array}$ & $5.16 \mathrm{E}-01$ & 658 & $6.22 \mathrm{E}-01$ & 386 & 4.47E-01 & $<0$ \\
\hline LG9.70.10.80B.11 & & 7.76E-05 & 24 & 0.020 & $4.30 \mathrm{E}-04$ & 111 & $5.60 \mathrm{E}-01$ & $\begin{array}{l}{[75.6]} \\
66^{*}\end{array}$ & $5.17 E-01$ & 627 & $5.93 E-01$ & 358 & $4.15 \mathrm{E}-01$ & $<0$ \\
\hline \multicolumn{15}{|c|}{ LG9 $70^{\circ} \mathrm{C} \mathrm{pH} 11$ Reactor $\# 1$} \\
\hline LG9.70.11.20.Blank1 & & $1.84 \mathrm{E}-05$ & --- & --- & --- & [49.4] & --- & [75.6] & --- & [164] & --- & [134] & --- & --- \\
\hline LG9.70.11.20.Blank2 & & $2.27 \mathrm{E}-05$ & --- & --- & --- & [49.4] & --- & [75.6] & --- & [164] & -.- & [134] & --- & -.- \\
\hline LG9.70.11.20.Blank3 & & $1.82 \mathrm{E}-05$ & --- & --- & --- & [49.4] & --- & [75.6] & --- & [164] & --- & [134] & --- & --- \\
\hline LG9.70.11.20.1 & & $3.02 \mathrm{E}-06$ & 2 & 0.751 & $1.48 \mathrm{E}-02$ & 1,090 & $6.23 \mathrm{E}-03$ & 889 & $6.88 \mathrm{E}-03$ & 5,770 & $6.19 \mathrm{E}-03$ & 6,640 & $8.72 \mathrm{E}-03$ & $9.25 \mathrm{E}-10$ \\
\hline LG9.70.11.20.2 & & $1.77 \mathrm{E}-06$ & 4 & 0.750 & $1.48 \mathrm{E}-02$ & 2,550 & $8.54 \mathrm{E}-03$ & 2,120 & $9.62 \mathrm{E}-03$ & 12,800 & $8.04 \mathrm{E}-03$ & 15,900 & $1.22 \mathrm{E}-02$ & $1.32 \mathrm{E}-09$ \\
\hline LG9.70.11.20.3 & & $2.75 \mathrm{E}-06$ & 7 & 0.749 & $1.48 \mathrm{E}-02$ & 3,090 & $1.61 \mathrm{E}-02$ & 2,680 & $1.89 \mathrm{E}-02$ & 15,600 & $1.52 \mathrm{E}-02$ & 19,900 & $2.38 \mathrm{E}-02$ & $2.47 \mathrm{E}-09$ \\
\hline LG9.70.11.20.5 & & $1.63 \mathrm{E}-06$ & 11 & 0.748 & $1.47 \mathrm{E}-02$ & 4,520 & $1.40 \mathrm{E}-02$ & 4,010 & $1.68 \mathrm{E}-02$ & 23,600 & $1.37 \mathrm{E}-02$ & 22,800 & $1.62 \mathrm{E}-02$ & $<0$ \\
\hline LG9.70.11.20.8 & & $5.96 \mathrm{E}-07$ & 18 & 0.748 & $1.47 \mathrm{E}-02$ & 4,470 & $5.05 \mathrm{E}-03$ & 4,490 & $6.87 E-03$ & 23,900 & $5.07 \mathrm{E}-03$ & 29,900 & $7.76 \mathrm{E}-03$ & 4.49E-10 \\
\hline LG9.70.11.20.9 & & $5.82 \mathrm{E}-07$ & 21 & 0.748 & $1.47 \mathrm{E}-02$ & 4,400 & $4.86 \mathrm{E}-03$ & 4,550 & $6.81 \mathrm{E}-03$ & 24,000 & $4.97 \mathrm{E}-03$ & 30,000 & $7.61 \mathrm{E}-03$ & $4.06 \mathrm{E}-10$ \\
\hline LG9.70.11.20.10 & & $1.08 \mathrm{E}-05$ & 23 & 0.742 & $1.47 \mathrm{E}-02$ & 4,420 & $9.11 \mathrm{E}-02$ & 4,660 & $1.30 \mathrm{E}-01$ & 24,200 & $9.36 \mathrm{E}-02$ & 31,300 & $1.48 \mathrm{E}-01$ & $9.13 \mathrm{E}-09$ \\
\hline LG9.70.11.20.11 & & $8.28 \mathrm{E}-07$ & 24 & 0.744 & $1.47 \mathrm{E}-02$ & 4,640 & $7.31 \mathrm{E}-03$ & 5,020 & $1.07 E-02$ & 25,600 & $7.57 \mathrm{E}-03$ & 32,700 & $1.18 \mathrm{E}-02$ & $5.67 \mathrm{E}-10$ \\
\hline \multicolumn{15}{|c|}{ LG9 $70^{\circ} \mathrm{C} \mathrm{pH} 11$ Reactor \#2 } \\
\hline LG9.70.11.40.Blank1 & & 4.07E-05 & --- & --- & --- & [49.4] & --- & {$[75.6]$} & --- & [164] & --- & [134] & --- & --- \\
\hline LG9.70.11.40.Blank2 & & $5.11 \mathrm{E}-05$ & --- & --- & --- & [49.4] & --- & [75.6] & --- & [164] & --- & [134] & --- & --- \\
\hline
\end{tabular}

B.18 


\begin{tabular}{|c|c|c|c|c|c|c|c|c|c|c|c|c|c|c|}
\hline Sample ID & $\begin{array}{l}\text { Influent } \\
{[\mathrm{Si}]} \\
(\mathrm{ppm})\end{array}$ & $\begin{array}{l}\text { Flow } \\
\text { Rate, q } \\
\left(\mathrm{m}^{3} \mathrm{~d}^{-1}\right)\end{array}$ & $\begin{array}{l}\text { Time } \\
\text { (d) }\end{array}$ & $\begin{array}{l}\text { Glass } \\
\text { Mass } \\
\text { (g) }\end{array}$ & $\begin{array}{l}\text { Surface } \\
\text { Area, S } \\
\left(\mathrm{m}^{2}\right)\end{array}$ & $\begin{array}{l}{[\mathrm{Al}]} \\
\left(\mu \mathrm{L} \mathrm{L}^{-1}\right)\end{array}$ & $\begin{array}{l}\text { Al Norm. } \\
\text { Diss. Rate } \\
\left(\mathrm{g} \mathrm{m}^{-2} \mathrm{~d}^{-1}\right)\end{array}$ & $\begin{array}{l}{[\mathrm{B}]} \\
\left(\mu \mathrm{g} \mathrm{L}^{-1}\right)\end{array}$ & $\begin{array}{l}\text { B Norm. } \\
\text { Diss. Rate } \\
\left(\mathrm{g} \mathrm{m}^{-2} \mathrm{~d}^{-1}\right)\end{array}$ & $\begin{array}{l}{[\mathrm{Si}]} \\
\left(\mu \mathrm{g} \mathrm{L}^{-1}\right)\end{array}$ & $\begin{array}{l}\text { Si Norm. } \\
\text { Diss. Rate } \\
\left(\mathrm{g} \mathrm{m}^{-2} \mathrm{~d}^{-1}\right)\end{array}$ & $\begin{array}{l}{[\mathrm{Na}]} \\
(\mu \mathrm{g} \mathrm{L} \\
\left.{ }^{1}\right)\end{array}$ & $\begin{array}{l}\text { Na Norm. } \\
\text { Diss. Rate, } \\
\mathrm{r} \\
\left(\mathrm{g} \mathrm{m}^{-2} \mathrm{~d}^{-1}\right)\end{array}$ & $\begin{array}{l}\mathrm{r}_{\mathrm{IEX}} \\
\left(\mathrm{mol} \mathrm{Na} \mathrm{No}^{-1}\right)\end{array}$ \\
\hline LG9.70.11.40.Blank3 & & $4.18 \mathrm{E}-05$ & -- & --- & --- & {$[49.4]$} & --- & {$[75.6]$} & -- & [164] & --- & [134] & --- & --- \\
\hline LG9.70.11.40.1 & & $1.99 \mathrm{E}-05$ & 1 & 0.375 & $7.37 \mathrm{E}-03$ & 654 & $4.93 \mathrm{E}-02$ & 198 & $2.02 \mathrm{E}-02$ & 3,420 & 4.83E-02 & 3,390 & $5.86 \mathrm{E}-02$ & $1.94 \mathrm{E}-08$ \\
\hline LG9.70.11.40.2 & & $2.37 \mathrm{E}-05$ & 1 & 0.375 & $7.37 \mathrm{E}-03$ & [49.4] & $4.43 E-03$ & {$[75.6]$} & $9.18 \mathrm{E}-03$ & [164] & 2.76E-03 & [134] & $2.76 \mathrm{E}-03$ & $<0$ \\
\hline LG9.70.11.40.3 & & $2.58 \mathrm{E}-05$ & 2 & 0.374 & $7.36 \mathrm{E}-03$ & 1,190 & $1.16 \mathrm{E}-01$ & 968 & $1.28 \mathrm{E}-01$ & 6,190 & 1.14E-01 & 6,460 & $1.45 \mathrm{E}-01$ & 8.53E-09 \\
\hline LG9.70.11.40.4 & & $2.41 \mathrm{E}-05$ & 4 & 0.371 & $7.32 \mathrm{E}-03$ & 1,360 & $1.25 \mathrm{E}-01$ & 1,100 & $1.37 \mathrm{E}-01$ & 7,060 & $1.22 \mathrm{E}-01$ & 6,880 & $1.46 \mathrm{E}-01$ & 4.22E-09 \\
\hline LG9.70.11.40.5 & & $2.41 \mathrm{E}-05$ & 7 & 0.367 & $7.28 \mathrm{E}-03$ & 1,410 & $1.31 \mathrm{E}-01$ & 1,120 & 1.40E-01 & 7,250 & 1.26E-01 & 6,500 & $1.38 \mathrm{E}-01$ & $<0$ \\
\hline LG9.70.11.40.7 & & $2.41 \mathrm{E}-05$ & 11 & 0.363 & $7.22 \mathrm{E}-03$ & 1,410 & $1.31 \mathrm{E}-01$ & 1,120 & $1.41 \mathrm{E}-01$ & 7,250 & 1.27E-01 & 6,270 & $1.34 \mathrm{E}-01$ & $<0$ \\
\hline LG9.70.11.40.9 & & $2.30 \mathrm{E}-05$ & 16 & 0.359 & $7.16 \mathrm{E}-03$ & 1,390 & $1.25 \mathrm{E}-01$ & 1,120 & $1.36 \mathrm{E}-01$ & 7,220 & 1.22E-01 & 6,180 & $1.28 \mathrm{E}-01$ & $<0$ \\
\hline LG9.70.11.40.11 & & $2.34 \mathrm{E}-05$ & 21 & 0.353 & $7.09 \mathrm{E}-03$ & 1,390 & $1.28 \mathrm{E}-01$ & 1,140 & $1.43 \mathrm{E}-01$ & 7,300 & 1.26E-01 & 6,100 & $1.29 \mathrm{E}-01$ & $<0$ \\
\hline LG9.70.11.40.12 & & $2.01 \mathrm{E}-05$ & 23 & 0.352 & $7.07 \mathrm{E}-03$ & 1,430 & $1.13 \mathrm{E}-01$ & 1,150 & $1.23 \mathrm{E}-01$ & 7,420 & 1.10E-01 & 6,400 & $1.17 \mathrm{E}-01$ & $<0$ \\
\hline LG9.70.11.40.13 & & $2.53 \mathrm{E}-05$ & 24 & 0 & 0 & 1,410 & $1.41 \mathrm{E}-01$ & 1,130 & 1.53E-01 & 7,260 & 1.37E-01 & 6,380 & $1.47 \mathrm{E}-01$ & $<0$ \\
\hline \multicolumn{15}{|c|}{ LG9 $70^{\circ} \mathrm{C} \mathrm{pH} 11$ Reactor $\# 3$} \\
\hline LG9.70.11.80A.Blank1 & & $7.76 \mathrm{E}-05$ & --- & --- & --- & [49.4] & --- & {$[75.6]$} & --- & [164] & --- & [134] & --- & --- \\
\hline LG9.70.11.80A.Blank2 & & $7.62 \mathrm{E}-05$ & --- & --- & --- & [49.4] & --- & {$[75.6]$} & -- & [164] & --- & [134] & --- & --- \\
\hline LG9.70.11.80A.Blank3 & & $7.76 \mathrm{E}-05$ & --- & --- & --- & [49.4] & -- & {$[75.6]$} & --- & [164] & --- & [134] & --- & --- \\
\hline LG9.70.11.80A.1 & & 3.47E-05 & 1 & 0.150 & $2.95 \mathrm{E}-03$ & 374 & $1.23 \mathrm{E}-01$ & {$[75.6]$} & $3.37 \mathrm{E}-02$ & 1,970 & 1.22E-01 & 1,680 & $1.27 \mathrm{E}-01$ & $4.69 \mathrm{E}-08$ \\
\hline LG9.70.11.80A.2 & & 3.87E-05 & 1 & 0.150 & $2.95 E-03$ & {$[49.4]$} & $1.81 \mathrm{E}-02$ & {$[75.6]$} & $3.76 \mathrm{E}-02$ & [164] & 1.13E-02 & [134] & $1.13 E-02$ & $<0$ \\
\hline LG9.70.11.80A.3 & & 3.96E-05 & 2 & 0.149 & $2.94 \mathrm{E}-03$ & 607 & $2.29 \mathrm{E}-01$ & 516 & $2.64 \mathrm{E}-01$ & 3,710 & 2.62E-01 & 3,010 & $2.61 \mathrm{E}-01$ & $<0$ \\
\hline LG9.70.11.80A.5 & & 3.82E-05 & 7 & 0.144 & $2.88 \mathrm{E}-03$ & 672 & $2.50 \mathrm{E}-01$ & 520 & 2.62E-01 & 3,650 & $2.54 \mathrm{E}-01$ & 2,900 & $2.47 \mathrm{E}-01$ & $<0$ \\
\hline LG9.70.11.80A.7 & & $3.86 \mathrm{E}-05$ & 11 & 0.141 & $2.83 \mathrm{E}-03$ & 727 & $2.77 \mathrm{E}-01$ & 565 & $2.91 \mathrm{E}-01$ & 3,920 & $2.80 \mathrm{E}-01$ & 3,030 & $2.65 \mathrm{E}-01$ & $<0$ \\
\hline LG9.70.11.80A.9 & & 3.90E-05 & 16 & 0.138 & $2.79 \mathrm{E}-03$ & 604 & $2.36 \mathrm{E}-01$ & 451 & $2.39 \mathrm{E}-01$ & 3,410 & $2.50 \mathrm{E}-01$ & 2,790 & $2.50 \mathrm{E}-01$ & $5.95 \mathrm{E}-09$ \\
\hline LG9.70.11.80A.11 & & 3.95E-05 & 21 & 0.134 & $2.73 \mathrm{E}-03$ & 670 & $2.71 \mathrm{E}-01$ & 518 & $2.83 \mathrm{E}-01$ & 3,760 & $2.85 \mathrm{E}-01$ & 2,750 & $2.55 \mathrm{E}-01$ & $<0$ \\
\hline LG9.70.11.80A.12 & & 3.96E-05 & 23 & 0.133 & $2.72 \mathrm{E}-03$ & 644 & 2.62E-01 & 486 & $2.68 \mathrm{E}-01$ & 3,590 & $2.74 \mathrm{E}-01$ & 2,760 & $2.58 \mathrm{E}-01$ & $<0$ \\
\hline LG9.70.11.80A.13 & & 3.96E-05 & 24 & 0.132 & $2.71 \mathrm{E}-03$ & 681 & $2.78 \mathrm{E}-01$ & 528 & $2.92 \mathrm{E}-01$ & 3,810 & $2.92 \mathrm{E}-01$ & 2,840 & $2.66 \mathrm{E}-01$ & $<0$ \\
\hline \multicolumn{15}{|c|}{ LG9 $70{ }^{\circ} \mathrm{C} \mathrm{pH} 11$ Reactor $\# 4$} \\
\hline LG9.70.11.80B.Blank1 & & $7.50 \mathrm{E}-05$ & --- & --- & --- & [49.4] & --- & {$[75.6]$} & -- & [164] & --- & [134] & --- & --- \\
\hline LG9.70.11.80B.Blank2 & & $7.56 \mathrm{E}-05$ & --- & --- & --- & [49.4] & --- & {$[75.6]$} & --- & [164] & --- & [134] & --- & -.- \\
\hline LG9.70.11.80B.Blank3 & & 7.66E-05 & -- & --- & --- & [49.4] & --- & {$[75.6]$} & -- & [164] & --- & [134] & --- & --- \\
\hline LG9.70.11.80B.1 & & $6.84 \mathrm{E}-05$ & 1 & 0.051 & $1.01 \mathrm{E}-03$ & 111 & $2.11 \mathrm{E}-01$ & {$[75.6]$} & $1.95 \mathrm{E}-01$ & 593 & $2.11 \mathrm{E}-01$ & 433 & $1.89 \mathrm{E}-01$ & $<0$ \\
\hline LG9.70.11.80B.2 & & $8.18 \mathrm{E}-05$ & 1 & 0.051 & $1.01 \mathrm{E}-03$ & 180 & 4.09E-01 & {$[75.6]$} & $2.33 \mathrm{E}-01$ & 997 & 4.25E-01 & 696 & $3.63 \mathrm{E}-01$ & $6.58 \mathrm{E}-08$ \\
\hline LG9.70.11.80B.3 & & $7.68 \mathrm{E}-05$ & 2 & 0.051 & $1.00 \mathrm{E}-03$ & 180 & 3.87E-01 & 138 & 4.01E-01 & 1,060 & 4.27E-01 & 759 & $3.74 \mathrm{E}-01$ & $<0$ \\
\hline LG9.70.11.80B.4 & & $7.76 \mathrm{E}-05$ & 4 & 0.050 & $9.86 \mathrm{E}-04$ & 171 & $3.76 \mathrm{E}-01$ & 122 & $3.63 \mathrm{E}-01$ & 967 & 3.99E-01 & 729 & $3.68 \mathrm{E}-01$ & $2.51 \mathrm{E}-09$ \\
\hline LG9.70.11.80B.5 & & 7.76E-05 & 7 & 0.048 & $9.67 \mathrm{E}-04$ & 191 & 4.29E-01 & 147 & 4.46E-01 & 1,090 & 4.59E-01 & 710 & $3.66 \mathrm{E}-01$ & $<0$ \\
\hline LG9.70.11.80B.7 & & $7.74 \mathrm{E}-05$ & 11 & 0.047 & $9.49 \mathrm{E}-04$ & 177 & 4.03E-01 & 123 & $3.79 \mathrm{E}-01$ & 1,020 & 4.36E-01 & 756 & $3.96 \mathrm{E}-01$ & $8.16 \mathrm{E}-09$ \\
\hline LG9.70.11.80B.11 & & $7.64 \mathrm{E}-05$ & 21 & 0.043 & $8.99 \mathrm{E}-04$ & 170 & 4.04E-01 & 125 & $4.02 \mathrm{E}-01$ & 964 & 4.29E-01 & 598 & $3.26 \mathrm{E}-01$ & $<0$ \\
\hline LG9.70.11.80B.12 & & $7.65 \mathrm{E}-05$ & 23 & 0.043 & $8.90 \mathrm{E}-04$ & 182 & 4.37E-01 & 134 & 4.35E-01 & 1,040 & $4.68 \mathrm{E}-01$ & 631 & $3.48 \mathrm{E}-01$ & $<0$ \\
\hline LG9.70.11.80B.13 & & $7.64 \mathrm{E}-05$ & 24 & 0.042 & $8.87 \mathrm{E}-04$ & 177 & $4.26 \mathrm{E}-01$ & 134 & 4.37E-01 & 1,080 & 4.87E-01 & 608 & $3.36 \mathrm{E}-01$ & $<0$ \\
\hline \multicolumn{15}{|c|}{ LG9 $70^{\circ} \mathrm{C} \mathrm{pH} 12$ Reactor $\# 1$} \\
\hline LG9.70.12.20.Blank1 & & $2.28 \mathrm{E}-05$ & -- & --- & --- & [49.4] & --- & {$[75.6]$} & -- & [164] & --- & 622 & --- &.-- \\
\hline LG9.70.12.20.Blank2 & & $1.76 \mathrm{E}-05$ & -.- & --- & --- & [49.4] & --- & {$[75.6]$} & --- & [164] & --- & 593 & --- & -.- \\
\hline LG9.70.12.20.Blank3 & & $1.94 \mathrm{E}-05$ & --- & --- & --- & {$[49.4]$} & --- & {$[75.6]$} & --- & 190 & --- & 605 & --- & --- \\
\hline LG9.70.12.20.1 & & $1.00 \mathrm{E}-05$ & 2 & 0.748 & $1.47 \mathrm{E}-02$ & 3,110 & $5.90 \mathrm{E}-02$ & 2,220 & $5.70 \mathrm{E}-02$ & 14,500 & 5.16E-02 & 15,100 & $6.58 \mathrm{E}-02$ & 4.42E-09 \\
\hline LG9.70.12.20.2 & & $1.06 \mathrm{E}-05$ & 4 & 0.743 & $1.47 \mathrm{E}-02$ & 6,440 & 1.30E-01 & 4,890 & 1.34E-01 & 29,900 & 1.13E-01 & 29,500 & $1.37 \mathrm{E}-01$ & $1.58 \mathrm{E}-09$ \\
\hline
\end{tabular}

B.19 


\begin{tabular}{|c|c|c|c|c|c|c|c|c|c|c|c|c|c|c|}
\hline Sample ID & $\begin{array}{l}\text { Influent } \\
\text { [Si] } \\
\text { (ppm) }\end{array}$ & $\begin{array}{l}\text { Flow } \\
\text { Rate, } \mathrm{q} \\
\left(\mathrm{m}^{3} \mathrm{~d}^{-1}\right)\end{array}$ & $\begin{array}{l}\text { Time } \\
\text { (d) }\end{array}$ & $\begin{array}{l}\text { Glass } \\
\text { Mass } \\
\text { (g) }\end{array}$ & $\begin{array}{l}\text { Surface } \\
\text { Area, S } \\
\left(\mathrm{m}^{2}\right)\end{array}$ & $\begin{array}{l}{[\mathrm{Al}]} \\
\left(\mu \mathrm{L} \mathrm{L}^{-1}\right)\end{array}$ & $\begin{array}{l}\text { Al Norm. } \\
\text { Diss. Rate } \\
\left(\mathrm{g} \mathrm{m}^{-2} \mathrm{~d}^{-1}\right)\end{array}$ & $\begin{array}{l}{[\mathrm{B}]} \\
\left(\mu \mathrm{g} \mathrm{L}^{-1}\right)\end{array}$ & $\begin{array}{l}\text { B Norm. } \\
\text { Diss. Rate } \\
\left(\mathrm{g} \mathrm{m}^{-2} \mathrm{~d}^{-1}\right)\end{array}$ & $\begin{array}{l}{[\mathrm{Si}]} \\
\left(\mu \mathrm{g} \mathrm{L}^{-1}\right)\end{array}$ & $\begin{array}{l}\text { Si Norm. } \\
\text { Diss. Rate } \\
\left(\mathrm{g} \mathrm{m}^{-2} \mathrm{~d}^{-1}\right)\end{array}$ & $\begin{array}{l}{[\mathrm{Na}]} \\
(\mu \mathrm{g} \mathrm{L}- \\
\left.{ }^{1}\right)\end{array}$ & $\begin{array}{l}\text { Na Norm. } \\
\text { Diss. Rate, } \\
\mathrm{r} \\
\left(\mathrm{g} \mathrm{m}^{-2} \mathrm{~d}^{-1}\right)\end{array}$ & $\begin{array}{l}\mathrm{r}_{\mathrm{IEX}} \\
\left(\mathrm{mol} \mathrm{Na} \mathrm{s}^{-2} \mathrm{~s}^{-1}\right)\end{array}$ \\
\hline LG9.70.12.20.3 & & $8.64 \mathrm{E}-06$ & 7 & 0.736 & $1.46 \mathrm{E}-02$ & 8,230 & $1.36 \mathrm{E}-01$ & 6,450 & $1.45 \mathrm{E}-01$ & 37,900 & $1.18 \mathrm{E}-01$ & 37,100 & $1.41 \mathrm{E}-01$ & $<0$ \\
\hline LG9.70.12.20.5 & & $3.24 \mathrm{E}-06$ & 11 & 0.733 & $1.45 \mathrm{E}-02$ & 10,500 & $6.54 \mathrm{E}-02$ & 8,580 & $7.24 \mathrm{E}-02$ & 49,200 & $5.75 \mathrm{E}-02$ & 47,300 & $6.77 \mathrm{E}-02$ & $<0$ \\
\hline LG9.70.12.20.7 & & $9.74 \mathrm{E}-06$ & 16 & 0.716 & $1.43 \mathrm{E}-02$ & 9,850 & $1.87 \mathrm{E}-01$ & 8,350 & $2.15 \mathrm{E}-01$ & 46,500 & $1.66 \mathrm{E}-01$ & 45,300 & $1.98 \mathrm{E}-01$ & $<0$ \\
\hline LG9.70.12.20.9 & & $9.56 \mathrm{E}-06$ & 21 & 0.701 & $1.41 \mathrm{E}-02$ & 9,160 & $1.73 \mathrm{E}-01$ & 7,840 & $2.01 \mathrm{E}-01$ & 43,900 & $1.56 \mathrm{E}-01$ & 43,800 & $1.90 \mathrm{E}-01$ & $<0$ \\
\hline LG9.70.12.20.10 & & $5.12 \mathrm{E}-07$ & 23 & 0.705 & $1.42 \mathrm{E}-02$ & 9,290 & $9.39 \mathrm{E}-03$ & 7,880 & $1.08 \mathrm{E}-02$ & 44,400 & $8.41 \mathrm{E}-03$ & 43,100 & $1.00 \mathrm{E}-02$ & $<0$ \\
\hline LG9.70.12.20.11 & & $1.13 \mathrm{E}-05$ & 24 & 0.700 & $1.41 \mathrm{E}-02$ & 9,320 & $2.09 \mathrm{E}-01$ & 7,800 & $2.37 \mathrm{E}-01$ & 44,700 & $1.88 \mathrm{E}-01$ & 43,400 & $2.24 \mathrm{E}-01$ & $<0$ \\
\hline \multicolumn{15}{|c|}{ LG9 $70{ }^{\circ} \mathrm{C} \mathrm{pH} 12$ Reactor $\# 2$} \\
\hline LG9.70.12.40.Blank1 & & $3.94 \mathrm{E}-05$ & --- & --- & --- & [49.4] & --- & [75.6] & --- & 199 & --- & 597 & --- & --. \\
\hline LG9.70.12.40.Blank2 & & 3.85E-05 & --- & --- & --- & [49.4] & --- & [75.6] & --- & 170 & --- & 521 & --- & --- \\
\hline LG9.70.12.40.Blank3 & & 3.87E-05 & -.- & --- & -.- & [49.4] & -.- & [75.6] & -.- & 210 & ..- & 487 & -.- & -.- \\
\hline LG9.70.12.40.1 & & $1.83 \mathrm{E}-05$ & 1 & 0.368 & $7.24 \mathrm{E}-03$ & 1,540 & $1.09 \mathrm{E}-01$ & 812 & $7.78 \mathrm{E}-02$ & 7,910 & $1.05 \mathrm{E}-01$ & 7,470 & $1.21 \mathrm{E}-01$ & $2.20 \mathrm{E}-08$ \\
\hline LG9.70.12.40.2 & & 2.35E-05 & 1 & 0.368 & 7.24E-03 & 2,540 & $2.30 \mathrm{E}-01$ & 1,510 & $1.85 \mathrm{E}-01$ & 13,000 & $2.21 \mathrm{E}-01$ & 12,200 & 2.54E-01 & $3.46 \mathrm{E}-08$ \\
\hline LG9.70.12.40.3 & & $2.14 \mathrm{E}-05$ & 2 & 0.366 & $7.22 \mathrm{E}-03$ & 2,980 & $2.47 \mathrm{E}-01$ & 2,160 & $2.42 \mathrm{E}-01$ & 14,100 & $2.19 \mathrm{E}-01$ & 14,000 & $2.66 \mathrm{E}-01$ & $1.21 \mathrm{E}-08$ \\
\hline LG9.70.12.40.5 & & $2.17 \mathrm{E}-05$ & 7 & 0.349 & $7.00 \mathrm{E}-03$ & 4,360 & 3.79E-01 & 3,230 & 3.80E-01 & 20,300 & $3.31 \mathrm{E}-01$ & 17,800 & $3.55 \mathrm{E}-01$ & $<0$ \\
\hline LG9.70.12.40.7 & & $2.63 \mathrm{E}-05$ & 11 & 0.337 & $6.84 \mathrm{E}-03$ & 4,050 & $4.36 \mathrm{E}-01$ & 2,940 & $4.29 \mathrm{E}-01$ & 19,100 & $3.86 \mathrm{E}-01$ & 17,900 & 4.43E-01 & $7.10 \mathrm{E}-09$ \\
\hline LG9.70.12.40.9 & & $2.11 \mathrm{E}-05$ & 16 & 0.325 & $6.67 \mathrm{E}-03$ & 4,220 & $3.72 \mathrm{E}-01$ & 3,210 & 3.83E-01 & 20,000 & $3.31 \mathrm{E}-01$ & 17,900 & 3.63E-01 & $<0$ \\
\hline LG9.70.12.40.10 & & $1.96 \mathrm{E}-05$ & 18 & 0.321 & $6.61 \mathrm{E}-03$ & 4,360 & $3.62 \mathrm{E}-01$ & 3,260 & 3.67E-01 & 20,600 & $3.21 \mathrm{E}-01$ & 18,300 & 3.49E-01 & $<0$ \\
\hline LG9.70.12.40.11 & & $2.00 \mathrm{E}-05$ & 21 & 0.312 & $6.50 \mathrm{E}-03$ & 4,360 & $3.75 \mathrm{E}-01$ & 3,310 & 3.85E-01 & 20,700 & $3.34 \mathrm{E}-01$ & 18,600 & $3.67 \mathrm{E}-01$ & $<0$ \\
\hline LG9.70.12.40.12 & & $2.37 \mathrm{E}-05$ & 23 & 0.307 & $6.42 \mathrm{E}-03$ & 4,400 & 4.53E-01 & 3,420 & $4.77 \mathrm{E}-01$ & 21,100 & 4.07E-01 & 20,600 & $4.87 \mathrm{E}-01$ & $5.25 \mathrm{E}-09$ \\
\hline LG9.70.12.40.13 & & $1.96 \mathrm{E}-05$ & 24 & 0 & 0 & 4,460 & $3.81 \mathrm{E}-01$ & 3,480 & $4.02 \mathrm{E}-01$ & 21,500 & $3.44 \mathrm{E}-01$ & 18,200 & $3.57 \mathrm{E}-01$ & $<0$ \\
\hline \multicolumn{15}{|c|}{ LG9 $70^{\circ} \mathrm{C} \mathrm{pH} 12$ Reactor $\# 3$} \\
\hline LG9.70.12.80A.Blank1 & & $7.64 \mathrm{E}-05$ & --- & --- & --. & [49.4] & --- & [75.6] & --- & 205 & --. & 495 & --. & -.- \\
\hline LG9.70.12.80A.Blank2 & & $7.88 \mathrm{E}-05$ & --- & --- & --- & [49.4] & --- & [75.6] & --- & 192 & --- & 507 & --- & --- \\
\hline LG9.70.12.80A.Blank3 & & 7.82E-05 & --- & --- & --- & [49.4] & --- & [75.6] & --- & 214 & --- & 544 & --- &.-- \\
\hline LG9.70.12.80A.1 & & $3.52 E-05$ & 1 & 0.149 & $2.94 \mathrm{E}-03$ & 494 & $1.65 \mathrm{E}-01$ & 102 & 4.62E-02 & 2,630 & 1.65E-01 & 2,800 & $2.15 \mathrm{E}-01$ & $8.51 \mathrm{E}-08$ \\
\hline LG9.70.12.80A.2 & & 3.96E-05 & 1 & 0.149 & $2.94 \mathrm{E}-03$ & 886 & $3.34 \mathrm{E}-01$ & 391 & $2.00 \mathrm{E}-01$ & 4,630 & $3.28 \mathrm{E}-01$ & 4,420 & 3.83E-01 & $9.23 E-08$ \\
\hline LG9.70.12.80A.3 & & 4.09E-05 & 2 & 0.148 & $2.92 \mathrm{E}-03$ & 1,100 & $4.31 \mathrm{E}-01$ & 796 & 4.22E-01 & 5,360 & $3.94 \mathrm{E}-01$ & 4,810 & 4.33E-01 & $5.34 \mathrm{E}-09$ \\
\hline LG9.70.12.80A.5 & & 4.05E-05 & 7 & 0.138 & $2.79 \mathrm{E}-03$ & 1,360 & $5.51 \mathrm{E}-01$ & 1,010 & $5.54 \mathrm{E}-01$ & 6,690 & $5.08 \mathrm{E}-01$ & 5,720 & $5.32 \mathrm{E}-01$ & $<0$ \\
\hline LG9.70.12.80A.7 & & 4.05E-05 & 11 & 0.132 & $2.71 \mathrm{E}-03$ & 1,340 & $5.60 \mathrm{E}-01$ & 1,020 & $5.77 \mathrm{E}-01$ & 6,740 & $5.28 \mathrm{E}-01$ & 5,680 & $5.45 \mathrm{E}-01$ & $<0$ \\
\hline LG9.70.12.80A.9 & & 4.00E-05 & 16 & 0.126 & 2.62E-03 & 1,220 & $5.21 \mathrm{E}-01$ & 905 & $5.23 E-01$ & 6,090 & 4.87E-01 & 5,030 & 4.93E-01 & $<0$ \\
\hline LG9.70.12.80A.10 & & 4.05E-05 & 18 & 0.123 & $2.58 \mathrm{E}-03$ & 1,190 & $5.21 \mathrm{E}-01$ & 893 & $5.30 \mathrm{E}-01$ & 5,940 & $4.88 \mathrm{E}-01$ & 4,920 & 4.95E-01 & $<0$ \\
\hline LG9.70.12.80A.11 & & 4.03E-05 & 21 & 0.119 & $2.52 \mathrm{E}-03$ & 1,180 & $5.27 \mathrm{E}-01$ & 889 & $5.38 \mathrm{E}-01$ & 5,920 & $4.96 \mathrm{E}-01$ & 4,890 & $5.02 \mathrm{E}-01$ & $<0$ \\
\hline LG9.70.12.80A.12 & & 3.93E-05 & 23 & 0.116 & 2.49E-03 & 1,190 & $5.25 \mathrm{E}-01$ & 903 & $5.40 \mathrm{E}-01$ & 6,010 & $4.98 \mathrm{E}-01$ & 4,890 & 4.96E-01 & $<0$ \\
\hline LG9.70.12.80A.13 & & 3.84E-05 & 24 & 0 & 0 & 1,220 & $5.29 \mathrm{E}-01$ & 940 & $5.52 \mathrm{E}-01$ & 6,150 & $5.00 \mathrm{E}-01$ & 4,970 & 4.95E-01 & $<0$ \\
\hline \multicolumn{15}{|c|}{ LG9 $70^{\circ} \mathrm{C}$ pH 12 Reactor $\# 4$} \\
\hline LG9.70.12.80B.Blank1 & & 8.05E-05 & --- & --- & --- & [49.4] & --- & [75.6] & --- & 294 & --- & 448 & --- & --- \\
\hline LG9.70.12.80B.Blank2 & & 7.91E-05 & --- & --- & --- & [49.4] & --- & {$[75.6]$} & --- & 283 & --- & 475 & --- & --- \\
\hline LG9.70.12.80B.Blank3 & & $7.79 \mathrm{E}-05$ & --- & --- & --- & [49.4] & --- & [75.6] & --- & 221 & --- & 395 & --- & --- \\
\hline LG9.70.12.80B.1 & & $6.32 \mathrm{E}-05$ & 1 & 0.049 & $9.65 \mathrm{E}-04$ & 505 & $9.25 \mathrm{E}-01$ & 116 & $2.88 \mathrm{E}-01$ & 2,800 & $9.61 \mathrm{E}-01$ & 2,520 & $1.06 \mathrm{E}+00$ & 3.89E-07 \\
\hline LG9.70.12.80B.2 & & $6.68 \mathrm{E}-05$ & 1 & 0.049 & $9.60 \mathrm{E}-04$ & 936 & $1.82 \mathrm{E}+00$ & 435 & $1.14 \mathrm{E}+00$ & 5,260 & $1.92 \mathrm{E}+00$ & 4,630 & $2.07 E+00$ & 4.64E-07 \\
\hline LG9.70.12.80B.3 & & $6.81 \mathrm{E}-05$ & 2 & 0.047 & $9.33 E-04$ & 987 & $2.01 \mathrm{E}+00$ & 725 & $2.00 \mathrm{E}+00$ & 5,180 & $1.98 \mathrm{E}+00$ & 4,010 & $1.88 \mathrm{E}+00$ & $<0$ \\
\hline LG9.70.12.80B.5 & & $6.79 \mathrm{E}-05$ & 7 & 0.035 & 7.74E-04 & 979 & $2.40 E+00$ & 727 & $2.41 \mathrm{E}+00$ & 5,240 & $2.41 E+00$ & 3,890 & $2.19 E+00$ & $<0$ \\
\hline LG9.70.12.80B.7 & & $7.95 \mathrm{E}-05$ & 11 & 0.028 & $6.69 \mathrm{E}-04$ & 814 & $2.71 \mathrm{E}+00$ & 600 & $2.70 E+00$ & 4,350 & $2.71 \mathrm{E}+00$ & 3,210 & $2.45 E+00$ & $<0$ \\
\hline
\end{tabular}

B. 20 


\begin{tabular}{|c|c|c|c|c|c|c|c|c|c|c|c|c|c|c|}
\hline Sample ID & $\begin{array}{l}\text { Influent } \\
{[\mathrm{Si}]} \\
(\mathrm{ppm})\end{array}$ & $\begin{array}{l}\text { Flow } \\
\text { Rate, } \mathrm{q} \\
\left(\mathrm{m}^{3} \mathrm{~d}^{-1}\right)\end{array}$ & $\begin{array}{l}\text { Time } \\
\text { (d) }\end{array}$ & $\begin{array}{l}\text { Glass } \\
\text { Mass } \\
\text { (g) }\end{array}$ & $\begin{array}{l}\text { Surface } \\
\text { Area, S } \\
\left(\mathrm{m}^{2}\right)\end{array}$ & $\begin{array}{l}{[\mathrm{Al}]} \\
\left(\mu \mathrm{L} \mathrm{L}^{-1}\right)\end{array}$ & $\begin{array}{l}\text { Al Norm. } \\
\text { Diss. Rate } \\
\left(\mathrm{g} \mathrm{m}^{-2} \mathrm{~d}^{-1}\right)\end{array}$ & $\begin{array}{l}{[\mathrm{B}]} \\
\left(\mu \mathrm{g} \mathrm{L}^{-1}\right)\end{array}$ & $\begin{array}{l}\text { B Norm. } \\
\text { Diss. Rate } \\
\left(\mathrm{g} \mathrm{m}^{-2} \mathrm{~d}^{-1}\right)\end{array}$ & $\begin{array}{l}{[\mathrm{Si}]} \\
\left(\mu \mathrm{g} \mathrm{L}^{-1}\right)\end{array}$ & $\begin{array}{l}\text { Si Norm. } \\
\text { Diss. Rate } \\
\left(\mathrm{g} \mathrm{m}^{-2} \mathrm{~d}^{-1}\right)\end{array}$ & $\begin{array}{l}{[\mathrm{Na}]} \\
(\mu \mathrm{g} \mathrm{L} \\
\left.{ }^{1}\right)\end{array}$ & $\begin{array}{l}\text { Na Norm. } \\
\text { Diss. Rate, } \\
\mathrm{r} \\
\left(\mathrm{g} \mathrm{m}^{-2} \mathrm{~d}^{-1}\right)\end{array}$ & $\begin{array}{l}\mathrm{r}_{\mathrm{IEX}} \\
\left(\mathrm{mol} \mathrm{Na} \mathrm{No}^{-1}\right)\end{array}$ \\
\hline LG9.70.12.80B.9 & & $7.94 \mathrm{E}-05$ & 16 & 0.022 & $5.66 \mathrm{E}-04$ & 621 & 2.43E +00 & 458 & $2.43 \mathrm{E}+00$ & 3,350 & $2.46 \mathrm{E}+00$ & 2,840 & $2.56 \mathrm{E}+00$ & $6.36 \mathrm{E}-08$ \\
\hline LG9.70.12.80B.10 & & 7.97E-05 & 18 & 0.019 & $5.10 \mathrm{E}-04$ & 685 & $2.99 \mathrm{E}+00$ & 505 & $2.98 \mathrm{E}+00$ & 3,650 & $2.99 \mathrm{E}+00$ & 2,720 & $2.73 E+00$ & $<0$ \\
\hline LG9.70.12.80B.11 & & $7.81 \mathrm{E}-05$ & 21 & 0.015 & 4.34E-04 & 613 & $3.09 \mathrm{E}+00$ & 447 & $3.05 \mathrm{E}+00$ & 3,430 & $3.24 \mathrm{E}+00$ & 2,540 & $2.94 \mathrm{E}+00$ & $<0$ \\
\hline LG9.70.12.80B.12 & & $8.04 \mathrm{E}-05$ & 23 & 0.013 & $3.91 \mathrm{E}-04$ & 585 & $3.36 \mathrm{E}+00$ & 444 & $3.46 \mathrm{E}+00$ & 3,410 & $3.68 \mathrm{E}+00$ & 2,420 & $3.19 E+00$ & $<0$ \\
\hline LG9.70.12.80B.13 & & $7.68 \mathrm{E}-05$ & 24 & 0 & 0 & 544 & $3.10 \mathrm{E}+00$ & 419 & $3.23 \mathrm{E}+00$ & 3,220 & $3.44 \mathrm{E}+00$ & 2,060 & $2.70 E+00$ & $<0$ \\
\hline \multicolumn{15}{|c|}{ LG9 $90^{\circ} \mathrm{C}$ pH 9 Reactor \#1 } \\
\hline LG9.90.9.20.Blank3 & & 4.96E-06 & --- & --- & --- & [49.4] & --- & {$[75.6]$} & --- & [164] & --- & [134] & --- & --. \\
\hline LG9.90.9.20.1 & & $6.52 \mathrm{E}-06$ & 2.3 & 0 & 0 & 1,060 & 0 & 4,470 & 0 & 6,670 & 0 & 32,800 & $1.76 \mathrm{E}-01$ & $1.74 \mathrm{E}-08$ \\
\hline LG9.90.9.20.2 & & 8.07E-06 & 4.3 & 0 & 0 & 1,690 & 0 & 6,790 & 0 & 10,800 & 0 & 48,300 & $3.24 \mathrm{E}-01$ & $2.79 \mathrm{E}-08$ \\
\hline LG9.90.9.20.3 & & $7.20 \mathrm{E}-06$ & 7.0 & 0.384 & $7.65 \mathrm{E}-03$ & 1,810 & $4.76 \mathrm{E}-02$ & 7,080 & $2.52 \mathrm{E}-01$ & 11,500 & 5.67E-02 & 53,200 & $3.21 \mathrm{E}-01$ & $3.49 \mathrm{E}-08$ \\
\hline LG9.90.9.20.4 & & $7.29 \mathrm{E}-06$ & 9.1 & 0.381 & $7.60 \mathrm{E}-03$ & 1,790 & $4.80 \mathrm{E}-02$ & 6,920 & $2.51 \mathrm{E}-01$ & 11,600 & 5.83E-02 & 48,500 & $2.98 \mathrm{E}-01$ & $2.39 \mathrm{E}-08$ \\
\hline LG9.90.9.20.5 & & $8.80 \mathrm{E}-06$ & 11.3 & 0.376 & $7.54 \mathrm{E}-03$ & 1,730 & $5.65 \mathrm{E}-02$ & 6,230 & $2.75 \mathrm{E}-01$ & 11,100 & $6.79 \mathrm{E}-02$ & 46,400 & $3.48 \mathrm{E}-01$ & $3.65 \mathrm{E}-08$ \\
\hline LG9.90.9.20.6 & & 8.17E-06 & 14.0 & 0.371 & 7.47E-03 & 1,670 & $5.10 \mathrm{E}-02$ & 5,620 & $2.32 \mathrm{E}-01$ & 10,800 & $6.19 \mathrm{E}-02$ & 42,700 & $3.00 \mathrm{E}-01$ & $3.38 \mathrm{E}-08$ \\
\hline LG9.90.9.20.7 & & $8.12 \mathrm{E}-06$ & 16.2 & 0.368 & $7.44 \mathrm{E}-03$ & 1,590 & $4.85 \mathrm{E}-02$ & 4,880 & $2.01 \mathrm{E}-01$ & 10,300 & $5.89 \mathrm{E}-02$ & 39,500 & $2.77 \mathrm{E}-01$ & $3.79 E-08$ \\
\hline LG9.90.9.20.8 & & $7.28 \mathrm{E}-06$ & 18.2 & 0.366 & $7.41 \mathrm{E}-03$ & 1,580 & $4.34 \mathrm{E}-02$ & 4,510 & $1.68 \mathrm{E}-01$ & 10,300 & $5.31 \mathrm{E}-02$ & 37,300 & $2.35 \mathrm{E}-01$ & $3.40 \mathrm{E}-08$ \\
\hline LG9.90.9.20.9 & & $8.28 \mathrm{E}-06$ & 21.0 & 0.362 & $7.35 \mathrm{E}-03$ & 1,690 & $5.32 \mathrm{E}-02$ & 4,340 & $1.85 \mathrm{E}-01$ & 11,100 & $6.55 \mathrm{E}-02$ & 34,500 & $2.49 \mathrm{E}-01$ & $3.25 \mathrm{E}-08$ \\
\hline \multicolumn{15}{|c|}{ LG9 $90^{\circ} \mathrm{C}$ pH 9 Reactor $\# 2$} \\
\hline LG9.90.9.40.Blank1 & & $1.94 \mathrm{E}-05$ & --- & --- & --- & {$[49.4]$} & -- & {$[75.6]$} & -- & [164] & --- & [134] & --- & --- \\
\hline LG9.90.9.40.Blank2 & & $1.83 \mathrm{E}-05$ & --- & --- & --- & [49.4] & --- & [75.6] & --- & [164] & --- & [134] & --- & -.- \\
\hline LG9.90.9.40.Blank3 & & $1.95 \mathrm{E}-05$ & -- & --- & --- & {$[49.4]$} & --- & {$[75.6]$} & -- & [164] & --- & [134] & --- & --- \\
\hline LG9.90.9.40.1 & & $1.88 \mathrm{E}-05$ & 2.3 & 0.147 & $2.92 \mathrm{E}-03$ & 545 & $9.83 \mathrm{E}-02$ & 1,940 & 4.73E- 01 & 3,350 & 1.13E-01 & 14,000 & $5.80 \mathrm{E}-01$ & $5.35 \mathrm{E}-08$ \\
\hline LG9.90.9.40.2 & & $1.93 \mathrm{E}-05$ & 4.3 & 0.143 & $2.87 \mathrm{E}-03$ & 809 & $1.52 \mathrm{E}-01$ & 2,330 & $5.93 \mathrm{E}-01$ & 5,100 & $1.80 \mathrm{E}-01$ & 15,700 & $6.77 \mathrm{E}-01$ & $4.27 \mathrm{E}-08$ \\
\hline LG9.90.9.40.3 & & $1.94 \mathrm{E}-05$ & 7.0 & 0.139 & $2.81 \mathrm{E}-03$ & 771 & $1.49 \mathrm{E}-01$ & 1,970 & $5.15 \mathrm{E}-01$ & 4,880 & 1.77E-01 & 14,200 & $6.30 \mathrm{E}-01$ & $5.78 \mathrm{E}-08$ \\
\hline LG9.90.9.40.4 & & $1.91 \mathrm{E}-05$ & 9.1 & 0.137 & $2.79 \mathrm{E}-03$ & 706 & $1.35 \mathrm{E}-01$ & 1,550 & $4.02 \mathrm{E}-01$ & 4,470 & 1.61E-01 & 11,700 & $5.15 \mathrm{E}-01$ & $5.68 \mathrm{E}-08$ \\
\hline LG9.90.9.40.5 & & 1.87E-05 & 11.3 & 0.136 & $2.77 \mathrm{E}-03$ & 672 & 1.27E-01 & 1,320 & $3.38 \mathrm{E}-01$ & 4,260 & 1.51E-01 & 10,400 & $4.52 \mathrm{E}-01$ & $5.73 \mathrm{E}-08$ \\
\hline LG9.90.9.40.6 & & $1.87 \mathrm{E}-05$ & 14.0 & 0.133 & $2.74 \mathrm{E}-03$ & 639 & $1.22 \mathrm{E}-01$ & 1,120 & $2.90 \mathrm{E}-01$ & 4,090 & $1.46 \mathrm{E}-01$ & 8,990 & $3.94 \mathrm{E}-01$ & $5.27 E-08$ \\
\hline LG9.90.9.40.7 & & $1.91 \mathrm{E}-05$ & 16.2 & 0.132 & $2.72 \mathrm{E}-03$ & 612 & $1.20 \mathrm{E}-01$ & 939 & 2.49E-01 & 3,920 & 1.44E-01 & 7,880 & $3.54 \mathrm{E}-01$ & $5.31 \mathrm{E}-08$ \\
\hline LG9.90.9.40.8 & & $1.89 \mathrm{E}-05$ & 18.2 & 0.131 & $2.71 \mathrm{E}-03$ & 517 & $1.01 \mathrm{E}-01$ & 749 & $1.98 \mathrm{E}-01$ & 3,380 & $1.24 \mathrm{E}-01$ & 6,990 & $3.13 \mathrm{E}-01$ & $5.80 \mathrm{E}-08$ \\
\hline LG9.90.9.40.9 & & 1.87E-05 & 21.0 & 0.130 & $2.68 \mathrm{E}-03$ & 577 & $1.13 \mathrm{E}-01$ & 773 & $2.04 \mathrm{E}-01$ & 3,820 & 1.40E-01 & 6,340 & $2.84 \mathrm{E}-01$ & $4.02 \mathrm{E}-08$ \\
\hline \multicolumn{15}{|c|}{ LG9 $90^{\circ} \mathrm{C}$ pH 9 Reactor \#3 } \\
\hline LG9.90.9.80.Blank1 & & $5.86 \mathrm{E}-05$ & --- & --- & --- & [49.4] & --- & {$[75.6]$} & --- & [164] & --- & [134] & --- & --- \\
\hline LG9.90.9.80.Blank2 & & $7.56 \mathrm{E}-05$ & --- & --- & --- & [49.4] & --- & [75.6] & -- & [164] & --- & [134] & --- & --- \\
\hline LG9.90.9.80.Blank3 & & $7.45 \mathrm{E}-05$ & --. & --- & --- & [49.4] & --- & {$[75.6]$} & --- & [164] & --- & [134] & --- & --- \\
\hline LG9.90.9.80.1 & & $7.10 \mathrm{E}-05$ & 2.3 & 0.092 & $1.86 \mathrm{E}-03$ & 227 & $2.42 \mathrm{E}-01$ & 831 & $1.20 \mathrm{E}+00$ & 1,380 & $2.76 \mathrm{E}-01$ & 5,580 & $1.36 \mathrm{E}+00$ & $8.39 \mathrm{E}-08$ \\
\hline LG9.90.9.80.2 & & $7.55 \mathrm{E}-05$ & 4.3 & 0.090 & $1.83 \mathrm{E}-03$ & 212 & $2.45 \mathrm{E}-01$ & 583 & $9.11 \mathrm{E}-01$ & 1,370 & $2.96 \mathrm{E}-01$ & 3,890 & $1.03 E+00$ & $6.05 \mathrm{E}-08$ \\
\hline LG9.90.9.80.3 & & 7.45E-05 & 7.0 & 0.087 & $1.79 \mathrm{E}-03$ & 191 & $2.23 \mathrm{E}-01$ & 416 & $6.56 \mathrm{E}-01$ & 1,120 & $2.45 \mathrm{E}-01$ & 2,860 & $7.65 \mathrm{E}-01$ & $5.49 \mathrm{E}-08$ \\
\hline LG9.90.9.80.4 & & 7.53E-05 & 9.1 & 0.085 & $1.77 \mathrm{E}-03$ & 175 & $2.08 \mathrm{E}-01$ & 335 & $5.40 \mathrm{E}-01$ & 1,010 & $2.25 \mathrm{E}-01$ & 2,330 & $6.37 \mathrm{E}-01$ & $4.89 \mathrm{E}-08$ \\
\hline LG9.90.9.80.5 & & $7.48 \mathrm{E}-05$ & 11.3 & 0.084 & $1.75 \mathrm{E}-03$ & 162 & $1.94 \mathrm{E}-01$ & 275 & $4.46 \mathrm{E}-01$ & 910 & $2.04 \mathrm{E}-01$ & 1,980 & $5.44 \mathrm{E}-01$ & 4.97E-08 \\
\hline LG9.90.9.80.6 & & $7.56 \mathrm{E}-05$ & 14.0 & 0.082 & $1.72 \mathrm{E}-03$ & 155 & $1.90 \mathrm{E}-01$ & 230 & 3.83E-01 & 833 & 1.92E-01 & 1,760 & 4.97E-01 & $5.74 \mathrm{E}-08$ \\
\hline LG9.90.9.80.7 & & 7.44E-05 & 16.2 & 0.081 & $1.71 \mathrm{E}-03$ & 154 & $1.88 \mathrm{E}-01$ & 199 & $3.28 \mathrm{E}-01$ & 818 & 1.87E-01 & 1,570 & $4.39 \mathrm{E}-01$ & $5.59 \mathrm{E}-08$ \\
\hline LG9.90.9.80.8 & & $7.55 \mathrm{E}-05$ & 18.2 & 0.080 & $1.69 \mathrm{E}-03$ & 150 & $1.87 \mathrm{E}-01$ & 184 & $3.10 \mathrm{E}-01$ & 796 & $1.86 \mathrm{E}-01$ & 1,430 & $4.09 \mathrm{E}-01$ & $4.98 \mathrm{E}-08$ \\
\hline LG9.90.9.80.9 & & 7.47E-05 & 21.0 & 0.078 & $1.67 \mathrm{E}-03$ & 161 & $2.01 \mathrm{E}-01$ & 185 & 3.13E-01 & 901 & 2.11E-01 & 1,590 & $4.56 \mathrm{E}-01$ & $7.22 \mathrm{E}-08$ \\
\hline \multicolumn{15}{|c|}{ LG9 $90^{\circ} \mathrm{C}$ pH 9 Reactor $\# 4$} \\
\hline
\end{tabular}




\begin{tabular}{|c|c|c|c|c|c|c|c|c|c|c|c|c|c|c|}
\hline Sample ID & $\begin{array}{l}\text { Influent } \\
{[\mathrm{Si}]} \\
(\mathrm{ppm})\end{array}$ & $\begin{array}{l}\text { Flow } \\
\text { Rate, } q \\
\left(\mathrm{~m}^{3} \mathrm{~d}^{-1}\right)\end{array}$ & $\begin{array}{l}\text { Time } \\
\text { (d) }\end{array}$ & $\begin{array}{l}\text { Glass } \\
\text { Mass } \\
\text { (g) }\end{array}$ & $\begin{array}{l}\text { Surface } \\
\text { Area, S } \\
\left(\mathrm{m}^{2}\right)\end{array}$ & $\begin{array}{l}{[\mathrm{Al}]} \\
\left(\mu \mathrm{L} \mathrm{L}^{-1}\right)\end{array}$ & $\begin{array}{l}\text { Al Norm. } \\
\text { Diss. Rate } \\
\left(\mathrm{g} \mathrm{m}^{-2} \mathrm{~d}^{-1}\right)\end{array}$ & $\begin{array}{l}{[\mathrm{B}]} \\
\left(\mu \mathrm{g} \mathrm{L}^{-1}\right)\end{array}$ & $\begin{array}{l}\text { B Norm. } \\
\text { Diss. Rate } \\
\left(\mathrm{g} \mathrm{m}^{-2} \mathrm{~d}^{-1}\right)\end{array}$ & $\begin{array}{l}{[\mathrm{Si}]} \\
\left(\mu \mathrm{g} \mathrm{L}^{-1}\right)\end{array}$ & $\begin{array}{l}\text { Si Norm. } \\
\text { Diss. Rate } \\
\left(\mathrm{g} \mathrm{m}^{-2} \mathrm{~d}^{-1}\right)\end{array}$ & $\begin{array}{l}{[\mathrm{Na}]} \\
\left.{ }_{1}{ }^{1}\right)\end{array}$ & $\begin{array}{l}\text { Na Norm. } \\
\text { Diss. Rate, } \\
\mathrm{r} \\
\left(\mathrm{g} \mathrm{m}^{-2} \mathrm{~d}^{-1}\right)\end{array}$ & $\begin{array}{l}\mathrm{r}_{\mathrm{IEX}} \\
\left(\mathrm{mol} \mathrm{Na} \mathrm{No}^{-1}\right)\end{array}$ \\
\hline LG9.90.9.160.Blank1 & & $8.77 \mathrm{E}-05$ & --- & --- & --- & [49.4] & $\cdots$ & {$[75.6]$} & --- & [164] & --- & [134] & --- & --- \\
\hline LG9.90.9.160.Blank2 & & $2.15 \mathrm{E}-05$ & --- & --- & --- & [49.4] & --- & {$[75.6]$} & --- & [164] & --- & [134] & --- & --- \\
\hline LG9.90.9.160.Blank3 & & $1.52 \mathrm{E}-04$ & --- & --- & --- & [49.4] & --- & {$[75.6]$} & --- & [164] & -- & [134] & --- & -.- \\
\hline LG9.90.9.160.1 & & $1.48 \mathrm{E}-04$ & 2.3 & 0.043 & $8.88 \mathrm{E}-04$ & 90 & 4.19E-01 & 301 & $1.90 \mathrm{E}+00$ & 313 & $2.74 \mathrm{E}-01$ & 2,110 & $2.26 \mathrm{E}+00$ & $1.81 \mathrm{E}-07$ \\
\hline LG9.90.9.160.2 & & $1.51 \mathrm{E}-04$ & 4.3 & 0.042 & $8.69 \mathrm{E}-04$ & 80 & $3.90 \mathrm{E}-01$ & 187 & $1.23 \mathrm{E}+00$ & 262 & $2.39 \mathrm{E}-01$ & 1,200 & $1.34 \mathrm{E}+00$ & $5.49 \mathrm{E}-08$ \\
\hline LG9.90.9.160.3 & & $1.51 \mathrm{E}-04$ & 7.0 & 0.040 & $8.40 \mathrm{E}-04$ & 70 & $3.51 \mathrm{E}-01$ & 138 & $9.41 \mathrm{E}-01$ & 198 & 1.87E-01 & 894 & $1.03 \mathrm{E}+00$ & 4.69E- -08 \\
\hline LG9.90.9.160.4 & & $1.52 E-04$ & 9.1 & 0.039 & $8.31 E-04$ & 61 & $3.13 E-01$ & 94 & $6.47 E-01$ & $\begin{array}{l}{[164]} \\
123^{\star}\end{array}$ & 1.57E-01 & 727 & $8.51 E-01$ & 1.03E-07 \\
\hline LG9.90.9.160.5 & & $1.51 \mathrm{E}-04$ & 11.3 & 0.038 & $8.17 E-04$ & 50 & 2.57E-01 & 82 & $5.72 \mathrm{E}-01$ & $\begin{array}{l}{[164]} \\
126^{*}\end{array}$ & 1.59E-01 & 603 & $7.15 E-01$ & $7.22 \mathrm{E}-08$ \\
\hline LG9.90.9.160.6 & & $1.51 \mathrm{E}-04$ & 14.0 & 0.037 & $7.99 \mathrm{E}-04$ & 60 & $3.17 \mathrm{E}-01$ & $\begin{array}{l}{[75.6]} \\
73^{\star}\end{array}$ & $5.42 \mathrm{E}-01$ & $\begin{array}{l}{[164]} \\
129^{\star}\end{array}$ & 1.63E-01 & 491 & $5.98 \mathrm{E}-01$ & $2.78 \mathrm{E}-08$ \\
\hline LG9.90.9.160.7 & & $1.41 \mathrm{E}-04$ & 16.4 & 0.036 & 7.87E-04 & 59 & 2.94E-01 & $\begin{array}{l}{[75.6]} \\
70^{\star}\end{array}$ & $5.12 \mathrm{E}-01$ & $\begin{array}{l}{[164]} \\
126^{*}\end{array}$ & 1.54E-01 & 469 & $5.39 \mathrm{E}-01$ & $1.35 \mathrm{E}-08$ \\
\hline LG9.90.9.160.8 & & $1.66 \mathrm{E}-04$ & 18.2 & 0.035 & $7.75 \mathrm{E}-04$ & 59 & 3.54E-01 & $\begin{array}{l}{[75.6]} \\
62^{\star}\end{array}$ & $6.12 \mathrm{E}-01$ & $\begin{array}{l}{[164]} \\
109^{\star}\end{array}$ & 1.84E-01 & 405 & $5.56 \mathrm{E}-01$ & $<0$ \\
\hline LG9.90.9.160.9 & & $1.52 \mathrm{E}-04$ & 21.0 & 0.034 & $7.55 \mathrm{E}-04$ & 58 & 3.27E-01 & $\begin{array}{l}{[75.6]} \\
65^{\star}\end{array}$ & $5.74 \mathrm{E}-01$ & $\begin{array}{l}{[164]} \\
132^{\star}\end{array}$ & 1.73E-01 & 403 & $5.19 \mathrm{E}-01$ & $<0$ \\
\hline \multicolumn{15}{|c|}{ LG9 $90^{\circ} \mathrm{C} \mathrm{pH} 10$ Reactor $\# 1$} \\
\hline LG9.90.10.20.Blank1 & & $8.01 \mathrm{E}-06$ & --- & --- & --- & [49.4] & --- & {$[75.6]$} & --- & [164] & --- & [134] & --- & --- \\
\hline LG9.90.10.20.Blank2 & & $7.99 \mathrm{E}-06$ & --- & --- & --- & {$[49.4]$} & --- & {$[75.6]$} & --- & [164] & --- & [134] & --- & -.- \\
\hline LG9.90.10.20.Blank3 & & $7.90 \mathrm{E}-06$ & --- & --- & --- & [49.4] & --- & {$[75.6]$} & --- & [164] & --- & [134] & --- & --- \\
\hline LG9.90.10.20.1 & & 7.74E-06 & 2.3 & 0.399 & $7.85 \mathrm{E}-03$ & 1,380 & $3.80 \mathrm{E}-02$ & 1,100 & $4.10 \mathrm{E}-02$ & 8,040 & 4.15E-02 & 14,600 & $9.23 \mathrm{E}-02$ & $2.58 \mathrm{E}-08$ \\
\hline LG9.90.10.20.2 & & $7.48 \mathrm{E}-06$ & 4.3 & 0.398 & $7.84 \mathrm{E}-03$ & 2,060 & $5.49 \mathrm{E}-02$ & 1,690 & $6.10 \mathrm{E}-02$ & 12,100 & $6.05 \mathrm{E}-02$ & 21,800 & $1.33 \mathrm{E}-01$ & $3.65 \mathrm{E}-08$ \\
\hline LG9.90.10.20.3 & & $8.01 \mathrm{E}-06$ & 7.0 & 0.396 & $7.82 \mathrm{E}-03$ & 2,200 & $6.31 \mathrm{E}-02$ & 1,840 & 7.14E-02 & 13,000 & 6.99E-02 & 24,400 & $1.61 \mathrm{E}-01$ & 4.49E-08 \\
\hline LG9.90.10.20.4 & & $7.74 \mathrm{E}-06$ & 9.1 & 0.395 & $7.80 \mathrm{E}-03$ & 2,240 & $6.21 \mathrm{E}-02$ & 1,890 & $7.09 \mathrm{E}-02$ & 13,300 & $6.91 \mathrm{E}-02$ & 24,300 & $1.55 \mathrm{E}-01$ & $4.22 \mathrm{E}-08$ \\
\hline LG9.90.10.20.5 & & $7.49 \mathrm{E}-06$ & 11.2 & 0.394 & $7.79 \mathrm{E}-03$ & 2,240 & $6.02 \mathrm{E}-02$ & 1,870 & $6.80 \mathrm{E}-02$ & 13,200 & $6.65 \mathrm{E}-02$ & 22,300 & $1.38 \mathrm{E}-01$ & $3.50 \mathrm{E}-08$ \\
\hline LG9.90.10.20.6 & & $7.52 \mathrm{E}-06$ & 14.0 & 0.392 & 7.77E-03 & 2,180 & $5.90 \mathrm{E}-02$ & 1,790 & $6.56 \mathrm{E}-02$ & 13,000 & $6.60 \mathrm{E}-02$ & 21,700 & $1.35 \mathrm{E}-01$ & $3.49 \mathrm{E}-08$ \\
\hline LG9.90.10.20.7 & & $6.96 \mathrm{E}-06$ & 16.2 & 0.391 & $7.75 \mathrm{E}-03$ & 2,310 & $5.80 \mathrm{E}-02$ & 1,970 & $6.69 \mathrm{E}-02$ & 13,700 & $6.45 \mathrm{E}-02$ & 25,700 & $1.48 \mathrm{E}-01$ & $4.09 E-08$ \\
\hline LG9.90.10.20.8 & & $6.95 \mathrm{E}-06$ & 18.2 & 0.390 & $7.74 \mathrm{E}-03$ & 2,260 & $5.67 \mathrm{E}-02$ & 1,950 & $6.63 \mathrm{E}-02$ & 13,800 & $6.50 \mathrm{E}-02$ & 20,700 & $1.19 \mathrm{E}-01$ & 2.67E-08 \\
\hline LG9.90.10.20.9 & & $6.89 \mathrm{E}-06$ & 21.0 & 0.389 & $7.72 \mathrm{E}-03$ & 2,130 & $5.31 \mathrm{E}-02$ & 1,840 & $6.21 \mathrm{E}-02$ & 13,000 & $6.08 \mathrm{E}-02$ & 19,600 & $1.12 \mathrm{E}-01$ & $2.52 \mathrm{E}-08$ \\
\hline \multicolumn{15}{|c|}{ LG9 $90^{\circ} \mathrm{C} \mathrm{pH} 10$ Reactor $\# 2$} \\
\hline LG9.90.10.40.Blank1 & & $1.99 \mathrm{E}-05$ & --- & --- & --- & [49.4] & --- & {$[75.6]$} & --- & [164] & --- & [134] & --- & --- \\
\hline LG9.90.10.40.Blank2 & & $1.82 \mathrm{E}-05$ & --- & --- & --- & [49.4] & --- & {$[75.6]$} & --- & [164] & --- & [134] & --- & -.- \\
\hline LG9.90.10.40.Blank3 & & $1.89 \mathrm{E}-05$ & --- & --- & --- & {$[49.4]$} & -- & {$[75.6]$} & --- & [164] & --- & [134] & --- & --- \\
\hline LG9.90.10.40.1 & & $1.78 \mathrm{E}-05$ & 2.3 & 0.147 & $2.91 \mathrm{E}-03$ & 830 & $1.42 \mathrm{E}-01$ & 657 & $1.52 \mathrm{E}-01$ & 5,020 & 1.61E-01 & 7,190 & $2.83 \mathrm{E}-01$ & $6.57 \mathrm{E}-08$ \\
\hline LG9.90.10.40.2 & & $1.83 \mathrm{E}-05$ & 4.3 & 0.146 & $2.89 \mathrm{E}-03$ & 1,140 & $2.02 \mathrm{E}-01$ & 897 & $2.15 \mathrm{E}-01$ & 6,820 & $2.27 \mathrm{E}-01$ & 8,840 & $3.60 \mathrm{E}-01$ & $7.28 \mathrm{E}-08$ \\
\hline LG9.90.10.40.3 & & $1.96 \mathrm{E}-05$ & 7.0 & 0.144 & $2.86 \mathrm{E}-03$ & 1,230 & $2.36 \mathrm{E}-01$ & 982 & $2.55 \mathrm{E}-01$ & 7,430 & 2.67E-01 & 8,260 & $3.63 \mathrm{E}-01$ & $5.47 \mathrm{E}-08$ \\
\hline LG9.90.10.40.4 & & $1.83 \mathrm{E}-05$ & 9.1 & 0.143 & $2.85 \mathrm{E}-03$ & 1,230 & $2.21 \mathrm{E}-01$ & 963 & $2.34 \mathrm{E}-01$ & 7,520 & $2.54 \mathrm{E}-01$ & 8,490 & $3.51 \mathrm{E}-01$ & $5.85 \mathrm{E}-08$ \\
\hline LG9.90.10.40.5 & & $1.94 \mathrm{E}-05$ & 11.2 & 0.141 & $2.83 \mathrm{E}-03$ & 1,180 & $2.26 \mathrm{E}-01$ & 946 & $2.45 \mathrm{E}-01$ & 7,180 & $2.58 \mathrm{E}-01$ & 7,260 & $3.19 \mathrm{E}-01$ & $3.73 \mathrm{E}-08$ \\
\hline LG9.90.10.40.6 & & $1.80 \mathrm{E}-05$ & 14.0 & 0.139 & $2.80 \mathrm{E}-03$ & 1,200 & $2.16 \mathrm{E}-01$ & 947 & 2.30E-01 & 7,260 & $2.45 \mathrm{E}-01$ & 7,000 & $2.89 \mathrm{E}-01$ & 2.95E-08 \\
\hline LG9.90.10.40.7 & & $1.78 \mathrm{E}-05$ & 16.2 & 0.138 & $2.78 \mathrm{E}-03$ & 1,230 & $2.20 \mathrm{E}-01$ & 984 & $2.38 \mathrm{E}-01$ & 7,540 & 2.53E-01 & 7,090 & $2.91 \mathrm{E}-01$ & 2.67E-08 \\
\hline LG9.90.10.40.8 & & 1.77E-05 & 18.2 & 0.137 & $2.77 \mathrm{E}-03$ & 1,160 & 2.07E-01 & 908 & 2.19E-01 & 7,060 & $2.36 \mathrm{E}-01$ & 6,580 & $2.69 \mathrm{E}-01$ & $2.53 \mathrm{E}-08$ \\
\hline LG9.90.10.40.9 & & $1.79 \mathrm{E}-05$ & 21.0 & 0.135 & $2.74 \mathrm{E}-03$ & 1,220 & 2.23E-01 & 968 & $2.39 \mathrm{E}-01$ & 7,430 & $2.55 \mathrm{E}-01$ & 6,440 & $2.70 \mathrm{E}-01$ & $1.55 \mathrm{E}-08$ \\
\hline
\end{tabular}




\begin{tabular}{|c|c|c|c|c|c|c|c|c|c|c|c|c|c|c|}
\hline Sample ID & $\begin{array}{l}\text { Influent } \\
\text { [Si] } \\
\text { (ppm) }\end{array}$ & $\begin{array}{l}\text { Flow } \\
\text { Rate, } q \\
\left(\mathrm{~m}^{3} \mathrm{~d}^{-1}\right)\end{array}$ & $\begin{array}{l}\text { Time } \\
\text { (d) }\end{array}$ & $\begin{array}{l}\text { Glass } \\
\text { Mass } \\
\text { (g) }\end{array}$ & $\begin{array}{l}\text { Surface } \\
\text { Area, S } \\
\left(\mathrm{m}^{2}\right)\end{array}$ & $\begin{array}{l}{[\mathrm{Al}]} \\
\left(\mu \mathrm{g} \mathrm{L}^{-1}\right)\end{array}$ & $\begin{array}{l}\text { Al Norm. } \\
\text { Diss. Rate } \\
\left(\mathrm{g} \mathrm{m}^{-2} \mathrm{~d}^{-1}\right)\end{array}$ & $\begin{array}{l}{[\mathrm{B}]} \\
\left(\mu \mathrm{L} \mathrm{L}^{-1}\right)\end{array}$ & $\begin{array}{l}\text { B Norm. } \\
\text { Diss. Rate } \\
\left(\mathrm{g} \mathrm{m}^{-2} \mathrm{~d}^{-1}\right)\end{array}$ & $\begin{array}{l}{[\mathrm{Si}]} \\
\left(\mu \mathrm{L} \mathrm{L}^{-1}\right)\end{array}$ & $\begin{array}{l}\text { Si Norm. } \\
\text { Diss. Rate } \\
\left(\mathrm{g} \mathrm{m}^{-2} \mathrm{~d}^{-1}\right)\end{array}$ & $\begin{array}{l}{[\mathrm{Na}]} \\
(\mu \mathrm{g} \mathrm{L} \\
\left.{ }^{1}\right)\end{array}$ & $\begin{array}{l}\text { Na Norm. } \\
\text { Diss. Rate, } \\
\mathrm{r} \\
\left(\mathrm{g} \mathrm{m}^{-2} \mathrm{~d}^{-1}\right)\end{array}$ & $\begin{array}{l}\mathrm{r}_{\mathrm{IEX}} \\
\left(\mathrm{mol} \mathrm{Na} \mathrm{s}^{-2} \mathrm{~s}^{-1}\right)\end{array}$ \\
\hline \multicolumn{15}{|c|}{ LG9 $90^{\circ} \mathrm{C}$ pH 10 Reactor $\# 3$} \\
\hline LG9.90.10.80.Blank1 & & $7.56 \mathrm{E}-05$ & --- & --- & --- & [49.4] & --- & {$[75.6]$} & --- & [164] & --- & [134] & --- & --- \\
\hline LG9.90.10.80.Blank2 & & $7.59 \mathrm{E}-05$ & --- & --- & --- & [49.4] & --- & {$[75.6]$} & --- & [164] & --- & [134] & --- & --- \\
\hline LG9.90.10.80.Blank3 & & $7.59 \mathrm{E}-05$ & --- & --- & --- & [49.4] & --- & {$[75.6]$} & --- & {$[164]$} & --- & [134] & --- & --- \\
\hline LG9.90.10.80.1 & & $7.36 \mathrm{E}-05$ & 2.3 & 0.097 & $1.93 E-03$ & 471 & $5.03 \mathrm{E}-01$ & 369 & $5.34 \mathrm{E}-01$ & 2,850 & $5.71 \mathrm{E}-01$ & 3,680 & $9.03 \mathrm{E}-01$ & $1.86 \mathrm{E}-07$ \\
\hline LG9.90.10.80.2 & & 7.53E-05 & 4.3 & 0.094 & $1.89 \mathrm{E}-03$ & 560 & $6.24 \mathrm{E}-01$ & 444 & $6.69 \mathrm{E}-01$ & 3,420 & 7.14E-01 & 3,070 & $7.85 \mathrm{E}-01$ & $5.83 \mathrm{E}-08$ \\
\hline LG9.90.10.80.3 & & $7.56 \mathrm{E}-05$ & 7.0 & 0.091 & $1.84 \mathrm{E}-03$ & 518 & $5.94 \mathrm{E}-01$ & 401 & $6.22 \mathrm{E}-01$ & 3,160 & $6.79 \mathrm{E}-01$ & 3,110 & $8.18 \mathrm{E}-01$ & $9.89 E-08$ \\
\hline LG9.90.10.80.4 & & $7.50 \mathrm{E}-05$ & 9.1 & 0.089 & $1.82 \mathrm{E}-03$ & 517 & $5.97 \mathrm{E}-01$ & 413 & $6.46 \mathrm{E}-01$ & 3,150 & $6.82 \mathrm{E}-01$ & 2,760 & $7.32 \mathrm{E}-01$ & 4.35E-08 \\
\hline LG9.90.10.80.5 & & $7.55 \mathrm{E}-05$ & 11.2 & 0.086 & $1.78 \mathrm{E}-03$ & 510 & $6.04 \mathrm{E}-01$ & 396 & $6.35 \mathrm{E}-01$ & 3,090 & $6.87 \mathrm{E}-01$ & 2,680 & $7.29 \mathrm{E}-01$ & $4.74 \mathrm{E}-08$ \\
\hline LG9.90.10.80.6 & & $7.49 \mathrm{E}-05$ & 14.0 & 0.083 & $1.73 \mathrm{E}-03$ & 505 & $6.10 \mathrm{E}-01$ & 400 & $6.54 \mathrm{E}-01$ & 3,130 & $7.09 E-01$ & 2,690 & $7.46 \mathrm{E}-01$ & $4.64 \mathrm{E}-08$ \\
\hline LG9.90.10.80.7 & & $7.29 \mathrm{E}-05$ & 16.2 & 0.081 & $1.70 \mathrm{E}-03$ & 505 & $6.04 \mathrm{E}-01$ & 395 & $6.40 \mathrm{E}-01$ & 3,090 & $6.93 \mathrm{E}-01$ & 2,710 & $7.44 \mathrm{E}-01$ & $5.28 \mathrm{E}-08$ \\
\hline LG9.90.10.80.8 & & $7.22 \mathrm{E}-05$ & 18.2 & 0.079 & $1.68 \mathrm{E}-03$ & 495 & $5.96 \mathrm{E}-01$ & 380 & $6.19 \mathrm{E}-01$ & 3,030 & $6.84 \mathrm{E}-01$ & 2,560 & 7.07E-01 & $4.45 \mathrm{E}-08$ \\
\hline LG9.90.10.80.9 & & $7.27 \mathrm{E}-05$ & 21.0 & 0.075 & $1.63 \mathrm{E}-03$ & 513 & $6.41 \mathrm{E}-01$ & 403 & $6.81 \mathrm{E}-01$ & 3,150 & 7.37E-01 & 2,700 & $7.74 \mathrm{E}-01$ & $4.68 \mathrm{E}-08$ \\
\hline \multicolumn{15}{|c|}{ LG9 $90^{\circ} \mathrm{C} \mathrm{pH} 10$ Reactor \#4 } \\
\hline LG9.90.10.160.Blank1 & & $1.49 \mathrm{E}-04$ & --- & --- & --- & [49.4] & --- & {$[75.6]$} & --- & [164] & --- & [134] & --- & --- \\
\hline LG9.90.10.160.Blank2 & & $1.47 \mathrm{E}-04$ & --- & --- & --- & [49.4] & -.- & {$[75.6]$} & --- & [164] & -.- & [134] & --- & --- \\
\hline LG9.90.10.160.Blank3 & & $1.49 \mathrm{E}-04$ & --- & --- & --- & [49.4] & --- & {$[75.6]$} & --- & [164] & --. & [134] & --- & --- \\
\hline LG9.90.10.160.1 & & $1.46 \mathrm{E}-04$ & 2.3 & 0.046 & $9.22 \mathrm{E}-04$ & 228 & $1.01 \mathrm{E}+00$ & 161 & $9.63 \mathrm{E}-01$ & 1,320 & $1.09 \mathrm{E}+00$ & 1,360 & $1.38 \mathrm{E}+00$ & $2.10 \mathrm{E}-07$ \\
\hline LG9.90.10.160.2 & & $1.50 \mathrm{E}-04$ & 4.3 & 0.044 & $8.94 \mathrm{E}-04$ & 228 & $1.07 \mathrm{E}+00$ & 180 & $1.14 \mathrm{E}+00$ & 1,440 & $1.27 \mathrm{E}+00$ & 1,240 & $1.34 \mathrm{E}+00$ & $9.72 \mathrm{E}-08$ \\
\hline LG9.90.10.160.3 & & $1.50 \mathrm{E}-04$ & 7.0 & 0.041 & $8.58 \mathrm{E}-04$ & 227 & $1.11 \mathrm{E}+00$ & 158 & $1.05 E+00$ & 1,290 & $1.18 \mathrm{E}+00$ & 1,320 & $1.49 \mathrm{E}+00$ & $2.20 \mathrm{E}-07$ \\
\hline LG9.90.10.160.4 & & $1.51 \mathrm{E}-04$ & 9.1 & 0.040 & $8.35 \mathrm{E}-04$ & 215 & $1.09 \mathrm{E}+00$ & 160 & $1.10 E+00$ & 1,320 & $1.25 \mathrm{E}+00$ & 1,190 & $1.38 \mathrm{E}+00$ & $1.45 \mathrm{E}-07$ \\
\hline LG9.90.10.160.5 & & $1.50 \mathrm{E}-04$ & 11.2 & 0.038 & $8.09 E-04$ & 202 & $1.04 \mathrm{E}+00$ & 151 & $1.06 \mathrm{E}+00$ & 1,250 & $1.21 \mathrm{E}+00$ & 1,160 & $1.38 \mathrm{E}+00$ & $1.61 \mathrm{E}-07$ \\
\hline LG9.90.10.160.6 & & $1.48 \mathrm{E}-04$ & 14.0 & 0.035 & $7.67 \mathrm{E}-04$ & 211 & $1.13 E+00$ & 164 & $1.19 E+00$ & 1,330 & $1.34 E+00$ & 1,160 & $1.43 E+00$ & $1.20 \mathrm{E}-07$ \\
\hline LG9.90.10.160.7 & & $1.47 \mathrm{E}-04$ & 16.2 & 0.033 & 7.44E-04 & 209 & $1.15 \mathrm{E}+00$ & 153 & $1.14 \mathrm{E}+00$ & 1,280 & $1.32 \mathrm{E}+00$ & 1,120 & $1.42 \mathrm{E}+00$ & $1.39 \mathrm{E}-07$ \\
\hline LG9.90.10.160.8 & & $1.48 \mathrm{E}-04$ & 18.2 & 0.032 & $7.24 \mathrm{E}-04$ & 186 & $1.06 \mathrm{E}+00$ & 139 & $1.08 \mathrm{E}+00$ & 1,130 & $1.21 \mathrm{E}+00$ & 1,000 & $1.31 \mathrm{E}+00$ & $1.19 \mathrm{E}-07$ \\
\hline LG9.90.10.160.9 & & 1.47E-04 & 21.0 & 0.029 & $6.83 E-04$ & 197 & $1.18 \mathrm{E}+00$ & 148 & $1.20 \mathrm{E}+00$ & 1,180 & $1.33 E+00$ & 1,080 & $1.49 \mathrm{E}+00$ & 1.44E-07 \\
\hline \multicolumn{15}{|c|}{ LG9 $90^{\circ} \mathrm{C} \mathrm{pH} 11$ Reactor $\# 1$} \\
\hline LG9.90.11.20.Blank1 & & $1.81 E-05$ & --- & --- & --- & [49.4] & --- & [75.6] & --- & [164] & --- & [134] & --- & --- \\
\hline LG9.90.11.20.Blank2 & & $1.48 \mathrm{E}-05$ & --. & --- & -.- & [49.4] & --- & {$[75.6]$} & --- & [164] & --. & [134] & --- & --. \\
\hline LG9.90.11.20.Blank3 & & $1.66 \mathrm{E}-05$ & --- & --- & --- & [49.4] & --- & {$[75.6]$} & --- & [164] & --- & [134] & --- & --- \\
\hline LG9.90.11.20.1 & & $1.55 \mathrm{E}-05$ & 2.3 & 0.746 & $1.47 \mathrm{E}-02$ & 1,300 & $3.83 \mathrm{E}-02$ & 1,110 & 4.43E-02 & 4,460 & $2.46 \mathrm{E}-02$ & 4,370 & $2.96 \mathrm{E}-02$ & $<0$ \\
\hline LG9.90.11.20.2 & & 1.64E-05 & 4.3 & 0.742 & $1.46 \mathrm{E}-02$ & 2,730 & $8.56 \mathrm{E}-02$ & 2,690 & 1.14E-01 & 9,920 & $5.83 \mathrm{E}-02$ & 10,400 & 7.49E-02 & $<0$ \\
\hline LG9.90.11.20.3 & & $1.52 \mathrm{E}-05$ & 7.0 & 0.736 & $1.46 \mathrm{E}-02$ & 2,680 & $7.79 \mathrm{E}-02$ & 2,910 & $1.15 \mathrm{E}-01$ & 10,200 & $5.56 \mathrm{E}-02$ & 11,200 & $7.48 \mathrm{E}-02$ & $<0$ \\
\hline LG9.90.11.20.5 & & $1.62 \mathrm{E}-05$ & 11.2 & 0.732 & $1.45 \mathrm{E}-02$ & 1,870 & $5.83 E-02$ & 2,200 & $9.29 \mathrm{E}-02$ & 7,430 & $4.34 \mathrm{E}-02$ & 8,410 & $6.02 \mathrm{E}-02$ & $<0$ \\
\hline LG9.90.11.20.6 & & $1.49 \mathrm{E}-05$ & 14.0 & 0.729 & $1.45 \mathrm{E}-02$ & 1,350 & $3.89 \mathrm{E}-02$ & 1,650 & $6.44 \mathrm{E}-02$ & 5,450 & $2.95 \mathrm{E}-02$ & 7,240 & $4.79 \mathrm{E}-02$ & $<0$ \\
\hline LG9.90.11.20.7 & & $1.48 \mathrm{E}-05$ & 16.2 & 0.728 & $1.44 \mathrm{E}-02$ & 1,240 & $3.55 \mathrm{E}-02$ & 1,520 & $5.89 \mathrm{E}-02$ & 5,130 & $2.76 \mathrm{E}-02$ & 6,500 & $4.28 \mathrm{E}-02$ & $<0$ \\
\hline LG9.90.11.20.8 & & $1.59 \mathrm{E}-05$ & 18.2 & 0.726 & $1.44 \mathrm{E}-02$ & 1,230 & $3.79 \mathrm{E}-02$ & 1,550 & $6.46 \mathrm{E}-02$ & 5,150 & $2.97 \mathrm{E}-02$ & 5,940 & $4.20 \mathrm{E}-02$ & $<0$ \\
\hline LG9.90.11.20.9 & & $1.73 \mathrm{E}-05$ & 21.0 & 0.723 & $1.44 \mathrm{E}-02$ & 999 & 3.36E-02 & 1,260 & $5.74 \mathrm{E}-02$ & 4,240 & 2.68E-02 & 5,100 & 3.94E-02 & $<0$ \\
\hline \multicolumn{15}{|c|}{ LG9 $90^{\circ} \mathrm{C} \mathrm{pH} 11$ Reactor $\# 2$} \\
\hline LG9.90.11.40.Blank1 & & $1.30 \mathrm{E}-05$ & --- & --- & --- & [49.4] & --- & {$[75.6]$} & --- & [164] & --- & [134] & --- & --- \\
\hline LG9.90.11.40.Blank3 & & $6.32 \mathrm{E}-06$ & --- & --- & --- & [49.4] & --- & {$[75.6]$} & --- & {$[164]$} & --- & [134] & --- & --- \\
\hline LG9.90.11.40.1 & & $2.08 \mathrm{E}-05$ & 0.9 & 0 & 0 & 1,090 & 0 & 835 & 0 & 6,020 & 0 & 7,260 & $1.31 \mathrm{E}-01$ & 2.13E-08 \\
\hline LG9.90.11.40.2 & & $2.20 \mathrm{E}-05$ & 1.4 & 0.375 & $7.38 \mathrm{E}-03$ & 1,550 & $1.29 \mathrm{E}-01$ & 1,220 & $1.37 \mathrm{E}-01$ & 8,450 & $1.32 \mathrm{E}-01$ & 10,900 & $2.08 \mathrm{E}-01$ & $3.57 \mathrm{E}-08$ \\
\hline
\end{tabular}




\begin{tabular}{|c|c|c|c|c|c|c|c|c|c|c|c|c|c|c|}
\hline Sample ID & $\begin{array}{l}\text { Influent } \\
\text { [Si] } \\
\text { (ppm) }\end{array}$ & $\begin{array}{l}\text { Flow } \\
\text { Rate, } \mathrm{q} \\
\left(\mathrm{m}^{3} \mathrm{~d}^{-1}\right)\end{array}$ & $\begin{array}{l}\text { Time } \\
\text { (d) }\end{array}$ & $\begin{array}{l}\text { Glass } \\
\text { Mass } \\
\text { (g) }\end{array}$ & $\begin{array}{l}\text { Surface } \\
\text { Area, S } \\
\left(\mathrm{m}^{2}\right)\end{array}$ & $\begin{array}{l}{[\mathrm{Al}]} \\
\left(\mu \mathrm{g} \mathrm{L}^{-1}\right)\end{array}$ & $\begin{array}{l}\text { Al Norm. } \\
\text { Diss. Rate } \\
\left(\mathrm{g} \mathrm{m}^{-2} \mathrm{~d}^{-1}\right)\end{array}$ & $\begin{array}{l}{[B]} \\
\left(\mu g L^{-1}\right)\end{array}$ & $\begin{array}{l}\text { B Norm. } \\
\text { Diss. Rate } \\
\left(\mathrm{g} \mathrm{m}^{-2} \mathrm{~d}^{-1}\right)\end{array}$ & $\begin{array}{l}{[\mathrm{Si}]} \\
\left(\mu \mathrm{L} \mathrm{L}^{-1}\right)\end{array}$ & $\begin{array}{l}\text { Si Norm. } \\
\text { Diss. Rate } \\
\left(\mathrm{g} \mathrm{m}^{-2} \mathrm{~d}^{-1}\right)\end{array}$ & $\begin{array}{l}{[\mathrm{Na}]} \\
(\mu \mathrm{gg} \mathrm{L} \\
\left.{ }^{1}\right)\end{array}$ & $\begin{array}{l}\text { Na Norm. } \\
\text { Diss. Rate, } \\
\mathrm{r} \\
\left(\mathrm{g} \mathrm{m}^{-2} \mathrm{~d}^{-1}\right)\end{array}$ & $\begin{array}{l}\mathrm{r}_{\mathrm{IEX}} \\
\left(\mathrm{mol} \mathrm{Na} \mathrm{No}^{-1}\right)\end{array}$ \\
\hline LG9.90.11.40.3 & & $2.31 \mathrm{E}-05$ & 2.3 & 0.373 & $7.36 \mathrm{E}-03$ & 1,980 & $1.74 \mathrm{E}-01$ & 1,610 & $1.91 \mathrm{E}-01$ & 10,900 & $1.79 \mathrm{E}-01$ & 12,100 & $2.43 \mathrm{E}-01$ & $2.64 \mathrm{E}-08$ \\
\hline LG9.90.11.40.4 & & $2.23 \mathrm{E}-05$ & 4.3 & 0.369 & $7.30 \mathrm{E}-03$ & 2,330 & $1.99 \mathrm{E}-01$ & 1,940 & $2.24 \mathrm{E}-01$ & 12,700 & $2.03 \mathrm{E}-01$ & 13,300 & $2.60 \mathrm{E}-01$ & $1.84 \mathrm{E}-08$ \\
\hline LG9.90.11.40.5 & & $2.36 \mathrm{E}-05$ & 7.0 & 0.362 & $7.21 \mathrm{E}-03$ & 2,600 & $2.38 \mathrm{E}-01$ & 2,330 & $2.89 \mathrm{E}-01$ & 14,700 & $2.52 \mathrm{E}-01$ & 14,500 & $3.05 \mathrm{E}-01$ & $8.11 \mathrm{E}-09$ \\
\hline LG9.90.11.40.6 & & $2.26 \mathrm{E}-05$ & 9.1 & 0.358 & $7.16 \mathrm{E}-03$ & 2,730 & $2.41 \mathrm{E}-01$ & 2,520 & $3.02 \mathrm{E}-01$ & 15,700 & $2.60 \mathrm{E}-01$ & 15,900 & $3.23 \mathrm{E}-01$ & $1.07 \mathrm{E}-08$ \\
\hline LG9.90.11.40.7 & & $2.17 \mathrm{E}-05$ & 11.2 & 0.353 & 7.09E-03 & 2,920 & $2.50 \mathrm{E}-01$ & 2,760 & $3.19 \mathrm{E}-01$ & 17,100 & $2.74 \mathrm{E}-01$ & 16,200 & $3.18 \mathrm{E}-01$ & $<0$ \\
\hline LG9.90.11.40.8 & & $2.39 \mathrm{E}-05$ & 14.0 & 0.345 & $6.98 \mathrm{E}-03$ & 2,940 & $2.81 \mathrm{E}-01$ & 2,830 & $3.66 \mathrm{E}-01$ & 17,400 & $3.12 \mathrm{E}-01$ & 17,500 & $3.84 \mathrm{E}-01$ & $9.05 \mathrm{E}-09$ \\
\hline LG9.90.11.40.9 & & $2.35 \mathrm{E}-05$ & 16.2 & 0.340 & $6.91 \mathrm{E}-03$ & 2,950 & $2.80 \mathrm{E}-01$ & 2,920 & $3.75 \mathrm{E}-01$ & 17,800 & $3.17 \mathrm{E}-01$ & 18,000 & $3.92 \mathrm{E}-01$ & $8.65 \mathrm{E}-09$ \\
\hline \multicolumn{15}{|c|}{ LG9 $90^{\circ} \mathrm{C} \mathrm{pH} 11$ Reactor $\# 3$} \\
\hline LG9.90.11.80.Blank1 & & 3.72E-05 & --- & --- & --- & [49.4] & --- & [75.6] & --- & [164] & --- & [134] & --- & --- \\
\hline LG9.90.11.80.Blank3 & & $2.35 \mathrm{E}-05$ & -.- & --- & --- & [49.4] & --. & {$[75.6]$} & --- & [164] & --- & [134] & --- & --- \\
\hline LG9.90.11.80.1 & & $7.09 \mathrm{E}-05$ & 0.9 & 0.296 & $5.84 \mathrm{E}-03$ & 712 & $2.42 \mathrm{E}-01$ & 525 & $2.41 \mathrm{E}-01$ & 3,840 & $2.44 \mathrm{E}-01$ & 4,740 & $3.69 \mathrm{E}-01$ & $6.45 \mathrm{E}-08$ \\
\hline LG9.90.11.80.2 & & $7.25 \mathrm{E}-05$ & 1.4 & 0.295 & $5.83 \mathrm{E}-03$ & 1,100 & $3.82 \mathrm{E}-01$ & 853 & $4.01 \mathrm{E}-01$ & 6,000 & $3.91 \mathrm{E}-01$ & 6,820 & $5.45 \mathrm{E}-01$ & $7.20 \mathrm{E}-08$ \\
\hline LG9.90.11.80.3 & & $7.56 \mathrm{E}-05$ & 2.3 & 0.292 & $5.79 \mathrm{E}-03$ & 1,150 & 4.19E-01 & 885 & 4.37E-01 & 6,250 & 4.27E-01 & 6,130 & $5.13 \mathrm{E}-01$ & $3.85 \mathrm{E}-08$ \\
\hline LG9.90.11.80.4 & & $7.41 \mathrm{E}-05$ & 4.3 & 0.286 & $5.71 \mathrm{E}-03$ & 1,140 & $4.14 \mathrm{E}-01$ & 884 & $4.34 \mathrm{E}-01$ & 6,200 & $4.22 \mathrm{E}-01$ & 5,830 & $4.86 \mathrm{E}-01$ & $2.60 \mathrm{E}-08$ \\
\hline LG9.90.11.80.5 & & $7.44 \mathrm{E}-05$ & 7.0 & 0.278 & $5.60 \mathrm{E}-03$ & 1,130 & $4.20 \mathrm{E}-01$ & 912 & $4.59 \mathrm{E}-01$ & 6,210 & 4.33E-01 & 6,220 & $5.31 \mathrm{E}-01$ & $3.63 E-08$ \\
\hline LG9.90.11.80.6 & & $7.54 \mathrm{E}-05$ & 9.1 & 0.272 & $5.52 \mathrm{E}-03$ & 1,300 & 4.97E-01 & 1,080 & $5.59 \mathrm{E}-01$ & 7,370 & $5.28 \mathrm{E}-01$ & 6,800 & $5.97 \mathrm{E}-01$ & $1.92 \mathrm{E}-08$ \\
\hline LG9.90.11.80.7 & & $7.49 \mathrm{E}-05$ & 11.2 & 0.266 & $5.43 \mathrm{E}-03$ & 1,270 & $4.90 \mathrm{E}-01$ & 1,050 & $5.48 \mathrm{E}-01$ & 7,180 & $5.19 \mathrm{E}-01$ & 7,060 & $6.25 \mathrm{E}-01$ & $3.88 \mathrm{E}-08$ \\
\hline LG9.90.11.80.8 & & $7.41 \mathrm{E}-05$ & 14.0 & 0.254 & $5.28 \mathrm{E}-03$ & 1,430 & $5.61 \mathrm{E}-01$ & 1,230 & $6.54 \mathrm{E}-01$ & 8,270 & $6.09 \mathrm{E}-01$ & 7,780 & $7.01 \mathrm{E}-01$ & $2.40 \mathrm{E}-08$ \\
\hline LG9.90.11.80.9 & & $7.41 \mathrm{E}-05$ & 16.2 & 0.248 & $5.18 \mathrm{E}-03$ & 1,430 & $5.72 \mathrm{E}-01$ & 1,240 & $6.71 \mathrm{E}-01$ & 8,290 & $6.21 \mathrm{E}-01$ & 8,130 & $7.46 \mathrm{E}-01$ & $3.79 E-08$ \\
\hline \multicolumn{15}{|c|}{ LG9 $90{ }^{\circ} \mathrm{C} \mathrm{pH} 11$ Reactor \#4 } \\
\hline LG9.90.11.160.Blank1 & & 7.33E-05 & --- & --- & --- & [49.4] & --- & [75.6] & --- & [164] & --- & [134] & --- & --- \\
\hline LG9.90.11.160.Blank3 & & $4.66 \mathrm{E}-05$ & --- & --- & --- & [49.4] & --- & {$[75.6]$} & --- & [164] & --- & [134] & --- & --- \\
\hline LG9.90.11.160.1 & & 1.42E-04 & 0.9 & 0.099 & $1.96 \mathrm{E}-03$ & 346 & $6.98 \mathrm{E}-01$ & 241 & $6.58 \mathrm{E}-01$ & 2,000 & $7.56 \mathrm{E}-01$ & 1,970 & $9.13 \mathrm{E}-01$ & $1.28 \mathrm{E}-07$ \\
\hline LG9.90.11.160.2 & & $1.49 \mathrm{E}-04$ & 1.4 & 0.098 & $1.95 \mathrm{E}-03$ & 520 & $1.11 \mathrm{E}+00$ & 371 & $1.07 \mathrm{E}+00$ & 3,030 & $1.21 \mathrm{E}+00$ & 2,540 & $1.24 \mathrm{E}+00$ & $8.70 \mathrm{E}-08$ \\
\hline LG9.90.11.160.3 & & $1.50 \mathrm{E}-04$ & 2.3 & 0.096 & $1.92 \mathrm{E}-03$ & 481 & $1.05 E+00$ & 341 & $1.00 \mathrm{E}+00$ & 2,790 & $1.14 \mathrm{E}+00$ & 2,430 & $1.21 \mathrm{E}+00$ & $1.06 \mathrm{E}-07$ \\
\hline LG9.90.11.160.4 & & $1.50 \mathrm{E}-04$ & 4.3 & 0.091 & $1.85 \mathrm{E}-03$ & 506 & $1.14 \mathrm{E}+00$ & 359 & $1.10 \mathrm{E}+00$ & 2,960 & $1.25 \mathrm{E}+00$ & 2,410 & $1.25 \mathrm{E}+00$ & $7.68 \mathrm{E}-08$ \\
\hline LG9.90.11.160.5 & & $1.48 \mathrm{E}-04$ & 7.0 & 0.084 & $1.76 \mathrm{E}-03$ & 507 & $1.19 \mathrm{E}+00$ & 366 & $1.17 E+00$ & 3,040 & $1.34 \mathrm{E}+00$ & 2,360 & $1.27 E+00$ & $5.51 \mathrm{E}-08$ \\
\hline LG9.90.11.160.6 & & $1.50 \mathrm{E}-04$ & 9.1 & 0.081 & $1.72 \mathrm{E}-03$ & 493 & $1.20 E+00$ & 359 & $1.18 \mathrm{E}+00$ & 2,850 & $1.30 \mathrm{E}+00$ & 2,410 & $1.35 \mathrm{E}+00$ & $8.28 \mathrm{E}-08$ \\
\hline LG9.90.11.160.7 & & $1.49 \mathrm{E}-04$ & 11.2 & 0.076 & $1.65 \mathrm{E}-03$ & 500 & $1.27 \mathrm{E}+00$ & 368 & $1.26 \mathrm{E}+00$ & 2,930 & $1.39 \mathrm{E}+00$ & 2,380 & $1.38 \mathrm{E}+00$ & $6.17 \mathrm{E}-08$ \\
\hline LG9.90.11.160.8 & & $1.48 \mathrm{E}-04$ & 14.0 & 0.071 & $1.56 \mathrm{E}-03$ & 484 & $1.28 \mathrm{E}+00$ & 348 & $1.25 \mathrm{E}+00$ & 2,820 & $1.40 \mathrm{E}+00$ & 2,270 & $1.38 \mathrm{E}+00$ & $6.70 \mathrm{E}-08$ \\
\hline LG9.90.11.160.9 & & $1.49 \mathrm{E}-04$ & 16.2 & 0.066 & $1.50 \mathrm{E}-03$ & 490 & $1.36 \mathrm{E}+00$ & 363 & $1.36 \mathrm{E}+00$ & 2,900 & $1.50 \mathrm{E}+00$ & 2,310 & $1.47 E+00$ & $5.45 \mathrm{E}-08$ \\
\hline \multicolumn{15}{|c|}{ LG9 $90^{\circ} \mathrm{C} \mathrm{pH} 12$ Reactor \#1 } \\
\hline LG9.90.12.20.Blank1 & & $1.74 \mathrm{E}-05$ & --. & --- & --- & [49.4] & --- & [75.6] & --- & [164] & --- & 1,090 & --- & --- \\
\hline LG9.90.12.20.Blank2 & & $8.50 \mathrm{E}-06$ & --- & --- & --- & [49.4] & --- & {$[75.6]$} & --- & [164] & --- & 1,190 & --- & --- \\
\hline LG9.90.12.20.Blank3 & & $6.28 \mathrm{E}-06$ & --- & --- & --- & [49.4] & --- & [75.6] & --- & [164] & --- & 1,180 & --- & -- \\
\hline LG9.90.12.20.1 & & $7.98 \mathrm{E}-06$ & 2.2 & 0.744 & $1.47 \mathrm{E}-02$ & 7,540 & $1.15 \mathrm{E}-01$ & 5,960 & $1.23 \mathrm{E}-01$ & 36,400 & $1.04 \mathrm{E}-01$ & 40,400 & $1.41 \mathrm{E}-01$ & $9.26 \mathrm{E}-09$ \\
\hline LG9.90.12.20.2 & & $6.27 \mathrm{E}-06$ & 4.3 & 0.737 & $1.46 \mathrm{E}-02$ & 14,300 & $1.72 \mathrm{E}-01$ & 12,300 & $2.00 \mathrm{E}-01$ & 72,000 & $1.62 \mathrm{E}-01$ & 81,600 & $2.25 \mathrm{E}-01$ & $1.26 \mathrm{E}-08$ \\
\hline LG9.90.12.20.3 & & 8.77E-06 & 7.0 & 0.720 & $1.44 E-02$ & 14,900 & $2.54 \mathrm{E}-01$ & 14,400 & 3.33E-01 & 80,700 & $2.58 \mathrm{E}-01$ & 83,200 & $3.26 \mathrm{E}-01$ & $<0$ \\
\hline LG9.90.12.20.4 & & $4.58 \mathrm{E}-06$ & 9.1 & 0.719 & $1.43 E-02$ & 15,000 & $1.34 \mathrm{E}-01$ & 15,500 & $1.87 \mathrm{E}-01$ & 84,200 & $1.41 \mathrm{E}-01$ & 95,500 & $1.96 \mathrm{E}-01$ & $4.27 \mathrm{E}-09$ \\
\hline LG9.90.12.20.5 & & $3.35 \mathrm{E}-06$ & 11.2 & 0.714 & $1.43 E-02$ & 15,600 & $1.02 \mathrm{E}-01$ & 17,400 & $1.54 \mathrm{E}-01$ & 91,000 & $1.12 \mathrm{E}-01$ & $\begin{array}{l}106,00 \\
0\end{array}$ & $1.60 \mathrm{E}-01$ & 2.60E-09 \\
\hline LG9.90.12.20.6 & & $5.79 \mathrm{E}-06$ & 14.0 & 0.700 & 1.41E-02 & 15,500 & $1.78 \mathrm{E}-01$ & 18,800 & 2.92E-01 & 96,200 & $2.07 E-01$ & $\begin{array}{l}115,00 \\
0\end{array}$ & $3.04 \mathrm{E}-01$ & $5.56 \mathrm{E}-09$ \\
\hline LG9.90.12.20.7 & & $7.04 \mathrm{E}-06$ & 16.2 & 0.689 & $1.39 \mathrm{E}-02$ & 14,800 & $2.09 \mathrm{E}-01$ & 18,800 & $3.60 \mathrm{E}-01$ & 95,100 & $2.52 \mathrm{E}-01$ & 110,00 & $3.57 \mathrm{E}-01$ & $<0$ \\
\hline
\end{tabular}




\begin{tabular}{|c|c|c|c|c|c|c|c|c|c|c|c|c|c|c|}
\hline Sample ID & $\begin{array}{l}\text { Influent } \\
\text { [Si] } \\
\text { (ppm) }\end{array}$ & $\begin{array}{l}\text { Flow } \\
\text { Rate, q } \\
\left(\mathrm{m}^{3} \mathrm{~d}^{-1}\right)\end{array}$ & $\begin{array}{l}\text { Time } \\
\text { (d) }\end{array}$ & $\begin{array}{l}\text { Glass } \\
\text { Mass } \\
\text { (g) }\end{array}$ & $\begin{array}{l}\text { Surface } \\
\text { Area, } \mathrm{S} \\
\left(\mathrm{m}^{2}\right)\end{array}$ & $\begin{array}{l}{[\mathrm{Al}]} \\
\left(\mu \mathrm{g} \mathrm{L}^{-1}\right)\end{array}$ & $\begin{array}{l}\text { Al Norm. } \\
\text { Diss. Rate } \\
\left(\mathrm{g} \mathrm{m}^{-2} \mathrm{~d}^{-1}\right)\end{array}$ & $\begin{array}{l}{[\mathrm{B}]} \\
\left(\mu \mathrm{g} \mathrm{L}^{-1}\right)\end{array}$ & $\begin{array}{l}\text { B Norm. } \\
\text { Diss. Rate } \\
\left(\mathrm{g} \mathrm{m}^{-2} \mathrm{~d}^{-1}\right)\end{array}$ & $\begin{array}{l}{[\mathrm{Si}]} \\
\left(\mu \mathrm{g} \mathrm{L}^{-1}\right)\end{array}$ & $\begin{array}{l}\text { Si Norm. } \\
\text { Diss. Rate } \\
\left(\mathrm{g} \mathrm{m}^{-2} \mathrm{~d}^{-1}\right)\end{array}$ & $\begin{array}{l}{[\mathrm{Na}]} \\
\left(\mu \mathrm{gg} \mathrm{L}^{-}\right. \\
\left.{ }^{1}\right)\end{array}$ & $\begin{array}{l}\text { Na Norm. } \\
\text { Diss. Rate, } \\
\mathrm{r} \\
\left(\mathrm{g} \mathrm{m}^{-2} \mathrm{~d}^{-1}\right)\end{array}$ & $\begin{array}{l}\mathrm{r}_{\mathrm{IEX}} \\
\left(\mathrm{mol} \mathrm{Na} \mathrm{N}^{-2} \mathrm{~s}^{-1}\right)\end{array}$ \\
\hline & & & & & & & & & & & & 0 & & \\
\hline LG9.90.12.20.8 & & $5.83 \mathrm{E}-06$ & 18.2 & 0.681 & $1.38 \mathrm{E}-02$ & 14,600 & $1.72 \mathrm{E}-01$ & 19,300 & $3.08 \mathrm{E}-01$ & 95,800 & $2.11 \mathrm{E}-01$ & $\begin{array}{l}115,00 \\
0\end{array}$ & $3.11 \mathrm{E}-01$ & $1.68 \mathrm{E}-09$ \\
\hline LG9.90.12.20.9 & & $8.23 \mathrm{E}-06$ & 21.0 & 0.686 & $1.39 \mathrm{E}-02$ & [49.4] & $8.18 \mathrm{E}-04$ & {$[75.6]$} & $1.69 \mathrm{E}-03$ & [164] & $5.09 E-04$ & 285 & $1.08 \mathrm{E}-03$ & $<0$ \\
\hline \multicolumn{15}{|c|}{ LG9 $90^{\circ} \mathrm{C} \mathrm{pH} 12$ Reactor $\# 2$} \\
\hline LG9.90.12.40.Blank1 & & $1.83 \mathrm{E}-05$ & --- & --- & -.- & [49.4] & --- & {$[75.6]$} & --- & [164] & --- & 1,190 & --- & --- \\
\hline LG9.90.12.40.Blank2 & & $1.66 \mathrm{E}-05$ & --. & --- & --- & [49.4] & --- & [75.6] & --- & [164] & --- & 1,200 & --- & --- \\
\hline LG9.90.12.40.Blank3 & & $1.84 \mathrm{E}-05$ & --. & --- & -.- & [49.4] & --- & {$[75.6]$} & --- & [164] & --- & 1,280 & --- & --- \\
\hline LG9.90.12.40.1 & & $1.67 \mathrm{E}-05$ & 0.9 & 0.374 & 7.37E-03 & 2,540 & $1.61 \mathrm{E}-01$ & 1,890 & $1.62 \mathrm{E}-01$ & 12,700 & $1.51 \mathrm{E}-01$ & 13,000 & $1.89 \mathrm{E}-01$ & $1.36 \mathrm{E}-08$ \\
\hline LG9.90.12.40.2 & & $1.63 \mathrm{E}-05$ & 1.4 & 0.373 & $7.36 \mathrm{E}-03$ & 5,110 & $3.16 \mathrm{E}-01$ & 3,920 & $3.28 \mathrm{E}-01$ & 25,400 & $2.94 \mathrm{E}-01$ & 24,700 & $3.50 \mathrm{E}-01$ & $1.14 \mathrm{E}-08$ \\
\hline LG9.90.12.40.3 & & $1.83 \mathrm{E}-05$ & 2.2 & 0.369 & $7.31 \mathrm{E}-03$ & 6,360 & $4.45 \mathrm{E}-01$ & 5,040 & $4.78 \mathrm{E}-01$ & 31,600 & 4.15E-01 & 31,100 & $5.00 \mathrm{E}-01$ & $1.13 \mathrm{E}-08$ \\
\hline LG9.90.12.40.4 & & $1.84 \mathrm{E}-05$ & 4.3 & 0.357 & 7.14E-03 & 7,940 & $5.72 \mathrm{E}-01$ & 6,690 & $6.53 \mathrm{E}-01$ & 40,000 & $5.41 \mathrm{E}-01$ & 40,800 & $6.76 \mathrm{E}-01$ & $1.14 \mathrm{E}-08$ \\
\hline LG9.90.12.40.5 & & $1.80 \mathrm{E}-05$ & 7.0 & 0.338 & $6.89 \mathrm{E}-03$ & 9,270 & $6.78 \mathrm{E}-01$ & 8,220 & $8.14 \mathrm{E}-01$ & 48,300 & $6.62 \mathrm{E}-01$ & 50,000 & $8.40 \mathrm{E}-01$ & $1.31 \mathrm{E}-08$ \\
\hline LG9.90.12.40.6 & & $1.42 \mathrm{E}-05$ & 9.1 & 0.329 & $6.77 \mathrm{E}-03$ & 10,300 & $6.05 \mathrm{E}-01$ & 9,950 & $7.92 \mathrm{E}-01$ & 56,000 & $6.17 \mathrm{E}-01$ & 57,000 & $7.69 \mathrm{E}-01$ & $<0$ \\
\hline LG9.90.12.40.7 & & $1.84 \mathrm{E}-05$ & 11.2 & 0.314 & $6.56 \mathrm{E}-03$ & 8,980 & $7.05 \mathrm{E}-01$ & 9,200 & $9.77 \mathrm{E}-01$ & 49,800 & 7.33E-01 & 62,300 & $1.12 \mathrm{E}+00$ & $7.32 \mathrm{E}-08$ \\
\hline LG9.90.12.40.8 & & $1.57 \mathrm{E}-05$ & 14.0 & 0.295 & $6.29 \mathrm{E}-03$ & 10,300 & $7.17 \mathrm{E}-01$ & 10,700 & $1.01 \mathrm{E}+00$ & 58,300 & $7.60 \mathrm{E}-01$ & 61,400 & $9.81 \mathrm{E}-01$ & $<0$ \\
\hline \multicolumn{15}{|c|}{ LG9 $90^{\circ} \mathrm{C} \mathrm{pH} 12$ Reactor $\# 3$} \\
\hline LG9.90.12.80.Blank1 & & $7.29 \mathrm{E}-05$ & -.- & --- & --- & [49.4] & --- & {$[75.6]$} & --- & [164] & --- & 1,190 & $\cdots$ & --- \\
\hline LG9.90.12.80.Blank2 & & $7.30 \mathrm{E}-05$ & -.- & --- & -.- & [49.4] & --- & [75.6] & --- & [164] & --- & 1,240 & --- & -.- \\
\hline LG9.90.12.80.Blank3 & & $7.16 \mathrm{E}-05$ & --- & --- & --- & [49.4] & --- & {$[75.6]$} & --- & [164] & --- & 1,270 & --- & --- \\
\hline LG9.90.12.80.1 & & $6.58 \mathrm{E}-05$ & 0.9 & 0.295 & $5.85 \mathrm{E}-03$ & 2,090 & $6.57 \mathrm{E}-01$ & 1,510 & $6.43 \mathrm{E}-01$ & 10,600 & $6.25 \mathrm{E}-01$ & 10,800 & $7.80 \mathrm{E}-01$ & $6.91 \mathrm{E}-08$ \\
\hline LG9.90.12.80.2 & & 7.06E-05 & 1.4 & 0.293 & $5.82 \mathrm{E}-03$ & 3,150 & $1.07 E+00$ & 2,370 & $1.09 E+00$ & 15,800 & $1.00 \mathrm{E}+00$ & 14,900 & $1.16 \mathrm{E}+00$ & $3.64 \mathrm{E}-08$ \\
\hline LG9.90.12.80.3 & & $7.28 \mathrm{E}-05$ & 2.2 & 0.285 & $5.72 \mathrm{E}-03$ & 3,420 & $1.22 \mathrm{E}+00$ & 2,520 & $1.21 \mathrm{E}+00$ & 17,000 & $1.13 E+00$ & 15,500 & $1.27 \mathrm{E}+00$ & $2.66 \mathrm{E}-08$ \\
\hline LG9.90.12.80.4 & & $7.29 \mathrm{E}-05$ & 4.3 & 0.266 & $5.46 \mathrm{E}-03$ & 3,560 & $1.33 \mathrm{E}+00$ & 2,670 & $1.35 E+00$ & 17,800 & $1.25 \mathrm{E}+00$ & 17,600 & $1.51 \mathrm{E}+00$ & $8.04 \mathrm{E}-08$ \\
\hline LG9.90.12.80.5 & & 7.34E-05 & 7.0 & 0.240 & $5.09 \mathrm{E}-03$ & 3,900 & $1.57 E+00$ & 2,910 & $1.59 \mathrm{E}+00$ & 19,700 & 1. $49 \mathrm{E}+00$ & 15,700 & $1.45 \mathrm{E}+00$ & $<0$ \\
\hline LG9.90.12.80.6 & & 7.16E-05 & 9.1 & 0.229 & 4.93E-03 & 3,640 & $1.48 \mathrm{E}+00$ & 2,730 & $1.50 \mathrm{E}+00$ & 18,300 & $1.39 E+00$ & 17,400 & $1.62 \mathrm{E}+00$ & $6.14 \mathrm{E}-08$ \\
\hline LG9.90.12.80.7 & & 7.31E-05 & 11.2 & 0.212 & 4.69E-03 & 3,520 & $1.53 \mathrm{E}+00$ & 2,700 & $1.59 \mathrm{E}+00$ & 17,800 & $1.45 \mathrm{E}+00$ & 17,700 & $1.77 \mathrm{E}+00$ & $8.99 \mathrm{E}-08$ \\
\hline LG9.90.12.80.8 & & $6.94 \mathrm{E}-05$ & 14.0 & 0.192 & 4.39E-03 & 3,300 & $1.46 \mathrm{E}+00$ & 2,560 & $1.53 E+00$ & 16,900 & $1.40 \mathrm{E}+00$ & 17,100 & $1.73 \mathrm{E}+00$ & $1.03 E-07$ \\
\hline LG9.90.12.80.9 & & $6.95 \mathrm{E}-05$ & 16.2 & 0.181 & 4.22E-03 & 3,010 & $1.39 \mathrm{E}+00$ & 2,350 & $1.46 \mathrm{E}+00$ & 15,700 & $1.36 \mathrm{E}+00$ & 15,600 & $1.65 \mathrm{E}+00$ & $9.31 E-08$ \\
\hline \multicolumn{15}{|c|}{ LG9 $90^{\circ} \mathrm{C} \mathrm{pH} 12$ Reactor $\# 4$} \\
\hline LG9.90.12.160.Blank1 & & $1.44 \mathrm{E}-04$ & --. & --- & -- & [49.4] & --- & [75.6] & --- & [164] & --- & 1,030 & --- & --- \\
\hline LG9.90.12.160.Blank2 & & $1.45 \mathrm{E}-04$ & --. & --- & --- & [49.4] & --- & [75.6] & --- & [164] & --- & 1,160 & --- & -.- \\
\hline LG9.90.12.160.Blank3 & & $1.44 \mathrm{E}-04$ & --- & --- & --- & [49.4] & --- & {$[75.6]$} & --- & [164] & --- & 924 & --- & --- \\
\hline LG9.90.12.160.1 & & $1.32 \mathrm{E}-04$ & 0.9 & 0.094 & $1.89 \mathrm{E}-03$ & 989 & $1.94 \mathrm{E}+00$ & 702 & $1.86 \mathrm{E}+00$ & 5,190 & $1.91 \mathrm{E}+00$ & 5,460 & $2.46 \mathrm{E}+00$ & $3.00 \mathrm{E}-07$ \\
\hline LG9.90.12.160.2 & & $1.47 \mathrm{E}-04$ & 1.4 & 0.093 & $1.87 \mathrm{E}-03$ & 1,340 & $2.94 \mathrm{E}+00$ & 966 & $2.87 E+00$ & 7,030 & $2.89 E+00$ & 6,970 & $3.51 \mathrm{E}+00$ & $3.24 \mathrm{E}-07$ \\
\hline LG9.90.12.160.3 & & $1.48 \mathrm{E}-04$ & 2.2 & 0.086 & $1.78 \mathrm{E}-03$ & 1,370 & $3.19 E+00$ & 988 & $3.11 \mathrm{E}+00$ & 7,140 & $3.12 \mathrm{E}+00$ & 7,200 & $3.85 \mathrm{E}+00$ & $3.70 \mathrm{E}-07$ \\
\hline LG9.90.12.160.4 & & $1.46 \mathrm{E}-04$ & 4.3 & 0.073 & $1.59 \mathrm{E}-03$ & 1,320 & $3.38 \mathrm{E}+00$ & 946 & $3.28 \mathrm{E}+00$ & 6,950 & $3.34 \mathrm{E}+00$ & 6,680 & $3.93 E+00$ & 3.27E-07 \\
\hline LG9.90.12.160.5 & & $1.47 \mathrm{E}-04$ & 7.0 & 0.057 & $1.35 \mathrm{E}-03$ & 1,250 & $3.79 E+00$ & 917 & $3.76 \mathrm{E}+00$ & 6,650 & $3.78 \mathrm{E}+00$ & 6,500 & $4.52 \mathrm{E}+00$ & $3.84 \mathrm{E}-07$ \\
\hline LG9.90.12.160.6 & & $1.47 \mathrm{E}-04$ & 9.1 & 0.050 & $1.23 \mathrm{E}-03$ & 1,180 & $3.92 \mathrm{E}+00$ & 856 & $3.85 E+00$ & 6,230 & $3.88 \mathrm{E}+00$ & 6,080 & $4.64 E+00$ & $3.98 \mathrm{E}-07$ \\
\hline LG9.90.12.160.7 & & $1.45 E-04$ & 11.2 & 0.040 & 1.07E-03 & 1,100 & $4.16 \mathrm{E}+00$ & 797 & $4.08 \mathrm{E}+00$ & 5,800 & $4.11 \mathrm{E}+00$ & 5,680 & $4.93 E+00$ & 4.29E-07 \\
\hline LG9.90.12.160.8 & & $1.41 \mathrm{E}-04$ & 14.0 & 0.030 & 8.73E-04 & 959 & $4.32 E+00$ & 704 & $4.29 \mathrm{E}+00$ & 5,170 & $4.36 \mathrm{E}+00$ & 4,920 & $5.08 \mathrm{E}+00$ & $4.01 E-07$ \\
\hline LG9.90.12.160.9 & & $1.41 \mathrm{E}-04$ & 16.2 & 0.024 & 7.59E-04 & 821 & $4.25 \mathrm{E}+00$ & 606 & $4.25 \mathrm{E}+00$ & 4,470 & $4.34 E+00$ & 4,340 & $5.16 \mathrm{E}+00$ & 4.60E-07 \\
\hline \multicolumn{15}{|c|}{ LG9 $23^{\circ} \mathrm{C}$ pH 9 Si Reactor \#1 } \\
\hline LG9.23.9.SI5.Blank1 & 5 & $3.04 \mathrm{E}-05$ & --. & --- & -.- & [49.4] & --- & {$[75.6]$} & --- & 4,260 & --- & [134] & --- & --- \\
\hline
\end{tabular}




\begin{tabular}{|c|c|c|c|c|c|c|c|c|c|c|c|c|c|c|}
\hline Sample ID & $\begin{array}{l}\text { Influent } \\
{[\mathrm{Si}]} \\
(\mathrm{ppm})\end{array}$ & $\begin{array}{l}\text { Flow } \\
\text { Rate, } \mathrm{q} \\
\left(\mathrm{m}^{3} \mathrm{~d}^{-1}\right)\end{array}$ & $\begin{array}{l}\text { Time } \\
\text { (d) }\end{array}$ & $\begin{array}{l}\text { Glass } \\
\text { Mass } \\
\text { (g) }\end{array}$ & $\begin{array}{l}\text { Surface } \\
\text { Area, S } \\
\left(\mathrm{m}^{2}\right)\end{array}$ & $\begin{array}{l}{[\mathrm{Al}]} \\
\left(\mu \mathrm{L} \mathrm{L}^{-1}\right)\end{array}$ & $\begin{array}{l}\text { Al Norm. } \\
\text { Diss. Rate } \\
\left(\mathrm{g} \mathrm{m}^{-2} \mathrm{~d}^{-1}\right)\end{array}$ & $\begin{array}{l}{[\mathrm{B}]} \\
\left(\mu \mathrm{g} \mathrm{L}^{-1}\right)\end{array}$ & $\begin{array}{l}\text { B Norm. } \\
\text { Diss. Rate } \\
\left(\mathrm{g} \mathrm{m}^{-2} \mathrm{~d}^{-1}\right)\end{array}$ & $\begin{array}{l}{[\mathrm{Si}]} \\
\left(\mu \mathrm{g} \mathrm{L}^{-1}\right)\end{array}$ & $\begin{array}{l}\text { Si Norm. } \\
\text { Diss. Rate } \\
\left(\mathrm{g} \mathrm{m}^{-2} \mathrm{~d}^{-1}\right)\end{array}$ & $\begin{array}{l}{[\mathrm{Na}]} \\
(\mu \mathrm{g} \mathrm{L} \\
\left.{ }^{1}\right)\end{array}$ & $\begin{array}{l}\text { Na Norm. } \\
\text { Diss. Rate, } \\
\mathrm{r} \\
\left(\mathrm{g} \mathrm{m}^{-2} \mathrm{~d}^{-1}\right)\end{array}$ & $\begin{array}{l}\mathrm{r}_{\mathrm{IEX}} \\
\left(\mathrm{mol} \mathrm{Na} \mathrm{No}^{-1}\right)\end{array}$ \\
\hline LG9.23.9.S15.Blank2 & 5 & $1.91 \mathrm{E}-05$ & --- & --- & --- & {$[49.4]$} & --- & {$[75.6]$} & --- & 3,930 & --- & [134] & --- & --- \\
\hline LG9.23.9.S15.Blank3 & 5 & $1.88 \mathrm{E}-05$ & --- & --- & --- & {$[49.4]$} & --- & {$[75.6]$} & --- & 4,420 & --- & [134] & --- & --- \\
\hline LG9.23.9.SI5.2 & 5 & $1.94 \mathrm{E}-05$ & 7.0 & 4.004 & $7.88 \mathrm{E}-02$ & [49.4] & 3.40E-04 & 137 & $1.28 \mathrm{E}-03$ & 4,030 & --- & 3,590 & $5.68 \mathrm{E}-03$ & $2.21 \mathrm{E}-09$ \\
\hline LG9.23.9.SI5.4 & 5 & $1.97 \mathrm{E}-05$ & 14.0 & 4.004 & $7.88 \mathrm{E}-02$ & 59 & $4.15 \mathrm{E}-04$ & 124 & $1.17 \mathrm{E}-03$ & 4,770 & --- & 1,980 & $3.17 \mathrm{E}-03$ & $1.01 E-09$ \\
\hline LG9.23.9.SI5.6 & 5 & $1.95 \mathrm{E}-05$ & 21.0 & 4.003 & $7.88 \mathrm{E}-02$ & 57 & 3.97E-04 & 94 & $8.75 \mathrm{E}-04$ & 4,750 & --- & 1,690 & $2.68 \mathrm{E}-03$ & $9.10 \mathrm{E}-10$ \\
\hline LG9.23.9.SI5.8 & 5 & $1.98 \mathrm{E}-05$ & 27.8 & 4.003 & $7.88 \mathrm{E}-02$ & $\begin{array}{l}{[49.4]} \\
44^{\star}\end{array}$ & 3.48E-04 & $\begin{array}{l}{[75.6]} \\
72^{\star}\end{array}$ & 7.20E-04 & 4,700 & --- & 1,180 & $1.91 \mathrm{E}-03$ & $5.98 \mathrm{E}-10$ \\
\hline LG9.23.9.S15.10 & 5 & $2.01 \mathrm{E}-05$ & 34.9 & 4.003 & $7.88 \mathrm{E}-02$ & $\begin{array}{l}{[49.4]} \\
41^{\star}\end{array}$ & 3.52E-04 & $\begin{array}{l}{[75.6]} \\
74^{\star}\end{array}$ & 7.30E-04 & 4,700 & --- & 1,150 & $1.88 \mathrm{E}-03$ & $5.81 \mathrm{E}-10$ \\
\hline LG9.23.9.S15.11 & 5 & $2.01 \mathrm{E}-05$ & 37.9 & 4.002 & $7.88 \mathrm{E}-02$ & 29 & $2.08 \mathrm{E}-04$ & 71 & $6.85 \mathrm{E}-04$ & 17,700 & --- & 843 & $1.38 \mathrm{E}-03$ & $3.51 \mathrm{E}-10$ \\
\hline LG9.23.9.S15.13 & 5 & $1.99 \mathrm{E}-05$ & 45.0 & 4.002 & $7.88 \mathrm{E}-02$ & 21 & $1.46 \mathrm{E}-04$ & 68 & $6.47 \mathrm{E}-04$ & 7,950 & -.- & 771 & $1.25 \mathrm{E}-03$ & $3.04 \mathrm{E}-10$ \\
\hline LG9.23.9.S15.14 & 5 & $1.92 \mathrm{E}-05$ & 48.9 & 4.002 & $7.88 \mathrm{E}-02$ & 23 & $1.54 \mathrm{E}-04$ & 68 & $6.24 \mathrm{E}-04$ & 7,790 & --- & 742 & $1.16 \mathrm{E}-03$ & $2.71 \mathrm{E}-10$ \\
\hline LG9.23.9.S15.19 & 5 & $1.97 \mathrm{E}-05$ & 66.0 & 3.999 & $7.87 \mathrm{E}-02$ & 25 & $1.75 \mathrm{E}-04$ & 61 & $5.73 \mathrm{E}-04$ & 6,290 & -.- & 626 & $1.00 \mathrm{E}-03$ & $2.16 \mathrm{E}-10$ \\
\hline LG9.23.9.S15.20 & 5 & 2.03E-05 & 69.9 & 3.999 & $7.87 \mathrm{E}-02$ & 26 & $1.86 \mathrm{E}-04$ & 58 & $5.70 \mathrm{E}-04$ & 5,920 & --- & 681 & $1.13 \mathrm{E}-03$ & $2.82 \mathrm{E}-10$ \\
\hline LG9.23.9.S15.21 & 5 & $1.98 \mathrm{E}-05$ & 73.0 & 3.999 & $7.87 \mathrm{E}-02$ & 28 & $1.98 \mathrm{E}-04$ & 57 & $5.47 \mathrm{E}-04$ & 5,500 & -.- & 569 & $9.20 \mathrm{E}-04$ & $1.88 \mathrm{E}-10$ \\
\hline LG9.23.9.S15.22 & 5 & 2.01E-05 & 76.9 & 3.998 & 7.87E-02 & 30 & $2.10 \mathrm{E}-04$ & 57 & $5.52 \mathrm{E}-04$ & 5,510 & --- & 568 & $9.29 \mathrm{E}-04$ & $1.90 \mathrm{E}-10$ \\
\hline \multicolumn{15}{|c|}{ LG9 $23^{\circ} \mathrm{C}$ pH 9 Si Reactor $\# 2$} \\
\hline LG9.23.9.SI20.Blank1 & 20 & $1.75 \mathrm{E}-05$ & --- & --- & --- & [49.4] & --- & [75.6] & --- & 17,500 & --- & 389 & --- & --- \\
\hline LG9.23.9.SI20.Blank2 & 20 & $1.79 \mathrm{E}-05$ & --- & --. & --- & [49.4] & -.- & [75.6] & --- & 31,300 & -.- & 262 & --- & -.- \\
\hline LG9.23.9.S120.Blank3 & 20 & $1.97 \mathrm{E}-05$ & --- & --- & --- & [49.4] & --- & [75.6] & --- & 17,800 & --- & 242 & --- & --- \\
\hline LG9.23.9.S120.2 & 20 & $1.97 \mathrm{E}-05$ & 7.0 & 3.993 & $7.86 \mathrm{E}-02$ & $\begin{array}{l}{[49.4]} \\
39^{\star}\end{array}$ & $3.47 \mathrm{E}-04$ & 145 & $1.38 \mathrm{E}-03$ & 17,700 & -.- & 3,370 & $5.43 \mathrm{E}-03$ & 2.04E-09 \\
\hline LG9.23.9.S120.4 & 20 & $1.82 \mathrm{E}-05$ & 14.0 & 3.993 & $7.86 \mathrm{E}-02$ & $\begin{array}{l}{[49.4]} \\
35^{\star}\end{array}$ & $3.20 \mathrm{E}-04$ & 114 & $1.00 \mathrm{E}-03$ & 17,700 & --- & 2,200 & $3.27 \mathrm{E}-03$ & 1.14E-09 \\
\hline LG9.23.9.SI20.6 & 20 & $1.83 \mathrm{E}-05$ & 21.0 & 3.992 & $7.86 \mathrm{E}-02$ & $\begin{array}{l}{[49.4]} \\
33^{\star}\end{array}$ & 3.22E-04 & 100 & $8.82 \mathrm{E}-04$ & 17,800 & --- & 1,350 & $2.02 \mathrm{E}-03$ & $5.73 \mathrm{E}-10$ \\
\hline LG9.23.9.SI20.8 & 20 & $1.77 \mathrm{E}-05$ & 27.8 & 3.992 & $7.86 \mathrm{E}-02$ & $\begin{array}{l}{[49.4]} \\
31^{\star}\end{array}$ & $3.12 \mathrm{E}-04$ & 89 & 7.59E-04 & 18,000 & --- & 943 & $1.37 \mathrm{E}-03$ & $3.06 \mathrm{E}-10$ \\
\hline LG9.23.9.SI20.10 & 20 & $1.74 \mathrm{E}-05$ & 34.9 & 3.992 & $7.86 \mathrm{E}-02$ & $\begin{array}{l}{[49.4]} \\
35^{\star}\end{array}$ & $3.05 \mathrm{E}-04$ & 83 & $6.94 \mathrm{E}-04$ & 18,200 & --- & 807 & $1.15 \mathrm{E}-03$ & $2.27 \mathrm{E}-10$ \\
\hline LG9.23.9.SI20.11 & 20 & $1.79 \mathrm{E}-05$ & 37.9 & 3.992 & $7.86 \mathrm{E}-02$ & 29 & $1.85 \mathrm{E}-04$ & 85 & $7.32 \mathrm{E}-04$ & 18,600 & --- & 1,020 & $1.49 \mathrm{E}-03$ & $3.82 \mathrm{E}-10$ \\
\hline LG9.23.9.SI20.13 & 20 & $1.83 \mathrm{E}-05$ & 45.0 & 3.991 & $7.86 \mathrm{E}-02$ & 28 & $1.81 \mathrm{E}-04$ & 87 & 7.64E-04 & 19,100 & --- & 863 & $1.29 \mathrm{E}-03$ & $2.65 \mathrm{E}-10$ \\
\hline LG9.23.9.SI20.14 & 20 & $1.83 \mathrm{E}-05$ & 48.9 & 3.991 & $7.85 \mathrm{E}-02$ & 29 & $1.87 \mathrm{E}-04$ & 90 & $7.95 \mathrm{E}-04$ & 19,300 & --- & 824 & $1.23 \mathrm{E}-03$ & $2.19 \mathrm{E}-10$ \\
\hline LG9.23.9.SI20.19 & 20 & $1.69 \mathrm{E}-05$ & 66.0 & 3.987 & $7.85 \mathrm{E}-02$ & 28 & $1.70 \mathrm{E}-04$ & 93 & $7.60 \mathrm{E}-04$ & 19,000 & -.- & 774 & $1.07 E-03$ & $1.56 \mathrm{E}-10$ \\
\hline LG9.23.9.SI20.20 & 20 & $1.72 E-05$ & 69.9 & 3.987 & $7.85 E-02$ & 29 & $1.77 \mathrm{E}-04$ & 90 & 7.48E-04 & 19,200 & --- & 696 & $9.77 \mathrm{E}-04$ & 1.15E-10 \\
\hline LG9.23.9.SI20.21 & 20 & $1.74 \mathrm{E}-05$ & 73.0 & 3.987 & $7.85 \mathrm{E}-02$ & 29 & $1.79 \mathrm{E}-04$ & 90 & $7.52 \mathrm{E}-04$ & 18,400 & -.- & 680 & $9.69 \mathrm{E}-04$ & $1.09 \mathrm{E}-10$ \\
\hline LG9.23.9.SI20.22 & 20 & $1.71 \mathrm{E}-05$ & 76.9 & 3.986 & $7.85 \mathrm{E}-02$ & 28 & $1.73 \mathrm{E}-04$ & 95 & $7.86 \mathrm{E}-04$ & 18,800 & --- & 770 & $1.08 \mathrm{E}-03$ & $1.46 \mathrm{E}-10$ \\
\hline \multicolumn{15}{|c|}{ LG9 $23^{\circ} \mathrm{C}$ pH 9 Si Reactor $\# 3$} \\
\hline LG9.23.9.SI35.Blank1 & 35 & 2.23E-05 & --- & --- & --- & [49.4] & --- & [75.6] & --- & 31,200 & --- & [134] & --- & --- \\
\hline LG9.23.9.SI35.Blank2 & 35 & $2.21 \mathrm{E}-05$ & --- & --. & --- & [49.4] & -.- & [75.6] & --- & 31,000 & -.- & [134] & --- & -.- \\
\hline LG9.23.9.SI35.Blank3 & 35 & 2.00E-05 & --- & --- & --- & [49.4] & --- & [75.6] & --- & 31,200 & --- & [134] & --- & --- \\
\hline LG9.23.9.S135.2 & 35 & $1.89 \mathrm{E}-05$ & 7.0 & 3.993 & $7.86 \mathrm{E}-02$ & $\begin{array}{l}{[49.4]} \\
45^{\star}\end{array}$ & $3.32 \mathrm{E}-04$ & 231 & $2.10 \mathrm{E}-03$ & 31,000 & --- & 6,580 & $1.01 \mathrm{E}-02$ & 4.05E-09 \\
\hline LG9.23.9.S135.4 & 35 & $1.94 \mathrm{E}-05$ & 14.0 & 3.992 & $7.86 \mathrm{E}-02$ & $\begin{array}{l}{[49.4]} \\
38^{\star}\end{array}$ & $3.41 \mathrm{E}-04$ & 119 & $1.11 \mathrm{E}-03$ & 31,300 & --- & 2,460 & $3.90 \mathrm{E}-03$ & 1.41E-09 \\
\hline
\end{tabular}




\begin{tabular}{|c|c|c|c|c|c|c|c|c|c|c|c|c|c|c|}
\hline Sample ID & $\begin{array}{l}\text { Influent } \\
\text { [Si] } \\
\text { (ppm) }\end{array}$ & $\begin{array}{l}\text { Flow } \\
\text { Rate, } \mathrm{q} \\
\left(\mathrm{m}^{3} \mathrm{~d}^{-1}\right)\end{array}$ & $\begin{array}{l}\text { Time } \\
\text { (d) }\end{array}$ & $\begin{array}{l}\text { Glass } \\
\text { Mass } \\
\text { (g) }\end{array}$ & $\begin{array}{l}\text { Surface } \\
\text { Area, S } \\
\left(\mathrm{m}^{2}\right)\end{array}$ & $\begin{array}{l}{[\mathrm{Al}]} \\
\left(\mu \mathrm{g} \mathrm{L}^{-1}\right)\end{array}$ & $\begin{array}{l}\text { Al Norm. } \\
\text { Diss. Rate } \\
\left(\mathrm{g} \mathrm{m}^{-2} \mathrm{~d}^{-1}\right)\end{array}$ & $\begin{array}{l}{[\mathrm{B}]} \\
\left(\mu \mathrm{g} \mathrm{L}^{-1}\right)\end{array}$ & $\begin{array}{l}\text { B Norm. } \\
\text { Diss. Rate } \\
\left(\mathrm{g} \mathrm{m}^{-2} \mathrm{~d}^{-1}\right)\end{array}$ & $\begin{array}{l}{[\mathrm{Si}]} \\
\left(\mu \mathrm{L} \mathrm{L}^{-1}\right)\end{array}$ & $\begin{array}{l}\text { Si Norm. } \\
\text { Diss. Rate } \\
\left(\mathrm{g} \mathrm{m}^{-2} \mathrm{~d}^{-1}\right)\end{array}$ & $\begin{array}{l}{[\mathrm{Na}]} \\
(\mu \mathrm{g} \mathrm{L} \\
\left.{ }^{1}\right)\end{array}$ & $\begin{array}{l}\text { Na Norm. } \\
\text { Diss. Rate, } \\
\mathrm{r} \\
\left(\mathrm{g} \mathrm{m}^{-2} \mathrm{~d}^{-1}\right)\end{array}$ & $\begin{array}{l}\mathrm{r}_{\mathrm{IEX}} \\
\left(\mathrm{mol} \mathrm{Na} \mathrm{s}^{-1}\right)\end{array}$ \\
\hline LG9.23.9.S135.6 & 35 & $1.82 E-05$ & 21.0 & 3.992 & 7.86E-02 & $\begin{array}{l}{[49.4]} \\
31^{\star}\end{array}$ & 3.19E-04 & 100 & $8.75 E-04$ & 31,800 & --- & 1,380 & $2.05 E-03$ & $5.91 \mathrm{E}-10$ \\
\hline LG9.23.9.SI35.8 & 35 & $1.92 E-05$ & 27.8 & 3.992 & $7.86 \mathrm{E}-02$ & $\begin{array}{l}{[49.4]} \\
33^{*}\end{array}$ & $3.38 \mathrm{E}-04$ & 84 & 7.79E-04 & 32,400 & --- & 1,070 & $1.68 \mathrm{E}-03$ & 4.55E-10 \\
\hline LG9.23.9.SI35.10 & 35 & $1.99 \mathrm{E}-05$ & 34.9 & 3.991 & $7.86 E-02$ & $\begin{array}{l}{[49.4]} \\
31^{*}\end{array}$ & $3.51 \mathrm{E}-04$ & 87 & $8.36 \mathrm{E}-04$ & 32,200 & --- & 1,060 & $1.73 \mathrm{E}-03$ & 4.49E-10 \\
\hline LG9.23.9.SI35.11 & 35 & $2.02 \mathrm{E}-05$ & 37.9 & 3.991 & $7.85 \mathrm{E}-02$ & 33 & $2.38 \mathrm{E}-04$ & 84 & $8.20 \mathrm{E}-04$ & 34,100 & --- & 885 & $1.46 \mathrm{E}-03$ & $3.21 \mathrm{E}-10$ \\
\hline LG9.23.9.SI35.13 & 35 & $1.90 \mathrm{E}-05$ & 45.0 & 3.991 & $7.85 \mathrm{E}-02$ & 34 & $2.27 \mathrm{E}-04$ & 91 & $8.38 \mathrm{E}-04$ & 33,800 & --- & 952 & $1.48 \mathrm{E}-03$ & $3.24 \mathrm{E}-10$ \\
\hline LG9.23.9.SI35.14 & 35 & $1.91 \mathrm{E}-05$ & 48.9 & 3.990 & $7.85 \mathrm{E}-02$ & 33 & $2.21 \mathrm{E}-04$ & 88 & $8.12 \mathrm{E}-04$ & 32,600 & --- & 820 & $1.28 \mathrm{E}-03$ & $2.37 \mathrm{E}-10$ \\
\hline LG9.23.9.SI35.19 & 35 & $1.94 \mathrm{E}-05$ & 66.0 & 3.986 & $7.85 \mathrm{E}-02$ & 31 & $2.17 \mathrm{E}-04$ & 93 & $8.70 \mathrm{E}-04$ & 34,200 & --- & 727 & $1.15 \mathrm{E}-03$ & 1.42E-10 \\
\hline LG9.23.9.SI35.20 & 35 & $1.89 \mathrm{E}-05$ & 69.9 & 3.985 & $7.85 \mathrm{E}-02$ & 31 & $2.05 \mathrm{E}-04$ & 91 & $8.27 \mathrm{E}-04$ & 33,000 & --- & 701 & $1.08 \mathrm{E}-03$ & $1.28 \mathrm{E}-10$ \\
\hline LG9.23.9.SI35.21 & 35 & $1.87 \mathrm{E}-05$ & 73.0 & 3.985 & $7.85 \mathrm{E}-02$ & 31 & $2.04 \mathrm{E}-04$ & 96 & $8.65 E-04$ & 33,400 & --- & 697 & $1.07 E-03$ & 1.02E-10 \\
\hline LG9.23.9.SI35.22 & 35 & $1.88 \mathrm{E}-05$ & 77 & 4 & 0 & 31 & $2.09 \mathrm{E}-04$ & 98 & $8.85 E-04$ & 34,600 & --- & 686 & $1.06 \mathrm{E}-03$ & $8.63 \mathrm{E}-11$ \\
\hline \multicolumn{15}{|c|}{ LG9 $40^{\circ} \mathrm{C} \mathrm{pH} 9 \mathrm{Si}$ Reactor $\# 1$} \\
\hline LG9.40.9.Sl10.Blank1 & 10 & $1.99 \mathrm{E}-05$ & --- & --- & --- & [49.4] & --- & {$[75.6]$} & --- & 8,480 & --- & [134] & --- & --- \\
\hline LG9.40.9.S110.Blank3 & 10 & $2.00 \mathrm{E}-05$ & --- & --- & --- & [49.4] & --- & [75.6] & --- & 8,560 & --- & [134] & --- & --- \\
\hline LG9.40.9.S110.2 & 10 & $1.87 \mathrm{E}-05$ & 4.9 & 1.994 & $3.92 \mathrm{E}-02$ & 82 & $1.09 \mathrm{E}-03$ & 439 & $7.91 \mathrm{E}-03$ & 9,770 & --- & 5,650 & $1.73 \mathrm{E}-02$ & $4.71 \mathrm{E}-09$ \\
\hline LG9.40.9.S110.4 & 10 & $1.94 \mathrm{E}-05$ & 9.0 & 1.993 & $3.92 \mathrm{E}-02$ & 98 & $1.35 \mathrm{E}-03$ & 376 & $7.04 \mathrm{E}-03$ & 9,190 & --- & 3,430 & $1.09 E-02$ & $1.94 \mathrm{E}-09$ \\
\hline LG9.40.9.S110.5 & 10 & $1.90 \mathrm{E}-05$ & 11.8 & 1.992 & $3.92 \mathrm{E}-02$ & 83 & $1.13 \mathrm{E}-03$ & 348 & $6.37 \mathrm{E}-03$ & 9,290 & --- & 2,990 & $9.28 \mathrm{E}-03$ & $1.47 \mathrm{E}-09$ \\
\hline LG9.40.9.S110.6 & 10 & $1.81 \mathrm{E}-05$ & 14.1 & 1.992 & $3.92 \mathrm{E}-02$ & 82 & $1.05 \mathrm{E}-03$ & 316 & $5.52 \mathrm{E}-03$ & 8,940 & --- & 2,680 & $7.95 \mathrm{E}-03$ & $1.22 \mathrm{E}-09$ \\
\hline LG9.40.9.S110.7 & 10 & $1.79 \mathrm{E}-05$ & 16.0 & 1.991 & $3.92 \mathrm{E}-02$ & 91 & $1.16 \mathrm{E}-03$ & 382 & $6.61 \mathrm{E}-03$ & 9,400 & --- & 2,820 & $8.27 \mathrm{E}-03$ & $8.39 \mathrm{E}-10$ \\
\hline LG9.40.9.S110.8 & 10 & $1.81 \mathrm{E}-05$ & 18.8 & 1.990 & 3.92E-02 & 93 & $1.20 \mathrm{E}-03$ & 406 & 7.09E-03 & 9,280 & --- & 2,880 & $8.53 E-03$ & $7.26 \mathrm{E}-10$ \\
\hline LG9.40.9.S110.9 & 10 & $1.82 \mathrm{E}-05$ & 21.0 & 1.990 & 3.92E-02 & 94 & $1.22 \mathrm{E}-03$ & 416 & $7.29 \mathrm{E}-03$ & 9,460 & --- & 3,180 & $9.46 \mathrm{E}-03$ & 1.09E-09 \\
\hline LG9.40.9.SI10.10 & 10 & $1.89 \mathrm{E}-05$ & 23.1 & 1.989 & 3.92E-02 & 92 & $1.24 \mathrm{E}-03$ & 395 & $7.22 \mathrm{E}-03$ & 9,300 & --- & 2,520 & $7.82 \mathrm{E}-03$ & $2.99 \mathrm{E}-10$ \\
\hline LG9.40.9.SI10.11 & 10 & $1.89 \mathrm{E}-05$ & 25.8 & 1.988 & $3.92 \mathrm{E}-02$ & 95 & $1.28 \mathrm{E}-03$ & 409 & 7.45E-03 & 9,530 & --- & 2,450 & $7.58 \mathrm{E}-03$ & $6.09 E-11$ \\
\hline LG9.40.9.S110.12 & 10 & $1.97 \mathrm{E}-05$ & 28.1 & 1.988 & 3.92E-02 & 91 & $1.28 \mathrm{E}-03$ & 391 & $7.45 \mathrm{E}-03$ & 9,200 & --- & 2,360 & 7.63E-03 & $8.99 E-11$ \\
\hline LG9.40.9.SI10.13 & 10 & $1.83 E-05$ & 30.1 & 1.987 & 3.92E-02 & 92 & $1.20 \mathrm{E}-03$ & 371 & $6.57 \mathrm{E}-03$ & 9,100 & --- & 2,690 & $8.08 \mathrm{E}-03$ & $7.61 \mathrm{E}-10$ \\
\hline LG9.40.9.SI10.15 & 10 & $1.73 \mathrm{E}-05$ & 35.0 & 1.986 & $3.91 \mathrm{E}-02$ & 93 & $1.15 \mathrm{E}-03$ & 405 & $6.79 \mathrm{E}-03$ & 9,400 & --- & 2,640 & $7.51 \mathrm{E}-03$ & $3.62 \mathrm{E}-10$ \\
\hline LG9.40.9.SI10.17 & 10 & $1.74 \mathrm{E}-05$ & 39.8 & 1.985 & $3.91 \mathrm{E}-02$ & 88 & $1.09 \mathrm{E}-03$ & 369 & $6.21 \mathrm{E}-03$ & 9,440 & --- & 2,540 & $7.25 \mathrm{E}-03$ & $5.24 \mathrm{E}-10$ \\
\hline LG9.40.9.S110.18 & 10 & $1.72 \mathrm{E}-05$ & 42.0 & 1.984 & $3.91 \mathrm{E}-02$ & 85 & $1.05 \mathrm{E}-03$ & 344 & $5.72 \mathrm{E}-03$ & 9,190 & --- & 2,600 & 7.34E-03 & 8.13E-10 \\
\hline \multicolumn{15}{|c|}{ LG9 $40^{\circ} \mathrm{C} \mathrm{pH} 9 \mathrm{Si}$ Reactor $\# 2$} \\
\hline LG9.40.9.S130.Blank1 & 30 & $1.43 E-05$ & --- & --- & --- & [49.4] & --- & {$[75.6]$} & --- & 25,300 & --- & [134] & --- & --- \\
\hline LG9.40.9.S130.Blank3 & 30 & $9.85 \mathrm{E}-06$ & --- & --- & --- & [49.4] & --- & {$[75.6]$} & --- & 26,100 & --- & [134] & --- & --- \\
\hline LG9.40.9.SI30.2 & 30 & $1.77 \mathrm{E}-05$ & 4.9 & 1.976 & 3.89E-02 & 83 & $1.05 \mathrm{E}-03$ & 616 & $1.06 \mathrm{E}-02$ & 26,500 & --- & 7,660 & 2.23E-02 & $5.92 \mathrm{E}-09$ \\
\hline LG9.40.9.S130.4 & 30 & $1.65 \mathrm{E}-05$ & 9.0 & 1.975 & $3.89 \mathrm{E}-02$ & 68 & $8.04 \mathrm{E}-04$ & 558 & $8.94 \mathrm{E}-03$ & 26,500 & --- & 5,390 & $1.47 \mathrm{E}-02$ & $2.88 \mathrm{E}-09$ \\
\hline LG9.40.9.S130.5 & 30 & 7.07E-06 & 11.8 & 1.975 & $3.89 E-02$ & 68 & 3.47E-04 & 604 & $4.16 \mathrm{E}-03$ & 26,300 & --- & 4,610 & $5.38 \mathrm{E}-03$ & $6.17 \mathrm{E}-10$ \\
\hline LG9.40.9.SI30.10 & 30 & $5.24 \mathrm{E}-08$ & 23.1 & 1.975 & $3.89 \mathrm{E}-02$ & [49.4] & $1.86 \mathrm{E}-06$ & [75.6] & $3.85 \mathrm{E}-06$ & 2,110 & --- & 515 & $4.45 \mathrm{E}-06$ & $3.02 \mathrm{E}-13$ \\
\hline LG9.40.9.SI30.11 & 30 & $1.22 \mathrm{E}-05$ & 25.8 & 1.971 & $3.88 \mathrm{E}-02$ & 114 & $1.00 \mathrm{E}-03$ & 2,110 & $2.51 \mathrm{E}-02$ & 26,700 & --- & 13,700 & $2.77 \mathrm{E}-02$ & $1.28 \mathrm{E}-09$ \\
\hline LG9.40.9.SI30.12 & 30 & $1.57 \mathrm{E}-05$ & 28.1 & 1.970 & $3.88 \mathrm{E}-02$ & 76 & $8.58 \mathrm{E}-04$ & 1,370 & $2.10 \mathrm{E}-02$ & 26,600 & --- & 8,140 & $2.12 \mathrm{E}-02$ & $8.45 E-11$ \\
\hline LG9.40.9.SI30.13 & 30 & 1.13E-05 & 30.1 & 1.969 & $3.88 \mathrm{E}-02$ & 65 & $5.32 \mathrm{E}-04$ & 1,060 & $1.17 \mathrm{E}-02$ & 26,400 & --- & 7,300 & $1.37 \mathrm{E}-02$ & $9.94 \mathrm{E}-10$ \\
\hline LG9.40.9.SI30.15 & 30 & $9.23 \mathrm{E}-06$ & 35.0 & 1.968 & $3.88 \mathrm{E}-02$ & 63 & 4.19E-04 & 994 & $8.95 \mathrm{E}-03$ & 25,800 & --- & 6,710 & $1.03 E-02$ & $6.54 \mathrm{E}-10$ \\
\hline LG9.40.9.SI30.17 & 30 & $1.41 \mathrm{E}-05$ & 39.8 & 1.965 & $3.88 \mathrm{E}-02$ & 57 & $5.84 \mathrm{E}-04$ & 860 & $1.19 \mathrm{E}-02$ & 26,400 & --- & 6,100 & $1.43 E-02$ & $1.21 \mathrm{E}-09$ \\
\hline LG9.40.9.SI30.18 & 30 & $1.32 \mathrm{E}-05$ & 42.0 & 1.965 & $3.87 \mathrm{E}-02$ & 54 & $5.13 E-04$ & 753 & $9.70 \mathrm{E}-03$ & 26,900 & --- & 4,880 & $1.07 \mathrm{E}-02$ & $4.86 \mathrm{E}-10$ \\
\hline \multicolumn{15}{|c|}{ LG9 $40^{\circ} \mathrm{C} \mathrm{pH} 9$ Si Reactor $\# 3$} \\
\hline
\end{tabular}

B. 27 


\begin{tabular}{|c|c|c|c|c|c|c|c|c|c|c|c|c|c|c|}
\hline Sample ID & $\begin{array}{l}\text { Influent } \\
{[\mathrm{Si}]} \\
(\mathrm{ppm})\end{array}$ & $\begin{array}{l}\text { Flow } \\
\text { Rate, q } \\
\left(\mathrm{m}^{3} \mathrm{~d}^{-1}\right)\end{array}$ & $\begin{array}{l}\text { Time } \\
\text { (d) }\end{array}$ & $\begin{array}{l}\text { Glass } \\
\text { Mass } \\
\text { (g) }\end{array}$ & $\begin{array}{l}\text { Surface } \\
\text { Area, S } \\
\left(\mathrm{m}^{2}\right)\end{array}$ & $\begin{array}{l}{[\mathrm{Al}]} \\
\left(\mu \mathrm{L} \mathrm{L}^{-1}\right)\end{array}$ & $\begin{array}{l}\text { Al Norm. } \\
\text { Diss. Rate } \\
\left(\mathrm{g} \mathrm{m}^{-2} \mathrm{~d}^{-1}\right)\end{array}$ & $\begin{array}{l}{[B]} \\
\left(\mu \mathrm{L} \mathrm{L}^{-1}\right)\end{array}$ & $\begin{array}{l}\text { B Norm. } \\
\text { Diss. Rate } \\
\left(\mathrm{g} \mathrm{m}^{-2} \mathrm{~d}^{-1}\right)\end{array}$ & $\begin{array}{l}{[\mathrm{Si}]} \\
\left(\mu g \mathrm{~L}^{-1}\right)\end{array}$ & $\begin{array}{l}\text { Si Norm. } \\
\text { Diss. Rate } \\
\left(\mathrm{g} \mathrm{m}^{-2} \mathrm{~d}^{-1}\right)\end{array}$ & $\begin{array}{l}{[\mathrm{Na}]} \\
(\mu \mathrm{g} \mathrm{L} \\
\left.{ }^{1}\right)\end{array}$ & $\begin{array}{l}\text { Na Norm. } \\
\text { Diss. Rate, } \\
\mathrm{r} \\
\left(\mathrm{g} \mathrm{m}^{-2} \mathrm{~d}^{-1}\right)\end{array}$ & $\begin{array}{l}\mathrm{r}_{\mathrm{IEX}} \\
\left(\mathrm{mol} \mathrm{Na} \mathrm{s}^{-1}\right)\end{array}$ \\
\hline LG9.40.9.S150.Blank1 & 50 & 1.95E-05 & $-\cdots$ & --- & --- & [49.4] & -.- & {$[75.6]$} & --- & 44,200 & --- & 181 & -.- & $-\ldots$ \\
\hline LG9.40.9.S150.Blank3 & 50 & $1.96 \mathrm{E}-05$ & --- & --- & --- & [49.4] & --- & {$[75.6]$} & --- & 42,500 & --- & 197 & --- & --- \\
\hline LG9.40.9.S150.2 & 50 & $3.29 \mathrm{E}-06$ & 4.9 & 1.992 & $3.92 \mathrm{E}-02$ & 69 & $1.61 \mathrm{E}-04$ & 497 & $1.58 \mathrm{E}-03$ & 40,000 & -- & 8,210 & $4.43 \mathrm{E}-03$ & $1.43 \mathrm{E}-09$ \\
\hline LG9.40.9.S150.4 & 50 & $6.75 \mathrm{E}-08$ & 9.0 & 1.992 & $3.92 \mathrm{E}-02$ & {$[49.4]$} & $2.38 \mathrm{E}-06$ & {$[75.6]$} & 4.92E-06 & 5,870 & --- & 1,300 & $1.44 \mathrm{E}-05$ & $4.75 \mathrm{E}-12$ \\
\hline LG9.40.9.S150.5 & 50 & $1.98 \mathrm{E}-05$ & 11.8 & 1.988 & $3.91 \mathrm{E}-02$ & 60 & 8.47E-04 & 1,060 & 2.03E-02 & 43,400 & --- & 10,400 & $3.37 E-02$ & $6.78 \mathrm{E}-09$ \\
\hline LG9.40.9.S150.6 & 50 & 2.02E-05 & 14.1 & 1.988 & $3.91 \mathrm{E}-02$ & 144 & $2.08 \mathrm{E}-03$ & 687 & $1.34 \mathrm{E}-02$ & 44,700 & --- & 5,820 & $1.93 \mathrm{E}-02$ & $2.95 \mathrm{E}-09$ \\
\hline LG9.40.9.S150.7 & 50 & 2.01E-05 & 16.0 & 1.987 & $3.91 \mathrm{E}-02$ & [49.4] & $7.10 \mathrm{E}-04$ & 617 & $1.20 \mathrm{E}-02$ & 44,700 & --- & 4,580 & $1.51 \mathrm{E}-02$ & $1.57 \mathrm{E}-09$ \\
\hline LG9.40.9.S150.8 & 50 & $2.01 \mathrm{E}-05$ & 18.8 & 1.986 & $3.91 \mathrm{E}-02$ & [49.4] & $7.09 \mathrm{E}-04$ & 584 & 1.13E-02 & 44,000 & --- & 3,930 & $1.30 \mathrm{E}-02$ & 8.09E-10 \\
\hline LG9.40.9.S150.9 & 50 & $1.95 \mathrm{E}-05$ & 21.0 & 1.985 & $3.91 \mathrm{E}-02$ & 55 & $7.61 \mathrm{E}-04$ & 545 & $1.03 \mathrm{E}-02$ & 43,000 & --- & 3,490 & $1.12 \mathrm{E}-02$ & $4.48 \mathrm{E}-10$ \\
\hline LG9.40.9.SI50.10 & 50 & $2.01 \mathrm{E}-05$ & 23.1 & 1.984 & $3.91 \mathrm{E}-02$ & 51 & $7.34 \mathrm{E}-04$ & 529 & 1.03E-02 & 42,700 & --- & 3,830 & $1.26 \mathrm{E}-02$ & $1.18 \mathrm{E}-09$ \\
\hline LG9.40.9.SI50.11 & 50 & 2.01E-05 & 25.8 & 1.983 & $3.91 \mathrm{E}-02$ & 54 & $7.73 \mathrm{E}-04$ & 557 & $1.09 \mathrm{E}-02$ & 43,500 & --- & 3,260 & $1.08 \mathrm{E}-02$ & $<0$ \\
\hline LG9.40.9.SI50.12 & 50 & 2.09E-05 & 28.1 & 1.982 & $3.91 \mathrm{E}-02$ & [49.4] & $7.38 \mathrm{E}-04$ & 572 & 1.16E-02 & 45,200 & --- & 3,260 & $1.12 \mathrm{E}-02$ & $<0$ \\
\hline LG9.40.9.SI50.13 & 50 & 1.87E-05 & 30.1 & 1.981 & $3.91 \mathrm{E}-02$ & [49.4] & $6.61 \mathrm{E}-04$ & 507 & $9.19 \mathrm{E}-03$ & 43,100 & --- & 3,390 & $1.04 \mathrm{E}-02$ & $6.21 \mathrm{E}-10$ \\
\hline LG9.40.9.SI50.15 & 50 & $1.98 \mathrm{E}-05$ & 35.0 & 1.979 & $3.90 \mathrm{E}-02$ & [49.4] & $7.01 E-04$ & 533 & $1.02 \mathrm{E}-02$ & 43,700 & -- & 3,410 & $1.11 \mathrm{E}-02$ & $4.40 \mathrm{E}-10$ \\
\hline LG9.40.9.SI50.17 & 50 & $2.01 \mathrm{E}-05$ & 39.8 & 1.978 & $3.90 E-02$ & {$[49.4]$} & 7.12E-04 & 427 & $8.33 E-03$ & 40,000 & --- & 3,610 & $1.19 \mathrm{E}-02$ & $1.82 E-09$ \\
\hline LG9.40.9.SI50.18 & 50 & $1.99 \mathrm{E}-05$ & 42.0 & 1.977 & $3.90 \mathrm{E}-02$ & [49.4] & $7.04 \mathrm{E}-04$ & 450 & $8.68 \mathrm{E}-03$ & 43,700 & --- & 2,990 & $9.79 \mathrm{E}-03$ & $5.56 \mathrm{E}-10$ \\
\hline \multicolumn{15}{|c|}{ LG9 $70^{\circ} \mathrm{C}$ pH 9 Si Reactor $\# 1$} \\
\hline LG9.70.9.SI30.Blank1 & 30 & $1.93 \mathrm{E}-08$ & --- & --- & --- & [49.4] & --- & {$[75.6]$} & --- & [164] & --- & [134] & --- & --- \\
\hline LG9.70.9.S130.Blank2 & 30 & 1.95E-06 & --- & --- & --- & {$[49.4]$} & --- & {$[75.6]$} & -- & 24,800 & --- & [134] & --- & --- \\
\hline LG9.70.9.S130.Blank3 & 30 & 4.70E-05 & --- & --- & --- & [49.4] & --- & {$[75.6]$} & --- & 26,900 & --- & [134] & --- & --- \\
\hline LG9.70.9.S130.2 & 30 & 1.77E-05 & 5.0 & 1.959 & $3.87 \mathrm{E}-02$ & 274 & $3.51 \mathrm{E}-03$ & 7,200 & $1.25 \mathrm{E}-01$ & 24,800 & --- & 49,000 & $1.44 \mathrm{E}-01$ & $9.71 \mathrm{E}-09$ \\
\hline LG9.70.9.SI30.4 & 30 & $1.75 \mathrm{E}-05$ & 9.0 & 1.947 & $3.86 \mathrm{E}-02$ & 195 & $2.47 E-03$ & 5,640 & 9.67E-02 & 26,600 & --- & 41,300 & $1.20 \mathrm{E}-01$ & $1.18 \mathrm{E}-08$ \\
\hline LG9.70.9.SI30.10 & 30 & 1.69E-05 & 23.1 & 1.907 & $3.81 \mathrm{E}-02$ & 199 & $2.46 \mathrm{E}-03$ & 4,540 & 7.61E-02 & 26,300 & --- & 30,400 & $8.65 \mathrm{E}-02$ & $5.21 \mathrm{E}-09$ \\
\hline LG9.70.9.SI30.11 & 30 & $1.62 \mathrm{E}-05$ & 25.8 & 1.898 & $3.79 \mathrm{E}-02$ & 208 & $2.48 \mathrm{E}-03$ & 4,830 & $7.80 \mathrm{E}-02$ & 27,500 & --- & 30,500 & $8.36 \mathrm{E}-02$ & $2.80 \mathrm{E}-09$ \\
\hline LG9.70.9.S|30.12 & 30 & $1.41 \mathrm{E}-05$ & 28.1 & 1.893 & $3.79 \mathrm{E}-02$ & 228 & $2.37 \mathrm{E}-03$ & 5,140 & $7.24 \mathrm{E}-02$ & 27,200 & --- & 33,200 & $7.94 \mathrm{E}-02$ & $3.49 \mathrm{E}-09$ \\
\hline LG9.70.9.SI30.13 & 30 & $1.36 \mathrm{E}-05$ & 30.0 & 1.888 & $3.78 \mathrm{E}-02$ & 233 & $2.34 \mathrm{E}-03$ & 5,350 & $7.27 \mathrm{E}-02$ & 26,700 & --- & 39,100 & $9.02 E-02$ & $8.78 \mathrm{E}-09$ \\
\hline LG9.70.9.SI30.14 & 30 & $1.41 \mathrm{E}-05$ & 32.8 & 1.880 & $3.77 \mathrm{E}-02$ & 213 & $2.22 \mathrm{E}-03$ & 4,760 & $6.73 E-02$ & 26,600 & --- & 31,900 & $7.65 E-02$ & 4.64E-09 \\
\hline \multicolumn{15}{|c|}{ LG9 $70^{\circ} \mathrm{C}$ pH 9 Si Reactor $\# 2$} \\
\hline LG9.70.9.SI60.Blank1 & 60 & $1.58 \mathrm{E}-05$ & -.- & --- & --- & {$[49.4]$} & --- & {$[75.6]$} & --- & 53,700 & --- & [134] & --- & --- \\
\hline LG9.70.9.S160.Blank2 & 60 & $2.00 \mathrm{E}-05$ & --- & --- & --- & [49.4] & --- & {$[75.6]$} & --- & 54,800 & --- & [134] & --- & --- \\
\hline LG9.70.9.S160.Blank3 & 60 & $2.16 \mathrm{E}-05$ & -.- & --. & --- & {$[49.4]$} & --- & {$[75.6]$} & --- & 55,500 & --- & [134] & --- & --- \\
\hline LG9.70.9.S160.2 & 60 & 1.91E-05 & 4.9 & 1.969 & $3.90 \mathrm{E}-02$ & 104 & $1.42 \mathrm{E}-03$ & 7,850 & $1.46 \mathrm{E}-01$ & 56,000 & --- & 52,900 & $1.66 \mathrm{E}-01$ & $1.05 \mathrm{E}-08$ \\
\hline LG9.70.9.S160.4 & 60 & $1.91 \mathrm{E}-05$ & 9.0 & 1.958 & $3.88 \mathrm{E}-02$ & 66 & $9.00 \mathrm{E}-04$ & 5,400 & $1.00 \mathrm{E}-01$ & 55,600 & --- & 35,900 & $1.13 \mathrm{E}-01$ & $6.46 \mathrm{E}-09$ \\
\hline LG9.70.9.S160.6 & 60 & $1.94 \mathrm{E}-05$ & 14.0 & 1.942 & $3.86 \mathrm{E}-02$ & 59 & $8.28 \mathrm{E}-04$ & 4,430 & $8.41 \mathrm{E}-02$ & 55,100 & --- & 29,400 & $9.47 E-02$ & 5.33E-09 \\
\hline LG9.70.9.S160.8 & 60 & 2.07E-05 & 18.8 & 1.920 & $3.83 \mathrm{E}-02$ & 64 & $9.60 \mathrm{E}-04$ & 5,320 & $1.09 \mathrm{E}-01$ & 57,600 & -- & 34,800 & $1.20 \mathrm{E}-01$ & $5.99 \mathrm{E}-09$ \\
\hline LG9.70.9.S160.10 & 60 & 2.18E-05 & 23.1 & 1.906 & $3.81 \mathrm{E}-02$ & 62 & $9.94 \mathrm{E}-04$ & 4,510 & $9.76 \mathrm{E}-02$ & 54,500 & --- & 30,900 & $1.13 \mathrm{E}-01$ & 7.97E-09 \\
\hline LG9.70.9.S160.12 & 60 & 2.39E-05 & 28.0 & 1.885 & $3.79 \mathrm{E}-02$ & 63 & $1.12 \mathrm{E}-03$ & 4,480 & $1.07 \mathrm{E}-01$ & 55,400 & --- & 31,100 & $1.26 \mathrm{E}-01$ & $9.56 \mathrm{E}-09$ \\
\hline LG9.70.9.S160.13 & 60 & $2.25 \mathrm{E}-05$ & 30.0 & 1.879 & $3.78 \mathrm{E}-02$ & 63 & $1.04 \mathrm{E}-03$ & 4,310 & $9.70 \mathrm{E}-02$ & 56,000 & --- & 28,200 & $1.08 \mathrm{E}-01$ & $5.37 E-09$ \\
\hline \multicolumn{15}{|c|}{ LG9 $70^{\circ} \mathrm{C}$ pH 9 Si Reactor $\# 3$} \\
\hline LG9.70.9.S190.Blank1 & 90 & 1.66E-05 & $-\cdots$ & --- & --- & 51 & -- & {$[75.6]$} & --- & 79,700 & -- & 154 & --- & --- \\
\hline LG9.70.9.S190.Blank2 & 90 & $1.89 \mathrm{E}-05$ & -- & --- & --- & 55 & --- & {$[75.6]$} & -- & 85,400 & --- & 178 & --- & --- \\
\hline LG9.70.9.S190.Blank3 & 90 & $1.91 \mathrm{E}-05$ & -- & --- & --- & 52 & --- & [75.6] & -- & 81,200 & --- & 146 & --- & --- \\
\hline LG9.70.9.S190.2 & 90 & $1.86 \mathrm{E}-05$ & 4.9 & 1.439 & $2.85 \mathrm{E}-02$ & 84 & $1.54 \mathrm{E}-03$ & 8,100 & $2.00 \mathrm{E}-01$ & 83,000 & --- & 55,500 & $2.33 \mathrm{E}-01$ & $1.64 \mathrm{E}-08$ \\
\hline
\end{tabular}




\begin{tabular}{|c|c|c|c|c|c|c|c|c|c|c|c|c|c|c|}
\hline Sample ID & $\begin{array}{l}\text { Influent } \\
\text { [Si] } \\
\text { (ppm) }\end{array}$ & $\begin{array}{l}\text { Flow } \\
\text { Rate, q } \\
\left(\mathrm{m}^{3} \mathrm{~d}^{-1}\right)\end{array}$ & $\begin{array}{l}\text { Time } \\
\text { (d) }\end{array}$ & $\begin{array}{l}\text { Glass } \\
\text { Mass } \\
\text { (g) }\end{array}$ & $\begin{array}{l}\text { Surface } \\
\text { Area, } \mathrm{S} \\
\left(\mathrm{m}^{2}\right)\end{array}$ & $\begin{array}{l}{[\mathrm{Al}]} \\
\left(\mu \mathrm{L} \mathrm{L}^{-1}\right)\end{array}$ & $\begin{array}{l}\text { Al Norm. } \\
\text { Diss. Rate } \\
\left(\mathrm{g} \mathrm{m}^{-2} \mathrm{~d}^{-1}\right)\end{array}$ & $\begin{array}{l}{[B]} \\
\left(\mu \mathrm{L} \mathrm{L}^{-1}\right)\end{array}$ & $\begin{array}{l}\text { B Norm. } \\
\text { Diss. Rate } \\
\left(\mathrm{g} \mathrm{m}^{-2} \mathrm{~d}^{-1}\right)\end{array}$ & $\begin{array}{l}{[\mathrm{Si}]} \\
\left(\mu \mathrm{L} \mathrm{L}^{-1}\right)\end{array}$ & $\begin{array}{l}\text { Si Norm. } \\
\text { Diss. Rate } \\
\left(\mathrm{g} \mathrm{m}^{-2} \mathrm{~d}^{-1}\right)\end{array}$ & $\begin{array}{l}{[\mathrm{Na}]} \\
(\mu \mathrm{g} \mathrm{L} \\
\left.{ }^{1}\right)\end{array}$ & $\begin{array}{l}\text { Na Norm. } \\
\text { Diss. Rate, } \\
\mathrm{r} \\
\left(\mathrm{g} \mathrm{m}^{-2} \mathrm{~d}^{-1}\right)\end{array}$ & $\begin{array}{l}\mathrm{r}_{\mathrm{IEX}} \\
\left(\mathrm{mol} \mathrm{Na}{ }^{-2} \mathrm{~s}^{-1}\right)\end{array}$ \\
\hline LG9.70.9.S190.4 & 90 & $1.85 \mathrm{E}-05$ & 9.0 & 1.427 & $2.84 \mathrm{E}-02$ & 85 & $1.54 \mathrm{E}-03$ & 5,660 & $1.39 \mathrm{E}-01$ & 85,100 & --- & 44,000 & $1.84 \mathrm{E}-01$ & 2.24E-08 \\
\hline LG9.70.9.S190.6 & 90 & 2.03E-05 & 14.0 & 1.410 & $2.82 \mathrm{E}-02$ & 60 & $1.21 \mathrm{E}-03$ & 4,550 & $1.24 \mathrm{E}-01$ & 83,000 & -- & 30,900 & $1.43 \mathrm{E}-01$ & $9.51 \mathrm{E}-09$ \\
\hline LG9.70.9.S190.8 & 90 & $1.71 \mathrm{E}-05$ & 18.8 & 1.392 & $2.79 \mathrm{E}-02$ & 60 & $1.03 E-03$ & 5,520 & $1.28 \mathrm{E}-01$ & 83,300 & --- & 37,200 & 1.47E-01 & $9.25 \mathrm{E}-09$ \\
\hline LG9.70.9.SI90.10 & 90 & $1.62 \mathrm{E}-05$ & 23.1 & 1.378 & $2.77 \mathrm{E}-02$ & 58 & $9.43 \mathrm{E}-04$ & 5,680 & $1.26 \mathrm{E}-01$ & 81,800 & -- & 38,400 & $1.44 \mathrm{E}-01$ & $9.30 \mathrm{E}-09$ \\
\hline LG9.70.9.S190.11 & 90 & 1.73E-05 & 25.7 & 1.367 & $2.76 \mathrm{E}-02$ & 70 & $1.22 \mathrm{E}-03$ & 5,520 & $1.31 \mathrm{E}-01$ & 86,500 & -.- & 38,000 & $1.53 \mathrm{E}-01$ & $1.11 \mathrm{E}-08$ \\
\hline LG9.70.9.SI90.12 & 90 & $1.88 \mathrm{E}-05$ & 28.0 & 1.359 & $2.75 \mathrm{E}-02$ & 69 & $1.32 \mathrm{E}-03$ & 5,180 & $1.34 \mathrm{E}-01$ & 80,900 & -- & 36,500 & $1.60 \mathrm{E}-01$ & $1.32 \mathrm{E}-08$ \\
\hline LG9.70.9.S190.13 & 90 & $1.59 \mathrm{E}-05$ & 30.0 & 1.354 & $2.74 \mathrm{E}-02$ & 72 & $1.17 \mathrm{E}-03$ & 5,330 & 1.17E-01 & 85,900 & --- & 35,700 & $1.33 \mathrm{E}-01$ & $8.01 E-09$ \\
\hline LG9.70.9.S190.14 & 90 & $1.72 \mathrm{E}-05$ & 32.8 & 1.344 & $2.73 E-02$ & 71 & 1.25E-03 & 4,830 & $1.15 \mathrm{E}-01$ & 84,700 & --- & 33,400 & $1.35 \mathrm{E}-01$ & $1.01 \mathrm{E}-08$ \\
\hline \multicolumn{15}{|c|}{ LG9 $90^{\circ} \mathrm{C}$ pH 9 Si Reactor $\# 1$} \\
\hline LG9.90.9.Si40.Blank1 & 40 & 2.49E-06 & --- & --- & --- & [49.4] & --- & {$[75.6]$} & -- & 37,600 & --- & 269 & --- & --- \\
\hline LG9.90.9.Si40.Blank2 & 40 & 3.75E-06 & --- & --- & --- & [49.4] & --- & {$[75.6]$} & --- & 36,600 & --- & 221 & --- & --- \\
\hline LG9.90.9.Si40.Blank3 & 40 & $3.00 \mathrm{E}-06$ & --- & --- & -- & [49.4] & --- & {$[75.6]$} & -- & 38,000 & --- & 227 & --. & -- \\
\hline LG9.90.9.Si40.2 & 40 & $3.17 \mathrm{E}-06$ & 5.0 & 1.298 & $2.57 \mathrm{E}-02$ & 504 & $1.74 \mathrm{E}-03$ & 17,500 & $8.16 \mathrm{E}-02$ & 40,400 & --- & $\begin{array}{l}113,00 \\
0\end{array}$ & $8.94 E-02$ & $3.92 \mathrm{E}-09$ \\
\hline LG9.90.9.Si40.4 & 40 & $5.70 \mathrm{E}-06$ & 9.0 & 1.282 & $2.55 \mathrm{E}-02$ & 468 & $2.93 E-03$ & 17,800 & $1.51 \mathrm{E}-01$ & 41,600 & --- & $\begin{array}{l}115,00 \\
0\end{array}$ & $1.65 \mathrm{E}-01$ & $7.28 \mathrm{E}-09$ \\
\hline LG9.90.9.Si40.5 & 40 & $6.14 \mathrm{E}-06$ & 11.8 & 1.270 & $2.53 \mathrm{E}-02$ & 451 & $3.06 \mathrm{E}-03$ & 15,400 & $1.41 \mathrm{E}-01$ & 39,900 & --- & $\begin{array}{l}104,00 \\
0\end{array}$ & $1.62 \mathrm{E}-01$ & $1.04 \mathrm{E}-08$ \\
\hline LG9.90.9.Si40.6 & 40 & $5.98 \mathrm{E}-06$ & 14.0 & 1.265 & 2.53E-02 & 426 & $2.82 E-03$ & 13,700 & 1.23E-01 & 40,500 & --- & 96,400 & $1.47 E-01$ & $1.20 \mathrm{E}-08$ \\
\hline LG9.90.9.Si40.7 & 40 & 7.15E-06 & 16.0 & 1.258 & $2.52 \mathrm{E}-02$ & 447 & $3.55 \mathrm{E}-03$ & 12,700 & 1.37E-01 & 42,500 & --- & 84,500 & $1.54 \mathrm{E}-01$ & $8.85 \mathrm{E}-09$ \\
\hline \multicolumn{15}{|c|}{ LG9 $90^{\circ} \mathrm{C}$ pH 9 Si Reactor $\# 2$} \\
\hline LG9.90.9.S170.Blank1 & 70 & 1.63E-05 & --- & --- & --- & 61 & --- & {$[75.6]$} & --- & 64,700 & --- & 168 & --- & --- \\
\hline LG9.90.9.S170.Blank2 & 70 & $1.82 \mathrm{E}-05$ & --- & --- & -- & 60 & --- & {$[75.6]$} & -- & 66,800 & --- & [134] & --- & --- \\
\hline LG9.90.9.S170.Blank3 & 70 & 1.80E-05 & --- & --- & -- & 57 & --- & {$[75.6]$} & --- & 63,400 & --- & 227 & --- & --- \\
\hline LG9.90.9.S170.2 & 70 & 1.61E-05 & 5.0 & 1.243 & $2.49 \mathrm{E}-02$ & 159 & $2.88 \mathrm{E}-03$ & 14,500 & 3.55E-01 & 69,300 & --- & 91,400 & $3.80 \mathrm{E}-01$ & $1.24 \mathrm{E}-08$ \\
\hline LG9.90.9.S170.4 & 70 & $1.61 \mathrm{E}-05$ & 9.0 & 1.221 & $2.46 \mathrm{E}-02$ & 106 & $1.94 \mathrm{E}-03$ & 11,900 & $2.95 \mathrm{E}-01$ & 76,100 & --- & 79,700 & $3.35 \mathrm{E}-01$ & $2.02 \mathrm{E}-08$ \\
\hline LG9.90.9.S170.5 & 70 & $1.82 \mathrm{E}-05$ & 11.8 & 1.197 & 2.43E-02 & 97 & $2.04 \mathrm{E}-03$ & 10,600 & $3.01 \mathrm{E}-01$ & 75,400 & --- & 71,000 & $3.42 \mathrm{E}-01$ & 2.06E-08 \\
\hline LG9.90.9.S170.6 & 70 & 1.82E-05 & 14.0 & 1.186 & $2.41 \mathrm{E}-02$ & 106 & $2.24 \mathrm{E}-03$ & 9,330 & 2.67E-01 & 72,800 & -- & 64,000 & $3.11 \mathrm{E}-01$ & $2.20 \mathrm{E}-08$ \\
\hline LG9.90.9.S170.7 & 70 & 1.86E-05 & 16.0 & 1.175 & $2.40 \mathrm{E}-02$ & 105 & $2.28 \mathrm{E}-03$ & 8,410 & 2.47E-01 & 73,700 & --- & 65,100 & $3.25 \mathrm{E}-01$ & $3.90 E-08$ \\
\hline LG9.90.9.S170.8 & 70 & 1.83E-05 & 18.8 & 1.160 & $2.38 \mathrm{E}-02$ & 101 & $2.17 E-03$ & 7,290 & $2.12 \mathrm{E}-01$ & 72,900 & --- & 53,600 & $2.64 \mathrm{E}-01$ & $2.64 \mathrm{E}-08$ \\
\hline LG9.90.9.S170.9 & 70 & $1.84 \mathrm{E}-05$ & 21.0 & 1.152 & 2.37E-02 & 112 & $2.43 E-03$ & 6,620 & 1.95E-01 & 74,000 & --- & 56,400 & $2.81 E-01$ & 4.37E-08 \\
\hline \multicolumn{15}{|c|}{ LG9 $90^{\circ} \mathrm{C}$ pH 9 Si Reactor \#3 } \\
\hline LG9.90.9.20SI100.Blank1 & 100 & $9.05 E-06$ & --- & --- & -- & 71 & --- & {$[75.6]$} & -- & 94,300 & --- & 288 & --- & --- \\
\hline LG9.90.9.20SI100.Blank2 & 100 & 1.49E-05 & --- & --- & -- & 74 & --- & [75.6] & -- & 94,500 & --- & 372 & --- & --- \\
\hline LG9.90.9.20SI100.Blank3 & 100 & 1.79E-05 & -.- & --- & --- & 50 & --- & {$[75.6]$} & --- & 91,100 & --- & 265 & --- & -.- \\
\hline LG9.90.9.20SI100.2 & 100 & 1.79E-05 & 5.0 & 1.222 & $2.45 \mathrm{E}-02$ & 112 & $2.28 \mathrm{E}-03$ & 14,800 & $4.08 \mathrm{E}-01$ & 105,000 & --- & $\begin{array}{l}108,00 \\
0\end{array}$ & $5.05 E-01$ & 4.89E- 08 \\
\hline LG9.90.9.20SI100.4 & 100 & $1.78 \mathrm{E}-05$ & 9.0 & 1.198 & $2.42 \mathrm{E}-02$ & 90 & $1.86 \mathrm{E}-03$ & 11,700 & $3.26 \mathrm{E}-01$ & 112,000 & --- & 77,400 & $3.66 \mathrm{E}-01$ & $2.01 E-08$ \\
\hline LG9.90.9.20SI100.5 & 100 & $1.82 \mathrm{E}-05$ & 11.8 & 1.177 & $2.39 \mathrm{E}-02$ & 83 & $1.77 \mathrm{E}-03$ & 9,860 & 2.84E-01 & 109,000 & --- & 69,100 & $3.37 E-01$ & $2.70 \mathrm{E}-08$ \\
\hline LG9.90.9.20SI100.6 & 100 & $1.82 \mathrm{E}-05$ & 14.0 & 1.167 & $2.38 \mathrm{E}-02$ & 83 & $1.78 \mathrm{E}-03$ & 8,440 & $2.44 \mathrm{E}-01$ & 109,000 & --- & 61,000 & $2.99 \mathrm{E}-01$ & $2.78 \mathrm{E}-08$ \\
\hline LG9.90.9.20SI100.7 & 100 & 1.79E-05 & 16.0 & 1.159 & $2.37 \mathrm{E}-02$ & 82 & $1.73 E-03$ & 7,450 & 2.14E-01 & 109,000 & --- & 55,700 & $2.71 E-01$ & 2.89E- 08 \\
\hline LG9.90.9.20SI100.9 & 100 & $1.76 \mathrm{E}-05$ & 21.0 & 1.139 & $2.34 \mathrm{E}-02$ & 84 & $1.77 \mathrm{E}-03$ & 5,850 & $1.67 \mathrm{E}-01$ & 106,000 & --- & 47,300 & $2.29 \mathrm{E}-01$ & $3.12 \mathrm{E}-08$ \\
\hline \multicolumn{15}{|c|}{ LG9 $90^{\circ} \mathrm{C} \mathrm{pH} 9 \mathrm{Si}$ Reactor $\# 4$} \\
\hline LG9.90.9.40Si100.Blank1 & 100 & 1.97E-05 & -- & -.- & -.- & 70 & -- & [75.6] & --- & 95,300 & --- & 317 & --- & --. \\
\hline LG9.90.9.40Si100.Blank2 & 100 & $3.50 \mathrm{E}-05$ & -- & --- & -- & 69 & --- & [75.6] & --- & 95,800 & -- & 316 & --- & --- \\
\hline
\end{tabular}




\begin{tabular}{|c|c|c|c|c|c|c|c|c|c|c|c|c|c|c|}
\hline Sample ID & $\begin{array}{l}\text { Influent } \\
\text { [Si] } \\
\text { (ppm) }\end{array}$ & $\begin{array}{l}\text { Flow } \\
\text { Rate, } q \\
\left(\mathrm{~m}^{3} \mathrm{~d}^{-1}\right)\end{array}$ & $\begin{array}{l}\text { Time } \\
\text { (d) }\end{array}$ & $\begin{array}{l}\text { Glass } \\
\text { Mass } \\
\text { (g) }\end{array}$ & $\begin{array}{l}\text { Surface } \\
\text { Area, S } \\
\left(\mathrm{m}^{2}\right)\end{array}$ & $\begin{array}{l}{[\mathrm{Al}]} \\
\left(\mu \mathrm{g} \mathrm{L}^{-1}\right)\end{array}$ & $\begin{array}{l}\text { Al Norm. } \\
\text { Diss. Rate } \\
\left(\mathrm{g} \mathrm{m}^{-2} \mathrm{~d}^{-1}\right)\end{array}$ & $\begin{array}{l}{[\mathrm{B}]} \\
\left(\mu \mathrm{g} \mathrm{L}^{-1}\right)\end{array}$ & $\begin{array}{l}\text { B Norm. } \\
\text { Diss. Rate } \\
\left(\mathrm{g} \mathrm{m}^{-2} \mathrm{~d}^{-1}\right)\end{array}$ & $\begin{array}{l}{[\mathrm{Si}]} \\
\left(\mu \mathrm{g} \mathrm{L}^{-1}\right)\end{array}$ & $\begin{array}{l}\text { Si Norm. } \\
\text { Diss. Rate } \\
\left(\mathrm{g} \mathrm{m}^{-2} \mathrm{~d}^{-1}\right)\end{array}$ & $\begin{array}{l}{[\mathrm{Na}]} \\
(\mu \mathrm{g} \mathrm{L} \\
\left.{ }^{1}\right)\end{array}$ & $\begin{array}{l}\text { Na Norm. } \\
\text { Diss. Rate, } \\
\mathrm{r} \\
\left(\mathrm{g} \mathrm{m}^{-2} \mathrm{~d}^{-1}\right)\end{array}$ & $\begin{array}{l}\mathrm{r}_{\mathrm{IEX}} \\
\left(\mathrm{mol} \mathrm{Na}{ }^{-2} \mathrm{~s}^{-1}\right)\end{array}$ \\
\hline LG9.90.9.40Si100.Blank3 & 100 & $3.93 E-05$ & --- & --- & --- & 72 & --- & {$[75.6]$} & --- & 97,700 & --- & 304 & --- & --- \\
\hline LG9.90.9.40Si100.2 & 100 & $3.78 \mathrm{E}-05$ & 5.0 & 1.211 & $2.45 \mathrm{E}-02$ & 101 & $4.36 \mathrm{E}-03$ & 10,600 & $6.20 \mathrm{E}-01$ & 114,000 & --- & 67,200 & $6.67 \mathrm{E}-01$ & $2.36 \mathrm{E}-08$ \\
\hline LG9.90.9.40Si100.4 & 100 & $3.74 \mathrm{E}-05$ & 9.0 & 1.189 & $2.42 \mathrm{E}-02$ & 86 & $3.71 \mathrm{E}-03$ & 6,400 & $3.75 \mathrm{E}-01$ & 115,000 & --- & 46,000 & $4.57 \mathrm{E}-01$ & $4.14 \mathrm{E}-08$ \\
\hline LG9.90.9.40Si100.5 & 100 & $3.79 \mathrm{E}-05$ & 11.8 & 1.166 & $2.39 \mathrm{E}-02$ & 82 & $3.62 \mathrm{E}-03$ & 5,190 & $3.12 \mathrm{E}-01$ & 108,000 & --- & 45,300 & $4.62 \mathrm{E}-01$ & $7.55 \mathrm{E}-08$ \\
\hline LG9.90.9.40Si100.6 & 100 & $3.77 E-05$ & 14.0 & 1.156 & $2.37 \mathrm{E}-02$ & 85 & $3.78 \mathrm{E}-03$ & 4,310 & 2.59E-01 & 106,000 & --- & 37,300 & $3.80 \mathrm{E}-01$ & $6.11 \mathrm{E}-08$ \\
\hline LG9.90.9.40Si100.7 & 100 & $3.69 \mathrm{E}-05$ & 16.0 & 1.148 & $2.36 \mathrm{E}-02$ & 87 & $3.78 \mathrm{E}-03$ & 3,740 & $2.21 \mathrm{E}-01$ & 107,000 & --- & 31,600 & $3.17 \mathrm{E}-01$ & $4.82 \mathrm{E}-08$ \\
\hline LG9.90.9.40Si100.8 & 100 & $3.76 \mathrm{E}-05$ & 18.8 & 1.133 & $2.34 \mathrm{E}-02$ & 75 & $3.37 \mathrm{E}-03$ & 3,300 & $2.01 \mathrm{E}-01$ & 97,500 & --- & 29,500 & 3.04E-01 & $5.22 \mathrm{E}-08$ \\
\hline LG9.90.9.40Si100.9 & 100 & $3.64 \mathrm{E}-05$ & 21.0 & 1.126 & $2.33 \mathrm{E}-02$ & 86 & $3.76 \mathrm{E}-03$ & 2,940 & $1.74 \mathrm{E}-01$ & 104,000 & --- & 25,100 & $2.52 \mathrm{E}-01$ & $3.92 \mathrm{E}-08$ \\
\hline \multicolumn{15}{|c|}{ LG9 $90^{\circ} \mathrm{C} \mathrm{pH} 9 \mathrm{Si}$ Reactor $\# 5$} \\
\hline LG9.90.9.80Si100.Blank1 & 100 & $3.71 \mathrm{E}-05$ & --- & --- & -.- & 69 & --- & {$[75.6]$} & --- & 95,800 & --. & 340 & --- & --- \\
\hline LG9.90.9.80Si100.Blank2 & 100 & $6.90 \mathrm{E}-05$ & --- & --- & --- & 72 & --- & {$[75.6]$} & --- & 95,000 & --- & 581 & --- & --- \\
\hline LG9.90.9.80Si100.Blank3 & 100 & $7.34 \mathrm{E}-05$ & --- & --- & --- & 73 & --- & {$[75.6]$} & --- & 95,700 & --- & 558 & --- & --- \\
\hline LG9.90.9.80Si100.2 & 100 & $6.89 E-05$ & 5.0 & 1.175 & $2.38 \mathrm{E}-02$ & 93 & $7.52 \mathrm{E}-03$ & 6,590 & $7.21 \mathrm{E}-01$ & 116,000 & --- & 42,300 & $7.85 \mathrm{E}-01$ & $3.23 \mathrm{E}-08$ \\
\hline LG9.90.9.80Si100.4 & 100 & $6.46 \mathrm{E}-05$ & 9.0 & 1.152 & $2.35 \mathrm{E}-02$ & 89 & $6.83 \mathrm{E}-03$ & 3,980 & 4.14E-01 & 121,000 & --- & 27,500 & $4.85 \mathrm{E}-01$ & $3.59 \mathrm{E}-08$ \\
\hline LG9.90.9.80Si100.5 & 100 & $5.17 \mathrm{E}-05$ & 11.8 & 1.132 & $2.32 \mathrm{E}-02$ & 80 & $4.97 \mathrm{E}-03$ & 3,580 & $3.01 \mathrm{E}-01$ & 109,000 & --- & 27,700 & 3.95E-01 & $4.74 \mathrm{E}-08$ \\
\hline LG9.90.9.80Si100.6 & 100 & $6.18 \mathrm{E}-05$ & 14.0 & 1.119 & $2.31 \mathrm{E}-02$ & 71 & $5.32 \mathrm{E}-03$ & 2,890 & $2.93 E-01$ & 94,900 & --- & 22,800 & $3.92 \mathrm{E}-01$ & $4.99 \mathrm{E}-08$ \\
\hline LG9.90.9.80Si100.7 & 100 & $6.07 \mathrm{E}-05$ & 16.0 & 1.111 & $2.30 \mathrm{E}-02$ & 83 & $6.15 \mathrm{E}-03$ & 2,250 & $2.25 \mathrm{E}-01$ & 106,000 & --- & 22,100 & $3.75 \mathrm{E}-01$ & $7.55 \mathrm{E}-08$ \\
\hline LG9.90.9.80Si100.8 & 100 & $6.35 \mathrm{E}-05$ & 18.8 & 1.096 & $2.27 \mathrm{E}-02$ & 87 & $6.82 \mathrm{E}-03$ & 2,090 & $2.21 \mathrm{E}-01$ & 102,000 & --- & 18,600 & $3.33 \mathrm{E}-01$ & $5.67 \mathrm{E}-08$ \\
\hline \multicolumn{15}{|c|}{ A161 $23^{\circ} \mathrm{C} \mathrm{pH} 9$ Reactor \#1 } \\
\hline A161.23.9.20.Blank1 & & $1.04 \mathrm{E}-05$ & --- & --- & --- & [49.4] & --- & {$[75.6]$} & --- & [164] & --- & [134] & --- & --- \\
\hline A161.23.9.20.Blank2 & & $1.07 E-05$ & --- & --- & --- & [49.4] & --- & {$[75.6]$} & --- & [164] & --- & [134] & --- & --- \\
\hline A161.23.9.20.Blank3 & & $1.12 \mathrm{E}-05$ & 0.0 & --- & --- & [49.4] & --- & {$[75.6]$} & --- & [164] & --- & [134] & --- & --- \\
\hline A161.23.9.20.2 & & $1.31 \mathrm{E}-05$ & 5.9 & 0.748 & $1.51 \mathrm{E}-02$ & 300 & $4.90 \mathrm{E}-03$ & 644 & $1.33 \mathrm{E}-02$ & 895 & $4.61 \mathrm{E}-03$ & 2,790 & $1.58 \mathrm{E}-02$ & $1.25 \mathrm{E}-09$ \\
\hline A161.23.9.20.4 & & $1.22 \mathrm{E}-05$ & 12.9 & 0.747 & $1.51 \mathrm{E}-02$ & 420 & $6.41 \mathrm{E}-03$ & 561 & $1.08 \mathrm{E}-02$ & 1,170 & $5.63 \mathrm{E}-03$ & 2,220 & $1.17 \mathrm{E}-02$ & $4.55 \mathrm{E}-10$ \\
\hline A161.23.9.20.6 & & $1.41 \mathrm{E}-05$ & 19.9 & 0.746 & $1.51 \mathrm{E}-02$ & 407 & $7.17 \mathrm{E}-03$ & 385 & $8.60 \mathrm{E}-03$ & 1,020 & $5.67 \mathrm{E}-03$ & 1,510 & $9.23 \mathrm{E}-03$ & $3.19 \mathrm{E}-10$ \\
\hline A161.23.9.20.8 & & $1.34 \mathrm{E}-05$ & 26.9 & 0.745 & $1.51 \mathrm{E}-02$ & 402 & $6.73 \mathrm{E}-03$ & 323 & $6.85 \mathrm{E}-03$ & 977 & $5.16 \mathrm{E}-03$ & 1,210 & $7.02 \mathrm{E}-03$ & $8.80 \mathrm{E}-11$ \\
\hline A161.23.9.20.12 & & $1.22 \mathrm{E}-05$ & 40.9 & 0.744 & $1.51 \mathrm{E}-02$ & 320 & $4.86 \mathrm{E}-03$ & 266 & $5.12 \mathrm{E}-03$ & 1,070 & $5.13 \mathrm{E}-03$ & 1,190 & $6.27 \mathrm{E}-03$ & $5.79 \mathrm{E}-10$ \\
\hline A161.23.9.20.14 & & $1.27 \mathrm{E}-05$ & 47.9 & 0.743 & $1.51 \mathrm{E}-02$ & 340 & $5.41 \mathrm{E}-03$ & 277 & $5.59 \mathrm{E}-03$ & 1,170 & $5.87 \mathrm{E}-03$ & 1,110 & $6.13 \mathrm{E}-03$ & 2.73E-10 \\
\hline A161.23.9.20.16 & & $1.26 \mathrm{E}-05$ & 54.8 & 0.743 & $1.51 \mathrm{E}-02$ & 315 & $4.95 \mathrm{E}-03$ & 247 & $4.91 \mathrm{E}-03$ & 1,040 & $5.15 \mathrm{E}-03$ & 999 & $5.44 \mathrm{E}-03$ & $2.65 \mathrm{E}-10$ \\
\hline A161.23.9.20.18 & & $1.29 \mathrm{E}-05$ & 61.8 & 0.743 & $1.50 \mathrm{E}-02$ & 295 & 4.77E-03 & 234 & 4.79E-03 & 996 & $5.08 \mathrm{E}-03$ & 911 & $5.10 \mathrm{E}-03$ & $1.59 \mathrm{E}-10$ \\
\hline A161.23.9.20.20 & & $1.32 \mathrm{E}-05$ & 68.9 & 0.742 & $1.50 \mathrm{E}-02$ & 301 & $4.98 \mathrm{E}-03$ & 245 & $5.14 \mathrm{E}-03$ & 1,040 & $5.43 \mathrm{E}-03$ & 979 & $5.62 \mathrm{E}-03$ & 2.43E-10 \\
\hline A161.23.9.20.22 & & $1.31 \mathrm{E}-05$ & 75.9 & 0.741 & $1.50 \mathrm{E}-02$ & 314 & $5.15 \mathrm{E}-03$ & 260 & $5.40 \mathrm{E}-03$ & 1,120 & $5.79 \mathrm{E}-03$ & 964 & $5.48 \mathrm{E}-03$ & 4.08E-11 \\
\hline A161.23.9.20.23 & & $1.29 \mathrm{E}-05$ & 78.9 & 0.741 & $1.50 \mathrm{E}-02$ & 321 & $5.18 \mathrm{E}-03$ & 307 & $6.27 \mathrm{E}-03$ & 1,180 & $6.00 \mathrm{E}-03$ & 1,170 & $6.54 \mathrm{E}-03$ & $1.37 \mathrm{E}-10$ \\
\hline A161.23.9.20.24 & & $1.37 \mathrm{E}-05$ & 82.9 & 0.741 & $1.50 \mathrm{E}-02$ & 297 & $5.11 \mathrm{E}-03$ & 261 & 5.69E-03 & 1,040 & $5.64 \mathrm{E}-03$ & 999 & $5.96 \mathrm{E}-03$ & $1.37 \mathrm{E}-10$ \\
\hline \multicolumn{15}{|c|}{ A161 $23^{\circ} \mathrm{C}$ pH 9 Reactor \#2 } \\
\hline A161.23.9.40.Blank1 & & 2.04E-05 & --- & --- & --- & [49.4] & --- & {$[75.6]$} & --- & [164] & --- & [134] & --- & --- \\
\hline A161.23.9.40.Blank2 & & $2.02 \mathrm{E}-05$ & --- & --- & --- & {$[49.4]$} & --- & {$[75.6]$} & --- & [164] & --- & [134] & --- & --- \\
\hline A161.23.9.40.Blank3 & & 2.07E-05 & 0.0 & --- & --- & [49.4] & --- & [75.6] & --- & [164] & --- & [134] & --- & --- \\
\hline A161.23.9.40.2 & & $2.00 \mathrm{E}-05$ & 5.9 & 0.374 & $7.56 \mathrm{E}-03$ & 153 & $7.61 \mathrm{E}-03$ & 244 & $1.54 \mathrm{E}-02$ & 230 & $3.61 \mathrm{E}-03$ & 1,060 & $1.83 \mathrm{E}-02$ & $1.46 \mathrm{E}-09$ \\
\hline A161.23.9.40.4 & & $1.97 E-05$ & 12.9 & 0.374 & $7.56 \mathrm{E}-03$ & 211 & $1.03 E-02$ & 192 & $1.19 \mathrm{E}-02$ & 369 & $5.70 \mathrm{E}-03$ & 735 & $1.25 \mathrm{E}-02$ & $2.88 \mathrm{E}-10$ \\
\hline A161.23.9.40.6 & & $1.58 \mathrm{E}-05$ & 19.9 & 0.373 & $7.55 \mathrm{E}-03$ & 208 & $8.17 \mathrm{E}-03$ & 156 & $7.76 \mathrm{E}-03$ & 341 & $4.23 \mathrm{E}-03$ & 581 & $7.92 \mathrm{E}-03$ & $7.65 \mathrm{E}-11$ \\
\hline A161.23.9.40.8 & & 2.02E-05 & 26.9 & 0.373 & 7.55E-03 & 188 & $9.48 \mathrm{E}-03$ & 125 & 7.98E-03 & 280 & 4.45E-03 & 540 & $9.44 \mathrm{E}-03$ & 7.34E-10 \\
\hline A161.23.9.40.10 & & $1.88 \mathrm{E}-05$ & 33.9 & 0.372 & $7.54 \mathrm{E}-03$ & 253 & 1.19E-02 & 197 & $1.17 \mathrm{E}-02$ & 534 & $7.89 \mathrm{E}-03$ & 770 & $1.25 \mathrm{E}-02$ & 4.12E-10 \\
\hline
\end{tabular}

B.30 


\begin{tabular}{|c|c|c|c|c|c|c|c|c|c|c|c|c|c|c|}
\hline Sample ID & $\begin{array}{l}\text { Influent } \\
{[\mathrm{Si}]} \\
(\mathrm{ppm})\end{array}$ & $\begin{array}{l}\text { Flow } \\
\text { Rate, } \mathrm{q} \\
\left(\mathrm{m}^{3} \mathrm{~d}^{-1}\right)\end{array}$ & $\begin{array}{l}\text { Time } \\
\text { (d) }\end{array}$ & $\begin{array}{l}\text { Glass } \\
\text { Mass } \\
\text { (g) }\end{array}$ & $\begin{array}{l}\text { Surface } \\
\text { Area, } \mathrm{S} \\
\left(\mathrm{m}^{2}\right)\end{array}$ & $\begin{array}{l}{[\mathrm{Al}]} \\
\left(\mu \mathrm{g} \mathrm{L}^{-1}\right)\end{array}$ & $\begin{array}{l}\text { Al Norm. } \\
\text { Diss. Rate } \\
\left(\mathrm{g} \mathrm{m}^{-2} \mathrm{~d}^{-1}\right)\end{array}$ & $\begin{array}{l}{[\mathrm{B}]} \\
\left(\mu \mathrm{g} \mathrm{L}^{-1}\right)\end{array}$ & $\begin{array}{l}\text { B Norm. } \\
\text { Diss. Rate } \\
\left(\mathrm{g} \mathrm{m}^{-2} \mathrm{~d}^{-1}\right)\end{array}$ & $\begin{array}{l}{[\mathrm{Si}]} \\
\left(\mu g \mathrm{~L}^{-1}\right)\end{array}$ & $\begin{array}{l}\text { Si Norm. } \\
\text { Diss. Rate } \\
\left(\mathrm{g} \mathrm{m}^{-2} \mathrm{~d}^{-1}\right)\end{array}$ & $\begin{array}{l}{[\mathrm{Na}]} \\
(\mu \mathrm{g} \mathrm{L} \\
\left.{ }^{1}\right)\end{array}$ & $\begin{array}{l}\text { Na Norm. } \\
\text { Diss. Rate, } \\
\mathrm{r} \\
\left(\mathrm{g} \mathrm{m}^{-2} \mathrm{~d}^{-1}\right)\end{array}$ & $\begin{array}{l}\mathrm{r}_{\mathrm{IEX}} \\
\left(\mathrm{mol} \mathrm{Na} \mathrm{s}^{-1}\right)\end{array}$ \\
\hline A161.23.9.40.12 & & 2.01E-05 & 40.9 & 0.372 & 7.53E-03 & 179 & $9.01 E-03$ & 143 & $9.12 \mathrm{E}-03$ & 542 & $8.60 E-03$ & 549 & $9.58 \mathrm{E}-03$ & 2.34E-10 \\
\hline A161.23.9.40.14 & & 2.03E-05 & 47.9 & 0.371 & 7.53E-03 & 173 & $8.79 \mathrm{E}-03$ & 137 & 8.82E-03 & 510 & $8.17 E-03$ & 548 & $9.65 \mathrm{E}-03$ & 4.22E-10 \\
\hline A161.23.9.40.16 & & $1.94 \mathrm{E}-05$ & 54.8 & 0.371 & $7.52 \mathrm{E}-03$ & 164 & $7.95 \mathrm{E}-03$ & 126 & $7.74 \mathrm{E}-03$ & 482 & $7.37 E-03$ & 526 & $8.84 \mathrm{E}-03$ & $5.56 \mathrm{E}-10$ \\
\hline A161.23.9.40.18 & & 2.06E-05 & 61.8 & 0.371 & 7.52E-03 & 154 & 7.93E-03 & 105 & $6.85 \mathrm{E}-03$ & 413 & $6.71 \mathrm{E}-03$ & 472 & 8.43E-03 & $7.95 \mathrm{E}-10$ \\
\hline A161.23.9.40.20 & & 2.04E-05 & 68.9 & 0.370 & $7.51 \mathrm{E}-03$ & 154 & $7.89 \mathrm{E}-03$ & 123 & $7.98 \mathrm{E}-03$ & 447 & $7.22 \mathrm{E}-03$ & 499 & $8.86 \mathrm{E}-03$ & 4.44E-10 \\
\hline A161.23.9.40.22 & & $2.04 \mathrm{E}-05$ & 75.9 & 0.370 & 7.50E-03 & 153 & $7.84 \mathrm{E}-03$ & 117 & $7.59 \mathrm{E}-03$ & 429 & $6.93 \mathrm{E}-03$ & 501 & $8.90 \mathrm{E}-03$ & $6.58 \mathrm{E}-10$ \\
\hline A161.23.9.40.23 & & 2.04E-05 & 78.9 & 0.370 & $7.50 \mathrm{E}-03$ & 153 & $7.84 \mathrm{E}-03$ & 137 & $8.89 E-03$ & 489 & $7.90 \mathrm{E}-03$ & 613 & $1.09 \mathrm{E}-02$ & $1.01 E-09$ \\
\hline A161.23.9.40.24 & & $2.01 \mathrm{E}-05$ & 82.9 & 0.369 & $7.50 \mathrm{E}-03$ & 158 & $7.96 \mathrm{E}-03$ & 111 & $7.09 \mathrm{E}-03$ & 444 & $7.06 \mathrm{E}-03$ & 495 & $8.65 \mathrm{E}-03$ & $7.88 \mathrm{E}-10$ \\
\hline \multicolumn{15}{|c|}{ A161 $23^{\circ} \mathrm{C} \mathrm{pH} 9$ Reactor $\# 3$} \\
\hline A161.23.9.80A.Blank1 & & $3.80 \mathrm{E}-05$ & --- & --- & --- & [49.4] & --- & {$[75.6]$} & --- & [164] & -- & [134] & --- & --- \\
\hline A161.23.9.80A.Blank2 & & 3.72E-05 & --- & --- & --- & [49.4] & --- & [75.6] & --- & [164] & --- & [134] & --- & --- \\
\hline A161.23.9.80A.Blank3 & & 3.63E-05 & 0.0 & --- & --- & [49.4] & --- & {$[75.6]$} & --- & [164] & --- & [134] & --- & -.- \\
\hline A161.23.9.80A.2 & & 3.65E-05 & 5.9 & 0.149 & $3.02 \mathrm{E}-03$ & 57 & $1.29 \mathrm{E}-02$ & $\begin{array}{l}{[75.6]} \\
55^{\star}\end{array}$ & $2.17 \mathrm{E}-02$ & [164] & 1.17E-02 & 259 & $2.04 \mathrm{E}-02$ & $<0$ \\
\hline A161.23.9.80A.4 & & 3.70E-05 & 12.9 & 0.149 & $3.01 E-03$ & 53 & $1.22 \mathrm{E}-02$ & $\begin{array}{l}{[75.6]} \\
26^{\star}\end{array}$ & $2.21 \mathrm{E}-02$ & [164] & 1.19E-02 & 147 & $1.18 \mathrm{E}-02$ & $<0$ \\
\hline A161.23.9.80A.6 & & 3.67E-05 & 19.9 & 0.148 & $3.01 \mathrm{E}-03$ & $\begin{array}{l}{[49.4]} \\
44^{\star}\end{array}$ & 1.13E-02 & {$[75.6]$} & $2.20 \mathrm{E}-02$ & [164] & 1.19E-02 & 152 & $1.21 \mathrm{E}-02$ & $<0$ \\
\hline A161.23.9.80A.8 & & 3.58E-05 & 26.9 & 0.148 & $3.00 \mathrm{E}-03$ & $\begin{array}{l}{[49.4]} \\
40^{\star}\end{array}$ & $1.11 \mathrm{E}-02$ & $\begin{array}{l}{[75.6]} \\
27^{\star}\end{array}$ & $2.15 \mathrm{E}-02$ & [164] & $1.16 \mathrm{E}-02$ & 152 & $1.18 \mathrm{E}-02$ & $<0$ \\
\hline A161.23.9.80A.10 & & 3.41E-05 & 33.9 & 0.148 & $3.00 \mathrm{E}-03$ & 56 & 1.19E-02 & $\begin{array}{l}{[75.6]} \\
45^{\star}\end{array}$ & $2.05 \mathrm{E}-02$ & [164] & $1.11 \mathrm{E}-02$ & 206 & $1.53 \mathrm{E}-02$ & $<0$ \\
\hline A161.23.9.80A.12 & & 3.65E-05 & 40.9 & 0.147 & 2.99E-03 & 57 & $1.31 \mathrm{E}-02$ & $\begin{array}{l}{[75.6]} \\
39^{\star}\end{array}$ & $2.20 \mathrm{E}-02$ & [164] & 1.19E-02 & 194 & $1.54 \mathrm{E}-02$ & $<0$ \\
\hline A161.23.9.80A.14 & & 3.59E-05 & 47.9 & 0.147 & $2.98 \mathrm{E}-03$ & $\begin{array}{l}{[49.4]} \\
31^{\star}\end{array}$ & 1.12E-02 & $\begin{array}{l}{[75.6]} \\
41^{\star}\end{array}$ & $2.17 \mathrm{E}-02$ & [164] & $1.17 \mathrm{E}-02$ & 181 & $1.42 \mathrm{E}-02$ & $<0$ \\
\hline A161.23.9.80A.16 & & 3.64E-05 & 54.8 & 0.146 & $2.98 \mathrm{E}-03$ & $\begin{array}{l}{[49.4]} \\
30^{*}\end{array}$ & $1.14 \mathrm{E}-02$ & $\begin{array}{l}{[75.6]} \\
31^{\star}\end{array}$ & $2.20 \mathrm{E}-02$ & {$[164]$} & $1.19 E-02$ & 176 & $1.41 \mathrm{E}-02$ & $<0$ \\
\hline A161.23.9.80A.18 & & 3.55E-05 & 61.8 & 0.146 & 2.97E-03 & $\begin{array}{l}{[49.4]} \\
28^{\star}\end{array}$ & 1.11E-02 & $\begin{array}{l}{[75.6]} \\
27^{\star}\end{array}$ & $2.15 \mathrm{E}-02$ & [164] & 1.16E-02 & 157 & $1.22 \mathrm{E}-02$ & $<0$ \\
\hline A161.23.9.80A.20 & & 3.54E-05 & 68.9 & 0.145 & 2.97E-03 & $\begin{array}{l}{[49.4]} \\
25^{\star}\end{array}$ & $1.11 \mathrm{E}-02$ & $\begin{array}{l}{[75.6]} \\
26^{\star}\end{array}$ & $2.15 \mathrm{E}-02$ & {$[164]$} & 1.16E-02 & 164 & $1.28 \mathrm{E}-02$ & $<0$ \\
\hline A161.23.9.80A.22 & & 3.56E-05 & 75.9 & 0.145 & $2.96 \mathrm{E}-03$ & $\begin{array}{l}{[49.4]} \\
28^{\star}\end{array}$ & 1.12E-02 & $\begin{array}{l}{[75.6]} \\
25^{\star}\end{array}$ & $2.17 \mathrm{E}-02$ & [164] & $1.17 E-02$ & 154 & $1.21 \mathrm{E}-02$ & $<0$ \\
\hline A161.23.9.80A.23 & & 3.54E-05 & 78.9 & 0.145 & $2.96 \mathrm{E}-03$ & $\begin{array}{l}{[49.4]} \\
30^{*}\end{array}$ & $1.11 \mathrm{E}-02$ & $\begin{array}{l}{[75.6]} \\
32^{\star}\end{array}$ & $2.15 \mathrm{E}-02$ & [164] & $1.16 \mathrm{E}-02$ & 190 & $1.48 \mathrm{E}-02$ & $<0$ \\
\hline A161.23.9.80A.24 & & $3.51 \mathrm{E}-05$ & 82.9 & 0.144 & $2.95 \mathrm{E}-03$ & $\begin{array}{l}{[49.4]} \\
24^{\star}\end{array}$ & $1.11 \mathrm{E}-02$ & $\begin{array}{l}{[75.6]} \\
37^{\star}\end{array}$ & $2.14 \mathrm{E}-02$ & [164] & $1.16 \mathrm{E}-02$ & 172 & $1.34 \mathrm{E}-02$ & $<0$ \\
\hline \multicolumn{15}{|c|}{ A161 $23^{\circ} \mathrm{C}$ pH 9 Reactor \#4 } \\
\hline A161.23.9.80B.Blank1 & & 4.06E-05 & --- & --- & --- & [49.4] & --- & [75.6] & --- & [164] & --- & [134] & --- & --- \\
\hline A161.23.9.80B.Blank2 & & 4.05E-05 & --- & --- & --- & [49.4] & --- & {$[75.6]$} & --- & [164] & --- & [134] & --- & --- \\
\hline A161.23.9.80B.Blank3 & & 4.01E-05 & 0.0 & --- & --- & [49.4] & --- & [75.6] & --- & [164] & --- & [134] & --- & --- \\
\hline A161.23.9.80B.2 & & 4.04E-05 & 5.9 & 0.029 & $5.94 \mathrm{E}-04$ & [49.4] & $6.32 \mathrm{E}-02$ & {$[75.6]$} & $1.22 \mathrm{E}-01$ & $\begin{array}{l}{[164]} \\
44^{*}\end{array}$ & $6.61 \mathrm{E}-02$ & $\begin{array}{l}{[134]} \\
44^{*}\end{array}$ & $5.94 \mathrm{E}-02$ & $<0$ \\
\hline A161.23.9.80B.4 & & 4.05E-05 & 12.9 & 0.029 & 5.87E-04 & [49.4] & $6.41 \mathrm{E}-02$ & {$[75.6]$} & $1.24 \mathrm{E}-01$ & [164] & $6.71 E-02$ & [134] & $6.03 \mathrm{E}-02$ & $<0$ \\
\hline A161.23.9.80B.6 & & $4.08 \mathrm{E}-05$ & 19.9 & 0.028 & $5.80 \mathrm{E}-04$ & [49.4] & $6.54 \mathrm{E}-02$ & {$[75.6]$} & 1.27E-01 & [164] & $6.85 \mathrm{E}-02$ & [134] & $6.15 \mathrm{E}-02$ & $<0$ \\
\hline
\end{tabular}




\begin{tabular}{|c|c|c|c|c|c|c|c|c|c|c|c|c|c|c|}
\hline Sample ID & $\begin{array}{l}\text { Influent } \\
\text { [Si] } \\
(\mathrm{ppm})\end{array}$ & $\begin{array}{l}\text { Flow } \\
\text { Rate, } q \\
\left(\mathrm{~m}^{3} \mathrm{~d}^{-1}\right)\end{array}$ & $\begin{array}{l}\text { Time } \\
\text { (d) }\end{array}$ & $\begin{array}{l}\text { Glass } \\
\text { Mass } \\
\text { (g) }\end{array}$ & $\begin{array}{l}\text { Surface } \\
\text { Area, S } \\
\left(\mathrm{m}^{2}\right)\end{array}$ & $\begin{array}{l}{[\mathrm{Al}]} \\
\left(\mu \mathrm{L}^{-1}\right)\end{array}$ & $\begin{array}{l}\text { Al Norm. } \\
\text { Diss. Rate } \\
\left(\mathrm{g} \mathrm{m}^{-2} \mathrm{~d}^{-1}\right)\end{array}$ & $\begin{array}{l}{[\mathrm{B}]} \\
\left(\mu \mathrm{g} \mathrm{L}^{-1}\right)\end{array}$ & $\begin{array}{l}\text { B Norm. } \\
\text { Diss. Rate } \\
\left(\mathrm{g} \mathrm{m}^{-2} \mathrm{~d}^{-1}\right)\end{array}$ & $\begin{array}{l}{[\mathrm{Si}]} \\
\left(\mu \mathrm{g} \mathrm{L}^{-1}\right)\end{array}$ & $\begin{array}{l}\text { Si Norm. } \\
\text { Diss. Rate } \\
\left(\mathrm{g} \mathrm{m}^{-2} \mathrm{~d}^{-1}\right)\end{array}$ & $\begin{array}{l}{[\mathrm{Na}]} \\
(\mu \mathrm{g} \mathrm{L} \\
\left.{ }^{1}\right)\end{array}$ & $\begin{array}{l}\text { Na Norm. } \\
\text { Diss. Rate, } \\
\mathrm{r} \\
\left(\mathrm{g} \mathrm{m}^{-2} \mathrm{~d}^{-1}\right)\end{array}$ & $\begin{array}{l}\mathrm{r}_{\mathrm{IEX}} \\
\left(\mathrm{mol} \mathrm{Na} \mathrm{s}^{-2}\right)\end{array}$ \\
\hline A161.23.9.80B.8 & & 3.99E-05 & 26.9 & 0.028 & $5.73 \mathrm{E}-04$ & [49.4] & $6.47 \mathrm{E}-02$ & {$[75.6]$} & $1.25 \mathrm{E}-01$ & [164] & $6.77 \mathrm{E}-02$ & [134] & $6.08 \mathrm{E}-02$ & $<0$ \\
\hline A161.23.9.80B.10 & & 3.88E-05 & 33.9 & 0.027 & 5.67E-04 & {$[49.4]$} & $6.37 \mathrm{E}-02$ & [75.6] & $1.23 \mathrm{E}-01$ & [164] & $6.66 \mathrm{E}-02$ & [134] & $5.99 E-02$ & $<0$ \\
\hline A161.23.9.80B.12 & & 4.06E-05 & 40.9 & 0.027 & $5.59 \mathrm{E}-04$ & {$[49.4]$} & $6.75 \mathrm{E}-02$ & [75.6] & $1.31 \mathrm{E}-01$ & [164] & 7.07E-02 & $\begin{array}{l}{[134]} \\
46^{*}\end{array}$ & $6.35 \mathrm{E}-02$ & $<0$ \\
\hline A161.23.9.80B.14 & & $4.11 \mathrm{E}-05$ & 47.9 & 0.026 & $5.52 \mathrm{E}-04$ & {$[49.4]$} & $6.92 \mathrm{E}-02$ & {$[75.6]$} & $1.34 \mathrm{E}-01$ & [164] & 7.24E-02 & $\begin{array}{l}{[134]} \\
66^{\star}\end{array}$ & $6.51 \mathrm{E}-02$ & $<0$ \\
\hline A161.23.9.80B.16 & & 4.11E-05 & 54.8 & 0.026 & $5.45 \mathrm{E}-04$ & [49.4] & $7.02 \mathrm{E}-02$ & [75.6] & $1.36 \mathrm{E}-01$ & [164] & $7.35 \mathrm{E}-02$ & $\begin{array}{l}{[134]} \\
50^{*}\end{array}$ & $6.60 \mathrm{E}-02$ & $<0$ \\
\hline A161.23.9.80B.18 & & $4.12 \mathrm{E}-05$ & 61.8 & 0.025 & $5.38 \mathrm{E}-04$ & {$[49.4]$} & $7.12 \mathrm{E}-02$ & {$[75.6]$} & $1.38 \mathrm{E}-01$ & [164] & 7.45E-02 & $\begin{array}{l}{[134]} \\
74^{\star}\end{array}$ & $6.69 \mathrm{E}-02$ & $<0$ \\
\hline A161.23.9.80B.20 & & 4.13E-05 & 68.9 & 0.025 & $5.30 \mathrm{E}-04$ & {$[49.4]$} & 7.24E-02 & {$[75.6]$} & 1.40E-01 & [164] & $7.58 \mathrm{E}-02$ & $\begin{array}{l}{[134]} \\
68^{\star}\end{array}$ & $6.81 E-02$ & $<0$ \\
\hline A161.23.9.80B.22 & & $4.11 \mathrm{E}-05$ & 75.9 & 0.024 & $5.22 \mathrm{E}-04$ & {$[49.4]$} & $7.31 E-02$ & {$[75.6]$} & $1.42 \mathrm{E}-01$ & [164] & 7.65E-02 & $\begin{array}{l}{[134]} \\
79^{\star}\end{array}$ & $6.87 \mathrm{E}-02$ & $<0$ \\
\hline A161.23.9.80B.23 & & 4.12E-05 & 78.9 & 0.024 & $5.20 \mathrm{E}-04$ & [49.4] & $7.38 \mathrm{E}-02$ & [75.6] & $1.43 \mathrm{E}-01$ & [164] & 7.72E-02 & $\begin{array}{l}{[134]} \\
77^{\star}\end{array}$ & $6.94 \mathrm{E}-02$ & $<0$ \\
\hline A161.23.9.80B.24 & & 4.16E-05 & 82.9 & 0.024 & 5.15E-04 & {$[49.4]$} & 7.51E-02 & {$[75.6]$} & 1.45E-01 & {$[164]$} & $7.86 \mathrm{E}-02$ & $\begin{array}{l}{[134]} \\
67^{\star}\end{array}$ & $7.06 \mathrm{E}-02$ & $<0$ \\
\hline \multicolumn{15}{|c|}{ A161 $23^{\circ} \mathrm{C}$ pH 9 Reactor \#5 } \\
\hline A161.23.9.C.Blank1 & & $1.96 \mathrm{E}-05$ & --- & --- & --- & {$[49.4]$} & --- & [75.6] & --- & [164] & --- & [134] & --- & --- \\
\hline A161.23.9.C.Blank2 & & $1.85 \mathrm{E}-05$ & --- & --- & --- & {$[49.4]$} & -- & [75.6] & --- & [164] & --- & [134] & --- & --- \\
\hline A161.23.9.C.Blank3 & & 1.83E-05 & --- & --- & -- & {$[49.4]$} & -- & {$[75.6]$} & --- & [164] & --- & [134] & --- & --- \\
\hline A161.23.9.C.2 & & 3.57E-05 & 5.2 & 0.020 & $7.31 \mathrm{E}-04$ & {$[49.4]$} & 4.54E-02 & [75.6] & $8.79 E-02$ & [164] & 4.75E-02 & $\begin{array}{l}{[134]} \\
90^{*}\end{array}$ & 4.27E- 02 & $<0$ \\
\hline A161.23.9.C.4 & & 3.61E-05 & 12.2 & 0.019 & $7.25 \mathrm{E}-04$ & {$[49.4]$} & 4.63E-02 & {$[75.6]$} & $8.97 E-02$ & [164] & $4.84 \mathrm{E}-02$ & $\begin{array}{l}{[134]} \\
52^{*}\end{array}$ & 4.35E-02 & $<0$ \\
\hline A161.23.9.C.6 & & 3.38E-05 & 19.2 & 0.019 & 7.19E-04 & [49.4] & 4.37E-02 & {$[75.6]$} & $8.47 E-02$ & [164] & 4.57E-02 & $\begin{array}{l}{[134]} \\
24^{\star}\end{array}$ & $4.11 \mathrm{E}-02$ & $<0$ \\
\hline A161.23.9.C.8 & & 3.45E-05 & 26.2 & 0.019 & 7.13E-04 & {$[49.4]$} & $4.50 \mathrm{E}-02$ & {$[75.6]$} & $8.73 E-02$ & [164] & $4.71 \mathrm{E}-02$ & $\begin{array}{l}{[134]} \\
58^{\star}\end{array}$ & 4.23E-02 & $<0$ \\
\hline A161.23.9.C.10 & & 3.36E-05 & 33.2 & 0.018 & 7.07E-04 & [49.4] & $4.41 \mathrm{E}-02$ & [75.6] & $8.55 E-02$ & [164] & 4.62E-02 & $\begin{array}{l}{[134]} \\
65^{\star}\end{array}$ & $4.15 \mathrm{E}-02$ & $<0$ \\
\hline A161.23.9.C.12 & & $3.70 \mathrm{E}-05$ & 40.2 & 0.018 & $7.01 \mathrm{E}-04$ & {$[49.4]$} & $4.91 \mathrm{E}-02$ & {$[75.6]$} & $9.51 \mathrm{E}-02$ & {$[164]$} & $5.14 \mathrm{E}-02$ & $\begin{array}{l}{[134]} \\
95^{\star}\end{array}$ & 4.62E-02 & $<0$ \\
\hline A161.23.9.C.14 & & 3.66E-05 & 47.2 & 0.017 & $6.94 \mathrm{E}-04$ & [49.4] & $4.90 \mathrm{E}-02$ & [75.6] & $9.50 \mathrm{E}-02$ & [164] & $5.13 \mathrm{E}-02$ & $\begin{array}{l}{[134]} \\
100^{*}\end{array}$ & $4.61 \mathrm{E}-02$ & $<0$ \\
\hline A161.23.9.C.16 & & 3.69E-05 & 54.1 & 0.017 & $6.88 \mathrm{E}-04$ & {$[49.4]$} & 4.99E-02 & {$[75.6]$} & $9.67 \mathrm{E}-02$ & [164] & $5.22 \mathrm{E}-02$ & $\begin{array}{l}{[134]} \\
79^{\star}\end{array}$ & 4.69E-02 & $<0$ \\
\hline A161.23.9.C.18 & & 3.64E-05 & 61.1 & 0.016 & $6.81 \mathrm{E}-04$ & [49.4] & $4.96 \mathrm{E}-02$ & [75.6] & $9.62 \mathrm{E}-02$ & [164] & $5.20 \mathrm{E}-02$ & $\begin{array}{l}{[134]} \\
65^{\star}\end{array}$ & 4.67E-02 & $<0$ \\
\hline A161.23.9.C.20 & & $3.62 \mathrm{E}-05$ & 68.2 & 0.016 & $6.74 \mathrm{E}-04$ & {$[49.4]$} & 4.99E-02 & {$[75.6]$} & $9.68 \mathrm{E}-02$ & [164] & $5.23 \mathrm{E}-02$ & $\begin{array}{l}{[134]} \\
69^{\star}\end{array}$ & 4.70E-02 & $<0$ \\
\hline A161.23.9.C.22 & & 3.63E-05 & 75.2 & 0.015 & 6.67E-04 & [49.4] & $5.06 \mathrm{E}-02$ & {$[75.6]$} & $9.80 \mathrm{E}-02$ & [164] & $5.29 \mathrm{E}-02$ & $\begin{array}{l}{[134]} \\
65^{\star}\end{array}$ & $4.76 \mathrm{E}-02$ & $<0$ \\
\hline A161.23.9.C.23 & & 3.68E-05 & 78.2 & 0.015 & $6.65 \mathrm{E}-04$ & {$[49.4]$} & $5.14 \mathrm{E}-02$ & {$[75.6]$} & $9.96 \mathrm{E}-02$ & [164] & $5.38 \mathrm{E}-02$ & $\begin{array}{l}{[134]} \\
78^{\star}\end{array}$ & 4.83E-02 & $<0$ \\
\hline A161.23.9.C.24 & & 3.53E-05 & 82.2 & 0.015 & $6.61 \mathrm{E}-04$ & [49.4] & 4.97E-02 & [75.6] & 9.63E-02 & [164] & 5.20E-02 & [134] & 4.67E-02 & $<0$ \\
\hline
\end{tabular}




\begin{tabular}{|c|c|c|c|c|c|c|c|c|c|c|c|c|c|c|}
\hline Sample ID & $\begin{array}{l}\text { Influent } \\
{[\mathrm{Si}]} \\
(\mathrm{ppm})\end{array}$ & $\begin{array}{l}\text { Flow } \\
\text { Rate, } q \\
\left(\mathrm{~m}^{3} \mathrm{~d}^{-1}\right)\end{array}$ & $\begin{array}{l}\text { Time } \\
\text { (d) }\end{array}$ & $\begin{array}{l}\text { Glass } \\
\text { Mass } \\
\text { (g) }\end{array}$ & $\begin{array}{l}\text { Surface } \\
\text { Area, S } \\
\left(\mathrm{m}^{2}\right)\end{array}$ & $\begin{array}{l}{[\mathrm{Al}]} \\
\left(\mu \mathrm{g} \mathrm{L}^{-1}\right)\end{array}$ & $\begin{array}{l}\text { Al Norm. } \\
\text { Diss. Rate } \\
\left(\mathrm{g} \mathrm{m}^{-2} \mathrm{~d}^{-1}\right)\end{array}$ & $\begin{array}{l}{[\mathrm{B}]} \\
\left(\mu \mathrm{g} \mathrm{L}^{-1}\right)\end{array}$ & $\begin{array}{l}\text { B Norm. } \\
\text { Diss. Rate } \\
\left(\mathrm{g} \mathrm{m}^{-2} \mathrm{~d}^{-1}\right)\end{array}$ & $\begin{array}{l}{[\mathrm{Si}]} \\
\left(\mu \mathrm{g} \mathrm{L}^{-1}\right)\end{array}$ & $\begin{array}{l}\text { Si Norm. } \\
\text { Diss. Rate } \\
\left(\mathrm{g} \mathrm{m}^{-2} \mathrm{~d}^{-1}\right)\end{array}$ & $\begin{array}{l}{[\mathrm{Na}]} \\
(\mu \mathrm{gg} \mathrm{L} \\
\left.{ }^{1}\right)\end{array}$ & $\begin{array}{l}\text { Na Norm. } \\
\text { Diss. Rate, } \\
\mathrm{r} \\
\left(\mathrm{g} \mathrm{m}^{-2} \mathrm{~d}^{-1}\right)\end{array}$ & $\begin{array}{l}\mathrm{r}_{\mathrm{IEX}} \\
\left(\mathrm{mol} \mathrm{Na} \mathrm{No}^{-1}\right)\end{array}$ \\
\hline & & & & & & & & & & & & $81^{\star}$ & & \\
\hline \multicolumn{15}{|c|}{ A161 $23^{\circ} \mathrm{C}$ pH 10 Reactor \#1 } \\
\hline A161.23.10.80A.Blank1 & & 3.67E-05 & --- & $-\cdots$ & --- & [49.4] & --- & [75.6] & --- & [164] & --- & [134] & --- & --- \\
\hline A161.23.10.80A.Blank2 & & $2.07 E-05$ & --- & --- & --- & {$[49.4]$} & --- & {$[75.6]$} & --- & {$[164]$} & -- & [134] & --- & --- \\
\hline A161.23.10.80A.Blank3 & & 3.70E-05 & 0.0 & --- & --- & [49.4] & --- & [75.6] & --- & [164] & --- & [134] & --- & --- \\
\hline A161.23.10.80A.2 & & 3.99E-05 & 5.9 & 0.029 & $5.98 \mathrm{E}-04$ & 98 & $1.24 \mathrm{E}-01$ & {$[75.6]$} & $1.20 \mathrm{E}-01$ & $\begin{array}{l}{[164]} \\
76^{*}\end{array}$ & $6.49 E-02$ & 258 & $1.12 \mathrm{E}-01$ & $<0$ \\
\hline A161.23.10.80A.4 & & 4.00E-05 & 12.9 & 0.029 & $5.91 E-04$ & 101 & $1.29 \mathrm{E}-01$ & {$[75.6]$} & $1.22 \mathrm{E}-01$ & $\begin{array}{l}{[164]} \\
160^{*}\end{array}$ & $6.58 E-02$ & 262 & $1.16 \mathrm{E}-01$ & $<0$ \\
\hline A161.23.10.80A.6 & & $3.92 E-05$ & 19.9 & 0.028 & 5.85E-04 & 97 & $1.22 \mathrm{E}-01$ & {$[75.6]$} & $1.21 \mathrm{E}-01$ & $\begin{array}{l}{[164]} \\
105^{*}\end{array}$ & $6.52 E-02$ & 244 & $1.07 E-01$ & $<0$ \\
\hline A161.23.10.80A.8 & & 3.80E-05 & 26.9 & 0.028 & $5.78 \mathrm{E}-04$ & 91 & 1.12E-01 & {$[75.6]$} & 1.19E-01 & $\begin{array}{l}{[164]} \\
126^{*}\end{array}$ & $6.40 E-02$ & 253 & 1.09E-01 & $<0$ \\
\hline A161.23.10.80A.10 & & 3.74E-05 & 33.9 & 0.027 & $5.72 \mathrm{E}-04$ & 90 & 1.10E-01 & {$[75.6]$} & $1.18 \mathrm{E}-01$ & $\begin{array}{l}{[164]} \\
89^{\star}\end{array}$ & $6.37 E-02$ & 257 & 1.10E-01 & $<0$ \\
\hline A161.23.10.80A.12 & & 3.70E-05 & 40.9 & 0.027 & 5.65E-04 & 80 & $9.80 \mathrm{E}-02$ & $\begin{array}{l}{[75.6]} \\
70^{\star}\end{array}$ & $1.18 \mathrm{E}-01$ & 266 & $1.03 E-01$ & 311 & 1.33E-01 & 7.49E-09 \\
\hline A161.23.10.80A.14 & & 4.24E-05 & 47.9 & 0.026 & 5.57E-04 & 76 & $1.08 \mathrm{E}-01$ & $\begin{array}{l}{[75.6]} \\
63^{\star}\end{array}$ & $1.37 \mathrm{E}-01$ & 259 & $1.17 E-01$ & 297 & $1.47 \mathrm{E}-01$ & $5.20 \mathrm{E}-09$ \\
\hline A161.23.10.80A.16 & & 4.22E-05 & 54.8 & 0.026 & 5.50E-04 & 67 & $9.73 E-02$ & $\begin{array}{l}{[75.6]} \\
56^{\star}\end{array}$ & $1.38 \mathrm{E}-01$ & 207 & $9.42 E-02$ & 250 & $1.25 \mathrm{E}-01$ & $<0$ \\
\hline A161.23.10.80A.18 & & 4.18E-05 & 61.8 & 0.025 & $5.43 E-04$ & 67 & $9.64 \mathrm{E}-02$ & $\begin{array}{l}{[75.6]} \\
57^{\star}\end{array}$ & 1.39E-01 & 290 & $1.33 E-01$ & 250 & $1.26 \mathrm{E}-01$ & $<0$ \\
\hline A161.23.10.80A.20 & & 4.30E-05 & 68.9 & 0.025 & $5.35 \mathrm{E}-04$ & 66 & 1.00E-01 & $\begin{array}{l}{[75.6]} \\
47^{\star}\end{array}$ & 1.45E-01 & 202 & 9.63E-02 & 253 & 1.33E-01 & $<0$ \\
\hline A161.23.10.80A.22 & & 4.77E-05 & 75.9 & 0.024 & $5.26 \mathrm{E}-04$ & 66 & $1.12 \mathrm{E}-01$ & $\begin{array}{l}{[75.6]} \\
54^{\star}\end{array}$ & 1.64E-01 & 197 & $1.06 \mathrm{E}-01$ & 232 & $1.37 \mathrm{E}-01$ & $<0$ \\
\hline A161.23.10.80A.23 & & 4.65E-05 & 78.9 & 0.024 & $5.23 E-04$ & 67 & $1.13 \mathrm{E}-01$ & $\begin{array}{l}{[75.6]} \\
58^{\star}\end{array}$ & 1.60E-01 & 194 & $1.02 \mathrm{E}-01$ & 237 & $1.38 \mathrm{E}-01$ & $<0$ \\
\hline A161.23.10.80A.24 & & 4.61E-05 & 82.9 & 0.024 & $5.17 \mathrm{E}-04$ & 56 & $9.36 \mathrm{E}-02$ & $\begin{array}{l}{[75.6]} \\
43^{\star}\end{array}$ & $1.61 \mathrm{E}-01$ & 202 & $1.07 E-01$ & 221 & $1.29 \mathrm{E}-01$ & $<0$ \\
\hline \multicolumn{15}{|c|}{ A161 $23^{\circ} \mathrm{C}$ pH 10 Reactor \#2 } \\
\hline A161.23.10.C.Blank1 & & $4.02 \mathrm{E}-05$ & -- & --- & --- & [49.4] & --- & {$[75.6]$} & --- & [164] & -- & [134] & --- & -- \\
\hline A161.23.10.C.Blank2 & & 3.95E-05 & --- & --- & --- & [49.4] & --- & [75.6] & --- & [164] & -- & [134] & --- & --- \\
\hline A161.23.10.C.Blank3 & & $2.39 \mathrm{E}-05$ & -.- & --- & --- & [49.4] & -.- & {$[75.6]$} & --- & [164] & -.- & [134] & --- & --- \\
\hline A161.23.10.C. 2 & & 4.05E-05 & 5.2 & 0.021 & 7.23E-04 & 82 & 8.64E-02 & {$[75.6]$} & $1.01 \mathrm{E}-01$ & $\begin{array}{l}{[164]} \\
123^{*}\end{array}$ & $5.45 E-02$ & 219 & 8.00E-02 & $<0$ \\
\hline A161.23.10.C.4 & & 4.05E-05 & 12.2 & 0.021 & $7.16 \mathrm{E}-04$ & 89 & $9.44 E-02$ & {$[75.6]$} & 1.02E-01 & $\begin{array}{l}{[164]} \\
102^{\star}\end{array}$ & $5.51 E-02$ & 223 & $8.24 \mathrm{E}-02$ & $<0$ \\
\hline A161.23.10.C. 6 & & 4.04E-05 & 19.2 & 0.020 & 7.09E-04 & 84 & 8.96E-02 & {$[75.6]$} & 1.03E-01 & $\begin{array}{l}{[164]} \\
66^{\star}\end{array}$ & $5.55 E-02$ & 206 & 7.67E-02 & $<0$ \\
\hline A161.23.10.C.8 & & $4.24 \mathrm{E}-05$ & 26.2 & 0.020 & 7.01E-04 & 74 & $8.36 \mathrm{E}-02$ & {$[75.6]$} & $1.09 \mathrm{E}-01$ & $\begin{array}{l}{[164]} \\
92^{\star}\end{array}$ & $5.88 \mathrm{E}-02$ & 190 & 7.49E-02 & $<0$ \\
\hline A161.23.10.C.10 & & 4.33E- 05 & 33.2 & 0.019 & $6.94 \mathrm{E}-04$ & 70 & $8.22 \mathrm{E}-02$ & {$[75.6]$} & 1.13E-01 & $\begin{array}{l}{[164]} \\
60^{*}\end{array}$ & $6.08 E-02$ & 177 & $7.21 \mathrm{E}-02$ & $<0$ \\
\hline A161.23.10.C.12 & & 4.25E-05 & 40.2 & 0.019 & $6.86 \mathrm{E}-04$ & 56 & $6.52 E-02$ & $\begin{array}{l}{[75.6]} \\
53^{\star}\end{array}$ & 1.12E-01 & 167 & $6.14 E-02$ & 245 & $9.90 \mathrm{E}-02$ & $<0$ \\
\hline
\end{tabular}




\begin{tabular}{|c|c|c|c|c|c|c|c|c|c|c|c|c|c|c|}
\hline Sample ID & $\begin{array}{l}\text { Influent } \\
{[\mathrm{Si}]} \\
(\mathrm{ppm})\end{array}$ & $\begin{array}{l}\text { Flow } \\
\text { Rate, } q \\
\left(\mathrm{~m}^{3} \mathrm{~d}^{-1}\right)\end{array}$ & $\begin{array}{l}\text { Time } \\
\text { (d) }\end{array}$ & $\begin{array}{l}\text { Glass } \\
\text { Mass } \\
\text { (g) }\end{array}$ & $\begin{array}{l}\text { Surface } \\
\text { Area, S } \\
\left(\mathrm{m}^{2}\right)\end{array}$ & $\begin{array}{l}{[\mathrm{Al}]} \\
\left(\mu \mathrm{L}^{-1}\right)\end{array}$ & $\begin{array}{l}\text { Al Norm. } \\
\text { Diss. Rate } \\
\left(\mathrm{g} \mathrm{m}^{-2} \mathrm{~d}^{-1}\right)\end{array}$ & $\begin{array}{l}{[\mathrm{B}]} \\
\left(\mu \mathrm{g} \mathrm{L}^{-1}\right)\end{array}$ & $\begin{array}{l}\text { B Norm. } \\
\text { Diss. Rate } \\
\left(\mathrm{g} \mathrm{m}^{-2} \mathrm{~d}^{-1}\right)\end{array}$ & $\begin{array}{l}{[\mathrm{Si}]} \\
\left(\mu \mathrm{g} \mathrm{L}^{-1}\right)\end{array}$ & $\begin{array}{l}\text { Si Norm. } \\
\text { Diss. Rate } \\
\left(\mathrm{g} \mathrm{m}^{-2} \mathrm{~d}^{-1}\right)\end{array}$ & $\begin{array}{l}{[\mathrm{Na}]} \\
(\mu \mathrm{g} \mathrm{L} \\
\left.{ }^{1}\right)\end{array}$ & $\begin{array}{l}\text { Na Norm. } \\
\text { Diss. Rate, } \\
\mathrm{r} \\
\left(\mathrm{g} \mathrm{m}^{-2} \mathrm{~d}^{-1}\right)\end{array}$ & $\begin{array}{l}\mathrm{r}_{\mathrm{IEX}} \\
\left(\mathrm{mol} \mathrm{Na} \mathrm{s}^{-1}\right)\end{array}$ \\
\hline A161.23.10.C.14 & & 4.00E-05 & 47.2 & 0.018 & $6.79 \mathrm{E}-04$ & 65 & $7.21 \mathrm{E}-02$ & $\begin{array}{l}{[75.6]} \\
62^{\star}\end{array}$ & $1.06 \mathrm{E}-01$ & 183 & $6.40 \mathrm{E}-02$ & 260 & $1.00 \mathrm{E}-01$ & $<0$ \\
\hline A161.23.10.C.16 & & 3.95E-05 & 54.1 & 0.018 & $6.72 \mathrm{E}-04$ & 57 & $6.27 \mathrm{E}-02$ & $\begin{array}{l}{[75.6]} \\
48^{\star}\end{array}$ & $1.06 \mathrm{E}-01$ & 164 & $5.73 E-02$ & 248 & $9.52 \mathrm{E}-02$ & $<0$ \\
\hline A161.23.10.C.18 & & $3.90 \mathrm{E}-05$ & 61.1 & 0.017 & $6.65 \mathrm{E}-04$ & $\begin{array}{l}{[49.4]} \\
48^{\star}\end{array}$ & $5.46 \mathrm{E}-02$ & $\begin{array}{l}{[75.6]} \\
42^{\star}\end{array}$ & $1.06 \mathrm{E}-01$ & $\begin{array}{l}{[164]} \\
146^{*}\end{array}$ & $5.71 \mathrm{E}-02$ & 228 & $8.73 \mathrm{E}-02$ & $<0$ \\
\hline A161.23.10.C.20 & & 3.93E-05 & 68.2 & 0.017 & $6.57 \mathrm{E}-04$ & 62 & $6.97 E-02$ & $\begin{array}{l}{[75.6]} \\
53^{\star}\end{array}$ & $1.08 \mathrm{E}-01$ & $\begin{array}{l}{[164]} \\
148^{*}\end{array}$ & $5.82 \mathrm{E}-02$ & 262 & $1.02 \mathrm{E}-01$ & $<0$ \\
\hline A161.23.10.C.22 & & 3.96E-05 & 75.2 & 0.016 & $6.50 \mathrm{E}-04$ & 61 & $6.97 \mathrm{E}-02$ & $\begin{array}{l}{[75.6]} \\
48^{\star}\end{array}$ & $1.10 \mathrm{E}-01$ & 175 & $6.32 \mathrm{E}-02$ & 269 & $1.07 E-01$ & $<0$ \\
\hline A161.23.10.C.23 & & 3.95E-05 & 78.2 & 0.016 & $6.47 \mathrm{E}-04$ & 68 & $7.80 \mathrm{E}-02$ & $\begin{array}{l}{[75.6]} \\
63^{\star}\end{array}$ & $1.10 \mathrm{E}-01$ & 518 & $1.88 \mathrm{E}-01$ & 366 & $1.46 \mathrm{E}-01$ & $1.80 \mathrm{E}-08$ \\
\hline A161.23.10.C.24 & & 3.95E-05 & 82.2 & 0.016 & $6.43 \mathrm{E}-04$ & 59 & $6.86 \mathrm{E}-02$ & $\begin{array}{l}{[75.6]} \\
47^{\star}\end{array}$ & $1.11 \mathrm{E}-01$ & $\begin{array}{l}{[164]} \\
162^{*}\end{array}$ & $5.98 \mathrm{E}-02$ & 239 & $9.59 \mathrm{E}-02$ & $<0$ \\
\hline \multicolumn{15}{|c|}{ A161 $23^{\circ} \mathrm{C} \mathrm{pH} 11$ Reactor \#1 } \\
\hline A161.23.11.80A.Blank1 & & 2.00E-05 & --- & --- & --- & {$[49.4]$} & -- & [75.6] & --- & [164] & -- & [134] & --- & --- \\
\hline A161.23.11.80A.Blank2 & & $1.98 \mathrm{E}-05$ & --- & --- & --- & [49.4] & -- & [75.6] & --- & [164] & --- & [134] & -- & --- \\
\hline A161.23.11.80A.Blank3 & & $1.94 \mathrm{E}-05$ & 0.0 & -- & -- & [49.4] & -- & [75.6] & --- & [164] & -- & [134] & -- & --- \\
\hline A161.23.11.80A.2 & & $1.15 \mathrm{E}-05$ & 1.0 & 0.050 & $1.01 \mathrm{E}-03$ & 116 & $2.48 \mathrm{E}-02$ & {$[75.6]$} & 2.05E-02 & 369 & $2.49 \mathrm{E}-02$ & 484 & $3.59 E-02$ & $7.76 \mathrm{E}-09$ \\
\hline A161.23.11.80A.4 & & $2.10 \mathrm{E}-05$ & 1.8 & 0.050 & $1.01 \mathrm{E}-03$ & 249 & $9.73 \mathrm{E}-02$ & [75.6] & $3.74 \mathrm{E}-02$ & 696 & $8.58 \mathrm{E}-02$ & 779 & $1.06 \mathrm{E}-01$ & $3.43 E-08$ \\
\hline A161.23.11.80A.6 & & 2.33E-05 & 8.8 & 0.050 & $1.01 \mathrm{E}-03$ & 229 & $9.98 \mathrm{E}-02$ & {$[75.6]$} & 4.17E-02 & 604 & $8.30 \mathrm{E}-02$ & 648 & $9.79 \mathrm{E}-02$ & $2.83 \mathrm{E}-08$ \\
\hline A161.23.11.80A.8 & & $1.57 \mathrm{E}-05$ & 15.9 & 0.050 & $1.00 \mathrm{E}-03$ & 229 & $6.75 \mathrm{E}-02$ & [75.6] & 2.82E-02 & 632 & 5.87E-02 & 655 & $6.69 \mathrm{E}-02$ & 1.95E-08 \\
\hline A161.23.11.80A.10 & & $1.34 \mathrm{E}-05$ & 22.6 & 0.049 & $1.00 \mathrm{E}-03$ & 258 & 6.47E-02 & [75.6] & 2.40E-02 & 705 & 5.57E-02 & 722 & $6.28 \mathrm{E}-02$ & $1.95 E-08$ \\
\hline A161.23.11.80A.12 & & $9.88 \mathrm{E}-06$ & 29.8 & 0.049 & $9.96 \mathrm{E}-04$ & 249 & $4.65 \mathrm{E}-02$ & 232 & $5.49 \mathrm{E}-02$ & 948 & $5.58 \mathrm{E}-02$ & 1,040 & $6.73 \mathrm{E}-02$ & $6.27 \mathrm{E}-09$ \\
\hline A161.23.11.80A.14 & & $1.26 \mathrm{E}-05$ & 36.8 & 0.048 & $9.90 \mathrm{E}-04$ & 244 & $5.87 \mathrm{E}-02$ & 219 & $6.67 \mathrm{E}-02$ & 912 & $6.91 \mathrm{E}-02$ & 974 & $8.12 \mathrm{E}-02$ & 7.30E-09 \\
\hline A161.23.11.80A.16 & & 1.20E-05 & 43.9 & 0.048 & 9.84E-04 & 239 & $5.50 \mathrm{E}-02$ & 208 & $6.06 \mathrm{E}-02$ & 846 & $6.14 \mathrm{E}-02$ & 954 & 7.61E-02 & 7.80E-09 \\
\hline A161.23.11.80A.18 & & 1.45E-05 & 50.8 & 0.047 & $9.76 \mathrm{E}-04$ & 235 & $6.55 \mathrm{E}-02$ & 223 & 7.87E-02 & 856 & 7.53E-02 & 920 & $8.89 E-02$ & $5.13 E-09$ \\
\hline A161.23.11.80A.20 & & $1.18 \mathrm{E}-05$ & 57.6 & 0.047 & $9.72 \mathrm{E}-04$ & 202 & $4.62 \mathrm{E}-02$ & 186 & $5.39 \mathrm{E}-02$ & 688 & 4.96E-02 & 831 & $6.59 \mathrm{E}-02$ & $6.05 \mathrm{E}-09$ \\
\hline A161.23.11.80A.22 & & 1.16E-05 & 64.9 & 0.047 & 9.67E-04 & 203 & $4.60 \mathrm{E}-02$ & 176 & $5.05 E-02$ & 651 & 4.65E-02 & 831 & $6.53 E-02$ & 7.44E-09 \\
\hline A161.23.11.80A.24 & & $1.28 \mathrm{E}-05$ & 71.9 & 0.046 & 9.62E-04 & 185 & $4.62 \mathrm{E}-02$ & 157 & 4.96E-02 & 483 & 3.80E-02 & 827 & 7.15E-02 & 1.10E-08 \\
\hline A161.23.11.80A.25 & & $1.31 \mathrm{E}-05$ & 75.8 & 0.046 & $9.59 \mathrm{E}-04$ & 204 & $5.25 \mathrm{E}-02$ & 175 & $5.71 E-02$ & 693 & $5.63 \mathrm{E}-02$ & 882 & $7.87 \mathrm{E}-02$ & $1.09 \mathrm{E}-08$ \\
\hline A161.23.11.80A.26 & & $1.29 \mathrm{E}-05$ & 78.9 & 0.046 & 9.57E-04 & 194 & $4.94 \mathrm{E}-02$ & 169 & $5.45 \mathrm{E}-02$ & 569 & 4.56E-02 & 892 & 7.87E-02 & $1.22 \mathrm{E}-08$ \\
\hline \multicolumn{15}{|c|}{ A161 $23^{\circ} \mathrm{C} \mathrm{pH} 11$ Reactor $\# 2$} \\
\hline A161.23.11.C.Blank1 & & $6.86 \mathrm{E}-06$ & --- & --- & --- & {$[49.4]$} & -- & [75.6] & --- & [164] & --- & [134] & --- & --- \\
\hline A161.23.11.C.Blank2 & & $7.08 \mathrm{E}-06$ & -- & -- & -- & {$[49.4]$} & -- & {$[75.6]$} & --- & {$[164]$} & -- & [134] & -- & --- \\
\hline A161.23.11.C.Blank3 & & $7.50 \mathrm{E}-06$ & --- & -- & -- & {$[49.4]$} & -- & [75.6] & --- & [164] & -- & [134] & -- & --- \\
\hline A161.23.11.C.2 & & 4.07E-05 & 5.1 & 0.039 & $1.08 \mathrm{E}-03$ & 193 & $1.37 \mathrm{E}-01$ & {$[75.6]$} & $6.78 \mathrm{E}-02$ & 501 & $1.12 \mathrm{E}-01$ & 602 & $1.48 \mathrm{E}-01$ & $4.03 E-08$ \\
\hline A161.23.11.C.4 & & $4.08 \mathrm{E}-05$ & 12.1 & 0.039 & $1.07 \mathrm{E}-03$ & 170 & $1.21 \mathrm{E}-01$ & [75.6] & $6.83 \mathrm{E}-02$ & 417 & $9.38 \mathrm{E}-02$ & 516 & $1.28 \mathrm{E}-01$ & 2.99E- 08 \\
\hline A161.23.11.C. 6 & & 4.19E-05 & 19.1 & 0.038 & 1.07E-03 & 151 & $1.12 \mathrm{E}-01$ & [75.6] & $7.08 \mathrm{E}-02$ & 367 & 8.55E-02 & 462 & $1.18 \mathrm{E}-01$ & $2.40 \mathrm{E}-08$ \\
\hline A161.23.11.C.8 & & 4.24E- 05 & 26.2 & 0.038 & $1.06 \mathrm{E}-03$ & 153 & $1.15 \mathrm{E}-01$ & [75.6] & $7.21 \mathrm{E}-02$ & 369 & 8.77E-02 & 478 & 1.25E-01 & 2.65E-08 \\
\hline A161.23.11.C.10 & & $4.30 \mathrm{E}-05$ & 33.1 & 0.037 & $1.05 \mathrm{E}-03$ & 149 & $1.15 \mathrm{E}-01$ & {$[75.6]$} & 7.37E-02 & 368 & 8.93E-02 & 483 & $1.29 \mathrm{E}-01$ & $2.78 \mathrm{E}-08$ \\
\hline A161.23.11.C.12 & & 4.24E-05 & 40.2 & 0.036 & $1.04 \mathrm{E}-03$ & 134 & 1.03E-01 & 113 & 1.10E-01 & 385 & $9.30 \mathrm{E}-02$ & 667 & $1.77 \mathrm{E}-01$ & 3.40E-08 \\
\hline A161.23.11.C.14 & & 4.30E-05 & 47.1 & 0.036 & $1.03 \mathrm{E}-03$ & 117 & $9.19 \mathrm{E}-02$ & 107 & $1.06 \mathrm{E}-01$ & 323 & $8.00 \mathrm{E}-02$ & 608 & $1.66 \mathrm{E}-01$ & $2.98 \mathrm{E}-08$ \\
\hline A161.23.11.C.16 & & $4.08 \mathrm{E}-05$ & 54.1 & 0.035 & $1.02 \mathrm{E}-03$ & 117 & $8.80 \mathrm{E}-02$ & 102 & $9.71 \mathrm{E}-02$ & 319 & 7.56E-02 & 581 & $1.51 \mathrm{E}-01$ & $2.73 \mathrm{E}-08$ \\
\hline
\end{tabular}




\begin{tabular}{|c|c|c|c|c|c|c|c|c|c|c|c|c|c|c|}
\hline Sample ID & $\begin{array}{l}\text { Influent } \\
\text { [Si] } \\
\text { (ppm) }\end{array}$ & $\begin{array}{l}\text { Flow } \\
\text { Rate, } \mathrm{q} \\
\left(\mathrm{m}^{3} \mathrm{~d}^{-1}\right)\end{array}$ & $\begin{array}{l}\text { Time } \\
\text { (d) }\end{array}$ & $\begin{array}{l}\text { Glass } \\
\text { Mass } \\
\text { (g) }\end{array}$ & $\begin{array}{l}\text { Surface } \\
\text { Area, S } \\
\left(\mathrm{m}^{2}\right)\end{array}$ & $\begin{array}{l}{[\mathrm{Al}]} \\
\left(\mu \mathrm{L} \mathrm{L}^{-1}\right)\end{array}$ & $\begin{array}{l}\text { Al Norm. } \\
\text { Diss. Rate } \\
\left(\mathrm{g} \mathrm{m}^{-2} \mathrm{~d}^{-1}\right)\end{array}$ & $\begin{array}{l}{[\mathrm{B}]} \\
\left(\mu \mathrm{g} \mathrm{L}^{-1}\right)\end{array}$ & $\begin{array}{l}\text { B Norm. } \\
\text { Diss. Rate } \\
\left(\mathrm{g} \mathrm{m}^{-2} \mathrm{~d}^{-1}\right)\end{array}$ & $\begin{array}{l}{[\mathrm{Si}]} \\
\left(\mu \mathrm{g} \mathrm{L}^{-1}\right)\end{array}$ & $\begin{array}{l}\text { Si Norm. } \\
\text { Diss. Rate } \\
\left(\mathrm{g} \mathrm{m}^{-2} \mathrm{~d}^{-1}\right)\end{array}$ & $\begin{array}{l}{[\mathrm{Na}]} \\
(\mu \mathrm{g} \mathrm{L}- \\
\left.{ }^{1}\right)\end{array}$ & $\begin{array}{l}\text { Na Norm. } \\
\text { Diss. Rate, } \\
\mathrm{r} \\
\left(\mathrm{g} \mathrm{m}^{-2} \mathrm{~d}^{-1}\right)\end{array}$ & $\begin{array}{l}\mathrm{r}_{\mathrm{IEX}} \\
\left(\mathrm{mol} \mathrm{Na} \mathrm{s}^{-2} \mathrm{~s}^{-1}\right)\end{array}$ \\
\hline A161.23.11.C.18 & & $4.29 \mathrm{E}-05$ & 61.0 & 0.034 & $1.01 \mathrm{E}-03$ & 94 & $7.53 \mathrm{E}-02$ & 93 & $9.41 \mathrm{E}-02$ & 195 & $4.91 \mathrm{E}-02$ & 570 & $1.58 \mathrm{E}-01$ & $3.20 \mathrm{E}-08$ \\
\hline A161.23.11.C.20 & & $4.01 \mathrm{E}-05$ & 68.1 & 0.034 & $1.00 \mathrm{E}-03$ & 106 & $8.00 \mathrm{E}-02$ & 107 & $1.02 \mathrm{E}-01$ & 289 & $6.88 \mathrm{E}-02$ & 556 & $1.45 \mathrm{E}-01$ & $2.18 \mathrm{E}-08$ \\
\hline A161.23.11.C.22 & & 4.11E-05 & 75.1 & 0.033 & $9.91 \mathrm{E}-04$ & 115 & $8.97 \mathrm{E}-02$ & 106 & $1.05 \mathrm{E}-01$ & 338 & $8.31 \mathrm{E}-02$ & 576 & $1.56 \mathrm{E}-01$ & $2.57 \mathrm{E}-08$ \\
\hline A161.23.11.C.23 & & 3.97E-05 & 78.2 & 0.033 & $9.86 \mathrm{E}-04$ & 120 & $9.09 \mathrm{E}-02$ & 119 & $1.14 \mathrm{E}-01$ & 413 & $9.87 \mathrm{E}-02$ & 615 & $1.62 \mathrm{E}-01$ & $2.39 \mathrm{E}-08$ \\
\hline A161.23.11.C.24 & & $3.88 \mathrm{E}-05$ & 82.1 & 0.032 & $9.79 \mathrm{E}-04$ & 119 & $8.88 \mathrm{E}-02$ & 117 & $1.11 \mathrm{E}-01$ & 354 & 8.33E-02 & 624 & $1.61 \mathrm{E}-01$ & $2.56 \mathrm{E}-08$ \\
\hline \multicolumn{15}{|c|}{ A161 $23^{\circ} \mathrm{C}$ pH 12 Reactor \#1 } \\
\hline A161.23.12.80A.Blank1 & & $1.12 \mathrm{E}-05$ & --- & -.- & -.- & [49.4] & --- & {$[75.6]$} & --- & [164] & --- & 436 & --- & -.- \\
\hline A161.23.12.80A.Blank2 & & $1.30 \mathrm{E}-05$ & --- & --- & --- & {$[49.4]$} & --- & {$[75.6]$} & --- & [164] & --- & 460 & --- & --- \\
\hline A161.23.12.80A.Blank3 & & $1.15 \mathrm{E}-05$ & 0.0 & --- & -.- & [49.4] & --- & {$[75.6]$} & --- & [164] & -.- & 445 & --- & -.- \\
\hline A161.23.12.80A.2 & & $9.45 \mathrm{E}-06$ & 1.0 & 0.050 & $1.01 \mathrm{E}-03$ & 203 & $3.57 \mathrm{E}-02$ & 109 & $2.43 \mathrm{E}-02$ & 594 & $3.29 \mathrm{E}-02$ & 883 & $5.38 \mathrm{E}-02$ & $1.49 \mathrm{E}-08$ \\
\hline A161.23.12.80A.4 & & $3.15 \mathrm{E}-05$ & 1.8 & 0.050 & $1.01 \mathrm{E}-03$ & 379 & $2.23 \mathrm{E}-01$ & 227 & 1.69E-01 & 1,230 & $2.28 \mathrm{E}-01$ & 1,390 & $2.83 \mathrm{E}-01$ & $5.75 \mathrm{E}-08$ \\
\hline A161.23.12.80A.6 & & $3.68 \mathrm{E}-05$ & 8.8 & 0.047 & $9.77 \mathrm{E}-04$ & 488 & $3.46 \mathrm{E}-01$ & 328 & 2.95E-01 & 1,570 & $3.51 \mathrm{E}-01$ & 1,850 & $4.55 \mathrm{E}-01$ & $8.07 E-08$ \\
\hline A161.23.12.80A.8 & & $3.65 \mathrm{E}-05$ & 15.9 & 0.047 & $9.69 \mathrm{E}-04$ & 315 & $2.24 \mathrm{E}-01$ & 135 & $1.21 \mathrm{E}-01$ & 910 & $2.04 \mathrm{E}-01$ & 1,400 & $3.44 \mathrm{E}-01$ & $1.12 \mathrm{E}-07$ \\
\hline A161.23.12.80A.10 & & 3.69E-05 & 22.6 & 0.045 & $9.44 \mathrm{E}-04$ & 417 & $3.07 \mathrm{E}-01$ & 271 & 2.53E-01 & 1,420 & $3.30 \mathrm{E}-01$ & 1,050 & $2.68 \mathrm{E}-01$ & $7.72 \mathrm{E}-09$ \\
\hline A161.23.12.80A.14 & & 3.58E-05 & 36.8 & 0.040 & $8.75 \mathrm{E}-04$ & 496 & $3.82 \mathrm{E}-01$ & 388 & $3.79 \mathrm{E}-01$ & 2,200 & $5.35 \mathrm{E}-01$ & 1,940 & $5.19 \mathrm{E}-01$ & 7.03E-08 \\
\hline A161.23.12.80A.16 & & $3.58 \mathrm{E}-05$ & 43.9 & 0.038 & 8.46E-04 & 451 & 3.59E-01 & 337 & 3.40E-01 & 2,040 & $5.13 \mathrm{E}-01$ & 1,640 & 4.53E-01 & $5.69 \mathrm{E}-08$ \\
\hline A161.23.12.80A.18 & & $3.61 \mathrm{E}-05$ & 50.8 & 0.037 & 8.19E-04 & 410 & 3.40E-01 & 309 & $3.25 \mathrm{E}-01$ & 1,880 & 4.92E-01 & 1,560 & 4.49E-01 & $6.24 \mathrm{E}-08$ \\
\hline A161.23.12.80A.20 & & 3.83E-05 & 57.6 & 0.035 & 7.95E-04 & 357 & $3.24 \mathrm{E}-01$ & 270 & 3.10E-01 & 1,700 & 4.86E-01 & 1,390 & 4.37E-01 & $6.39 \mathrm{E}-08$ \\
\hline A161.23.12.80A.22 & & 3.83E-05 & 64.9 & 0.033 & 7.66E-04 & 365 & $3.43 \mathrm{E}-01$ & 274 & 3.26E-01 & 1,530 & $4.54 \mathrm{E}-01$ & 1,290 & $4.21 \mathrm{E}-01$ & $4.75 \mathrm{E}-08$ \\
\hline A161.23.12.80A.24 & & $4.21 \mathrm{E}-05$ & 71.9 & 0.031 & 7.42E-04 & 322 & 3.44E-01 & 232 & 3.14E-01 & 1,260 & 4.25E-01 & 1,200 & 4.45E-01 & $6.58 \mathrm{E}-08$ \\
\hline A161.23.12.80A.25 & & $4.18 \mathrm{E}-05$ & 75.8 & 0.030 & $7.25 \mathrm{E}-04$ & 331 & $3.59 \mathrm{E}-01$ & 236 & $3.24 \mathrm{E}-01$ & 1,280 & $4.38 \mathrm{E}-01$ & 1,220 & 4.59E-01 & $6.78 \mathrm{E}-08$ \\
\hline A161.23.12.80A.26 & & 4.14E-05 & 78.9 & 0.030 & 7.14E-04 & 346 & $3.77 \mathrm{E}-01$ & 254 & 3.50E-01 & 1,370 & 4.71E-01 & 1,210 & 4.57E-01 & $5.37 \mathrm{E}-08$ \\
\hline \multicolumn{15}{|c|}{ A161 $23{ }^{\circ} \mathrm{C}$ pH 12 Reactor \#2 } \\
\hline A161.23.12.C.Blank1 & & $1.08 \mathrm{E}-05$ & -.- & -.- & -.- & [49.4] & -.- & {$[75.6]$} & --- & [164] & --. & 532 & --. & -.- \\
\hline A161.23.12.C.Blank2 & & $1.01 \mathrm{E}-05$ & --- & --- & --- & {$[49.4]$} & --- & {$[75.6]$} & --- & [164] & -- & 478 & --- & --- \\
\hline A161.23.12.C.Blank3 & & $9.62 \mathrm{E}-06$ & -.- & -.- & --- & [49.4] & --. & {$[75.6]$} & --- & [164] & --- & 477 & --. & -.- \\
\hline A161.23.12.C.2 & & $4.27 \mathrm{E}-05$ & 5.2 & 0.039 & $1.08 \mathrm{E}-03$ & 410 & $3.05 \mathrm{E}-01$ & 254 & $2.39 \mathrm{E}-01$ & 1,190 & $2.79 \mathrm{E}-01$ & 1,540 & $3.97 \mathrm{E}-01$ & $7.95 \mathrm{E}-08$ \\
\hline A161.23.12.C.4 & & $4.27 \mathrm{E}-05$ & 12.1 & 0.037 & $1.05 \mathrm{E}-03$ & 462 & $3.54 \mathrm{E}-01$ & 300 & $2.91 \mathrm{E}-01$ & 1,410 & $3.41 \mathrm{E}-01$ & 1,800 & $4.78 \mathrm{E}-01$ & $9.42 \mathrm{E}-08$ \\
\hline A161.23.12.C.6 & & $4.29 \mathrm{E}-05$ & 19.1 & 0.035 & $1.02 \mathrm{E}-03$ & 454 & $3.60 \mathrm{E}-01$ & 302 & 3.03E-01 & 1,350 & $3.37 E-01$ & 1,750 & $4.80 \mathrm{E}-01$ & $8.94 \mathrm{E}-08$ \\
\hline A161.23.12.C.8 & & $4.68 \mathrm{E}-07$ & 26.1 & 0.035 & $1.02 \mathrm{E}-03$ & 463 & $3.98 \mathrm{E}-03$ & 317 & 3.45E-03 & 1,410 & $3.82 \mathrm{E}-03$ & 1,890 & $5.63 \mathrm{E}-03$ & $1.10 \mathrm{E}-09$ \\
\hline A161.23.12.C.10 & & $2.78 \mathrm{E}-05$ & 33.1 & 0.031 & $9.62 \mathrm{E}-04$ & 1,060 & $5.76 \mathrm{E}-01$ & 745 & $5.12 \mathrm{E}-01$ & 3,400 & $5.82 E-01$ & 3,530 & $6.65 \mathrm{E}-01$ & $7.66 \mathrm{E}-08$ \\
\hline A161.23.12.C.12 & & $4.48 \mathrm{E}-05$ & 40.2 & 0.029 & $9.34 \mathrm{E}-04$ & 398 & $3.59 \mathrm{E}-01$ & 300 & 3.43E-01 & 1,490 & 4.24E-01 & 1,570 & $4.91 \mathrm{E}-01$ & $7.46 \mathrm{E}-08$ \\
\hline A161.23.12.C.14 & & $4.35 \mathrm{E}-05$ & 47.1 & 0.027 & 9.03E-04 & 399 & $3.61 \mathrm{E}-01$ & 292 & 3.35E-01 & 1,410 & 4.03E-01 & 1,590 & $4.99 \mathrm{E}-01$ & $8.28 \mathrm{E}-08$ \\
\hline A161.23.12.C.16 & & $4.20 \mathrm{E}-05$ & 54.1 & 0.025 & $8.71 \mathrm{E}-04$ & 385 & $3.49 \mathrm{E}-01$ & 290 & 3.33E-01 & 1,430 & 4.09E-01 & 1,390 & 4.37E-01 & $5.23 \mathrm{E}-08$ \\
\hline A161.23.12.C.18 & & 4.24E-05 & 61.0 & 0.023 & 8.40E-04 & 386 & $3.67 \mathrm{E}-01$ & 279 & 3.36E-01 & 1,420 & 4.25E-01 & 1,290 & $4.25 \mathrm{E}-01$ & 4.49E-08 \\
\hline A161.23.12.C.20 & & $4.13 \mathrm{E}-05$ & 68.1 & 0.021 & $8.06 \mathrm{E}-04$ & 379 & $3.65 \mathrm{E}-01$ & 290 & 3.54E-01 & 1,370 & 4.16E-01 & 1,430 & $4.77 \mathrm{E}-01$ & $6.23 \mathrm{E}-08$ \\
\hline A161.23.12.C.22 & & 4.02E-05 & 75.1 & 0.019 & 7.75E-04 & 383 & $3.74 \mathrm{E}-01$ & 277 & $3.42 \mathrm{E}-01$ & 1,360 & 4.19E-01 & 1,440 & 4.87E-01 & 7.29E-08 \\
\hline A161.23.12.C. 23 & & $4.08 \mathrm{E}-05$ & 78.1 & 0.018 & 7.62E-04 & 385 & $3.88 \mathrm{E}-01$ & 283 & $3.61 \mathrm{E}-01$ & 1,300 & 4.13E-01 & 1,380 & $4.82 E-01$ & $6.09 \mathrm{E}-08$ \\
\hline A161.23.12.C.24 & & $4.04 \mathrm{E}-05$ & 82.1 & 0.017 & 7.43E-04 & 348 & $3.57 \mathrm{E}-01$ & 269 & $3.49 \mathrm{E}-01$ & 1,240 & 4.01E-01 & 1,180 & 4.19E-01 & $3.53 E-08$ \\
\hline \multicolumn{15}{|c|}{ A161 $40^{\circ} \mathrm{C} \mathrm{pH} 9$ Reactor \#1 } \\
\hline A161.40.9.10.Blank1 & & $1.88 \mathrm{E}-05$ & -.- & -.- & --- & [49.4] & --- & {$[75.6]$} & --- & [164] & --- & [134] & --- & -.- \\
\hline A161.40.9.10.Blank2 & & $4.15 \mathrm{E}-05$ & --- & --- & --- & [49.4] & --- & {$[75.6]$} & --- & [164] & -- & [134] & --- & --- \\
\hline A161.40.9.10.Blank3 & & $1.82 \mathrm{E}-05$ & --- & -.- & --- & [49.4] & -.- & {$[75.6]$} & --- & [164] & --- & [134] & --- & -.- \\
\hline
\end{tabular}




\begin{tabular}{|c|c|c|c|c|c|c|c|c|c|c|c|c|c|c|}
\hline Sample ID & $\begin{array}{l}\text { Influent } \\
{[\mathrm{Si}]} \\
(\mathrm{ppm})\end{array}$ & $\begin{array}{l}\text { Flow } \\
\text { Rate, q } \\
\left(\mathrm{m}^{3} \mathrm{~d}^{-1}\right)\end{array}$ & $\begin{array}{l}\text { Time } \\
\text { (d) }\end{array}$ & $\begin{array}{l}\text { Glass } \\
\text { Mass } \\
\text { (g) }\end{array}$ & $\begin{array}{l}\text { Surface } \\
\text { Area, S } \\
\left(\mathrm{m}^{2}\right)\end{array}$ & $\begin{array}{l}{[\mathrm{Al}]} \\
\left(\mu \mathrm{L} \mathrm{L}^{-1}\right)\end{array}$ & $\begin{array}{l}\text { Al Norm. } \\
\text { Diss. Rate } \\
\left(\mathrm{g} \mathrm{m}^{-2} \mathrm{~d}^{-1}\right)\end{array}$ & $\begin{array}{l}{[B]} \\
\left(\mu g L^{-1}\right)\end{array}$ & $\begin{array}{l}\text { B Norm. } \\
\text { Diss. Rate } \\
\left(\mathrm{g} \mathrm{m}^{-2} \mathrm{~d}^{-1}\right)\end{array}$ & $\begin{array}{l}{[\mathrm{Si}]} \\
\left(\mu \mathrm{g} \mathrm{L}^{-1}\right)\end{array}$ & $\begin{array}{l}\text { Si Norm. } \\
\text { Diss. Rate } \\
\left(\mathrm{g} \mathrm{m}^{-2} \mathrm{~d}^{-1}\right)\end{array}$ & $\begin{array}{l}{[\mathrm{Na}]} \\
(\mu \mathrm{g} \mathrm{L} \\
\left.{ }^{1}\right)\end{array}$ & $\begin{array}{l}\text { Na Norm. } \\
\text { Diss. Rate, } \\
\mathrm{r} \\
\left(\mathrm{g} \mathrm{m}^{-2} \mathrm{~d}^{-1}\right)\end{array}$ & $\begin{array}{l}\mathrm{r}_{\mathrm{IEX}} \\
\left(\mathrm{mol} \mathrm{Na} \mathrm{No}^{-1}\right)\end{array}$ \\
\hline A161.40.9.10.2 & & $2.86 \mathrm{E}-06$ & 4.0 & 3.665 & $7.40 \mathrm{E}-02$ & 578 & $4.20 \mathrm{E}-04$ & 6,470 & $5.95 \mathrm{E}-03$ & 4,990 & $1.14 \mathrm{E}-03$ & 26,500 & $6.67 \mathrm{E}-03$ & $3.63 \mathrm{E}-10$ \\
\hline A161.40.9.10.4 & & 7.47E-06 & 8.8 & 3.658 & $7.39 \mathrm{E}-02$ & 565 & $1.07 \mathrm{E}-03$ & 7,490 & $1.80 \mathrm{E}-02$ & 5,180 & $3.11 \mathrm{E}-03$ & 29,500 & $1.94 \mathrm{E}-02$ & $7.10 \mathrm{E}-10$ \\
\hline A161.40.9.10.6 & & $7.49 \mathrm{E}-06$ & 13.9 & 3.651 & 7.39E-02 & 600 & $1.15 \mathrm{E}-03$ & 7,190 & $1.74 \mathrm{E}-02$ & 5,040 & $3.03 \mathrm{E}-03$ & 27,500 & $1.82 \mathrm{E}-02$ & $4.12 \mathrm{E}-10$ \\
\hline A161.40.9.10.8 & & $7.48 \mathrm{E}-06$ & 18.1 & 3.646 & $7.38 \mathrm{E}-02$ & 643 & $1.23 \mathrm{E}-03$ & 6,700 & $1.62 \mathrm{E}-02$ & 4,800 & $2.89 \mathrm{E}-03$ & 25,400 & $1.68 \mathrm{E}-02$ & $3.08 \mathrm{E}-10$ \\
\hline A161.40.9.10.10 & & $6.76 \mathrm{E}-06$ & 23.8 & 3.640 & 7.37E-02 & 703 & $1.21 \mathrm{E}-03$ & 6,420 & 1.40E-02 & 4,670 & $2.54 \mathrm{E}-03$ & 23,800 & 1.42E-02 & 1.05E-10 \\
\hline A161.40.9.10.12 & & $7.80 \mathrm{E}-06$ & 28.0 & 3.635 & 7.36E-02 & 745 & $1.48 \mathrm{E}-03$ & 6,250 & $1.58 \mathrm{E}-02$ & 4,560 & $2.87 \mathrm{E}-03$ & 22,000 & $1.52 \mathrm{E}-02$ & $<0$ \\
\hline A161.40.9.10.14 & & $7.46 \mathrm{E}-06$ & 32.0 & 3.631 & 7.36E-02 & 775 & $1.48 \mathrm{E}-03$ & 5,940 & 1.43E-02 & 4,430 & $2.66 \mathrm{E}-03$ & 21,900 & $1.45 \mathrm{E}-02$ & $6.72 \mathrm{E}-11$ \\
\hline A161.40.9.10.15 & & $7.95 \mathrm{E}-06$ & 34.8 & 3.628 & $7.35 \mathrm{E}-02$ & 800 & 1.63E-03 & 5,920 & $1.52 \mathrm{E}-02$ & 4,410 & 2.83E-03 & 21,500 & $1.52 \mathrm{E}-02$ & $<0$ \\
\hline A161.40.9.10.16 & & 7.65E-06 & 36.7 & 3.626 & 7.35E-02 & 809 & $1.58 \mathrm{E}-03$ & 5,560 & $1.38 \mathrm{E}-02$ & 4,230 & $2.61 \mathrm{E}-03$ & 20,900 & 1.42E- 02 & 2.02E-10 \\
\hline \multicolumn{15}{|c|}{ A161 $40^{\circ} \mathrm{C}$ pH 9 Reactor $\# 2$} \\
\hline A161.40.9.20.Blank1 & & $1.90 \mathrm{E}-05$ & --- & --- & --- & [49.4] & --- & [75.6] & --- & [164] & --- & [134] & --- & --- \\
\hline A161.40.9.20.Blank2 & & $1.93 \mathrm{E}-05$ & --- & -.- & --- & {$[49.4]$} & --- & [75.6] & --- & [164] & --- & [134] & --- & --. \\
\hline A161.40.9.20.Blank3 & & 4.02E-05 & --- & --- & --- & [49.4] & --- & [75.6] & --- & [164] & --- & [134] & --- & --- \\
\hline A161.40.9.20.2 & & $1.99 \mathrm{E}-05$ & 4.0 & 1.487 & $3.01 \mathrm{E}-02$ & 681 & $8.50 \mathrm{E}-03$ & 2,600 & $4.11 \mathrm{E}-02$ & 3,390 & $1.33 \mathrm{E}-02$ & 9,800 & $4.24 \mathrm{E}-02$ & $6.59 \mathrm{E}-10$ \\
\hline A161.40.9.20.4 & & $1.91 \mathrm{E}-05$ & 8.8 & 1.482 & 3.00E-02 & 792 & $9.50 \mathrm{E}-03$ & 2,130 & $3.24 \mathrm{E}-02$ & 3,290 & $1.24 \mathrm{E}-02$ & 7,620 & 3.17E-02 & $<0$ \\
\hline A161.40.9.20.6 & & $1.90 \mathrm{E}-05$ & 13.9 & 1.478 & $3.00 \mathrm{E}-02$ & 831 & $9.91 \mathrm{E}-03$ & 1,830 & $2.76 \mathrm{E}-02$ & 3,120 & 1.17E-02 & 6,920 & $2.86 \mathrm{E}-02$ & $4.90 \mathrm{E}-10$ \\
\hline A161.40.9.20.8 & & $1.94 \mathrm{E}-05$ & 18.1 & 1.475 & $2.99 \mathrm{E}-02$ & 824 & $1.01 \mathrm{E}-02$ & 1,670 & $2.58 \mathrm{E}-02$ & 2,820 & $1.08 \mathrm{E}-02$ & 6,110 & $2.58 \mathrm{E}-02$ & 2.06E-11 \\
\hline A161.40.9.20.10 & & $1.93 \mathrm{E}-05$ & 23.8 & 1.471 & $2.99 \mathrm{E}-02$ & 831 & $1.01 \mathrm{E}-02$ & 1,550 & $2.38 \mathrm{E}-02$ & 2,720 & $1.04 \mathrm{E}-02$ & 5,680 & $2.39 \mathrm{E}-02$ & $3.82 \mathrm{E}-11$ \\
\hline A161.40.9.20.12 & & $1.88 \mathrm{E}-05$ & 28.0 & 1.468 & $2.98 \mathrm{E}-02$ & 809 & $9.59 \mathrm{E}-03$ & 1,490 & 2.24E-02 & 2,650 & $9.91 \mathrm{E}-03$ & 5,210 & $2.14 \mathrm{E}-02$ & $<0$ \\
\hline A161.40.9.20.14 & & $1.93 \mathrm{E}-05$ & 32.0 & 1.466 & $2.98 \mathrm{E}-02$ & 787 & $9.60 \mathrm{E}-03$ & 1,410 & $2.18 \mathrm{E}-02$ & 2,560 & $9.85 \mathrm{E}-03$ & 5,190 & $2.19 E-02$ & $8.40 \mathrm{E}-11$ \\
\hline A161.40.9.20.15 & & $1.92 \mathrm{E}-05$ & 34.8 & 1.464 & $2.98 \mathrm{E}-02$ & 786 & 9.55E-03 & 1,420 & $2.19 \mathrm{E}-02$ & 2,530 & $9.70 \mathrm{E}-03$ & 5,020 & $2.12 E-02$ & $<0$ \\
\hline A161.40.9.20.16 & & $1.88 \mathrm{E}-05$ & 36.7 & 1.463 & 2.97E-02 & 783 & 9.33E-03 & 1,410 & $2.13 E-02$ & 2,510 & $9.43 \mathrm{E}-03$ & 5,070 & 2.09E-02 & $<0$ \\
\hline \multicolumn{15}{|c|}{ A161 $40^{\circ} \mathrm{C}$ pH 9 Reactor $\# 3$} \\
\hline A161.40.9.40.Blank1 & & $3.82 \mathrm{E}-05$ & --- & -- & --- & {$[49.4]$} & -- & [75.6] & --- & [164] & -- & [134] & --- & --- \\
\hline A161.40.9.40.Blank2 & & 3.94E-05 & --- & -- & --- & {$[49.4]$} & -- & [75.6] & --- & {$[164]$} & -- & [134] & --- & --- \\
\hline A161.40.9.40.Blank3 & & 3.81E-05 & --- & -- & --- & {$[49.4]$} & -- & {$[75.6]$} & --- & {$[164]$} & -- & [134] & -- & --- \\
\hline A161.40.9.40.2 & & $3.85 \mathrm{E}-05$ & 4.0 & 0.754 & 1.53E-02 & 351 & 1.67E-02 & 833 & $5.01 E-02$ & 1,420 & $2.13 \mathrm{E}-02$ & 3,210 & $5.29 \mathrm{E}-02$ & $1.39 \mathrm{E}-09$ \\
\hline A161.40.9.40.4 & & 3.87E-05 & 8.8 & 0.751 & $1.52 \mathrm{E}-02$ & 375 & $1.80 \mathrm{E}-02$ & 584 & $3.54 \mathrm{E}-02$ & 1,270 & 1.92E-02 & 2,170 & $3.60 \mathrm{E}-02$ & $3.07 E-10$ \\
\hline A161.40.9.40.6 & & 3.89E-05 & 13.9 & 0.749 & $1.52 \mathrm{E}-02$ & 340 & $1.64 \mathrm{E}-02$ & 500 & $3.05 \mathrm{E}-02$ & 1,110 & $1.69 \mathrm{E}-02$ & 1,870 & $3.13 E-02$ & 3.67E-10 \\
\hline A161.40.9.40.8 & & 3.87E-05 & 18.1 & 0.747 & $1.52 \mathrm{E}-02$ & 321 & $1.54 \mathrm{E}-02$ & 495 & $3.01 \mathrm{E}-02$ & 943 & $1.43 E-02$ & 1,640 & $2.73 \mathrm{E}-02$ & $<0$ \\
\hline A161.40.9.40.10 & & 3.86E-05 & 23.8 & 0.745 & $1.51 \mathrm{E}-02$ & 312 & 1.50E-02 & 459 & $2.79 \mathrm{E}-02$ & 885 & $1.34 \mathrm{E}-02$ & 1,520 & $2.53 \mathrm{E}-02$ & $<0$ \\
\hline A161.40.9.40.12 & & 3.83E-05 & 28.0 & 0.743 & $1.51 \mathrm{E}-02$ & 307 & 1.47E-02 & 439 & $2.65 \mathrm{E}-02$ & 869 & 1.31E-02 & 1,540 & $2.55 \mathrm{E}-02$ & $<0$ \\
\hline A161.40.9.40.14 & & 3.85E-05 & 32.0 & 0.742 & $1.51 \mathrm{E}-02$ & 302 & $1.45 \mathrm{E}-02$ & 417 & $2.54 \mathrm{E}-02$ & 845 & $1.28 \mathrm{E}-02$ & 1,410 & $2.35 \mathrm{E}-02$ & $<0$ \\
\hline A161.40.9.40.15 & & 3.86E-05 & 34.8 & 0.741 & $1.51 \mathrm{E}-02$ & 299 & $1.44 \mathrm{E}-02$ & 413 & $2.52 \mathrm{E}-02$ & 808 & $1.23 \mathrm{E}-02$ & 1,500 & $2.50 \mathrm{E}-02$ & $<0$ \\
\hline A161.40.9.40.16 & & 3.63E-05 & 36.7 & 0.740 & $1.51 \mathrm{E}-02$ & 289 & $1.31 \mathrm{E}-02$ & 417 & $2.39 \mathrm{E}-02$ & 814 & $1.16 \mathrm{E}-02$ & 1,400 & $2.20 \mathrm{E}-02$ & $<0$ \\
\hline \multicolumn{15}{|c|}{ A161 $40{ }^{\circ} \mathrm{C}$ pH 9 Reactor $\# 4$} \\
\hline A161.40.9.80A.Blank1 & & 7.47E-05 & --- & -- & -- & {$[49.4]$} & -- & {$[75.6]$} & --- & [164] & --- & [134] & --- & -- \\
\hline A161.40.9.80A.Blank2 & & 7.86E-05 & --- & --- & -- & {$[49.4]$} & -- & {$[75.6]$} & --- & {$[164]$} & -- & [134] & -- & --- \\
\hline A161.40.9.80A.Blank3 & & $7.62 \mathrm{E}-05$ & --- & --- & --- & {$[49.4]$} & -- & [75.6] & --- & [164] & --- & [134] & --- & --- \\
\hline A161.40.9.80A.2 & & 7.67E-05 & 4.0 & 0.310 & $6.27 \mathrm{E}-03$ & 281 & $6.47 \mathrm{E}-02$ & 278 & $8.10 \mathrm{E}-02$ & 862 & $6.25 \mathrm{E}-02$ & 1,000 & $7.98 \mathrm{E}-02$ & $<0$ \\
\hline A161.40.9.80A.4 & & $7.65 \mathrm{E}-05$ & 8.8 & 0.308 & $6.25 \mathrm{E}-03$ & 222 & $5.12 \mathrm{E}-02$ & 211 & $6.16 \mathrm{E}-02$ & 635 & $4.61 \mathrm{E}-02$ & 777 & $6.21 \mathrm{E}-02$ & $2.51 \mathrm{E}-10$ \\
\hline A161.40.9.80A.6 & & 7.63E-05 & 13.9 & 0.306 & $6.22 \mathrm{E}-03$ & 214 & 4.94E-02 & 218 & $6.38 \mathrm{E}-02$ & 642 & $4.68 \mathrm{E}-02$ & 756 & $6.06 \mathrm{E}-02$ & $<0$ \\
\hline A161.40.9.80A.8 & & $7.66 \mathrm{E}-05$ & 18.1 & 0.304 & $6.20 \mathrm{E}-03$ & 212 & 4.93E-02 & 222 & $6.54 \mathrm{E}-02$ & 535 & $3.92 \mathrm{E}-02$ & 684 & $5.51 \mathrm{E}-02$ & $<0$ \\
\hline
\end{tabular}

B.36 


\begin{tabular}{|c|c|c|c|c|c|c|c|c|c|c|c|c|c|c|}
\hline Sample ID & $\begin{array}{l}\text { Influent } \\
{[\mathrm{Si}]} \\
(\mathrm{ppm})\end{array}$ & $\begin{array}{l}\text { Flow } \\
\text { Rate, } q \\
\left(\mathrm{~m}^{3} \mathrm{~d}^{-1}\right)\end{array}$ & $\begin{array}{l}\text { Time } \\
\text { (d) }\end{array}$ & $\begin{array}{l}\text { Glass } \\
\text { Mass } \\
\text { (g) }\end{array}$ & $\begin{array}{l}\text { Surface } \\
\text { Area, S } \\
\left(\mathrm{m}^{2}\right)\end{array}$ & $\begin{array}{l}{[\mathrm{Al}]} \\
\left(\mu \mathrm{L} \mathrm{L}^{-1}\right)\end{array}$ & $\begin{array}{l}\text { Al Norm. } \\
\text { Diss. Rate } \\
\left(\mathrm{g} \mathrm{m}^{-2} \mathrm{~d}^{-1}\right)\end{array}$ & $\begin{array}{l}{[\mathrm{B}]} \\
\left(\mu \mathrm{L} \mathrm{L}^{-1}\right)\end{array}$ & $\begin{array}{l}\text { B Norm. } \\
\text { Diss. Rate } \\
\left(\mathrm{g} \mathrm{m}^{-2} \mathrm{~d}^{-1}\right)\end{array}$ & $\begin{array}{l}{[\mathrm{Si}]} \\
\left(\mu \mathrm{L} \mathrm{L}^{-1}\right)\end{array}$ & $\begin{array}{l}\text { Si Norm. } \\
\text { Diss. Rate } \\
\left(\mathrm{g} \mathrm{m}^{-2} \mathrm{~d}^{-1}\right)\end{array}$ & $\begin{array}{l}{[\mathrm{Na}]} \\
(\mu \mathrm{g} \mathrm{L} \\
\left.{ }^{1}\right)\end{array}$ & $\begin{array}{l}\text { Na Norm. } \\
\text { Diss. Rate, } \\
\mathrm{r} \\
\left(\mathrm{g} \mathrm{m}^{-2} \mathrm{~d}^{-1}\right)\end{array}$ & $\begin{array}{l}\mathrm{r}_{\mathrm{IEX}} \\
\left(\mathrm{mol} \mathrm{Na} \mathrm{s}^{-2}\right)\end{array}$ \\
\hline A161.40.9.80A.10 & & $7.65 \mathrm{E}-05$ & 23.8 & 0.302 & $6.16 \mathrm{E}-03$ & 215 & $5.02 \mathrm{E}-02$ & 227 & $6.71 \mathrm{E}-02$ & 562 & $4.14 \mathrm{E}-02$ & 691 & $5.59 \mathrm{E}-02$ & $<0$ \\
\hline A161.40.9.80A.12 & & 7.66E-05 & 28.0 & 0.300 & $6.14 \mathrm{E}-03$ & 204 & $4.79 E-02$ & 205 & $6.10 \mathrm{E}-02$ & 493 & 3.65E-02 & 669 & $5.45 \mathrm{E}-02$ & $<0$ \\
\hline A161.40.9.80A.14 & & $7.66 \mathrm{E}-05$ & 32.0 & 0.299 & $6.12 \mathrm{E}-03$ & 204 & $4.80 \mathrm{E}-02$ & 199 & $5.93 \mathrm{E}-02$ & 505 & 3.75E-02 & 650 & $5.30 \mathrm{E}-02$ & $<0$ \\
\hline A161.40.9.80A.15 & & $7.64 \mathrm{E}-05$ & 34.8 & 0.298 & $6.11 \mathrm{E}-03$ & 199 & $4.68 \mathrm{E}-02$ & 189 & $5.63 \mathrm{E}-02$ & 480 & 3.56E-02 & 668 & $5.45 \mathrm{E}-02$ & $<0$ \\
\hline A161.40.9.80A.16 & & $7.62 \mathrm{E}-05$ & 36.7 & 0.297 & $6.10 \mathrm{E}-03$ & 195 & $4.58 \mathrm{E}-02$ & 190 & $5.66 \mathrm{E}-02$ & 470 & $3.48 \mathrm{E}-02$ & 646 & $5.26 \mathrm{E}-02$ & $<0$ \\
\hline \multicolumn{15}{|c|}{ A161 $40^{\circ} \mathrm{C}$ pH 9 Reactor $\# 5$} \\
\hline A161.40.9.80B.Blank1 & & $7.55 \mathrm{E}-05$ & --- & --- & --- & {$[49.4]$} & --- & {$[75.6]$} & --- & [164] & -- & [134] & --- & --- \\
\hline A161.40.9.80B.Blank2 & & 7.77E-05 & --- & --- & --- & [49.4] & --- & [75.6] & --- & [164] & --- & [134] & --- & --- \\
\hline A161.40.9.80B.Blank3 & & $7.56 \mathrm{E}-05$ & --- & --- & --- & {$[49.4]$} & --- & {$[75.6]$} & --- & [164] & --- & [134] & --- & --- \\
\hline A161.40.9.80B.2 & & $7.58 \mathrm{E}-05$ & 4.0 & 0.064 & $1.30 \mathrm{E}-03$ & 68 & $7.48 \mathrm{E}-02$ & 103 & 1.43E-01 & 172 & $5.93 \mathrm{E}-02$ & 361 & $1.37 \mathrm{E}-01$ & $<0$ \\
\hline A161.40.9.80B.4 & & $7.62 \mathrm{E}-05$ & 8.8 & 0.064 & $1.29 \mathrm{E}-03$ & 51 & $5.69 \mathrm{E}-02$ & 86 & $1.20 \mathrm{E}-01$ & $\begin{array}{l}{[164]} \\
135^{*}\end{array}$ & $5.73 \mathrm{E}-02$ & 298 & $1.14 \mathrm{E}-01$ & $<0$ \\
\hline A161.40.9.80B.6 & & 7.63E-05 & 13.9 & 0.063 & $1.28 \mathrm{E}-03$ & $\begin{array}{l}{[49.4]} \\
43^{*}\end{array}$ & $5.52 E-02$ & $\begin{array}{l}{[75.6]} \\
74^{\star}\end{array}$ & 1.07E-01 & $\begin{array}{l}{[164]} \\
89^{*}\end{array}$ & $5.78 \mathrm{E}-02$ & 259 & 1.00E-01 & $<0$ \\
\hline A161.40.9.80B.8 & & 7.63E-05 & 18.1 & 0.062 & $1.28 \mathrm{E}-03$ & $\begin{array}{l}{[49.4]} \\
40^{*}\end{array}$ & $5.55 \mathrm{E}-02$ & $\begin{array}{l}{[75.6]} \\
75^{\star}\end{array}$ & $1.08 \mathrm{E}-01$ & {$[164]$} & $5.81 \mathrm{E}-02$ & 248 & $9.67 E-02$ & $<0$ \\
\hline A161.40.9.80B.10 & & $7.63 \mathrm{E}-05$ & 23.8 & 0.061 & $1.27 \mathrm{E}-03$ & $\begin{array}{l}{[49.4]} \\
36^{*}\end{array}$ & $5.61 \mathrm{E}-02$ & $\begin{array}{l}{[75.6]} \\
70^{\star}\end{array}$ & $1.09 \mathrm{E}-01$ & [164] & $5.87 \mathrm{E}-02$ & 206 & $8.10 \mathrm{E}-02$ & $<0$ \\
\hline A161.40.9.80B.12 & & $7.62 \mathrm{E}-05$ & 28.0 & 0.061 & $1.26 \mathrm{E}-03$ & $\begin{array}{l}{[49.4]} \\
33^{\star}\end{array}$ & $5.63 \mathrm{E}-02$ & $\begin{array}{l}{[75.6]} \\
75^{\star}\end{array}$ & $1.09 \mathrm{E}-01$ & [164] & $5.90 \mathrm{E}-02$ & 200 & $7.90 \mathrm{E}-02$ & $<0$ \\
\hline A161.40.9.80B.14 & & $7.62 \mathrm{E}-05$ & 32.0 & 0.060 & $1.25 \mathrm{E}-03$ & $\begin{array}{l}{[49.4]} \\
32^{\star}\end{array}$ & $5.66 \mathrm{E}-02$ & $\begin{array}{l}{[75.6]} \\
59^{\star}\end{array}$ & 1.10E-01 & [164] & 5.93E-02 & 190 & 7.55E-02 & $<0$ \\
\hline A161.40.9.80B.15 & & 7.63E-05 & 34.8 & 0.060 & $1.24 \mathrm{E}-03$ & $\begin{array}{l}{[49.4]} \\
28^{*}\end{array}$ & $5.70 \mathrm{E}-02$ & 83 & $1.21 \mathrm{E}-01$ & [164] & 5.96E-02 & 219 & $8.76 \mathrm{E}-02$ & $<0$ \\
\hline A161.40.9.80B.16 & & $7.61 \mathrm{E}-05$ & 36.7 & 0.060 & $1.24 \mathrm{E}-03$ & $\begin{array}{l}{[49.4]} \\
29^{\star}\end{array}$ & $5.70 \mathrm{E}-02$ & $\begin{array}{l}{[75.6]} \\
74^{\star}\end{array}$ & $1.10 \mathrm{E}-01$ & [164] & $5.96 \mathrm{E}-02$ & 177 & $7.08 \mathrm{E}-02$ & $<0$ \\
\hline \multicolumn{15}{|c|}{ A161 $40^{\circ} \mathrm{C}$ pH 9 Reactor \#6 } \\
\hline A161.40.9.C.Blank1 & & $9.29 \mathrm{E}-06$ & -.- & -.- & -.- & [49.4] & -.- & [75.6] & --- & [164] & -.- & [134] & --- & -.- \\
\hline A161.40.9.C.Blank2 & & $8.40 \mathrm{E}-06$ & --- & --- & --- & {$[49.4]$} & --- & {$[75.6]$} & --- & [164] & -- & [134] & --- & --- \\
\hline A161.40.9.C.Blank3 & & $7.99 \mathrm{E}-06$ & --- & -.- & --- & [49.4] & --- & {$[75.6]$} & --- & [164] & --- & [134] & --- & -.- \\
\hline A161.40.9.C.2 & & $1.89 \mathrm{E}-05$ & 4.0 & --- & $2.90 \mathrm{E}-04$ & $\begin{array}{l}{[49.4]} \\
35^{\star}\end{array}$ & $6.04 \mathrm{E}-02$ & $\begin{array}{l}{[75.6]} \\
72^{\star}\end{array}$ & $1.17 \mathrm{E}-01$ & [164] & $6.32 \mathrm{E}-02$ & 272 & $1.15 \mathrm{E}-01$ & $<0$ \\
\hline A161.40.9.C.4 & & $1.88 \mathrm{E}-05$ & 8.8 & --- & 2.90E-04 & $\begin{array}{l}{[49.4]} \\
42^{\star}\end{array}$ & $6.03 E-02$ & $\begin{array}{l}{[75.6]} \\
70^{\star}\end{array}$ & 1.17E-01 & [164] & $6.31 \mathrm{E}-02$ & 242 & $1.02 \mathrm{E}-01$ & $<0$ \\
\hline A161.40.9.C.6 & & $1.88 \mathrm{E}-05$ & 13.9 & --- & $2.90 \mathrm{E}-04$ & $\begin{array}{l}{[49.4]} \\
36^{\star}\end{array}$ & $6.03 E-02$ & $\begin{array}{l}{[75.6]} \\
65^{\star}\end{array}$ & 1.17E-01 & [164] & $6.31 \mathrm{E}-02$ & 184 & $7.79 E-02$ & $<0$ \\
\hline A161.40.9.C.8 & & $1.88 \mathrm{E}-05$ & 18.1 & --- & $2.90 \mathrm{E}-04$ & $\begin{array}{l}{[49.4]} \\
32^{\star}\end{array}$ & $6.03 E-02$ & $\begin{array}{l}{[75.6]} \\
54^{\star}\end{array}$ & $1.17 \mathrm{E}-01$ & [164] & $6.31 \mathrm{E}-02$ & 160 & $6.77 \mathrm{E}-02$ & $<0$ \\
\hline A161.40.9.C.10 & & $1.85 \mathrm{E}-05$ & 23.8 & --- & $2.90 \mathrm{E}-04$ & $\begin{array}{l}{[49.4]} \\
28^{*}\end{array}$ & $5.94 \mathrm{E}-02$ & $\begin{array}{l}{[75.6]} \\
63^{\star}\end{array}$ & $1.15 \mathrm{E}-01$ & [164] & $6.22 \mathrm{E}-02$ & $\begin{array}{l}{[134]} \\
119^{*}\end{array}$ & $5.59 \mathrm{E}-02$ & $<0$ \\
\hline A161.40.9.C.12 & & $1.89 \mathrm{E}-05$ & 28.0 & --- & 2.90E-04 & $\begin{array}{l}{[49.4]} \\
27^{\star}\end{array}$ & $6.05 E-02$ & $\begin{array}{l}{[75.6]} \\
53^{\star}\end{array}$ & 1.17E-01 & [164] & $6.34 \mathrm{E}-02$ & $\begin{array}{l}{[134]} \\
107^{*}\end{array}$ & 5.69E-02 & $<0$ \\
\hline A161.40.9.C.14 & & $1.84 \mathrm{E}-05$ & 32.0 & --- & $2.90 \mathrm{E}-04$ & $\begin{array}{l}{[49.4]} \\
24^{*}\end{array}$ & $5.89 \mathrm{E}-02$ & $\begin{array}{l}{[75.6]} \\
51^{\star}\end{array}$ & 1.14E-01 & [164] & 6.17E-02 & $\begin{array}{l}{[134]} \\
116^{*}\end{array}$ & $5.54 \mathrm{E}-02$ & $<0$ \\
\hline A161.40.9.C.15 & & $1.89 \mathrm{E}-05$ & 34.8 & --- & $2.90 \mathrm{E}-04$ & $\begin{array}{l}{[49.4]} \\
26^{*}\end{array}$ & $6.05 E-02$ & $\begin{array}{l}{[75.6]} \\
50^{*}\end{array}$ & 1.17E-01 & [164] & $6.33 \mathrm{E}-02$ & $\begin{array}{l}{[134]} \\
108^{\star}\end{array}$ & $5.69 \mathrm{E}-02$ & $<0$ \\
\hline
\end{tabular}




\begin{tabular}{|c|c|c|c|c|c|c|c|c|c|c|c|c|c|c|}
\hline Sample ID & $\begin{array}{l}\text { Influent } \\
\text { [Si] } \\
\text { (ppm) }\end{array}$ & $\begin{array}{l}\text { Flow } \\
\text { Rate, q } \\
\left(\mathrm{m}^{3} \mathrm{~d}^{-1}\right)\end{array}$ & $\begin{array}{l}\text { Time } \\
\text { (d) }\end{array}$ & $\begin{array}{l}\text { Glass } \\
\text { Mass } \\
\text { (g) }\end{array}$ & $\begin{array}{l}\text { Surface } \\
\text { Area, } \mathrm{S} \\
\left(\mathrm{m}^{2}\right)\end{array}$ & $\begin{array}{l}{[\mathrm{Al}]} \\
\left(\mu \mathrm{g} \mathrm{L}^{-1}\right)\end{array}$ & $\begin{array}{l}\text { Al Norm. } \\
\text { Diss. Rate } \\
\left(\mathrm{g} \mathrm{m}^{-2} \mathrm{~d}^{-1}\right)\end{array}$ & $\begin{array}{l}{[\mathrm{B}]} \\
\left(\mu \mathrm{g} \mathrm{L}^{-1}\right)\end{array}$ & $\begin{array}{l}\text { B Norm. } \\
\text { Diss. Rate } \\
\left(\mathrm{g} \mathrm{m}^{-2} \mathrm{~d}^{-1}\right)\end{array}$ & $\begin{array}{l}{[\mathrm{Si}]} \\
\left(\mu \mathrm{g} \mathrm{L}^{-1}\right)\end{array}$ & $\begin{array}{l}\text { Si Norm. } \\
\text { Diss. Rate } \\
\left(\mathrm{g} \mathrm{m}^{-2} \mathrm{~d}^{-1}\right)\end{array}$ & $\begin{array}{l}{[\mathrm{Na}]} \\
\left(\mu \mathrm{g} \mathrm{L}{ }^{-}\right. \\
\left.{ }^{1}\right)\end{array}$ & $\begin{array}{l}\text { Na Norm. } \\
\text { Diss. Rate, } \\
\mathrm{r} \\
\left(\mathrm{g} \mathrm{m}^{-2} \mathrm{~d}^{-1}\right)\end{array}$ & $\begin{array}{l}\mathrm{r}_{\mathrm{IEX}} \\
\left(\mathrm{mol} \mathrm{Na} \mathrm{a}^{-2} \mathrm{~s}^{-1}\right)\end{array}$ \\
\hline A161.40.9.C.16 & & $1.88 \mathrm{E}-05$ & 36.7 & $-\cdots$ & $2.90 E-04$ & $\begin{array}{l}{[49.4]} \\
23^{\star}\end{array}$ & $6.01 E-02$ & $\begin{array}{l}{[75.6]} \\
50^{*}\end{array}$ & $1.16 \mathrm{E}-01$ & {$[164]$} & $6.29 \mathrm{E}-02$ & $\begin{array}{l}{[134]} \\
131^{*}\end{array}$ & $5.65 \mathrm{E}-02$ & $<0$ \\
\hline \multicolumn{15}{|c|}{ A161 $40^{\circ} \mathrm{C}$ pH 10 Reactor \#1 } \\
\hline A161.40.10.80A.Blank1 & & 8.17E-05 & -.- & -.- & -.- & [49.4] & --- & [75.6] & --- & 164 & --- & [134] & -.- & --- \\
\hline A161.40.10.80A.Blank2 & & 8.02E-05 & -.- & -.- & --- & [49.4] & -.- & [75.6] & --- & [164] & --- & [134] & --- & --- \\
\hline A161.40.10.80A.Blank3 & & $3.98 \mathrm{E}-05$ & --. & -.- & -.- & [49.4] & -.- & [75.6] & --- & [164] & --- & [134] & --- &.-- \\
\hline A161.40.10.80A.2 & & $7.03 \mathrm{E}-05$ & 4.0 & 0.059 & $1.19 \mathrm{E}-03$ & 102 & $1.13 \mathrm{E}-01$ & 77 & $1.08 \mathrm{E}-01$ & 260 & $9.10 \mathrm{E}-02$ & 255 & $9.82 \mathrm{E}-02$ & $<0$ \\
\hline A161.40.10.80A.4 & & $7.52 \mathrm{E}-05$ & 8.8 & 0.058 & $1.18 \mathrm{E}-03$ & 101 & $1.21 \mathrm{E}-01$ & 93 & $1.41 \mathrm{E}-01$ & 503 & $1.90 \mathrm{E}-01$ & 442 & $1.84 \mathrm{E}-01$ & $2.17 \mathrm{E}-08$ \\
\hline A161.40.10.80A.6 & & $7.63 \mathrm{E}-05$ & 13.8 & 0.057 & $1.17 \mathrm{E}-03$ & 99 & $1.22 \mathrm{E}-01$ & 80 & $1.24 \mathrm{E}-01$ & 501 & $1.94 \mathrm{E}-01$ & 440 & $1.87 \mathrm{E}-01$ & $3.17 \mathrm{E}-08$ \\
\hline A161.40.10.80A.8 & & 7.53E-05 & 18.0 & 0.056 & $1.16 \mathrm{E}-03$ & 218 & $2.67 \mathrm{E}-01$ & 111 & $1.72 \mathrm{E}-01$ & 558 & $2.15 \mathrm{E}-01$ & 534 & $2.27 \mathrm{E}-01$ & $2.75 \mathrm{E}-08$ \\
\hline A161.40.10.80A.10 & & 7.73E-05 & 23.8 & 0.055 & $1.14 \mathrm{E}-03$ & 216 & $2.76 \mathrm{E}-01$ & 116 & $1.88 \mathrm{E}-01$ & 578 & $2.33 \mathrm{E}-01$ & 546 & $2.42 \mathrm{E}-01$ & $2.72 \mathrm{E}-08$ \\
\hline A161.40.10.80A.12 & & $7.39 \mathrm{E}-05$ & 27.9 & 0.054 & $1.13 \mathrm{E}-03$ & 205 & $2.52 \mathrm{E}-01$ & 91 & $1.42 \mathrm{E}-01$ & 516 & $2.00 \mathrm{E}-01$ & 529 & $2.26 \mathrm{E}-01$ & $4.21 \mathrm{E}-08$ \\
\hline A161.40.10.80A.14 & & $7.44 \mathrm{E}-05$ & 32.0 & 0.054 & $1.12 \mathrm{E}-03$ & 225 & $2.81 \mathrm{E}-01$ & 110 & $1.74 \mathrm{E}-01$ & 591 & $2.33 \mathrm{E}-01$ & 594 & $2.57 \mathrm{E}-01$ & 4.19E-08 \\
\hline A161.40.10.80A.16 & & $7.55 \mathrm{E}-05$ & 36.7 & 0.053 & $1.11 \mathrm{E}-03$ & 230 & $2.95 \mathrm{E}-01$ & 104 & $1.69 \mathrm{E}-01$ & 593 & $2.40 \mathrm{E}-01$ & 608 & $2.70 \mathrm{E}-01$ & $5.10 \mathrm{E}-08$ \\
\hline \multicolumn{15}{|c|}{ A161 $40^{\circ} \mathrm{C} \mathrm{pH} 10$ Reactor $\# 2$} \\
\hline A161.40.10.C.Blank1 & & $3.54 \mathrm{E}-08$ & --- & --- & --- & [49.4] & -.- & [75.6] & --- & [164] & --. & [134] & --- & --- \\
\hline A161.40.10.C.Blank2 & & $1.15 \mathrm{E}-05$ & --- & --- & --- & [49.4] & --- & [75.6] & --- & 224 & --- & [134] & --- & --- \\
\hline A161.40.10.C.Blank3 & & 7.95E-06 & --- & -.- & -.- & [49.4] & -.- & [75.6] & --- & 210 & -.- & [134] & --- & --- \\
\hline A161.40.10.C.2 & & $1.79 \mathrm{E}-05$ & 4.0 & --- & $2.88 \mathrm{E}-04$ & 71 & $8.33 \mathrm{E}-02$ & [75.6] & $1.12 \mathrm{E}-01$ & [164] & $6.03 \mathrm{E}-02$ & 177 & $7.16 \mathrm{E}-02$ & $<0$ \\
\hline A161.40.10.c.4 & & 2.16E-05 & 8.8 & -.- & $2.88 \mathrm{E}-04$ & 188 & $2.65 \mathrm{E}-01$ & 149 & $2.66 \mathrm{E}-01$ & 526 & $2.34 \mathrm{E}-01$ & 497 & $2.43 \mathrm{E}-01$ & $<0$ \\
\hline A161.40.10.C.6 & & 2.35E-05 & 13.8 & -.- & $2.88 \mathrm{E}-04$ & 175 & $2.68 \mathrm{E}-01$ & 140 & $2.72 \mathrm{E}-01$ & 511 & $2.47 \mathrm{E}-01$ & 459 & $2.44 \mathrm{E}-01$ & $<0$ \\
\hline A161.40.10.C.10 & & $2.31 \mathrm{E}-05$ & 23.8 & -.- & $2.88 \mathrm{E}-04$ & 157 & $2.37 \mathrm{E}-01$ & {$[75.6]$} & $1.44 \mathrm{E}-01$ & 393 & 1.87E-01 & 350 & $1.83 \mathrm{E}-01$ & $1.94 \mathrm{E}-08$ \\
\hline A161.40.10.C.12 & & $2.24 \mathrm{E}-05$ & 27.9 & --- & $2.88 \mathrm{E}-04$ & 152 & $2.22 \mathrm{E}-01$ & {$[75.6]$} & $1.40 \mathrm{E}-01$ & 358 & $1.65 \mathrm{E}-01$ & 341 & $1.73 \mathrm{E}-01$ & $1.66 \mathrm{E}-08$ \\
\hline A161.40.10.C.14 & & $2.39 \mathrm{E}-05$ & 32.0 & -.- & $2.88 \mathrm{E}-04$ & 159 & $2.49 \mathrm{E}-01$ & {$[75.6]$} & $1.50 \mathrm{E}-01$ & 403 & 1.99E-01 & 390 & $2.11 \mathrm{E}-01$ & $3.11 \mathrm{E}-08$ \\
\hline A161.40.10.C.16 & & 2.32E-05 & 36.7 & -.- & $2.88 \mathrm{E}-04$ & 166 & $2.52 \mathrm{E}-01$ & {$[75.6]$} & $1.45 \mathrm{E}-01$ & 393 & $1.88 \mathrm{E}-01$ & 381 & $2.00 \mathrm{E}-01$ & $2.78 \mathrm{E}-08$ \\
\hline \multicolumn{15}{|c|}{ A161 $40^{\circ} \mathrm{C} \mathrm{pH} 11$ Reactor \#1 } \\
\hline A161.40.11.80A.Blank1 & & $7.76 \mathrm{E}-05$ & -.- & -.- & --- & [49.4] & --- & [75.6] & --- & [164] & --- & [134] & --- & --. \\
\hline A161.40.11.80A.Blank2 & & $7.86 \mathrm{E}-05$ & --- & --- & --- & {$[49.4]$} & --- & [75.6] & --- & [164] & --- & [134] & -.- & --. \\
\hline A161.40.11.80A.Blank3 & & $7.85 \mathrm{E}-05$ & --- & -.- & -.- & [49.4] & --- & [75.6] & --- & [164] & --. & [134] & --. & --- \\
\hline A161.40.11.80A.1 & & $1.65 \mathrm{E}-06$ & 0.6 & 0.050 & $1.01 \mathrm{E}-03$ & 74 & $2.28 \mathrm{E}-03$ & [75.6] & $2.94 \mathrm{E}-03$ & 210 & $2.04 \mathrm{E}-03$ & 195 & $2.08 \mathrm{E}-03$ & $<0$ \\
\hline A161.40.11.80A.3 & & 4.00E-05 & 1.6 & 0.050 & $1.01 \mathrm{E}-03$ & 304 & $2.27 \mathrm{E}-01$ & 109 & $1.03 \mathrm{E}-01$ & 1,000 & $2.35 \mathrm{E}-01$ & 848 & $2.19 \mathrm{E}-01$ & $5.86 \mathrm{E}-08$ \\
\hline A161.40.11.80A.4 & & $3.61 \mathrm{E}-05$ & 3.1 & 0.049 & $1.00 \mathrm{E}-03$ & 410 & $2.78 \mathrm{E}-01$ & 335 & $2.87 \mathrm{E}-01$ & 1,480 & $3.16 \mathrm{E}-01$ & 1,300 & $3.05 \mathrm{E}-01$ & $9.01 \mathrm{E}-09$ \\
\hline A161.40.11.80A.5 & & 3.74E-05 & 5.7 & 0.048 & $9.87 E-04$ & 416 & $2.97 \mathrm{E}-01$ & 374 & 3.38E-01 & 1,620 & 3.64E-01 & 1,310 & $3.24 \mathrm{E}-01$ & $<0$ \\
\hline A161.40.11.80A.6 & & $3.73 \mathrm{E}-05$ & 8.7 & 0.047 & $9.73 E-04$ & 368 & $2.66 \mathrm{E}-01$ & 362 & $3.31 \mathrm{E}-01$ & 1,570 & $3.57 \mathrm{E}-01$ & 1,270 & $3.18 \mathrm{E}-01$ & $<0$ \\
\hline A161.40.11.80A.7 & & $3.73 \mathrm{E}-05$ & 9.9 & 0.047 & $9.72 \mathrm{E}-04$ & 422 & $3.05 \mathrm{E}-01$ & 373 & 3.42E-01 & 1,570 & $3.58 \mathrm{E}-01$ & 1,230 & $3.08 \mathrm{E}-01$ & $<0$ \\
\hline A161.40.11.80A.8 & & $3.76 \mathrm{E}-05$ & 12.9 & 0.046 & $9.57 \mathrm{E}-04$ & 347 & $2.57 \mathrm{E}-01$ & 322 & $3.02 \mathrm{E}-01$ & 1,380 & $3.22 \mathrm{E}-01$ & 1,350 & $3.47 \mathrm{E}-01$ & $2.25 \mathrm{E}-08$ \\
\hline A161.40.11.80A.9 & & $3.79 \mathrm{E}-05$ & 14.7 & 0.046 & $9.51 \mathrm{E}-04$ & 356 & $2.67 \mathrm{E}-01$ & 342 & $3.25 \mathrm{E}-01$ & 1,480 & $3.50 \mathrm{E}-01$ & 1,160 & $3.02 \mathrm{E}-01$ & $<0$ \\
\hline A161.40.11.80A.10 & & $3.58 \mathrm{E}-05$ & 17.0 & 0.045 & $9.40 \mathrm{E}-04$ & 447 & $3.20 \mathrm{E}-01$ & 358 & $3.25 \mathrm{E}-01$ & 1,570 & $3.55 \mathrm{E}-01$ & 1,230 & $3.06 \mathrm{E}-01$ & $<0$ \\
\hline A161.40.11.80A.11 & & $3.72 \mathrm{E}-05$ & 19.7 & 0.044 & $9.27 \mathrm{E}-04$ & 433 & $3.27 \mathrm{E}-01$ & 345 & $3.30 \mathrm{E}-01$ & 1,510 & $3.60 \mathrm{E}-01$ & 1,210 & 3.17E-01 & $<0$ \\
\hline A161.40.11.80A.12 & & $3.72 \mathrm{E}-05$ & 21.7 & 0.044 & $9.26 \mathrm{E}-04$ & 411 & $3.11 \mathrm{E}-01$ & 202 & $1.93 \mathrm{E}-01$ & 1,280 & $3.05 \mathrm{E}-01$ & 1,050 & $2.75 \mathrm{E}-01$ & $4.12 \mathrm{E}-08$ \\
\hline A161.40.11.80A.13 & & $3.91 \mathrm{E}-05$ & 24.0 & 0.043 & $9.16 \mathrm{E}-04$ & 367 & $2.95 \mathrm{E}-01$ & 267 & $2.72 \mathrm{E}-01$ & 1,220 & $3.09 \mathrm{E}-01$ & 1,040 & $2.90 \mathrm{E}-01$ & $9.07 \mathrm{E}-09$ \\
\hline A161.40.11.80A.14 & & $3.74 \mathrm{E}-05$ & 26.9 & 0.042 & $9.06 \mathrm{E}-04$ & 375 & $2.92 \mathrm{E}-01$ & 269 & $2.65 \mathrm{E}-01$ & 1,280 & $3.14 \mathrm{E}-01$ & 1,020 & $2.75 \mathrm{E}-01$ & 5.07E-09 \\
\hline A161.40.11.80A.15 & & $0.00 \mathrm{E}+00$ & 28.6 & 0.043 & $9.11 \mathrm{E}-04$ & 505 & $0.00 E+00$ & 361 & $0.00 \mathrm{E}+00$ & 1,720 & $0.00 E+00$ & 1,360 & $0.00 E+00$ & $<0$ \\
\hline
\end{tabular}

B.38 


\begin{tabular}{|c|c|c|c|c|c|c|c|c|c|c|c|c|c|c|}
\hline Sample ID & $\begin{array}{l}\text { Influent } \\
\text { [Si] } \\
\text { (ppm) }\end{array}$ & $\begin{array}{l}\text { Flow } \\
\text { Rate, q } \\
\left(\mathrm{m}^{3} \mathrm{~d}^{-1}\right)\end{array}$ & $\begin{array}{l}\text { Time } \\
\text { (d) }\end{array}$ & $\begin{array}{l}\text { Glass } \\
\text { Mass } \\
\text { (g) }\end{array}$ & $\begin{array}{l}\text { Surface } \\
\text { Area, } \mathrm{S} \\
\left(\mathrm{m}^{2}\right)\end{array}$ & $\begin{array}{l}{[\mathrm{Al}]} \\
\left(\mu \mathrm{g} \mathrm{L}^{-1}\right)\end{array}$ & $\begin{array}{l}\text { Al Norm. } \\
\text { Diss. Rate } \\
\left(\mathrm{g} \mathrm{m}^{-2} \mathrm{~d}^{-1}\right)\end{array}$ & $\begin{array}{l}{[\mathrm{B}]} \\
\left(\mu \mathrm{L} \mathrm{L}^{-1}\right)\end{array}$ & $\begin{array}{l}\text { B Norm. } \\
\text { Diss. Rate } \\
\left(\mathrm{g} \mathrm{m}^{-2} \mathrm{~d}^{-1}\right)\end{array}$ & $\begin{array}{l}{[\mathrm{Si}]} \\
\left(\mu \mathrm{L} \mathrm{L}^{-1}\right)\end{array}$ & $\begin{array}{l}\text { Si Norm. } \\
\text { Diss. Rate } \\
\left(\mathrm{g} \mathrm{m}^{-2} \mathrm{~d}^{-1}\right)\end{array}$ & $\begin{array}{l}{[\mathrm{Na}]} \\
(\mu \mathrm{g} \mathrm{L} \\
\left.{ }^{1}\right)\end{array}$ & $\begin{array}{l}\text { Na Norm. } \\
\text { Diss. Rate, } \\
\mathrm{r} \\
\left(\mathrm{g} \mathrm{m}^{-2} \mathrm{~d}^{-1}\right)\end{array}$ & $\begin{array}{l}\mathrm{r}_{\mathrm{IEX}} \\
\left(\mathrm{mol} \mathrm{Na} \mathrm{s}^{-2} \mathrm{~s}^{-1}\right)\end{array}$ \\
\hline \multicolumn{15}{|c|}{ A161 $40^{\circ} \mathrm{C} \mathrm{pH} 11$ Reactor \#2 } \\
\hline A161.40.11.C.Blank1 & & 4.04E-06 & -- & --- & --- & [49.4] & --- & [75.6] & --- & [164] & --- & [134] & --- & --- \\
\hline A161.40.11.C.Blank2 & & $9.14 \mathrm{E}-06$ & -- & --- & $\cdots$ & [49.4] & --- & {$[75.6]$} & -- & [164] & --- & [134] & --- & -.- \\
\hline A161.40.11.C.Blank3 & & $7.60 \mathrm{E}-06$ & --- & --- & --- & [49.4] & --- & [75.6] & --- & [164] & --- & [134] & --- & -.- \\
\hline A161.40.11.C.1 & & $6.56 \mathrm{E}-07$ & 1.1 & -- & $2.89 \mathrm{E}-04$ & 58 & $2.47 \mathrm{E}-03$ & {$[75.6]$} & 4.09E-03 & 171 & $2.30 \mathrm{E}-03$ & 178 & $2.64 \mathrm{E}-03$ & $<0$ \\
\hline A161.40.11.C.3 & & $8.51 \mathrm{E}-06$ & 5.7 & -.- & $2.89 \mathrm{E}-04$ & 339 & $1.88 \mathrm{E}-01$ & 153 & 1.07E-01 & 1,070 & $1.87 \mathrm{E}-01$ & 940 & $1.81 \mathrm{E}-01$ & $3.69 \mathrm{E}-08$ \\
\hline A161.40.11.C.5 & & $2.40 \mathrm{E}-06$ & 9.9 & --- & $2.89 \mathrm{E}-04$ & 411 & $6.43 \mathrm{E}-02$ & 228 & 4.52E-02 & 1,420 & $7.00 \mathrm{E}-02$ & 1,200 & $6.51 \mathrm{E}-02$ & $1.00 \mathrm{E}-08$ \\
\hline A161.40.11.C.7 & & 7.06E-06 & 14.7 & -.- & $2.89 \mathrm{E}-04$ & 333 & 1.53E-01 & 242 & 1.41E-01 & 1,440 & $2.09 \mathrm{E}-01$ & 1,230 & $1.96 \mathrm{E}-01$ & $2.78 \mathrm{E}-08$ \\
\hline A161.40.11.C.9 & & $8.74 \mathrm{E}-06$ & 19.7 & --- & $2.89 \mathrm{E}-04$ & 425 & $2.42 \mathrm{E}-01$ & 382 & $2.75 \mathrm{E}-01$ & 1,660 & $2.98 \mathrm{E}-01$ & 1,310 & $2.59 \mathrm{E}-01$ & $<0$ \\
\hline A161.40.11.C.10 & & $8.81 \mathrm{E}-06$ & 21.7 & -.- & $2.89 \mathrm{E}-04$ & 419 & $2.41 \mathrm{E}-01$ & 383 & $2.78 \mathrm{E}-01$ & 1,650 & $2.99 \mathrm{E}-01$ & 1,290 & $2.57 \mathrm{E}-01$ & $<0$ \\
\hline A161.40.11.C.11 & & $8.48 \mathrm{E}-06$ & 24.0 & --- & $2.89 \mathrm{E}-04$ & 371 & $2.05 \mathrm{E}-01$ & 354 & $2.48 \mathrm{E}-01$ & 1,510 & 2.63E-01 & 1,200 & $2.30 \mathrm{E}-01$ & $<0$ \\
\hline A161.40.11.C.12 & & $8.89 \mathrm{E}-06$ & 26.9 & --- & $2.89 \mathrm{E}-04$ & 404 & $2.34 \mathrm{E}-01$ & 351 & 2.57E-01 & 1,500 & $2.74 \mathrm{E}-01$ & 1,190 & $2.39 \mathrm{E}-01$ & $<0$ \\
\hline A161.40.11.C.13 & & $8.73 \mathrm{E}-06$ & 28.6 & --- & $2.89 \mathrm{E}-04$ & 438 & $2.49 \mathrm{E}-01$ & 330 & $2.38 \mathrm{E}-01$ & 1,960 & $3.51 \mathrm{E}-01$ & 1,160 & $2.29 \mathrm{E}-01$ & $<0$ \\
\hline \multicolumn{15}{|c|}{ A161 $40^{\circ} \mathrm{C}$ pH 12 Reactor \#1 } \\
\hline A161.40.12.80A.Blank1 & & $7.64 \mathrm{E}-05$ & --- & --- & --- & 74 & --- & [75.6] & --- & 306 & --- & 563 & --- & --- \\
\hline A161.40.12.80A.Blank2 & & $8.17 E-05$ & -- & -- & --- & {$[49.4]$} & --- & [75.6] & --- & 468 & --- & 634 & --- & --- \\
\hline A161.40.12.80A.Blank3 & & $7.76 \mathrm{E}-05$ & -- & --- & --- & {$[49.4]$} & --- & {$[75.6]$} & -- & 498 & --- & 573 & --- & -- \\
\hline A161.40.12.80A.1 & & $4.61 \mathrm{E}-06$ & 0.7 & 0.104 & $2.10 \mathrm{E}-03$ & 195 & 8.04E-03 & 183 & $9.55 \mathrm{E}-03$ & 1,280 & $1.66 \mathrm{E}-02$ & 988 & $1.41 \mathrm{E}-02$ & $2.30 \mathrm{E}-09$ \\
\hline A161.40.12.80A.2 & & 7.69E-05 & 1.0 & 0.104 & $2.10 \mathrm{E}-03$ & 327 & $2.25 \mathrm{E}-01$ & 271 & $2.36 \mathrm{E}-01$ & 1,530 & $3.32 E-01$ & 1,350 & $3.22 \mathrm{E}-01$ & 4.33E-08 \\
\hline A161.40.12.80A.3 & & $7.85 \mathrm{E}-05$ & 1.7 & 0.103 & $2.09 \mathrm{E}-03$ & 695 & $4.91 \mathrm{E}-01$ & 530 & 4.74E-01 & 2,600 & $5.79 \mathrm{E}-01$ & 2,380 & $5.83 \mathrm{E}-01$ & $5.48 \mathrm{E}-08$ \\
\hline A161.40.12.80A.4 & & $7.67 \mathrm{E}-05$ & 3.9 & 0.099 & $2.03 \mathrm{E}-03$ & 1,010 & 7.19E-01 & 775 & $6.99 \mathrm{E}-01$ & 3,550 & 7.97E-01 & 3,460 & $8.54 \mathrm{E}-01$ & 7.82E-08 \\
\hline A161.40.12.80A.5 & & $7.86 \mathrm{E}-05$ & 6.8 & 0.094 & $1.96 \mathrm{E}-03$ & 1,060 & 7.99E-01 & 805 & 7.68E-01 & 3,680 & $8.75 E-01$ & 3,510 & $9.17 E-01$ & 7.49E-08 \\
\hline A161.40.12.80A.7 & & 7.92E-05 & 11.1 & 0.088 & $1.88 \mathrm{E}-03$ & 1,010 & 8.02E-01 & 780 & 7.84E-01 & 3,500 & $8.76 \mathrm{E}-01$ & 3,360 & $9.25 \mathrm{E}-01$ & 7.08E-08 \\
\hline A161.40.12.80A.9 & & 7.82E-05 & 15.8 & 0.084 & $1.82 \mathrm{E}-03$ & 876 & 7.08E-01 & 537 & 5.49E-01 & 2,830 & $7.21 \mathrm{E}-01$ & 2,670 & 7.48E-01 & 9.99E-08 \\
\hline A161.40.12.80A.11 & & 7.97E-05 & 20.7 & 0.078 & $1.74 \mathrm{E}-03$ & 891 & 7.69E-01 & 560 & $6.12 \mathrm{E}-01$ & 2,840 & $7.73 \mathrm{E}-01$ & 2,830 & $8.47 \mathrm{E}-01$ & $1.18 \mathrm{E}-07$ \\
\hline A161.40.12.80A.13 & & $7.88 \mathrm{E}-05$ & 25.0 & 0.075 & $1.69 \mathrm{E}-03$ & 839 & 7.37E-01 & 508 & $5.65 \mathrm{E}-01$ & 2,700 & 7.47E-01 & 2,620 & 7.97E-01 & 1.17E-07 \\
\hline A161.40.12.80A.15 & & $7.80 \mathrm{E}-05$ & 29.7 & 0.071 & $1.63 \mathrm{E}-03$ & 769 & $6.94 \mathrm{E}-01$ & 451 & 5.15E-01 & 2,550 & $7.25 \mathrm{E}-01$ & 2,480 & $7.76 \mathrm{E}-01$ & $1.31 \mathrm{E}-07$ \\
\hline A161.40.12.80A.17 & & 7.48E-05 & 34.8 & 0.067 & $1.57 \mathrm{E}-03$ & 700 & $6.29 \mathrm{E}-01$ & 412 & 4.69E-01 & 2,340 & $6.64 \mathrm{E}-01$ & 2,160 & $6.73 E-01$ & 1.03E-07 \\
\hline A161.40.12.80A.18 & & $5.99 \mathrm{E}-05$ & 36.7 & 0.066 & $1.55 \mathrm{E}-03$ & 799 & $5.81 \mathrm{E}-01$ & 481 & 4.43E-01 & 2,630 & $6.03 \mathrm{E}-01$ & 2,630 & $6.63 \mathrm{E}-01$ & $1.11 \mathrm{E}-07$ \\
\hline \multicolumn{15}{|c|}{ A161 $40^{\circ} \mathrm{C} \mathrm{pH} 12$ Reactor $\# 2$} \\
\hline A161.40.12.C.Blank1 & & $2.11 \mathrm{E}-05$ & --- & -.- & --- & [49.4] & --. & [75.6] & --- & 322 & --- & 510 & --- & --- \\
\hline A161.40.12.C.Blank2 & & $1.93 E-05$ & --- & --- & --- & {$[49.4]$} & --- & [75.6] & --- & 417 & --- & 533 & --- & --- \\
\hline A161.40.12.C.Blank3 & & $1.91 \mathrm{E}-05$ & --- & -.- & --- & [49.4] & --- & [75.6] & --- & 484 & --- & 536 & --- & -.- \\
\hline A161.40.12.C.1 & & $1.82 E-06$ & 1.0 & -- & $3.10 \mathrm{E}-04$ & 101 & 1.11E-02 & 87 & $1.21 \mathrm{E}-02$ & 779 & $2.71 \mathrm{E}-02$ & 682 & 2.60E-02 & $6.99 \mathrm{E}-09$ \\
\hline A161.40.12.C.2 & & $2.06 \mathrm{E}-05$ & 1.9 & --- & $3.10 \mathrm{E}-04$ & 308 & 3.84E-01 & 252 & $3.98 \mathrm{E}-01$ & 1,380 & $5.43 \mathrm{E}-01$ & 1,240 & $5.36 \mathrm{E}-01$ & $6.95 \mathrm{E}-08$ \\
\hline A161.40.12.C. 3 & & $1.07 E-05$ & 3.9 & -- & $3.10 \mathrm{E}-04$ & 615 & 3.98E-01 & 477 & 3.91E-01 & 2,380 & $4.86 \mathrm{E}-01$ & 2,140 & $4.80 E-01$ & 4.49E-08 \\
\hline A161.40.12.C. 4 & & $9.70 E-06$ & 6.8 & -- & $3.10 E-04$ & 945 & 5.56E-01 & 740 & 5.51E-01 & 3,460 & $6.42 E-01$ & 3,030 & $6.18 \mathrm{E}-01$ & $3.36 \mathrm{E}-08$ \\
\hline A161.40.12.C. 5 & & $9.43 E-06$ & 8.8 & -- & $3.10 \mathrm{E}-04$ & 1,110 & $6.35 \mathrm{E}-01$ & 854 & $6.18 \mathrm{E}-01$ & 4,000 & $7.21 \mathrm{E}-01$ & 3,510 & $6.96 \mathrm{E}-01$ & $3.90 \mathrm{E}-08$ \\
\hline A161.40.12.C.7 & & $9.56 \mathrm{E}-06$ & 13.8 & -- & $3.10 E-04$ & 1,180 & $6.84 \mathrm{E}-01$ & 934 & 6.85E-01 & 4,190 & $7.65 E-01$ & 3,770 & $7.57 E-01$ & 3.62E-08 \\
\hline A161.40.12.C.9 & & $1.01 \mathrm{E}-05$ & 18.1 & -- & $3.10 \mathrm{E}-04$ & 1,050 & $6.43 \mathrm{E}-01$ & 665 & $5.16 \mathrm{E}-01$ & 3,470 & $6.70 \mathrm{E}-01$ & 3,260 & $6.92 \mathrm{E}-01$ & $8.88 \mathrm{E}-08$ \\
\hline A161.40.12.C.11 & & 8.43E-06 & 23.7 & -- & $3.10 \mathrm{E}-04$ & 1,170 & $5.98 \mathrm{E}-01$ & 766 & 4.96E-01 & 3,900 & $6.29 \mathrm{E}-01$ & 3,540 & $6.28 \mathrm{E}-01$ & $6.62 E-08$ \\
\hline A161.40.12.C.13 & & $9.16 \mathrm{E}-06$ & 27.9 & -- & $3.10 \mathrm{E}-04$ & 1,140 & 6.33E-01 & 739 & $5.20 \mathrm{E}-01$ & 3,790 & $6.64 \mathrm{E}-01$ & 3,380 & $6.51 \mathrm{E}-01$ & $6.60 E-08$ \\
\hline A161.40.12.C.15 & & $8.86 \mathrm{E}-06$ & 32.0 & -- & $3.10 \mathrm{E}-04$ & 1,110 & $5.96 \mathrm{E}-01$ & 728 & $4.95 \mathrm{E}-01$ & 3,660 & $6.20 \mathrm{E}-01$ & 3,370 & $6.27 \mathrm{E}-01$ & $6.66 \mathrm{E}-08$ \\
\hline
\end{tabular}

B.39 


\begin{tabular}{|c|c|c|c|c|c|c|c|c|c|c|c|c|c|c|}
\hline Sample ID & $\begin{array}{l}\text { Influent } \\
{[\mathrm{Si}]} \\
(\mathrm{ppm})\end{array}$ & $\begin{array}{l}\text { Flow } \\
\text { Rate, } \mathrm{q} \\
\left(\mathrm{m}^{3} \mathrm{~d}^{-1}\right)\end{array}$ & $\begin{array}{l}\text { Time } \\
\text { (d) }\end{array}$ & $\begin{array}{l}\text { Glass } \\
\text { Mass } \\
\text { (g) }\end{array}$ & $\begin{array}{l}\text { Surface } \\
\text { Area, } \mathrm{S} \\
\left(\mathrm{m}^{2}\right)\end{array}$ & $\begin{array}{l}{[\mathrm{Al}]} \\
\left(\mu \mathrm{g} \mathrm{L}^{-1}\right)\end{array}$ & $\begin{array}{l}\text { Al Norm. } \\
\text { Diss. Rate } \\
\left(\mathrm{g} \mathrm{m}^{-2} \mathrm{~d}^{-1}\right)\end{array}$ & $\begin{array}{l}{[\mathrm{B}]} \\
\left(\mu \mathrm{g} \mathrm{L}^{-1}\right)\end{array}$ & $\begin{array}{l}\text { B Norm. } \\
\text { Diss. Rate } \\
\left(\mathrm{g} \mathrm{m}^{-2} \mathrm{~d}^{-1}\right)\end{array}$ & $\begin{array}{l}{[\mathrm{Si}]} \\
\left(\mu \mathrm{g} \mathrm{L}^{-1}\right)\end{array}$ & $\begin{array}{l}\text { Si Norm. } \\
\text { Diss. Rate } \\
\left(\mathrm{g} \mathrm{m}^{-2} \mathrm{~d}^{-1}\right)\end{array}$ & $\begin{array}{l}{[\mathrm{Na}]} \\
(\mu \mathrm{g} \mathrm{L}- \\
\left.{ }^{1}\right)\end{array}$ & $\begin{array}{l}\text { Na Norm. } \\
\text { Diss. Rate, } \\
\mathrm{r} \\
\left(\mathrm{g} \mathrm{m}^{-2} \mathrm{~d}^{-1}\right)\end{array}$ & $\begin{array}{l}\mathrm{r}_{\mathrm{IEX}} \\
\left(\mathrm{mol} \mathrm{Na} \mathrm{s}^{-2} \mathrm{~s}^{-1}\right)\end{array}$ \\
\hline A161.40.12.C.17 & & $2.96 \mathrm{E}-06$ & 36.7 & $-\ldots$ & $3.10 \mathrm{E}-04$ & 1,170 & $2.10 \mathrm{E}-01$ & 774 & $1.76 \mathrm{E}-01$ & 3,960 & $2.24 \mathrm{E}-01$ & 3,520 & $2.19 \mathrm{E}-01$ & $2.17 E-08$ \\
\hline \multicolumn{15}{|c|}{ A161 $70^{\circ} \mathrm{C} \mathrm{pH} 9$ Reactor \#1 } \\
\hline A161.70.9.20.Blank1 & & $1.80 \mathrm{E}-05$ & --- & --- & --- & [49.4] & --- & {$[75.6]$} & --- & [164] & --- & [134] & --- & -- \\
\hline A161.70.9.20.Blank2 & & $2.00 \mathrm{E}-05$ & --- & --- & --- & [49.4] & --- & {$[75.6]$} & --- & [164] & --- & [134] & --- & --- \\
\hline A161.70.9.20.Blank3 & & $1.94 \mathrm{E}-05$ & --- & --- & --- & [49.4] & -.- & [75.6] & --- & [164] & -.- & [134] & --- & -.- \\
\hline A161.70.9.20.1 & & $2.73 \mathrm{E}-06$ & 2.0 & 0.398 & $8.05 \mathrm{E}-03$ & 1,110 & $7.08 \mathrm{E}-03$ & 5,070 & $4.10 \mathrm{E}-02$ & 4,620 & $9.29 \mathrm{E}-03$ & 18,800 & $4.16 \mathrm{E}-02$ & $3.12 \mathrm{E}-10$ \\
\hline A161.70.9.20.2 & & $6.46 \mathrm{E}-06$ & 4.2 & 0.395 & $8.00 \mathrm{E}-03$ & 1,580 & $2.40 \mathrm{E}-02$ & 7,670 & $1.48 \mathrm{E}-01$ & 6,130 & $2.94 \mathrm{E}-02$ & 25,600 & $1.35 \mathrm{E}-01$ & $<0$ \\
\hline A161.70.9.20.3 & & $4.72 \mathrm{E}-06$ & 7.0 & 0.392 & $7.96 \mathrm{E}-03$ & 1,750 & 1.95E-02 & 9,300 & 1.31E-01 & 6,400 & $2.25 \mathrm{E}-02$ & 32,600 & $1.26 \mathrm{E}-01$ & $<0$ \\
\hline A161.70.9.20.5 & & $5.20 \mathrm{E}-06$ & 11.4 & 0.386 & $7.88 \mathrm{E}-03$ & 1,860 & $2.31 \mathrm{E}-02$ & 10,500 & 1.65E-01 & 6,520 & $2.55 \mathrm{E}-02$ & 35,900 & $1.54 \mathrm{E}-01$ & $<0$ \\
\hline A161.70.9.20.7 & & $4.18 \mathrm{E}-06$ & 16.3 & 0.381 & $7.82 \mathrm{E}-03$ & 1,780 & $1.79 \mathrm{E}-02$ & 9,920 & 1.26E-01 & 6,090 & $1.93 \mathrm{E}-02$ & 35,600 & $1.24 \mathrm{E}-01$ & $<0$ \\
\hline A161.70.9.20.9 & & $3.10 \mathrm{E}-06$ & 21.1 & 0.378 & $7.78 \mathrm{E}-03$ & 1,820 & 1.37E-02 & 9,220 & 8.76E-02 & 6,070 & $1.44 \mathrm{E}-02$ & 34,500 & $8.97 \mathrm{E}-02$ & $1.07 \mathrm{E}-09$ \\
\hline A161.70.9.20.10 & & $2.43 \mathrm{E}-06$ & 23.1 & 0.377 & $7.77 \mathrm{E}-03$ & 2,010 & 1.18E-02 & 10,100 & 7.54E-02 & 6,670 & $1.24 \mathrm{E}-02$ & 36,500 & $7.46 \mathrm{E}-02$ & $<0$ \\
\hline A161.70.9.20.11 & & 4.57E-06 & 24.2 & 0.376 & $7.75 \mathrm{E}-03$ & 2,010 & $2.23 \mathrm{E}-02$ & 10,300 & $1.45 \mathrm{E}-01$ & 6,660 & $2.33 \mathrm{E}-02$ & 36,300 & $1.40 \mathrm{E}-01$ & $<0$ \\
\hline \multicolumn{15}{|c|}{ A161 $70^{\circ} \mathrm{C}$ pH 9 Reactor $\# 2$} \\
\hline A161.70.9.40.Blank1 & & $3.78 \mathrm{E}-05$ & --. & --- & --- & [49.4] & --- & [75.6] & --- & [164] & --- & [134] & --- & --- \\
\hline A161.70.9.40.Blank2 & & $3.89 \mathrm{E}-05$ & --- & --- & --- & [49.4] & --- & {$[75.6]$} & --- & [164] & --- & [134] & --- & --- \\
\hline A161.70.9.40.Blank3 & & $3.87 \mathrm{E}-05$ & --- & --- & --- & [49.4] & --- & {$[75.6]$} & --- & [164] & --- & [134] & --- & --- \\
\hline A161.70.9.40.1 & & $6.50 \mathrm{E}-06$ & 2.0 & 0.153 & $3.10 \mathrm{E}-03$ & 1,620 & $6.40 \mathrm{E}-02$ & 3,730 & 1.87E-01 & 6,010 & $7.49 \mathrm{E}-02$ & 13,600 & $1.86 \mathrm{E}-01$ & $<0$ \\
\hline A161.70.9.40.2 & & $1.99 \mathrm{E}-05$ & 4.2 & 0.147 & $3.02 E-03$ & 1,670 & $2.08 \mathrm{E}-01$ & 4,010 & $6.31 \mathrm{E}-01$ & 5,900 & $2.31 \mathrm{E}-01$ & 13,600 & $5.86 \mathrm{E}-01$ & $<0$ \\
\hline A161.70.9.40.3 & & $2.21 \mathrm{E}-05$ & 7.0 & 0.142 & $2.95 \mathrm{E}-03$ & 1,370 & 1.93E-01 & 3,190 & 5.69E-01 & 4,710 & $2.09 \mathrm{E}-01$ & 10,900 & $5.32 \mathrm{E}-01$ & $<0$ \\
\hline A161.70.9.40.5 & & $2.25 \mathrm{E}-05$ & 11.4 & 0.138 & $2.89 \mathrm{E}-03$ & 1,080 & $1.58 \mathrm{E}-01$ & 2,200 & 4.07E-01 & 3,880 & $1.79 \mathrm{E}-01$ & 7,560 & $3.83 \mathrm{E}-01$ & $<0$ \\
\hline A161.70.9.40.7 & & $2.24 \mathrm{E}-05$ & 16.3 & 0.134 & $2.84 \mathrm{E}-03$ & 993 & 1.47E-01 & 1,730 & 3.25E-01 & 3,560 & 1.67E-01 & 6,000 & $3.09 \mathrm{E}-01$ & $<0$ \\
\hline A161.70.9.40.9 & & $2.38 \mathrm{E}-05$ & 21.1 & 0.130 & $2.78 \mathrm{E}-03$ & 919 & $1.48 \mathrm{E}-01$ & 1,440 & 2.95E-01 & 3,310 & 1.69E-01 & 5,020 & $2.81 \mathrm{E}-01$ & $<0$ \\
\hline A161.70.9.40.10 & & $2.32 \mathrm{E}-05$ & 23.1 & 0.129 & $2.76 \mathrm{E}-03$ & 872 & 1.38E-01 & 1,310 & $2.62 \mathrm{E}-01$ & 3,180 & $1.58 \mathrm{E}-01$ & 4,600 & $2.52 \mathrm{E}-01$ & $<0$ \\
\hline A161.70.9.40.11 & & $2.25 \mathrm{E}-05$ & 24.2 & 0.128 & $2.76 \mathrm{E}-03$ & 863 & 1.33E-01 & 1,270 & 2.47E-01 & 3,210 & $1.56 \mathrm{E}-01$ & 4,220 & $2.25 \mathrm{E}-01$ & $<0$ \\
\hline \multicolumn{15}{|c|}{ A161 $70^{\circ} \mathrm{C} \mathrm{pH} 9$ Reactor $\# 3$} \\
\hline A161.70.9.80A.Blank1 & & $7.37 \mathrm{E}-05$ & --- & --- & --- & [49.4] & --- & {$[75.6]$} & --- & {$[164]$} & --- & [134] & --- & -.- \\
\hline A161.70.9.80A.Blank2 & & $7.68 \mathrm{E}-05$ & -.- & --- & --- & [49.4] & -.- & {$[75.6]$} & --- & [164] & --- & [134] & --- & -.- \\
\hline A161.70.9.80A.Blank3 & & $7.65 \mathrm{E}-05$ & --- & --- & -.- & [49.4] & --- & {$[75.6]$} & --- & [164] & --- & [134] & --- & --- \\
\hline A161.70.9.80A.1 & & $1.31 \mathrm{E}-05$ & 2.0 & 0.050 & $1.01 \mathrm{E}-03$ & 909 & $2.22 \mathrm{E}-01$ & 1,310 & 4.06E-01 & 3,340 & $2.58 \mathrm{E}-01$ & 4,040 & $3.43 \mathrm{E}-01$ & $<0$ \\
\hline A161.70.9.80A.2 & & $3.81 \mathrm{E}-05$ & 4.2 & 0.046 & $9.68 \mathrm{E}-04$ & 817 & $6.05 \mathrm{E}-01$ & 1,200 & $1.13 E+00$ & 2,950 & $6.89 \mathrm{E}-01$ & 4,170 & $1.07 E+00$ & $<0$ \\
\hline A161.70.9.80A.3 & & $3.79 \mathrm{E}-05$ & 7.0 & 0.044 & $9.37 \mathrm{E}-04$ & 624 & $4.75 \mathrm{E}-01$ & 916 & 8.82E-01 & 2,260 & $5.42 \mathrm{E}-01$ & 3,000 & $7.91 \mathrm{E}-01$ & $<0$ \\
\hline A161.70.9.80A.5 & & $3.76 \mathrm{E}-05$ & 11.4 & 0.042 & $9.06 \mathrm{E}-04$ & 478 & $3.74 \mathrm{E}-01$ & 661 & $6.54 \mathrm{E}-01$ & 1,730 & 4.26E-01 & 2,310 & $6.26 \mathrm{E}-01$ & $<0$ \\
\hline A161.70.9.80A.7 & & 3.83E-05 & 16.3 & 0.040 & $8.75 \mathrm{E}-04$ & 420 & $3.46 \mathrm{E}-01$ & 523 & $5.45 \mathrm{E}-01$ & 1,470 & $3.81 \mathrm{E}-01$ & 1,820 & $5.19 \mathrm{E}-01$ & $<0$ \\
\hline A161.70.9.80A.9 & & 3.82E-05 & 21.1 & 0.038 & $8.44 \mathrm{E}-04$ & 385 & $3.28 \mathrm{E}-01$ & 457 & 4.93E-01 & 1,410 & $3.79 \mathrm{E}-01$ & 1,510 & $4.46 \mathrm{E}-01$ & $<0$ \\
\hline A161.70.9.80A.10 & & $3.72 \mathrm{E}-05$ & 23.1 & 0.037 & $8.36 \mathrm{E}-04$ & 388 & $3.25 \mathrm{E}-01$ & 428 & 4.54E-01 & 1,380 & $3.64 \mathrm{E}-01$ & 1,440 & $4.18 \mathrm{E}-01$ & $<0$ \\
\hline A161.70.9.80A.11 & & 3.93E-05 & 24.2 & 0.037 & $8.32 \mathrm{E}-04$ & 378 & 3.36E-01 & 411 & 4.63E-01 & 1,350 & $3.79 E-01$ & 1,250 & $3.86 \mathrm{E}-01$ & $<0$ \\
\hline \multicolumn{15}{|c|}{ A161 $70^{\circ} \mathrm{C} \mathrm{pH} 9$ Reactor $\# 4$} \\
\hline A161.70.9.80B.Blank1 & & 7.46E-05 & -.- & -.- & -.- & [49.4] & -.- & [75.6] & -.- & [164] & -.- & [134] & -.- & -.- \\
\hline A161.70.9.80B.Blank2 & & $7.61 \mathrm{E}-05$ & --- & --- & -- & [49.4] & --- & [75.6] & --- & [164] & --- & [134] & --- & --- \\
\hline A161.70.9.80B.Blank3 & & $7.62 \mathrm{E}-05$ & --- & --. & --- & [49.4] & --- & {$[75.6]$} & --- & [164] & --- & [134] & --- & -.- \\
\hline A161.70.9.80B.1 & & $2.61 \mathrm{E}-05$ & 2.0 & 0.026 & $5.29 \mathrm{E}-04$ & 600 & $5.57 \mathrm{E}-01$ & 781 & $9.17 \mathrm{E}-01$ & 2,240 & $6.55 \mathrm{E}-01$ & 2,660 & $8.55 \mathrm{E}-01$ & $<0$ \\
\hline A161.70.9.80B.2 & & $7.70 \mathrm{E}-05$ & 4.2 & 0.023 & 4.97E-04 & 385 & $1.12 \mathrm{E}+00$ & 463 & $1.71 \mathrm{E}+00$ & 1,380 & $1.27 \mathrm{E}+00$ & 1,550 & $1.57 \mathrm{E}+00$ & $<0$ \\
\hline
\end{tabular}

B. 40 


\begin{tabular}{|c|c|c|c|c|c|c|c|c|c|c|c|c|c|c|}
\hline Sample ID & $\begin{array}{l}\text { Influent } \\
\text { [Si] } \\
\text { (ppm) }\end{array}$ & $\begin{array}{l}\text { Flow } \\
\text { Rate, q } \\
\left(\mathrm{m}^{3} \mathrm{~d}^{-1}\right)\end{array}$ & $\begin{array}{l}\text { Time } \\
\text { (d) }\end{array}$ & $\begin{array}{l}\text { Glass } \\
\text { Mass } \\
\text { (g) }\end{array}$ & $\begin{array}{l}\text { Surface } \\
\text { Area, S } \\
\left(\mathrm{m}^{2}\right)\end{array}$ & $\begin{array}{l}{[\mathrm{Al}]} \\
\left(\mu \mathrm{g} \mathrm{L}^{-1}\right)\end{array}$ & $\begin{array}{l}\text { Al Norm. } \\
\text { Diss. Rate } \\
\left(\mathrm{g} \mathrm{m}^{-2} \mathrm{~d}^{-1}\right)\end{array}$ & $\begin{array}{l}{[\mathrm{B}]} \\
\left(\mu \mathrm{g} \mathrm{L}^{-1}\right)\end{array}$ & $\begin{array}{l}\text { B Norm. } \\
\text { Diss. Rate } \\
\left(\mathrm{g} \mathrm{m}^{-2} \mathrm{~d}^{-1}\right)\end{array}$ & $\begin{array}{l}{[\mathrm{Si}]} \\
\left(\mu \mathrm{g} \mathrm{L}^{-1}\right)\end{array}$ & $\begin{array}{l}\text { Si Norm. } \\
\text { Diss. Rate } \\
\left(\mathrm{g} \mathrm{m}^{-2} \mathrm{~d}^{-1}\right)\end{array}$ & $\begin{array}{l}{[\mathrm{Na}]} \\
\left(\mu \mathrm{g} \mathrm{L}{ }^{-}\right. \\
\left.{ }^{1}\right)\end{array}$ & $\begin{array}{l}\text { Na Norm. } \\
\text { Diss. Rate, } \\
\mathrm{r} \\
\left(\mathrm{g} \mathrm{m}^{-2} \mathrm{~d}^{-1}\right)\end{array}$ & $\begin{array}{l}\mathrm{r}_{\mathrm{IEX}} \\
\left(\mathrm{mol} \mathrm{Na} \mathrm{No}^{-1}\right)\end{array}$ \\
\hline A161.70.9.80B.3 & & $7.81 \mathrm{E}-05$ & 7.0 & 0.022 & $4.73 \mathrm{E}-04$ & 298 & $9.27 \mathrm{E}-01$ & 346 & $1.36 \mathrm{E}+00$ & 1,110 & $1.09 \mathrm{E}+00$ & 1,040 & $1.12 E+00$ & $<0$ \\
\hline A161.70.9.80B.5 & & $7.81 \mathrm{E}-05$ & 11.4 & 0.020 & 4.47E-04 & 248 & $8.15 \mathrm{E}-01$ & 249 & $1.04 \mathrm{E}+00$ & 880 & $9.12 \mathrm{E}-01$ & 790 & $9.00 \mathrm{E}-01$ & $<0$ \\
\hline A161.70.9.80B.7 & & $7.82 \mathrm{E}-05$ & 16.3 & 0.018 & $4.20 \mathrm{E}-04$ & 206 & $7.22 \mathrm{E}-01$ & 214 & $9.49 \mathrm{E}-01$ & 747 & $8.25 \mathrm{E}-01$ & 632 & $7.68 \mathrm{E}-01$ & $<0$ \\
\hline A161.70.9.80B.9 & & 7.77E-05 & 21.1 & 0.017 & 3.97E-04 & 153 & $5.64 \mathrm{E}-01$ & 166 & 7.75E-01 & 591 & $6.87 \mathrm{E}-01$ & 548 & $7.01 \mathrm{E}-01$ & $<0$ \\
\hline A161.70.9.80B.10 & & $7.89 \mathrm{E}-05$ & 23.1 & 0.016 & $3.84 \mathrm{E}-04$ & 193 & $7.47 \mathrm{E}-01$ & 221 & $1.08 \mathrm{E}+00$ & 764 & $9.33 \mathrm{E}-01$ & 487 & $6.54 \mathrm{E}-01$ & $<0$ \\
\hline A161.70.9.80B.11 & & $7.76 \mathrm{E}-05$ & 24.2 & 0.016 & 3.83E-04 & 150 & $5.72 \mathrm{E}-01$ & 145 & $7.00 \mathrm{E}-01$ & 542 & $6.52 \mathrm{E}-01$ & 416 & $5.50 \mathrm{E}-01$ & $<0$ \\
\hline \multicolumn{15}{|c|}{ A161 $70^{\circ} \mathrm{C} \mathrm{pH} 9$ Reactor \#5 } \\
\hline A161.70.9.C.Blank1 & & $1.86 \mathrm{E}-05$ & --- & --. & --- & [49.4] & --- & {$[75.6]$} & --- & [164] & --- & [134] & --- & --- \\
\hline A161.70.9.C.Blank2 & & $1.82 \mathrm{E}-05$ & --- & --- & --- & [49.4] & --- & {$[75.6]$} & --- & [164] & --- & [134] & --- & --- \\
\hline A161.70.9.C.Blank3 & & $1.88 \mathrm{E}-05$ & --- & --. & --- & [49.4] & --- & {$[75.6]$} & --- & [164] & --- & [134] & --- & --- \\
\hline A161.70.9.C.1 & & $1.36 \mathrm{E}-05$ & 2.0 & --- & $2.42 \mathrm{E}-04$ & 208 & $2.19 \mathrm{E}-01$ & 310 & 4.14E-01 & 789 & $2.62 \mathrm{E}-01$ & 1,060 & $3.88 \mathrm{E}-01$ & $<0$ \\
\hline A161.70.9.C.2 & & 3.83E-05 & 4.2 & --- & 2.42E-04 & 199 & $5.93 \mathrm{E}-01$ & 283 & $1.07 E+00$ & 753 & $7.08 \mathrm{E}-01$ & 937 & $9.69 \mathrm{E}-01$ & $<0$ \\
\hline A161.70.9.C.3 & & $3.88 \mathrm{E}-05$ & 7.0 & --- & $2.42 \mathrm{E}-04$ & 153 & $4.62 \mathrm{E}-01$ & 212 & $8.10 \mathrm{E}-01$ & 563 & $5.36 \mathrm{E}-01$ & 668 & $6.99 \mathrm{E}-01$ & $<0$ \\
\hline A161.70.9.C.7 & & $3.87 \mathrm{E}-05$ & 16.3 & --- & $2.42 \mathrm{E}-04$ & 74 & $2.23 \mathrm{E}-01$ & 112 & 4.27E-01 & 318 & $3.02 \mathrm{E}-01$ & 298 & $3.11 \mathrm{E}-01$ & $<0$ \\
\hline A161.70.9.C.9 & & $3.89 \mathrm{E}-05$ & 21.1 & --- & $2.42 \mathrm{E}-04$ & 61 & $1.84 \mathrm{E}-01$ & {$[75.6]$} & $2.90 \mathrm{E}-01$ & 265 & $2.53 \mathrm{E}-01$ & 225 & $2.36 \mathrm{E}-01$ & $<0$ \\
\hline A161.70.9.C.10 & & $3.88 \mathrm{E}-05$ & 23.1 & --- & $2.42 \mathrm{E}-04$ & 56 & $1.68 \mathrm{E}-01$ & [75.6] & $2.89 \mathrm{E}-01$ & 239 & $2.28 \mathrm{E}-01$ & 190 & $1.99 \mathrm{E}-01$ & $<0$ \\
\hline A161.70.9.C.11 & & $3.85 \mathrm{E}-05$ & 24.2 & --- & $2.42 \mathrm{E}-04$ & 59 & $1.77 \mathrm{E}-01$ & {$[75.6]$} & $2.87 \mathrm{E}-01$ & 262 & $2.48 \mathrm{E}-01$ & 177 & $1.84 \mathrm{E}-01$ & $<0$ \\
\hline \multicolumn{15}{|c|}{ A161 $70^{\circ} \mathrm{C} \mathrm{pH} 10$ Reactor $\# 1$} \\
\hline A161.70.10.80A.Blank1 & & $1.44 \mathrm{E}-07$ & --- & --- & --- & [49.4] & --- & {$[75.6]$} & --- & [164] & --- & [134] & --- & --- \\
\hline A161.70.10.80A.Blank2 & & 7.19E-05 & --- & --- & --- & [49.4] & --- & [75.6] & --- & [164] & --- & [134] & --- & --- \\
\hline A161.70.10.80A.Blank3 & & $2.37 \mathrm{E}-05$ & -.- & --- & -.- & [49.4] & --- & {$[75.6]$} & --- & [164] & -.- & [134] & --- & -.- \\
\hline A161.70.10.80A.1 & & $1.47 \mathrm{E}-05$ & 2.0 & 0.024 & $4.95 \mathrm{E}-04$ & 687 & $3.83 \mathrm{E}-01$ & 535 & $3.77 \mathrm{E}-01$ & 2,430 & 4.27E-01 & 1,890 & $3.65 \mathrm{E}-01$ & $<0$ \\
\hline A161.70.10.80A.2 & & $7.18 \mathrm{E}-05$ & 4.2 & 0.021 & 4.55E-04 & 710 & $2.11 \mathrm{E}+00$ & 545 & $2.05 E+00$ & 2,460 & $2.30 \mathrm{E}+00$ & 1,940 & $2.00 E+00$ & $<0$ \\
\hline A161.70.10.80A.3 & & $1.36 \mathrm{E}-05$ & 7.0 & 0.022 & $4.61 \mathrm{E}-04$ & 571 & $3.18 \mathrm{E}-01$ & 443 & $3.12 \mathrm{E}-01$ & 2,000 & $3.51 \mathrm{E}-01$ & 1,550 & 2.99E-01 & $<0$ \\
\hline A161.70.10.80A.7 & & $7.68 \mathrm{E}-05$ & 16.3 & 0.015 & $3.61 \mathrm{E}-04$ & 481 & $1.93 E+00$ & 367 & $1.86 \mathrm{E}+00$ & 1,690 & $2.13 \mathrm{E}+00$ & 1,270 & $1.76 \mathrm{E}+00$ & $<0$ \\
\hline A161.70.10.80A.9 & & 7.67E-05 & 21.1 & 0.012 & $3.09 \mathrm{E}-04$ & 456 & $2.13 E+00$ & 338 & $2.00 \mathrm{E}+00$ & 1,620 & $2.39 E+00$ & 1,220 & $1.98 E+00$ & $<0$ \\
\hline A161.70.10.80A.10 & & $7.60 \mathrm{E}-05$ & 23.1 & 0.011 & $2.94 \mathrm{E}-04$ & 440 & $2.14 \mathrm{E}+00$ & 319 & $1.97 E+00$ & 1,510 & $2.32 \mathrm{E}+00$ & 1,160 & $1.96 \mathrm{E}+00$ & $<0$ \\
\hline A161.70.10.80A.11 & & 7.47E-05 & 24.2 & 0.011 & 2.87E-04 & 420 & $2.05 E+00$ & 322 & $1.99 \mathrm{E}+00$ & 1,490 & $2.30 \mathrm{E}+00$ & 1,090 & $1.85 \mathrm{E}+00$ & $<0$ \\
\hline \multicolumn{15}{|c|}{ A161 $70^{\circ} \mathrm{C} \mathrm{pH} 10$ Reactor $\# 2$} \\
\hline A161.70.10.C.Blank1 & & $-4.81 \mathrm{E}-09$ & --- & --- & --- & [49.4] & --- & [75.6] & --- & [164] & --- & [134] & --- & --- \\
\hline A161.70.10.C.Blank2 & & $1.78 \mathrm{E}-05$ & --- & --- & --- & [49.4] & --- & {$[75.6]$} & --- & [164] & --. & [134] & --- & --- \\
\hline A161.70.10.C.Blank3 & & $6.61 \mathrm{E}-06$ & --- & --- & --- & [49.4] & --- & {$[75.6]$} & --- & [164] & --- & [134] & --- & --- \\
\hline A161.70.10.C.1 & & $2.17 \mathrm{E}-06$ & 2.0 & --. & $1.51 \mathrm{E}-04$ & 292 & $7.86 \mathrm{E}-02$ & 207 & $7.06 \mathrm{E}-02$ & 968 & $8.22 \mathrm{E}-02$ & 750 & $7.00 \mathrm{E}-02$ & $<0$ \\
\hline A161.70.10.C. 2 & & $3.50 \mathrm{E}-05$ & 4.2 & --- & $1.51 \mathrm{E}-04$ & 481 & $2.09 E+00$ & 349 & $1.92 \mathrm{E}+00$ & 1,630 & $2.24 \mathrm{E}+00$ & 1,220 & $1.84 \mathrm{E}+00$ & $<0$ \\
\hline A161.70.10.C.3 & & 3.69E-06 & 7.0 & --- & $1.51 \mathrm{E}-04$ & [49.4] & $2.27 \mathrm{E}-02$ & {$[75.6]$} & 4.39E-02 & [164] & $2.37 \mathrm{E}-02$ & 1,590 & $2.53 \mathrm{E}-01$ & $1.05 \mathrm{E}-07$ \\
\hline A161.70.10.C.7 & & $3.92 \mathrm{E}-05$ & 16.3 & -.- & $1.51 \mathrm{E}-04$ & 485 & $2.36 \mathrm{E}+00$ & 366 & $2.26 \mathrm{E}+00$ & 1,680 & $2.58 \mathrm{E}+00$ & 1,280 & $2.16 \mathrm{E}+00$ & $<0$ \\
\hline A161.70.10.C.9 & & $3.29 \mathrm{E}-05$ & 21.1 & --- & $1.51 \mathrm{E}-04$ & 383 & $1.57 E+00$ & 281 & $1.46 \mathrm{E}+00$ & 1,340 & $1.73 \mathrm{E}+00$ & 964 & $1.37 E+00$ & $<0$ \\
\hline A161.70.10.C.10 & & $4.01 \mathrm{E}-05$ & 23.1 & -.- & $1.51 \mathrm{E}-04$ & 379 & $1.89 \mathrm{E}+00$ & 270 & $1.71 \mathrm{E}+00$ & 1,260 & $1.98 \mathrm{E}+00$ & 978 & $1.69 \mathrm{E}+00$ & $<0$ \\
\hline A161.70.10.C.11 & & $3.77 \mathrm{E}-05$ & 24.2 & --. & $1.51 \mathrm{E}-04$ & 350 & $1.64 \mathrm{E}+00$ & 265 & $1.57 E+00$ & 1,210 & $1.79 \mathrm{E}+00$ & 871 & $1.41 \mathrm{E}+00$ & $<0$ \\
\hline \multicolumn{15}{|c|}{ A161 $70^{\circ} \mathrm{C} \mathrm{pH} 11$ Reactor \#1 } \\
\hline A161.70.11.80A.Blank1 & & $7.72 \mathrm{E}-05$ & --- & --- & --- & [49.4] & --- & {$[75.6]$} & --- & {$[164]$} & -- & [134] & --- & --- \\
\hline A161.70.11.80A.Blank2 & & 7.67E-05 & -.- & --. & --- & [49.4] & -.- & {$[75.6]$} & --- & [164] & --- & [134] & --- & -.- \\
\hline A161.70.11.80A.Blank3 & & $7.60 \mathrm{E}-05$ & -.- & --- & -.- & [49.4] & -.- & {$[75.6]$} & --- & [164] & --- & [134] & --- & -.- \\
\hline
\end{tabular}




\begin{tabular}{|c|c|c|c|c|c|c|c|c|c|c|c|c|c|c|}
\hline Sample ID & $\begin{array}{l}\text { Influent } \\
{[\mathrm{Si}]} \\
(\mathrm{ppm})\end{array}$ & $\begin{array}{l}\text { Flow } \\
\text { Rate, q } \\
\left(\mathrm{m}^{3} \mathrm{~d}^{-1}\right)\end{array}$ & $\begin{array}{l}\text { Time } \\
\text { (d) }\end{array}$ & $\begin{array}{l}\text { Glass } \\
\text { Mass } \\
\text { (g) }\end{array}$ & $\begin{array}{l}\text { Surface } \\
\text { Area, S } \\
\left(\mathrm{m}^{2}\right)\end{array}$ & $\begin{array}{l}{[\mathrm{Al}]} \\
\left(\mu \mathrm{L} \mathrm{L}^{-1}\right)\end{array}$ & $\begin{array}{l}\text { Al Norm. } \\
\text { Diss. Rate } \\
\left(\mathrm{g} \mathrm{m}^{-2} \mathrm{~d}^{-1}\right)\end{array}$ & $\begin{array}{l}{[B]} \\
\left(\mu g L^{-1}\right)\end{array}$ & $\begin{array}{l}\text { B Norm. } \\
\text { Diss. Rate } \\
\left(\mathrm{g} \mathrm{m}^{-2} \mathrm{~d}^{-1}\right)\end{array}$ & $\begin{array}{l}{[\mathrm{Si}]} \\
\left(\mu \mathrm{L} \mathrm{L}^{-1}\right)\end{array}$ & $\begin{array}{l}\text { Si Norm. } \\
\text { Diss. Rate } \\
\left(\mathrm{g} \mathrm{m}^{-2} \mathrm{~d}^{-1}\right)\end{array}$ & $\begin{array}{l}{[\mathrm{Na}]} \\
(\mu \mathrm{g} \mathrm{L} \\
\left.{ }^{1}\right)\end{array}$ & $\begin{array}{l}\text { Na Norm. } \\
\text { Diss. Rate, } \\
\mathrm{r} \\
\left(\mathrm{g} \mathrm{m}^{-2} \mathrm{~d}^{-1}\right)\end{array}$ & $\begin{array}{l}\mathrm{r}_{\mathrm{IEX}} \\
\left(\mathrm{mol} \mathrm{Na} \mathrm{No}^{-1}\right)\end{array}$ \\
\hline A161.70.11.80A.1 & & $6.70 \mathrm{E}-05$ & 0.9 & 0.049 & $9.98 \mathrm{E}-04$ & 763 & 9.63E-01 & 319 & $5.10 \mathrm{E}-01$ & 2,500 & $9.95 \mathrm{E}-01$ & 2,080 & $9.10 \mathrm{E}-01$ & $2.02 \mathrm{E}-07$ \\
\hline A161.70.11.80A.2 & & $7.76 \mathrm{E}-05$ & 1.2 & 0.049 & $9.94 \mathrm{E}-04$ & 827 & $1.22 \mathrm{E}+00$ & 679 & $1.26 \mathrm{E}+00$ & 3,700 & $1.72 \mathrm{E}+00$ & 3,270 & $1.67 \mathrm{E}+00$ & $2.03 E-07$ \\
\hline A161.70.11.80A.3 & & $7.32 \mathrm{E}-05$ & 2.0 & 0.047 & $9.68 \mathrm{E}-04$ & 515 & 7.33E-01 & 1,030 & $1.86 \mathrm{E}+00$ & 3,690 & $1.66 \mathrm{E}+00$ & 3,970 & $1.96 \mathrm{E}+00$ & $5.15 \mathrm{E}-08$ \\
\hline A161.70.11.80A.4 & & $7.22 \mathrm{E}-05$ & 4.2 & 0.041 & 8.87E-04 & 1,350 & $2.07 E+00$ & 1,130 & $2.19 E+00$ & 4,510 & $2.18 \mathrm{E}+00$ & 3,760 & $2.00 \mathrm{E}+00$ & $<0$ \\
\hline A161.70.11.80A.5 & & 7.12E-05 & 7.1 & 0.033 & 7.72E-04 & 1,570 & $2.73 \mathrm{E}+00$ & 1,370 & $3.01 \mathrm{E}+00$ & 5,040 & $2.76 \mathrm{E}+00$ & 4,680 & $2.82 \mathrm{E}+00$ & $<0$ \\
\hline A161.70.11.80A.6 & & $7.03 E-05$ & 9.0 & 0.029 & 7.08E-04 & 1,580 & $2.95 \mathrm{E}+00$ & 1,490 & $3.53 E+00$ & 5,300 & $3.12 \mathrm{E}+00$ & 5,230 & $3.39 \mathrm{E}+00$ & $<0$ \\
\hline A161.70.11.80A.7 & & 7.18E-05 & 11.3 & 0.023 & $6.01 \mathrm{E}-04$ & 1,620 & $3.64 \mathrm{E}+00$ & 1,530 & $4.35 \mathrm{E}+00$ & 5,480 & $3.88 \mathrm{E}+00$ & 4,970 & $3.87 \mathrm{E}+00$ & $<0$ \\
\hline A161.70.11.80A.9 & & $6.98 \mathrm{E}-05$ & 16.2 & 0.016 & $4.72 \mathrm{E}-04$ & 1,100 & $3.06 \mathrm{E}+00$ & 997 & $3.51 E+00$ & 3,790 & $3.32 \mathrm{E}+00$ & 3,270 & $3.15 \mathrm{E}+00$ & $<0$ \\
\hline A161.70.11.80A.11 & & $7.32 \mathrm{E}-05$ & 21.1 & 0.010 & 3.53E-04 & 772 & $3.01 E+00$ & 678 & $3.35 \mathrm{E}+00$ & 2,900 & $3.57 E+00$ & 2,220 & $3.00 \mathrm{E}+00$ & $<0$ \\
\hline A161.70.11.80A.12 & & $7.76 \mathrm{E}-05$ & 23.0 & 0.009 & $3.25 \mathrm{E}-04$ & 595 & $2.68 \mathrm{E}+00$ & 525 & $2.99 \mathrm{E}+00$ & 2,350 & $3.33 \mathrm{E}+00$ & 1,640 & $2.56 \mathrm{E}+00$ & $<0$ \\
\hline A161.70.11.80A.13 & & 7.42E-05 & 24.1 & 0.009 & 3.19E-04 & 462 & $2.02 E+00$ & 408 & $2.26 \mathrm{E}+00$ & 1,920 & $2.65 \mathrm{E}+00$ & 1,350 & $2.05 \mathrm{E}+00$ & $<0$ \\
\hline \multicolumn{15}{|c|}{ A161 $70^{\circ} \mathrm{C} \mathrm{pH} 11$ Reactor $\# 2$} \\
\hline A161.70.11.C.Blank1 & & $5.16 \mathrm{E}-06$ & --- & --- & --- & [49.4] & --- & [75.6] & --- & [164] & --- & [134] & --- & --- \\
\hline A161.70.11.C.Blank2 & & $9.40 \mathrm{E}-06$ & -- & --- & -- & {$[49.4]$} & -- & {$[75.6]$} & --- & {$[164]$} & -- & [134] & -- & --- \\
\hline A161.70.11.C.Blank3 & & $8.65 E-06$ & --- & --- & --- & [49.4] & --- & [75.6] & --- & [164] & --- & [134] & --- & --- \\
\hline A161.70.11.C.1 & & $1.54 \mathrm{E}-05$ & 2.0 & --- & $2.64 \mathrm{E}-04$ & 420 & $4.59 \mathrm{E}-01$ & 357 & $4.94 \mathrm{E}-01$ & 1,520 & $5.24 \mathrm{E}-01$ & 1,090 & $4.13 E-01$ & $<0$ \\
\hline A161.70.11.C.2 & & $1.69 \mathrm{E}-05$ & 4.2 & -.- & $2.64 \mathrm{E}-04$ & 633 & 7.63E-01 & 674 & $1.03 \mathrm{E}+00$ & 2,690 & $1.02 \mathrm{E}+00$ & 2,140 & $8.95 \mathrm{E}-01$ & $<0$ \\
\hline A161.70.11.C.3 & & $1.69 \mathrm{E}-05$ & 7.1 & --- & 2.64E-04 & 922 & $1.11 \mathrm{E}+00$ & 801 & $1.22 \mathrm{E}+00$ & 3,270 & $1.24 \mathrm{E}+00$ & 2,480 & $1.03 E+00$ & $<0$ \\
\hline A161.70.11.C. 4 & & $1.71 \mathrm{E}-05$ & 9.0 & --- & 2.64E-04 & 1,010 & $1.23 \mathrm{E}+00$ & 887 & $1.37 \mathrm{E}+00$ & 3,570 & $1.37 \mathrm{E}+00$ & 2,870 & $1.21 \mathrm{E}+00$ & $<0$ \\
\hline A161.70.11.C.5 & & $1.63 E-05$ & 11.3 & --- & 2.64E-04 & 1,090 & $1.27 \mathrm{E}+00$ & 962 & $1.42 \mathrm{E}+00$ & 3,660 & $1.34 \mathrm{E}+00$ & 3,110 & $1.25 \mathrm{E}+00$ & $<0$ \\
\hline A161.70.11.C.7 & & $1.63 \mathrm{E}-05$ & 16.2 & -.- & 2.64E-04 & 1,110 & $1.29 \mathrm{E}+00$ & 1,010 & $1.48 \mathrm{E}+00$ & 3,850 & $1.41 \mathrm{E}+00$ & 3,500 & $1.41 \mathrm{E}+00$ & $<0$ \\
\hline A161.70.11.C.9 & & 1.83E-05 & 21.1 & --- & 2.64E-04 & 1,210 & $1.57 \mathrm{E}+00$ & 1,150 & $1.89 \mathrm{E}+00$ & 4,200 & $1.72 \mathrm{E}+00$ & 3,700 & $1.67 \mathrm{E}+00$ & $<0$ \\
\hline A161.70.11.C.10 & & $2.07 \mathrm{E}-05$ & 23.0 & -.- & 2.64E-04 & 1,160 & $1.71 \mathrm{E}+00$ & 1,190 & $2.22 \mathrm{E}+00$ & 4,170 & $1.94 \mathrm{E}+00$ & 3,670 & $1.88 \mathrm{E}+00$ & $<0$ \\
\hline A161.70.11.C.11 & & 1.77E-05 & 24.1 & --- & 2.64E-04 & 1,120 & $1.41 \mathrm{E}+00$ & 1,120 & $1.78 \mathrm{E}+00$ & 3,970 & $1.58 \mathrm{E}+00$ & 3,460 & $1.51 \mathrm{E}+00$ & $<0$ \\
\hline \multicolumn{15}{|c|}{ A161 $70^{\circ} \mathrm{C}$ pH 12 Reactor \#1 } \\
\hline A161.70.12.80A.Blank1 & & $7.58 \mathrm{E}-05$ & --- & --- & --- & {$[49.4]$} & -- & {$[75.6]$} & --- & 396 & -- & 417 & --- & --- \\
\hline A161.70.12.80A.Blank2 & & $1.35 \mathrm{E}-05$ & -.- & -.- & --- & [49.4] & --- & [75.6] & --- & 509 & --- & 405 & --- & -.- \\
\hline A161.70.12.80A.Blank3 & & 1.06E-05 & --- & --- & --- & {$[49.4]$} & -- & [75.6] & --- & 706 & --- & 412 & --- & --- \\
\hline A161.70.12.80A.1 & & $5.81 \mathrm{E}-05$ & 0.9 & 0.047 & $9.74 \mathrm{E}-04$ & 2,100 & $2.36 \mathrm{E}+00$ & 1,360 & $1.93 E+00$ & 7,090 & $2.51 \mathrm{E}+00$ & 6,170 & $2.40 \mathrm{E}+00$ & $2.36 \mathrm{E}-07$ \\
\hline A161.70.12.80A.2 & & $5.82 E-05$ & 1.2 & 0.047 & $9.65 \mathrm{E}-04$ & 3,200 & $3.63 E+00$ & 2,440 & $3.51 \mathrm{E}+00$ & 9,490 & $3.40 \mathrm{E}+00$ & 8,420 & $3.31 \mathrm{E}+00$ & $<0$ \\
\hline A161.70.12.80A.3 & & $6.13 \mathrm{E}-05$ & 2.0 & 0.043 & $9.13 \mathrm{E}-04$ & 3,300 & $4.17 \mathrm{E}+00$ & 2,500 & $4.00 \mathrm{E}+00$ & 9,610 & $3.83 \mathrm{E}+00$ & 8,310 & $3.64 \mathrm{E}+00$ & $<0$ \\
\hline A161.70.12.80A.4 & & $5.55 \mathrm{E}-05$ & 4.2 & 0.033 & 7.59E-04 & 3,510 & $4.83 E+00$ & 2,700 & $4.71 \mathrm{E}+00$ & 10,200 & $4.43 E+00$ & 9,320 & $4.45 \mathrm{E}+00$ & $<0$ \\
\hline A161.70.12.80A.5 & & $5.26 \mathrm{E}-05$ & 7.1 & 0.024 & $6.16 \mathrm{E}-04$ & 3,140 & $5.04 \mathrm{E}+00$ & 2,370 & $4.82 E+00$ & 8,870 & $4.49 \mathrm{E}+00$ & 7,940 & $4.42 \mathrm{E}+00$ & $<0$ \\
\hline \multicolumn{15}{|c|}{ A161 $70^{\circ} \mathrm{C}$ pH 20 Reactor \#2 } \\
\hline A161.70.12.C.Blank1 & & $9.27 \mathrm{E}-06$ & --- & --- & -- & {$[49.4]$} & -- & [75.6] & --- & 376 & -- & 380 & --- & -- \\
\hline A161.70.12.C.Blank2 & & $1.06 \mathrm{E}-05$ & --- & --- & -- & {$[49.4]$} & -- & {$[75.6]$} & --- & 560 & -- & 408 & -- & --- \\
\hline A161.70.12.C.Blank3 & & $1.04 \mathrm{E}-05$ & --- & --- & -- & {$[49.4]$} & -- & [75.6] & --- & 755 & --- & 410 & -- & --- \\
\hline A161.70.12.C.1 & & $1.41 \mathrm{E}-05$ & 2.0 & --- & 2.60E-04 & 1,460 & $1.49 \mathrm{E}+00$ & 1,140 & $1.47 \mathrm{E}+00$ & 4,990 & $1.61 \mathrm{E}+00$ & 3,820 & $1.35 \mathrm{E}+00$ & $<0$ \\
\hline A161.70.12.C. 2 & & $1.35 \mathrm{E}-05$ & 4.2 & --- & 2.60E-04 & 2,770 & $2.70 \mathrm{E}+00$ & 2,150 & $2.66 \mathrm{E}+00$ & 8,630 & $2.66 \mathrm{E}+00$ & 7,210 & $2.44 \mathrm{E}+00$ & $<0$ \\
\hline A161.70.12.C.3 & & $1.20 \mathrm{E}-05$ & 7.1 & -- & 2.60E-04 & 2,790 & $2.42 \mathrm{E}+00$ & 2,180 & $2.39 \mathrm{E}+00$ & 8,490 & $2.32 \mathrm{E}+00$ & 7,750 & $2.33 \mathrm{E}+00$ & $<0$ \\
\hline A161.70.12.C.5 & & $1.01 \mathrm{E}-05$ & 11.3 & -- & 2.60E-04 & 3,020 & $2.21 \mathrm{E}+00$ & 2,480 & $2.30 \mathrm{E}+00$ & 9,100 & $2.10 \mathrm{E}+00$ & 8,500 & $2.15 \mathrm{E}+00$ & $<0$ \\
\hline A161.70.12.C.7 & & 2.00E-05 & 16.2 & -- & 2.60E-04 & 2,100 & $3.04 \mathrm{E}+00$ & 1,740 & $3.19 \mathrm{E}+00$ & 6,300 & $2.88 \mathrm{E}+00$ & 5,840 & $2.93 \mathrm{E}+00$ & $<0$ \\
\hline A161.70.12.C.9 & & 2.03E-05 & 21.1 & --- & 2.60E-04 & 1,850 & $2.72 E+00$ & 1,550 & $2.89 \mathrm{E}+00$ & 5,630 & $2.61 \mathrm{E}+00$ & 5,070 & $2.58 \mathrm{E}+00$ & $<0$ \\
\hline
\end{tabular}




\begin{tabular}{|c|c|c|c|c|c|c|c|c|c|c|c|c|c|c|}
\hline Sample ID & $\begin{array}{l}\text { Influent } \\
{[\mathrm{Si}]} \\
(\mathrm{ppm})\end{array}$ & $\begin{array}{l}\text { Flow } \\
\text { Rate, } \mathrm{q} \\
\left(\mathrm{m}^{3} \mathrm{~d}^{-1}\right)\end{array}$ & $\begin{array}{l}\text { Time } \\
\text { (d) }\end{array}$ & $\begin{array}{l}\text { Glass } \\
\text { Mass } \\
\text { (g) }\end{array}$ & $\begin{array}{l}\text { Surface } \\
\text { Area, } \mathrm{S} \\
\left(\mathrm{m}^{2}\right)\end{array}$ & $\begin{array}{l}{[\mathrm{Al}]} \\
\left(\mu \mathrm{g} \mathrm{L}^{-1}\right)\end{array}$ & $\begin{array}{l}\text { Al Norm. } \\
\text { Diss. Rate } \\
\left(\mathrm{g} \mathrm{m}^{-2} \mathrm{~d}^{-1}\right)\end{array}$ & $\begin{array}{l}{[\mathrm{B}]} \\
\left(\mu \mathrm{g} \mathrm{L}^{-1}\right)\end{array}$ & $\begin{array}{l}\text { B Norm. } \\
\text { Diss. Rate } \\
\left(\mathrm{g} \mathrm{m}^{-2} \mathrm{~d}^{-1}\right)\end{array}$ & $\begin{array}{l}{[\mathrm{Si}]} \\
\left(\mu \mathrm{g} \mathrm{L}^{-1}\right)\end{array}$ & $\begin{array}{l}\text { Si Norm. } \\
\text { Diss. Rate } \\
\left(\mathrm{g} \mathrm{m}^{-2} \mathrm{~d}^{-1}\right)\end{array}$ & $\begin{array}{l}{[\mathrm{Na}]} \\
(\mu \mathrm{g} \mathrm{L}- \\
\left.{ }^{1}\right)\end{array}$ & $\begin{array}{l}\text { Na Norm. } \\
\text { Diss. Rate, } \\
\mathrm{r} \\
\left(\mathrm{g} \mathrm{m}^{-2} \mathrm{~d}^{-1}\right)\end{array}$ & $\begin{array}{l}\mathrm{r}_{\mathrm{IEX}} \\
\left(\mathrm{mol} \mathrm{Na} \mathrm{s}^{-2} \mathrm{~s}^{-1}\right)\end{array}$ \\
\hline A161.70.12.C.10 & & $1.71 \mathrm{E}-05$ & 23.0 & $\ldots$ & $2.60 \mathrm{E}-04$ & 1,800 & $2.22 \mathrm{E}+00$ & 1,460 & $2.28 \mathrm{E}+00$ & 5,400 & $2.10 \mathrm{E}+00$ & 5,020 & $2.15 \mathrm{E}+00$ & $<0$ \\
\hline A161.70.12.C.11 & & $2.01 \mathrm{E}-05$ & 24.1 & --- & $2.60 \mathrm{E}-04$ & 1,760 & $2.55 \mathrm{E}+00$ & 1,450 & $2.66 \mathrm{E}+00$ & 5,290 & $2.42 \mathrm{E}+00$ & 4,910 & $2.47 \mathrm{E}+00$ & $<0$ \\
\hline \multicolumn{15}{|c|}{ A161 $90^{\circ} \mathrm{C}$ pH 9 Reactor \#1 } \\
\hline A161.90.9.20.Blank1 & & $7.01 \mathrm{E}-06$ & --- & --- & --- & 105 & -- & {$[75.6]$} & --- & [164] & --- & [134] & --- & --- \\
\hline A161.90.9.20.Blank2 & & $7.19 \mathrm{E}-06$ & --- & -.- & --- & 139 & --- & {$[75.6]$} & --- & [164] & --- & [134] & -.- & -.- \\
\hline A161.90.9.20.Blank3 & & $6.89 \mathrm{E}-06$ & --- & --- & --- & 134 & -- & {$[75.6]$} & --- & [164] & --- & [134] & --- & --- \\
\hline A161.90.9.20.1 & & $7.98 \mathrm{E}-06$ & 2 & 0.393 & $7.98 \mathrm{E}-03$ & 2,710 & $5.10 \mathrm{E}-02$ & 11,300 & $2.69 \mathrm{E}-01$ & 11,100 & $6.59 \mathrm{E}-02$ & 41,700 & $2.72 \mathrm{E}-01$ & $1.39 \mathrm{E}-09$ \\
\hline A161.90.9.20.2 & & $8.42 E-06$ & 4 & 0.388 & 7.92E-03 & 3,010 & $6.02 \mathrm{E}-02$ & 12,300 & 3.12E-01 & 12,100 & 7.64E-02 & 50,200 & $3.48 \mathrm{E}-01$ & $1.84 \mathrm{E}-08$ \\
\hline A161.90.9.20.3 & & $8.74 \mathrm{E}-06$ & 7 & 0.381 & $7.82 \mathrm{E}-03$ & 2,960 & $6.23 \mathrm{E}-02$ & 11,400 & 3.04E-01 & 11,800 & 7.83E-02 & 44,400 & $3.24 \mathrm{E}-01$ & $1.01 \mathrm{E}-08$ \\
\hline A161.90.9.20.4 & & $7.35 \mathrm{E}-06$ & 9 & 0.379 & $7.79 E-03$ & 2,890 & 5.13E-02 & 9,700 & $2.18 \mathrm{E}-01$ & 11,500 & $6.44 \mathrm{E}-02$ & 38,100 & $2.35 \mathrm{E}-01$ & $8.27 \mathrm{E}-09$ \\
\hline A161.90.9.20.5 & & $8.92 \mathrm{E}-06$ & 11 & 0.374 & $7.73 \mathrm{E}-03$ & 2,860 & $6.21 \mathrm{E}-02$ & 8,570 & $2.36 \mathrm{E}-01$ & 11,500 & $7.88 \mathrm{E}-02$ & 34,900 & $2.63 \mathrm{E}-01$ & $1.36 \mathrm{E}-08$ \\
\hline A161.90.9.20.6 & & $9.16 \mathrm{E}-06$ & 14 & 0.370 & 7.67E-03 & 2,760 & $6.21 \mathrm{E}-02$ & 7,080 & 2.02E-01 & 11,200 & 7.94E- 02 & 28,500 & 2.22E-01 & $1.04 \mathrm{E}-08$ \\
\hline A161.90.9.20.7 & & $8.41 \mathrm{E}-06$ & 16 & 0.368 & 7.65E-03 & 2,720 & $5.63 \mathrm{E}-02$ & 5,920 & $1.55 \mathrm{E}-01$ & 11,200 & $7.31 \mathrm{E}-02$ & 24,900 & $1.79 \mathrm{E}-01$ & $1.18 \mathrm{E}-08$ \\
\hline A161.90.9.20.8 & & $8.89 \mathrm{E}-06$ & 18 & 0.366 & 7.62E-03 & 2,660 & $5.84 \mathrm{E}-02$ & 5,310 & $1.48 \mathrm{E}-01$ & 11,100 & $7.69 \mathrm{E}-02$ & 21,800 & $1.66 \mathrm{E}-01$ & $9.21 \mathrm{E}-09$ \\
\hline A161.90.9.20.9 & & $9.69 \mathrm{E}-06$ & 21 & 0.363 & $7.57 \mathrm{E}-03$ & 2,580 & $6.21 \mathrm{E}-02$ & 4,520 & 1.38E-01 & 10,900 & $8.27 \mathrm{E}-02$ & 18,800 & $1.57 \mathrm{E}-01$ & $9.62 \mathrm{E}-09$ \\
\hline \multicolumn{15}{|c|}{ A161 $90{ }^{\circ} \mathrm{C}$ pH 9 Reactor \#2 } \\
\hline A161.90.9.40.Blank1 & & $1.94 \mathrm{E}-05$ & --- & --- & --- & 58 & --- & {$[75.6]$} & --- & [164] & --- & [134] & --- & --- \\
\hline A161.90.9.40.Blank2 & & $1.82 \mathrm{E}-05$ & -.- & -.- & -.- & 65 & --- & [75.6] & -.- & [164] & -.- & [134] & --- & -.- \\
\hline A161.90.9.40.Blank3 & & $1.88 \mathrm{E}-05$ & --- & --- & --- & [49.4] & -- & {$[75.6]$} & --- & {$[164]$} & --- & [134] & --- & --- \\
\hline A161.90.9.40.1 & & $1.71 \mathrm{E}-05$ & 2 & 0.145 & $2.97 \mathrm{E}-03$ & 1,870 & 2.03E-01 & 4,760 & $6.55 \mathrm{E}-01$ & 7,800 & 2.67E-01 & 19,300 & 7.27E-01 & 3.63E-08 \\
\hline A161.90.9.40.2 & & $1.86 \mathrm{E}-05$ & 4 & 0.141 & $2.91 \mathrm{E}-03$ & 1,980 & $2.38 \mathrm{E}-01$ & 4,720 & 7.17E-01 & 8,220 & $3.11 \mathrm{E}-01$ & 18,600 & $7.74 \mathrm{E}-01$ & $2.85 \mathrm{E}-08$ \\
\hline A161.90.9.40.3 & & $1.83 \mathrm{E}-05$ & 7 & 0.137 & $2.86 \mathrm{E}-03$ & 1,820 & 2.19E-01 & 3,370 & $5.15 \mathrm{E}-01$ & 7,500 & $2.85 \mathrm{E}-01$ & 13,400 & $5.60 \mathrm{E}-01$ & $2.29 \mathrm{E}-08$ \\
\hline A161.90.9.40.4 & & $1.81 \mathrm{E}-05$ & 9 & 0.135 & $2.84 E-03$ & 1,730 & $2.07 \mathrm{E}-01$ & 2,490 & 3.78E-01 & 7,140 & $2.70 E-01$ & 9,900 & 4.12E-01 & $1.68 \mathrm{E}-08$ \\
\hline A161.90.9.40.5 & & $1.87 \mathrm{E}-05$ & 11 & 0.134 & $2.82 \mathrm{E}-03$ & 1,650 & 2.06E-01 & 1,950 & 3.08E-01 & 6,840 & $2.69 \mathrm{E}-01$ & 8,300 & $3.59 \mathrm{E}-01$ & $2.57 \mathrm{E}-08$ \\
\hline A161.90.9.40.6 & & $1.87 E-05$ & 14 & 0.132 & $2.79 E-03$ & 1,590 & $2.00 \mathrm{E}-01$ & 1,580 & $2.52 \mathrm{E}-01$ & 6,630 & 2.63E-01 & 6,480 & $2.83 E-01$ & $1.56 \mathrm{E}-08$ \\
\hline A161.90.9.40.7 & & $1.87 \mathrm{E}-05$ & 16 & 0.131 & $2.77 \mathrm{E}-03$ & 1,550 & 1.96E-01 & 1,360 & $2.18 \mathrm{E}-01$ & 6,510 & $2.60 \mathrm{E}-01$ & 5,240 & $2.30 \mathrm{E}-01$ & $6.01 \mathrm{E}-09$ \\
\hline A161.90.9.40.8 & & $1.90 \mathrm{E}-05$ & 18 & 0.130 & $2.76 \mathrm{E}-03$ & 1,530 & $1.98 \mathrm{E}-01$ & 1,300 & 2.13E-01 & 6,450 & 2.63E-01 & 5,020 & $2.25 \mathrm{E}-01$ & $6.13 E-09$ \\
\hline A161.90.9.40.9 & & $1.87 \mathrm{E}-05$ & 21 & 0.128 & $2.73 \mathrm{E}-03$ & 1,520 & 1.96E-01 & 1,240 & 2.02E-01 & 6,380 & $2.59 \mathrm{E}-01$ & 4,720 & $2.11 \mathrm{E}-01$ & $4.28 \mathrm{E}-09$ \\
\hline \multicolumn{15}{|c|}{ A161 $90^{\circ} \mathrm{C}$ pH 9 Reactor \#3 } \\
\hline A161.90.9.80.Blank1 & & 7.33E-05 & --- & --- & --- & [49.4] & --- & {$[75.6]$} & --- & [164] & --- & [134] & --- & --- \\
\hline A161.90.9.80.Blank2 & & $7.41 \mathrm{E}-05$ & --- & --- & --- & [49.4] & -- & {$[75.6]$} & --- & [164] & --- & [134] & --- & --- \\
\hline A161.90.9.80.Blank3 & & $7.41 \mathrm{E}-05$ & --- & -.- & -.- & [49.4] & --- & {$[75.6]$} & --- & [164] & --- & [134] & --- & --- \\
\hline A161.90.9.80.1 & & 7.26E-05 & 2 & 0.088 & $1.86 \mathrm{E}-03$ & 1,100 & 8.07E-01 & 2,110 & $1.96 \mathrm{E}+00$ & 4,520 & $1.05 E+00$ & 7,990 & $2.03 E+00$ & $3.62 \mathrm{E}-08$ \\
\hline A161.90.9.80.2 & & $7.52 \mathrm{E}-05$ & 4 & 0.087 & $1.84 \mathrm{E}-03$ & 861 & $6.62 \mathrm{E}-01$ & 1,090 & $1.06 \mathrm{E}+00$ & 3,470 & $8.41 E-01$ & 4,190 & $1.12 E+00$ & $2.80 \mathrm{E}-08$ \\
\hline A161.90.9.80.3 & & 7.46E-05 & 7 & 0.083 & $1.79 \mathrm{E}-03$ & 780 & $6.12 \mathrm{E}-01$ & 743 & 7.38E-01 & 3,350 & $8.29 \mathrm{E}-01$ & 2,810 & $7.64 \mathrm{E}-01$ & $1.31 \mathrm{E}-08$ \\
\hline A161.90.9.80.4 & & $7.48 \mathrm{E}-05$ & 9 & 0.081 & $1.76 \mathrm{E}-03$ & 756 & $6.03 \mathrm{E}-01$ & 645 & $6.51 \mathrm{E}-01$ & 3,180 & $8.00 E-01$ & 2,400 & $6.64 \mathrm{E}-01$ & $6.11 \mathrm{E}-09$ \\
\hline A161.90.9.80.5 & & $7.52 \mathrm{E}-05$ & 11 & 0.079 & $1.73 \mathrm{E}-03$ & 724 & $5.92 \mathrm{E}-01$ & 615 & 6.37E-01 & 3,090 & 7.97E-01 & 2,240 & $6.35 \mathrm{E}-01$ & $<0$ \\
\hline A161.90.9.80.6 & & 7.57E-05 & 14 & 0.076 & 1.68E-03 & 718 & $6.08 \mathrm{E}-01$ & 600 & $6.44 \mathrm{E}-01$ & 3,040 & $8.12 \mathrm{E}-01$ & 2,210 & $6.49 \mathrm{E}-01$ & $2.70 \mathrm{E}-09$ \\
\hline A161.90.9.80.7 & & 7.57E-05 & 16 & 0.073 & $1.65 \mathrm{E}-03$ & 710 & $6.14 \mathrm{E}-01$ & 615 & $6.73 \mathrm{E}-01$ & 3,060 & $8.34 \mathrm{E}-01$ & 2,210 & $6.62 \mathrm{E}-01$ & $<0$ \\
\hline A161.90.9.80.8 & & $7.55 \mathrm{E}-05$ & 18 & 0.072 & $1.62 \mathrm{E}-03$ & 693 & $6.08 \mathrm{E}-01$ & 589 & $6.55 \mathrm{E}-01$ & 2,940 & $8.14 \mathrm{E}-01$ & 2,170 & $6.60 \mathrm{E}-01$ & $2.83 \mathrm{E}-09$ \\
\hline A161.90.9.80.9 & & $7.58 \mathrm{E}-05$ & 21 & 0.068 & $1.57 \mathrm{E}-03$ & 693 & $6.31 \mathrm{E}-01$ & 608 & 7.01E-01 & 2,910 & $8.35 \mathrm{E}-01$ & 2,180 & $6.88 \mathrm{E}-01$ & $<0$ \\
\hline \multicolumn{15}{|c|}{ A161 $90^{\circ} \mathrm{C}$ pH 9 Reactor $\# 4$} \\
\hline A161.90.9.160.Blank1 & & $1.52 \mathrm{E}-04$ & --- & -.- & --- & [49.4] & --- & {$[75.6]$} & --- & [164] & --- & [134] & --- & -.- \\
\hline
\end{tabular}




\begin{tabular}{|c|c|c|c|c|c|c|c|c|c|c|c|c|c|c|}
\hline Sample ID & $\begin{array}{l}\text { Influent } \\
{[\mathrm{Si}]} \\
(\mathrm{ppm})\end{array}$ & $\begin{array}{l}\text { Flow } \\
\text { Rate, q } \\
\left(\mathrm{m}^{3} \mathrm{~d}^{-1}\right)\end{array}$ & $\begin{array}{l}\text { Time } \\
\text { (d) }\end{array}$ & $\begin{array}{l}\text { Glass } \\
\text { Mass } \\
\text { (g) }\end{array}$ & $\begin{array}{l}\text { Surface } \\
\text { Area, S } \\
\left(\mathrm{m}^{2}\right)\end{array}$ & $\begin{array}{l}{[\mathrm{Al}]} \\
\left(\mu \mathrm{L} \mathrm{L}^{-1}\right)\end{array}$ & $\begin{array}{l}\text { Al Norm. } \\
\text { Diss. Rate } \\
\left(\mathrm{g} \mathrm{m}^{-2} \mathrm{~d}^{-1}\right)\end{array}$ & $\begin{array}{l}{[B]} \\
\left(\mu g L^{-1}\right)\end{array}$ & $\begin{array}{l}\text { B Norm. } \\
\text { Diss. Rate } \\
\left(\mathrm{g} \mathrm{m}^{-2} \mathrm{~d}^{-1}\right)\end{array}$ & $\begin{array}{l}{[\mathrm{Si}]} \\
\left(\mu \mathrm{g} \mathrm{L}^{-1}\right)\end{array}$ & $\begin{array}{l}\text { Si Norm. } \\
\text { Diss. Rate } \\
\left(\mathrm{g} \mathrm{m}^{-2} \mathrm{~d}^{-1}\right)\end{array}$ & $\begin{array}{l}{[\mathrm{Na}]} \\
(\mu \mathrm{g} \mathrm{L} \\
\left.{ }^{1}\right)\end{array}$ & $\begin{array}{l}\text { Na Norm. } \\
\text { Diss. Rate, } \\
\mathrm{r} \\
\left(\mathrm{g} \mathrm{m}^{-2} \mathrm{~d}^{-1}\right)\end{array}$ & $\begin{array}{l}\mathrm{r}_{\mathrm{IEX}} \\
\left(\mathrm{mol} \mathrm{Na} \mathrm{No}^{-1}\right)\end{array}$ \\
\hline A161.90.9.160.Blank2 & & $1.51 \mathrm{E}-04$ & --- & -- & --- & {$[49.4]$} & --- & {$[75.6]$} & --- & [164] & --- & [134] & --- & --- \\
\hline A161.90.9.160.Blank3 & & $1.52 \mathrm{E}-04$ & --- & --- & --- & {$[49.4]$} & -- & {$[75.6]$} & --- & {$[164]$} & -- & {$[134]$} & --- & --- \\
\hline A161.90.9.160.1 & & $1.47 \mathrm{E}-04$ & 2 & 0.041 & $8.92 \mathrm{E}-04$ & 615 & $1.91 \mathrm{E}+00$ & 857 & $3.37 E+00$ & 2,550 & $2.50 \mathrm{E}+00$ & 3,200 & $3.44 E+00$ & $3.76 \mathrm{E}-08$ \\
\hline A161.90.9.160.2 & & $1.52 \mathrm{E}-04$ & 4 & 0.040 & $8.72 \mathrm{E}-04$ & 433 & $1.42 \mathrm{E}+00$ & 439 & $1.82 \mathrm{E}+00$ & 1,710 & $1.76 \mathrm{E}+00$ & 1,600 & $1.81 \mathrm{E}+00$ & $<0$ \\
\hline A161.90.9.160.3 & & $1.51 \mathrm{E}-04$ & 7 & 0.036 & $8.21 \mathrm{E}-04$ & 385 & $1.34 \mathrm{E}+00$ & 343 & $1.51 E+00$ & 1,500 & $1.64 E+00$ & 1,270 & $1.53 E+00$ & $1.03 E-08$ \\
\hline A161.90.9.160.4 & & $1.52 \mathrm{E}-04$ & 9 & 0.034 & 7.90E-04 & 379 & $1.37 \mathrm{E}+00$ & 328 & $1.50 E+00$ & 1,460 & $1.67 \mathrm{E}+00$ & 1,180 & $1.48 \mathrm{E}+00$ & $<0$ \\
\hline A161.90.9.160.5 & & $1.51 \mathrm{E}-04$ & 11 & 0.032 & 7.51E-04 & 369 & $1.40 \mathrm{E}+00$ & 316 & $1.52 E+00$ & 1,420 & $1.70 \mathrm{E}+00$ & 1,160 & $1.52 \mathrm{E}+00$ & 3.77E-09 \\
\hline A161.90.9.160.6 & & $1.52 \mathrm{E}-04$ & 14 & 0.028 & $6.96 \mathrm{E}-04$ & 372 & $1.53 \mathrm{E}+00$ & 308 & $1.60 \mathrm{E}+00$ & 1,400 & $1.81 \mathrm{E}+00$ & 1,150 & $1.64 \mathrm{E}+00$ & $1.78 \mathrm{E}-08$ \\
\hline A161.90.9.160.7 & & $1.52 \mathrm{E}-04$ & 16 & 0.026 & $6.64 \mathrm{E}-04$ & 355 & $1.53 E+00$ & 294 & $1.60 \mathrm{E}+00$ & 1,360 & $1.84 \mathrm{E}+00$ & 1,060 & $1.58 \mathrm{E}+00$ & $<0$ \\
\hline A161.90.9.160.8 & & $1.51 \mathrm{E}-04$ & 18 & 0.025 & $6.33 \mathrm{E}-04$ & 326 & $1.47 \mathrm{E}+00$ & 279 & $1.59 E+00$ & 1,210 & $1.72 \mathrm{E}+00$ & 997 & $1.55 \mathrm{E}+00$ & $<0$ \\
\hline A161.90.9.160.9 & & $1.52 \mathrm{E}-04$ & 21 & 0.022 & $5.80 \mathrm{E}-04$ & 317 & $1.56 \mathrm{E}+00$ & 265 & $1.65 \mathrm{E}+00$ & 1,170 & $1.81 \mathrm{E}+00$ & 944 & $1.61 \mathrm{E}+00$ & $<0$ \\
\hline \multicolumn{15}{|c|}{ A161 $90^{\circ} \mathrm{C} \mathrm{pH} 9$ Reactor $\# 5$} \\
\hline A161.90.9.C.Blank1 & & 2.89E-05 & -.- & -.- & -.- & [49.4] & --- & [75.6] & -.- & [164] & -.- & [134] & -.- & -.- \\
\hline A161.90.9.C.Blank2 & & $2.38 \mathrm{E}-05$ & -- & -- & -- & {$[49.4]$} & -- & {$[75.6]$} & --- & {$[164]$} & -- & [134] & -- & --- \\
\hline A161.90.9.C.Blank3 & & $3.78 \mathrm{E}-05$ & --- & --- & --- & [49.4] & --- & [75.6] & --- & [164] & --- & [134] & --- & --- \\
\hline A161.90.9.C.1 & & $3.10 \mathrm{E}-05$ & 2 & --- & $4.30 \mathrm{E}-04$ & 667 & $9.05 \mathrm{E}-01$ & 859 & $1.48 \mathrm{E}+00$ & 2,770 & $1.19 \mathrm{E}+00$ & 3,310 & $1.56 \mathrm{E}+00$ & $4.08 \mathrm{E}-08$ \\
\hline A161.90.9.C.2 & & $3.74 \mathrm{E}-05$ & 4 & -.- & 4.30E-04 & 634 & $1.04 \mathrm{E}+00$ & 717 & $1.49 \mathrm{E}+00$ & 2,650 & $1.37 \mathrm{E}+00$ & 2,710 & $1.54 \mathrm{E}+00$ & $2.60 \mathrm{E}-08$ \\
\hline A161.90.9.C.3 & & 3.13E-05 & 7 & --- & 4.30E-04 & 618 & $8.46 \mathrm{E}-01$ & 587 & $1.02 \mathrm{E}+00$ & 2,550 & $1.10 \mathrm{E}+00$ & 2,240 & $1.06 \mathrm{E}+00$ & $2.29 \mathrm{E}-08$ \\
\hline A161.90.9.C.4 & & $2.49 \mathrm{E}-05$ & 9 & --- & 4.30E-04 & 670 & 7.31E-01 & 620 & 8.57E-01 & 2,800 & 9.63E-01 & 2,280 & $8.62 \mathrm{E}-01$ & $2.90 \mathrm{E}-09$ \\
\hline A161.90.9.C.5 & & 3.50E-05 & 11 & --- & 4.30E-04 & 633 & $9.71 \mathrm{E}-01$ & 562 & $1.09 \mathrm{E}+00$ & 2,640 & $1.28 \mathrm{E}+00$ & 2,120 & $1.13 E+00$ & $1.80 \mathrm{E}-08$ \\
\hline A161.90.9.C.6 & & $3.80 \mathrm{E}-05$ & 14 & -.- & 4.30E-04 & 591 & $9.82 \mathrm{E}-01$ & 549 & $1.16 \mathrm{E}+00$ & 2,470 & $1.29 \mathrm{E}+00$ & 1,960 & $1.13 \mathrm{E}+00$ & $<0$ \\
\hline A161.90.9.C.7 & & 3.80E-05 & 16 & --- & 4.30E-04 & 593 & $9.85 \mathrm{E}-01$ & 544 & $1.14 \mathrm{E}+00$ & 2,450 & $1.28 \mathrm{E}+00$ & 1,960 & $1.13 E+00$ & $<0$ \\
\hline A161.90.9.C.8 & & $3.68 \mathrm{E}-05$ & 18 & -.- & 4.30E-04 & 594 & 9.57E-01 & 549 & $1.12 E+00$ & 2,510 & $1.28 \mathrm{E}+00$ & 1,820 & $1.02 \mathrm{E}+00$ & $<0$ \\
\hline A161.90.9.C.9 & & $3.62 E-05$ & 21 & --- & 4.30E-04 & 610 & $9.67 \mathrm{E}-01$ & 557 & $1.12 \mathrm{E}+00$ & 2,510 & $1.25 E+00$ & 1,970 & $1.08 \mathrm{E}+00$ & $<0$ \\
\hline \multicolumn{15}{|c|}{ A161 $90^{\circ} \mathrm{C}$ pH 10 Reactor \#1 } \\
\hline A161.90.10.160.Blank1 & & $1.56 \mathrm{E}-04$ & --- & --- & --- & {$[49.4]$} & -- & {$[75.6]$} & --- & {$[164]$} & --- & [134] & --- & --- \\
\hline A161.90.10.160.Blank2 & & $1.50 \mathrm{E}-04$ & -.- & -.- & --- & [49.4] & --- & [75.6] & --- & [164] & --- & [134] & --- & -.- \\
\hline A161.90.10.160.Blank3 & & $1.51 \mathrm{E}-04$ & --- & --- & --- & {$[49.4]$} & -- & {$[75.6]$} & --- & {$[164]$} & --- & {$[134]$} & --- & --- \\
\hline A161.90.10.160.1 & & $1.47 \mathrm{E}-04$ & 2 & 0.043 & 9.17E-04 & 777 & $2.34 \mathrm{E}+00$ & 652 & $2.49 \mathrm{E}+00$ & 2,790 & $2.65 \mathrm{E}+00$ & 3,170 & $3.31 \mathrm{E}+00$ & 4.14E-07 \\
\hline A161.90.10.160.2 & & 1.47E-04 & 4 & 0.038 & 8.52E-04 & 793 & $2.57 \mathrm{E}+00$ & 663 & $2.72 E+00$ & 2,820 & $2.88 \mathrm{E}+00$ & 2,810 & $3.16 \mathrm{E}+00$ & $2.20 \mathrm{E}-07$ \\
\hline A161.90.10.160.3 & & $1.48 \mathrm{E}-04$ & 7 & 0.032 & 7.57E-04 & 716 & $2.63 E+00$ & 599 & $2.78 E+00$ & 2,730 & $3.16 \mathrm{E}+00$ & 2,940 & $3.74 \mathrm{E}+00$ & 4.81E-07 \\
\hline A161.90.10.160.4 & & $1.47 \mathrm{E}-04$ & 9 & 0.029 & 7.09E-04 & 638 & $2.49 E+00$ & 547 & $2.70 \mathrm{E}+00$ & 2,460 & $3.03 E+00$ & 2,340 & $3.17 \mathrm{E}+00$ & 2.33E-07 \\
\hline A161.90.10.160.5 & & $1.48 \mathrm{E}-04$ & 11 & 0.026 & $6.51 \mathrm{E}-04$ & 592 & $2.54 \mathrm{E}+00$ & 474 & $2.58 \mathrm{E}+00$ & 2,180 & $2.95 \mathrm{E}+00$ & 2,100 & $3.12 \mathrm{E}+00$ & $2.76 \mathrm{E}-07$ \\
\hline A161.90.10.160.6 & & $1.48 \mathrm{E}-04$ & 14 & 0.021 & $5.70 \mathrm{E}-04$ & 513 & $2.51 E+00$ & 438 & $2.71 E+00$ & 1,950 & $3.01 \mathrm{E}+00$ & 1,820 & $3.09 \mathrm{E}+00$ & $1.88 \mathrm{E}-07$ \\
\hline A161.90.10.160.7 & & $1.53 E-04$ & 16 & 0.019 & $5.34 \mathrm{E}-04$ & 514 & $2.76 \mathrm{E}+00$ & 342 & $2.33 \mathrm{E}+00$ & 1,570 & $2.66 \mathrm{E}+00$ & 1,520 & $2.83 E+00$ & 2.54E-07 \\
\hline A161.90.10.160.8 & & $1.54 \mathrm{E}-04$ & 18 & 0.017 & 4.99E-04 & 449 & $2.61 E+00$ & 299 & $2.20 \mathrm{E}+00$ & 1,370 & $2.51 E+00$ & 1,300 & $2.62 \mathrm{E}+00$ & $2.11 \mathrm{E}-07$ \\
\hline A161.90.10.160.9 & & $1.54 \mathrm{E}-04$ & 21 & 0.014 & $4.31 \mathrm{E}-04$ & 347 & $2.34 \mathrm{E}+00$ & 290 & $2.47 \mathrm{E}+00$ & 1,340 & $2.84 \mathrm{E}+00$ & 1,440 & $3.36 \mathrm{E}+00$ & 4.47E-07 \\
\hline \multicolumn{15}{|c|}{ A161 $900^{\circ} \mathrm{C} \mathrm{pH} 10$ Reactor $\# 2$} \\
\hline A161.90.10.C.Blank1 & & 3.24E-05 & --- & --- & --- & {$[49.4]$} & --- & [75.6] & --- & [164] & --- & [134] & --- &.-- \\
\hline A161.90.10.C.Blank2 & & 3.54E-05 & --- & --- & --- & {$[49.4]$} & -- & {$[75.6]$} & --- & {$[164]$} & --- & {$[134]$} & -- & --- \\
\hline A161.90.10.C.Blank3 & & 3.76E-05 & --- & --- & --- & {$[49.4]$} & --- & [75.6] & --- & [164] & --- & {$[134]$} & --- & --- \\
\hline A161.90.10.C.1 & & 3.64E-05 & 2 & --- & 4.38E-04 & 1,000 & $1.56 \mathrm{E}+00$ & 825 & $1.63 E+00$ & 3,550 & $1.75 E+00$ & 3,570 & $1.94 \mathrm{E}+00$ & 1.52E-07 \\
\hline A161.90.10.C.2 & & 3.70E-05 & 4 & -.- & $4.38 \mathrm{E}-04$ & 1,240 & $1.97 \mathrm{E}+00$ & 1,040 & $2.09 \mathrm{E}+00$ & 4,500 & $2.26 \mathrm{E}+00$ & 4,920 & $2.71 \mathrm{E}+00$ & $3.11 E-07$ \\
\hline
\end{tabular}




\begin{tabular}{|c|c|c|c|c|c|c|c|c|c|c|c|c|c|c|}
\hline Sample ID & $\begin{array}{l}\text { Influent } \\
\text { [Si] } \\
\text { (ppm) }\end{array}$ & $\begin{array}{l}\text { Flow } \\
\text { Rate, } q \\
\left(\mathrm{~m}^{3} \mathrm{~d}^{-1}\right)\end{array}$ & $\begin{array}{l}\text { Time } \\
\text { (d) }\end{array}$ & $\begin{array}{l}\text { Glass } \\
\text { Mass } \\
\text { (g) }\end{array}$ & $\begin{array}{l}\text { Surface } \\
\text { Area, S } \\
\left(\mathrm{m}^{2}\right)\end{array}$ & $\begin{array}{l}{[\mathrm{Al}]} \\
\left(\mu \mathrm{g} \mathrm{L}^{-1}\right)\end{array}$ & $\begin{array}{l}\text { Al Norm. } \\
\text { Diss. Rate } \\
\left(\mathrm{g} \mathrm{m}^{-2} \mathrm{~d}^{-1}\right)\end{array}$ & $\begin{array}{l}{[\mathrm{B}]} \\
\left(\mu \mathrm{L}^{-1}\right)\end{array}$ & $\begin{array}{l}\text { B Norm. } \\
\text { Diss. Rate } \\
\left(\mathrm{g} \mathrm{m}^{-2} \mathrm{~d}^{-1}\right)\end{array}$ & $\begin{array}{l}{[\mathrm{Si}]} \\
\left(\mu \mathrm{g} \mathrm{L}^{-1}\right)\end{array}$ & $\begin{array}{l}\text { Si Norm. } \\
\text { Diss. Rate } \\
\left(\mathrm{g} \mathrm{m}^{-2} \mathrm{~d}^{-1}\right)\end{array}$ & $\begin{array}{l}{[\mathrm{Na}]} \\
(\mu \mathrm{g} \mathrm{L} \\
\left.{ }^{1}\right)\end{array}$ & $\begin{array}{l}\text { Na Norm. } \\
\text { Diss. Rate, } \\
\mathrm{r} \\
\left(\mathrm{g} \mathrm{m}^{-2} \mathrm{~d}^{-1}\right)\end{array}$ & $\begin{array}{l}\mathrm{r}_{\mathrm{IEX}} \\
\left(\mathrm{mol} \mathrm{Na}{ }^{-2} \mathrm{~s}^{-1}\right)\end{array}$ \\
\hline A161.90.10.C.3 & & $3.74 \mathrm{E}-05$ & 7 & --- & $4.38 \mathrm{E}-04$ & 1,160 & $1.86 \mathrm{E}+00$ & 937 & $1.91 \mathrm{E}+00$ & 4,110 & $2.08 \mathrm{E}+00$ & 4,290 & $2.39 \mathrm{E}+00$ & $2.43 \mathrm{E}-07$ \\
\hline A161.90.10.C.4 & & 3.73E-05 & 9 & --- & $4.38 \mathrm{E}-04$ & 1,180 & $1.89 \mathrm{E}+00$ & 999 & $2.03 E+00$ & 4,260 & $2.15 \mathrm{E}+00$ & 4,280 & $2.38 \mathrm{E}+00$ & $1.76 \mathrm{E}-07$ \\
\hline A161.90.10.C.5 & & $3.80 E-05$ & 11 & --- & $4.38 \mathrm{E}-04$ & 1,190 & $1.95 \mathrm{E}+00$ & 1,000 & $2.07 \mathrm{E}+00$ & 4,380 & $2.26 \mathrm{E}+00$ & 3,920 & $2.22 \mathrm{E}+00$ & $7.62 \mathrm{E}-08$ \\
\hline A161.90.10.C.6 & & $3.77 \mathrm{E}-05$ & 14 & -.- & $4.38 \mathrm{E}-04$ & 1,210 & $1.96 \mathrm{E}+00$ & 1,010 & $2.07 \mathrm{E}+00$ & 4,430 & $2.26 \mathrm{E}+00$ & 4,370 & $2.45 \mathrm{E}+00$ & $1.92 \mathrm{E}-07$ \\
\hline A161.90.10.c.7 & & 3.43E-05 & 16 & --- & $4.38 \mathrm{E}-04$ & 1,190 & $1.75 \mathrm{E}+00$ & 985 & $1.84 \mathrm{E}+00$ & 4,260 & $1.98 \mathrm{E}+00$ & 4,240 & $2.17 \mathrm{E}+00$ & $1.65 \mathrm{E}-07$ \\
\hline A161.90.10.C.8 & & $2.93 \mathrm{E}-05$ & 18 & -.- & $4.38 \mathrm{E}-04$ & 1,170 & 1.47E+00 & 972 & $1.55 \mathrm{E}+00$ & 4,210 & $1.67 \mathrm{E}+00$ & 4,110 & $1.79 \mathrm{E}+00$ & $1.23 \mathrm{E}-07$ \\
\hline A161.90.10.C.9 & & 3.99E-05 & 21 & --- & $4.38 \mathrm{E}-04$ & 1,160 & $1.99 \mathrm{E}+00$ & 956 & $2.08 \mathrm{E}+00$ & 4,150 & $2.24 E+00$ & 4,100 & $2.44 \mathrm{E}+00$ & $1.82 \mathrm{E}-07$ \\
\hline \multicolumn{15}{|c|}{ A161 $900^{\circ} \mathrm{C}$ pH 11 Reactor \#1 } \\
\hline A161.90.11.160.Blank1 & & 1.44E-04 & --- & --- & --- & [49.4] & --- & {$[75.6]$} & --- & [164] & --- & [134] & --- & --- \\
\hline A161.90.11.160.Blank2 & & $1.41 \mathrm{E}-04$ & --- & --- & -.- & [49.4] & --- & {$[75.6]$} & --- & [164] & --. & [134] & --- & --- \\
\hline A161.90.11.160.Blank3 & & $1.44 \mathrm{E}-04$ & --- & --- & --- & [49.4] & --- & {$[75.6]$} & --- & [164] & --- & [134] & --- & --- \\
\hline A161.90.11.160.1 & & $1.44 \mathrm{E}-04$ & 1 & 0.093 & $1.91 \mathrm{E}-03$ & 1,450 & $2.05 E+00$ & 1,260 & $2.26 \mathrm{E}+00$ & 4,890 & $2.19 E+00$ & 5,020 & $2.47 E+00$ & $1.03 \mathrm{E}-07$ \\
\hline A161.90.11.160.2 & & $1.51 \mathrm{E}-04$ & 1 & 0.090 & $1.87 \mathrm{E}-03$ & 2,250 & $3.43 E+00$ & 2,240 & $4.32 \mathrm{E}+00$ & 7,920 & $3.80 E+00$ & 8,520 & $4.50 E+00$ & 8.97E-08 \\
\hline A161.90.11.160.3 & & $1.48 \mathrm{E}-04$ & 2 & 0.080 & $1.73 \mathrm{E}-03$ & 2,380 & $3.82 \mathrm{E}+00$ & 2,460 & $5.01 \mathrm{E}+00$ & 8,550 & $4.33 E+00$ & 9,440 & $5.26 \mathrm{E}+00$ & $1.27 \mathrm{E}-07$ \\
\hline A161.90.11.160.4 & & $1.54 \mathrm{E}-04$ & 4 & 0.060 & $1.43 \mathrm{E}-03$ & 2,030 & $4.11 \mathrm{E}+00$ & 2,130 & $5.46 \mathrm{E}+00$ & 7,480 & $4.77 \mathrm{E}+00$ & 8,190 & $5.74 E+00$ & $1.45 \mathrm{E}-07$ \\
\hline A161.90.11.160.5 & & $1.54 \mathrm{E}-04$ & 7 & 0.047 & $1.22 \mathrm{E}-03$ & 1,250 & $2.97 E+00$ & 1,370 & $4.12 \mathrm{E}+00$ & 4,830 & $3.62 E+00$ & 5,250 & $4.33 \mathrm{E}+00$ & $1.02 \mathrm{E}-07$ \\
\hline A161.90.11.160.6 & & $1.52 E-04$ & 9 & 0.043 & $1.14 \mathrm{E}-03$ & 943 & $2.36 \mathrm{E}+00$ & 1,050 & $3.33 \mathrm{E}+00$ & 3,710 & $2.93 \mathrm{E}+00$ & 3,890 & $3.38 \mathrm{E}+00$ & $2.38 \mathrm{E}-08$ \\
\hline A161.90.11.160.7 & & $1.52 E-04$ & 11 & 0.036 & $1.02 \mathrm{E}-03$ & 784 & $2.20 \mathrm{E}+00$ & 866 & $3.08 \mathrm{E}+00$ & 3,330 & $2.95 \mathrm{E}+00$ & 3,220 & $3.14 \mathrm{E}+00$ & $2.78 \mathrm{E}-08$ \\
\hline A161.90.11.160.8 & & $1.53 \mathrm{E}-04$ & 14 & 0.029 & $8.76 \mathrm{E}-04$ & 680 & $2.23 \mathrm{E}+00$ & 718 & $2.98 \mathrm{E}+00$ & 2,900 & $3.00 \mathrm{E}+00$ & 2,720 & $3.09 \mathrm{E}+00$ & $5.57 \mathrm{E}-08$ \\
\hline A161.90.11.160.9 & & $1.53 \mathrm{E}-04$ & 16 & 0.025 & $8.08 \mathrm{E}-04$ & 577 & $2.05 E+00$ & 568 & $2.56 \mathrm{E}+00$ & 2,420 & $2.71 \mathrm{E}+00$ & 2,170 & $2.68 \mathrm{E}+00$ & $5.91 \mathrm{E}-08$ \\
\hline \multicolumn{15}{|c|}{ A161 $90^{\circ} \mathrm{C} \mathrm{pH} 11$ Reactor $\# 2$} \\
\hline A161.90.11.C.Blank1 & & $4.12 \mathrm{E}-06$ & --- & --- & --- & [49.4] & --- & {$[75.6]$} & --- & [164] & --- & [134] & --- & --- \\
\hline A161.90.11.C.Blank3 & & $1.04 \mathrm{E}-06$ & -.- & --. & --- & {$[49.4]$} & --- & {$[75.6]$} & --- & [164] & --- & [134] & --- & --. \\
\hline A161.90.11.C.1 & & $3.36 \mathrm{E}-06$ & 2 & --- & 0 & 2,220 & 0 & 1,910 & 0 & 12,200 & 1 & 16,700 & $8.55 \mathrm{E}-01$ & $2.51 \mathrm{E}-07$ \\
\hline A161.90.11.C.2 & & $2.73 \mathrm{E}-06$ & 4 & -.- & $4.28 \mathrm{E}-04$ & 4,230 & 5.07E-01 & 4,030 & $6.11 \mathrm{E}-01$ & 23,500 & 8.88E-01 & 31,200 & $1.30 \mathrm{E}+00$ & 3.45E-07 \\
\hline A161.90.11.C.3 & & $1.34 \mathrm{E}-06$ & 7 & --- & $4.28 \mathrm{E}-04$ & 5,100 & 3.01E-01 & 5,410 & 4.04E-01 & 29,400 & $5.47 \mathrm{E}-01$ & 42,700 & $8.73 E-01$ & $2.36 \mathrm{E}-07$ \\
\hline A161.90.11.C.4 & & $3.05 \mathrm{E}-06$ & 9 & -.- & $4.28 \mathrm{E}-04$ & 5,780 & 7.74E-01 & 6,660 & $1.13 E+00$ & 34,900 & $1.47 \mathrm{E}+00$ & 47,900 & $2.22 \mathrm{E}+00$ & $5.51 \mathrm{E}-07$ \\
\hline A161.90.11.C.5 & & $3.01 E-06$ & 11 & --- & $4.28 \mathrm{E}-04$ & 5,830 & 7.71E-01 & 6,990 & $1.17 \mathrm{E}+00$ & 35,900 & $1.50 E+00$ & 49,900 & $2.29 \mathrm{E}+00$ & $5.62 \mathrm{E}-07$ \\
\hline A161.90.11.C.6 & & $1.61 \mathrm{E}-06$ & 14 & --- & $4.28 \mathrm{E}-04$ & 6,040 & 4.28E-01 & 7,600 & $6.82 \mathrm{E}-01$ & 37,700 & 8.42E-01 & 53,500 & $1.31 E+00$ & $3.18 \mathrm{E}-07$ \\
\hline A161.90.11.C.7 & & 1.33E-06 & 16 & --- & $4.28 \mathrm{E}-04$ & 6,110 & 3.57E-01 & 8,090 & $5.98 \mathrm{E}-01$ & 39,000 & $7.18 \mathrm{E}-01$ & 57,400 & $1.16 \mathrm{E}+00$ & $2.84 \mathrm{E}-07$ \\
\hline A161.90.11.C.8 & & $1.10 \mathrm{E}-06$ & 18 & --- & $4.28 \mathrm{E}-04$ & 6,240 & 3.01E-01 & 8,320 & $5.08 \mathrm{E}-01$ & 40,100 & $6.10 \mathrm{E}-01$ & 58,900 & $9.85 \mathrm{E}-01$ & $2.40 \mathrm{E}-07$ \\
\hline A161.90.11.C.9 & & 4.22E-07 & 21 & --- & $4.28 \mathrm{E}-04$ & 6,600 & 1.22E-01 & 9,070 & $2.13 \mathrm{E}-01$ & 42,500 & $2.48 \mathrm{E}-01$ & 62,300 & $4.00 \mathrm{E}-01$ & $9.43 \mathrm{E}-08$ \\
\hline \multicolumn{15}{|c|}{ A161 $90^{\circ} \mathrm{C} \mathrm{pH} 12$ Reactor \#1 } \\
\hline A161.90.12.160.Blank1 & & $1.58 \mathrm{E}-04$ & --- & --- & --- & [49.4] & --- & {$[75.6]$} & --- & [164] & --- & 996 & --- & --- \\
\hline A161.90.12.160.Blank2 & & $1.57 E-04$ & -- & --- & --- & {$[49.4]$} & --- & {$[75.6]$} & --- & [164] & --- & 990 & --- & --- \\
\hline A161.90.12.160.Blank3 & & $1.58 \mathrm{E}-04$ & --- & --- & --- & {$[49.4]$} & --- & {$[75.6]$} & --- & [164] & --- & 986 & --- & --- \\
\hline A161.90.12.160.1 & & $1.47 E-04$ & 1 & 0.087 & $1.84 \mathrm{E}-03$ & 3,430 & $5.17 \mathrm{E}+00$ & 2,780 & $5.30 \mathrm{E}+00$ & 10,300 & $4.89 \mathrm{E}+00$ & 11,500 & $6.00 E+00$ & $3.54 \mathrm{E}-07$ \\
\hline A161.90.12.160.2 & & $1.56 \mathrm{E}-04$ & 1 & 0.083 & $1.79 \mathrm{E}-03$ & 4,530 & $7.46 \mathrm{E}+00$ & 3,810 & $7.95 \mathrm{E}+00$ & 13,700 & $7.12 \mathrm{E}+00$ & 15,900 & $9.08 \mathrm{E}+00$ & $5.70 \mathrm{E}-07$ \\
\hline A161.90.12.160.3 & & $1.58 \mathrm{E}-04$ & 2 & 0.067 & $1.55 \mathrm{E}-03$ & 4,310 & $8.25 \mathrm{E}+00$ & 3,770 & $9.13 \mathrm{E}+00$ & 13,200 & $7.96 \mathrm{E}+00$ & 14,900 & $9.88 \mathrm{E}+00$ & $3.77 \mathrm{E}-07$ \\
\hline A161.90.12.160.4 & & $1.58 \mathrm{E}-04$ & 4 & 0.039 & $1.09 \mathrm{E}-03$ & 3,240 & $8.83 E+00$ & 2,960 & $1.02 \mathrm{E}+01$ & 10,200 & $8.76 \mathrm{E}+00$ & 11,700 & $1.10 \mathrm{E}+01$ & $4.22 \mathrm{E}-07$ \\
\hline A161.90.12.160.5 & & $1.56 \mathrm{E}-04$ & 7 & 0.017 & $6.23 E-04$ & 2,440 & $1.15 \mathrm{E}+01$ & 2,180 & $1.30 \mathrm{E}+01$ & 7,910 & $1.17 \mathrm{E}+01$ & 8,880 & $1.45 \mathrm{E}+01$ & $7.53 \mathrm{E}-07$ \\
\hline A161.90.12.160.6 & & $1.56 \mathrm{E}-04$ & 9 & 0.013 & 5.09E-04 & 1,680 & $9.68 \mathrm{E}+00$ & 1,390 & $1.01 \mathrm{E}+01$ & 5,550 & $1.01 \mathrm{E}+01$ & 5,520 & $1.10 \mathrm{E}+01$ & $4.45 \mathrm{E}-07$ \\
\hline A161.90.12.160.7 & & $1.55 \mathrm{E}-04$ & 11 & 0.008 & $3.84 \mathrm{E}-04$ & 1,030 & $7.81 E+00$ & 798 & $7.67 \mathrm{E}+00$ & 3,760 & $8.99 \mathrm{E}+00$ & 3,370 & $8.86 \mathrm{E}+00$ & $6.02 \mathrm{E}-07$ \\
\hline
\end{tabular}




\begin{tabular}{|c|c|c|c|c|c|c|c|c|c|c|c|c|c|c|}
\hline Sample ID & $\begin{array}{l}\text { Influent } \\
{[\mathrm{Si}]} \\
(\mathrm{ppm})\end{array}$ & $\begin{array}{l}\text { Flow } \\
\text { Rate, q } \\
\left(\mathrm{m}^{3} \mathrm{~d}^{-1}\right)\end{array}$ & $\begin{array}{l}\text { Time } \\
\text { (d) }\end{array}$ & $\begin{array}{l}\text { Glass } \\
\text { Mass } \\
\text { (g) }\end{array}$ & $\begin{array}{l}\text { Surface } \\
\text { Area, S } \\
\left(\mathrm{m}^{2}\right)\end{array}$ & $\begin{array}{l}{[\mathrm{Al}]} \\
\left(\mu \mathrm{L} \mathrm{L}^{-1}\right)\end{array}$ & $\begin{array}{l}\text { Al Norm. } \\
\text { Diss. Rate } \\
\left(\mathrm{g} \mathrm{m}^{-2} \mathrm{~d}^{-1}\right)\end{array}$ & $\begin{array}{l}{[B]} \\
\left(\mu \mathrm{L} \mathrm{L}^{-1}\right)\end{array}$ & $\begin{array}{l}\text { B Norm. } \\
\text { Diss. Rate } \\
\left(\mathrm{g} \mathrm{m}^{-2} \mathrm{~d}^{-1}\right)\end{array}$ & $\begin{array}{l}{[\mathrm{Si}]} \\
\left(\mu g \mathrm{~L}^{-1}\right)\end{array}$ & $\begin{array}{l}\text { Si Norm. } \\
\text { Diss. Rate } \\
\left(\mathrm{g} \mathrm{m}^{-2} \mathrm{~d}^{-1}\right)\end{array}$ & $\begin{array}{l}{[\mathrm{Na}]} \\
(\mu \mathrm{g} \mathrm{L} \\
\left.{ }^{1}\right)\end{array}$ & $\begin{array}{l}\text { Na Norm. } \\
\text { Diss. Rate, } \\
\mathrm{r} \\
\left(\mathrm{g} \mathrm{m}^{-2} \mathrm{~d}^{-1}\right)\end{array}$ & $\begin{array}{l}\mathrm{r}_{\mathrm{IEX}} \\
\left(\mathrm{mol} \mathrm{Na} \mathrm{s}^{-1}\right)\end{array}$ \\
\hline A161.90.12.160.8 & & $1.55 \mathrm{E}-04$ & 14 & 0.006 & $3.02 \mathrm{E}-04$ & 560 & $5.42 \mathrm{E}+00$ & 371 & $4.55 E+00$ & 2,600 & $7.93 E+00$ & 1,750 & $5.87 E+00$ & $6.67 \mathrm{E}-07$ \\
\hline A161.90.12.160.9 & & $1.56 \mathrm{E}-04$ & 16 & 0.006 & $3.06 \mathrm{E}-04$ & 261 & $2.51 \mathrm{E}+00$ & 144 & $1.75 \mathrm{E}+00$ & 1,460 & $4.43 E+00$ & 1,010 & $3.37 \mathrm{E}+00$ & $8.13 \mathrm{E}-07$ \\
\hline \multicolumn{15}{|c|}{ A161 $90^{\circ} \mathrm{C} \mathrm{pH} 12$ Reactor \#2 } \\
\hline A161.90.12.C.Blank1 & & $3.11 \mathrm{E}-08$ & -- & --- & --- & {$[49.4]$} & -- & [75.6] & --- & {$[164]$} & -- & [134] & --- & --- \\
\hline A161.90.12.C.Blank2 & & 8.93E- 07 & -- & --- & --- & {$[49.4]$} & --- & [75.6] & --- & [164] & --- & 451 & --- & --- \\
\hline A161.90.12.C.Blank3 & & $1.26 \mathrm{E}-05$ & --- & --- & --- & {$[49.4]$} & -- & {$[75.6]$} & --- & {$[164]$} & -- & 423 & --- & --- \\
\hline A161.90.12.C.1 & & $1.50 \mathrm{E}-05$ & 2 & --- & 3.97E-04 & 2,880 & $2.04 E+00$ & 2,270 & $2.04 \mathrm{E}+00$ & 9,020 & $2.02 E+00$ & 8,500 & $2.09 E+00$ & $2.57 \mathrm{E}-08$ \\
\hline A161.90.12.C.2 & & $1.35 \mathrm{E}-05$ & 4 & --- & 3.97E-04 & 4,550 & $2.92 \mathrm{E}+00$ & 3,890 & $3.16 \mathrm{E}+00$ & 14,100 & $2.85 \mathrm{E}+00$ & 14,900 & $3.31 \mathrm{E}+00$ & $7.72 \mathrm{E}-08$ \\
\hline A161.90.12.C.3 & & $1.32 \mathrm{E}-05$ & 7 & --- & 3.97E-04 & 4,760 & $2.97 \mathrm{E}+00$ & 4,410 & $3.48 \mathrm{E}+00$ & 15,100 & $2.97 E+00$ & 17,000 & $3.67 \mathrm{E}+00$ & $9.69 \mathrm{E}-08$ \\
\hline A161.90.12.C.4 & & $1.62 \mathrm{E}-05$ & 9 & -.- & 3.97E-04 & 4,420 & $3.39 \mathrm{E}+00$ & 4,220 & $4.10 E+00$ & 14,400 & $3.48 \mathrm{E}+00$ & 17,300 & $4.60 \mathrm{E}+00$ & $2.52 \mathrm{E}-07$ \\
\hline A161.90.12.C.5 & & $1.49 \mathrm{E}-05$ & 11 & --- & 3.97E-04 & 4,160 & $2.94 E+00$ & 4,180 & $3.74 \mathrm{E}+00$ & 13,800 & $3.07 E+00$ & 15,700 & $3.85 E+00$ & $5.32 \mathrm{E}-08$ \\
\hline A161.90.12.C.6 & & $1.49 \mathrm{E}-05$ & 14 & --. & 3.97E-04 & 3,680 & $2.60 \mathrm{E}+00$ & 3,810 & $3.41 E+00$ & 12,400 & $2.76 \mathrm{E}+00$ & 14,500 & $3.55 \mathrm{E}+00$ & 7.19E-08 \\
\hline A161.90.12.C.7 & & $1.46 \mathrm{E}-05$ & 16 & --- & 3.97E-04 & 2,980 & $2.06 E+00$ & 3,250 & $2.85 E+00$ & 10,200 & $2.23 E+00$ & 13,600 & $3.27 \mathrm{E}+00$ & 2.09E-07 \\
\hline A161.90.12.C.8 & & $1.25 \mathrm{E}-05$ & 18 & -- & $3.97 \mathrm{E}-04$ & 3,540 & $2.09 \mathrm{E}+00$ & 3,810 & $2.84 \mathrm{E}+00$ & 12,200 & $2.27 \mathrm{E}+00$ & 13,700 & $2.80 \mathrm{E}+00$ & $<0$ \\
\hline A161.90.12.C.9 & & $1.25 \mathrm{E}-05$ & 21 & --- & 3.97E-04 & 3,450 & 2.03E+00 & 3,760 & $2.81 E+00$ & 12,000 & $2.23 \mathrm{E}+00$ & 12,600 & $2.58 \mathrm{E}+00$ & $<0$ \\
\hline \multicolumn{15}{|c|}{ A161 $23^{\circ} \mathrm{C}$ pH 9 Si Reactor \#1 } \\
\hline A161.23.9.SI5.Blank1 & 5 & 2.07E-05 & --- & --- & --- & [49.4] & --- & [75.6] & --- & 4,520 & --- & 174 & --- & --- \\
\hline A161.23.9.S15.Blank2 & 5 & 2.13E-05 & -- & -- & -- & {$[49.4]$} & -- & {$[75.6]$} & --- & 4,400 & -- & 142 & -- & --- \\
\hline A161.23.9.S15.Blank3 & 5 & 2.09E-05 & -- & -- & --- & {$[49.4]$} & -- & [75.6] & --- & 4,380 & --- & 143 & --- & --- \\
\hline A161.23.9.S15.2 & 5 & $2.09 \mathrm{E}-05$ & 7.0 & 3.999 & $8.08 \mathrm{E}-02$ & 123 & $5.98 \mathrm{E}-04$ & 1,340 & $8.26 \mathrm{E}-03$ & 5,340 & --- & 5,200 & $8.77 \mathrm{E}-03$ & $2.59 \mathrm{E}-10$ \\
\hline A161.23.9.SI5.4 & 5 & 2.07E-05 & 14.0 & 3.996 & $8.08 \mathrm{E}-02$ & 147 & 7.10E-04 & 986 & $6.03 \mathrm{E}-03$ & 5,180 & --- & 3,680 & $6.16 \mathrm{E}-03$ & $6.60 \mathrm{E}-11$ \\
\hline A161.23.9.S15.6 & 5 & 2.03E-05 & 21.0 & 3.993 & 8.07E-02 & 152 & 7.19E-04 & 707 & 4.23E-03 & 5,020 & -- & 2,640 & $4.33 E-03$ & $4.74 E-11$ \\
\hline A161.23.9.SI5.8 & 5 & 2.04E-05 & 27.8 & 3.992 & 8.07E-02 & 156 & 7.40E-04 & 523 & $3.14 \mathrm{E}-03$ & 4,820 & --- & 2,400 & $3.95 \mathrm{E}-03$ & 4.05E-10 \\
\hline A161.23.9.S15.10 & 5 & $2.01 \mathrm{E}-05$ & 34.9 & 3.990 & 8.07E-02 & 171 & 8.03E-04 & 571 & $3.40 \mathrm{E}-03$ & 5,140 & -- & 2,110 & $3.44 \mathrm{E}-03$ & $1.99 \mathrm{E}-11$ \\
\hline A161.23.9.S15.11 & 5 & $2.02 \mathrm{E}-05$ & 37.9 & 3.989 & 8.07E-02 & 134 & $6.31 \mathrm{E}-04$ & 528 & $3.15 \mathrm{E}-03$ & 5,300 & --- & 1,900 & $3.10 \mathrm{E}-03$ & $<0$ \\
\hline A161.23.9.SI5.13 & 5 & 2.03E-05 & 45.0 & 3.988 & 8.07E-02 & 138 & $6.55 \mathrm{E}-04$ & 512 & $3.08 \mathrm{E}-03$ & 5,210 & -- & 1,850 & $3.04 E-03$ & $<0$ \\
\hline A161.23.9.S15.14 & 5 & $2.11 \mathrm{E}-05$ & 48.9 & 3.987 & $8.06 \mathrm{E}-02$ & 138 & $6.79 \mathrm{E}-04$ & 490 & $3.06 \mathrm{E}-03$ & 5,260 & --- & 1,770 & $3.02 \mathrm{E}-03$ & $<0$ \\
\hline A161.23.9.S15.17 & 5 & $3.24 \mathrm{E}-05$ & 58.0 & 3.983 & $8.06 \mathrm{E}-02$ & 134 & $1.01 \mathrm{E}-03$ & 476 & $4.56 \mathrm{E}-03$ & 5,410 & --- & 1,630 & $4.28 \mathrm{E}-03$ & $<0$ \\
\hline A161.23.9.S15.19 & 5 & 2.07E-05 & 66.0 & 3.981 & $8.06 \mathrm{E}-02$ & 136 & $6.58 \mathrm{E}-04$ & 475 & $2.91 \mathrm{E}-03$ & 5,330 & --- & 1,620 & $2.72 \mathrm{E}-03$ & $<0$ \\
\hline A161.23.9.SI5.20 & 5 & $1.99 \mathrm{E}-05$ & 69.9 & 3.981 & 8.06E-02 & 140 & $6.49 \mathrm{E}-04$ & 482 & 2.83E-03 & 5,400 & --- & 1,810 & $2.91 \mathrm{E}-03$ & $3.99 E-11$ \\
\hline A161.23.9.S15.21 & 5 & $1.99 \mathrm{E}-05$ & 73.0 & 3.980 & 8.06E-02 & 136 & $6.33 \mathrm{E}-04$ & 468 & $2.76 \mathrm{E}-03$ & 5,120 & --- & 1,560 & $2.52 \mathrm{E}-03$ & $<0$ \\
\hline A161.23.9.S15.22 & 5 & $1.99 \mathrm{E}-05$ & 73.0 & 3.980 & $8.06 \mathrm{E}-02$ & 140 & $6.51 \mathrm{E}-04$ & 492 & $2.90 \mathrm{E}-03$ & 5,420 & --- & 1,670 & $2.69 \mathrm{E}-03$ & $<0$ \\
\hline \multicolumn{15}{|c|}{ A161 $23{ }^{\circ} \mathrm{C}$ pH 9 Si Reactor $\# 2$} \\
\hline A161.23.9.SI20.Blank1 & 20 & $2.04 \mathrm{E}-05$ & --- & --- & --- & {$[49.4]$} & -- & [75.6] & --- & 16,700 & --- & 287 & --- & --- \\
\hline A161.23.9.SI20.Blank2 & 20 & $2.02 \mathrm{E}-05$ & --- & --- & -- & {$[49.4]$} & -- & [75.6] & --- & 17,600 & -- & 180 & --- & -- \\
\hline A161.23.9.SI20.Blank3 & 20 & $2.03 \mathrm{E}-05$ & --- & -- & --- & {$[49.4]$} & -- & [75.6] & --- & 16,800 & -- & 200 & --- & --- \\
\hline A161.23.9.SI20.2 & 20 & $2.04 \mathrm{E}-05$ & 7.0 & 3.996 & 8.07E-02 & 57 & $2.70 \mathrm{E}-04$ & 1,020 & $6.13 \mathrm{E}-03$ & 17,800 & --- & 4,440 & $7.30 \mathrm{E}-03$ & $5.91 \mathrm{E}-10$ \\
\hline A161.23.9.SI20.4 & 20 & $2.20 \mathrm{E}-05$ & 14.0 & 3.994 & 8.07E-02 & {$[49.4]$} & $2.53 \mathrm{E}-04$ & 692 & $4.50 \mathrm{E}-03$ & 17,600 & --- & 2,670 & $4.75 \mathrm{E}-03$ & $1.27 \mathrm{E}-10$ \\
\hline A161.23.9.SI20.6 & 20 & $2.20 \mathrm{E}-05$ & 21.0 & 3.992 & 8.07E-02 & [49.4] & 2.53E-04 & 536 & $3.48 \mathrm{E}-03$ & 18,300 & --- & 1,960 & $3.48 \mathrm{E}-03$ & $1.84 \mathrm{E}-12$ \\
\hline A161.23.9.S120.8 & 20 & $2.26 \mathrm{E}-05$ & 27.8 & 3.990 & 8.07E-02 & 86 & $4.52 \mathrm{E}-04$ & 530 & $3.54 \mathrm{E}-03$ & 18,400 & --- & 2,060 & $3.77 \mathrm{E}-03$ & $1.14 \mathrm{E}-10$ \\
\hline A161.23.9.S120.10 & 20 & $2.30 \mathrm{E}-05$ & 34.9 & 3.988 & $8.06 \mathrm{E}-02$ & [49.4] & $2.66 \mathrm{E}-04$ & 515 & $3.51 \mathrm{E}-03$ & 18,300 & --- & 1,780 & $3.32 \mathrm{E}-03$ & $<0$ \\
\hline A161.23.9.SI20.11 & 20 & $2.20 \mathrm{E}-05$ & 37.9 & 3.987 & $8.06 \mathrm{E}-02$ & 36 & 1.82E-04 & 498 & $3.24 \mathrm{E}-03$ & 18,900 & --- & 1,770 & $3.15 \mathrm{E}-03$ & $<0$ \\
\hline A161.23.9.SI20.13 & 20 & $2.21 \mathrm{E}-05$ & 45.0 & 3.985 & $8.06 \mathrm{E}-02$ & 35 & $1.79 \mathrm{E}-04$ & 520 & $3.39 \mathrm{E}-03$ & 19,000 & --- & 1,810 & $3.23 E-03$ & $<0$ \\
\hline
\end{tabular}

B. 46 


\begin{tabular}{|c|c|c|c|c|c|c|c|c|c|c|c|c|c|c|}
\hline Sample ID & $\begin{array}{l}\text { Influent } \\
{[\mathrm{Si}]} \\
(\mathrm{ppm})\end{array}$ & $\begin{array}{l}\text { Flow } \\
\text { Rate, } \mathrm{q} \\
\left(\mathrm{m}^{3} \mathrm{~d}^{-1}\right)\end{array}$ & $\begin{array}{l}\text { Time } \\
\text { (d) }\end{array}$ & $\begin{array}{l}\text { Glass } \\
\text { Mass } \\
\text { (g) }\end{array}$ & $\begin{array}{l}\text { Surface } \\
\text { Area, S } \\
\left(\mathrm{m}^{2}\right)\end{array}$ & $\begin{array}{l}{[\mathrm{Al}]} \\
\left(\mu \mathrm{g} \mathrm{L}^{-1}\right)\end{array}$ & $\begin{array}{l}\text { Al Norm. } \\
\text { Diss. Rate } \\
\left(\mathrm{g} \mathrm{m}^{-2} \mathrm{~d}^{-1}\right)\end{array}$ & $\begin{array}{l}{[\mathrm{B}]} \\
\left(\mu \mathrm{g} \mathrm{L}^{-1}\right)\end{array}$ & $\begin{array}{l}\text { B Norm. } \\
\text { Diss. Rate } \\
\left(\mathrm{g} \mathrm{m}^{-2} \mathrm{~d}^{-1}\right)\end{array}$ & $\begin{array}{l}{[\mathrm{Si}]} \\
\left(\mu \mathrm{g} \mathrm{L}^{-1}\right)\end{array}$ & $\begin{array}{l}\text { Si Norm. } \\
\text { Diss. Rate } \\
\left(\mathrm{g} \mathrm{m}^{-2} \mathrm{~d}^{-1}\right)\end{array}$ & $\begin{array}{l}{[\mathrm{Na}]} \\
(\mu \mathrm{g} \mathrm{L}- \\
\left.{ }^{1}\right)\end{array}$ & $\begin{array}{l}\text { Na Norm. } \\
\text { Diss. Rate, } \\
\mathrm{r} \\
\left(\mathrm{g} \mathrm{m}^{-2} \mathrm{~d}^{-1}\right)\end{array}$ & $\begin{array}{l}\mathrm{r}_{\mathrm{IEX}} \\
\left(\mathrm{mol} \mathrm{Na} \mathrm{s}^{-2} \mathrm{~s}^{-1}\right)\end{array}$ \\
\hline A161.23.9.S120.14 & 20 & $2.20 \mathrm{E}-05$ & 48.9 & 3.984 & $8.06 \mathrm{E}-02$ & 34 & $1.74 \mathrm{E}-04$ & 502 & $3.27 \mathrm{E}-03$ & 19,200 & --- & 1,780 & $3.18 \mathrm{E}-03$ & $<0$ \\
\hline A161.23.9.S120.17 & 20 & 3.19E-05 & 58.0 & 3.981 & $8.05 \mathrm{E}-02$ & 33 & $2.42 \mathrm{E}-04$ & 520 & $4.91 \mathrm{E}-03$ & 19,200 & --- & 1,840 & $4.75 \mathrm{E}-03$ & $<0$ \\
\hline A161.23.9.S120.19 & 20 & $2.27 \mathrm{E}-05$ & 66.0 & 3.979 & 8.05E-02 & 31 & 1.64E-04 & 475 & 3.20E-03 & 18,600 & --- & 1,700 & $3.13 \mathrm{E}-03$ & $<0$ \\
\hline A161.23.9.S120.20 & 20 & $2.31 \mathrm{E}-05$ & 69.9 & 3.978 & $8.05 \mathrm{E}-02$ & 31 & $1.66 \mathrm{E}-04$ & 486 & $3.32 \mathrm{E}-03$ & 19,100 & --- & 1,720 & $3.22 \mathrm{E}-03$ & $<0$ \\
\hline A161.23.9.S120.21 & 20 & $2.29 \mathrm{E}-05$ & 73.0 & 3.977 & 8.05E-02 & 30 & 1.60E-04 & 493 & $3.35 \mathrm{E}-03$ & 19,000 & --- & 1,730 & $3.22 \mathrm{E}-03$ & $<0$ \\
\hline A161.23.9.SI20.22 & 20 & $1.84 \mathrm{E}-05$ & 76.9 & 3.976 & 8.05E-02 & 29 & 1.24E-04 & 448 & $2.45 \mathrm{E}-03$ & 17,700 & --. & 1,720 & $2.57 \mathrm{E}-03$ & $6.29 \mathrm{E}-11$ \\
\hline \multicolumn{15}{|c|}{ A161 $23^{\circ} \mathrm{C} \mathrm{pH} 9$ Si Reactor $\# 3$} \\
\hline A161.23.9.S135.Blank1 & 35 & $2.01 \mathrm{E}-05$ & --- & --- & --- & [49.4] & --- & {$[75.6]$} & --- & 31,300 & --- & 225 & --- & --- \\
\hline A161.23.9.S135.Blank2 & 35 & $2.02 \mathrm{E}-05$ & -.- & --- & -.- & [49.4] & -.- & {$[75.6]$} & --- & 17,600 & -.- & [134] & --- & -.- \\
\hline A161.23.9.SI35.Blank3 & 35 & $2.01 \mathrm{E}-05$ & -.- & -.- & -.- & [49.4] & -.- & {$[75.6]$} & --- & 29,500 & --- & 163 & --- & -.- \\
\hline A161.23.9.S135.2 & 35 & $2.11 \mathrm{E}-05$ & 7.0 & 3.991 & 8.07E-02 & 54 & $2.66 \mathrm{E}-04$ & 1,200 & $7.48 \mathrm{E}-03$ & 30,800 & --. & 5,620 & $9.58 \mathrm{E}-03$ & $1.06 \mathrm{E}-09$ \\
\hline A161.23.9.SI35.4 & 35 & $2.08 \mathrm{E}-05$ & 14.0 & 3.990 & 8.06E-02 & [49.4] & 2.40E-04 & 674 & 4.15E-03 & 31,200 & --- & 2,610 & $4.40 \mathrm{E}-03$ & $1.26 \mathrm{E}-10$ \\
\hline A161.23.9.SI35.6 & 35 & $2.20 \mathrm{E}-05$ & 21.0 & 3.988 & $8.06 \mathrm{E}-02$ & [49.4] & $2.54 \mathrm{E}-04$ & 499 & 3.25E-03 & 30,000 & --- & 2,130 & $3.80 \mathrm{E}-03$ & $2.76 \mathrm{E}-10$ \\
\hline A161.23.9.SI35.8 & 35 & $2.11 \mathrm{E}-05$ & 27.8 & 3.986 & $8.06 \mathrm{E}-02$ & [49.4] & 2.44E-04 & 519 & $3.24 \mathrm{E}-03$ & 31,400 & --- & 1,890 & $3.23 \mathrm{E}-03$ & $<0$ \\
\hline A161.23.9.SI35.10 & 35 & $1.99 \mathrm{E}-05$ & 34.9 & 3.984 & $8.06 \mathrm{E}-02$ & [49.4] & $2.30 \mathrm{E}-04$ & 576 & $3.39 \mathrm{E}-03$ & 32,300 & --- & 2,080 & $3.36 \mathrm{E}-03$ & $<0$ \\
\hline A161.23.9.SI35.11 & 35 & 2.04E-05 & 37.9 & 3.983 & 8.06E-02 & 32 & 1.54E-04 & 567 & 3.43E-03 & 33,000 & -.- & 2,040 & $3.38 \mathrm{E}-03$ & $<0$ \\
\hline A161.23.9.SI35.13 & 35 & $2.16 \mathrm{E}-05$ & 45.0 & 3.981 & 8.05E-02 & 31 & $1.56 \mathrm{E}-04$ & 541 & $3.46 \mathrm{E}-03$ & 33,800 & --- & 1,980 & $3.46 \mathrm{E}-03$ & $3.34 \mathrm{E}-12$ \\
\hline A161.23.9.SI35.14 & 35 & $2.10 \mathrm{E}-05$ & 48.9 & 3.980 & 8.05E-02 & 31 & $1.52 \mathrm{E}-04$ & 517 & 3.22E-03 & 33,500 & -.- & 1,930 & $3.29 \mathrm{E}-03$ & $3.56 \mathrm{E}-11$ \\
\hline A161.23.9.S135.17 & 35 & $3.18 \mathrm{E}-05$ & 58.0 & 3.977 & 8.05E-02 & 31 & $2.30 \mathrm{E}-04$ & 519 & $4.88 \mathrm{E}-03$ & 33,400 & --- & 2,160 & $5.56 \mathrm{E}-03$ & $3.42 \mathrm{E}-10$ \\
\hline A161.23.9.SI35.19 & 35 & 2.09E-05 & 66.0 & 3.975 & 8.04E-02 & 30 & 1.45E-04 & 540 & 3.34E-03 & 34,300 & -.- & 1,920 & $3.25 \mathrm{E}-03$ & $<0$ \\
\hline A161.23.9.SI35.20 & 35 & $2.18 \mathrm{E}-05$ & 69.9 & 3.974 & $8.04 \mathrm{E}-02$ & 29 & 1.47E-04 & 513 & $3.31 \mathrm{E}-03$ & 34,200 & -- & 1,900 & $3.36 \mathrm{E}-03$ & $2.32 \mathrm{E}-11$ \\
\hline A161.23.9.SI35.21 & 35 & $2.19 \mathrm{E}-05$ & 73.0 & 3.973 & $8.04 \mathrm{E}-02$ & 30 & $1.56 \mathrm{E}-04$ & 502 & 3.26E-03 & 33,000 & --- & 2,040 & $3.62 \mathrm{E}-03$ & $1.84 \mathrm{E}-10$ \\
\hline A161.23.9.S135.22 & 35 & $2.19 \mathrm{E}-05$ & 73.0 & 3.973 & $8.04 \mathrm{E}-02$ & 29 & $1.46 \mathrm{E}-04$ & 529 & 3.43E-03 & 33,600 & --- & 1,920 & $3.41 \mathrm{E}-03$ & $<0$ \\
\hline \multicolumn{15}{|c|}{ A161 $23^{\circ} \mathrm{C} \mathrm{pH} 9$ Si Reactor \#4 } \\
\hline A161.23.9.10Si1.Blank2 & 1 & & --- & --- & --- & [49.4] & --- & {$[75.6]$} & --- & 886 & -- & [134] & --- & --- \\
\hline A161.23.9.10Si1.Blank3 & 1 & & -.- & -.- & -.- & [49.4] & --- & [75.6] & --- & 897 & --- & [134] & --. & -.- \\
\hline A161.23.9.10Si1.Blank1 & 1 & $1.23 \mathrm{E}-05$ & --- & --- & --- & [49.4] & -- & {$[75.6]$} & --- & 867 & --- & 220 & --- & -.- \\
\hline A161.23.9.10Si1.1 & 1 & $1.15 \mathrm{E}-05$ & 1.9 & 2.007 & 4.06E-02 & 82 & 4.40E-04 & 634 & 4.30E-03 & 1,940 & --- & 3,650 & $6.77 \mathrm{E}-03$ & $1.25 \mathrm{E}-09$ \\
\hline A161.23.9.10Si1.2 & 1 & $1.19 \mathrm{E}-05$ & 2.9 & 2.007 & 4.05E-02 & 144 & 7.95E-04 & 1,040 & 7.27E-03 & 2,690 & --- & 5,220 & $9.99 \mathrm{E}-03$ & $1.37 \mathrm{E}-09$ \\
\hline A161.23.9.10Si1.3 & 1 & $1.19 \mathrm{E}-05$ & 4.9 & 2.006 & 4.05E-02 & 172 & 9.52E-04 & 1,140 & 7.99E-03 & 3,050 & --- & 5,540 & $1.06 \mathrm{E}-02$ & $1.33 \mathrm{E}-09$ \\
\hline A161.23.9.10Si1.4 & 1 & $1.19 \mathrm{E}-05$ & 6.8 & 2.006 & 4.05E-02 & 187 & 1.03E-03 & 1,170 & $8.20 \mathrm{E}-03$ & 3,230 & --- & 5,360 & $1.03 \mathrm{E}-02$ & $1.05 \mathrm{E}-09$ \\
\hline A161.23.9.10Si1.5 & 1 & $1.20 \mathrm{E}-05$ & 10.0 & 2.004 & 4.05E-02 & 202 & 1.12E-03 & 1,110 & $7.81 \mathrm{E}-03$ & 3,330 & --- & 4,840 & $9.33 \mathrm{E}-03$ & $7.62 \mathrm{E}-10$ \\
\hline A161.23.9.10Si1.6 & 1 & $1.19 \mathrm{E}-05$ & 13.0 & 2.003 & 4.05E-02 & 260 & $1.44 \mathrm{E}-03$ & 1,090 & 7.64E-03 & 3,470 & --- & 4,320 & $8.29 \mathrm{E}-03$ & 3.27E-10 \\
\hline A161.23.9.10Si1.7 & 1 & $1.19 \mathrm{E}-05$ & 16.9 & 2.003 & 4.05E-02 & 235 & $1.30 \mathrm{E}-03$ & 844 & $5.92 \mathrm{E}-03$ & 2,850 & --- & 3,710 & $7.12 \mathrm{E}-03$ & $6.06 \mathrm{E}-10$ \\
\hline A161.23.9.10Si1.8 & 1 & $1.19 \mathrm{E}-05$ & 19.9 & 2.002 & 4.05E-02 & 276 & $1.53 \mathrm{E}-03$ & 765 & $5.36 \mathrm{E}-03$ & 2,700 & --- & 2,820 & $5.41 \mathrm{E}-03$ & 2.47E-11 \\
\hline A161.23.9.10Si1.9 & 1 & $1.19 \mathrm{E}-05$ & 23.9 & 2.001 & 4.05E-02 & 189 & 1.05E-03 & 651 & 4.56E-03 & 3,670 & --- & 2,730 & $5.24 \mathrm{E}-03$ & $3.40 \mathrm{E}-10$ \\
\hline A161.23.9.10Si1.10 & 1 & $1.19 \mathrm{E}-05$ & 25.9 & 2.001 & 4.05E-02 & 319 & 1.77E-03 & 646 & 4.54E-03 & 2,490 & -.- & 2,400 & $4.62 \mathrm{E}-03$ & $3.90 \mathrm{E}-11$ \\
\hline A161.23.9.10Si1.11 & 1 & $1.19 \mathrm{E}-05$ & 26.8 & 2.001 & 4.05E-02 & 311 & 1.73E-03 & 603 & 4.24E-03 & 2,460 & --- & 2,320 & 4.47E-03 & $1.14 \mathrm{E}-10$ \\
\hline A161.23.9.10Si1.12 & 1 & $1.12 \mathrm{E}-05$ & 27.8 & 2.001 & 4.05E-02 & 334 & $1.74 \mathrm{E}-03$ & 624 & $4.12 \mathrm{E}-03$ & 2,530 & --- & 2,610 & $4.72 \mathrm{E}-03$ & $3.01 \mathrm{E}-10$ \\
\hline \multicolumn{15}{|c|}{ A161 $23^{\circ} \mathrm{C}$ pH 9 Si Reactor $\# 5$} \\
\hline A161.23.9.10Si3.Blank2 & 3 & & -.- & -.- & --- & [49.4] & --- & {$[75.6]$} & --- & 2,830 & --- & [134] & --- & -.- \\
\hline A161.23.9.10Si3.Blank3 & 3 & & --- & --- & --- & [49.4] & -- & [75.6] & --- & 2,910 & -- & [134] & --- & --- \\
\hline A161.23.9.10Si3.Blank1 & 3 & $1.68 \mathrm{E}-05$ & --- & -.- & --- & [49.4] & --- & {$[75.6]$} & --- & 2,730 & --- & 224 & --- & -.- \\
\hline
\end{tabular}




\begin{tabular}{|c|c|c|c|c|c|c|c|c|c|c|c|c|c|c|}
\hline Sample ID & $\begin{array}{l}\text { Influent } \\
{[\mathrm{Si}]} \\
(\mathrm{ppm})\end{array}$ & $\begin{array}{l}\text { Flow } \\
\text { Rate, q } \\
\left(\mathrm{m}^{3} \mathrm{~d}^{-1}\right)\end{array}$ & $\begin{array}{l}\text { Time } \\
\text { (d) }\end{array}$ & $\begin{array}{l}\text { Glass } \\
\text { Mass } \\
\text { (g) }\end{array}$ & $\begin{array}{l}\text { Surface } \\
\text { Area, S } \\
\left(\mathrm{m}^{2}\right)\end{array}$ & $\begin{array}{l}{[\mathrm{Al}]} \\
\left(\mu \mathrm{L} \mathrm{L}^{-1}\right)\end{array}$ & $\begin{array}{l}\text { Al Norm. } \\
\text { Diss. Rate } \\
\left(\mathrm{g} \mathrm{m}^{-2} \mathrm{~d}^{-1}\right)\end{array}$ & $\begin{array}{l}{[\mathrm{B}]} \\
\left(\mu \mathrm{g} \mathrm{L}^{-1}\right)\end{array}$ & $\begin{array}{l}\text { B Norm. } \\
\text { Diss. Rate } \\
\left(\mathrm{g} \mathrm{m}^{-2} \mathrm{~d}^{-1}\right)\end{array}$ & $\begin{array}{l}{[\mathrm{Si}]} \\
\left(\mu \mathrm{g} \mathrm{L}^{-1}\right)\end{array}$ & $\begin{array}{l}\text { Si Norm. } \\
\text { Diss. Rate } \\
\left(\mathrm{g} \mathrm{m}^{-2} \mathrm{~d}^{-1}\right)\end{array}$ & $\begin{array}{l}{[\mathrm{Na}]} \\
(\mu \mathrm{g} \mathrm{L} \\
\left.{ }^{1}\right)\end{array}$ & $\begin{array}{l}\text { Na Norm. } \\
\text { Diss. Rate, } \\
\mathrm{r} \\
\left(\mathrm{g} \mathrm{m}^{-2} \mathrm{~d}^{-1}\right)\end{array}$ & $\begin{array}{l}\mathrm{r}_{\mathrm{IEX}} \\
\left(\mathrm{mol} \mathrm{Na} \mathrm{No}^{-1}\right)\end{array}$ \\
\hline A161.23.9.10Si3.1 & 3 & 1.07E-05 & 1.9 & 2.000 & $4.04 \mathrm{E}-02$ & {$[49.4]$} & $2.46 \mathrm{E}-04$ & 520 & $3.28 \mathrm{E}-03$ & 3,200 & --- & 3,310 & $5.72 \mathrm{E}-03$ & $1.23 \mathrm{E}-09$ \\
\hline A161.23.9.10Si3.2 & 3 & 1.09E-05 & 2.9 & 1.999 & $4.04 \mathrm{E}-02$ & 72 & 3.67E-04 & 929 & $5.96 \mathrm{E}-03$ & 3,600 & -- & 5,200 & $9.13 \mathrm{E}-03$ & $1.60 \mathrm{E}-09$ \\
\hline A161.23.9.10Si3.3 & 3 & $1.11 \mathrm{E}-05$ & 4.9 & 1.999 & $4.04 \mathrm{E}-02$ & 96 & $4.95 \mathrm{E}-04$ & 1,090 & $7.14 \mathrm{E}-03$ & 3,730 & --- & 5,340 & $9.58 \mathrm{E}-03$ & $1.23 \mathrm{E}-09$ \\
\hline A161.23.9.10Si3.4 & 3 & 1.11E-05 & 6.8 & 1.998 & 4.04E-02 & 117 & $6.03 \mathrm{E}-04$ & 1,150 & $7.51 \mathrm{E}-03$ & 3,840 & --- & 5,370 & $9.60 \mathrm{E}-03$ & $1.05 \mathrm{E}-09$ \\
\hline A161.23.9.10Si3.5 & 3 & $1.16 \mathrm{E}-05$ & 10.0 & 1.997 & $4.04 \mathrm{E}-02$ & 124 & $6.71 \mathrm{E}-04$ & 1,070 & $7.33 \mathrm{E}-03$ & 3,800 & --- & 4,700 & $8.81 \mathrm{E}-03$ & $7.47 E-10$ \\
\hline A161.23.9.10Si3.6 & 3 & 1.16E-05 & 13.0 & 1.996 & 4.04E-02 & 133 & 7.17E-04 & 905 & $6.18 \mathrm{E}-03$ & 3,720 & --- & 4,030 & $7.53 \mathrm{E}-03$ & $6.81 \mathrm{E}-10$ \\
\hline A161.23.9.10Si3.7 & 3 & 1.16E-05 & 16.9 & 1.996 & 4.03E-02 & 133 & $7.22 \mathrm{E}-04$ & 744 & $5.11 \mathrm{E}-03$ & 3,570 & --- & 3,440 & $6.47 \mathrm{E}-03$ & $6.84 \mathrm{E}-10$ \\
\hline A161.23.9.10Si3.8 & 3 & 1.09E-05 & 19.9 & 1.995 & 4.03E-02 & 176 & $8.94 \mathrm{E}-04$ & 725 & 4.67E-03 & 3,840 & --- & 2,530 & $4.46 \mathrm{E}-03$ & $<0$ \\
\hline A161.23.9.10Si3.9 & 3 & $9.96 \mathrm{E}-06$ & 23.9 & 1.994 & 4.03E-02 & 291 & $1.35 \mathrm{E}-03$ & 712 & $4.19 \mathrm{E}-03$ & 2,690 & --- & 2,550 & $4.11 \mathrm{E}-03$ & $<0$ \\
\hline A161.23.9.10Si3.10 & 3 & $1.00 \mathrm{E}-05$ & 25.9 & 1.994 & 4.03E-02 & 186 & 8.69E-04 & 607 & $3.59 \mathrm{E}-03$ & 3,780 & --. & 2,220 & $3.59 \mathrm{E}-03$ & $2.20 \mathrm{E}-12$ \\
\hline A161.23.9.10Si3.11 & 3 & $9.86 \mathrm{E}-06$ & 26.8 & 1.994 & 4.03E-02 & 181 & 8.33E-04 & 593 & $3.46 \mathrm{E}-03$ & 3,730 & --- & 2,090 & $3.33 \mathrm{E}-03$ & $<0$ \\
\hline A161.23.9.10Si3.12 & 3 & $1.01 \mathrm{E}-05$ & 27.8 & 1.994 & 4.03E-02 & 184 & $8.65 \mathrm{E}-04$ & 597 & $3.55 \mathrm{E}-03$ & 3,770 & --- & 2,040 & $3.32 \mathrm{E}-03$ & $<0$ \\
\hline \multicolumn{15}{|c|}{ A161 $40^{\circ} \mathrm{C}$ pH 9 Si Reactor \#1 } \\
\hline A161.40.9.SI10.Blank1 & 10 & 2.03E-05 & --- & -- & -- & {$[49.4]$} & -- & [75.6] & --- & 8,440 & -- & [134] & --- & --- \\
\hline A161.40.9.SI10.Blank3 & 10 & 2.03E-05 & --- & --- & --- & [49.4] & --- & [75.6] & --- & 8,480 & -.- & [134] & --- & -.- \\
\hline A161.40.9.S110.2 & 10 & $2.04 \mathrm{E}-05$ & 4.9 & 1.994 & 4.03E-02 & 218 & 2.07E-03 & 2,740 & $3.30 \mathrm{E}-02$ & 10,000 & --- & 9,800 & $3.23 E-02$ & $<0$ \\
\hline A161.40.9.SI10.4 & 10 & 2.03E-05 & 9.0 & 1.990 & 4.03E-02 & 219 & $2.08 \mathrm{E}-03$ & 2,300 & 2.77E-02 & 9,540 & --- & 8,060 & $2.65 \mathrm{E}-02$ & $<0$ \\
\hline A161.40.9.SI10.5 & 10 & 2.03E-05 & 11.8 & 1.987 & 4.02E-02 & 219 & $2.08 \mathrm{E}-03$ & 2,100 & 2.53E-02 & 9,510 & -- & 6,970 & $2.30 \mathrm{E}-02$ & $<0$ \\
\hline A161.40.9.SI10.6 & 10 & 2.05E-05 & 14.1 & 1.985 & $4.02 \mathrm{E}-02$ & 234 & $2.24 \mathrm{E}-03$ & 2,020 & $2.45 \mathrm{E}-02$ & 9,640 & -.- & 6,790 & $2.26 \mathrm{E}-02$ & $<0$ \\
\hline A161.40.9.SI10.7 & 10 & 2.05E-05 & 16.0 & 1.983 & $4.02 \mathrm{E}-02$ & 255 & $2.44 \mathrm{E}-03$ & 2,230 & $2.71 \mathrm{E}-02$ & 9,780 & -- & 7,710 & $2.56 \mathrm{E}-02$ & $<0$ \\
\hline A161.40.9.SI10.8 & 10 & 2.05E-05 & 18.8 & 1.979 & $4.01 \mathrm{E}-02$ & 248 & $2.38 \mathrm{E}-03$ & 2,320 & $2.82 \mathrm{E}-02$ & 9,800 & --- & 7,740 & $2.57 \mathrm{E}-02$ & $<0$ \\
\hline A161.40.9.SI10.9 & 10 & 2.03E-05 & 21.0 & 1.978 & $4.01 \mathrm{E}-02$ & 252 & $2.40 \mathrm{E}-03$ & 2,180 & $2.63 \mathrm{E}-02$ & 9,710 & -- & 7,540 & $2.49 E-02$ & $<0$ \\
\hline A161.40.9.SI10.10 & 10 & $2.04 \mathrm{E}-05$ & 23.1 & 1.975 & $4.01 \mathrm{E}-02$ & 232 & $2.22 \mathrm{E}-03$ & 2,130 & $2.58 \mathrm{E}-02$ & 9,770 & --- & 7,190 & $2.38 \mathrm{E}-02$ & $<0$ \\
\hline A161.40.9.SI10.11 & 10 & $2.05 \mathrm{E}-05$ & 25.8 & 1.972 & $4.00 \mathrm{E}-02$ & 247 & $2.37 \mathrm{E}-03$ & 2,180 & $2.65 \mathrm{E}-02$ & 9,890 & -- & 7,390 & $2.46 \mathrm{E}-02$ & $<0$ \\
\hline A161.40.9.SI10.12 & 10 & 2.07E-05 & 28.1 & 1.970 & 4.00E-02 & 246 & $2.40 \mathrm{E}-03$ & 2,130 & $2.63 \mathrm{E}-02$ & 9,910 & --- & 7,070 & $2.39 \mathrm{E}-02$ & $<0$ \\
\hline A161.40.9.S110.13 & 10 & $1.95 \mathrm{E}-05$ & 30.1 & 1.968 & $4.00 \mathrm{E}-02$ & 245 & $2.25 \mathrm{E}-03$ & 2,030 & $2.36 \mathrm{E}-02$ & 9,770 & -- & 7,670 & $2.44 \mathrm{E}-02$ & $4.08 \mathrm{E}-10$ \\
\hline A161.40.9.SI10.15 & 10 & 2.04E-05 & 35.0 & 1.964 & $3.99 \mathrm{E}-02$ & 245 & $2.35 \mathrm{E}-03$ & 1,820 & $2.21 \mathrm{E}-02$ & 9,470 & --- & 6,980 & $2.32 \mathrm{E}-02$ & $5.56 \mathrm{E}-10$ \\
\hline A161.40.9.SI10.17 & 10 & 2.04E-05 & 39.8 & 1.960 & $3.99 \mathrm{E}-02$ & 236 & $2.28 \mathrm{E}-03$ & 1,750 & $2.14 \mathrm{E}-02$ & 9,600 & --- & 6,710 & $2.24 \mathrm{E}-02$ & $5.35 \mathrm{E}-10$ \\
\hline A161.40.9.SI10.18 & 10 & $2.04 \mathrm{E}-05$ & 42.0 & 1.958 & $3.99 \mathrm{E}-02$ & 239 & $2.31 \mathrm{E}-03$ & 1,640 & $2.00 \mathrm{E}-02$ & 9,590 & --- & 6,470 & $2.16 \mathrm{E}-02$ & 8.07E-10 \\
\hline \multicolumn{15}{|c|}{ A161 $40^{\circ} \mathrm{C}$ pH 9 Si Reactor $\# 2$} \\
\hline A161.40.9.SI30.Blank1 & 30 & $1.56 \mathrm{E}-05$ & --- & --- & --- & [49.4] & --- & [75.6] & --- & 25,200 & --- & 193 & --- & --- \\
\hline A161.40.9.SI30.Blank3 & 30 & $1.22 \mathrm{E}-05$ & --- & --- & --- & {$[49.4]$} & -- & [75.6] & --- & 25,600 & --- & 174 & --- & --- \\
\hline A161.40.9.SI30.2 & 30 & 1.87E-05 & 4.9 & 2.041 & 4.13E-02 & 81 & $6.92 \mathrm{E}-04$ & 2,220 & $2.40 \mathrm{E}-02$ & 25,300 & --- & 9,620 & $2.84 \mathrm{E}-02$ & 2.25E-09 \\
\hline A161.40.9.SI30.4 & 30 & 1.73E-05 & 9.0 & 2.037 & 4.12E-02 & 58 & 4.59E-04 & 2,430 & 2.43E-02 & 25,800 & --- & 7,890 & $2.16 \mathrm{E}-02$ & $<0$ \\
\hline A161.40.9.SI30.5 & 30 & $1.54 \mathrm{E}-05$ & 11.8 & 2.035 & 4.12E-02 & 72 & $5.09 \mathrm{E}-04$ & 2,440 & $2.18 \mathrm{E}-02$ & 25,900 & -- & 8,290 & $2.03 E-02$ & $<0$ \\
\hline A161.40.9.SI30.6 & 30 & 1.59E-05 & 14.1 & 2.033 & $4.12 \mathrm{E}-02$ & 54 & $3.91 \mathrm{E}-04$ & 2,390 & $2.20 \mathrm{E}-02$ & 25,800 & -.- & 7,970 & $2.01 \mathrm{E}-02$ & $<0$ \\
\hline A161.40.9.S130.7 & 30 & $1.77 \mathrm{E}-05$ & 16.0 & 2.031 & 4.11E-02 & 55 & $4.41 \mathrm{E}-04$ & 2,580 & $2.64 \mathrm{E}-02$ & 26,100 & --- & 8,880 & $2.49 \mathrm{E}-02$ & $<0$ \\
\hline A161.40.9.SI30.8 & 30 & 1.50E-05 & 18.8 & 2.028 & 4.11E-02 & 71 & 4.87E-04 & 2,680 & $2.33 \mathrm{E}-02$ & 25,800 & --- & 9,190 & $2.19 \mathrm{E}-02$ & $<0$ \\
\hline A161.40.9.S130.9 & 30 & $1.91 \mathrm{E}-05$ & 21.0 & 2.025 & $4.11 \mathrm{E}-02$ & 53 & 4.62E-04 & 2,370 & 2.62E-02 & 24,100 & --- & 8,710 & $2.64 \mathrm{E}-02$ & 8.03E-11 \\
\hline A161.40.9.SI30.10 & 30 & 1.90E-05 & 23.1 & 2.023 & 4.10E-02 & 51 & 4.45E-04 & 2,370 & $2.61 \mathrm{E}-02$ & 26,300 & --- & 8,060 & $2.43 \mathrm{E}-02$ & $<0$ \\
\hline A161.40.9.SI30.11 & 30 & 1.78E-05 & 25.8 & 2.020 & $4.10 \mathrm{E}-02$ & 52 & $4.21 \mathrm{E}-04$ & 2,310 & $2.39 \mathrm{E}-02$ & 26,100 & --- & 7,970 & $2.25 \mathrm{E}-02$ & $<0$ \\
\hline A161.40.9.SI30.12 & 30 & 1.63E-05 & 28.1 & 2.019 & 4.10E-02 & 64 & 4.82E-04 & 2,420 & $2.30 \mathrm{E}-02$ & 25,900 & --- & 9,150 & $2.38 \mathrm{E}-02$ & $4.06 \mathrm{E}-10$ \\
\hline A161.40.9.SI30.13 & 30 & 1.45E-05 & 30.1 & 2.017 & 4.09E-02 & 50 & $3.35 \mathrm{E}-04$ & 2,290 & $1.94 \mathrm{E}-02$ & 25,600 & --- & 8,920 & $2.07 E-02$ & $6.47 \mathrm{E}-10$ \\
\hline
\end{tabular}

B. 48 


\begin{tabular}{|c|c|c|c|c|c|c|c|c|c|c|c|c|c|c|}
\hline Sample ID & $\begin{array}{l}\text { Influent } \\
{[\mathrm{Si}]} \\
(\mathrm{ppm})\end{array}$ & $\begin{array}{l}\text { Flow } \\
\text { Rate, q } \\
\left(\mathrm{m}^{3} \mathrm{~d}^{-1}\right)\end{array}$ & $\begin{array}{l}\text { Time } \\
\text { (d) }\end{array}$ & $\begin{array}{l}\text { Glass } \\
\text { Mass } \\
\text { (g) }\end{array}$ & $\begin{array}{l}\text { Surface } \\
\text { Area, S } \\
\left(\mathrm{m}^{2}\right)\end{array}$ & $\begin{array}{l}{[\mathrm{Al}]} \\
\left(\mu \mathrm{g} \mathrm{L}^{-1}\right)\end{array}$ & $\begin{array}{l}\text { Al Norm. } \\
\text { Diss. Rate } \\
\left(\mathrm{g} \mathrm{m}^{-2} \mathrm{~d}^{-1}\right)\end{array}$ & $\begin{array}{l}{[\mathrm{B}]} \\
\left(\mu \mathrm{g} \mathrm{L}^{-1}\right)\end{array}$ & $\begin{array}{l}\text { B Norm. } \\
\text { Diss. Rate } \\
\left(\mathrm{g} \mathrm{m}^{-2} \mathrm{~d}^{-1}\right)\end{array}$ & $\begin{array}{l}{[\mathrm{Si}]} \\
\left(\mu \mathrm{g} \mathrm{L}^{-1}\right)\end{array}$ & $\begin{array}{l}\text { Si Norm. } \\
\text { Diss. Rate } \\
\left(\mathrm{g} \mathrm{m}^{-2} \mathrm{~d}^{-1}\right)\end{array}$ & $\begin{array}{l}{[\mathrm{Na}]} \\
(\mu \mathrm{g} \mathrm{L} \\
\left.{ }^{1}\right)\end{array}$ & $\begin{array}{l}\text { Na Norm. } \\
\text { Diss. Rate, } \\
\mathrm{r} \\
\left(\mathrm{g} \mathrm{m}^{-2} \mathrm{~d}^{-1}\right)\end{array}$ & $\begin{array}{l}\mathrm{r}_{\mathrm{IEX}} \\
\left(\mathrm{mol} \mathrm{Na}^{-2} \mathrm{~s}^{-1}\right)\end{array}$ \\
\hline A161.40.9.SI30.15 & 30 & $1.26 \mathrm{E}-05$ & 35.0 & 2.013 & $4.09 \mathrm{E}-02$ & 52 & $3.01 \mathrm{E}-04$ & 2,620 & $1.93 \mathrm{E}-02$ & 26,500 & --- & 9,650 & $1.94 \mathrm{E}-02$ & $8.06 \mathrm{E}-11$ \\
\hline A161.40.9.SI30.17 & 30 & $1.34 \mathrm{E}-05$ & 39.8 & 2.009 & $4.08 \mathrm{E}-02$ & $\begin{array}{l}{[49.4]} \\
46^{*}\end{array}$ & $3.06 \mathrm{E}-04$ & 2,390 & 1.87E-02 & 25,900 & -- & 10,700 & $2.30 \mathrm{E}-02$ & $2.13 E-09$ \\
\hline A161.40.9.SI30.18 & 30 & 1.54E-05 & 42.0 & 2.008 & $4.08 \mathrm{E}-02$ & $\begin{array}{l}{[49.4]} \\
45^{\star}\end{array}$ & $3.51 \mathrm{E}-04$ & 2,470 & $2.23 E-02$ & 26,800 & --- & 9,120 & $2.25 \mathrm{E}-02$ & $1.21 \mathrm{E}-10$ \\
\hline \multicolumn{15}{|c|}{ A161 $40^{\circ} \mathrm{C} \mathrm{pH} 9$ Si Reactor $\# 3$} \\
\hline A161.40.9.S150.Blank1 & 50 & 2.13E-05 & --- & -- & --- & {$[49.4]$} & -- & {$[75.6]$} & --- & 43,100 & --- & 297 & --- & --- \\
\hline A161.40.9.SI50.Blank3 & 50 & $2.10 \mathrm{E}-05$ & --- & -- & --- & [49.4] & -- & [75.6] & --- & 42,900 & --- & 326 & --- & --- \\
\hline A161.40.9.S150.2 & 50 & 3.32E-06 & 4.9 & 1.972 & $3.98 \mathrm{E}-02$ & 84 & $1.32 \mathrm{E}-04$ & 2,100 & 4.17E-03 & 42,900 & --- & 8,320 & 4.53E-03 & $1.78 \mathrm{E}-10$ \\
\hline A161.40.9.S150.4 & 50 & $2.59 \mathrm{E}-08$ & 9.0 & 1.972 & $3.98 \mathrm{E}-02$ & [49.4] & $6.05 \mathrm{E}-07$ & 143 & $2.22 \mathrm{E}-06$ & 2,720 & --- & 848 & $3.60 \mathrm{E}-06$ & $6.96 \mathrm{E}-13$ \\
\hline A161.40.9.S150.5 & 50 & $1.98 \mathrm{E}-05$ & 11.8 & 1.964 & 3.97E-02 & 74 & $6.94 \mathrm{E}-04$ & 4,400 & $5.23 \mathrm{E}-02$ & 42,700 & -.- & 17,600 & $5.73 \mathrm{E}-02$ & $2.50 \mathrm{E}-09$ \\
\hline A161.40.9.SI50.6 & 50 & 2.03E-05 & 14.1 & 1.962 & 3.97E-02 & 61 & $5.85 \mathrm{E}-04$ & 2,800 & $3.41 \mathrm{E}-02$ & 42,900 & --- & 10,000 & $3.33 \mathrm{E}-02$ & $<0$ \\
\hline A161.40.9.S150.7 & 50 & 2.03E-05 & 16.0 & 1.960 & 3.97E-02 & 58 & $5.61 \mathrm{E}-04$ & 2,520 & 3.07E-02 & 42,700 & --- & 9,250 & $3.08 \mathrm{E}-02$ & 7.51E-11 \\
\hline A161.40.9.SI50.8 & 50 & 2.02E-05 & 18.8 & 1.956 & $3.96 \mathrm{E}-02$ & 54 & $5.20 \mathrm{E}-04$ & 2,430 & $2.96 \mathrm{E}-02$ & 42,800 & --- & 8,550 & $2.85 \mathrm{E}-02$ & $<0$ \\
\hline A161.40.9.S150.9 & 50 & 2.03E-05 & 21.0 & 1.954 & $3.96 \mathrm{E}-02$ & 55 & $5.33 \mathrm{E}-04$ & 2,220 & $2.71 \mathrm{E}-02$ & 42,500 & --- & 7,580 & $2.53 \mathrm{E}-02$ & $<0$ \\
\hline A161.40.9.S150.10 & 50 & 2.02E-05 & 23.1 & 1.952 & $3.96 \mathrm{E}-02$ & $\begin{array}{l}{[49.4]} \\
40^{\star}\end{array}$ & $4.76 \mathrm{E}-04$ & 2,170 & $2.65 \mathrm{E}-02$ & 43,600 & -- & 7,770 & $2.59 \mathrm{E}-02$ & $<0$ \\
\hline A161.40.9.SI50.11 & 50 & 2.03E-05 & 25.8 & 1.949 & $3.95 \mathrm{E}-02$ & 52 & $5.05 \mathrm{E}-04$ & 2,180 & 2.67E-02 & 43,200 & --- & 8,790 & $2.95 \mathrm{E}-02$ & $1.40 \mathrm{E}-09$ \\
\hline A161.40.9.SI50.12 & 50 & 2.10E-05 & 28.1 & 1.947 & 3.95E-02 & $\begin{array}{l}{[49.4]} \\
40^{\star}\end{array}$ & 4.94E-04 & 2,170 & $2.75 \mathrm{E}-02$ & 43,900 & --- & 7,480 & $2.59 \mathrm{E}-02$ & $<0$ \\
\hline A161.40.9.SI50.13 & 50 & $1.94 \mathrm{E}-05$ & 30.1 & 1.945 & 3.95E-02 & $\begin{array}{l}{[49.4]} \\
37^{\star}\end{array}$ & 4.57E-04 & 1,970 & $2.31 E-02$ & 42,500 & -- & 7,800 & $2.50 E-02$ & $9.76 \mathrm{E}-10$ \\
\hline A161.40.9.S150.15 & 50 & 2.03E-05 & 35.0 & 1.940 & $3.94 \mathrm{E}-02$ & $\begin{array}{l}{[49.4]} \\
34^{*}\end{array}$ & $4.79 \mathrm{E}-04$ & 2,020 & $2.48 \mathrm{E}-02$ & 43,500 & --. & 7,750 & $2.61 \mathrm{E}-02$ & $6.28 \mathrm{E}-10$ \\
\hline A161.40.9.S150.17 & 50 & 2.01E-05 & 39.8 & 1.936 & $3.94 \mathrm{E}-02$ & $\begin{array}{l}{[49.4]} \\
33^{*}\end{array}$ & $4.76 \mathrm{E}-04$ & 1,850 & $2.26 \mathrm{E}-02$ & 43,100 & -- & 7,420 & $2.48 \mathrm{E}-02$ & $1.11 \mathrm{E}-09$ \\
\hline A161.40.9.SI50.18 & 50 & $2.01 \mathrm{E}-05$ & 42.0 & 1.934 & 3.93E-02 & $\begin{array}{l}{[49.4]} \\
33^{*}\end{array}$ & 4.74E-04 & 1,920 & 2.33E-02 & 44,100 & --- & 7,520 & $2.50 \mathrm{E}-02$ & $8.48 E-10$ \\
\hline \multicolumn{15}{|c|}{ A161 $40^{\circ} \mathrm{C}$ pH 9 Si Reactor $\# 4$} \\
\hline A161.40.9.20Si5.Blank2 & 5 & & --- & --- & --- & [49.4] & -- & [75.6] & --- & 4,830 & --- & [134] & --- & --- \\
\hline A161.40.9.20Si5.Blank3 & 5 & & --- & --- & --- & {$[49.4]$} & -- & {$[75.6]$} & --- & 4,820 & --- & [134] & --- & --- \\
\hline A161.40.9.20Si5.Blank1 & 5 & $1.16 \mathrm{E}-05$ & --- & --- & --- & [49.4] & -- & [75.6] & --- & 4,590 & --- & 344 & --- & --- \\
\hline A161.40.9.20Si5.1 & 5 & $1.92 \mathrm{E}-05$ & 1.9 & 0.999 & $2.02 \mathrm{E}-02$ & 205 & $3.66 \mathrm{E}-03$ & 1,280 & $2.90 \mathrm{E}-02$ & 5,050 & --- & 5,700 & $3.53 \mathrm{E}-02$ & $3.19 \mathrm{E}-09$ \\
\hline A161.40.9.20Si5.2 & 5 & 2.07E-05 & 2.9 & 0.999 & $2.02 \mathrm{E}-02$ & 342 & $6.60 \mathrm{E}-03$ & 1,640 & $4.01 \mathrm{E}-02$ & 5,160 & --- & 6,970 & $4.66 \mathrm{E}-02$ & $3.30 \mathrm{E}-09$ \\
\hline A161.40.9.20Si5.3 & 5 & 2.07E-05 & 4.9 & 0.997 & $2.02 \mathrm{E}-02$ & 401 & $7.73 \mathrm{E}-03$ & 1,670 & $4.08 \mathrm{E}-02$ & 5,100 & --- & 7,020 & $4.69 \mathrm{E}-02$ & $3.10 \mathrm{E}-09$ \\
\hline A161.40.9.20Si5.4 & 5 & 2.06E-05 & 6.8 & 0.995 & $2.01 \mathrm{E}-02$ & 432 & $8.32 \mathrm{E}-03$ & 1,510 & $3.68 \mathrm{E}-02$ & 5,180 & --- & 6,050 & $4.04 \mathrm{E}-02$ & $1.80 \mathrm{E}-09$ \\
\hline A161.40.9.20Si5.5 & 5 & $2.01 \mathrm{E}-05$ & 9.9 & 0.993 & $2.01 \mathrm{E}-02$ & 466 & $8.76 \mathrm{E}-03$ & 1,410 & $3.36 \mathrm{E}-02$ & 5,030 & -- & 5,540 & $3.61 \mathrm{E}-02$ & $1.28 \mathrm{E}-09$ \\
\hline A161.40.9.20Si5.6 & 5 & 2.07E-05 & 13.0 & 0.991 & $2.01 \mathrm{E}-02$ & 482 & $9.36 \mathrm{E}-03$ & 1,320 & $3.25 \mathrm{E}-02$ & 4,930 & --- & 5,190 & $3.49 E-02$ & $1.25 \mathrm{E}-09$ \\
\hline A161.40.9.20Si5.7 & 5 & 2.02E-05 & 16.8 & 0.988 & $2.00 \mathrm{E}-02$ & 484 & $9.18 \mathrm{E}-03$ & 1,260 & 3.03E-02 & 4,760 & --- & 5,060 & $3.33 \mathrm{E}-02$ & $1.51 \mathrm{E}-09$ \\
\hline A161.40.9.20Si5.8 & 5 & 2.09E-05 & 19.9 & 0.986 & $2.00 \mathrm{E}-02$ & 418 & $8.20 \mathrm{E}-03$ & 1,420 & $3.53 \mathrm{E}-02$ & 6,160 & --- & 4,840 & $3.29 \mathrm{E}-02$ & $<0$ \\
\hline \multicolumn{15}{|c|}{ A161 $40^{\circ} \mathrm{C}$ pH 9 Si Reactor \#5 } \\
\hline A161.40.9.20Si3.Blank2 & 3 & & --- & --- & --- & [49.4] & --- & [75.6] & --- & 2,890 & --- & [134] & --- & --- \\
\hline A161.40.9.20Si3.Blank3 & 3 & & -.- & --. & --- & [49.4] & --- & {$[75.6]$} & --- & 2,850 & -.- & [134] & -.- & -.- \\
\hline A161.40.9.20Si3.Blank1 & 3 & 8.95E-06 & --- & --- & --- & [49.4] & --- & [75.6] & --- & 3,120 & --- & 241 & --- & --- \\
\hline A161.40.9.20Si3.1 & 3 & $1.98 \mathrm{E}-05$ & 1.9 & 0.999 & $2.02 \mathrm{E}-02$ & 236 & $4.36 \mathrm{E}-03$ & 1,390 & $3.26 \mathrm{E}-02$ & 5,150 & --- & 6,070 & $3.89 \mathrm{E}-02$ & $3.20 \mathrm{E}-09$ \\
\hline
\end{tabular}




\begin{tabular}{|c|c|c|c|c|c|c|c|c|c|c|c|c|c|c|}
\hline Sample ID & $\begin{array}{l}\text { Influent } \\
{[\mathrm{Si}]} \\
(\mathrm{ppm})\end{array}$ & $\begin{array}{l}\text { Flow } \\
\text { Rate, q } \\
\left(\mathrm{m}^{3} \mathrm{~d}^{-1}\right)\end{array}$ & $\begin{array}{l}\text { Time } \\
\text { (d) }\end{array}$ & $\begin{array}{l}\text { Glass } \\
\text { Mass } \\
\text { (g) }\end{array}$ & $\begin{array}{l}\text { Surface } \\
\text { Area, S } \\
\left(\mathrm{m}^{2}\right)\end{array}$ & $\begin{array}{l}{[\mathrm{Al}]} \\
\left(\mu \mathrm{L} \mathrm{L}^{-1}\right)\end{array}$ & $\begin{array}{l}\text { Al Norm. } \\
\text { Diss. Rate } \\
\left(\mathrm{g} \mathrm{m}^{-2} \mathrm{~d}^{-1}\right)\end{array}$ & $\begin{array}{l}{[B]} \\
\left(\mu \mathrm{L} \mathrm{L}^{-1}\right)\end{array}$ & $\begin{array}{l}\text { B Norm. } \\
\text { Diss. Rate } \\
\left(\mathrm{g} \mathrm{m}^{-2} \mathrm{~d}^{-1}\right)\end{array}$ & $\begin{array}{l}{[\mathrm{Si}]} \\
\left(\mu g \mathrm{~L}^{-1}\right)\end{array}$ & $\begin{array}{l}\text { Si Norm. } \\
\text { Diss. Rate } \\
\left(\mathrm{g} \mathrm{m}^{-2} \mathrm{~d}^{-1}\right)\end{array}$ & $\begin{array}{l}{[\mathrm{Na}]} \\
(\mu \mathrm{g} \mathrm{L} \\
\left.{ }^{1}\right)\end{array}$ & $\begin{array}{l}\text { Na Norm. } \\
\text { Diss. Rate, } \\
\mathrm{r} \\
\left(\mathrm{g} \mathrm{m}^{-2} \mathrm{~d}^{-1}\right)\end{array}$ & $\begin{array}{l}\mathrm{r}_{\mathrm{IEX}} \\
\left(\mathrm{mol} \mathrm{Na} \mathrm{s}^{-1}\right)\end{array}$ \\
\hline A161.40.9.20Si3.2 & 3 & 2.09E-05 & 2.9 & 0.998 & $2.02 \mathrm{E}-02$ & 374 & $7.31 \mathrm{E}-03$ & 1,820 & $4.50 \mathrm{E}-02$ & 5,940 & --- & 7,830 & $5.30 \mathrm{E}-02$ & $4.03 E-09$ \\
\hline A161.40.9.20Si3.3 & 3 & 2.09E-05 & 4.9 & 0.996 & $2.01 \mathrm{E}-02$ & 375 & $7.31 \mathrm{E}-03$ & 1,680 & $4.15 \mathrm{E}-02$ & 5,810 & -- & 7,240 & $4.89 \mathrm{E}-02$ & $3.75 \mathrm{E}-09$ \\
\hline A161.40.9.20Si3.4 & 3 & $2.08 \mathrm{E}-05$ & 6.8 & 0.994 & $2.01 \mathrm{E}-02$ & 399 & $7.78 \mathrm{E}-03$ & 1,600 & $3.95 \mathrm{E}-02$ & 6,120 & --- & 6,640 & $4.49 E-02$ & $2.71 \mathrm{E}-09$ \\
\hline A161.40.9.20Si3.5 & 3 & 2.09E-05 & 9.9 & 0.991 & $2.01 \mathrm{E}-02$ & 409 & $8.01 \mathrm{E}-03$ & 1,510 & $3.74 \mathrm{E}-02$ & 6,170 & --- & 6,090 & 4.13E-02 & $1.96 \mathrm{E}-09$ \\
\hline A161.40.9.20Si3.6 & 3 & $2.08 \mathrm{E}-05$ & 13.0 & 0.989 & $2.01 \mathrm{E}-02$ & 403 & $7.88 \mathrm{E}-03$ & 1,430 & $3.54 \mathrm{E}-02$ & 6,010 & --- & 5,660 & $3.84 \mathrm{E}-02$ & 1.49E-09 \\
\hline A161.40.9.20Si3.7 & 3 & 2.09E-05 & 16.8 & 0.986 & $2.00 \mathrm{E}-02$ & 409 & $8.02 \mathrm{E}-03$ & 1,430 & $3.55 \mathrm{E}-02$ & 6,110 & --- & 5,650 & $3.84 \mathrm{E}-02$ & $1.46 \mathrm{E}-09$ \\
\hline A161.40.9.20Si3.8 & 3 & $2.06 \mathrm{E}-05$ & 19.9 & 0.985 & $2.00 \mathrm{E}-02$ & 500 & $9.71 \mathrm{E}-03$ & 1,250 & 3.07E-02 & 4,870 & --- & 4,300 & $2.90 \mathrm{E}-02$ & $<0$ \\
\hline A161.40.9.20Si3.9 & 3 & 2.06E-05 & 23.9 & 0.982 & $2.00 \mathrm{E}-02$ & 427 & $8.31 \mathrm{E}-03$ & 1,400 & $3.45 \mathrm{E}-02$ & 6,230 & --- & 4,950 & $3.34 \mathrm{E}-02$ & $<0$ \\
\hline A161.40.9.20Si3.10 & 3 & 2.09E-05 & 25.9 & 0.981 & $2.00 \mathrm{E}-02$ & 486 & $9.59 \mathrm{E}-03$ & 1,200 & $3.00 \mathrm{E}-02$ & 4,770 & --- & 4,050 & $2.77 \mathrm{E}-02$ & $<0$ \\
\hline A161.40.9.20Si3.11 & 3 & 2.07E-05 & 26.8 & 0.981 & 1.99E-02 & 431 & $8.42 \mathrm{E}-03$ & 1,080 & $2.67 \mathrm{E}-02$ & 4,560 & --. & 3,750 & $2.54 \mathrm{E}-02$ & $<0$ \\
\hline A161.40.9.20Si3.12 & 3 & $2.08 \mathrm{E}-05$ & 27.8 & 0.981 & $1.99 \mathrm{E}-02$ & 401 & $7.85 \mathrm{E}-03$ & 1,050 & 2.60E-02 & 4,430 & --- & 3,630 & $2.46 \mathrm{E}-02$ & $<0$ \\
\hline \multicolumn{15}{|c|}{ A161 $70^{\circ} \mathrm{C} \mathrm{pH} 9$ Si Reactor \#1 } \\
\hline A161.70.9.SI30.Blank2 & 30 & $2.36 \mathrm{E}-05$ & --- & --- & --- & {$[49.4]$} & -- & {$[75.6]$} & --- & 25,700 & --- & [134] & --- & --- \\
\hline A161.70.9.SI30.Blank3 & 30 & $6.93 \mathrm{E}-05$ & --- & --- & -- & {$[49.4]$} & -- & [75.6] & --- & 25,900 & -- & [134] & --- & -- \\
\hline A161.70.9.SI30.2 & 30 & $2.21 \mathrm{E}-05$ & 5.0 & 1.942 & $3.96 \mathrm{E}-02$ & 205 & $2.16 \mathrm{E}-03$ & 17,100 & $2.28 \mathrm{E}-01$ & 26,500 & --- & 69,700 & $2.54 \mathrm{E}-01$ & $1.33 \mathrm{E}-08$ \\
\hline A161.70.9.SI30.6 & 30 & $2.04 \mathrm{E}-05$ & 14.0 & 1.854 & $3.84 \mathrm{E}-02$ & 193 & $1.93 \mathrm{E}-03$ & 13,400 & $1.69 \mathrm{E}-01$ & 28,000 & --- & 53,200 & $1.84 \mathrm{E}-01$ & 7.41E-09 \\
\hline A161.70.9.SI30.8 & 30 & $1.67 \mathrm{E}-05$ & 18.9 & 1.839 & $3.82 \mathrm{E}-02$ & 171 & $1.41 \mathrm{E}-03$ & 8,950 & $9.32 \mathrm{E}-02$ & 28,100 & --- & 37,400 & $1.07 \mathrm{E}-01$ & $6.75 \mathrm{E}-09$ \\
\hline A161.70.9.S130.10 & 30 & $1.70 \mathrm{E}-05$ & 23.8 & 1.824 & $3.80 \mathrm{E}-02$ & 183 & $1.54 \mathrm{E}-03$ & 7,680 & $8.21 \mathrm{E}-02$ & 27,700 & --- & 33,000 & $9.66 \mathrm{E}-02$ & $7.29 \mathrm{E}-09$ \\
\hline A161.70.9.SI30.11 & 30 & $1.72 \mathrm{E}-05$ & 25.9 & 1.816 & 3.79E-02 & 208 & $1.77 \mathrm{E}-03$ & 9,510 & 1.03E-01 & 28,200 & --- & 37,900 & $1.12 \mathrm{E}-01$ & 4.70E-09 \\
\hline \multicolumn{15}{|c|}{ A161 $70^{\circ} \mathrm{C}$ pH 9 Si Reactor $\# 2$} \\
\hline A161.70.9.SI60.Blank1 & 60 & $1.16 \mathrm{E}-05$ & --- & --- & --- & [49.4] & --- & [75.6] & --- & 53,400 & --- & [134] & --- & --- \\
\hline A161.70.9.SI60.Blank2 & 60 & 1.97E-05 & --- & -.- & --- & {$[49.4]$} & --- & [75.6] & --- & 53,800 & --- & [134] & --- & --- \\
\hline A161.70.9.SI60.Blank3 & 60 & $2.06 \mathrm{E}-05$ & --- & --- & --- & {$[49.4]$} & -- & [75.6] & --- & 54,300 & --- & [134] & --- & --- \\
\hline A161.70.9.S160.2 & 60 & 1.66E-05 & 5.0 & 1.965 & 4.00E-02 & 86 & $6.73 \mathrm{E}-04$ & 16,200 & $1.60 \mathrm{E}-01$ & 56,400 & --- & 65,900 & $1.79 \mathrm{E}-01$ & $9.18 \mathrm{E}-09$ \\
\hline A161.70.9.S160.3 & 60 & $1.58 \mathrm{E}-05$ & 7.1 & 1.954 & $3.98 \mathrm{E}-02$ & 78 & $5.82 \mathrm{E}-04$ & 15,500 & 1.47E-01 & 56,000 & --- & 69,500 & $1.80 \mathrm{E}-01$ & $1.68 \mathrm{E}-08$ \\
\hline A161.70.9.S160.4 & 60 & 1.52E-05 & 9.8 & 1.939 & $3.96 \mathrm{E}-02$ & 71 & $5.17 \mathrm{E}-04$ & 14,800 & $1.36 \mathrm{E}-01$ & 53,400 & --- & 57,500 & $1.44 \mathrm{E}-01$ & $4.34 \mathrm{E}-09$ \\
\hline A161.70.9.S160.6 & 60 & $1.21 \mathrm{E}-05$ & 14.0 & 1.924 & $3.94 \mathrm{E}-02$ & 67 & $3.85 \mathrm{E}-04$ & 14,700 & $1.08 \mathrm{E}-01$ & 54,600 & --- & 56,400 & $1.13 \mathrm{E}-01$ & 2.73E-09 \\
\hline A161.70.9.S160.9 & 60 & 1.67E-05 & 20.9 & 1.831 & $3.81 \mathrm{E}-02$ & 56 & $4.60 \mathrm{E}-04$ & 11,100 & $1.16 \mathrm{E}-01$ & 53,800 & --- & 43,700 & $1.25 \mathrm{E}-01$ & 4.53E-09 \\
\hline A161.70.9.S160.10 & 60 & 1.71E-05 & 23.8 & 1.818 & 3.79E-02 & 56 & 4.77E-04 & 9,930 & 1.07E-01 & 55,000 & --- & 39,000 & $1.15 \mathrm{E}-01$ & 4.04E-09 \\
\hline A161.70.9.S160.11 & 60 & $1.61 \mathrm{E}-05$ & 25.9 & 1.812 & $3.79 \mathrm{E}-02$ & 57 & $4.54 \mathrm{E}-04$ & 9,950 & $1.01 \mathrm{E}-01$ & 54,500 & --- & 38,400 & $1.06 \mathrm{E}-01$ & $2.86 \mathrm{E}-09$ \\
\hline A161.70.9.S160.12 & 60 & $1.50 \mathrm{E}-05$ & 27.8 & 1.807 & $3.78 \mathrm{E}-02$ & 52 & 3.87E-04 & 9,040 & $8.58 \mathrm{E}-02$ & 56,100 & --- & 37,300 & $9.69 \mathrm{E}-02$ & $5.59 \mathrm{E}-09$ \\
\hline \multicolumn{15}{|c|}{ A161 $70^{\circ} \mathrm{C} \mathrm{pH} 9$ Si Reactor $\# 3$} \\
\hline A161.70.9.SI90.Blank1 & 90 & $1.83 \mathrm{E}-05$ & --- & -- & --- & 55 & --- & [75.6] & --- & 81,900 & --- & 168 & --- & --- \\
\hline A161.70.9.SI90.Blank2 & 90 & $1.87 \mathrm{E}-05$ & --- & --- & --- & 53 & --- & [75.6] & --- & 81,400 & --- & 164 & $\cdots$ & --- \\
\hline A161.70.9.SI90.Blank3 & 90 & 2.05E-05 & -.- & --. & -.- & 51 & --- & [75.6] & --- & 79,800 & --- & 168 & --- & -.- \\
\hline A161.70.9.S190.2 & 90 & $1.57 \mathrm{E}-05$ & 5.0 & 1.964 & 3.99E-02 & 70 & 5.17E-04 & 16,100 & $1.51 \mathrm{E}-01$ & 82,700 & -- & 72,400 & $1.85 \mathrm{E}-01$ & $1.75 \mathrm{E}-08$ \\
\hline A161.70.9.S190.4 & 90 & $1.83 \mathrm{E}-05$ & 9.8 & 1.933 & 3.95E-02 & 69 & $6.02 \mathrm{E}-04$ & 14,000 & 1.54E-01 & 84,500 & --- & 56,800 & $1.71 \mathrm{E}-01$ & $8.60 \mathrm{E}-09$ \\
\hline A161.70.9.S190.6 & 90 & $1.75 \mathrm{E}-05$ & 14.0 & 1.916 & 3.93E-02 & 75 & $6.27 \mathrm{E}-04$ & 11,800 & $1.26 \mathrm{E}-01$ & 83,700 & -- & 48,500 & $1.41 \mathrm{E}-01$ & $7.92 \mathrm{E}-09$ \\
\hline A161.70.9.S190.8 & 90 & 1.96E-05 & 18.9 & 1.896 & $3.90 \mathrm{E}-02$ & 57 & $5.37 \mathrm{E}-04$ & 8,750 & $1.05 \mathrm{E}-01$ & 82,400 & --- & 36,500 & $1.20 \mathrm{E}-01$ & $7.51 \mathrm{E}-09$ \\
\hline A161.70.9.S190.10 & 90 & $2.05 \mathrm{E}-05$ & 23.8 & 1.877 & 3.87E-02 & {$[49.4]$} & 4.93E-04 & 7,690 & $9.72 \mathrm{E}-02$ & 79,900 & --- & 31,700 & 1.10E-01 & $6.29 E-09$ \\
\hline A161.70.9.S190.11 & 90 & $2.00 \mathrm{E}-05$ & 25.9 & 1.869 & $3.86 \mathrm{E}-02$ & [49.4] & $4.81 \mathrm{E}-04$ & 8,780 & $1.08 \mathrm{E}-01$ & 82,500 & -- & 37,500 & $1.27 \mathrm{E}-01$ & $9.23 \mathrm{E}-09$ \\
\hline A161.70.9.S190.12 & 90 & 1.93E-05 & 27.8 & 1.862 & $3.85 \mathrm{E}-02$ & {$[49.4]$} & $4.66 \mathrm{E}-04$ & 8,990 & 1.07E-01 & 84,400 & --- & 36,600 & $1.20 \mathrm{E}-01$ & $6.20 \mathrm{E}-09$ \\
\hline
\end{tabular}




\begin{tabular}{|c|c|c|c|c|c|c|c|c|c|c|c|c|c|c|}
\hline Sample ID & $\begin{array}{l}\text { Influent } \\
\text { [Si] } \\
\text { (ppm) }\end{array}$ & $\begin{array}{l}\text { Flow } \\
\text { Rate, q } \\
\left(\mathrm{m}^{3} \mathrm{~d}^{-1}\right)\end{array}$ & $\begin{array}{l}\text { Time } \\
\text { (d) }\end{array}$ & $\begin{array}{l}\text { Glass } \\
\text { Mass } \\
\text { (g) }\end{array}$ & $\begin{array}{l}\text { Surface } \\
\text { Area, S } \\
\left(\mathrm{m}^{2}\right)\end{array}$ & $\begin{array}{l}{[\mathrm{Al}]} \\
\left(\mu \mathrm{g} \mathrm{L}^{-1}\right)\end{array}$ & $\begin{array}{l}\text { Al Norm. } \\
\text { Diss. Rate } \\
\left(\mathrm{g} \mathrm{m}^{-2} \mathrm{~d}^{-1}\right)\end{array}$ & $\begin{array}{l}{[\mathrm{B}]} \\
\left(\mu \mathrm{g} \mathrm{L}^{-1}\right)\end{array}$ & $\begin{array}{l}\text { B Norm. } \\
\text { Diss. Rate } \\
\left(\mathrm{g} \mathrm{m}^{-2} \mathrm{~d}^{-1}\right)\end{array}$ & $\begin{array}{l}{[\mathrm{Si}]} \\
\left(\mu \mathrm{g} \mathrm{L}^{-1}\right)\end{array}$ & $\begin{array}{l}\text { Si Norm. } \\
\text { Diss. Rate } \\
\left(\mathrm{g} \mathrm{m}^{-2} \mathrm{~d}^{-1}\right)\end{array}$ & $\begin{array}{l}{[\mathrm{Na}]} \\
\left(\mu \mathrm{g} \mathrm{L} \mathrm{L}^{-}\right. \\
\left.{ }^{1}\right)\end{array}$ & $\begin{array}{l}\text { Na Norm. } \\
\text { Diss. Rate, } \\
\mathrm{r} \\
\left(\mathrm{g} \mathrm{m}^{-2} \mathrm{~d}^{-1}\right)\end{array}$ & $\begin{array}{l}\mathrm{r}_{\mathrm{IEX}} \\
\left(\mathrm{mol} \mathrm{Na} \mathrm{s}^{-2} \mathrm{~s}^{-1}\right)\end{array}$ \\
\hline \multicolumn{15}{|c|}{ A161 $70^{\circ} \mathrm{C}$ pH 9 Si Reactor \#4 } \\
\hline A161.70.9.20Si5.Blank2 & 5 & & -- & --- & --- & {$[49.4]$} & --- & {$[75.6]$} & --- & 4,860 & --- & [134] & --- & -- \\
\hline A161.70.9.20Si5.Blank3 & 5 & & --- & --- & --- & [49.4] & --- & [75.6] & --- & 4,840 & -- & [134] & --- & --- \\
\hline A161.70.9.20Si5.Blank1 & 5 & $7.86 \mathrm{E}-06$ & --- & -- & --- & 68 & --- & [75.6] & --- & 4,500 & --- & 194 & --- & --- \\
\hline A161.70.9.20Si5.1 & 5 & $1.27 \mathrm{E}-05$ & 1.9 & 0.994 & $2.01 \mathrm{E}-02$ & 816 & $9.73 \mathrm{E}-03$ & 6,560 & $9.91 \mathrm{E}-02$ & 9,210 & --- & 25,800 & $1.07 \mathrm{E}-01$ & 3.82E-09 \\
\hline A161.70.9.20Si5.2 & 5 & $1.30 \mathrm{E}-05$ & 2.9 & 0.991 & $2.01 \mathrm{E}-02$ & 1,050 & $1.28 \mathrm{E}-02$ & 10,700 & $1.66 \mathrm{E}-01$ & 10,400 & --- & 41,200 & $1.75 \mathrm{E}-01$ & 4.51E-09 \\
\hline A161.70.9.20Si5.3 & 5 & $1.13 \mathrm{E}-05$ & 4.9 & 0.983 & 2.00E-02 & 1,110 & $1.18 \mathrm{E}-02$ & 12,100 & 1.63E-01 & 10,200 & -- & 46,300 & $1.70 \mathrm{E}-01$ & 3.89E-09 \\
\hline A161.70.9.20Si5.4 & 5 & $1.10 \mathrm{E}-05$ & 6.8 & 0.977 & $1.99 \mathrm{E}-02$ & 1,160 & $1.21 \mathrm{E}-02$ & 12,700 & 1.67E-01 & 10,100 & --- & 47,800 & $1.73 \mathrm{E}-01$ & $2.56 \mathrm{E}-09$ \\
\hline A161.70.9.20Si5.5 & 5 & $1.07 \mathrm{E}-05$ & 10.0 & 0.965 & 1.97E-02 & 1,170 & $1.19 \mathrm{E}-02$ & 11,800 & 1.52E-01 & 9,540 & -- & 45,000 & $1.59 \mathrm{E}-01$ & $3.36 \mathrm{E}-09$ \\
\hline A161.70.9.20Si5.6 & 5 & $1.08 \mathrm{E}-05$ & 11.9 & 0.962 & 1.97E-02 & 1,240 & $1.29 \mathrm{E}-02$ & 11,400 & 1.50E-01 & 9,560 & -- & 42,700 & $1.54 \mathrm{E}-01$ & $1.91 \mathrm{E}-09$ \\
\hline A161.70.9.20Si5.7 & 5 & $1.04 \mathrm{E}-05$ & 13.0 & 0.960 & 1.97E-02 & 1,240 & $1.23 \mathrm{E}-02$ & 10,700 & $1.35 \mathrm{E}-01$ & 9,290 & -- & 41,200 & $1.42 \mathrm{E}-01$ & 3.67E-09 \\
\hline A161.70.9.20Si5.8 & 5 & $1.18 \mathrm{E}-05$ & 16.8 & 0.946 & 0 & 1,250 & $1.43 \mathrm{E}-02$ & 9,740 & $1.41 \mathrm{E}-01$ & 9,030 & --- & 38,400 & $1.52 \mathrm{E}-01$ & 5.63E-09 \\
\hline A161.70.9.20Si5.9 & 5 & 1.16E-05 & 18.0 & 0.947 & 0 & 1,250 & 1.40E-02 & 8,880 & $1.26 \mathrm{E}-01$ & 8,960 & --- & 34,900 & $1.35 \mathrm{E}-01$ & 4.81E-09 \\
\hline \multicolumn{15}{|c|}{ A161 $70^{\circ} \mathrm{C}$ pH 9 Si Reactor $\# 5$} \\
\hline A161.70.9.20Si10.Blank2 & 10 & & -- & --- & --- & [49.4] & -- & [75.6] & --- & 9,040 & --- & [134] & --- & --- \\
\hline A161.70.9.20Si10.Blank3 & 10 & & -- & -- & --- & [49.4] & --- & [75.6] & --- & 9,080 & -- & [134] & --- & --- \\
\hline A161.70.9.20Si10.Blank1 & 10 & $9.68 \mathrm{E}-06$ & -.- & -.- & -.- & 221 & -.- & [75.6] & --- & 8,950 & --- & 172 & --- & -.- \\
\hline A161.70.9.20Si10.1 & 10 & $2.08 \mathrm{E}-05$ & 1.9 & 0.992 & $2.01 \mathrm{E}-02$ & 560 & $1.09 \mathrm{E}-02$ & 6,160 & 1.52E-01 & 11,800 & -- & 24,300 & $1.64 \mathrm{E}-01$ & $6.12 \mathrm{E}-09$ \\
\hline A161.70.9.20Si10.2 & 10 & $2.11 \mathrm{E}-05$ & 2.9 & 0.987 & $2.00 \mathrm{E}-02$ & 666 & $1.32 \mathrm{E}-02$ & 9,300 & $2.34 \mathrm{E}-01$ & 12,700 & --- & 35,200 & $2.43 \mathrm{E}-01$ & 4.26E-09 \\
\hline A161.70.9.20Si10.3 & 10 & $2.12 \mathrm{E}-05$ & 4.9 & 0.976 & $1.99 \mathrm{E}-02$ & 687 & $1.38 \mathrm{E}-02$ & 9,430 & $2.40 \mathrm{E}-01$ & 12,500 & -- & 36,100 & $2.51 \mathrm{E}-01$ & $5.79 \mathrm{E}-09$ \\
\hline A161.70.9.20Si10.4 & 10 & 2.03E-05 & 6.8 & 0.968 & $1.98 \mathrm{E}-02$ & 714 & $1.38 \mathrm{E}-02$ & 8,910 & $2.18 \mathrm{E}-01$ & 12,200 & --- & 34,200 & $2.29 \mathrm{E}-01$ & $5.56 \mathrm{E}-09$ \\
\hline A161.70.9.20Si10.5 & 10 & $2.09 E-05$ & 10.0 & 0.953 & $1.96 \mathrm{E}-02$ & 743 & $1.49 \mathrm{E}-02$ & 7,930 & 2.02E-01 & 12,200 & -- & 30,000 & $2.09 \mathrm{E}-01$ & $3.62 \mathrm{E}-09$ \\
\hline A161.70.9.20Si10.6 & 10 & 2.04E-05 & 11.9 & 0.950 & $1.95 \mathrm{E}-02$ & 761 & $1.50 \mathrm{E}-02$ & 6,920 & 1.73E-01 & 11,900 & --- & 26,800 & $1.83 \mathrm{E}-01$ & $5.24 \mathrm{E}-09$ \\
\hline A161.70.9.20Si10.7 & 10 & $2.12 \mathrm{E}-05$ & 13.0 & 0.947 & $1.95 \mathrm{E}-02$ & 784 & $1.61 \mathrm{E}-02$ & 6,640 & 1.73E-01 & 12,200 & -- & 25,100 & $1.79 \mathrm{E}-01$ & 3.03E-09 \\
\hline A161.70.9.20Si10.8 & 10 & $2.04 \mathrm{E}-05$ & 16.8 & 0.933 & $1.93 \mathrm{E}-02$ & 760 & $1.51 \mathrm{E}-02$ & 5,900 & 1.49E-01 & 11,800 & --- & 22,700 & $1.57 \mathrm{E}-01$ & $3.99 \mathrm{E}-09$ \\
\hline A161.70.9.20Si10.9 & 10 & $2.07 E-05$ & 18.0 & 0.934 & $1.93 \mathrm{E}-02$ & 753 & $1.52 \mathrm{E}-02$ & 5,270 & $1.35 \mathrm{E}-01$ & 11,500 & -- & 21,300 & $1.49 \mathrm{E}-01$ & $7.21 \mathrm{E}-09$ \\
\hline \multicolumn{15}{|c|}{ A161 $90^{\circ} \mathrm{C}$ pH 9 Si Reactor $\# 1$} \\
\hline A161.90.9.20S1100.Blank1 & 100 & $9.47 \mathrm{E}-06$ & --- & --. & --- & 69 & --- & [75.6] & --- & 95,200 & --- & 444 & --- & --- \\
\hline A161.90.9.20SI100.Blank2 & 100 & $1.56 \mathrm{E}-05$ & --- & --- & -.- & 66 & --- & [75.6] & --- & 94,500 & --- & 505 & --- & --- \\
\hline A161.90.9.20SI100.Blank3 & 100 & $2.01 \mathrm{E}-05$ & -.- & -.- & -.- & 53 & -.- & [75.6] & -.- & 96,500 & -.- & 448 & -.- & -.- \\
\hline A161.90.9.20S1100.2 & 100 & $1.83 E-05$ & 5.0 & 1.189 & $2.45 \mathrm{E}-02$ & 65 & $9.11 E-04$ & 23,200 & 4.14E-01 & 105,000 & -- & 85,100 & $4.15 \mathrm{E}-01$ & $8.68 \mathrm{E}-10$ \\
\hline A161.90.9.20SI100.4 & 100 & $1.80 \mathrm{E}-05$ & 9.0 & 1.169 & $2.42 \mathrm{E}-02$ & 61 & $8.55 \mathrm{E}-04$ & 16,500 & 2.92E-01 & 113,000 & -.- & 63,200 & $3.07 \mathrm{E}-01$ & 7.15E-09 \\
\hline A161.90.9.20S1100.5 & 100 & $2.01 \mathrm{E}-05$ & 11.8 & 1.150 & 2.39E-02 & 60 & $9.52 \mathrm{E}-04$ & 13,100 & 2.63E-01 & 112,000 & -- & 53,600 & $2.94 \mathrm{E}-01$ & $1.59 \mathrm{E}-08$ \\
\hline A161.90.9.20SI100.6 & 100 & $1.89 \mathrm{E}-05$ & 14.0 & 1.143 & $2.38 \mathrm{E}-02$ & 64 & $9.60 \mathrm{E}-04$ & 10,400 & 1.96E-01 & 107,000 & --- & 42,400 & $2.19 \mathrm{E}-01$ & $1.15 \mathrm{E}-08$ \\
\hline A161.90.9.20S1100.7 & 100 & $1.85 \mathrm{E}-05$ & 16.0 & 1.136 & $2.37 \mathrm{E}-02$ & 68 & $1.00 \mathrm{E}-03$ & 9,100 & 1.69E-01 & 107,000 & -- & 39,400 & $2.01 \mathrm{E}-01$ & $1.58 \mathrm{E}-08$ \\
\hline A161.90.9.20SI100.8 & 100 & $1.88 \mathrm{E}-05$ & 18.8 & 1.126 & $2.36 \mathrm{E}-02$ & 68 & $1.01 \mathrm{E}-03$ & 7,690 & 1.46E-01 & 104,000 & --- & 34,900 & $1.81 \mathrm{E}-01$ & $1.78 \mathrm{E}-08$ \\
\hline A161.90.9.20S1100.9 & 100 & $1.79 \mathrm{E}-05$ & 21.0 & 1.121 & $2.35 \mathrm{E}-02$ & 70 & $9.97 \mathrm{E}-04$ & 6,870 & $1.25 \mathrm{E}-01$ & 103,000 & -- & 28,000 & $1.39 \mathrm{E}-01$ & $7.26 \mathrm{E}-09$ \\
\hline \multicolumn{15}{|c|}{ A161 $90^{\circ} \mathrm{C}$ pH 9 Si Reactor \#2 } \\
\hline A161.90.9.S140.Blank1 & 40 & $2.04 \mathrm{E}-06$ & -- & --- & --- & 52 & --- & [75.6] & --- & 38,800 & -- & 361 & --- & --- \\
\hline A161.90.9.SI40.Blank2 & 40 & $1.03 \mathrm{E}-05$ & --- & --- & -.- & 50 & --- & [75.6] & --- & 37,000 & --- & 326 & --- & -.- \\
\hline A161.90.9.S140.Blank3 & 40 & $5.12 \mathrm{E}-06$ & --- & --- & --- & [49.4] & --- & [75.6] & --- & 38,700 & --- & 369 & --- & --- \\
\hline A161.90.9.S140.2 & 40 & $1.58 \mathrm{E}-05$ & 5.0 & 1.209 & $2.48 \mathrm{E}-02$ & 341 & 4.09E-03 & 20,900 & $3.18 \mathrm{E}-01$ & 39,200 & --- & 78,400 & $3.26 \mathrm{E}-01$ & 4.31E-09 \\
\hline A161.90.9.S140.4 & 40 & $1.45 \mathrm{E}-05$ & 9.0 & 1.197 & $2.46 \mathrm{E}-02$ & 384 & $4.26 \mathrm{E}-03$ & 14,300 & $2.01 \mathrm{E}-01$ & 39,900 & --- & 55,500 & $2.13 \mathrm{E}-01$ & $6.32 \mathrm{E}-09$ \\
\hline
\end{tabular}




\begin{tabular}{|c|c|c|c|c|c|c|c|c|c|c|c|c|c|c|}
\hline Sample ID & $\begin{array}{l}\text { Influent } \\
\text { [Si] } \\
\text { (ppm) }\end{array}$ & $\begin{array}{l}\text { Flow } \\
\text { Rate, q } \\
\left(\mathrm{m}^{3} \mathrm{~d}^{-1}\right)\end{array}$ & $\begin{array}{l}\text { Time } \\
\text { (d) }\end{array}$ & $\begin{array}{l}\text { Glass } \\
\text { Mass } \\
\text { (g) }\end{array}$ & $\begin{array}{l}\text { Surface } \\
\text { Area, S } \\
\left(\mathrm{m}^{2}\right)\end{array}$ & $\begin{array}{l}{[\mathrm{Al}]} \\
\left(\mu \mathrm{L} \mathrm{L}^{-1}\right)\end{array}$ & $\begin{array}{l}\text { Al Norm. } \\
\text { Diss. Rate } \\
\left(\mathrm{g} \mathrm{m}^{-2} \mathrm{~d}^{-1}\right)\end{array}$ & $\begin{array}{l}{[\mathrm{B}]} \\
\left(\mu \mathrm{g} \mathrm{L}^{-1}\right)\end{array}$ & $\begin{array}{l}\text { B Norm. } \\
\text { Diss. Rate } \\
\left(\mathrm{g} \mathrm{m}^{-2} \mathrm{~d}^{-1}\right)\end{array}$ & $\begin{array}{l}{[\mathrm{Si}]} \\
\left(\mu \mathrm{g} \mathrm{L}^{-1}\right)\end{array}$ & $\begin{array}{l}\text { Si Norm. } \\
\text { Diss. Rate } \\
\left(\mathrm{g} \mathrm{m}^{-2} \mathrm{~d}^{-1}\right)\end{array}$ & $\begin{array}{l}{[\mathrm{Na}]} \\
(\mu \mathrm{g} \mathrm{L} \\
\left.{ }^{1}\right)\end{array}$ & $\begin{array}{l}\text { Na Norm. } \\
\text { Diss. Rate, } \\
\mathrm{r} \\
\left(\mathrm{g} \mathrm{m}^{-2} \mathrm{~d}^{-1}\right)\end{array}$ & $\begin{array}{l}\mathrm{r}_{\mathrm{IEX}} \\
\left(\mathrm{mol} \mathrm{Na} \mathrm{No}^{-1}\right)\end{array}$ \\
\hline A161.90.9.S140.5 & 40 & $1.61 \mathrm{E}-05$ & 11.8 & 1.181 & $2.44 \mathrm{E}-02$ & 406 & $5.03 \mathrm{E}-03$ & 12,500 & $1.96 \mathrm{E}-01$ & 40,200 & --- & 47,300 & $2.03 \mathrm{E}-01$ & $3.55 \mathrm{E}-09$ \\
\hline A161.90.9.SI40.6 & 40 & $1.62 \mathrm{E}-05$ & 14.0 & 1.174 & 2.43E-02 & 465 & $5.84 \mathrm{E}-03$ & 11,100 & $1.77 \mathrm{E}-01$ & 43,000 & --- & 40,500 & $1.76 \mathrm{E}-01$ & $<0$ \\
\hline A161.90.9.S140.7 & 40 & $1.67 E-05$ & 16.0 & 1.168 & $2.42 \mathrm{E}-02$ & 443 & $5.74 \mathrm{E}-03$ & 9,050 & $1.49 \mathrm{E}-01$ & 40,100 & --- & 36,000 & $1.62 \mathrm{E}-01$ & $6.65 \mathrm{E}-09$ \\
\hline A161.90.9.S140.8 & 40 & 1.64E-05 & 18.8 & 1.159 & $2.41 \mathrm{E}-02$ & 445 & $5.69 \mathrm{E}-03$ & 7,770 & $1.26 \mathrm{E}-01$ & 39,700 & --- & 30,800 & $1.37 \mathrm{E}-01$ & $5.39 \mathrm{E}-09$ \\
\hline A161.90.9.S140.9 & 40 & $1.61 \mathrm{E}-05$ & 21.0 & 1.154 & $2.40 \mathrm{E}-02$ & 468 & $5.89 \mathrm{E}-03$ & 6,890 & $1.10 \mathrm{E}-01$ & 40,500 & -- & 28,000 & $1.22 \mathrm{E}-01$ & $6.23 \mathrm{E}-09$ \\
\hline \multicolumn{15}{|c|}{ A161 $90^{\circ} \mathrm{C}$ pH 9 Si Reactor \#3 } \\
\hline A161.90.9.S170.Blank1 & 70 & $1.69 \mathrm{E}-05$ & -- & --- & -- & 59 & -- & [75.6] & -- & 66,900 & -- & 424 & -- & -- \\
\hline A161.90.9.S170.Blank2 & 70 & $1.64 \mathrm{E}-05$ & --- & -.- & --- & 58 & --- & [75.6] & --- & 64,400 & --- & 453 & --- & --- \\
\hline A161.90.9.S170.Blank3 & 70 & $1.81 \mathrm{E}-05$ & --- & --- & --- & 61 & -- & {$[75.6]$} & --- & 67,900 & --- & 436 & --- & --- \\
\hline A161.90.9.S170.2 & 70 & $1.82 \mathrm{E}-05$ & 5.0 & 1.197 & $2.46 \mathrm{E}-02$ & 132 & $1.84 \mathrm{E}-03$ & 20,500 & $3.63 \mathrm{E}-01$ & 66,300 & --- & 79,100 & $3.83 \mathrm{E}-01$ & $1.03 \mathrm{E}-08$ \\
\hline A161.90.9.S170.4 & 70 & $1.87 \mathrm{E}-05$ & 9.0 & 1.178 & $2.43 \mathrm{E}-02$ & 159 & $2.30 \mathrm{E}-03$ & 14,500 & $2.66 \mathrm{E}-01$ & 70,200 & --- & 55,800 & $2.80 \mathrm{E}-01$ & 7.15E-09 \\
\hline A161.90.9.S170.5 & 70 & $1.61 \mathrm{E}-05$ & 11.8 & 1.164 & $2.41 \mathrm{E}-02$ & 164 & $2.06 \mathrm{E}-03$ & 12,600 & $2.01 \mathrm{E}-01$ & 70,800 & --- & 47,600 & $2.08 \mathrm{E}-01$ & $3.46 \mathrm{E}-09$ \\
\hline A161.90.9.S170.6 & 70 & $1.55 \mathrm{E}-05$ & 14.0 & 1.158 & $2.40 \mathrm{E}-02$ & 163 & $1.98 \mathrm{E}-03$ & 9,930 & $1.53 \mathrm{E}-01$ & 69,000 & --- & 40,700 & $1.71 \mathrm{E}-01$ & $9.38 \mathrm{E}-09$ \\
\hline A161.90.9.S170.7 & 70 & $1.58 \mathrm{E}-05$ & 16.0 & 1.152 & $2.39 \mathrm{E}-02$ & 175 & $2.17 \mathrm{E}-03$ & 8,990 & $1.41 \mathrm{E}-01$ & 73,800 & --- & 35,300 & $1.52 \mathrm{E}-01$ & $5.33 \mathrm{E}-09$ \\
\hline A161.90.9.S170.8 & 70 & $1.60 \mathrm{E}-05$ & 18.8 & 1.143 & $2.38 \mathrm{E}-02$ & 177 & $2.24 \mathrm{E}-03$ & 7,780 & $1.25 \mathrm{E}-01$ & 74,500 & --- & 30,700 & $1.35 \mathrm{E}-01$ & $5.04 \mathrm{E}-09$ \\
\hline A161.90.9.S170.9 & 70 & $1.55 \mathrm{E}-05$ & 21.0 & 1.139 & $2.38 \mathrm{E}-02$ & 184 & $2.25 \mathrm{E}-03$ & 6,830 & $1.06 \mathrm{E}-01$ & 73,500 & --- & 27,300 & $1.16 \mathrm{E}-01$ & $5.03 E-09$ \\
\hline \multicolumn{15}{|c|}{ A161 $90^{\circ} \mathrm{C} \mathrm{pH} 9$ Si Reactor \#4 } \\
\hline A161.90.9.20Si10.Blank2 & 10 & $0.00 \mathrm{E}+00$ & 0.0 & -- & --- & [49.4] & -- & [75.6] & --- & 8,960 & --- & [134] & --- & --- \\
\hline A161.90.9.20Si10.Blank3 & 10 & $0.00 \mathrm{E}+00$ & 0.0 & --- & --- & {$[49.4]$} & -- & 776 & --- & 9,070 & --- & [134] & --- & --- \\
\hline A161.90.9.20Si10.2 & 10 & $1.58 \mathrm{E}-05$ & 2.9 & 0.601 & $1.23 \mathrm{E}-02$ & 1,550 & $3.76 \mathrm{E}-02$ & 18,400 & $5.65 \mathrm{E}-01$ & 16,500 & $1.26 \mathrm{E}-01$ & 70,200 & $5.90 \mathrm{E}-01$ & $1.26 \mathrm{E}-08$ \\
\hline A161.90.9.20Si10.3 & 10 & $2.05 \mathrm{E}-05$ & 4.9 & 0.584 & $1.21 \mathrm{E}-02$ & 1,440 & $4.60 \mathrm{E}-02$ & 14,300 & $5.78 \mathrm{E}-01$ & 16,000 & $1.61 \mathrm{E}-01$ & 53,600 & $5.93 \mathrm{E}-01$ & $7.60 \mathrm{E}-09$ \\
\hline A161.90.9.20Si10.4 & 10 & $2.00 \mathrm{E}-05$ & 6.8 & 0.578 & $1.20 \mathrm{E}-02$ & 1,390 & $4.37 \mathrm{E}-02$ & 9,550 & $3.80 \mathrm{E}-01$ & 14,700 & $1.46 \mathrm{E}-01$ & 38,300 & $4.18 \mathrm{E}-01$ & $1.87 \mathrm{E}-08$ \\
\hline A161.90.9.20Si10.5 & 10 & 2.04E-05 & 10.0 & 0.566 & $1.18 \mathrm{E}-02$ & 1,440 & $4.68 \mathrm{E}-02$ & 6,920 & $2.85 \mathrm{E}-01$ & 14,700 & $1.51 \mathrm{E}-01$ & 27,900 & $3.14 \mathrm{E}-01$ & $1.49 \mathrm{E}-08$ \\
\hline A161.90.9.20Si10.6 & 10 & $1.95 \mathrm{E}-05$ & 11.9 & 0.565 & $1.18 \mathrm{E}-02$ & 1,440 & $4.48 \mathrm{E}-02$ & 5,170 & $2.04 \mathrm{E}-01$ & 14,200 & $1.39 \mathrm{E}-01$ & 21,000 & $2.27 \mathrm{E}-01$ & $1.15 \mathrm{E}-08$ \\
\hline A161.90.9.20Si10.7 & 10 & 2.03E-05 & 13.0 & 0.563 & $1.18 \mathrm{E}-02$ & 1,360 & $4.41 \mathrm{E}-02$ & 4,500 & $1.85 \mathrm{E}-01$ & 14,100 & 1.44E-01 & 18,700 & $2.10 \mathrm{E}-01$ & $1.28 \mathrm{E}-08$ \\
\hline A161.90.9.20Si10.8 & 10 & $1.97 \mathrm{E}-05$ & 16.9 & 0.555 & 1.17E-02 & 1,240 & $3.95 \mathrm{E}-02$ & 3,630 & $1.47 \mathrm{E}-01$ & 13,400 & $1.35 \mathrm{E}-01$ & 16,300 & $1.80 \mathrm{E}-01$ & $1.69 \mathrm{E}-08$ \\
\hline A161.90.9.20Si10.9 & 10 & $1.98 \mathrm{E}-05$ & 18.0 & 0.555 & 1.17E-02 & 1,270 & 4.05E-02 & 3,230 & $1.31 \mathrm{E}-01$ & 13,600 & 1.37E-01 & 14,200 & $1.57 \mathrm{E}-01$ & $1.34 \mathrm{E}-08$ \\
\hline \multicolumn{15}{|c|}{ A161 $90^{\circ} \mathrm{C} \mathrm{pH} 9$ Si Reactor \#5 } \\
\hline A161.90.9.20Si20.Blank2 & 20 & & -- & --. & --- & {$[49.4]$} & -- & {$[75.6]$} & --- & 18,100 & --- & [134] & --- & --- \\
\hline A161.90.9.20Si20.Blank3 & 20 & & --- & --- & --- & [49.4] & --- & {$[75.6]$} & --- & 18,000 & --- & [134] & --- & --- \\
\hline A161.90.9.20Si20.Blank1 & 20 & $1.06 \mathrm{E}-05$ & --- & --. & --- & {$[49.4]$} & --- & {$[75.6]$} & --- & 18,200 & --- & [134] & --- & --- \\
\hline A161.90.9.20Si20.1 & 20 & $1.96 \mathrm{E}-05$ & 1.9 & 0.610 & $1.24 \mathrm{E}-02$ & 745 & $2.22 \mathrm{E}-02$ & 11,400 & 4.30E-01 & 21,600 & 2.03E-01 & 42,500 & $4.38 \mathrm{E}-01$ & 4.45E-09 \\
\hline A161.90.9.20Si20.2 & 20 & $2.06 \mathrm{E}-05$ & 2.9 & 0.606 & $1.24 \mathrm{E}-02$ & 775 & $2.43 \mathrm{E}-02$ & 11,500 & 4.57E-01 & 19,600 & 1.94E-01 & 48,300 & $5.25 \mathrm{E}-01$ & $3.44 \mathrm{E}-08$ \\
\hline A161.90.9.20Si20.3 & 20 & $2.00 E-05$ & 4.9 & 0.594 & $1.22 \mathrm{E}-02$ & 812 & $2.50 \mathrm{E}-02$ & 10,800 & 4.22E-01 & 21,800 & $2.12 \mathrm{E}-01$ & 42,100 & 4.50E-01 & 1.43E-08 \\
\hline A161.90.9.20Si20.4 & 20 & $2.12 \mathrm{E}-05$ & 6.8 & 0.588 & $1.21 \mathrm{E}-02$ & 829 & $2.73 \mathrm{E}-02$ & 7,990 & $3.33 \mathrm{E}-01$ & 20,800 & $2.16 \mathrm{E}-01$ & 32,600 & $3.72 \mathrm{E}-01$ & $1.96 \mathrm{E}-08$ \\
\hline A161.90.9.20Si20.5 & 20 & $2.05 E-05$ & 10.0 & 0.577 & $1.20 \mathrm{E}-02$ & 870 & $2.81 \mathrm{E}-02$ & 6,220 & $2.54 \mathrm{E}-01$ & 21,300 & 2.17E-01 & 25,100 & $2.81 \mathrm{E}-01$ & $1.34 \mathrm{E}-08$ \\
\hline A161.90.9.20Si20.6 & 20 & $2.08 \mathrm{E}-05$ & 11.9 & 0.575 & $1.19 \mathrm{E}-02$ & 933 & $3.06 \mathrm{E}-02$ & 4,830 & $2.01 E-01$ & 21,600 & $2.24 \mathrm{E}-01$ & 20,400 & $2.32 \mathrm{E}-01$ & $1.58 \mathrm{E}-08$ \\
\hline A161.90.9.20Si20.7 & 20 & $2.12 \mathrm{E}-05$ & 13.0 & 0.574 & 1.19E-02 & 867 & $2.90 \mathrm{E}-02$ & 4,160 & $1.76 \mathrm{E}-01$ & 20,900 & $2.20 \mathrm{E}-01$ & 17,400 & $2.01 \mathrm{E}-01$ & $1.28 \mathrm{E}-08$ \\
\hline A161.90.9.20Si20.8 & 20 & $2.07 E-05$ & 16.9 & 0.565 & $1.18 \mathrm{E}-02$ & 833 & $2.74 \mathrm{E}-02$ & 3,410 & $1.42 \mathrm{E}-01$ & 20,900 & $2.17 \mathrm{E}-01$ & 13,900 & $1.59 \mathrm{E}-01$ & $8.30 \mathrm{E}-09$ \\
\hline A161.90.9.20Si20.9 & 20 & $2.02 \mathrm{E}-05$ & 18.0 & 0.566 & $1.18 \mathrm{E}-02$ & 854 & $2.74 \mathrm{E}-02$ & 2,910 & $1.18 \mathrm{E}-01$ & 20,800 & $2.11 \mathrm{E}-01$ & 12,900 & $1.44 \mathrm{E}-01$ & $1.27 \mathrm{E}-08$ \\
\hline
\end{tabular}


PNNL-26169

RPT-IGTP-013 Rev. 0.0

\section{Distribution}

No. of

Copies

OFFSITE

U.S. Department of Energy

Office of River Protection

AA Kruger

GL Pyles

Washington River Protection Solutions

EE Brown

PA Cavanah

SE Kelly

KP Lee (AREVA)

WG Ramsey

KH Subramanian

DJ Swanberg

LE Thompson

Savannah River National Laboratory

AD Cozzi

GP Flach

KM Fox

CC Herman

CM Jantzen

Vitreous State Laboratory

H Gan

W Kot

I Muller

IL Pegg

\section{Energy Solutions}

I Joseph

Intera

$\mathrm{R}$ Andrews

*All distribution will be made electronically.
No. of

Copies

ONSITE

Pacific Northwest National Laboratory

DH Bacon

VL Freedman

JJ Neeway

DK Peeler

JV Ryan

GL Smith

RM Asmussen

JD Vienna

JH Westsik, Jr

Distr. 1 


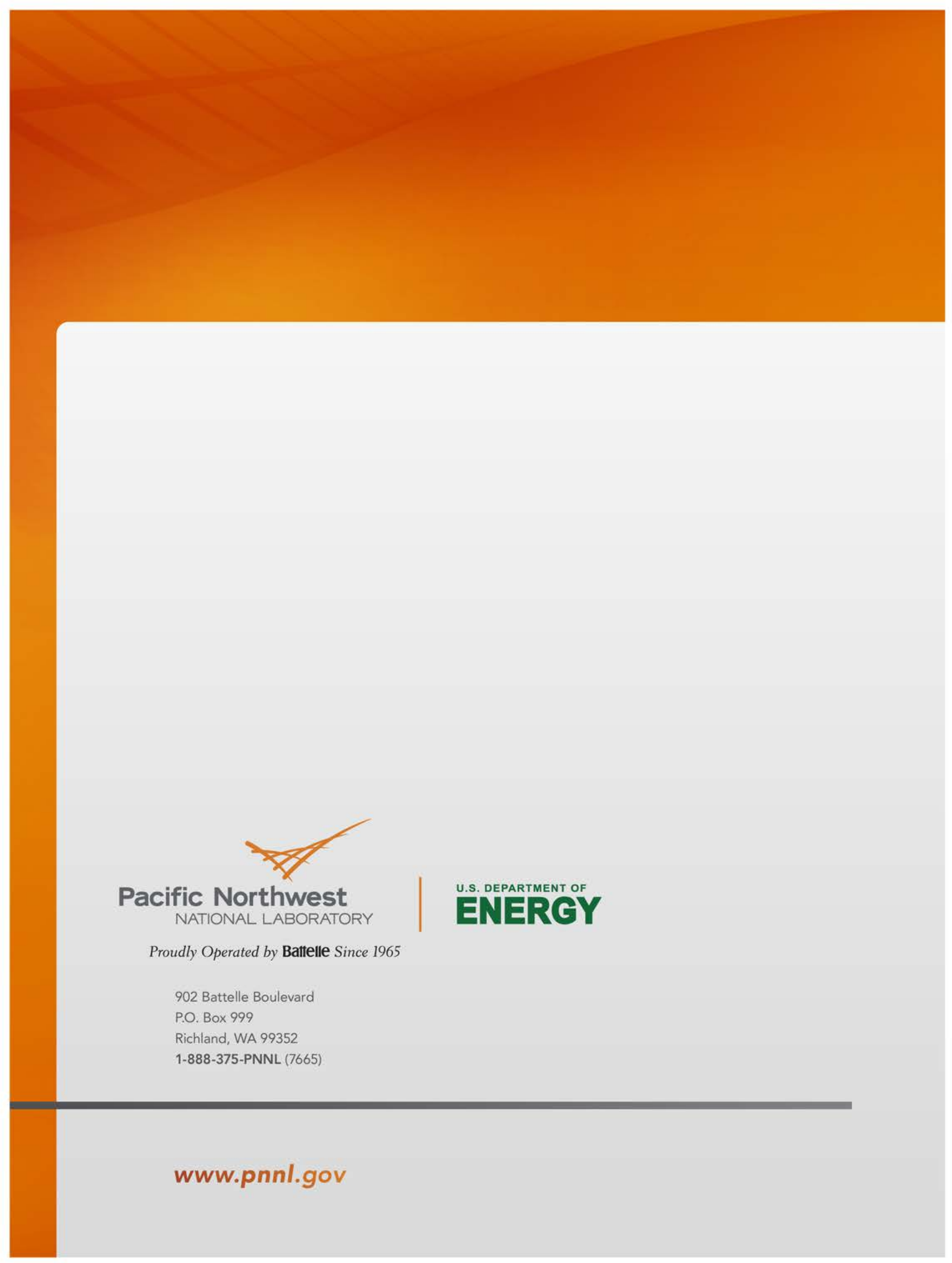

